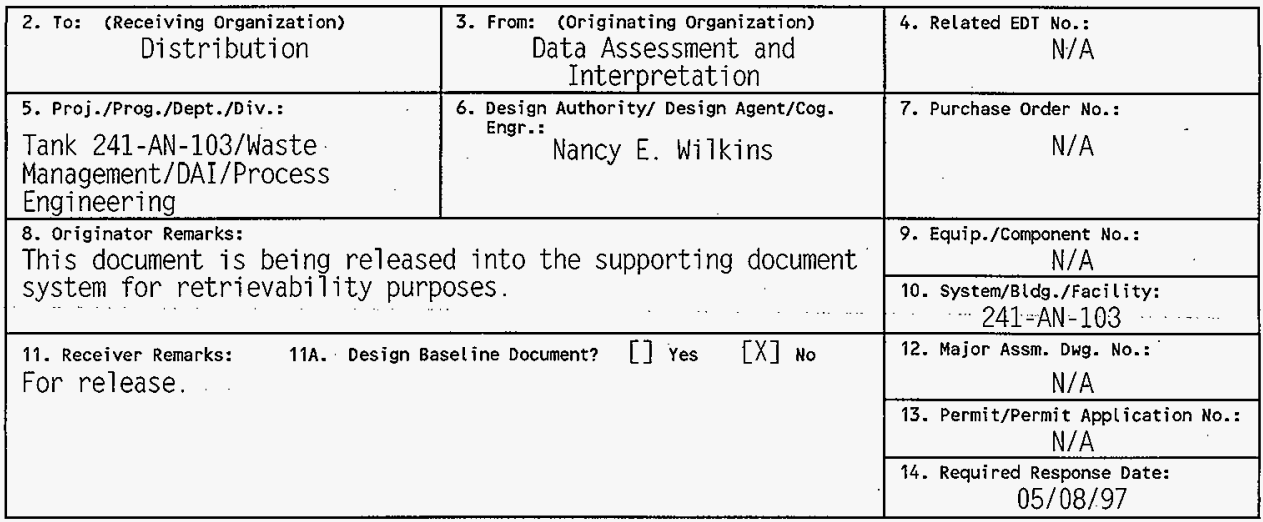

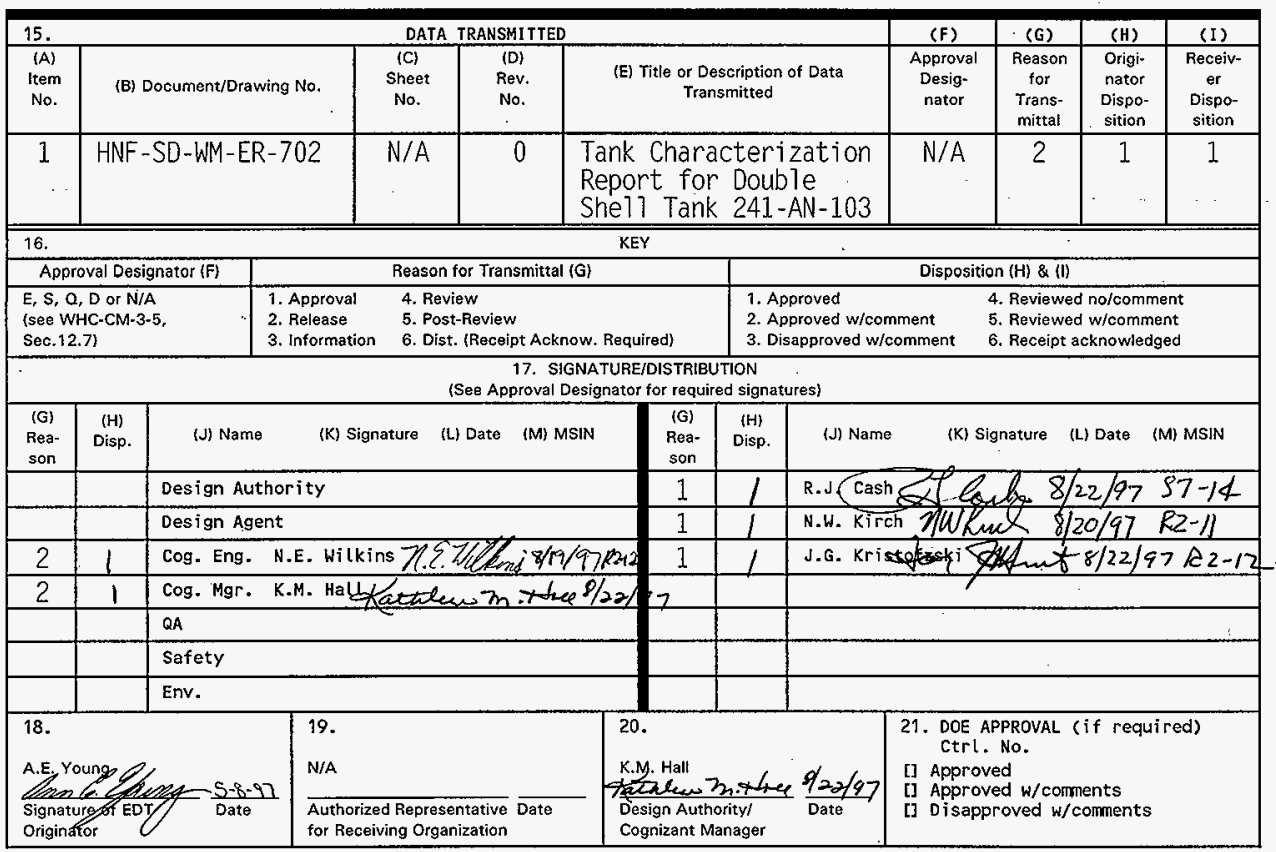

BD-7400-172-2 (05/96) GEF097 
BLOCK IITLE

(1)* EDT

12) To: (Receiving Organization)

(3) From: (Originating Organization)

(4) Related EDT No.

(5) * Proj.JPtog.JDept./Div.

(6) * Design Authority (for Design Baseline Documents)/Cognizant Engineer (for all others)/Design Agent

(7) Purchase Order No.

(8) * Otiginator Remarks

(9) Equipment/Component No.

(10) System/Bldg./Facility

(11) Receiver Remarks

(11A) Design Baseline Document

(12) Major Assm. Dwg. No.

(13) Parmit/Permit Application No.

(14) Required Response Date

(15) * Data Transmitted

(A) * Item Number

(B) * Document/Drawing No.

(C) * Sheet No.

(D)* Rev. No.

(E) Titie or Description of Data Tranemitted

[F]* Approval Designator

(G) Reason for Transmittal

(H) Originator Disposition

(i) Receiver Disposition

(16) Key

(17) Signature/Distribution

(G) Reason
(H) Disposition
(J) Name
(K)* Signature
(L)* Date
(M)* MSIN

Signature of EDT Originator

(19) Authorized Representative for Receiving Organization

(20)* Cognizant Manager

(21)* OOE Approval
- Pro-assigned EDT number.

- Enter the individual's name, titie of the organization, or entity (e.g.. Distribution) that the EDT is being transmitted to.

- Enter the title of the organization originating and transmitting the EDT.

- Enter EDT numbers which relate to the dats being transmitted.

- Enter the Project/Program/Department/Division title or Project/Program acronym or Project Number, Work Order Number or Organization Code.

- Enter the name of the individual identified as being responsible for coordinating disposition of the EDT.

- Enter related Purchase Order (P.O.) Number, if available.

- Enter special or additional comments concerning transmittal, or "Key" rotrieval words may be entered.

- Enter equipment/component number of affected item, if appropriate.

- Enter applicable system, building or facility number, if appropriate.

- Enter special or additional comments concerning transmittal.

- Enter an " $X$ " in the appropriate box. Consult with Design Authority for identification of Design Baseline Documents, if required.

- Enter applicable drawing number of major assembly, if appropriate.

- Enter applicable permit or permit application number, if appropriate.

- Enter the date a response is required from individuals identified in Block 17 (Signature/Distribution).

- Enter sequential number, beginning with 1 , of the information listed on EDT.

- Enter the unique identification number assigned to the document or drawing being transmitted.

- Enter the sheet number of the information being transmitted. If no sheet number, leave blank.

- Enter the revision number of the information being transmitted. If no revision number, leave blank.

- Enter the title of the document or drawing or a brief description of the subject if no title is identified.

- Enter the appropriate Approval Designator (Block 15). Also, indicate the appropriate approvals for each item listed, i.e., \$Q, EsQ, etc.

- Enter the appropriate code to identify the purpose of the data transmittal (see Block 16).

- Enter the appropriato disposition codo (see Block 16).

- Enter the appropriate disposition code (see Block 16).

- Number codes used in completion of Blocks 15 (G), (H), and (I), and 17 (G), (H) (Signature/Distribution).

- Enter the code of the reason for transmittal (Block 16).

- Enter the code for the disposition (Block 16).

- Enter the signature of the individual completing the Disposition $17(\mathrm{H})$ and the Transmittal.

- Obtain appropriate signature(s).

- Enter date signature is obtained.

- Enter MSIN. Note: If Distribution Sheet is used, show entire distribution (including that indicated on Page 1 of the EDT on the Distribution Sheet.

- Enter the signature and date of the individual originating the EDT lentered prior to transmittal to Receiving Organization). If the EDT originator is the Design Authority (for Design Baseline Documents)/Cognizant Engineer (for all others) or Design Agent, sign both Blocks 17 and 18 .

- Enter the signature and date of the individual identified by the Receiving Organization Design Authority (for Design Baseline Documents)/Cognizant Engineer (for all others) as authorized to approve disposition of the EDT and acceptance of the data transmitted, as applicable.

Enter the gignature and date of the cognizant manager. (This signature is authorization for release.) This signature is not required if the Design Authority is approving the document.

- Enter DOE approval (if required) by signature or control number that tracks the approval to a signature, and Indicato DOE action. 


\section{Tank Characterization Report for Double-Shell Tank 241-AN-103}

Nancy E. WiTkins

Lockheed Martin Hanford Corp. .. Richland, WA 99352

U.S. Department of Energy Contract DE-AC06-87RL10930

EDT/ECN: , EDT-617663

$\begin{array}{lll}\text { Org Code: } & 74620 & \text { Charge Code: } \\ \text { B\&R Code: } & \text { EW } 3120074 & \text { Total Pages: } 320\end{array}$

UC: 2070

Key Words: Waste Characterization, Double-Shell Tank, DST, Tank 241-AN103. Tank AN-103, AN-103, AN Farm, Tank Characterization Report, TCR, Waste Inventory, TPA Milestone M-44

Abstract: This document summarizes the information on the historical uses, present status; and the sampling and analysis results of waste stored in Tank 241-AN-103. This report supports the requirements of the Tri-Party Agreement Milestone M-44-10.

TRADEMARK DISCLAIMER. Reference herein to any specific comercial product, process, or service by trade name, trademark, manufacturer, or otherwise, does not necessarily constitute or imply its endorsement, recommendation, or favoring by the United States Government or any agency thereof or its contractors or subcontractors.

Printed in the United States of America. To obtain copies of this document, contact: WHC/BCS Document Control Services, P.O. Box 1970, Mailstop H6-08, Richland WA 99352, Phone (509) 372-2420; Fax (509) 376-4989.
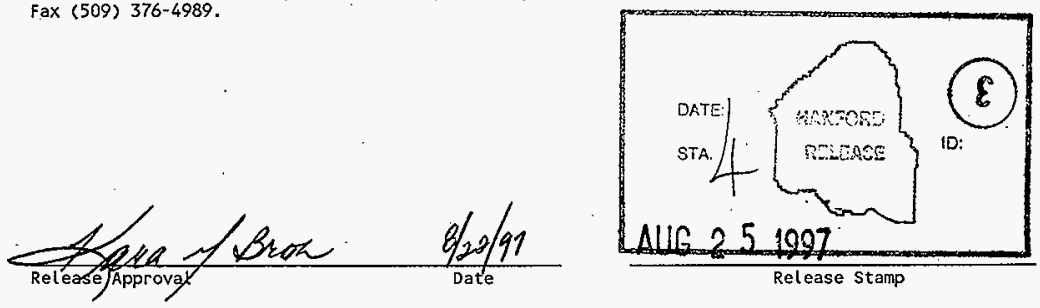

Approved for Public Release 


\title{
Tank Characterization Report for Double-Shell Tank 241-AN-103
}

\author{
N. E. Wilkins \\ J. G. Field \\ L. W. Shelton \\ Lockheed Martin Hanford Corporation \\ T. L. Welsch \\ B\&W Protec, Inc.
}

Date Published

August 1997

Prepared for the U.S. Department of Energy

Assistant Secretary for Environmental Management

Project Hanford Management Contractor for the

U.S. Department of Energy under Contract DE-ACO6-96RL13200

Approved for public release; distribution is unlimited. 


\section{CONTENTS}

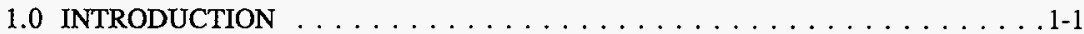

1.1 SCOPE . . . . . . . . . . . . . . . . . . . . . .1-1

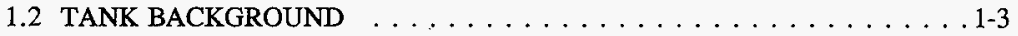

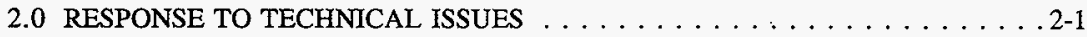

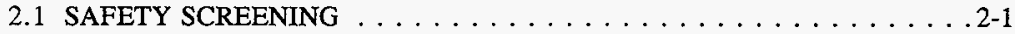

2.1.1 Exothermic Conditions (Energetics) $\ldots \ldots \ldots \ldots \ldots \ldots \ldots .2-2$

2.1.2 Flammable Gas . . . . . . . . . . . . . . . . . 2-2

2.1 .3 Criticality $\ldots \ldots \ldots \ldots \ldots \ldots \ldots \ldots \ldots \ldots \ldots \ldots \ldots \ldots \ldots \ldots \ldots .2-2$

2.2 FLAMMABLE GAS TANK SAFETY EVALUATION . . . . . . . 2-3

2.3 ORGANIC SOLVENTS SCREENING $\ldots \ldots \ldots \ldots \ldots \ldots \ldots . \ldots \ldots$

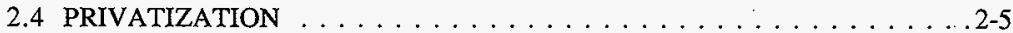

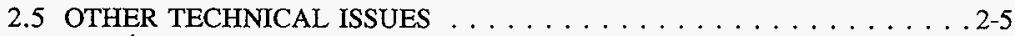

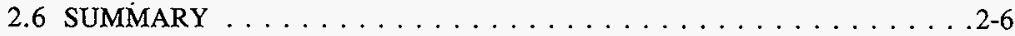

3.0 BEST BASIS STANDARD INVENTORY ESTIMATE $\ldots \ldots \ldots \ldots \ldots \ldots$ 3-1

4.0 RECOMMENDATIONS $\ldots \ldots \ldots \ldots \ldots \ldots \ldots \ldots \ldots \ldots \ldots$. $\ldots \ldots \ldots$

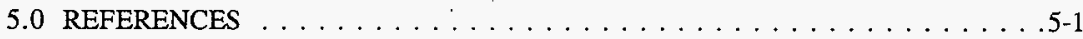

APPENDIXES

APPENDIX A: HISTORICAL TANK INFORMATION $\ldots \ldots \ldots \ldots \ldots \ldots$ A-1

A1.0 CURRENT TANK STATUS $\ldots \ldots \ldots \ldots \ldots \ldots \ldots \ldots \ldots$ A-3

A2.0 TANK DESIGN AND BACKGROUND $\ldots \ldots \ldots \ldots \ldots \ldots \ldots$ A-4

A3.0 PROCESS KNOWLEDGE . . . . . . . . . . . . . . . . . A-9

A3.1 WASTE TRANSFER HISTORY $\ldots \ldots \ldots \ldots \ldots \ldots \ldots$ A-9

A3.2 HISTORICAL ESTIMATION OF TANK CONTENTS . . . . . . A-11

A4.0 SURVEILLANCE DATA $\ldots \ldots \ldots \ldots \ldots \ldots \ldots \ldots \ldots \ldots \ldots$ A-17

A4.1 SURFACE LEVEL READINGS $\ldots \ldots \ldots \ldots \ldots \ldots \ldots \ldots$ A-17

A4.2 INTERNAL TANK TEMPERATURES $\ldots \ldots \ldots \ldots \ldots \ldots \ldots \ldots$ A-17

A4.3 TANK 241 -AN-103 VIDEO . . . . . . . . . . . . A-18

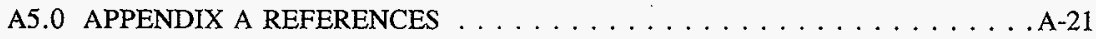




\section{TAELE OF CONTENTS (Continued)}

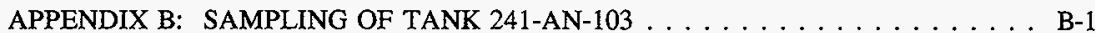

B1.0 TANK SAMPLING OVERVIEW $\ldots \ldots \ldots \ldots \ldots \ldots \ldots$ B-3

B2.0 SAMPLING EVENTS $\ldots \ldots \ldots \ldots \ldots \ldots \ldots \ldots \ldots \ldots$ B-4

B2.1 1996 PUSH CORE SAMPLE $\ldots \ldots \ldots \ldots \ldots \ldots \ldots \ldots \ldots$ B-4

B2.1.1 SAMPLE HANDLING $\ldots \ldots \ldots \ldots \ldots \ldots \ldots \ldots$

B2.1.2 SAMPLE ANALYSIS $\ldots \ldots \ldots \ldots \ldots \ldots \ldots$ B-18

B2.1.3 ANALYTICAL RESULTS . . . . . . . . . . . . . B-29

B2.2 VAPOR PHASE MEASUREMENT $\ldots \ldots \ldots \ldots \ldots \ldots \ldots \ldots$. . . . . . .

B2.3 HISTORICAL SAMPLES . . . . . . . . . . . . . . . . . . . B-34

B3.0 ASSESSMENT OF CHARACTERIZATION RESULTS . . . . . . . . . . . B-189

B3.1 FIELD OBSERVATIONS . . . . . . . . . . . . . . . . . . . . . . B-189

B3.2 QUALITY CONTROL ASSESSMENT . . . . . . . . . . . . B B-189

B3.3 DATA CONSISTENCY CHECKS . . . . . . . . . . . . . B-191

B3.3.1 Comparison of Results from Different Analytical Methods ... B-191

B3.3.2 Mass and Charge Balance . . . . . . . . . . . . . B-192

B3.4 Mean Concentrations and Confidence Intervals . . . . . . . . . . . B-196

B3.4.1 Mean Concentrations . . . . . . . . . . . . . . B-197

B3.4.2 Analysis of Variance Model . . . . . . . . . . . . B-206

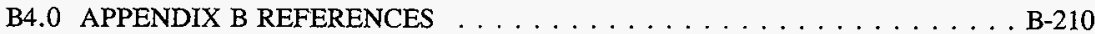

APPENDIX C: STATISTICAL ANALYSIS FOR ISSUE RESOLUTION $\ldots \ldots \ldots$ C-1

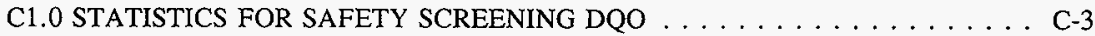

C2.0 APPENDIX C REFERENCES $\ldots \ldots \ldots \ldots \ldots \ldots \ldots \ldots \ldots$ C-9

APPENDIX D: EVALUATION TO ESTABLISH BEST-BASIS INVENTORY FOR DOUBLE-SHELL TANK $241-A N-103 \ldots \ldots \ldots \ldots \ldots$ D-1

D1.0 CHEMICAL INFORMATION SOURCES $\ldots \ldots \ldots \ldots \ldots \ldots \ldots \ldots$ D-3

D2.0 COMPARISON OF COMPONENT INVENTORY VALUES $\ldots \ldots \ldots \ldots \ldots$ D-3

D3.0 COMPONENT INVENTORY EVALUATION $\ldots \ldots \ldots \ldots \ldots \ldots$ D-8

D4.0 DEFINE THE BEST-BASIS AND ESTABLISH COMPONENT

INVENTORIES 


\section{TABLE OF CONTENTS (Continued)}

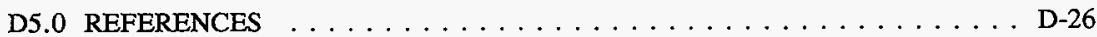
APPENDIX E: BIBLIOGRAPHY FOR TANK $241-A N-103 \ldots \ldots \ldots \ldots$ E-1

\section{LIST OF FIGURES}

A2-1 Riser Configuration for Tank $241-\mathrm{AN}-103 \ldots \ldots \ldots \ldots \ldots$ A .6 . . . . .

A2-2 Tank 241-AN-103 Cross Section and Schematic . . . . . . . . . . . . A-8

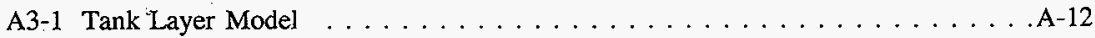

A4-1 Tank 241-AN-103 Level History . . . . . . . . . . . . . . . . . . . . A-19

A4-2 Tank 241-AN-103 Weekly High Temperature Plot . . . . . . . . . . . . . A-20 


\section{LIST OF TABLES}

1-1 Summary of Recent Sampling $\ldots \ldots \ldots \ldots \ldots \ldots \ldots \ldots \ldots \ldots \ldots \ldots \ldots$

1-2 Description of Tank $241-\mathrm{AN}-103 \ldots \ldots \ldots \ldots \ldots$. . . . . . . . . . . . . .

2-1 Heat Load for Tank 241-AN-103 Based on Radionuclide Content . . . . . . . 2-6

2-2 Summary of Safety Screening, Organic Solvents Screening and Flammable Gas Results . . . . . . . . . . . . . . . . . . . . 2-7

3-1 Best-Basis Total Inventory Estimates for Nonradioactive Components in Tank 241-AN-103 as of May 31, 1997. . . . . . . . . . . . . . 3-2

3-2 Best-Basis Total Inventory Estimates for Nonradioactie Components in Tank 241-AN-103 as of May 31, $1997 \ldots \ldots \ldots \ldots$. . . . . . . . . . . 3-4

4-1 Acceptance of Tank 241-AN-103 Sampling and Analysis . . . . . . . . . . 4-1

4-2 Acceptance of Evaluation of Characterization Data and Information for Tank $241-\mathrm{AN}-103 \ldots \ldots \ldots \ldots$. . . . . . . . . . . . . . . . .

A1-1 Tank Contents Status Summary $\ldots \ldots \ldots \ldots \ldots \ldots$ A-4

A2-1 Tank 241-AN-103 Risers . . . . . . . . . . . . . . . A-7

A3-1 Tank 241-AN-103 Major Waste Transfers . . . . . . . . . . . . . . A-10

A3-2 Historical Tank Inventory Estimate Analytes $\ldots \ldots \ldots \ldots \ldots \ldots$ A-13

A3-3 Historical Tank Inventory Estimate Radionuclides . . . . . . . . . . . . A-15

B1-1 Integrated Data Quality Objective Requirements for Tank 241-AN-103 . . . . B B-4

B2-1a Sample Receipt and Extrusion Information for Tank 241-AN-103, Core $166 \ldots$ B-6

B2-1b Sample Receipt and Extrusion Information for Tank 241-AN-103, Core 167 . . B-12

B2-2 Tank 241-AN-103 Sample Analysis Summary . . . . . . . . . . . . . . B-19

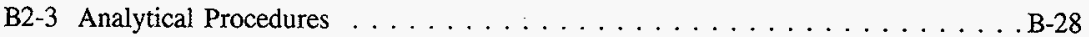

B2-4 Analytical Presentation Tables . . . . . . . . . . . . . . . . . B-29 


\section{LIST OF TABLES (Continued)}

B2-5 Tank 241-AN-103 Analytical Results: Total Alpha (Alpha Rad) . . . . . . . . B-34 B2-6 Tank 241-AN-103 Analytical Results: Total Beta . . . . . . . . . . . . B-36 B2-7 Tank 241-AN-103 Analytical Results: Strontium-89/90 . . . . . . . . . . B-37 B2-8 Tank 241-AN-103 Analytical Results: Americium-241 (GEA) $\ldots \ldots \ldots \ldots$. B-37 B2-9 Tank 241-AN-103 Analytical Results: Cesium-137 (GEA) . . . . . . . . . B-37 B2-10 Tank 241-AN-103 Analytical Results: Cobalt-60 (GEA) . . . . . . . . . . . B B-37 B2-11 Tank 241-AN-103 Analytical Results: Europium-154 (GEA) . . . . . . . . . B-38 B2-12 Tank 241-AN-103 Analytical Results: Europium-155 (GEA) . . . . . . . . . . B-38 B2-13 Tank 241-AN-103 Analytical Results: Iodine-129 . . . . . . . . . . . B-38 B2-14 Tank 241-AN-103 Analytical Results: Technetium-99 . . . . . . . . . . . . B-39 B2-15 Tank 241-AN-103 Analytical Results: Americium-241 . . . . . . . . . . . B-39 B2-16 Tank 241-AN-103 Analytical Results: Curium-243/244 . . . . . . . . . . . B-39 B2-17 Tank 241-AN-103 Analytical Results: Plutonium-239/240 . . . . . . . . . B-39 B2-18 Tank 241-AN-103 Analytical Results: Tritium (Liquid Scintillation) . . . . . B-39 B2-19 Tank 241-AN-103 Analytical Results: Percent Water (TGA) . . . . . . . . . B-41 B2-20 Corrected Thermogravimetric Analysis Measurements . . . . . . . . B-43

B2-21 Tank 241-AN-103 Analytical Results: Exotherm (Differential Scanning Calorimetry) . . . . . . . . . . . . . . . . . . B-44

B2-22 Tank 241-AN-103 Analytical Results: Bulk density . . . . . . . . . . . B-44

B2-23 Tank 241-AN-103 Analytical Results: Specific Gravity . . . . . . . . . . . . B-46 B2-24 Tank 241-AN-103 Analytical Results: Aluminum (ICP) . . . . . . . . . . . B-47 B2-25 Tank 241-AN-103 Analytical Results: Antimony (ICP) $\ldots \ldots \ldots \ldots$. . . . B-51 


\section{LIST OF TABLES (Continued)}

B2-26 Tank 241-AN-103 Analytical Results: Beryllium (ICP) $\ldots \ldots \ldots \ldots$. . . B-55

B2-27 Tank 241-AN-103 Analytical Results: Boron (ICP) $\ldots \ldots \ldots \ldots \ldots \ldots$. . . . .59

B2-28 Tank 241-AN-103 Analytical Results: Cadmium (ICP) $\ldots \ldots \ldots \ldots \ldots$. . B-63

B2-29 Tank 241-AN-103 Analytical Results: Calcium (ICP) . . . . . . . . . . . B-67

B2-30 Tank 241-AN-103 Analytical Results: Chromium (ICP) . . . . . . . . . . . B-71

B2-31 Tank 241-AN-103 Analytical Results: Cobalt (ICP) . . . . . . . . . . B-75

B2-32 Tank 241-AN-103 Analytical Results: Copper (ICP) . . . . . . . . . . . . . B-79

B2-33 Tank 241-AN-103 Analytical Results: Iron (ICP) . . . . . . . . . . . . B-83

B2-34 Tank 241-AN-103 Analytical Results: Lanthanum (ICP) . . . . . . . . . . . B-87

B2-35 Tank 241-AN-103 Analytical Results: Lead (ICP) . . . . . . . . . . . B-91

B2-36 Tank 241-AN-103 Analytical Results: Lithium (ICP) $\ldots \ldots \ldots \ldots \ldots$. B-95

B2-37 Tank 241-AN-103 Analytical Results: Manganese (ICP) . . . . . . . . . . . B-99

B2-38 Tank 241-AN-103 Analytical Results: Molybdenum (ICP) . . . . . . . . . B-103

B2-39 Tank 241-AN-103 Analytical Results: Nickel (ICP) . . . . . . . . . . . . B-107

B2-40 Tank 241-AN-103 Analytical Results: Phosphorous (ICP) $\ldots \ldots \ldots \ldots$. . B-111

B2-41 Tank 241-AN-103 Analytical Results: Potassium (ICP) $\ldots \ldots \ldots$. . . B-115

B2-42 Tank 241-AN-103 Analytical Results: Selenium (ICP) . . . . . . . . . B B-118

B2-43 Tank 241-AN-103 Analytical Results: Silicon (ICP) . . . . . . . . . B-122

B2-44 Tank 241-AN-103 Analytical Results: Silver (ICP) . . . . . . . . . . . B-126

B2-45 Tank 241-AN-103 Analytical Results: Sodium (ICP) . . . . . . . . . . . . B-130

B2-46 Tank 241-AN-103 Analytical Results: Sulfur (ICP) $\ldots \ldots \ldots \ldots \ldots$ B-134 


\section{LIST OF TABLES (Continued)}

B2-47 Tank 241-AN-103 Analytical Results: Titanium (ICP) . . . . . . . . . . B-138

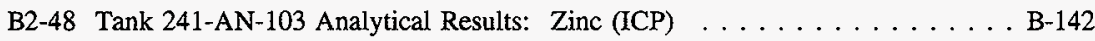

B2-49 Tank 241-AN-103 Analytical Results: Zirconium (ICP) . . . . . . . . . . B-146

B2-50 Tank 241-AN-103 Inductively Coupled Plasma Results with

Less Than Value . . . . . . . . . . . . . . . . . B-150

B2-51 Tank 241-AN-103 Analytical Results: Bromide (IC) . . . . . . . . . . . B B-151

B2-52 Tank 241-AN-103 Analytical Results: Chloride (IC) . . . . . . . . . . . B-153

B2-53 Tank 241-AN-103 Analytical Results: Fluoride (IC) . . . . . . . . . . . B-156

B2-54 Tank 241-AN-103 Analytical Results: Formate (IC) . . . . . . . . . . . . B-159

B2-55 Tank 241-AN-103 Analytical Results: Nitrate (IC) . . . . . . . . . . . . B-162

B2-56 Tank 241-AN-103 Analytical Results: Nitrite (IC) $\ldots \ldots \ldots \ldots \ldots$. . . B-165

B2-57 Tank 241-AN-103 Analytical Results: Phosphate (IC) . . . . . . . . . . B-168

B2-58 Tank 241-AN-103 Analytical Results: Sulfate (IC) . . . . . . . . . . . . B-171

B2-59 Tank 241-AN-103 Analytical Results: Acetate (IC) . . . . . . . . . . . . B-174

B2-60 Tank 241-AN-103 Analytical Results: Oxalate (IC) . . . . . . . . . . . B-177

B2-61 Tank 241-AN-103 Analytical Results: Total Uranium . . . . . . . . . . . B-179

B2-62 Tank 241-AN-103 Analytical Results: Hydroxide . . . . . . . . . . . . B-179

B2-63 Tank 241-AN-103 Analytical Results: Hexavalent Chromium $(\mathrm{Cr}+6) \ldots$. . B-180

B2-64 Tank 241-AN-103 Analytical Results: Total Organic Carbon . . . . . . . . . B-180

B2-65 Tank 241-AN-103 Analytical Results: Total Inorganic Carbon . . . . . . . . B-180

B2-66 Tank 241-AN-103 Vapor Sampling Results $\ldots \ldots \ldots \ldots \ldots \ldots \ldots$ B-181

B2-67 Composition of Sludge Sample $\mathrm{R}-8190 \ldots \ldots \ldots \ldots \ldots$. . . . . . . . . . . . 


\section{LIST OF TABLES (Continued)}

B2-68 Main Constituents of Slurry Samples Taken During Campaign 86-2 . . . . . B B-184

B2-69 Inorganic Analyses of 241-AN-103 Core Composite $\ldots \ldots \ldots \ldots \ldots$ B-185

B2-70 Radiochemical Analyses of Tank 241-AN-103 . . . . . . . . . . . . B-187

B3-1 Phosphate versus Phosphorous. . . . . . . . . . . . . . B-190

B3-2 Sulfate versus Sulfur $\ldots \ldots \ldots \ldots \ldots \ldots \ldots \ldots \ldots$. . . . . . . . . . . .

B3-3 Supernatant Cation Mass and Charge Data . . . . . . . . . . . . . . B-192

B3-4 Supernatant Anion Mass and Charge Data . . . . . . . . . . . . . . . . B-193

B3-5 Mass Balance Totals . . . . . . . . . . . . . . . . . . . . B-193

B3-6 Salt Slurry Cation Mass and Charge Data . . . . . . . . . . . . . B-194

B3-7 Salt Slurry Anion Mass and Charge Data . . . . . . . . . . . . . . . B-194

B3-8 Mass Balance Totals . . . . . . . . . . . . . . . . . . . . B-195

B3-9 Summary Statistics: Convective Layer - Liquid Data Only (one-way ANOVA) . . . . . . . . . . . . . . . . . . . . . . . . . B-197

B3-10 Summary Statistics: Convective Layer-Calculated . . . . . . . . . . . . . B-198

B3-11 Summary Statistics: Liquid Composite (Core 166, segments 3-12) . . . . . B-200

B3-12 Summary Statistics: Nonconvective Layer (Slurry) (nested ANOVA

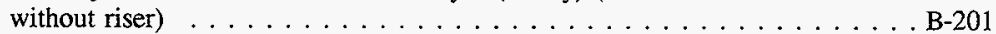

B3-13 Summary Statistics: Slurry Composite (Core 166, segments 1 to 19) . . . . B-202

B3-14 Summary Statistics: Crust Layer (nested ANOVA without the riser term) . . . B-204

C1-1 Tank 241-AN-103 Differential Scanning Calorimetry Results . . . . . . . C-4

C1-2 Tank 241-AN-103 Total Alpha Results . . . . . . . . . . . . . C-7

D2-1 Sampling and Hanford Defined Waste Model Inventory Estimates for Nonradioactive Components in Double-Shell Tank 241-AN-103 . . . . . . . D-5 


\section{LIST OF TABLES (Continued)}

D2-2 Sampling and Hanford Defined Waste Model Inventory Estimates for Radioactive Components in Double-Shell Tank 241-AN-103 . . . . . . . D-6

D3-1 Comparison of September 1996 Push Core Sample Concentrations . . . . . . . D-9

D4-1 Best-Basis Inventory Estimates for Nonradioactive Components in Tank 241-AN-103 Supernatant as of May 31, $1997 \ldots \ldots \ldots \ldots$ D-13

D4-2 Best-Basis Inventory Estimates for Radioactive Components in Tank 241-AN-103 Supernatant as of May 31, 1997

D4-3 Best-Basis Inventory Estimates for Nonradioactive Components in Tank 241-AN-103 Salt Slurry as of May $31,1997 \ldots \ldots \ldots \ldots$. . . . . . . . . .

D4-4 Best-Basis Inventory Estimates for Radioactive Components in Tank 241-AN-103 Salt Slurry as of May 31, $1997 \ldots \ldots \ldots \ldots$ D-18 . . . . . .

D4-5 Best-Basis Inventory Estimates for Nonradioactive Components in Tank 241-AN-103 Crust as of May 31, $1997 \ldots \ldots \ldots \ldots$. . . . . . . . . . . .

D4-6 Best-Basis Inventory Estimates for Radioactive Components in Tank 241-AN-103 Crust as of May 31, $1997 \ldots \ldots \ldots \ldots$ D-21 . . . . . . . .

D4-7 Best-Basis Total Inventory Estimates for Nonradioactive Components in Tank 241-AN-103 as of May 31, $1997 \ldots \ldots \ldots \ldots \ldots$. . . . . . . . . . . . .

D4-8 Best-Basis Inventory Estimates for Radioactive Components in Tank 241-AN-103 as of May $31,1997 \ldots \ldots \ldots \ldots$ D-24 


\section{LIST OF TERMS}

$1 \mathrm{C}$

AEA

ANOVA

AT

$\mathrm{Btu} / \mathrm{hr}$

$\mathrm{Ci}$

$\mathrm{Ci} / \mathrm{L}$

CI

$\mathrm{cm}$

$\mathrm{cm} / \mathrm{s}$

$\mathrm{cP}$

DQO

DSC

DSSF

DSS

FIC

$\mathrm{ft}$

GEA

$\mathrm{g}$

$\mathrm{g} / \mathrm{cc}$

$\mathrm{g} / \mathrm{L}$

$\mathrm{g} / \mathrm{mL}$

HDW

HTCE

IC

ICP

in.

$\mathrm{J} / \mathrm{g}$

$\mathrm{kg}$

kgal

$\mathrm{kL}$

$\mathrm{kW}$

LANL

LAW

LEL

LFL

LL

m

$M$

$\mathrm{M}^{3}$

mig

MIT first cycle decontamination waste

alpha energy analysis

analysis of variance

alpha total

British thermal units per hour

curies

curies per liter

confidence interval

centimeter

centimeters per second

centipoise

data quality objectives

differential scanning calorimetry

double-shell slurry feed

double-sheil slurry

Food Instrument Corporation

feet

gramma energy analysis

gram

grams per cubic centimeter

grams per liter

grams per milliliter

Hanford defined waste

historical tank content estimate

ion chromatography

inductively coupled plasma spectroscopy

inch

joules per gram

kilogram

kilogallon

kiloliter

kilowatt

Los Alamos National Laboratory

low activity waste

lower explosive limit

lower flammability limit

lower limit

meter

moles per liter

cubic meters

milligram

Multifunctional Instrument Tree 


\section{LIST OF TERMS (Continued)}

\begin{tabular}{|c|c|}
\hline $\mathrm{mL}$ & milliliter \\
\hline $\mathrm{mm}$ & millimeter \\
\hline mol\% & mole percent \\
\hline NA & not available \\
\hline $\mathrm{n} / \mathbf{a}$ & not applicable \\
\hline $\mathrm{n} / \mathrm{d}$ & not detected \\
\hline ND & not determined \\
\hline NR & not reported \\
\hline PHMC & Project Hanford Management Contractor \\
\hline ppm & parts per million \\
\hline ppmv & parts per million volume \\
\hline QC & quality control \\
\hline REML & restricted maximum likelihood estimation methods \\
\hline RGS & retained gas sampler \\
\hline RPD & relative percent difference \\
\hline SACS & Surveiliance Analysis Computer System \\
\hline SAP & sampling and analysis plan \\
\hline Seg. & segment \\
\hline SMM & supernatant mixing model \\
\hline STP & standard temperature and pressure \\
\hline TB & total beta \\
\hline $\mathrm{TCD}$ & Tank Characterization Database \\
\hline TCP & tank characterization plan \\
\hline TCR & tank characterization report \\
\hline TGA & thermogravimetric analysis \\
\hline $\mathrm{TIC}$ & total inorganic carbon \\
\hline TLM & tank layer model \\
\hline TOC & total organic carbon \\
\hline TSAP & Tank Sampling and Analysis Plan \\
\hline TWRS & Tank Waste Remediation System \\
\hline UL & upper limit \\
\hline VFI & void fraction instrument \\
\hline W & watts \\
\hline $\mathrm{W} / \mathrm{Ci}$ & watts per curies \\
\hline WSTRS & waste status and transaction record summary \\
\hline wt\% & weight percent \\
\hline${ }^{\circ} \mathrm{C}$ & degrees Centigrade \\
\hline${ }^{\circ} \mathrm{F}$ & degrees Fahrenheit \\
\hline
\end{tabular}


HNF-SD-WM-ER-702 Rev. 0

\section{LIST OF TERMS (Continued)}

$\begin{array}{ll}\mu \mathrm{Ci} / \mathrm{g} & \text { microcuries per gram } \\ \mu \mathrm{Ci} / \mathrm{mL} & \text { microcuries per milliliter } \\ \mu \mathrm{eq} / \mathrm{g} & \text { microequivalents per gram } \\ \mu \mathrm{g} / \mathrm{mL} & \text { micrograms per milliliter } \\ \mu \mathrm{g} / \mathrm{g} & \text { micrograms per gram } \\ \mu \mathrm{mole} / \mathrm{g} & \text { micromole per gram }\end{array}$




\subsection{INTRODUCTION}

A major function of the Tank Waste Remediation System (TWRS) is to characterize wastes in support of waste management and disposal activities at the Hanford Site. Analytical data from sampling and analysis, along with other available information about a tank, are compiled and maintained in a tank characterization report (TCR). This report and its appendixes serve as the TCR for double-shell tank 241-AN-103.

The objectives of this report are: 1) to use characterization data in response to technical issues associated with tank 241-AN-103 waste; and 2) to provide a standard characterization of this waste in terms of a best-basis inventory estimate. The response to technical issues is summarized in Section 2.0, and the best-basis inventory estimate is presented in Section 3.0. Recommendations regarding safety status and additional sampling needs are provided in Section 4.0. Supporting data and information are contained in the appendixes. This report also supports the requirements of the Hanford Federal Facility Agreement and Consent Order (Ecology et al. 1996) milestone M-44-10.

\subsection{SCOPE}

Characterization information presented in this report originated from sample analyses and known historical sources. While only the results of recent sample events will be used to fulfill the requirements of the data quality objectives (DQOs), other information can be used to support (or question) conclusions derived from these results. Historical information for tank 241-AN-103, provided in Appendix A, includes surveillance information, records pertaining to waste transfers and tank operations, and expected tank contents derived from a process knowledge model. The recent sampling events listed in Table 1-1, as well as sample data obtained before 1989, are summarized in Appendix B. The results of the 1996 sampling event, also reported in the laboratory data package (Steen 1997), satisfied the data requirements specified in the tank characterization plan (TCP) for this tank (Kruger and Winkelman 1996). The statistical analysis of data used in issue resolution are reported in Appendixes B and C. Appendix D contains the evaluation to establish the best basis for the inventory estimate. A bibliography that resulted from an in-depth literature search of all known information sources applicable to tank 241-AN-103 and its respective waste types is contained in Appendix E. 


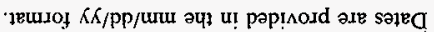
. ә

:solon

\begin{tabular}{|c|c|c|c|c|}
\hline $\mathrm{E} / \mathrm{U}$ & $\mathrm{e} / \mathrm{U}$ & 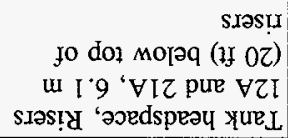 & SED & 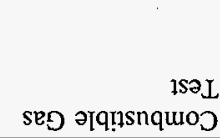 \\
\hline นuอวrอd 00 L - $6 L$ & 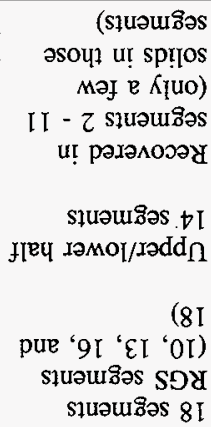 & VIZ Iəs!̣ & $\begin{array}{l}\text { punbĩ } \\
\text { /p!̣os }\end{array}$ & 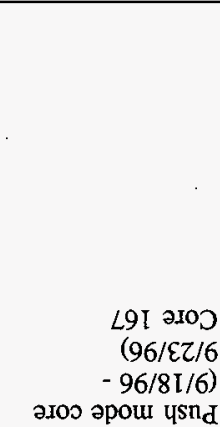 \\
\hline fuəəدəd 00I - 6L & 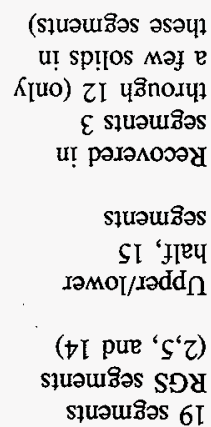 & 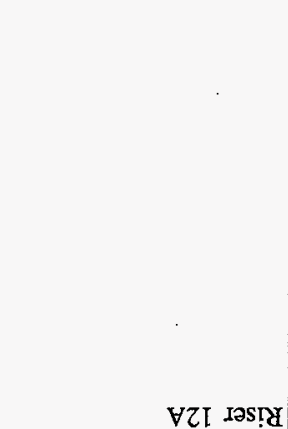 & $\begin{array}{l}\text { p!Ios } \\
\text { punbị } \\
\text { jpı̣os }\end{array}$ & 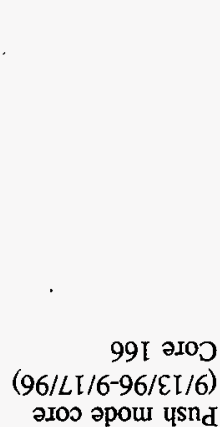 \\
\hline 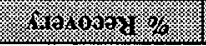 & gom & 17ingens & 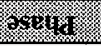 & ojmproldinis: \\
\hline
\end{tabular}

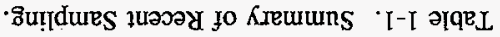




\subsection{TANK BACKGROUND}

Tank 241-AN-103 is located in the 200 East Area AN Tank Farm on the Hanford Site. The tank went into service in the first quarter of 1982, receiving raw water. The tank received non-complexed waste from tank 241-SY-102 and water from miscellaneous sources during the fourth quarter of 1982 . Waste was sent to tank 241-AW-102 from tank 241-AN-103 during the fourth quarter of 1982 and the first quarter of 1983. Non-complexed waste from the 300 and 400 Areas was sent to tank 241-AN-103 during the third quarter of 1983. In the third quarter of 1983 and 1984, the tank received liquid from the salt well pumping of several single-shell tanks in the 200 East and West Areas. The tank also received non-complexed waste from B Plant cesium processing during the first quarter of 1984. Non-complexed waste from 241-AN-104 and water from miscellaneous sources was sent to tank 241-AN-103 during the third quarter of 1984. Tank 241-AN-103 sent waste to tank 241-AN-101 during the fourth quarter of 1984. Tank 241-AN-103 exchanged non-complexed waste with tank 241-AW-102 (the 242-A Evaporator feed tank) during the first and fourth quarters of 1984 and the first quarter of 1986 . No further waste transfers have involved tank 241-AN-103 from the third quarter of 1986 to the present.

A description of tank 241-AN-103 is summarized in Table 1-2. The tank has an operating capacity of 4,390 kL $(1,160 \mathrm{kgal})$, and presently contains an estimated $3,615 \mathrm{~kL}$ (955 kgal) of double-shell slurry waste (Hanlon 1997) made up of three layers: a floating crust, a convective layer, and a non-convective layer. The tank is on the Flammable Gas Watch List (Public Law 101-510). 
Table 1-2. Description of Tank 241-AN-103.,

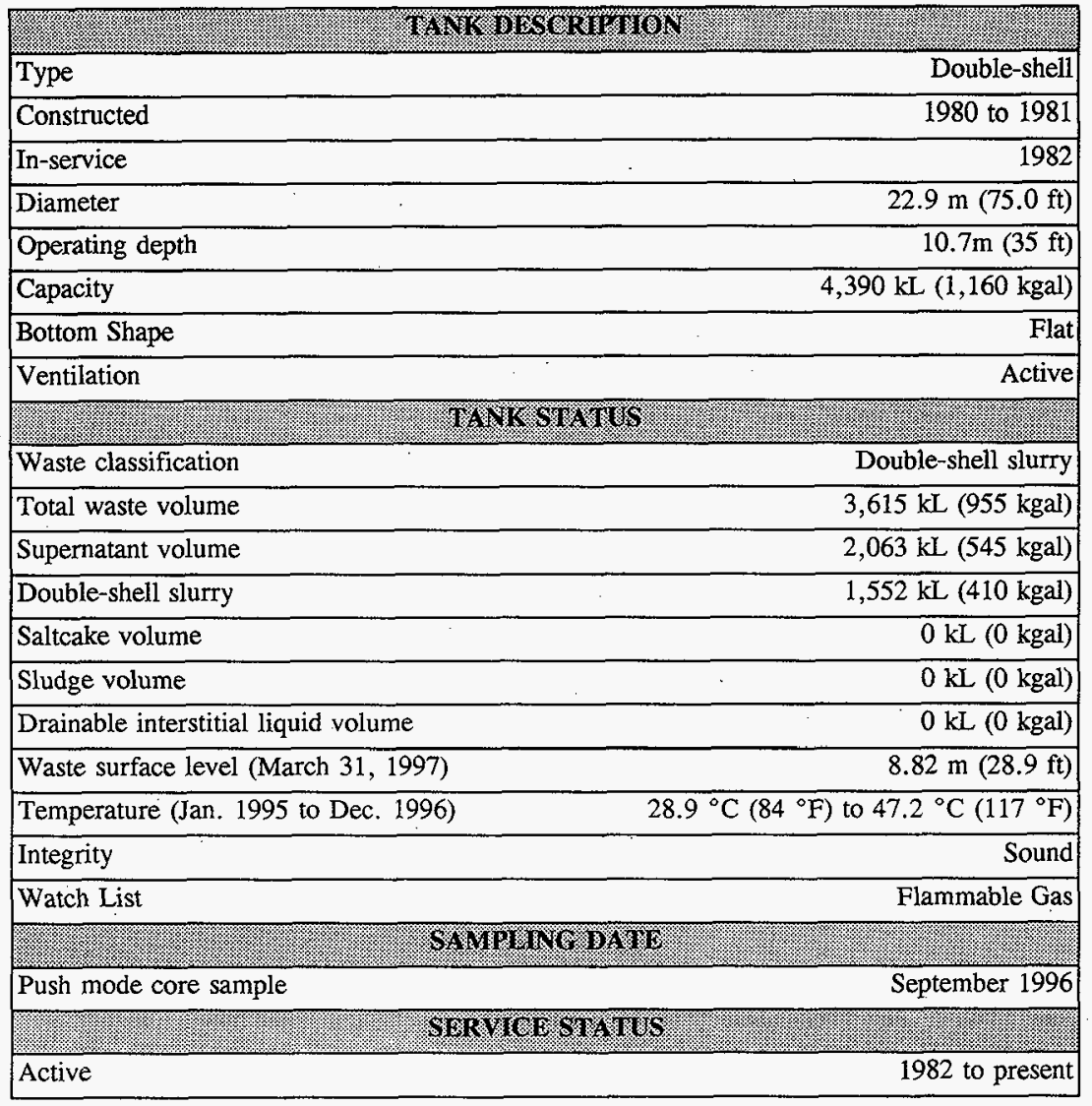

Notes:

${ }^{1}$ Waste volumes are based on Hanlon (1997).

${ }^{2}$ This is an active tank, any new waste transferred into this tank may change the data presented in this table. 


\subsection{RESPONSE TO TECHNICAL ISSUES}

Four technical issues have been identified for tank 241-AN-103 (Brown et al. 1996). They are:

- Safety Screening: Does the waste pose or contribute to any recognized potential safety problems?

- Flammable Gas: Data from the push core samples are needed to provide an understanding of the tank contents so that: (1) insight may be obtained on the mechanisms within the waste for gas generation, retention, and release, (2) models of the waste behavior can be developed to support safety analysis and development of mitigation methods, (3) simulants for waste studies can be developed, and (4) modeling of the release of gases into the dome space, and subsequent potential for ignition in the dome space, can be done to support hazard analyses.

- Organic Solvents Screening: Does an organic solvent pool exist that may cause a fire or ignition of organic solvents in entrained waste solids?

- Privatization: Do the September 1996 push core samples taken from tank 241-AN-103, and the subsequent laboratory analysis, meet the needs of the privatization low activity waste (LAW) DQO (Jones and Wiemers 1996)?

The Tank Characterization Plan (Kruger and Winkelman 1996) and the Tank Safety and Analysis Plan (TSAP) (Kruger 1996) provide the types of sampling and analysis used to address the above issues. Data from the recent analysis of two push core samples and tank vapor space flammability measurements, as well as available historical information, provided the means to respond to these two issues. This response is detailed in the following sections. See Appendix B for sample and analysis data for tank 241-AN-103.

\subsection{SAFETY SCREENING}

The data needed to screen the waste in tank 241-AN-103 for potential safety problems are documented in Tank Safery Screening Data Quality Objective (Dukelow et al. 1995). These potential safety problems are exothermic conditions in the waste; flammable gases in the waste and/or tank headspace; and criticality conditions in the waste. Each of these conditions is addressed separately below. 


\subsubsection{Exothermic Conditions (Energetics)}

The first requirement outlined in the safety screening DQO (Dukelow et al. 1995) is to ensure that there are not sufficient exothermic constituents in tank 241-AN-103 to cause a safety hazard. Because of this requirement, energetics in the tank 241-AN-103 waste were evaluated. The safety screening DQO required that solid samples be tested for energetics every $24 \mathrm{~cm}(9.5 \mathrm{in}$.) to determine if the energetics exceed the safety threshold limit. Note that the first 12 segments from core 166 and the first 9 segments from core 167 were mostly composed of drainable liquid and therefore, were not subdivided into upper and lower samples (see Table B1-2a).

Results obtained using differential scanning calorimetry (DSC) indicated that exotherms were well below notification limits of $480 \mathrm{~J} / \mathrm{g}$ on a dry weight basis. The maximum DSC value measured was $268 \mathrm{~J} / \mathrm{g}$ (dry). The upper limits of the one-sided 95 percent confidence interval for the DSC results were below the notification limit ( $480 \mathrm{~J} / \mathrm{g}$ dry) except for core 167 , segment 7 , drainable liquid, which had an upper limit of $629 \mathrm{~J} / \mathrm{g}$ (dry). The upper limit was high because of difference in the exotherms found for the two samples (result: $0 \mathrm{~J} / \mathrm{g}$, duplicate $172.0 \mathrm{~J} / \mathrm{g}$ ). The total organic carbon (TOC) results for this sample were $2,500 \mu \mathrm{g} / \mathrm{g}$ and $2,720 \mu \mathrm{g} / \mathrm{g}$, well below the limit of $30,000 \mu \mathrm{g} / \mathrm{g}$. Therefore, energetics is not a concern for this tank.

\subsubsection{Flammable Gas}

Vapor phase measurements, taken in the tank headspace prior to the push core samples in September 1996, indicated that no flammable gas was detected (0 percent of the lower flammability limit [LFL]). Data from these vapor phase measurements are presented in Appendix B. However, before this sampling event there has been one gas release event and the resulting peak hydrogen concentration was $0.3 \mathrm{vol} \%$, which is less than 25 percent of the LFL (Wilkins et al. 1997). Tank 241-AN-103 is on the flammable gas watch list. Additional information on the flammable gas tank safety evaluation is provided in Section 2.2 .

\subsubsection{Criticality}

The safety threshold limit is $1 \mathrm{~g}{ }^{239} \mathrm{Pu}$ per liter of waste. As part of the safety screening $\mathrm{DQO}$, the potential for a criticality event in a tank is assessed using the total alpha activity data. The safety screening DQO identifies the total alpha activity safety threshold limit as 1 gram of fissile material per liter of waste. This limit was converted into the laboratory-reported solid units of microcuries per gram by assuming that all fissile material (alpha activity) exists as ${ }^{239} \mathrm{Pu}$ and by using the maximum bulk density of $1.93 \mathrm{~g} / \mathrm{mL}$ found from the tank $241-\mathrm{AN}-103$ samples. The assumption that all fissile material exists as ${ }^{239} \mathrm{Pu}$ was made because ${ }^{239} \mathrm{Pu}$ is the radioisotope of concern with respect to the criticality issue. The maximum density value was used because it produces the minimum threshold limit and 
is therefore the most conservative estimate. For tank $241-\mathrm{AN}-103,1$ gram of ${ }^{239} \mathrm{Pu}$ per liter of waste is equivalent to $32.1 \mu \mathrm{Ci} / \mathrm{g}$ of alpha activity for solid subsamples. There is no density correction for liquids because the results are reported by the laboratory in microcuries per milliliter. The gram to microcurie conversion for liquid samples, assuming all alpha activity is from ${ }^{239} \mathrm{Pu}$, results in a safety threshold limit of $61.5 \mu \mathrm{Ci} / \mathrm{mL}$.

Waste samples were tested for total alpha activity for each push core sample. Concentrations in all samples were well below the threshold limit with a maximum value of $0.18 \mu \mathrm{Ci} / \mathrm{mL}$. Additionally, the upper limit of the one-sided 95 percent confidence interval for these results was less than $61.5 \mu \mathrm{Ci} / \mathrm{mL}$, with a maximum value of $0.534 \mu \mathrm{Ci} / \mathrm{mL}$. The method used to calculate confidence limits is contained in Appendix $C$. The data show that there is no criticality concern with this tank.

\subsection{FLAMMABLE GAS TANK SAFETY EVALUATION}

The purpose of the flammable gas tank safety evaluation is to obtain data to develop mitigation methods, to support tank behavior models needed for making safety analyses, and to support evaluations of chemical mechanisms for gas production and release. These data needs were met by the sampling event and retained gas sampler (RGS) analysis in September 1996, and by the ball rheometer and void fraction instrument (VFI) deployed in May 1996.

Tank 241-AN-103 was the fifth double-shell tank sampled for retained gases. The RGS was used in two risers within this tank to obtain seven segments. The waste level at time of - sampling was about $884 \mathrm{~cm}$ (348 in.) and consisted of a nonconvective layer of about $378 \pm 29 \mathrm{~cm}(149 \pm 12$ in.) in depth, $414 \pm 37 \mathrm{~cm}(163 \pm 14$ in.) of convective liquid, and a floating crust $92 \pm 8 \mathrm{~cm}(36 \pm 3$ in.) thick. The head space volume in tank 241-AN-103 is about $1,723 \mathrm{~m}^{3}\left(61,000 \mathrm{ft}^{3}\right)$ (Shekarriz et al. 1997).

Retained gas measurements and estimated solubilities show three major constituents in the gas/vapor phase (free gas) in the waste. The major components of gas in the nonconvective layer are hydrogen $(62.0 \pm 6.6 \mathrm{~mol} \%)$, nitrogen $(33.1 \pm 3.5 \mathrm{~mol} \%)$ and nitrous oxide $(3.8 \pm 0.4 \mathrm{~mol} \%)$. Gas in the convective layer consists of mostly nitrogen $(76.3 \pm 47 \mathrm{~mol} \%)$, hydrogen $(18.4 \pm 10 \mathrm{~mol} \%)$ and nitrous oxide $(2.2 \pm 1.2 \mathrm{~mol} \%)$. The remainder of the gas in both layers is composed of ammonia, methane, and other hydrocarbons (Shekarriz et al. 1997).

The measured local ammonia concentrations in tank $241-\mathrm{AN}-103$ ranged from $1260 \pm 350$ to $3820 \pm 3150 \mu \mathrm{mole} / \mathrm{liter}$ of waste. More than 99.9 percent of the ammonia is dissolved in the waste. The RGS analysis of ammonia is believed to underestimate the actual ammonia content in the tank by a factor of 2 to 3 (Shekarriz et al. 1997).

The extraction results show that the insoluble gases were primarily retained in the lower, nonconvective layer. Based on the estimated solubilities and RGS measurements of gas concentrations, about 7.7 percent by volume (in-situ) of the nonconvective layer was filled 
with free gas, while 0.4 percent by volume (in-situ) of the convective (upper) layer was filled with free gas. Local calculated void fractions based on RGS data did not agree closely with the VFI results. The VFI data show a maximum of approximately 0.15 void fraction at an elevation of $200 \mathrm{~cm}$ ( $80 \mathrm{in}$.). The maximum void fraction found with the RGS in the non-convective layer is 0.094 . The calculated hydrogen inventory in both phases of the nonconvective and convective layers of tank 241-AN-103 is $136 \mathrm{~m}^{3}\left(4,800 \mathrm{ft}^{3}\right)$, based on integrated RGS measurements (Shekarriz et al. 1997). The total stored gas inventory is $380 \mathrm{~m}^{3}$, the highest of any of the double-shell tanks.

X-rays of the RGS segments, prior to extrusion, showed large gas pockets in tank 241-AN-103 that account for a large portion of the measured void fraction. This contrasts with the observations made for tank $241-\mathrm{AW}-101$ waste, where the major portion of the gas was observed to be smaller than the detection threshold of the $x$-ray imaging system $(<0.5 \mathrm{~mm})$. No fractures or irregularly shaped bubbles were observed in this tank, unlike what was observed in tank 241-A-101 waste. Estimated densities of the five segments in the nonconvective layer in this tank, based on $\mathrm{x}$-ray. images, ranged from 1.85 to $2.06 \mathrm{~g} / \mathrm{cc}$ (Shekarriz et al. 1997).

The ball rheometer and VFI were run in tank $241-\mathrm{AN}-103$ in riser 16B on May 7 and 14, 1996, and in riser IB on May 9 and 16, 1995, respectively. Three traverses were accomplished with the VFI in riser $1 \mathrm{~B}$ and two in $16 \mathrm{~B}$. The ball sank to within about $2 \mathrm{~m}$

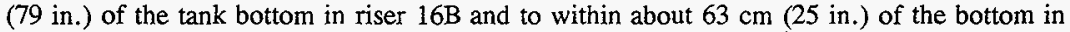
IB. No significant gas releases were observed on the waste surface or on the gas monitoring equipment during VFI operation (Stewart et al. 1996).

Tank 241-AN-103 has the highest void fraction of the six flammable gas Watch List tanks tested with the VFI. It also shows the least scatter in the void measurements. The data show a trend of almost linear decreasing void fraction at waste depths below about $250 \mathrm{~cm}$ (Stewart et al. 1996).

The viscosity of the convective layer in risers $1 \mathrm{~B}$ and $16 \mathrm{~B}$ was calculated to be 10 centipoise (cP), bounded above by $60 \mathrm{cP}$ and below by $2 \mathrm{cP}$. At all the elevations from which data could be obtained, the yield stress in riser $16 \mathrm{~B}$ is slightly higher than that in riser $1 \mathrm{~B}$. The apparent viscosities were also higher in riser 16B than in riser 1B (Stewart et al. 1996).

Preliminary evaluations of flammable gas analytical results for tank 241-AN-103 were conducted by Meyer et al. (1997). Additional evaluations are in progress. Preliminary indications are that gas is still being generated, and future releases are possible because of the large volume of gas stored in the convective layer and the thick layer of crust which prevents gasses from escaping to the tank dome space. 


\subsection{ORGANIC SOLVENTS SCREENING}

The data required to support the organic solvent screening issue are documented in the 93-5 implementation plan (DOE-RL 1996). A new DQO is currently being developed to address the organic solvent issue. In the interim, tanks are to be sampled for total non-methane hydrocarbon, to determine if an organic extractant pool greater than $1 \mathrm{~m}^{2}$ $\left(10.8 \mathrm{ft}^{2}\right.$ ) exists (Cash 1996). The purpose of this assessment is to ensure that an organic solvent pool fire or ignition of organic solvents cannot occur. Vapor analyses for total non-methane organic hydrocarbon have not been conducted. Vapor samples are scheduled for FY 1998.

\subsection{PRIVATIZATION}

Tank 241-AN-103 is in the scope of the privatization LAW DQO (Jones and Wiemers 1996). However, sampling was performed before issuing this DQO. The sampling and analytical results are being assessed by the privatization program to determine whether the 1996 sample meets the needs of the privatization LAW DQO. Results will be reported in a future revision to this report or additional sampling and analyses may be required.

\subsection{OTHER TECHNICAL ISSUES}

A factor in assessing tank safety is the heat generation and temperature of the waste. Heat is generated in the tanks from radioactive decay. An estimate of the tank heat load can be calculated based on the results from the 1996 sample event. The heat load value calculated from the 1996 sample events was $10.6 \mathrm{~kW}(36,300 \mathrm{Btu} / \mathrm{hr})$, as shown in Table 2-1. The heat load estimate based on the tank process history was $6.69 \mathrm{~kW}(22,800 \mathrm{Btu} / \mathrm{hr})$ (Agnew et al. 1997). The heat load estimate based on the tank headspace temperature was $13.2 \mathrm{~kW}(45,000 \mathrm{Btu} / \mathrm{hr}$ ) (Kummerer 1995). These estimates are well below the limit of the maximum heat load, $20.5 \mathrm{~kW}(70,000 \mathrm{Btu} / \mathrm{hr})$, allowed for double-shell tanks (Heubach 1996). 
Table 2-1. Heat Load for Tank 241-AN-103 Based on Radionuclide Content.

\begin{tabular}{|c|c|c|c|c|}
\hline & 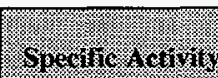 & moresind & 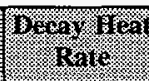 & 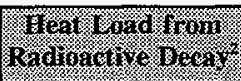 \\
\hline Rarionucture & 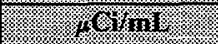 & 4 & . $17 \%$ & $1 \%$ \\
\hline${ }^{137} \mathrm{Cs}$ & 638 & $2.31 \mathrm{E}+06$ & 0.00472 & 10,900 \\
\hline Total & & & & 10,900 \\
\hline
\end{tabular}

Notes:

${ }^{1}$ Include daughter radionuclides.

${ }^{2}$ Only analytes contributing to the heat generation rate above $100 \mathrm{~W}$ are included.

\subsection{SUMMARY}

The results from all analyses performed to address potential safety issues showed that no primary analyte exceeded safety decision threshold limits with the exception of two samples. These samples exceeded the notification limit at the upper limit of the 95 percent confidence interval. Total organic carbon analyses are pending. The analyses for the flammable gas were completed to obtain data to develop mitigation methods, to support tank behavior models needed for making safety analyses, and to support evaluations of chemical mechanisms for gas production and release. The analyses results are summarized in Table 2-2. 
Table 2-2. Summary of Safety Screening, Organic Solvents Screening and Flammable Gas Results.

\begin{tabular}{|c|c|c|}
\hline 1ssive & 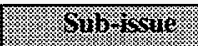 & (4) \\
\hline \multirow[t]{3}{*}{$\begin{array}{l}\text { Safety } \\
\text { screening }\end{array}$} & Energetics & $\begin{array}{l}\text { All exothermic reactions below } 480 \mathrm{~J} / \mathrm{g} \text {. Highest } 95 \\
\text { percent confidence interval upper limit }=629 \mathrm{~J} / \mathrm{g} \text {. The } \\
\text { average TOC for this sample was } 2 ; 610 \text {, well below } \\
30,000 \mu \mathrm{g} / \mathrm{g} \text {. }\end{array}$ \\
\hline & Flammable gas & $\begin{array}{l}\text { Vapor measurement reported } 0 \text { percent of lower } \\
\text { flammability limit. (Combustible gas meter). }\end{array}$ \\
\hline & Criticality & $\begin{array}{l}\text { All analyses well below } 32 \mu \mathrm{Ci} / \mathrm{g} \text { total alpha (within } \\
95 \text { percent confidence limit on each sample). }\end{array}$ \\
\hline $\begin{array}{l}\text { Flammable } \\
\text { Gas }\end{array}$ & $\begin{array}{l}\text { Mechanisms for } \\
\text { generation } \\
\text { retention and } \\
\text { release } \\
\text { Waste Models }\end{array}$ & $\begin{array}{l}7.7 \text { percent void filled with retained gases in the } \\
\text { nonconvective layer }\left(314 \mathrm{~m}^{3}\right), 62 \text { percent hydrogen } \\
\text { content. High ammonia concentration in the waste. } \\
\text { Preliminary assessments of flammable gas generation, } \\
\text { retention and release mechanisms, and waste behavior } \\
\text { modeling results are reported in Meyer et al. (1997). } \\
\text { Additional evaluations to assess potential impacts and } \\
\text { waste behavior in tank } 241-\mathrm{AN}-103 \text { are in progress. }\end{array}$ \\
\hline $\begin{array}{l}\text { Organic } \\
\text { Solvent }\end{array}$ & $\begin{array}{l}\text { Total } \\
\text { Non-Methane } \\
\text { Hydrocarbon }\end{array}$ & Evaluation in progress based on TOC sniff test results. \\
\hline Privatization & $\begin{array}{l}\text { Low Activity } \\
\text { Waste }\end{array}$ & $\begin{array}{l}\text { Results of } 1996 \text { push core sample are being evaluated to } \\
\text { determine if they meet the needs of the privatization } \\
\text { (LAW) DQO (Jones and Wiemers 1996). }\end{array}$ \\
\hline
\end{tabular}


HNF-SD-WM-ER-702 Rev. 0

This page intentionally left blank. 


\subsection{BEST BASIS STANDARD INVENTORY ESTIMATE}

Information about the chemical and/or physical properties of tank wastes is used to perform safety analyses, engineering evaluations, and risk assessments associated with waste management activities, as well as to address regulatory issues. Waste management activities include overseeing tank farm operations and identifying, monitoring, and resolving safety issues associated with these operations and with the tank wastes. Disposal activities involve designing equipment, processes, and facilities for retrieving wastes and processing the wastes into a form that is suitable for long-term storage.

Chemical inventory information generally is derived using two approaches: 1) component inventories are estimated using the results of sample analyses; and 2) component inventories are predicted using a model based on process knowledge and historical information. The most recent model was developed by Los Alamos National Laboratory (LANL) (Agnew et al. 1997). Information derived from these two different approaches is often inconsistent.

An effort is underway to provide waste inventory estimates that will serve as standard characterization information for the various waste management activities (Hodgson and LeClair 1996). As part of this effort, an evaluation of available information for tank 241-AN-103 was performed:

- A report by Steen (1997) and Appendix B of this report provide characterization results from the September 1996 core sampling event.

- A feed projection document that supported grout treatment facility studies provides estimates of the waste in tank 24l-AN-103 based on a 1986 push core sample. (Hendrickson 1994).

- The HDW model document (Agnew et al. 1997) provides tank content estimates derived from the LANL model, in terms of component concentrations and inventories.

Inventories based on the September 1996 sampling event should serve as the basis for the best estimate inventory to tank $241-\mathrm{AN}-103$ for the following reasons:

- The September 1996 sampling event provides the most recent data for the waste.

- Estimates based on the 1986 core sampling event agree with the September 1996 data.

- The HDW model estimates, although in reasonable agreement with the 1996 sampling data, do not compare as well with the 1986 data. 
Best-basis total inventory estimates for tank 241-AN-103 are presented in Tables 3-1 and 3-2. Best-basis inventory estimates for the supernatant, salt slurry, and crust layers are included in Appendix B (Tables D4-1 through D4-6). Radionuclide values are decayed to January 1 , 1994.

The inventory values reported in Tables 3-1 and 3-2 are subject to change. Refer to the Tank Characterization Database (TCD) for the most current inventory values.

Best-basis tank inventory values are derived for 46 key radionuclides (as defined in Section 3.1 of Kupfer et al. 1997), all decayed to a common report date of January 1, 1994. Often, waste sample analyses have only reported ${ }^{90} \mathrm{Sr},{ }^{137} \mathrm{Cs},{ }^{239 / 240} \mathrm{Pu}$, and total uranium, or (total beta and total alpha) while other key radionuclides such as ${ }^{60} \mathrm{Co},{ }^{99} \mathrm{Tc},{ }^{129} \mathrm{I},{ }^{154} \mathrm{Eu},{ }^{155} \mathrm{Eu}$, and ${ }^{241} \mathrm{Am}$, and so forth, have been infrequently reported. For this reason it has been necessary to derive most of the 46 key radionuclides by computer models. These models estimate radionuclide activity in batches of reactor fuel, account for the split of radionuclides to various separations plant waste streams, and track their movement with tank waste transactions. (These computer models are described in Kupfer et al. 1997, Section 6.1 and in Watrous and Wootan 1997.) Model generated values for radionuclides in any of 177 tanks are reported in the Hanford Defined Waste Rev. 4 model results (Agnew et al. 1997). The best-basis value for any one analyte may be either a model result or a sample or engineering assessment-based result if available. (No attempt has been made to ratio or normalize model results for all 46 radionuclides when values for measured radionuclides disagree with the model.) For a discussion of typical error between model derived values and sample derived values, see Kupfer et al. 1997, Section 6.1.10.

Table 3-1. Best-Basis Total Inventory Estimates for Nonradioactive Components in Tank 241-AN-103 as of May 31, 1997. (2 sheets)

\begin{tabular}{|c|c|c|c|}
\hline (nallice & 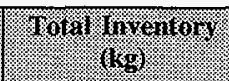 & 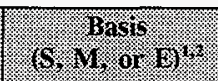 & Cominan \\
\hline $\mathrm{Al}$ & 239,000 & $S$ & \\
\hline $\mathrm{Bi}$ & $<1,120$ & $S$ & \\
\hline $\mathrm{Ca}$ & $<6,100$ & $S$ & \\
\hline $\mathrm{Cl}$ & 27,500 & $S$ & \\
\hline $\mathrm{TIC}$ as $\mathrm{CO}_{3}$ & 84,600 & $S$ & \\
\hline $\mathrm{Cr}$ & 2,820 & $S$ & \\
\hline $\mathrm{F}$ & 3,860 & $S$ & \\
\hline $\mathrm{Fe}$ & $<774$ & $\mathrm{~S}$ & \\
\hline $\mathrm{K}$ & 46,300 & $S$ & \\
\hline $\mathrm{La}$ & $<811$ & $S$ & \\
\hline
\end{tabular}


Table 3-1. Best-Basis Total Inventory Estimates for Nonradioactive Components in Tank 241-AN-103 as of May 31, 1997. (2 sheets)

\begin{tabular}{|c|c|c|c|}
\hline 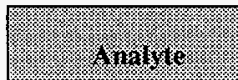 & 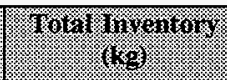 & 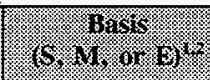 & Somingeri \\
\hline $\mathrm{Mn}$ & $<112$ & $S$ & \\
\hline $\mathrm{Na}$ & $1.27 \mathrm{E}+06$ & $S$ & \\
\hline $\mathrm{Ni}$ & $<225$ & $\mathrm{~S}$ & . \\
\hline $\mathrm{NO}_{2}$ & 494,000 & $S$ & \\
\hline $\mathrm{NO}_{3}$ & 771,000 & $S$ & \\
\hline $\mathrm{OH}$ & 999,000 & $S$ & \\
\hline $\mathrm{Pb}$ & $<795$ & $S$ & \\
\hline $\mathrm{PO}_{4}$ & 9,800 & $s$ & \\
\hline $\mathrm{Si}$ & 2,350 & $S$ & \\
\hline $\mathrm{SO}_{4}$ & 13,200 & $S$ & \\
\hline $\mathrm{Sr}$ & $<112$ & $S$ & \\
\hline TOC & 12,000 & $S$ & \\
\hline $\mathbf{U}_{\text {TOTAL }}$ & $<5,620$ & $S$ & \\
\hline $\mathrm{Zr}$ & $<113$ & $S$ & \\
\hline$\%$ Water & 48.0 & $S$ & \\
\hline Density, $\mathrm{g} / \mathrm{mL}$ & 1.54 & $S$ & \\
\hline
\end{tabular}

Notes:

TIC $=$ total inorganic carbon

${ }^{1} \dot{S}=$ Sample-based, $M=$ HDW model-based, and $\mathrm{E}=$ Engineering assessment-based.

2 Based on a sample volume of $3,615 \mathrm{~kL}$ (995 Kgal). 
HNF-SD-WM-ER-702 Rev. 0

Table 3-2. Best-Basis Total Inventory Estimates for Radioactive Components in

Tank 241-AN-103 as of May 31,.1997. (Decayed to January 1, 1994) (2 Sheets)

\begin{tabular}{|c|c|c|c|}
\hline . & 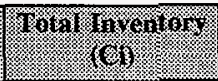 & 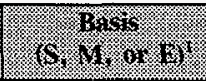 & Cormingin \\
\hline${ }^{3} \mathrm{H}$ & 9.31 & S & Slurry/Crust only \\
\hline${ }^{14} \mathrm{C}$ & 112 & $S$ & \\
\hline${ }^{59} \mathrm{Ni}$ & \begin{tabular}{|l|}
7.58 \\
\end{tabular} & $M$ & \\
\hline${ }^{60} \mathrm{Co}$ & $<112$ & $S$ & \\
\hline${ }^{63} \mathrm{Ni}$ & 747 & $\mathbf{M}$ & \\
\hline${ }^{79} \mathrm{Se}$ & 12.3 & $M$ & \\
\hline${ }^{90} \mathrm{Sr}$ & 8,030 & $S$ & \\
\hline${ }^{90} \mathrm{Y}$ & 8,030 & $S$ & Referenced to ${ }^{90} \mathrm{Sr}$ \\
\hline${ }^{93} \mathrm{Zr}$ & 59.6 & $\mathrm{M}$ & \\
\hline${ }^{93 m} \mathrm{Nb}$ & 43.2 & $M$ & \\
\hline${ }^{99} \mathrm{Tc}$ & 456 & $s$ & \\
\hline${ }^{106} \mathrm{Ru}$ & 0.0533 & $\mathrm{M}$ & \\
\hline${ }^{113 \mathrm{~m}} \mathrm{Cd}$ & 302 & $\mathrm{M}$ & \\
\hline${ }^{125} \mathrm{Sb}$ & 865 & $\mathrm{M}$ & \\
\hline${ }^{126} \mathrm{Sn}$ & 18.8 & $M$ & \\
\hline${ }^{129} \mathrm{I}$ & 8.32 & $S$ & \\
\hline${ }^{134} \mathrm{Cs}$ & 189 & $M$ & \\
\hline${ }^{137} \mathrm{Cs}$ & $2.31 \mathrm{E}+06$ & $S$ & \\
\hline${ }^{137 \mathrm{~m}} \mathrm{Ba}$ & $2.19 \mathrm{E}+06$ & $\mathrm{~S}$ & Referenced to ${ }^{137} \mathrm{Cs}$ \\
\hline${ }^{151} \mathrm{Sm}$ & 43,600 & $\mathrm{M}$ & \\
\hline${ }^{152} \mathrm{Eu}$ & 15.8 & $\mathbf{M}$ & \\
\hline${ }^{154} \mathrm{Eu}$ & $<604$ & $S$ & \\
\hline${ }^{155} \mathrm{Eu}$ & $<3,300$ & $S$ & \\
\hline${ }^{226} \mathrm{Ra}$ & $5.81 \mathrm{E}-04$ & $M$ & \\
\hline${ }^{227} \mathrm{AC}$ & 0.00355 & $M$ & \\
\hline${ }^{228} \mathrm{Ra}$ & 1.05 & $\mathrm{M}$ & \\
\hline${ }^{229} \mathrm{Th}$ & 0.0243 & $\mathrm{M}$ & \\
\hline${ }^{231} \mathrm{~Pa}$ & 0.0143 & $M$ & \\
\hline${ }^{232} \mathrm{Th}$ & 0.103 & $M$ & \\
\hline
\end{tabular}


Table 3-2. Best-Basis Total Inventory Estimates for Radioactive Components in Tank 241-AN-103 as of May 31,.1997. (Decayed to January 1, 1994) (2 Sheets)

\begin{tabular}{|c|c|c|c|}
\hline 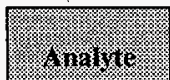 & 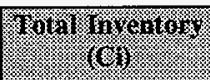 & 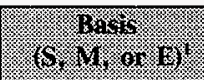 & Coninent. \\
\hline${ }^{232} \mathrm{U}$ & 3.59 & M & \\
\hline${ }^{233} \mathrm{U}$ & 13.8 & $\mathbf{M}$ & \\
\hline${ }^{234} \mathrm{U}$ & 4.87 & M & \\
\hline${ }^{235} \mathrm{U}$ & 0.189 & M & \\
\hline${ }^{236} \mathrm{U}$ & 0.285 & $\mathrm{M}$ & \\
\hline${ }^{237} \mathrm{~Np}$ & $<31.0$ & $S$ & \\
\hline${ }^{238} \mathrm{Pu}$ & 12.6 & $\mathrm{M}$ & \\
\hline${ }^{238} \mathrm{U}$ & 4.84 & $M$ & \\
\hline${ }^{239 / 240} \mathrm{Pu}$ & 12.3 & $S$ & \\
\hline${ }^{241} \mathrm{Am}$ & 21.7 & $S$ & \\
\hline${ }^{241} \mathrm{Pu}$ & 1,060 & $\mathrm{M}$ & \\
\hline${ }^{242} \mathrm{Cm}$. & 0.623 & $M$ & \\
\hline${ }^{242} \mathrm{Pu}$ & 0.00497 & $\mathrm{M}$ & \\
\hline${ }^{243} \mathrm{Am}$ & 0.00209 & $\mathbf{M}$ & \\
\hline${ }^{243} \mathrm{Cm}$ & $<4.85$ & $S$ & \\
\hline${ }^{244} \mathrm{Cm}$ & $<13.0$ & $S$ & \\
\hline
\end{tabular}

Note:

'S = Sample-based, $M=$ HDW model-based, and $E=$ Engineering assessment-based. 
HNF-SD-WM-ER-702 Rev. 0

This page intentionally left blank. 


\subsection{RECOMMENDATIONS}

With the exception of two samples, analytical results for the safety screening DQO (Dukelow et al. 1995) were within the safety notification limits. One sample had upper limits of the one-sided 95 percent confidence interval above the notification limit for DSC results. The average TOC for this sample was $2,610 \mu \mathrm{g} / \mathrm{g}$. Well below the limit of 30,000 $\mu \mathrm{g} / \mathrm{g}$ for TOC, indicating that energetics is not an issue for this tank. The sampling and analysis activities performed for tank 241-AN-103 have met all requirements for the safety screening DQOs. Retained Gas Sampler samples were obtained to address the flammable gas DQO (McDuffie and Johnson 1995). Large gas pockets were found in the non-convective layer of the tank waste. Additional evaluation of RGS results and flammable gas issues are in progress to determine the safety status for the tank. Vapor samples to address the organic solvents screening DQO have not been obtained, but are scheduled for FY 1998.

The privatization LAW DQO (Jones and Wiemers 1996) aiso applies to tank 241-AN-103. Sampling and analytical results are being assessed by the privatization program to determine whether the 1996 push core sample meets the needs of the privatization LAW DQO.

Table 4-1 summarizes the status of the Project Hanford Management Contractor (PMHC) TWRS Program review and acceptance of the sampling and analysis results reported in this tank characterization report. All DQO issues required to be addressed by sampling and analysis are listed in column one of Table 4-1. The second column indicates whether the requirements of the DQO were met by the sampling and analysis activities performed and is answered with a "yes" or a "no." The third column indicates concurrence and acceptance by the program in TWRS that is responsible for the DQO that the sampling and analysis activities performed adequately meet the needs of the DQO. A "yes" or "no" in column three indicates acceptance or disapproval of the sampling and analysis information presented in the TCR.

Table 4-1. Acceptance of Tank 241-AN-103 Sampling and Analysis.

\begin{tabular}{|c|c|c|}
\hline issue & Saming and diansisis & 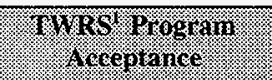 \\
\hline Safety Screening DQO & Yes & Yes \\
\hline Flammable Gas Tank Safety Program & Yes & Yes \\
\hline Organic Solvents Screening & No & No \\
\hline Privatization LAW DQO & ND & ND \\
\hline
\end{tabular}

Notes:

$\mathrm{ND}=$ not determined

' PHMC Program Office. 


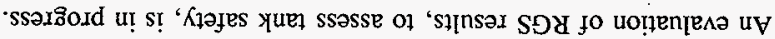

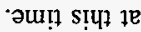

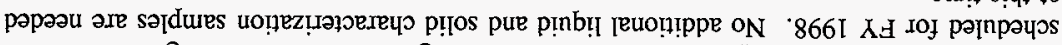

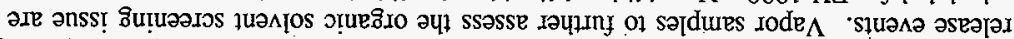

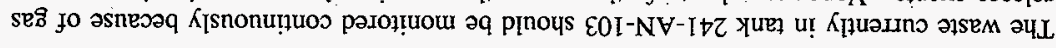

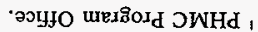

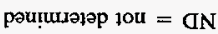

$: \because 0 \mathrm{~N}$

\begin{tabular}{|c|c|c|}
\hline aN & ssarso $_{d}$ uI & 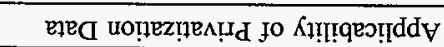 \\
\hline aN & $\mathrm{ON}_{\mathrm{N}}$ & 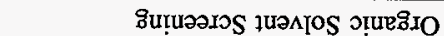 \\
\hline aN & ssərôox $u_{\mathrm{I}}$ & 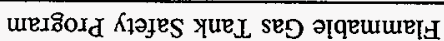 \\
\hline aN & $\mathrm{ON}_{\mathrm{N}}$ & 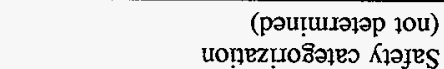 \\
\hline 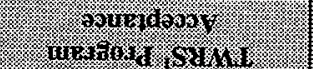 & 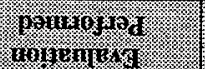 & Innт: \\
\hline
\end{tabular}

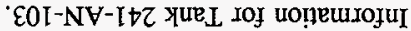

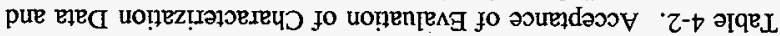

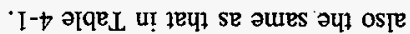

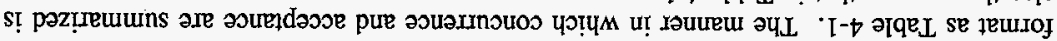

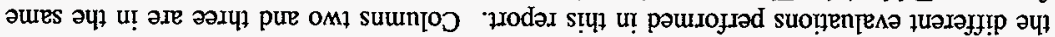

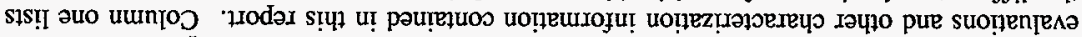

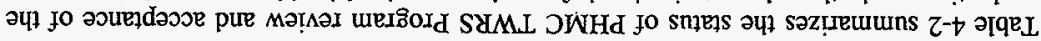




\subsection{REFERENCES}

Agnew, S. F., J. Boyer, R. A. Corbin, T. B. Duran, J. R. Fitzpatrick, K. A. Jurgensen, T. P. Ortiz, and B. L. Young, 1997, Hanford Tank Chemical and Radionuclide Inventories: HDW Model Revision 4, LA-UR-96-3680, Los Alamos National Laboratory, Los Alamos, New Mexico.

Brown, T. M., S. J. Eberlein, J. W. Hunt, and L. J. Fergestrom, 1997, Tank Characterization Sampling Basis, WHC-SD-WM-TA-164, Rev. 2, Westinghouse Hanford Company, Richland, Washington.

Cash, R. J., 1996, Scope Increase of "Data Quality Objective to Support Resolution of the Organic Complexant Safery Issue" Rev. 2, (internal memo 79300-96-029 to S. J. Eberlein, July 12), Westinghouse Hanford Company, Richland, Washington.

DOE-RL, 1996, Recommendation 93-5, DOE/RL-94-0001, Rev. 1, U. S. Department of Energy, Richland, Washington.

Dukelow, G. T., J. W. Hunt, H. Babad, and J. E. Meacham, 1995, Tank Safety Screening Data Quality Objective, HNF-SD-WM-SP-004, Rev. 3, Lockheed Martin Hanford Corporation, Richland, Washington.

Ecology, EPA and DOE, 1996, Hanford Federal Facility Agreement and Consent Order, as amended, Washington State Department of Ecology, U.S. Environmental Protection Agency, and U.S. Department of Energy, Olympia, Washington.

Hanlon, B. M., 1997, Waste Tank Summary Report for Month Ending March 31, 1997, HNF-EP-0182-108, Lockheed Martin Hanford Corporation, Richland, Washington.

Heubach, E. C., 1996, Double-Shell Tank Interim Operational Safety Requirements, WHC-SD-WM-OSR-016, Rev. 0E, Westinghouse Hanford Company, Richland Washington.

Hendrickson, D. W., 1994, Grout Treatment Facility Waste Feed Projections, WHC-SD-WM-TI-528, Rev. 1, Westinghouse Hanford Company, Richland, Washington.

Hodgson, K. M., and M. D. LeClair, 1996, Work Plan for Defining a Standard Inventory Estimate for Wastes Stored in Hanford Site Underground Tanks, WHC-SD-WM-WP-311, Rev. 1, Lockheed Martin Hanford Corporation for Fluor Daniel Hanford, Inc., Richland, Washington. 
Jones, T. E., and K. D. Wiemers, 1996, Data Requirements for TWRS Privatization Characterization of Potential Low Activity Waste Feed, WHC-SD-WM-SP-023, Rev. 0, Westinghouse Hanford Company, Richland, Washington.

Kruger, A. A., 1996, Tank 241-AN-103 Push Mode Core Sampling and Analysis Plan, WHC-SD-WM-TSAP-105, Rev. 0, Westinghouse Hanford Company, Richland, Washington.

Kruger A. A., and Winkelman, W. D., 1996, Tank 24I-AN-103 Tank Characterization Plan, WHC-SD-WM-TP-383, Rev. 3, Lockheed Martin Hanford Corporation, Richland, Washington.

Kummerer, M., 1995, Topical Report on Heat Removal Characteristics of Waste Storage Tanks, WHC-SD-WM-SARR-010, Rev. 1, Westinghouse Hanford Company, Richland, Washington.

Kupfer, M. J., A. L. Boldt, B. A. Higley, K. M. Hodgson, L. W. Shelton, R. A. Watrous, S. L. Lambert, D. E. Place, R. M. Orme, G. L. Borsheim, N. G. Colton, M. D. LeClair, R. T. Winward, and W. W. Schulz, 1997, Standard Inventories of Chemicals and Radionuclides in Hanford Site Tank Wastes,

HNF-SD-WM-TI-740, Rev. 0, Lockheed Martin Hanford Corporation for Fluor Daniel Hanford, Inc., Richland, Washington.

Meyer, P. A., M. E. Brewster, S. A. Bryan, G. Chen, L. R. Pederson, C. W. Stewart, and G. Terrones, 1997, Gas Retention and Release Behavior in Hanford Double-Shell Waste Tanks, PNNL-11536, Rev. 1, Pacific Northwest National Laboratory, Richland, Washington

Public Law 101-510, 1990, "Safety Measures for. Waste Tanks at Hanford Nuclear Reservation," Section 3137 of National Defense Authorization Act for Fiscal Year 1991.

Shekarriz, A., R. E. Bauer, D. R. Rector, N. S. Cannon, L. A. Mahoney, B. E. Hey, M. A. Chieda, C. G. Linschooten, J. M. Bates, F. J. Reitz, and E. R. Siciliano, 1997, Composition and Quantities of Retained Gas Measured in Hanford Waste Tanks 241-AW-101, A-101, AN-105, AN-104, and AN-103, PNNL-11450, Rev. 1, Pacific Northwest National Laboratory, Richland, Washington.

Steen, F. H., 1997, Tank 241-AN-103, Cores 166 and 167, Analytical Results for the Final Report, HNF-SD-WM-DP-223, Rev. 0, Rust Federal Service of Hanford, Inc. for Fluor Daniel Hanford, Inc., Richland, Washington. 
Stewart, C. W., J. M. Alzheimer, M. E. Brewster, G. Chen, R. E. Mendoza, H. C. Reid, C. L. Shepard, and G. Terrones, 1996, In Situ Rheology and Gas Volume in Hanford Double-Shell Waste Tanks, PNNL-11296, Pacific Northwest National Laboratory, Richland, Washington.

Watrous, R. A., and D. W. Wootan, 1997, Activity of Fuel Batches Processed Through Hanford Separations Plants, 1944 Through 1989, HNF-SD-WM-TI-794, Rev. 0, Lockheed Martin Hanford Corp. for Fluor Daniel Hanford, Inc., Richland, Washington.

Wilkins, N. E., R. E. Bauer, and D. M. Ogden, 1997, Results of Vapor Space Monitoring of Flammable Gas Watch List Tanks, HNF-SD-WM-TI-797, Rev. 1, Lockheed Martin Hanford Corporation, Richland, Washington. 
HNF-SD-WM-ER-702 Rev. 0

This page intentionally left blank. 
APPENDIX A

\section{HISTORICAL TANK INFORMATION}


HNF-SD-WM-ER-702 Rev. 0

This page intentionally left blank. 


\section{APPENDIX A}

\section{HISTORICAL TANK INFORMATION}

Appendix A describes tank 241-AN-103 based on historical information. For this report, historical information includes any information about the fill history, waste types, surveillance, or modeling data about the tank. This information is necessary for providing a balanced assessment of the sampling and analytical results.

This appendix contains the following information:

- Section A1: Current status of the tank, including the current waste levels as well as the stabilization and isolation status of the tank.

- Section A2: Information about the design of the tank.

- Section A3: Process knowledge of the tank, that is, the waste transfer history and the estimated contents of the tank based on modeling data.

- Section A4: Surveillance data for tank 241-AN-103, including surface-level readings, temperatures, and a description of the waste surface based on photographs.

- Section A5: References for Appendix A.

Historical sampling results (results from samples obtained prior to 1989) are included in Appendix B.

\section{A1.0 CURRENT TANK STATUS}

As of March 31, 1997, tank 241-AN-103 contained an estimated $3615 \mathrm{~kL}$ (955 kgal) of waste classified as double-shell slurry (Hanlon 1997). Liquid waste volumes are estimated using a level measurement gauge. The solid waste volumes are estimated using a sludge level measurement device. The solid waste volume was last updated on March 31, 1997. The amounts of various waste phases in the tank are presented in Table A1-1.

Tank 241-AN-103 is categorized as sound and is actively ventilated. The tank is classified as a concentrated waste holding tank. The tank is on the Hydrogen/Flammable Gas Watch List (Hanlon 1997). All monitoring systems were in compliance with documented standards as of March 31, 1997 (Hanlon 1997). 
Table A1-1. Tank Contents Status Summary. ${ }^{1}$

\begin{tabular}{|l|l|}
\hline Total waste & $3615(955)$ \\
\hline Supernatant liquid & $2,063(545)$ \\
\hline Double-shell slurry & $1,552(410)$ \\
\hline Sludge & $0(0)$ \\
\hline Saltcake & $0(0)$ \\
\hline Drainable interstitial liquid & $0(0)$ \\
\hline Drainable liquid remaining & $2,063(545)$ \\
\hline Pumpable liquid remaining & $2,063(545)$ \\
\hline
\end{tabular}

Note:

${ }^{1}$ Hanlon (1997).

\section{A2.0 TANK DESIGN AND BACKGROUND}

The 241-AN Tank Farm was constructed from 1980 to 1981 in the 200 East Area. The tank farm contains seven double-shell tanks. These tanks have a capacity of 4,390 kL $(1,160$ $\mathrm{kgal})$ and a diameter of $22.9 \mathrm{~m}(75 \mathrm{ft})$. These tanks were designed to hold boiling waste with a maximum design temperature of $177^{\circ} \mathrm{C}\left(350^{\circ} \mathrm{F}\right)$ (Brevick et al. 1997).

Tank 241-AN-103 was constructed with a primary carbon steel liner (heat-treated and stress-relieved), a secondary carbon steel liner (not heat-treated), and a reinforced concrete shell. The bottom of the primary liner is $13 \mathrm{~mm}(0.5 \mathrm{in}$.) thick, the lower portion of the sides is $19 \mathrm{~mm}(0.75 \mathrm{in}$.) thick, the upper portion of the sides is $13 \mathrm{~mm}(0.5 \mathrm{in}$.) thick, and the dome liner is $9.5 \mathrm{~mm}(0.375 \mathrm{in}$.) thick. The secondary liner is $9.5 \mathrm{~mm}(0.375 \mathrm{in}$.) thick. The concrete walls are $457 \mathrm{~mm}$ ( $1.5 \mathrm{ft})$ thick and the dome is $381 \mathrm{~mm}(1.25 \mathrm{ft})$ thick (Brevick et al. 1997). The tank has a flat bottom. The bottoms of the primary and secondary liners are separated by an insulating concrete layer. There is a grid of drain slots in the concrete foundation beneath the secondary steel liner. The grid's function is to collect any waste that may leak from the tank and divert it to the leak detection well. 
Tank 241-AN-103 has 22 risers ranging in diameter from $102 \mathrm{~mm} \mathrm{(4} \mathrm{in.)} \mathrm{to} 1.07 \mathrm{~m} \mathrm{(42} \mathrm{in.)}$ that provide access to the tank and 37 risers that provide access to the annulus. Table A2-1 shows numbers, diameters, and descriptions of the risers (annular risers are not included). A plan view that depicts the riser configuration is shown as Figure A2-1. Risers $10 \mathrm{~A}$ and $21 \mathrm{~A}$ (each $102 \mathrm{~mm}$ [4 in.] in diameter) and risers 7B and 12A (each $305 \mathrm{~mm}$ [12 in.] in diameter) are available for use in sampling (Lipnicki 1997). A tank cross-section showing the approximate waste level along with a schematic of the tank equipment is in Figure A2-2. 
Figure A2-1. Riser Configuration for Tank 241-AN-103.

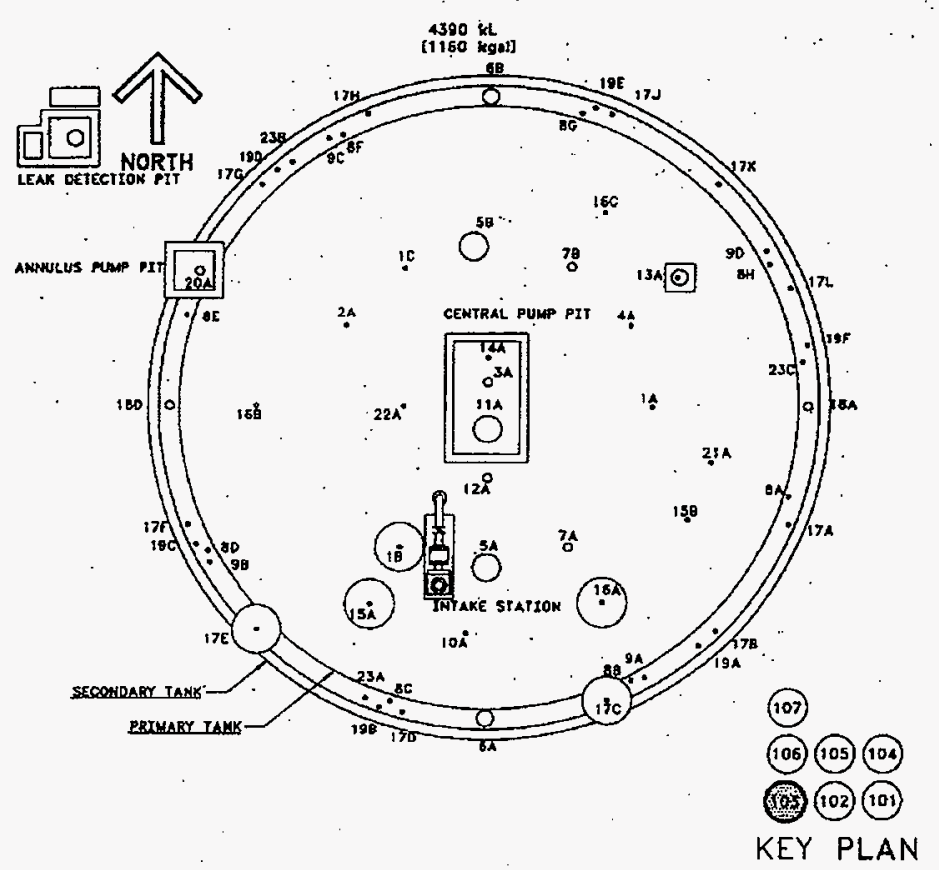


HNF-SD-WM-ER-702 Rev. 0

Table A2-1. Tank 241-AN-103 Risers. ${ }^{1,2,3,4}$

\begin{tabular}{|c|c|c|c|}
\hline Metrist & nords & $\begin{array}{l}\text { Gianetor } \\
\text { (Mnches) }\end{array}$ & 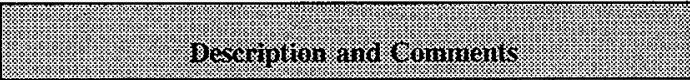 \\
\hline 102 & R1A & 4 & Level Indicator (Manual Tape) ${ }^{5}$ \\
\hline 103 & R1B & 4 & Sludge Measurement Port \\
\hline 101 & R1C & 4 & Sludge Measurement Port, (12 inch cover) \\
\hline 104 & R2A & 4 & ENRAF $^{6}$ \\
\hline 105 & R3A & 12 & Supernatant Pump, Central Pump Pit (Pit) \\
\hline 106 & R4A & 4 & Thermocouple Tree \\
\hline 108 & $\mathrm{R5A}$ & 42 & Manhole \\
\hline 107 & $\mathrm{R} 5 \mathrm{~B}$ & 42 & Color video camera $^{7}$ \\
\hline 112 & R7A & 12 & Tank Ventilation (Standard Hydrogen Monitoring System ${ }^{8}$ ) \\
\hline $111^{10}$ & R7B & 12 & Spare \\
\hline $125^{10}$ & RI0A & 4 & Spare \\
\hline 126 & RIIA & 42 & Slurry Distributor, Central Pump Pit \\
\hline $127^{10}$ & R12A & 12 & Observation Port, Spare \\
\hline 128 & R13A & 4 & Tank Pressure \\
\hline 129 & R14A & 4 & Supernatant Return, Central Pump Pit \\
\hline 131 & $\mathrm{R} 15 \mathrm{~A}$ & 4 & Multifunctional Instrument Tree ${ }^{9}$ \\
\hline 130 & R15B & 4 & High Liquid Level Sensor \\
\hline 134 & R16A & 4 & Sludge Measurement Port \\
\hline 132 & $\mathrm{R} 16 \mathrm{~B}$ & 4 & Sludge Measurement Port \\
\hline 133 & $\mathrm{R} 16 \mathrm{C}$ & 4 & Siudge Measurement Port, (12 inch cover) \\
\hline $155^{10}$ & R21A & 4 & Spare \\
\hline 156 & $\mathrm{R} 22 \mathrm{~A}$ & 4 & Sludge Measurement Port \\
\hline
\end{tabular}

Notes:

${ }^{1}$ Salazar 1994

${ }^{2}$ Tran 1993

${ }^{3}$ WHC (1996a)

${ }^{4} \mathrm{WHC}(1996 \mathrm{~b})$

${ }^{5}$ RHO (1983)

${ }^{6} \mathrm{KEH}(1996)$

7WHC (1995b)

${ }^{8}$ WHC (1995a)

WHC (1995c)

${ }^{10}$ Denotes risers tentatively available for sampling (Lipnicki 1997). 


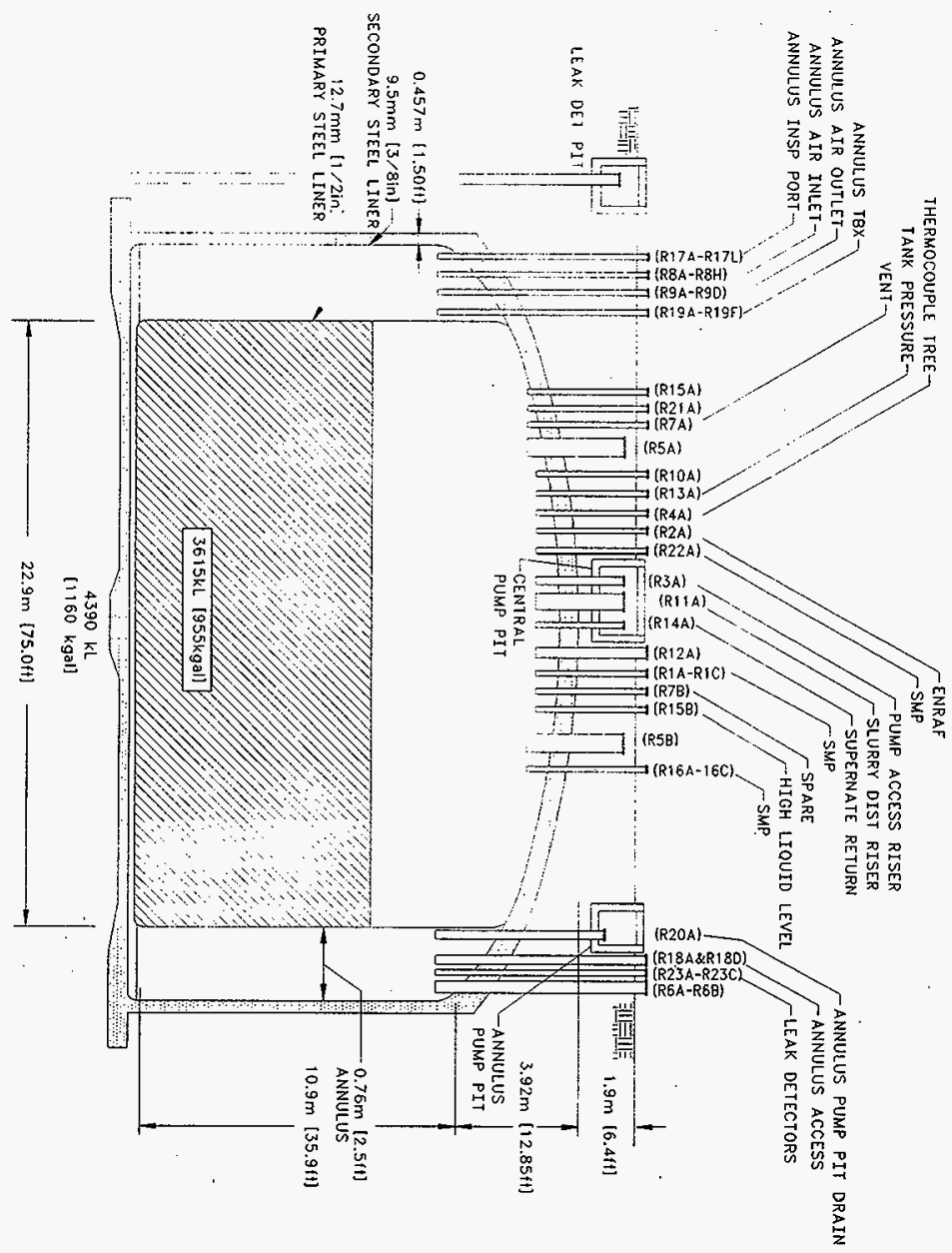

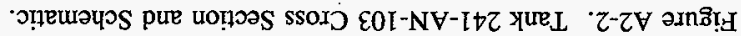




\section{A3.0 PROCESS KNOWLEDGE}

The sections below: 1) provide information about the history of the major waste transfers that involved tank 241-AN-103,2) describe the process wastes that were transferred, and 3) estimate current tank contents based on waste transfer history.

\section{A3.1 WASTE TRANSFER HISTORY}

Table A3-1 summarizes the waste transfer history of tank 241-AN-103 (Agnew et al. 1997b). Waste was initially added to tank 241-AN-103 in the second quarter of 1982 with the addition of water to test the tank's integrity. In the fourth quarter of 1982 , more water was added and non-complexed waste was sent from tank 241-SY-102. During the fourth quarter of 1982 and the first quarter of 1983, waste was sent to tank 241-AW-102 from tank 241-AN-103. Dilute, non-complexed waste from the 300 and 400 Areas was transferred to tank 241-AN-103 during the third quarter of 1983.

During the fourth quarter of 1984 , tank $241-\mathrm{AN}-103$ began receiving waste from the single-shell salt well pumping occurring in the 200 East and West Areas. Salt well liquor was received from various single-shell tanks listed in Table A3-1. Tanks 241-S-106 and 241-B-102 sent the salt well pumped waste to tank 241-AN-103 during the first and third quarters of 1984, respectively. Tank 241-AN-103 received dilute, non-complexed waste from B Plant cesium processing and wash water during the first quarter of 1984.

Tank 241-AN-104 sent non-complexed waste to tank 241-AN-103 during the third quarter of 1984. Tank 241-AN-103 sent waste to tank 241-AN-101 during the fourth quarter of 1984 . Tank 241-AN-103 exchanged non-complexed waste with tank 241-AW-102 (242-A Evaporator Feed Tank) during the first and fourth quarters of 1984 and the first quarter of 1986.

No further waste transfers have involved tank 241-AN-103 since the third quarter of 1986. Level changes that have occurred since 1986 are due to water additions and slurry fluctuations caused by gas generation within the waste. 


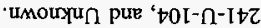

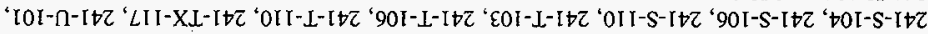

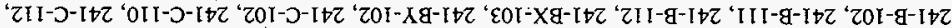
:SMOIIOJ se alem Buṭdund IIəM

aum[on ajsem

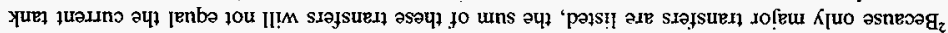

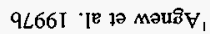

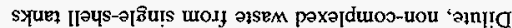

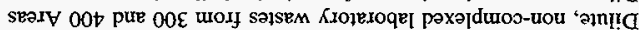
(T/B) OI) \%IM I > JOL Ч!IM әISEM

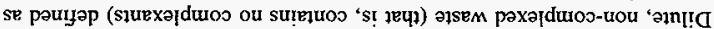

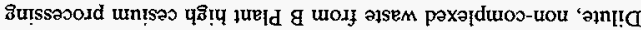

$$
\begin{aligned}
& =\text { OITMS } \\
& =\quad \forall \nabla V E T \\
& =\quad N G \\
& =\text { STTdg }
\end{aligned}
$$

\begin{tabular}{|c|c|c|c|c|c|}
\hline \$89- & $\varepsilon 6 \mathcal{S Z}^{-}$ & $986 I$ & $\mathrm{Na}$ & $Z 0 I-M \forall-I \nabla Z$ & \\
\hline$\varepsilon 99$ & $60 S z$ & $986 \mathrm{I}$ & $\mathrm{Na}$ & & $Z 0 I-M V-I t Z$ \\
\hline OZI- & $t S t-$ & $786 \mathrm{I}$ & $\mathrm{NG}$ & - IOI-NV-I $t+Z$ & \\
\hline E6I & {$[\varepsilon L$} & $586 \mathrm{I}$ & $\mathrm{Na}$ & & tOI-NV-ItZ \\
\hline ZL9I & $62 \varepsilon 9$ & $586 \mathrm{I}$ & $\mathrm{Na}$ & & $Z 0 I-M \forall-I+Z$ \\
\hline$\nabla I$ & $\varepsilon S$ & $\$ 861$ & тәฺุ $_{M}$ & & saomos os!n \\
\hline$\varepsilon 9$ & $8 \mathcal{E Z}$ & $\downarrow 86 \mathrm{I}$ & SDTdg & & Iueld \& \\
\hline $98 \mathcal{E}$ & I9tI & $\$ 86 I-\varepsilon 86 I$ & OITMS & & морәс әәs * \\
\hline$\nabla \mathcal{S Z}$ & I96 & $\varepsilon 86 \mathrm{I}$ & $\forall \triangleright \forall E I$ & & $\begin{array}{r}\text { seəry } \\
\text { 00t pue } 00 \varepsilon\end{array}$ \\
\hline $6 S 6 z-$ & 00ZII- & $\downarrow 86 I-Z 86 I$ & $\mathrm{Na}$ & $Z 0 I-M V-I t z$ & \\
\hline \$96 & $\varepsilon \subseteq 9 \varepsilon$ & 2861 & $\mathrm{Na}$ & & $z 01-x s-I t z$ \\
\hline $86 \mathrm{I}$ & $O S L$ & $886 \mathrm{I}-286 \mathrm{I}$ & $\operatorname{səte}_{M}$ & . & sooInos 'OSIL \\
\hline 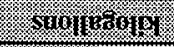 & crogurous. & 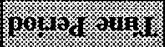 & (w) & 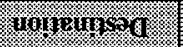 & $200310 \%$ \\
\hline 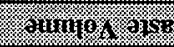 & 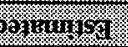 & & $45 \%$ & \% $10 \% 114$ & ing114. \\
\hline
\end{tabular}

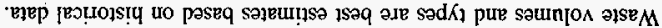

:SOJON

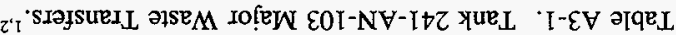




\section{A3.2 HISTORICAL ESTIMATION OF TANK CONTENTS}

The historical transfer data used for this estimate are from the following sources:

- Waste Status and Transaction Record Summary (WSTRS) (Agnew et al. 1997b). WSTRS is a tank-by-tank quarterly summary spreadsheet of waste transactions.

- Hanford Tank Chemical and Radionuclide Inventories: HDW Model Rev. 4 (Agnew et al. 1997a). This document contains the Hanford Defined Waste [HDW] list, the Supernatant Mixing Model [SMM], the Tank Layer Model [TLM] and the Historical Tank Inventory Estimates.

- Hanford Defined Waste List (HDW List): The HDW list is comprised of approximately 50 waste types defined by concentration for major analytes/compounds for both sludge and supernatant layers.

- Tank Layer Model (TLM). The TLM defines the sludge and saltcake layers in each tank using waste composition and waste transfer information.

- Supernatant Mixing Model (SMM). This is a subroutine within the HDW model that calculates the volume and composition of certain supernatant blends and concentrates.

Using these records, the TLM defines the sludge and saltcake layers in each tank. The SMM uses information from the WSTRS, the TLM and the HDW list to describe the supernatants and concentrates in each tank. Together the WSTRS, HDW, TLM, and SMM determine each tank's inventory estimate. These model predictions are considered estimates that require further evaluation using analytical data.

Based on the HDW model, tank $241-\mathrm{AN}-103$ contains $3607 \mathrm{~kL}$ (953 kgal) of waste comprised of a bottom solids layer of $8 \mathrm{~kL}$ ( $2 \mathrm{kgal}$ ) of B Plant low level waste (BL) with a top layer of 3,540 kL (935 kgal) of concentrated supernatant solids waste (SMMA2) and a $61 \mathrm{~kL}$ (16 kgal) layer of supernatant (SU) liquid above the solids waste. Figure A3-1 shows a graphical representation of the estimated solids waste types and volumes for each tank layer. Note that this is inconsistent with Hanlon (1997) which reports a total waste volume of $3615 \mathrm{~kL}$ ( $955 \mathrm{kgal}$ ) and separates the top layer reported by Agnew (1997a) into supernatant and double-shell siurry (DSS) waste, based on current tank sample information and surface level adjustments.

Tables A3-3 and A3-4 show estimates of the expected analyte and radionuclide waste constituents and concentrations, respectively. 
HNF-SD-WM-ER-702 Rev. 0

Figure A3-1. Tank Layer Model.

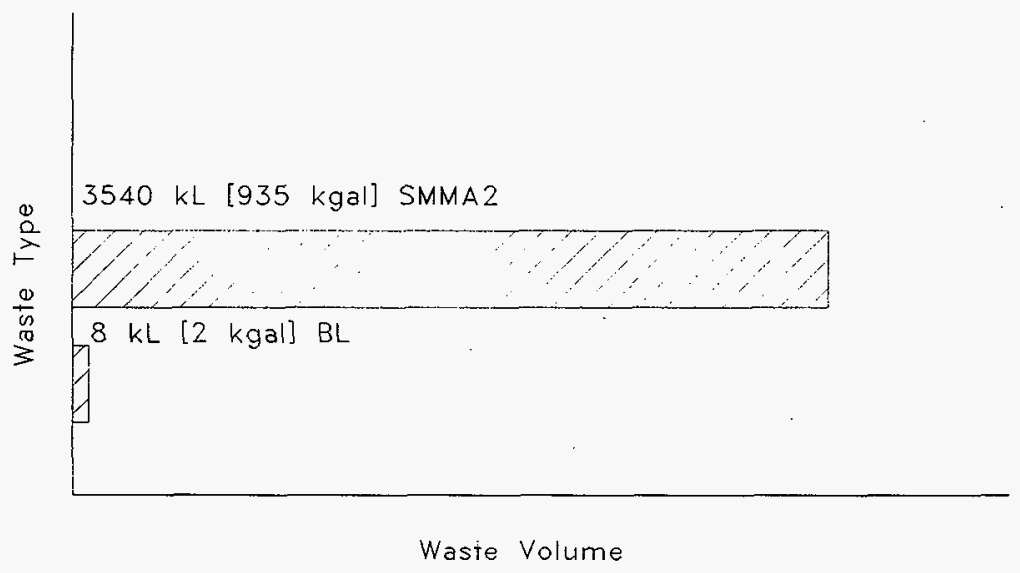


HNF-SD-WM-ER-702 Rev. 0

Table A3-2. Historical Tank Inventory Estimate Analytes. ${ }^{1,2}$ (2 sheets)

\begin{tabular}{|c|c|c|c|c|c|}
\hline \multirow{2}{*}{ 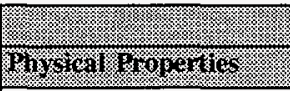 } & \multicolumn{3}{|c|}{ 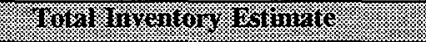 } & 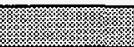 & 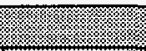 \\
\hline & & & & 96.9 & 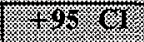 \\
\hline Total Waste & \multicolumn{5}{|c|}{$5.79 \mathrm{E}+06(\mathrm{~kg})(953 \mathrm{kgal})$} \\
\hline Heat Load & \multicolumn{3}{|c|}{$6.69(\mathrm{~kW})(2.28 \mathrm{E}+04 \mathrm{Btu} / \mathrm{hr})$} & 6.30 & 7.13 \\
\hline Bulk Density & \multicolumn{3}{|c|}{$1.60(\mathrm{~g} / \mathrm{cc})$} & 1.55 & 1.66 \\
\hline Water wt\% & \multicolumn{3}{|l|}{32.6} & 29.0 & 36.1 \\
\hline TOC wt\% C (wet) & \multicolumn{3}{|l|}{1.04} & 0.740 & 1.33 \\
\hline 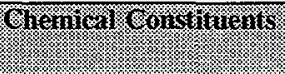 & mold & 3010 & 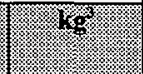 & (mos) & (1001) \\
\hline $\mathrm{Na}^{+}$ & 14.2 & $2.04 \mathrm{E}+05$ & $1.18 \mathrm{E}+06$ & 12.8 & 15.6 \\
\hline $\mathrm{Al}^{3+}$ & 1.57 & $2.63 \mathrm{E}+04$ & $1.52 \mathrm{E}+05$ & 1.39 & 1.68 \\
\hline $\mathrm{Fe}^{3+}($ total $\mathrm{Fe})$ & $1.71 \mathrm{E}-02$ & 595 & $3.44 \mathrm{E}+03$ & $1.61 \mathrm{E}-02$ & $1.81 \mathrm{E}-02$ \\
\hline $\mathrm{Cr}^{3+}$ & 0.111 & $3.59 \mathrm{E}+03$ & $2.08 \mathrm{E}+04$ & $9.70 \mathrm{E}-02$ & 0.119 \\
\hline $\mathrm{Bi}^{3+}$ & $1.16 \mathrm{E}-03$ & 151 & 874 & $1.09 \mathrm{E}-03$ & $1.23 \mathrm{E}-03$ \\
\hline $\mathrm{La}^{3+}$ & $1.24 \mathrm{E}-05$ & 1.07 & 6.19 & $9.13 \mathrm{E}-06$ & $1.56 \mathrm{E}-05$ \\
\hline $\mathrm{Hg}^{2+}$ & $1.38 \mathrm{E}-05$ & 1.73 & 10.0 & $1.03 \mathrm{E}-05$ & $1.44 \mathrm{E}-05$ \\
\hline $\mathrm{Zr}\left(\right.$ as $\left.\mathrm{ZrO}(\mathrm{OH})_{2}\right)$ & $1.72 \mathrm{E}-03$ & 97.8 & 566 & $8.91 \mathrm{E}-04$ & $1.93 \mathrm{E}-03$ \\
\hline $\mathrm{Pb}^{2+}$ & $1.15 \mathrm{E}-03$ & 149 & 862 & $9.68 \mathrm{E}-04$ & $1.34 \mathrm{E}-03$ \\
\hline $\mathrm{Ni}^{2+}$ & $1.06 \mathrm{E}-02$ & 389 & $2.25 \mathrm{E}+03$ & $9.96 \mathrm{E}-03$ & $1.08 \mathrm{E}-02$ \\
\hline $\mathrm{Sr}^{2+}$ & 0 & 0 & 0 & 0 & 0 \\
\hline $\mathrm{Mn}^{4+}$ & $9.24 \mathrm{E}-03$ & 316 & $1.83 \mathrm{E}+03$ & $5.75 \mathrm{E}-03$ & $1.22 \mathrm{E}-02$ \\
\hline $\mathrm{Ca}^{2+}$ & $4.71 \mathrm{E}-02$ & $1.18 \mathrm{E}+03$ & $6.81 \mathrm{E}+03$ & $4.25 \mathrm{E}-02$ & $5.06 \mathrm{E}-02$ \\
\hline $\mathrm{K}^{+}$ & 0.185 & $4.50 \mathrm{E}+03$ & $2.61 \mathrm{E}+04$ & $6.90 \mathrm{E}-02$ & 0.305 \\
\hline $\mathrm{OH}^{-}$ & 9.40 & $9.96 \mathrm{E}+04$ & $5.77 \mathrm{E}+05$ & 8.41 & 10.4 \\
\hline $\mathrm{NO}_{3}^{-}$ & 4.97 & $1.92 \mathrm{E}+05$ & $1.11 E+06$ & 4.62 & 5.33 \\
\hline $\mathrm{NO}_{2}^{-}$ & 1.99 & $5.70 E+04$ & $3.30 \mathrm{E}+05$ & 1.69 & 2.23 \\
\hline $\mathrm{CO}_{3}^{2-}$ & 0.674 & $2.52 \mathrm{E}+04$ & $1.46 \mathrm{E}+05$ & 0.560 & 0.770 \\
\hline $\mathrm{PO}_{4}^{3-}$ & 0.162 & $9.62 \mathrm{E}+03$ & $5.57 \mathrm{E}+04$ & 0.122 & 0.203 \\
\hline $\mathrm{SO}_{4}{ }^{2-}$ & 0.245 & $1.47 \mathrm{E}+04$ & $8.50 \mathrm{E}+04$ & 0.210 & 0.273 \\
\hline $\mathrm{Si}\left(\right.$ as $\left.\mathrm{SiO}_{3}{ }^{2-}\right)$ & $8.22 \mathrm{E}-02$ & $1.44 \mathrm{E}+03$ & $8.33 \mathrm{E}+03$ & $6.84 \mathrm{E}-02$ & $8.98 \mathrm{E}-02$ \\
\hline $\mathrm{F}^{-}$ & 0.185 & $2.19 \mathrm{E}+03$ & $1.27 \mathrm{E}+04$ & $6.50 \mathrm{E}-02$ & 0.224 \\
\hline $\mathrm{Cl}^{-}$ & 0.242 & $5.34 \mathrm{E}+03$ & $3.09 \mathrm{E}+04$ & 0.215 & 0.269 \\
\hline $\mathrm{C}_{6} \mathrm{H}_{5} \mathrm{O}_{7}{ }^{3-}$ & $3.22 \mathrm{E}-02$ & $3.79 \mathrm{E}+03$ & $2.20 \mathrm{E}+04$ & $2.58 \mathrm{E}-02$ & $3.86 \mathrm{E}-02$ \\
\hline
\end{tabular}




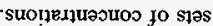

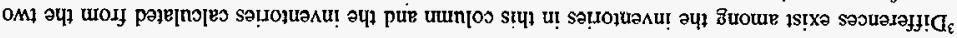
'Họ̣nes uฺ!

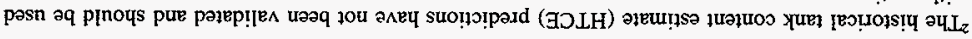

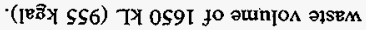

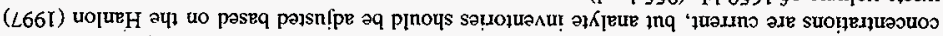

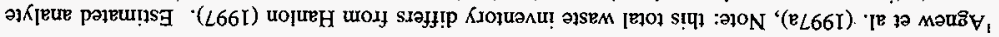

\begin{tabular}{|c|c|c|c|c|c|}
\hline 0 & 0 & 0 & 0 & 0 & ${ }_{-t}^{9}(\mathrm{NO}) \partial_{\mathrm{U}}$ \\
\hline$\varepsilon 88^{\circ} 0$ & $20-306^{\circ} \mathrm{s}$ & $\neq 0+\exists \varepsilon 8^{\circ} \tau$ & $\varepsilon 0+306 \%$ & $290^{\circ} 0$ & ${ }^{\varepsilon} \mathrm{HN}$ \\
\hline $20^{-}-3 \angle 9^{\circ} z$ & $20^{-} \exists 66^{\circ} \mathrm{I}$ & $\varepsilon 0+\exists \neq 0^{\circ} 9$ & $\varepsilon 0+\xi \rightarrow 0^{\circ} I$ & $20^{-}-39 z^{\circ} z$ & [oure]ng \\
\hline $20-3 \angle 9^{\circ} 2$ & $20^{-}-366^{\circ} \mathrm{I}$ & $t 0+G I L \cdot I$ & $\varepsilon 0+396^{\circ} \mathrm{Z}$ & $20-392 \cdot 2$ & dgव \\
\hline$\subseteq 0-96 L \cdot I$ & so-gtot I & $\nabla I^{\prime} \mathrm{S}$ & $888^{\circ} 0$ & s0-'ㄱ29' I & 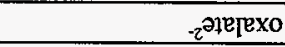 \\
\hline $20-320^{\circ} \mathrm{z}$ & $20-382.1$ & $\varepsilon 0+\exists \not \varepsilon \varepsilon$ & $8 \angle S$ & $20-\exists \angle S^{\cdot} \cdot I$ & әрецәое \\
\hline $182^{\circ} 0$ & OLI'O & $50+36 z^{\circ} \mathrm{s}$ & $\varepsilon 0+7+r \cdot 6$ & $96 \mathrm{I}^{\circ} 0$ & 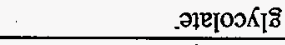 \\
\hline $20-39 s^{\circ} \mathrm{s}$ & $\varepsilon 0-326^{\circ} \mathrm{L}$ & $t 0+3 Z I^{\circ} \varepsilon$ & $\varepsilon 0+\exists 6 \mathcal{E} \subseteq$ & $20-39 I^{\circ} \varepsilon$ & ${ }_{\varepsilon} \forall \mathrm{LCO} H \mathrm{H}$ \\
\hline $20^{\circ}-\exists \varepsilon 0^{\circ} \varepsilon$ & $\varepsilon 0-\exists Z \nabla^{\circ} 9$ & $t 0+306^{\circ} I$ & $\mathcal{E} 0+\exists 8 \mathcal{Z}^{\circ} \varepsilon$ & $20-328^{\circ} I$ & ${ }_{-} \forall \mathrm{LG}$ \\
\hline 的 & (3) & 9 & 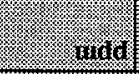 & $6 \%$ & 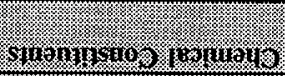 \\
\hline & & 288 & 15061011 & $1 / 2 y_{1}$ & \\
\hline
\end{tabular}

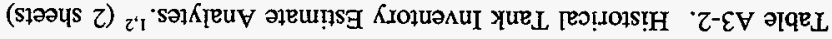


HNF-SD-WM-ER-702 Rev. 0

Table A3-3. Historical Tank Inventory Estimate Radionuclides. ${ }^{1.2}$ (2 sheets)

\begin{tabular}{|c|c|c|c|c|c|}
\hline s: & & (T) & 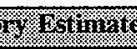 & & 1. \\
\hline $\begin{array}{l}\text { Thysien } \\
\text { eroperites }\end{array}$ & & 1. & & . $5 \%$ & . 95.91 \\
\hline Total Waste & $5.79 \mathrm{E}+06$ & $(953 \mathrm{kgal}$ & & & \\
\hline Heat Load & $6.69(\mathrm{~kW})$ & $28 \mathrm{E}+04 \mathrm{Bt}$ & & 6.30 & 7.13 \\
\hline Bulk Density & $1.60(\mathrm{~g} / \mathrm{cc})$ & & & 1.55 & 1.66 \\
\hline Water wt\% & 32.6 & & & 29.0 & 36.1 \\
\hline $\begin{array}{l}\text { TOC wt\% C } \\
\text { (wet) }\end{array}$ & 1.04 & & & 0.740 & 1.33 \\
\hline Rartanogerf & 6 & & & (c) & 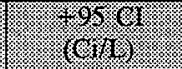 \\
\hline $\mathrm{H}-3$ & $2.23 \mathrm{E}-04$ & 0.139 & 805 & $1.59 \mathrm{E}-04$ & $2.49 \mathrm{E}-04$ \\
\hline C-14 & $2.91 \mathrm{E}-05$ & $1.82 \mathrm{E}-02$ & 105 & $1.57 \mathrm{E}-05$ & $3.00 \mathrm{E}-05$ \\
\hline Ni-59 & $2.10 \mathrm{E}-06$ & $1.31 \mathrm{E}-03$ & 7.58 & $1.47 \mathrm{E}-06$ & $2.16 \mathrm{E}-06$ \\
\hline $\mathrm{Ni}-63$ & $2.07 \mathrm{E}-04$ & 0.129 & 747 & $1.45 \mathrm{E}-04$ & $2.13 \mathrm{E}-04$ \\
\hline Co-60 & $3.85 \mathrm{E}-05$ & $2: 40 \mathrm{E}-02$ & 139 & $2.31 \mathrm{E}-05$ & $4.06 \mathrm{E}-05$ \\
\hline Se-79 & $3.42 \mathrm{E}-06$ & $2.13 \mathrm{E}-03$ & 12.3 & $2.56 \mathrm{E}-06$ & $4.02 \mathrm{E}-06$ \\
\hline Sr-90 & 0.123 & 76.7 & $4.44 \mathrm{E}+05$ & 0.110 & 0.127 \\
\hline Y-90 & 0.123 & 76.8 & $4.44 \mathrm{E}+05$ & $9.36 \mathrm{E}-02$ & 0.127 \\
\hline $\mathrm{Zr}-93$ & $1.65 \mathrm{E}-05$ & $1.03 \mathrm{E}-02$ & 59.6 & $1.23 \mathrm{E}-05$ & $1.96 \mathrm{E}-05$ \\
\hline $\mathrm{Nb}-93 \mathrm{~m}$ & $1.21 \mathrm{E}-05$ & $7.56 \mathrm{E}-03$ & 43.8 & $9.14 \mathrm{E}-06$ & $1.43 \mathrm{E}-05$ \\
\hline Tc-99 & 2.17E-04 & 0.135 & 784 & $1.67 \mathrm{E}-04$ & $2.71 \mathrm{E}-04$ \\
\hline Ru-106 & $1.48 \mathrm{E}-08$ & $9.20 \mathrm{E}-06$ & $5.33 \mathrm{E}-02$ & $1.28 \mathrm{E}-08$ & $1.66 \mathrm{E}-08$ \\
\hline $\mathrm{Cd}-113 \mathrm{~m}$ & $8.37 \mathrm{E}-05$ & $5.22 \mathrm{E}-02$ & 302 & $5.80 \mathrm{E}-05$ & $1.02 \mathrm{E}-04$ \\
\hline Sb-125 & $2.40 \mathrm{E}-04$ & 0.149 & 865 & $1.73 \mathrm{E}-04$ & $2.90 \mathrm{E}-04$ \\
\hline Sn-126 & $5.20 \mathrm{E}-06$ & $3.24 \mathrm{E}-03$ & 18.8 & $3.92 \mathrm{E}-06$ & $6.11 \mathrm{E}-06$ \\
\hline \begin{tabular}{|l|}
$\mathrm{I}-129$ \\
\end{tabular} & $4.20 \mathrm{E}-07$ & $2.62 \mathrm{E}-04$ & 1.51 & $3.23 \mathrm{E}-07$ & $5.24 \mathrm{E}-07$ \\
\hline Cs-134 & $5.23 \mathrm{E}-05$ & $3.26 \mathrm{E}-02$ & 189 & $3.07 \mathrm{E}-05$ & $7.48 \mathrm{E}-05$ \\
\hline Cs-137 & 0.219 & 136 & $7.89 \mathrm{E}+05$ & 0.198 & 0.245 \\
\hline $\mathrm{Ba}-137 \mathrm{~m}$ & 0.207 & 129 & $7.46 \mathrm{E}+05$ & 0.177 & 0.225 \\
\hline $\mathrm{Sm}-151$ & $1.21 \mathrm{E}-02$ & 7.53 & $4.36 \mathrm{E}+04$ & $9.09 \mathrm{E}-03$ & $1.42 \mathrm{E}-02$ \\
\hline Eu-152 & $4.39 \mathrm{E}-06$ & $2.74 \mathrm{E}-03$ & 15.8 & $3.33 \mathrm{E}-06$ & $5.04 \mathrm{E}-06$ \\
\hline Eu-154 & $6.10 \mathrm{E}-04$ & 0.380 & $2.20 \mathrm{E}+03$ & $3.96 \mathrm{E}-04$ & $7.18 \mathrm{E}-04$ \\
\hline Eu-155 & $2.75 \mathrm{E}-04$ & 0.171 & 991 & $2.12 \mathrm{E}-04$ & $3.14 \mathrm{E}-04$ \\
\hline
\end{tabular}


Table A3-3. Historical Tank Inventory Estimate Radionuclides. ${ }^{1,2}$ (2 sheets)

\begin{tabular}{|c|c|c|c|c|c|}
\hline r. & & 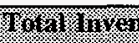 & M 1 in w & & \\
\hline 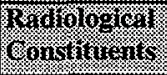 & (c) & \% & (3) & 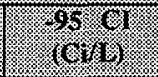 & 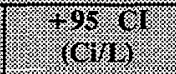 \\
\hline Ra-226 & $1.61 \mathrm{E}-10$ & $1.00 \mathrm{E}-07$ & $5.79 \mathrm{E}-04$ & $1.34 \mathrm{E}-10$ & $1.79 \mathrm{E}-10$ \\
\hline Ra-228 & $2.90 \mathrm{E}-07$ & $1.81 \mathrm{E}-04$ & 1.05 & $1.24 \mathrm{E}-07$ & $3.81 \mathrm{E}-07$ \\
\hline Ac-227 & $9.83 \mathrm{E}-10$ & $6.12 \mathrm{E}-07$ & $3.54 \mathrm{E}-03$ & $8.29 \mathrm{E}-10$ & $1.09 \mathrm{E}-09$ \\
\hline $\mathrm{Pa}-231$ & $3.97 \mathrm{E}-09$ & $2.47 \mathrm{E}-06$ & $1.43 \mathrm{E}-02$ & $3.11 \mathrm{E}-09$ & $4.58 \mathrm{E}-09$ \\
\hline Th-229 & $6.73 \mathrm{E}-09$ & $4.20 \mathrm{E}-06$ & $2.43 \mathrm{E}-02$ & $2.89 \mathrm{E}-09$ & $8.73 \mathrm{E}-09$ \\
\hline Th-232 & $2.85 \mathrm{E}-08$ & $1.77 \mathrm{E}-05$ & 0.103 & $7.89 \mathrm{E}-09$ & $3.95 \mathrm{E}-08$ \\
\hline $\mathrm{U}-232$ & $9.97 \mathrm{E}-07$ & $6.21 \mathrm{E}-04$ & 3.59 & $6.99 \mathrm{E}-07$ & $1.36 \mathrm{E}-06$ \\
\hline $\mathrm{U}-233$ & $3.82 \mathrm{E}-06$ & $2.38 \mathrm{E}-03$ & 13.8 & $2.68 \mathrm{E}-06$ & $5.21 \mathrm{E}-06$ \\
\hline $\mathrm{U}-234$ & $1.35 \mathrm{E}-06$ & $8.42 \mathrm{E}-04$ & 4.87 & $1.12 \mathrm{E}-06$ & $1.40 \mathrm{E}-06$ \\
\hline U-235 & $5.24 \mathrm{E}-08$ & $3.27 \mathrm{E}-05$ & 0.189 & $4.35 \mathrm{E}-08$ & $5.42 \mathrm{E}-08$ \\
\hline$\overline{\mathrm{U}}-236$ & $7.89 \mathrm{E}-08$ & $4.92 \mathrm{E}-05$ & 0.285 & $5.96 \mathrm{E}-08$ & $8.27 \mathrm{E}-08$ \\
\hline U-238 & $1.34 \mathrm{E}-06$ & $8.37 \mathrm{E}-04$ & 4.84 & $1.18 \mathrm{E}-06$ & $1.37 \mathrm{E}-06$ \\
\hline $\mathrm{Np}-237$ & $7.82 \mathrm{E}-07$ & $4.87 \mathrm{E}-04$ & 2.82 & $6.18 \mathrm{E}-07$ & $9.56 \mathrm{E}-07$ \\
\hline Pu-238 & $3.48 \mathrm{E}-06$ & $2.17 \mathrm{E}-03$ & 12.6 & $2.74 \mathrm{E}-06$ & $4.22 \mathrm{E}-06$ \\
\hline Pu-239 & $6.51 \mathrm{E}-05$ & $4.06 \mathrm{E}-02$ & 235 & $5.91 \mathrm{E}-05$ & $7.11 \mathrm{E}-05$ \\
\hline $\mathrm{Pu}-240$ & $1.35 \mathrm{E}-05$ & $8.44 \mathrm{E}-03$ & 48.9 & 1.17E-05 & $1.54 \mathrm{E}-05$ \\
\hline $\mathrm{Pu}-241$ & $2.93 \mathrm{E}-04$ & 0.183 & $1.06 \mathrm{E}+03$ & $2.18 \mathrm{E}-04$ & $3.69 \mathrm{E}-04$ \\
\hline $\mathrm{Pu}-242$ & $1.38 \mathrm{E}-09$ & $8.59 \mathrm{E}-07$ & $4.97 \mathrm{E}-03$ & $1.10 \mathrm{E}-09$ & $1.66 \mathrm{E}-09$ \\
\hline $\mathrm{Am}-241$ & $8.55 \mathrm{E}-05$ & $5.33 \mathrm{E}-02$ & 308 & $7.40 \mathrm{E}-05$ & $9.46 \mathrm{E}-05$ \\
\hline Am-243 & $5.79 \mathrm{E}-09$ & $3.61 \mathrm{E}-06$ & $2.09 \mathrm{E}-02$ & $3.68 \mathrm{E}-09$ & $6.95 \mathrm{E}-09$ \\
\hline $\mathrm{Cm}-242$ & $1.73 \mathrm{E}-07$ & $1.08 \mathrm{E}-04$ & 0.623 & $1.29 \mathrm{E}-07$ & $1.97 \mathrm{E}-07$ \\
\hline $\mathrm{Cm}-243$ & $1.75 \mathrm{E}-08$ & $1.09 \mathrm{E}-05$ & $6.32 \mathrm{E}-02$ & $1.34 \mathrm{E}-08$ & 2.00E-08 \\
\hline $\mathrm{Cm}-244$ & $2.39 \mathrm{E}-07$ & $\overline{1.49 \mathrm{E}-04}$ & 0.863 & $1.96 \mathrm{E}-07$ & $2.70 \mathrm{E}-07$ \\
\hline Wotuls. & M & \%kg & 16 & (5) & (1) \\
\hline $\mathrm{Pu}$ & $9.35 \mathrm{E}-04(\mathrm{~g} / \mathrm{L})$ & --- & 3.37 & $8.30 \mathrm{E}-04$ & $1.04 \mathrm{E}-03$ \\
\hline $\mathrm{U}$ & $1.33 \mathrm{E}-02$ & $1.98 \mathrm{E}+03$ & $1.14 \mathrm{E}+04$ & $1.13 \mathrm{E}-02$ & $1.37 \mathrm{E}-02$ \\
\hline
\end{tabular}

Notes:

'Agnew et al. (1997b)

${ }^{2}$ The HTCE predictions have not been validated and should be used with caution. 


\section{A4.0 SURVEILLANCE DATA}

Tank 241-AN-103 surveillance includes surface level measurements and temperature monitoring inside the tank (waste and vapor space). The tank annulus is equipped with leak-detection instrumentation consisting of continuous air monitors and conductivity probes (Johnson 1995). Surveillance data provide the basis for determining tank integrity.

For double-shell tanks, the leak detection instruments are the primary means of detecting a leak from the primary tank; liquid level measurements may be used to confirm a possible leak detected by the annulus instruments (Johnson 1995).

\section{A4.1 SURFACE LEVEL READINGS}

A Food Instrument Corporation (FIC) gauge was used to monitor the waste surface level in tank 241-AN-103 through riser 2A until April 14, 1995. The FIC was replaced with a manual ENRAF system that began recording on August 2, 1995. A manual tape was installed in riser 1A and began recording on April 9, 1995. On March 31, 1997, the waste surface level was $8.82 \mathrm{~m}$ (347 in.), as measured by the ENRAF ${ }^{1}$ system and was $8.84 \mathrm{~m}$ (348 in.) as measured by the manual tape. A graphical representation of the volume measurements is presented as a level history graph in Figure A4-1.

\section{A4.2 INTERNAL TANK TEMPERATURES}

Tank 241-AN-103 has a thermocouple tree, located in riser 4A, with 18 thermocouples to monitor the waste temperature. Temperature data recorded from January 1, 1995 through December 23, 1996 were obtained from the Surveillance Analysis Computer System (SACS) (LMHC 1997) for all thermocouples. The average temperature of the SACS data is $40.6^{\circ} \mathrm{C}$ $\left(105^{\circ} \mathrm{F}\right)$, the minimum is $28.9^{\circ} \mathrm{C}\left(84^{\circ} \mathrm{F}\right)$, and the maximum is $47.2{ }^{\circ} \mathrm{C}\left(117^{\circ} \mathrm{F}\right)$. The average temperature of the SACS data over the last year (December 1995 through December 1996) was $40.0^{\circ} \mathrm{C}\left(104^{\circ} \mathrm{F}\right)$, the minimum was $28.9^{\circ} \mathrm{C}\left(84^{\circ} \mathrm{F}\right)$, and the maximum was $45.6^{\circ} \mathrm{C}\left(114^{\circ} \mathrm{F}\right)$. The maximum temperature on December 23,1996 was $44.4^{\circ} \mathrm{C}\left(112^{\circ} \mathrm{F}\right)$ on thermocouple 5 and the minimum was $29.4^{\circ} \mathrm{C}\left(85^{\circ} \mathrm{F}\right)$ on thermocouples 17 and 18 . Thermocouple 5 is located in the waste while thermocouples 17 and 18 are in the vapor space. A graph of the weekly high temperatures can be found in Figure A4-2. Plots of the individual thermocouple readings can be found in the AN Tank Farm Supporting Document for the HTCE (Brevick et al. 1997).

The tank also has a Multifunctional Instrument Tree (MIT), located in riser 15A, that has 22 thermocouples. Temperature data from July 22, 1996 through March 10, 1997 were

${ }^{1}$ ENRAF is a trademark of ENRAF Corporation, Houston Texas. 
recorded manually once per week. The averas temperature of the MIT data is $38.3^{\circ} \mathrm{C}$ $\left(101^{\circ} \mathrm{F}\right)$, the minimum is $\left.25.0^{\circ} \mathrm{C} 77^{\circ} \mathrm{F}\right)$, ari ne maximum is $43.9^{\circ} \mathrm{C}\left(111^{\circ} \mathrm{F}\right)$. The maximum temperature on March 10,1997 , was $42.8^{\circ} \mathrm{C}\left(109^{\circ} \mathrm{F}\right)$ on thermocouple 8 , and the minimum was $27.2^{\circ} \mathrm{C}\left(81^{\circ} \mathrm{F}\right)$ on thermocouples 20 and 22 . Thermocouple 8 is located in the waste, and thermocouples 20 and 22 are located in the vapor space.

\section{A4.3 TANK 241-AN-103 VIDEO}

A video camera is located in riser 5B. A video was recorded on February 27, 1996, that shows a light, cream colored crust (Harding 1996). The crust is broken in some areas, and a dark brown liquid can be seen underneath the chunks of crust. 
Figure A4-1. Tank 241-AN-103 Level History.

竞

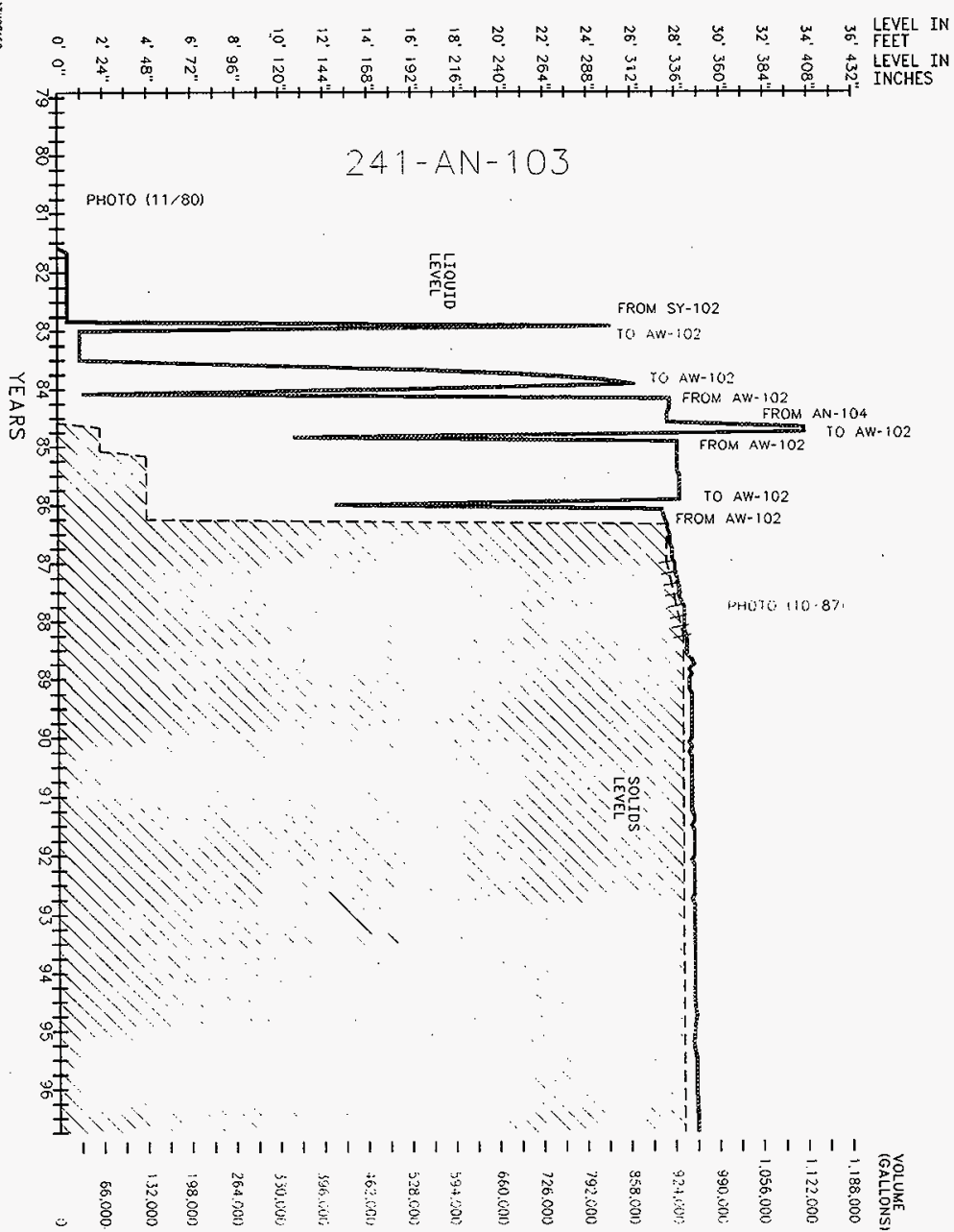


HNF-SD-WM-ER-702 Rev, 0

Figure A4-2. Tank 241-AN-103 Weekly High Temperature Plot.

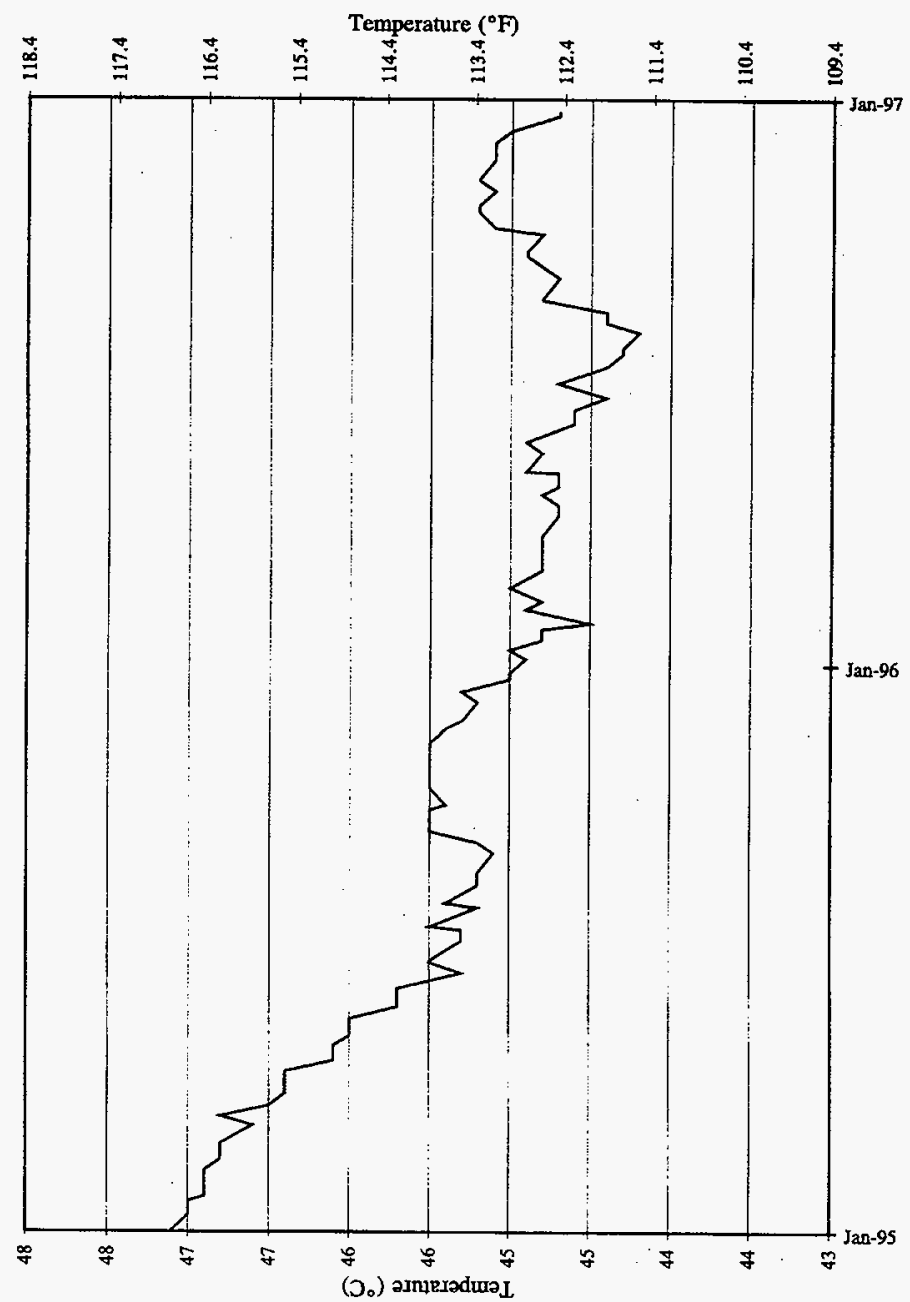




\section{A5.0 APPENDIX A REFERENCES}

Agnew, S.F., J. Boyer, R. A. Corbin, T. B. Duran, J. R. Fitzpatrick, K. A. Jurgensen, T. P. Ortiz, and B. L. Young, 1997a, Hanford Tank Chemical and Radionuclide Inventories: HDW Model Rev. 4, LA-UR-96-3860, Rev. 0, Los Alamos National Laboratory, Los Alamos, New Mexico.

Agnew, S.F., P. Baca, R. A. Corbin, T. B. Duran, and K. A. Jurgensen, 1997b, Waste Status and Transaction Record Summary: WSTRS Rev. 4, LA-UR-97-311, Rev. 4, Los Alamos National Laboratory, Los Alamos, New Mexico.

Brevick, C. H., L. A. Gaddis, and S. D. Consort, 1997, Supporting Document for the Historical Tank Content Estimate for AN Tank Farm, WHC-SD-WM-ER-314, Rev. 0, ICF Kaiser Hanford Company, Richland, Washington.

Harding, R. A., 1996, "AN-103, 104, and 105 Tank Surface Scan," Videograph \#200-1470, Westinghouse Hanford Company, Richland, Washington.

Hanlon, B. M., 1997, Waste Tank Summary Report for Month Ending March 31, 1997, WHC-EP-0182-108, Lockheed Martin Hanford Corporation, Richland, Washington.

Johnson, M. G., 1995, Technical Bases for Leak Detection Surveillance of Waste Storage Tanks, WHC-SD-WM-TI-573, Rev. 1, Westinghouse Hanford Company, Richland, Washington.

KEH, 1996, Instrument Enraf-Nonius Assembly, Installation and Riser Schedule, Drawing H-2-817634, Rev. 3, Kaiser Engineers Hanford Company, Richland, Washington.

Lipnicki, J., 1997, Waste Tank Risers Available for Sampling, WHC-SD-WM-TI-710, Rev. 4, Westinghouse Hanford Company, Richland, Washington.

LMHC, 1997, Surveillance Analysis Computer System database, In:SQL Server/Visual Basic [Mainframe], available: HLAN, Lockheed Martin Hanford Company, Richland, Washington.

RHO, 1983, Manual Liquid Level Tape - Primary Tank, Drawing H-2-92324, Rev. 0, Rockwell Hanford Operations, Richland, Washington.

Salazar, B. E., 1994, Double-Shell Underground Waste Storage Tanks Riser Survey, WHC-SD-RE-TI-093, Rev, 4, Westinghouse Hanford Company, Richland, Washington. 
Tran, T. T., 1993, Thermocouple Status Single-Shell \& Double-Shell Waste Tanks, WHC-SD-WM-TI-553, Rev. 0, Westinghouse Hanford Company, Richland, Washington.

WHC, 1995a, Electrical Power and Control Plans and Details, Drawing H-2-71925, Rev. 5, Westinghouse Hanford Company, Richland, Washington.

WHC, 1995b, AN Farm In-Tank Video System Installation, Drawing H-2-822810, Rev. 0, Westinghouse Hanford Company, Richland, Washington.

WHC, 1995c, Supplemental Change to Drawings to Install 3 Multifunctional Instrument Trees in 103AN, 104AN, \& 105AN, ECN-624472, Westinghouse Hanford Company, Richland, Washington.

WHC, 1996a, Piping Plan Tank 103, Drawing H-2-71993, Rev. 8, Westinghouse Hanford Company, Richland, Washington.

WHC, 1996b, Plan Tank Penetrations Tanks 101 - 106, Drawing H-14-010501, Rev. 0 , Westinghouse Hanford Company, Richland, Washington. 
HNF-SD-WM-ER-702 Rev. 0

\section{APPENDIX B}

SAMPLING OF TANK 241-AN-103 
HNF-SD-WM-ER-702 Rev. 0

This page intentionally left blank. 


\section{APPENDIX B \\ SAMPLING OF TANK 241-AN-103}

Appendix B provides sampling and analysis information for each known sampling event for tank 241-AN-103 and provides an assessment of the push core sample results.

- Section B1: Tank Sampling Overview

- Section B2: Sampling Events

B2.1: 1996 Push Core Samples

B2.2: 1995 Vapor Phase Measurement

B2.3: Historical samples

- $\quad$ Section B3: Assessment of Characterization Results

- Section B4: References for Appendix B.

Future sampling of tank 241-AN-103 will be appended to the above list.

\section{B1.0 TANK SAMPLING OVERVIEW}

This section describes the September 1996 sampling and analysis events for tank 241-AN-103. Push core samples were taken to satisfy the requirements of the Tank Safety Screening Data Quality Objective (Dukelow et al. 1995), and the Flammable Gas Safety Program: Data Requirements for Core Sample Analysis Developed through the Data Quality Objectives (DQO) Process (McDuffie and Johnson 1995). The sampling and analyses were performed in accordance with the Tank 241-AN-103 Push Mode Core Sampling and Analysis Plan (Kruger 1996). In addition, the Organic Solvent Screening Issue (DOE-RL 1996, Cash 1996a) and privatization LAW DQO (Jones and Wiemers 1996) apply to this tank. Vapor samples to address the organic solvent screening DQO are scheduled to be obtained in FY 1998. The Privatization LAW DQO has been applied since the 1996 push core sampling event.

Sampling and analytical requirements for tank 241-AN-103 are summarized in Table B1-1. 
Table B1-1. Integrated Data Quality Objective Requirements for Tank 241-AN-103.1

\begin{tabular}{|l|l|l|l|}
\hline Vapor sampling & $\begin{array}{l}\text {-Organic Solvent } \\
\text { Screening } \\
\text { (DOE-RL 1996a, } \\
\text { Cash 1996b) }\end{array}$ & Steel canisters & Organic Vapors \\
\hline $\begin{array}{l}\text { Push mode core } \\
\text { sample }\end{array}$ & $\begin{array}{l}\text {-Safety Screening } \\
\text { DQO } \\
\text {-Flammable Gas } \\
\text { DQO } \\
\text {-Privatization } \\
\text { LAW DQO }\end{array}$ & $\begin{array}{l}\text { Push core samples from 2 risers } \\
\text { separated radially to the } \\
\text { maximum extent possible (Grab } \\
\text { samples may be used to obtailn } \\
\text { supernatant. }\end{array}$ & $\begin{array}{l}\text { Flammability, } \\
\text { Energetics, } \\
\text { Moisture, Total } \\
\text { alpha activity, } \\
\text { Anions, Cations, } \\
\text { Radionuclides, } \\
\text { Density, Physical } \\
\text { Combustible Gas Measurementies, pH, } \\
\text { Total Organic } \\
\text { Carbon, Total } \\
\text { Inorganic Carbon, } \\
\text { Cr(VI) }\end{array}$ \\
\hline
\end{tabular}

Notes:

'Kruger 1996

\section{B2.0 SAMPLING EVENTS}

Sampling events are described in this section. Analytical results are presented in Tables B2-5 through B2-70. These include 1996 push core sample results, vapor phase measurements from during sniff tests, and historical sample results.

In September 1995, an attempt was made to obtain grab samples. However, no sample could be obtained because of the thick saltcake crust in the tank.

\section{B2.1 1996 PUSH CORE SAMPLE}

Nineteen push mode core segments were removed from tank 241-AN-103 riser 12A (core 166) between September 13, 1996, and September 17, 1996. Segments were received by the 222-S Laboratory between September 20,1996, and September 30, 1996. Selected segments (2, 5, and 14) were sampled using the Retained Gas Sampler (RGS) and extruded by the Process Chemistry and Statistical Analysis Group. The sample jar for the drainable 
liquid portion of segment 4 was dropped and broken during the subsampling process, and the sample was not retrievable.

Eighteen push mode core segments were removed from tank 241-AN-103 riser 21A (core 167) between September 18, 1996, and September 23, 1996. Tank Farm Operations were unsuccessful in obtaining segment 19 due to the high downforce encountered during sampling. Segments were received by the 222-S Laboratory between September 23, 1996, and September 30,1996. Selected segments (10,13, 16 and 18) were sampled using the Retained Gas Sampler and extruded by the Process Chemistry and Statistical Analysis Group.

The delay between the field sampling and sample extrusion in the lab was about a month. Solids were found throughout the convective layer, and these solids probably precipitated due to cooling of the samples.

A field blank was provided to the 222-S Laboratory with core 166 . It underwent the same analysis as the drainable liquid as instructed by the TSAP (Kruger 1996).

Lithium bromide solution, used as hydrostatic head fluid during sampling, was provided to the 222-S Laboratory with core 166. It underwent Inductively Coupled Plasma Spectroscopy (ICP) and Ion Chromatography (IC) analyses as instructed by the TSAP (Kruger 1996).

Safety screening analyses include: total alpha to determine criticality, differential scanning calorimetry (DSC) to ascertain the fuel energy value, and thermogravimetric analysis (TGA) to obtain the total moisture content. In addition, combustible gas meter readings in the tank headspace were performed to measure flammability.

Tank 241-AN-103 also was evaluated for the Flammable Gas Tank Safety Program. The specified analyses include: DSC, TGA, bulk density, viscosity, void fraction, TIC, TOC, hydroxide, formate, oxalate, ICP, IC, and radiochemistry.

\section{B2.1.1 SAMPLE HANDLING}

The push core samples were shipped to the 222-S Laboratory for subsampling and analysis. Samples were assigned LABCORE numbers and were subjected to visual inspection for color, clarity, and solids content. The radiation dose rate on contact was also measured. The salt slurry samples were divided into upper and lower half-segments. The material was homogenized and subsampled for laboratory analyses and archiving. Subsamples of each half-segment were then recombined and subsampled for core composite analyses. Tables B1-2a and B1-2b gives the subsampling scheme and sample description for core 166 and 167 , respectively. 
Table B2-1a. Sample Receipt and Extrusion Information for Tank 241-AN-103, Core 166. (6 sheets)

\begin{tabular}{|c|c|c|c|c|c|c|c|c|}
\hline Sample. & $\operatorname{seg}$ & $\begin{array}{l}\text { Wate } \\
\text { Sampled }\end{array}$ & . & $\begin{array}{l}\text { Bate } \\
\text { Propudra }\end{array}$ & mavol & 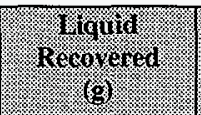 & 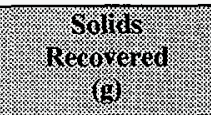 & Simple Descogoton \\
\hline $\begin{array}{l}\text { Field blank } \\
\left(\mathrm{H}_{2} \mathrm{O}\right)\end{array}$ & FB & $9 / 16 / 96$ & $9 / 27 / 96$ & $10 / 7 / 96$ & 0 & 252.0-Drainable & 0.0 & $\begin{array}{l}\text { The liquid was clear and } \\
\text { colorless. Collected } \\
250 \text { mL of liquid. No } \\
\text { organic layer was } \\
\text { observed. }\end{array}$ \\
\hline $96-445$ & 1 & $9 / 13 / 96$ & $9 / 26 / 96$ & $10 / 23 / 96$ & 2.5 & 0.0 & 58.0 - Lower half & $\begin{array}{l}\text { The solids were light } \\
\text { gray in color and } \\
\text { resembled a wet sait. }\end{array}$ \\
\hline $96-446$ & 2 & $9 / 13 / 96$ & $9 / 20 / 96$ & $n / a$ & $n / a$ & $\mathrm{n} / \mathrm{a}$ & $n / a$ & $\begin{array}{l}\text { This segment was } \\
\text { sampled using the } \\
\text { Retained Gas Sampler } \\
\text { and extruded by the } \\
\text { Process Chemistry and } \\
\text { Statistical Analysis } \\
\text { Group. }\end{array}$ \\
\hline $96-447$ & 3 & $9 / 13 / 96$ & $9 / 27 / 96$ & $10 / 21 / 96$ & 0.0 & 409.5-Drainable & 0.0 & $\begin{array}{l}\text { Collected } 260 \mathrm{~mL} \text { of } \\
\text { drainable liquid. The } \\
\text { liquid was blue-green in } \\
\text { color and opaque. No } \\
\text { organic layer was } \\
\text { observed. }\end{array}$ \\
\hline
\end{tabular}


Table B2-1a. Sample Receipt and Extrusion Information for Tank 241-AN-103, Core 166. (6 sheets)

\begin{tabular}{|c|c|c|c|c|c|c|c|c|}
\hline S dimpler & א. & finter & 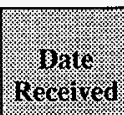 & 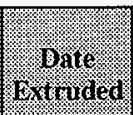 & $\begin{array}{l}\text { matis } \\
\text { manded }\end{array}$ & 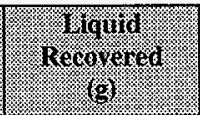 & gerovers! & Sample bescerpirin \\
\hline $96-448$ & 4 & $9 / 13 / 96$ & $9 / 26 / 96$ & $10 / 21 / 96$ & 2.0 & 368.6-Drainable & 64.2 - Lower half & $\begin{array}{l}\text { Collected } 250 \mathrm{~mL} \text { of } \\
\text { drainable liquid. The } \\
\text { liquid was blue-green in } \\
\text { color and opaque. The } \\
\text { solids were white in } \\
\text { color and resembled a } \\
\text { salt slurry. No organic } \\
\text { layer was observed: }\end{array}$ \\
\hline $96-449$ & 5 & $9 / 13 / 96$ & $9 / 20 / 96$ & $n / a$ & $n / a$ & $n / a$ & $n / a$ & $\begin{array}{l}\text { This segment was } \\
\text { sampled using the } \\
\text { Retained Gas Sampler } \\
\text { and extruded by the } \\
\text { Process Chemistry and } \\
\text { Statistical Analysis } \\
\text { Group. }\end{array}$ \\
\hline $96-450$ & 6 & $9 / 14 / 96$ & $9 / 26 / 96$ & $10 / 23 / 96$ & 2.0 & 357.8-Drainable & 62.9 - Lower half & $\begin{array}{l}\text { Collected } 250 \mathrm{~mL} \text { of } \\
\text { liquid. The liquid was } \\
\text { blue-green in color and } \\
\text { opaque. No organic } \\
\text { layer was observed. } \\
\text { The solids were white in } \\
\text { color and resembled a } \\
\text { salt slurry. }\end{array}$ \\
\hline
\end{tabular}


Table B2-1a. Sample Receipt and Extrusion Information for Tank 241-AN-103, Core 166. (6 sheets)

\begin{tabular}{|c|c|c|c|c|c|c|c|c|}
\hline Sample: & seg. & Sampleat & Recived & I Extroded & Inties & 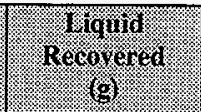 & 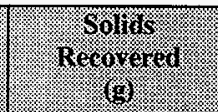 & Srmple Bestivition \\
\hline $96-451$ & 7 & $9 / 14 / 96$ & $9 / 26 / 96$ & $10 / 31 / 96$ & 4.0 & 368.9-Drainable & 64.7 - Lower half & $\begin{array}{l}\text { Collected } 250 \mathrm{~mL} \text { of } \\
\text { liquid. The liquid was } \\
\text { blue-green in color and } \\
\text { opaque. No organic } \\
\text { layer was observed. } \\
\text { The solids were white in } \\
\text { color and resembled a } \\
\text { salt slurry. }\end{array}$ \\
\hline $96-452$ & 8 & $9 / 14 / 96$ & $9 / 26 / 96$ & $10 / 31 / 96$ & 4.0 & 370.4-Drainable & 48.1 - Lower half & $\begin{array}{l}\text { Collected } 250 \mathrm{~mL} \text { of } \\
\text { liquid. The liquid was } \\
\text { blue-green in color and } \\
\text { opaque. No organic } \\
\text { layer was observed. } \\
\text { The solids were white in } \\
\text { color and resembled a } \\
\text { salt slurry. }\end{array}$ \\
\hline $96-453$ & 9 & $9 / 14 / 96$ & $9 / 26 / 96$ & $10 / 21 / 96$ & 3.0 & 383.4-Drainable & 59.5 - Lower half & $\begin{array}{l}\text { Collected } 260 \mathrm{~mL} \text { of } \\
\text { liquid. The liquid was } \\
\text { blue-green in color and } \\
\text { opaque. No organic } \\
\text { layer was observed. } \\
\text { The solids were white in } \\
\text { color and resembled a } \\
\text { salt slurry. }\end{array}$ \\
\hline
\end{tabular}


Table B2-1a. Sample Receipt and Extrusion Information for Tank 241-AN-103, Core 166. (6 sheets)

\begin{tabular}{|c|c|c|c|c|c|c|c|c|}
\hline Sample. & Seg. & 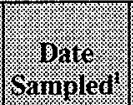 & mares & orito & $\begin{array}{l}\text { moros } \\
\text { tromied }\end{array}$ & 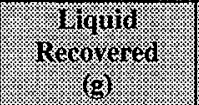 & 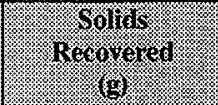 & 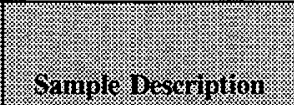 \\
\hline $96-454$ & 10 & $9 / 14 / 96$ & $9 / 26 / 96$ & $10 / 21 / 96$ & 2.0 & 387.5-Drainable & 58.1 - Lower half & $\begin{array}{l}\text { Collected } 260 \mathrm{~mL} \text { of } \\
\text { liquid. The liquid was } \\
\text { blue-green in color and } \\
\text { opaque. No organic } \\
\text { layer was observed. } \\
\text { The solids were white in } \\
\text { color and resembled a } \\
\text { salt slurry. }\end{array}$ \\
\hline $96-455$ & 11 & $9 / 14 / 96$ & $9 / 30 / 96$ & $10 / 23 / 96$ & 1.0 & 360.1-Drainable & 48.8 - Lower half & $\begin{array}{l}\text { Collected } 260 \mathrm{~mL} \text { of } \\
\text { liquid. The liquid was } \\
\text { blue-green in color and } \\
\text { opaque. No organic } \\
\text { layer was observed. } \\
\text { The solids were white in } \\
\text { color and resembled a } \\
\text { salt slurry. }\end{array}$ \\
\hline $96-456$ & 12 & $9 / 14 / 96$ & $9 / 30 / 96$ & $10 / 23 / 96$ & 3.0 & 344.0-Drainable & 59.9 - Lower half & $\begin{array}{l}\text { Collected } 250 \mathrm{~mL} \text { of } \\
\text { liquid. The liquid was } \\
\text { blue-green in color and } \\
\text { opaque. No organic } \\
\text { layer was observed. } \\
\text { The solids were white in } \\
\text { color and resembled a } \\
\text { salt slurry. }\end{array}$ \\
\hline
\end{tabular}


Table B2-1a. Sample Receipt and Extrusion Information for Tank 241-AN-103, Core 166. (6 sheets)

\begin{tabular}{|c|c|c|c|c|c|c|c|c|}
\hline hominger. & א. & (6anpled & 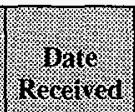 & 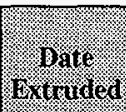 & 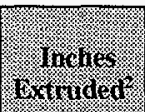 & Reromerod & 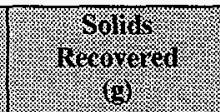 & 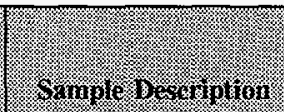 \\
\hline $96-457$ & 13 & $9 / 14 / 96$ & $9 / 30 / 96$ & $10 / 26 / 96$ & 19.0 & 0.0 & $\begin{array}{l}\text { 235.7-Upper half } \\
\text { 216.5-Lower half }\end{array}$ & $\begin{array}{l}\text { The solids were light } \\
\text { gray in color and } \\
\text { resembled a wet salt. }\end{array}$ \\
\hline $96-458$ & 14 & $9 / 16 / 96$ & $9 / 23 / 96$ & $n / a$ & $n / a$ & $n / a$ & $n / a$ & $\begin{array}{l}\text { This segment was } \\
\text { sampled using the } \\
\text { Retained Gas Sampler } \\
\text { and extruded by the } \\
\text { Process Chemistry and } \\
\text { Statistical Analysis } \\
\text { Group. }\end{array}$ \\
\hline $96-459$ & 15 & $9 / 16 / 96$ & $9 / 27 / 96$ & $10 / 7 / 96$ & 19.0 & 0.0 & $\begin{array}{l}\text { 240.6-Upper half } \\
\text { 216.8-Lower half }\end{array}$ & $\begin{array}{l}\text { The solids were light } \\
\text { gray in color and } \\
\text { resembled a wet salt. }\end{array}$ \\
\hline $96-460$ & 16 & $9 / 16 / 96$ & $9 / 30 / 96$ & $10 / 23 / 96$ & 19.0 & 0.0 & $\begin{array}{l}\text { 226.9-Upper half } \\
\text { 208.4-Lower half }\end{array}$ & $\begin{array}{l}\text { The solids were light } \\
\text { gray in color and } \\
\text { resembled a moist salt. }\end{array}$ \\
\hline $96-461$ & 17 & $9 / 16 / 96$ & $9 / 27 / 96$ & $10 / 7 / 96$ & 19.0 & 0.0 & $\begin{array}{l}\text { 201.8-Upper half } \\
\text { 202.8-Lower half }\end{array}$ & $\begin{array}{l}\text { The solids were light } \\
\text { gray in color and } \\
\text { resembled a moist salt. }\end{array}$ \\
\hline $96-462$ & 18 & $9 / 16 / 96$ & $9 / 26 / 96$ & $10 / 24 / 96$ & 19.0 & 0.0 & $\begin{array}{l}\text { 217.1-Upper half } \\
\text { 198.4-Lower half }\end{array}$ & $\begin{array}{l}\text { The solids were light } \\
\tan \text {-gray in color and } \\
\text { resembled a moist salt. }\end{array}$ \\
\hline
\end{tabular}


Table B2-1a. Sample Receipt and Extrusion Information for Tank 241-AN-103, Core 166. (6 sheets)

\begin{tabular}{|c|c|c|c|c|c|c|c|c|}
\hline $\begin{array}{l}\text { Samples } \\
\text { Number }\end{array}$ & St: & $\begin{array}{l}\text { Date } \\
\text { Sampled }\end{array}$ & $\begin{array}{l}\text { Oates } \\
\text { Received }\end{array}$ & Extrutad & Wamider? & $\begin{array}{l}\text { Mgurd } \\
\text { Requgered } \\
(0)\end{array}$ & $\begin{array}{l}\text { Soliais } \\
\text { Rracovered }\end{array}$ & Sampre Bescribtion \\
\hline $96-463$ & 19 & $9 / 17 / 96$ & $9 / 26 / 96$ & $10 / 31 / 96$ & 19.0 & 0.0 & $\begin{array}{l}\text { 216.9-Upper half } \\
\text { 173.7-Lower half }\end{array}$ & $\begin{array}{l}\text { The solids were gray in } \\
\text { color and resembled a } \\
\text { salt slurry. }\end{array}$ \\
\hline
\end{tabular}

Notes:

$\mathrm{n} / \mathrm{a}=$ not appiticable

Seg. $=$ segment

'Dates are provided in the $\mathrm{mm} / \mathrm{dd} / \mathrm{yy}$ format.

$\stackrel{\square}{\vdots}$

${ }^{2}$ Approximate inches extruded. 


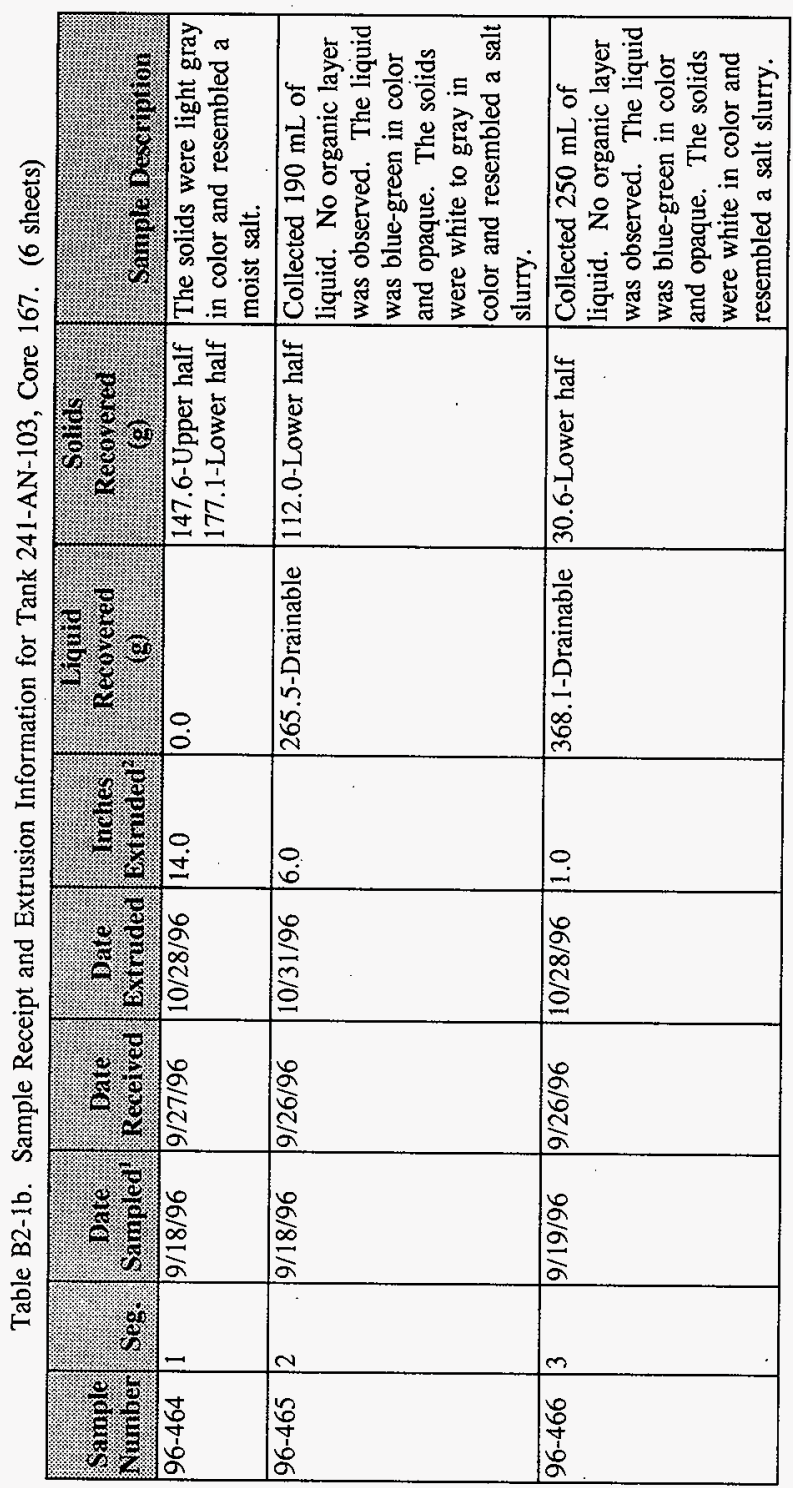


Table B2-1b. Sample Receipt and Extrusion Information for Tank 241-AN-103, Core 167. (6 sheets)

\begin{tabular}{|c|c|c|c|c|c|c|c|c|}
\hline Saming: & seg. & Sale. & Recentrit & Brate & 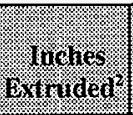 & (1) & 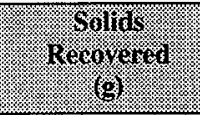 & 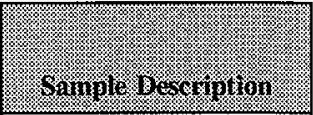 \\
\hline $96-467$ & 4 & $9 / 19 / 96$ & $9 / 26 / 96$ & $10 / 28 / 96$ & 1.0 & 359.9-Drainable & 29.9-Lower half & $\begin{array}{l}\text { Collected } 250 \mathrm{~mL} \text { of } \\
\text { liquid. No organic layer } \\
\text { was observed. The liquid } \\
\text { was blue-green in color } \\
\text { and opaque. The jar for } \\
\text { the drainable liquid } \\
\text { portion was dropped and } \\
\text { broken during the } \\
\text { subsampling process. } \\
\text { The solids were white in } \\
\text { color and resembled a salt } \\
\text { slurry. }\end{array}$ \\
\hline
\end{tabular}


Table B2-1b. Sample Receipt and Extrusion Information for Tank 241-AN-103, Core 167. (6 sheets)

\begin{tabular}{|c|c|c|c|c|c|c|c|c|}
\hline 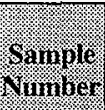 & Seg. & Sampied? & Bectired & 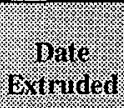 & 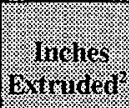 & 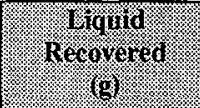 & Rritis: & 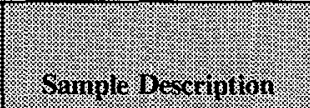 \\
\hline $96-469$ & 6 & $9 / 19 / 96$ & $9 / 27 / 96$ & $10 / 7 / 96$ & 3.0 & 348.6-Drainable & 74.6-Lower half & $\begin{array}{l}\text { Collected } 250 \mathrm{~mL} \text { of } \\
\text { liquid. No organic layer } \\
\text { was observed. The liquid } \\
\text { was blue-green in color } \\
\text { and opaque. The solids } \\
\text { were white in color and } \\
\text { resembled a wet salt. }\end{array}$ \\
\hline $96-470$ & 7 & $9 / 19 / 96$ & $9 / 27 / 96$ & $10 / 28 / 96$ & 2.0 & 370.8-Drainable & 42.7-Lower half & $\begin{array}{l}\text { Collected } 250 \mathrm{~mL} \text { of } \\
\text { liquid. No organic layer } \\
\text { was observed. The liquid } \\
\text { was blue-green in color } \\
\text { and opaque. The solids } \\
\text { were white in color and } \\
\text { resembled a salt slurry. }\end{array}$ \\
\hline $96-471$ & 8 & $9 / 19 / 96$ & $9 / 27 / 96$ & $10 / 29 / 96$ & 1.5 & 378.0-Drainable & 44.7-Lower half & $\begin{array}{l}\text { Collected } 260 \mathrm{~mL} \text { of } \\
\text { liquid. No organic layer } \\
\text { was observed. The liquid } \\
\text { was blue-green in color } \\
\text { and opaque. The solids } \\
\text { were gray in color and } \\
\text { resembled a salt slurry. }\end{array}$ \\
\hline
\end{tabular}


Table B2-1b. Sample Receipt and Extrusion Information for Tank 241-AN-103, Core 167. (6 sheets)

\begin{tabular}{|c|c|c|c|c|c|c|c|c|}
\hline Sample & seg. & U Sampled? & Dater & $\begin{array}{l}\text { Date } \\
\text { Extmidea }\end{array}$ & $\int$ Eximiries & 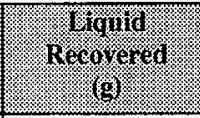 & ( & S Sample Description \\
\hline $96-472$ & 9 & $9 / 20 / 96$ & 9/26/96 & $10 / 31 / 96$ & 5.5 & 363.8-Drainable & 64.1-Lower half & $\begin{array}{l}\text { Collected } 250 \mathrm{~mL} \text { of } \\
\text { liquid. No organic layer } \\
\text { was observed. The liquid } \\
\text { was blue-green in color } \\
\text { and opaque. The solids } \\
\text { were white in color and } \\
\text { resembled a salt slurry. }\end{array}$ \\
\hline $96-473$ & 10 & $9 / 20 / 96$ & 9/24/96 & $n / a$ & $\mathrm{n} / \mathrm{a}$ & n/a & $\mathrm{n} / \mathrm{a}$ & $\begin{array}{l}\text { This segment was } \\
\text { sampled using the } \\
\text { Retained Gas Sampler and } \\
\text { extruded by the Process } \\
\text { Chemistry and Statistical } \\
\text { Analysis Group. }\end{array}$ \\
\hline $96-474$ & 11 & 9/20/96 & $9 / 26 / 96$ & $10 / 24 / 96$ & 16.0 & 52.5-Drainable & $\begin{array}{l}\text { 212.4-Upper half } \\
\text { 162.7-Lower half }\end{array}$ & $\begin{array}{l}\text { Collected } 40 \mathrm{~mL} \text { of } \\
\text { liquid. The liquid was } \\
\text { gray in color and opaque. } \\
\text { No organic layer was } \\
\text { observed. The solids } \\
\text { were light gray in color } \\
\text { and resembled a wet salt. }\end{array}$ \\
\hline $96-475$ & 12 & $9 / 20 / 96$ & $9 / 27 / 96$ & $10 / 2 / 96$ & 18.0 & 0.0 & $\begin{array}{l}\text { 270.3-Upper half } \\
\text { 181.7-Lower half }\end{array}$ & $\begin{array}{l}\text { The solids were light gray } \\
\text { in color and resembled a } \\
\text { salt slurry. }\end{array}$ \\
\hline
\end{tabular}


Table B2-1b. Sample Receipt and Extrusion Information for Tank 241-AN-103, Core 167. (6 sheets)

\begin{tabular}{|c|c|c|c|c|c|c|c|c|}
\hline Sumple & Seg & Sariol & 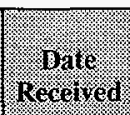 & 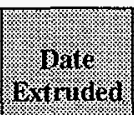 & 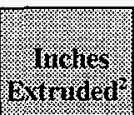 & 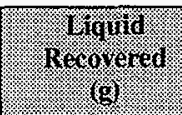 & 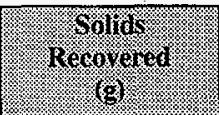 & 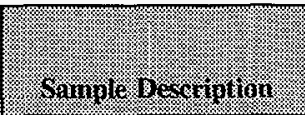 \\
\hline $96-476$ & 13 & $9 / 20 / 96$ & $9 / 23 / 96$ & $\mathrm{n} / \mathrm{a}$ & $n / a$ & $\mathrm{n} / \mathrm{a}$ & $n / a$ & $\begin{array}{l}\text { This segment was } \\
\text { sampled using the } \\
\text { Retained Gas Sampler and } \\
\text { extruded by the Process } \\
\text { Chemistry and Statistical } \\
\text { Analysis Group. }\end{array}$ \\
\hline $96-477$ & 14 & $9 / 20 / 96$ & $9 / 30 / 96$ & $10 / 7 / 96$ & 15.0 & 0.0 & $\begin{array}{l}\text { 210.8-Lower half } \\
\text { 195.1-Upper half }\end{array}$ & $\begin{array}{l}\text { The solids were light gray } \\
\text { in color and resembled a } \\
\text { wet salt. }\end{array}$ \\
\hline $96-478$ & 15 & $9 / 20 / 96$ & $9 / 27 / 96$ & $10 / 11 / 96$ & 18.0 & 0.0 & $\begin{array}{l}\text { 237.7-Upper half } \\
\text { 206.9-Lower half }\end{array}$ & $\begin{array}{l}\text { The solids were gray in } \\
\text { color and resembled a } \\
\text { moist salt. }\end{array}$ \\
\hline $96-479$ & 16 & $9 / 20 / 96$ & $9 / 24 / 96$ & $n / a$ & $\mathrm{n} / \mathrm{a}$ & $n / a$ & $n / a$ & $\begin{array}{l}\text { This segment was } \\
\text { sampled using the } \\
\text { Retained Gas Sampler and } \\
\text { extruded by the Process } \\
\text { Chemistry and Statistical } \\
\text { Analysis Group. }\end{array}$ \\
\hline $96-480$ & 17 & $9 / 20 / 96$ & $9 / 30 / 96$ & $10 / 15 / 96$ & 14.0 & 0.0 & $\begin{array}{l}\text { 154.5-Upper half } \\
\text { 144.3-Lower half }\end{array}$ & $\begin{array}{l}\text { The solids were light gray } \\
\text { in color and resembled a } \\
\text { moist salt. }\end{array}$ \\
\hline
\end{tabular}


Table B2-1b. Sample Receipt and Extrusion Information for Tank 241-AN-103, Core 167. (6 sheets)

\begin{tabular}{|c|c|c|c|c|c|c|c|c|}
\hline Sample & Seg: & Sumpled & Rectived & Extrided & Hoxmuded & 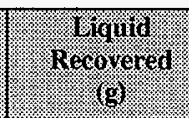 & 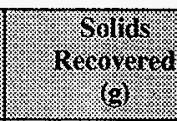 & Sample Desctiption \\
\hline $96-481$ & 18 & 9/23/96 & $9 / 24 / 96$ & n/a & $n / a$ & $n / a$ & $n / a$ & $\begin{array}{l}\text { This segment was } \\
\text { sampled using the } \\
\text { Retained Gas Sampler and } \\
\text { extruded by the Process } \\
\text { Chemistry and Statistical } \\
\text { Analysis Group. }\end{array}$ \\
\hline
\end{tabular}

Notes:

'Dates are provided in the $\mathrm{mm} / \mathrm{dd} / \mathrm{yy}$ format.

'Approximate inches extruded. 


\section{B2.1.2 SAMPLE ANALYSIS}

The analyses performed on the push core samples were limited to those required by the safety screening $D Q O$ and the flammable gas DQO. The analyses required by the safety screening DQO included analyses for thermal properties by DSC, moisture content by TGA, and content of fissile material by total alpha activity analysis. The flammable gas DQO required a full set of analytes to be analyzed by IC and ICP as well as TOC, TIC and various radionuclides.

Differential scanning calorimetry and TGA were performed on $7.887-\mathrm{mg}$ to $61.272-\mathrm{mg}$ samples. Quality control tests included performing the analyses in duplicate, and the use of standards. Moisture content was also measured by a gravimetric method.

Total alpha activity measurements were performed on samples that had been fused in a matrix of potassium hydroxide and then dissolved in acid. The resulting solution was then dried on a counting planchet and counted in an alpha proportional counter. Quality control tests included standards, spikes, blanks, and duplicate analyses.

Ion chromatography was performed on samples that had been prepared by water digestion. Quality control tests included standards, spikes, blanks, and duplicate analyses. The sampling and analysis plan (SAP) required that the full suite of IC analytes be measured.

Inductively coupled plasma spectrometry was performed on samples that had been prepared by a fusion procedure, followed by dissolution in acid. Quality control tests included standards, blanks, spikes, and duplicate analyses. The SAP required that the full suite of ICP elements be analyzed.

All reported analyses were performed in accordance with approved laboratory procedures. A list of the sample numbers and applicable analyses is presented in Table B2-2. The sample preparation procedure numbers and analysis procedure numbers are presented in Table B2-3. 


\begin{tabular}{|c|c|c|c|c|}
\hline 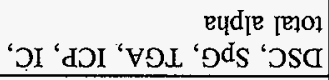 & 918S00L96S & p!̣nb!̣ & pụnb!̣l əqeu!̣exa & $L: 991$ \\
\hline $\mathrm{OI}$ & $\varepsilon \neq 8500 \mathrm{~L} 96 \mathrm{~S}$ & \multirow[b]{4}{*}{ sptros } & \multirow[b]{4}{*}{ I[ЕЧ دОМОТ } & \multirow[b]{4}{*}{$L: 991$} \\
\hline dDI & $6 \varepsilon 8500 \mathrm{~L} 96 \mathrm{~S}$ & & & \\
\hline eudfe [eרol'dor & SE8S00L96S & & & \\
\hline VDL'DSC & $028 S 00 L 96 S$ & & & \\
\hline 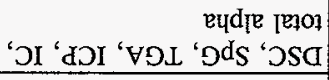 & $66 \mathrm{SS} 00 \mathrm{~L} 96 \mathrm{~S}$ & pinbị & 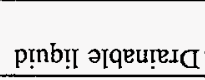 & 9:991 \\
\hline गI & 6I9\$00L96S & \multirow[b]{4}{*}{ SpIIOS } & \multirow[b]{4}{*}{ গ[еч гәмот } & \multirow[b]{4}{*}{ 9:99I } \\
\hline dDI & SI9S00L96S & & & \\
\hline eųdre [e]01'dDI & I I9S00L96S & & & \\
\hline VOL'OSA & 6LSS00L96S & & & \\
\hline SOY & & & & s:99I \\
\hline כI & E9I000LL6S & \multirow[b]{2}{*}{ pụnby } & \multirow[b]{2}{*}{ pịnb!̣ әqвuाexa } & \multirow[b]{2}{*}{$\forall: 99 I$} \\
\hline 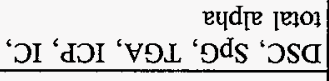 & $0 t \subseteq \$ 00 L 96 \mathrm{~S}$ & & & \\
\hline DI & ZLSS00.L96S & \multirow[b]{4}{*}{ spịos } & \multirow[b]{4}{*}{ IןE хәмоТ } & \multirow[b]{4}{*}{$\downarrow: 991$} \\
\hline dכI & $0 L S \$ 00 L 96 \mathrm{~S}$ & & & \\
\hline eYdIF IETOL 'dDI & 89SS00L96S & & & \\
\hline VDI ‘OS & $z+S S 00 \mathrm{~L} 96 \mathrm{~S}$ & & & \\
\hline 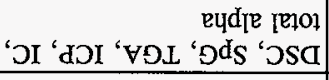 & 6ESS00L96S & p!nb!̃ & p̣̣nb!̣া ә|qвu!̣exa & $\varepsilon: 991$ \\
\hline SOd & & & & $z: 99 \mathrm{I}$ \\
\hline OI & 0I9S00L96S & & & \\
\hline dOI & 609 S00L96S & & & \\
\hline zudrV [E]OL 'dDI & $809500 \perp 96 \mathrm{~S}$ & & & \\
\hline VOL 'OSA & $8 L S S 00 \mathrm{~L} 96 \mathrm{~S}$ & spt!os & J[Еप دәMOT & [:99[ \\
\hline Saripul: & magringe & $x+19410190$ & 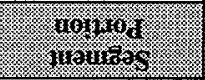 & 1970180 \\
\hline
\end{tabular}

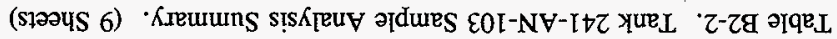


HNF-SD-WM-ER-702 Rev. 0

Table B2-2. Tank 241-AN-103 Sample Analysis Summary. (9 Sheets)

\begin{tabular}{|c|c|c|c|c|}
\hline 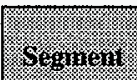 & $\begin{array}{l}\text { Signenit } \\
\text { eqution: }\end{array}$ & 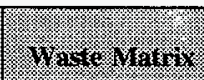 & Sumple & : \\
\hline \multirow[t]{4}{*}{$166: 8$} & \multirow[t]{4}{*}{ Lower half } & \multirow[t]{4}{*}{ Solids } & S96T005821 & DSC, TGA \\
\hline & & & S96T005836 & ICP, total alpha \\
\hline & & & S96T005840 & $\overline{I C P}$ \\
\hline & & & S96T005844 & IC \\
\hline $166: 8$ & Drainable liquid & Liquid & S96T005817 & $\begin{array}{l}\text { DSC, SpG, TGA, ICP, IC, } \\
\text { total alpha }\end{array}$ \\
\hline \multirow[t]{4}{*}{$166: 9$} & \multirow[t]{4}{*}{ Lower half } & \multirow[t]{4}{*}{ Solids } & S96T005543 & DSC, TGA \\
\hline & & & S96T005574 & ICP, total alpha \\
\hline & & & S96T005575 & $\mathrm{ICP}$ \\
\hline & & & S96T005576 & $\mathrm{IC}$ \\
\hline $166: 9$ & Drainable liquid & Liquid & S96T005541 & $\begin{array}{l}\text { DSC, SpG, TGA, ICP, IC, } \\
\text { total alpha }\end{array}$ \\
\hline \multirow[t]{4}{*}{ 166: 10} & \multirow[t]{4}{*}{ Lower half } & \multirow[t]{4}{*}{ Solids } & S96T005564 & $\mathrm{DSC}, \mathrm{TGA}$ \\
\hline & & & S96T005569 & ICP, total alpha \\
\hline & & & S96T005571 & ICP \\
\hline & & & S96T005573 & IC \\
\hline 166: 10 & Drainable liquid & Liquid & S96T005566 & $\begin{array}{l}\mathrm{DSC}, \mathrm{SpG}, \mathrm{TGA}, \mathrm{ICP}, \mathrm{IC} \text {, } \\
\text { total alpha }\end{array}$ \\
\hline \multirow[t]{5}{*}{ 166: 11} & \multirow[t]{5}{*}{ Lower half } & \multirow[t]{5}{*}{ Solids } & S96T005580 & DSC, TGA \\
\hline & & & S96T005586 & bulk density \\
\hline & & & S96T005612 & ICP, total alpha \\
\hline & & & S96T005616 & $\mathrm{ICP}$ \\
\hline & & & S96T005620 & IC \\
\hline 166: 11 & Drainable liquid & Liquid & S96T005600 & $\begin{array}{l}\text { DSC, TGA, SpG, ICP, IC, } \\
\text { total alpha }\end{array}$ \\
\hline \multirow[t]{4}{*}{ 166: 12} & \multirow[t]{4}{*}{ Lower half } & \multirow[t]{4}{*}{ Solids } & S96T005581 & DSC, TGA \\
\hline & & & S96T005613 & ICP, total alpha \\
\hline & & & S96T005617 & ICP \\
\hline & & & S96T005621 & IC \\
\hline
\end{tabular}


HNF-SD-WM-ER-702 Rev. 0

Table B2-2. Tank 241-AN-103 Sample Analysis Summary. ( 9 Sheets)

\begin{tabular}{|c|c|c|c|c|}
\hline sienging & 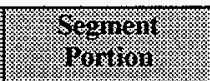 & 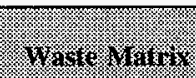 & 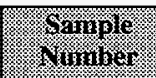 & 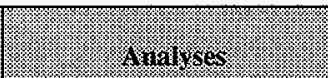 \\
\hline 166: 12 & Drainable liquid & Liquid & S96T005601 & $\begin{array}{l}\text { DSC, SpG, TGA, ICP, IC, } \\
\text { total alpha }\end{array}$ \\
\hline \multirow[t]{5}{*}{$166: 13$} & \multirow[t]{5}{*}{ Upper half } & \multirow[t]{5}{*}{ Solids } & \$96T005629 & bulk density \\
\hline & & & S96T005636 & $\mathrm{DSC}, \mathrm{TGA}$ \\
\hline & & & S96T005714 & ICP \\
\hline & & & S96T005716 & ICP \\
\hline & & & S96T005718 & IC \\
\hline \multirow{5}{*}{ 166: 13} & \multirow[t]{5}{*}{ Lower half } & \multirow[t]{5}{*}{ Solids } & \$96T005632 & bulk density \\
\hline & & & $\$ 96 \mathrm{~T} 005637$ & DSC, TGA \\
\hline & & & \$96T005720 & ICP, total alpha \\
\hline & & & S96T005722 & ICP \\
\hline & & & S96T005724 & IC \\
\hline $166: 14$ & & & & RGS \\
\hline \multirow[t]{5}{*}{$166: 15$} & \multirow[t]{5}{*}{ Upper half } & \multirow[t]{5}{*}{ Solids } & S96T005371 & bulk density \\
\hline & & & S96T005398 & DSC, TGA \\
\hline & & & S96T005414 & ICP \\
\hline & & & S96T005416 & ICP \\
\hline & & & \$96T005418 & IC \\
\hline \multirow[t]{5}{*}{$166: 15$} & \multirow[t]{5}{*}{ Lower half } & \multirow[t]{5}{*}{ Solids } & \$96T005369 & bulk density \\
\hline & & & \$96T005399 & DSC, TGA \\
\hline & & & S96T005408 & ICP, total alpha \\
\hline & & & \$96T005412 & IC \\
\hline & & & S97T000459 & ICP \\
\hline \multirow[t]{5}{*}{$166: 16$} & \multirow[t]{5}{*}{ Upper half } & \multirow[t]{5}{*}{ Solids } & S96T005582 & DSC, TGA \\
\hline & & & S96T005588 & bulk density \\
\hline & & & S96T005623 & $\mathrm{ICP}$ \\
\hline & & & S96T005624 & ICP \\
\hline & & & S96T005625 & IC \\
\hline
\end{tabular}


Table B2-2. Tank 241-AN-103 Sample Analysis Summary. (9 Sheets)

\begin{tabular}{|c|c|c|c|c|}
\hline Scginient & $\begin{array}{l}\text { Segment } \\
\text { Tonitor }\end{array}$ & W & Sample & andilses \\
\hline \multirow[t]{5}{*}{$166: 16$} & \multirow[t]{5}{*}{ Lower half } & \multirow[t]{5}{*}{ Solids } & S96T005583 & DSC, TGA \\
\hline & & & S96T005589 & bulk density \\
\hline & & & $\overline{\text { S96T005614 }}$ & ICP, total alpha \\
\hline & & & \$96T005618 & ICP \\
\hline & & & S96T005622 & IC \\
\hline \multirow[t]{5}{*}{$166: 17$} & \multirow[t]{5}{*}{ Upper half } & \multirow[t]{5}{*}{ Solids } & S96T005372 & bulk density \\
\hline & & & S96T005400 & DSC, TGA \\
\hline & & & S96T005415 & $\mathrm{ICP}$ \\
\hline & & & S96T005417 & ICP \\
\hline & & & S96T005419 & IC \\
\hline \multirow[t]{5}{*}{ 166: 17} & \multirow[t]{5}{*}{ Lower half } & \multirow[t]{5}{*}{ Solids } & S96T005370 & bulk density \\
\hline & & & S96T005401 & DSC, TGA \\
\hline & & & S96T005409 & ICP, total alpha \\
\hline & & & S96T005411 & ICP \\
\hline & & & S96T005413 & IC \\
\hline \multirow[t]{5}{*}{ 166: 18} & \multirow[t]{5}{*}{ Upper half } & \multirow[t]{5}{*}{ Solids } & S96T005631 & bulk density \\
\hline & & & S96T005638 & DSC, TGA \\
\hline & & & S96T005715 & ICP \\
\hline & & & S96T005717 & ICP \\
\hline & & & S96T005719 & IC \\
\hline \multirow[t]{5}{*}{$166: 18$} & \multirow[t]{5}{*}{ Lower half } & \multirow[t]{5}{*}{ Solids } & \$96T005634 & bulk density \\
\hline & & & S96T005639 & DSC, TGA \\
\hline & & & S96T005721 & ICP \\
\hline & & & S96T005723 & ICP \\
\hline & & & S96T005725 & IC \\
\hline \multirow[t]{5}{*}{ 166: 19} & \multirow[t]{5}{*}{ Upper half } & \multirow[t]{5}{*}{ Solids } & S96T005812 & bulk density \\
\hline & & & S96T005822 & DSC, TGA \\
\hline & & & S96T005837 & ICP \\
\hline & & & S96T005841 & $\overline{I C P}$ \\
\hline & & & S96T005845 & IC \\
\hline
\end{tabular}


Table B2-2: Tank 241-AN-103 Sample Analysis Summary. (9 Sheets)

\begin{tabular}{|c|c|c|c|c|}
\hline Seganen & (3) & 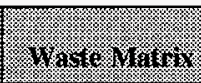 & hampos & angalyses \\
\hline \multirow[t]{5}{*}{ 166: 19} & \multirow[t]{5}{*}{ Lower half } & \multirow[t]{5}{*}{ Solids } & \$96T005813 & bulk density \\
\hline & & & \$96T005823 & DSC, TGA \\
\hline & & & S96T005838 & ICP \\
\hline & & & S96T005842 & ICP \\
\hline & & & S96T005846 & $\mathrm{IC}$ \\
\hline \multirow[t]{6}{*}{166} & \multirow[t]{6}{*}{ Core composite } & \multirow[t]{6}{*}{ Solids } & S96T005983 & bulk density \\
\hline & & & S96T005984 & DSC, TGA, TIC, TOC \\
\hline & & & S96T005986 & $\begin{array}{l}{ }^{99} \mathrm{Tc},{ }^{89 / 90} \mathrm{Sr},{ }^{239 / 240} \mathrm{Pu}, \mathrm{GEA}, \\
{ }^{241} \mathrm{Am},{ }^{243 / 244} \mathrm{Cm} \text {, total } \\
\text { alpha, total beta }\end{array}$ \\
\hline & & & S96T005988 & $\mathrm{Cr}(\mathrm{VI}), \mathrm{OH}$ \\
\hline & & & S97T000023 & $\mathrm{IC}, \mathrm{I} 129, \mathrm{H} 3$ \\
\hline & & & $\$ 97 T 000460$ & ICP \\
\hline \multirow[t]{6}{*}{166} & \multirow{6}{*}{$\begin{array}{l}\text { Drainable liquid } \\
\text { composite }\end{array}$} & \multirow[t]{6}{*}{ Liquid } & S96T005990 & DSC, TGA, TIC, TOC \\
\hline & & & S96T005991 & ICP, IC \\
\hline & & & S96T005992 & $\begin{array}{l}\mathrm{Cr}(\mathrm{VI}),{ }^{239 / 240} \mathrm{Pu},{ }^{241} \mathrm{Am}, \\
{ }^{243 / 244} \mathrm{Cm}\end{array}$ \\
\hline & & & S96T005993 & $\mathrm{OH}, \mathrm{SpG}, \mathrm{U}$ \\
\hline & & & S97T000020 & ${ }^{99} \mathrm{Tc},{ }^{90} \mathrm{Sr},{ }^{129} \mathrm{I}$ \\
\hline & & & S97T000388 & $\mathrm{H} 3$ \\
\hline FB & Field blank & Liquid & S96T005406 & $\begin{array}{l}\text { DSC, SpG, TGA, ICP, IC, } \\
\text { total alpha }\end{array}$ \\
\hline HHF & $\begin{array}{l}\text { Hydrostatic head } \\
\text { fluid }\end{array}$ & Liquid & S96T005235 & ICP, IC \\
\hline \multirow[t]{5}{*}{ 167: 1} & \multirow[t]{5}{*}{ Upper half } & \multirow[t]{5}{*}{ Solids } & S96T005660 & bulk density \\
\hline & & & S96T005667 & DSC, TGA \\
\hline & & & S96T005705 & ICP \\
\hline & & & \$96T005708 & ICP \\
\hline & & & S96T005711 & IC \\
\hline
\end{tabular}


Table B2-2. Tank 241-AN-103 Sample Analysis Summary. (9 Sheets)

\begin{tabular}{|c|c|c|c|c|}
\hline Sezinent & $\begin{array}{l}\text { Sognerit } \\
\text { bontion }\end{array}$ & wite. natro & Simple: & Inillser \\
\hline \multirow[t]{5}{*}{$167: 1$} & \multirow[t]{5}{*}{ Lower half } & \multirow[t]{5}{*}{ Solids } & S96T005661 & bulk density \\
\hline & & & S96T005668 & DSC, TGA \\
\hline & & & S96T005687 & ICP, total alpha \\
\hline & & & S96T005693 & ICP \\
\hline & & & S96T005699 & IC \\
\hline \multirow[t]{5}{*}{$167: 2$} & \multirow[t]{5}{*}{ Lower half } & \multirow[t]{5}{*}{ Solids } & S96T005847 & bulk density \\
\hline & & & S96T005851 & DSC, TGA \\
\hline & & & S96T005855 & ICP, total alpha \\
\hline & & & S96T005857 & ICP \\
\hline & & & S96T005859 & IC \\
\hline $167: 2$ & Drainable liquid & Liquid & S96T005861 & $\begin{array}{l}\text { DSC, SpG, TGA, ICP, IC, } \\
\text { total alpha }\end{array}$ \\
\hline \multirow[t]{4}{*}{$167: 3$} & \multirow[t]{4}{*}{ Lower half } & \multirow[t]{4}{*}{ Solids } & S96T005669 & DSC, TGA \\
\hline & & & S96T005688 & ICP, total alpha \\
\hline & & & S96T005694 & $\mathrm{ICP}$ \\
\hline & & & S96T005700 & IC \\
\hline $167: 3$ & Drainable liquid & Liquid & S96T005673 & $\begin{array}{l}\text { DSC, SpG, TGA, ICP, IC, } \\
\text { total alpha }\end{array}$ \\
\hline \multirow[t]{4}{*}{$167: 4$} & \multirow[t]{4}{*}{ Lower half } & \multirow[t]{4}{*}{ Solids } & S96T005675 & $\mathrm{DSC}, \mathrm{TGA}$ \\
\hline & & & S96T005689 & ICP, total alpha \\
\hline & & & S96T005695 & ICP \\
\hline & & & \$96T005701 & $\mathrm{IC}$ \\
\hline \multirow[t]{5}{*}{$167: 5$} & \multirow[t]{5}{*}{ Lower half } & \multirow[t]{5}{*}{ Solids } & \$96T005374 & bulk density \\
\hline & & & S96T005424 & DSC, TGA \\
\hline & & & S96T005436 & ICP, total alpha \\
\hline & & & S96T005438 & ICP \\
\hline & & & S96T005440 & IC \\
\hline $167: 5$ & Drainable liquid & Liquid & S96T005420 & $\begin{array}{l}\text { DSC, SpG, TGA, ICP, IC, } \\
\text { total alpha }\end{array}$ \\
\hline
\end{tabular}


Table B2-2. Tank 241-AN-103 Sample Analysis Summary. (9 Sheets)

\begin{tabular}{|c|c|c|c|c|}
\hline Segritint. & $\begin{array}{l}\text { soment } \\
\text { rontion }\end{array}$ & 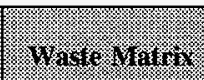 & $\begin{array}{l}\text { Sample: } \\
\text { Nanber }\end{array}$ & (2) \\
\hline \multirow[t]{5}{*}{$167: 6$} & \multirow[t]{5}{*}{ Lower half } & \multirow[t]{5}{*}{ Solids } & S96T005375 & bulk density \\
\hline & & & S96T005425 & DSC, TGA \\
\hline & & & S96T005448 & ICP, total alpha \\
\hline & & & S96T005450 & ICP \\
\hline & & & S96T005452 & IC \\
\hline $167: 6$ & Drainable liquid & Liquid & S96T005421 & $\begin{array}{l}\text { DSC, SpG, TGA, ICP, IC, } \\
\text { total alpha }\end{array}$ \\
\hline \multirow[t]{4}{*}{$167: 7$} & \multirow[t]{4}{*}{ Lower half } & \multirow[t]{4}{*}{ Solids } & S96T005685 & DSC, TGA \\
\hline & & & S96T005690 & ICP, total alpha \\
\hline & & & S96T005696 & ICP \\
\hline & & & S96T005702 & IC \\
\hline $167: 7$ & Drainable liquid & Liquid & S96T005683 & $\begin{array}{l}\text { DSC, SpG, TGA, ICP, IC, } \\
\text { total alpha }\end{array}$ \\
\hline \multirow[t]{4}{*}{$167: 8$} & \multirow[t]{4}{*}{ Lower half } & \multirow[t]{4}{*}{ Solids } & S96T005734 & DSC, TGA \\
\hline & & & S96T005736 & ICP, total alpha \\
\hline & & & S96T005737 & ICP \\
\hline & & & S96T005738 & IC \\
\hline $167: 8$ & Drainable liquid & Liquid & S96T005732 & $\begin{array}{l}\text { DSC, TGA, SpG, ICP, IC, } \\
\text { total alpha }\end{array}$ \\
\hline \multirow[t]{4}{*}{$167: 9$} & \multirow[t]{4}{*}{ Lower half } & \multirow[t]{4}{*}{ Solids } & S96T005852 & DSC, TGA, SpG \\
\hline & & & S96T005856 & ICP, total alpha \\
\hline & & & S96T005858 & ICP \\
\hline & & & S96T005860 & IC \\
\hline $167: 9$ & Drainable liquid & Liquid & S96T005862 & $\begin{array}{l}\text { DSC, SpG, TGA, ICP, IC, } \\
\text { total alpha }\end{array}$ \\
\hline $167: 10$ & & & & RGS \\
\hline \multirow[t]{5}{*}{ 167: 11} & \multirow[t]{5}{*}{ Upper half } & \multirow[t]{5}{*}{ Solids } & S96T005644 & bulk density \\
\hline & & & S96T005646 & DSC, TGA \\
\hline & & & S96T005706 & ICP \\
\hline & & & \$96T005709 & ICP \\
\hline & & & S96T005712 & IC \\
\hline
\end{tabular}


HNF-SD-WM-ER-702 Rev. 0

Table B2-2. Tank 241-AN-103 Sample Analysis Summary. (9 Sheets)

\begin{tabular}{|c|c|c|c|c|}
\hline (6) & 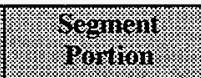 & \% & oranger & is: \\
\hline \multirow[t]{5}{*}{$167: 11$} & \multirow[t]{5}{*}{ Lower half } & \multirow[t]{5}{*}{ Solids } & S96T005645 & bulk density \\
\hline & & & S96T005647 & DSC, TGA \\
\hline & & & S96T005691 & ICP, total alpha \\
\hline & & & S96T005697 & ICP \\
\hline & & & S96T005703 & IC \\
\hline 167: 11 & Drainable liquid & Liquid & S96T005650 & $\begin{array}{l}\text { DSC, SpG, TGA, ICP, IC, } \\
\text { total alpha }\end{array}$ \\
\hline \multirow[t]{5}{*}{ 167: 12} & \multirow[t]{5}{*}{ Upper half } & \multirow[t]{5}{*}{ Solids } & S96T005378 & bulk density \\
\hline & & & S96T005432 & $\mathrm{DSC}, \mathrm{TGA}$ \\
\hline & & & S96T005442 & $\mathrm{ICP}$ \\
\hline & & & S96T005444 & ICP \\
\hline & & & S96T005446 & IC \\
\hline \multirow[t]{5}{*}{ 167: 12} & \multirow[t]{5}{*}{ Lower half } & \multirow[t]{5}{*}{ Solids } & S96T005376 & bulk density \\
\hline & & & S96T005426 & DSC, TGA \\
\hline & & & S96T005449 & ICP, total alpha \\
\hline & & & S96T005451 & ICP \\
\hline & & & S96T005453 & $\mathrm{IC}$ \\
\hline $167: 13$ & & & & RGS \\
\hline \multirow[t]{5}{*}{$167: 14$} & \multirow[t]{5}{*}{ Upper half } & \multirow[t]{5}{*}{ Solids } & S96T005379 & bulk density \\
\hline & & & S96T005433 & DSC, TGA \\
\hline & & & S96T005443 & ICP \\
\hline & & & S96T005445 & ICP \\
\hline & & & S96T005447 & IC \\
\hline \multirow[t]{5}{*}{ 167: 14} & \multirow[t]{5}{*}{ Lower half } & \multirow[t]{5}{*}{ Solids } & S96T005377 & bulk density \\
\hline & & & S96T005427 & DSC, TGA \\
\hline & & & S96T005437 & ICP, total alpha \\
\hline & & & S96T005439 & ICP \\
\hline & & & S96T005441 & IC \\
\hline
\end{tabular}


HNF-SD-WM-ER-702 Rev. 0

Table B2-2. Tank 241-AN-103 Sample Analysis Summary. (9 Sheets)

\begin{tabular}{|c|c|c|c|c|}
\hline Gognent. & for & $46980.1146 \%$ & Manple & 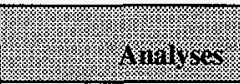 \\
\hline \multirow[t]{5}{*}{$167: 15$} & \multirow[t]{5}{*}{ Upper half } & \multirow[t]{5}{*}{ Solids } & S96T005653 & bulk density \\
\hline & & & S96T005655 & DSC, TGA \\
\hline & & & \$96T005707 & ICP \\
\hline & & & S96T005710 & $\mathrm{ICP}$ \\
\hline & & & S96T005713 & IC \\
\hline \multirow[t]{5}{*}{ 167: 15} & \multirow[t]{5}{*}{ Lower half } & \multirow[t]{5}{*}{ Solids } & \$96T005654 & bulk density \\
\hline & & & S96T005656 & DSC, TGA \\
\hline & & & S96T005692 & ICP, total alpha \\
\hline & & & S96T005698 & ICP \\
\hline & & & S96T005704 & IC \\
\hline $167: 16$ & & & & RGS \\
\hline \multirow[t]{5}{*}{$167: 17$} & \multirow[t]{5}{*}{ Upper half } & \multirow[t]{5}{*}{ Solids } & \$96T005490 & bulk density \\
\hline & & & S96T005492 & DSC, TGA \\
\hline & & & S96T005496 & ICP \\
\hline & & & S96T005498 & ICP \\
\hline & & & S96T005500 & IC \\
\hline \multirow[t]{5}{*}{ 167: 17} & \multirow[t]{5}{*}{ Lower half } & \multirow[t]{5}{*}{ Solids } & S96T005491 & bulk density \\
\hline & & & S96T005493 & DSC, TGA \\
\hline & & & S96T005497 & ICP, total alpha \\
\hline & & & S96T005499 & ICP \\
\hline & & & S96T005501 & $\mathrm{IC}$ \\
\hline
\end{tabular}

Notes:

$\mathrm{SpG}=$ specific gravity

GEA = gamma evergy analysis 
Table B2-3. Analytical Procedures. (2 sheets)

\begin{tabular}{|c|c|c|c|}
\hline ( & 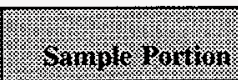 & $\begin{array}{l}\text { Provaration } \\
\text { Procedino }\end{array}$ & Mnathit \\
\hline DSC & Solid/liquid & $\mathrm{n} / \mathrm{a}$ & $\begin{array}{l}\text { LA-514-114 Rev. D-0 } \\
\text { LA-514-113 Rev. C-1 }\end{array}$ \\
\hline TGA & Solid/liquid & $n / a$ & $\begin{array}{l}\text { LA-514-114 Rev. D-0 } \\
\text { LA-560-112 Rev. C-0 }\end{array}$ \\
\hline Bulk Density & Solid & $\mathrm{n} / \mathrm{a}$ & LO-160-103 Rev. B-0 \\
\hline SpG & Liquid & $\mathrm{n} / \mathrm{a}$ & LA-510-112 Rev, C-3 \\
\hline$\overline{\mathrm{U}}$ & Solid/liquid & $\begin{array}{l}\text { LA-549-141 Rev. F-0 } \\
\text { n/a }\end{array}$ & LA-925-009 Rev. A-1 \\
\hline$\overline{\mathrm{IC}}$ & Solid/liquid & $\begin{array}{l}\text { LA-504-101 Rev. E-0 } \\
\text { n/a }\end{array}$ & LA-533-105 Rev. D-1 \\
\hline ICP & Solid/liquid & $\begin{array}{l}\text { LA-505-159 Rev. D-0 } \\
\text { LA-549-141 Rev. F-0 } \\
\text { n/a }\end{array}$ & $\begin{array}{l}\text { LA-505-151 Rev. D-3 } \\
\text { LA-505-161 Rev. B-1 }\end{array}$ \\
\hline OH- & Solid/liquid & $\begin{array}{l}\text { LA-504-101 Rev. E-0 } \\
\text { n/a }\end{array}$ & LA-211-102 Rev. C-0 \\
\hline TIC/TOC & Solid/liquid & $\mathrm{n} / \mathrm{a}$ & LA-342-100 Rev, E-0 \\
\hline $\mathrm{Cr}(\mathrm{VI})$ & Solid/liquid & $\begin{array}{l}\text { LA-504-101 Rev. E-0 } \\
\text { n/a }\end{array}$ & LA-265-101 Rev. B-0 \\
\hline${ }^{3} \mathrm{H}$ & Solid/liquid & $\begin{array}{l}\text { LA-504-101 Rev. E-0 } \\
\text { n/a }\end{array}$ & LA-218-114 Rev. B-0 \\
\hline${ }^{129} \mathrm{I}$ & Solid/liquid & $\begin{array}{l}\begin{array}{l}\text { LA-504-101 Rev. E-0 } \\
\text { n/a }\end{array} \\
\end{array}$ & LA-378-103 Rev. C-0 \\
\hline${ }^{99} \mathrm{Tc}$ & Solid/liquid & $\begin{array}{l}\text { LA-549-141 Rev. F-0 } \\
\text { n/a }\end{array}$ & LA-438-101 Rev. D-2 \\
\hline $\mathrm{AT} / \mathrm{TB}$ & Solid/liquid & $\begin{array}{l}\text { LA-549-101 Rev. F-0 } \\
\mathrm{n} / \mathrm{a}\end{array}$ & LA-508-101 Rev. E-1 \\
\hline GEA & Solid/liquid & $\begin{array}{l}\begin{array}{l}\text { LA-549-101 Rev. F-0 } \\
\text { n/a }\end{array} \\
\end{array}$ & LA-548-121 Rev, E-0 \\
\hline${ }^{241} \mathrm{Am} /{ }^{244} \mathrm{Cm}$ & Solid/liquid & $\begin{array}{l}\text { LA-549-101 Rev. F-0 } \\
\text { n/a }\end{array}$ & LA-953-103 Rev. B-0 \\
\hline
\end{tabular}


primary/duplicate pair, the sample number is for the primary result. The second column lists the core from which the samples were derived. The third column lists the sample portion from which the aliquots were taken. The final three columns display the primary and duplicate analytical values and a mean for each sample/duplicate pair.

The four QC parameters assessed in conjunction with the tank 241-AN-103 samples were standard recoveries, spike recoveries, duplicate analyses (relative percent difference [RPDs]), and blanks. The QC criteria were as specified in the TSAP (Kruger 1996). The only QC parameter for which limits were not specified in the TSAP is blank contamination. The limits for blanks are set forth in guidelines followed by the laboratory, and all data results presented in this report have met those guidelines. Sample and duplicate pairs in which any of the QC parameters were outside of these limits are footnoted in the sample mean column of the following data summary tables with an $a, b, c, d, e$, or $f$ as follows:

- $\quad \mathrm{a}$ " indicates that the standard recovery was below the QC range.

- $\quad$ " $b "$ indicates that the standard recovery was above the QC range.

- $\quad c$ " indicates that the spike recovery was below the QC range.

- $\quad \mathrm{d}$ ". indicates that the spike recovery was above the QC range.

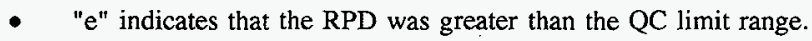

- $\quad f$ " indicates that there was blank contamination.

B2.1.3.1 Total Alpha Activity. Total alpha activity analyses were measured on the solids and liquids using alpha spectroscopy. The analyses were performed according to procedure LA-508-101. Total alpha activities ranged from 0.0048 to $0.0429 \mu \mathrm{Ci} / \mathrm{g}$ for the solids and from 0.0039 to $0.18 \mu \mathrm{Ci} / \mathrm{mL}(0.023$ to $0.12 \mu \mathrm{Ci} / \mathrm{g})$ for the liquids. Mostly the results were at or below detection limits. There was also a result of $<6.71 \mathrm{E}-04 \mu \mathrm{Ci} / \mathrm{g}$ in core 167 , segment 7 solids.

B2.1.3.2 Total Beta. Total beta activity was measured on the core composite samples according to procedure LA-508-101. Total beta activities were $622 \mu \mathrm{Ci} / \mathrm{mL}(418 \mu \mathrm{Ci} / \mathrm{g})$ for the liquid and $29.55 \mu \mathrm{Ci} / \mathrm{g}$ for the solid. The sample results are in Table B2-3. These results do not agree well with ${ }^{137} \mathrm{Cs}$ concentrations, the only beta emitter of significant magnitude present. ${ }^{137} \mathrm{Cs}$ concentrations were $739 \mu \mathrm{Ci} / \mathrm{mL}$ in the liquid composite and $274 \mu \mathrm{Ci} / \mathrm{g}$ in the solid composite.

B2.1.3.3 Strontium 90. Strontium-90 was measured in the core composite using procedure LA-220-101. The activities were $0.0208 \mu \mathrm{Ci} / \mathrm{mL}(0.014 \mu \mathrm{Ci} / \mathrm{g})$ in the liquid and $2.66 \mu \mathrm{Ci} / \mathrm{g}$ in the solid. 
B2.1.3.4 Gamma Energy Analysis. Gamma energy analysis (GEA), procedure number LA-548-121, was used to measure the activity of ${ }^{241} \mathrm{Am},{ }^{137} \mathrm{Cs},{ }^{60} \mathrm{Co}{ }^{154} \mathrm{Eu}$, and ${ }^{155} \mathrm{Eu}$ in the core composite. ${ }^{137} \mathrm{Cs}$ was the dominant radionuclide.

B2.1.3.5 Iodine 129. ${ }^{129} \mathrm{I}$ analyses were performed on the core composite using procedure LA-378-103. The mean results were $3.790 \mathrm{E}-04 \mu \mathrm{Ci} / \mathrm{mL}(2.545 \mathrm{E}-04 \mu \mathrm{Ci} / \mathrm{g})$ in the liquid and $<0.0027 \mu \mathrm{Ci} / \mathrm{g}$ in the solid.

B2.1.3.6 Technetium-99. ${ }^{99} \mathrm{Tc}$ analyses were performed on the core composite using procedure LA-438-101. The mean results were $1.675 \mathrm{E}-4 \mu \mathrm{Ci} / \mathrm{mL}(1.125 \mathrm{E}-4 \mu \mathrm{Ci} / \mathrm{g})$ in the liquid and $0.1445 \mu \mathrm{Ci} / \mathrm{g}$ in the solid.

B2.1.3.7. Alpha Energy Analysis. ${ }^{241} \mathrm{Am},{ }^{243 / 244} \mathrm{Cm}$, and ${ }^{239 / 240} \mathrm{Pu}$ were measured on core composite samples using alpha energy analysis (AEA). All results were below detection limits.

B2.1.3.8 Tritium. Tritium was measured using liquid scintillation (procedure LA-218-114). The mean results were $0.00284 \mu \mathrm{Ci} / \mathrm{g}$ in the solid and $0.0015675 \mu \mathrm{Ci} / \mathrm{mL}(1.0527 \mathrm{E}-3 \mu \mathrm{Ci} / \mathrm{g})$ in the liquid.

B2.13.9 Thermogravimetric Analysis. Thermogravimetric analysis measures the mass of a sample while its temperature is increased at a constant rate. Nitrogen is passed over the sample during heating to remove any released gases. Any decrease in the weight of a sample during TGA represents a loss of gaseous matter from the sample, either through evaporation or through a reaction that forms gas phase products. The moisture content is estimated by assuming that all TGA sample weight loss up to a certain temperature (typically 150 to $200{ }^{\circ} \mathrm{C}$ ) is because of water evaporation. The temperature limit for moisture loss is chosen by the operator at an inflection point on the TGA plot. Other volatile matter fractions can often be differentiated by inflection points as well.

Tank 241-AN-103 samples were analyzed by TGA using either a Perkin-Elmer ${ }^{1}$ TGA 7 instrument, or a Mettler ${ }^{2}$ TG 50 instrument. Typically, TGA results are determined by summing the weight loss steps that occur below $250{ }^{\circ} \mathrm{C}$; weight loss steps above this are not used to determine the result. However, for tank 241-AN-103, approximately 40 percent of the thermograms showed continuous weight loss beyond $250^{\circ} \mathrm{C}$. This weight loss was not the result of additional weight loss steps above $250^{\circ} \mathrm{C}$ and was included in the calculation of the results.

The results for thirty-two of the fifty-eight subsamples were the sum of two or more weight loss steps. The weight percent water values for the tank supernatant and salt slurry were

\footnotetext{
${ }^{1}$ Perkin-Elmer is a trademark of Perkins Research \& Manufacturing Company, Incorporated, Canoga Park, California.

${ }^{2}$ Mettler is a trademark of Mettler Instrument Corporation, Anaheim, California.
} 
between 14.3 and 50.1. Relative percent differences greater than 30 percent were reported for five of the sixty-three subsamples. Selected samples were reanalyzed. The reruns resulted in RPDs of less than 30 percent. Several thermograms showed small sharp peaks. These were the result of instrument vibration and were not used in the calculation of the result. The standard recoveries for this analysis were within the required limits.

Eight segments were contaminated by the hydrostatic head fluid (see Tables B2-36 and B2-51 for the lithium and bromide results). The solid portion from four additional segments in the convective layer showed contamination, but the liquid portion did not. Corrected TGA results for the affected subsamples are listed in Table B2-20.

B2.1.3.10 Differential Scanning Calorimetry. In a DSC analysis, heat absorbed or emitted by a substance is measured while the temperature of the sample is heated at a constant rate. Nitrogen is passed over the sample material to remove any gases being released. The onset temperature for an endothermic or exothermic event is determined graphically. The DSC analyses for tank 24I-AN-103 were performed using either procedure LA-514-113 on a Mettler ${ }^{T M}$ DSC 20 instrument or procedure LA-514-114 on a Perkin-Elmer ${ }^{\mathrm{TM}}$ DSC 7 instrument.

One sample exceeded the safety screening DQO decision criteria threshold of $480 \mathrm{~J} / \mathrm{g}$ (dry weight basis). The lower half of segment 2 (solids) from core 167 had a sample result of $160 \mathrm{~J} / \mathrm{g}$ and a duplicate of $586 \mathrm{~J} / \mathrm{g}$. Because the difference between the sample and duplicate results was large (Relative Percent Difference: 114 percent), the sample and duplicate were reanalyzed and found to exhibit exotherms of 152.0 and $83.7 \mathrm{~J} / \mathrm{g}$, respectively. This sample had the highest exothermic results for solids. The total organic carbon from this core was $2,440 \mu \mathrm{g} / \mathrm{g}$. The highest individual sample exothermic results for liquids (dry weight basis) was $268 \mathrm{~J} / \mathrm{g}$.

High RPDs ( $>30$ percent) were reported for twenty-seven of the sixty-one subsamples. The RPDs can be attributed to the small exotherms and the heterogenous nature of the samples. Thermograms for several subsamples showed small sharp peaks near $200{ }^{\circ} \mathrm{C}$ that indicated a decomposition of a pure compound. The standard recoveries for this analysis were within the required limits.

B2.1.3.11 Density and Specific Gravity. Density/specific gravity measurements were performed on all subsegments. The subsegment-level results for salt slurry densities ranged from 1.59 to $1.93 \mathrm{~g} / \mathrm{mL}$. The specific gravity measurements for the liquid samples ranged from 1.34 to 1.54 . The initial specific gravity measurements for the drainable liquid from segment 6 of core 167 was 2.7 for the result and 2.8 for the duplicate. This sample was rerun and the results were 1.462 for the result and 1.475 for the duplicate ${ }^{3}$. The statistical analysis (Section B.4) was performed prior to the rerun. In the analysis, the specific gravity measurement for the drainable liquid from core 166 , segment 7 was substituted for the

\footnotetext{
${ }^{3}$ Electronic mail message from F. H. Steen, RFSH, June 17, 1997, "AN-103 DSC Results."
} 
measurements from segment 6 of core 167. These segments were sampled from about the same elevation in the tank. The specific gravity measurements for core 166, segment 7 are 1.459 and 1.458 .

B2.1.3.12 Inductively Coupled Plasma. The ICP analyses were performed per procedures LA-505-161, or LA-505-151, depending on the ICP instrument used. A full suite of analytes were reported. Phosphorus was analyzed as a cross-check for the phosphate results reported from IC analyses. The liquid subsamples were prepared for analysis by an acid adjustment of the direct subsample. Solid subsamples were prepared for analysis by performing both an acid digest and a fusion. The results from two preparation methods, fusion and acid, are presented for the metals.

The results indicate that there are three phases in the tank (crust, liquid, and solid). Aluminum and chromium concentrations show a linear correlation of 0.74 and a quadratic correlation of 0.94 . Changes in $\mathrm{Al}$ and $\mathrm{Cr}$ concentration correspond with changing regions in the tank. The concentrations change near the bottom of the tank, indicating that the bottom solids may be two distinct materials. Several analytes (Al, Cr, Pb, F, Si, and P) appear to be at saturation concentrations in the liquid.

B2.1.3.13 Ion Chromatography. The IC analyses were performed on direct subsamples of liquid samples. The solid subsamples were prepared for analysis by performing a water digest. Samples for ion chromatography were performed in duplicate per procedure LA-533-105. Chloride concentrations were relatively high ( $>1$ percent in the solids).

B2.1.3.14 Total Uranium. Uranium was measured in the core composite using procedure LA-925-009. The mean results were $1.835 \mu \mathrm{g} / \mathrm{mL}(1.23 \mu \mathrm{g} / \mathrm{g})$ in the liquid and $45.1 \mu \mathrm{g} / \mathrm{g}$ in the solid.

B2.1.3.15 OH. The $\mathrm{OH}$ analyses were performed on the core composite using procedure LA-211-102. The mean results were $98,200 \mu \mathrm{g} / \mathrm{mL}(65,950 \mu \mathrm{g} / \mathrm{g})$ in the liquid and $32,900 \mu \mathrm{g} / \mathrm{g}$ in the solid.

B2.1.3.16 Chromium VI. The Cr(VI) analyses were performed on the core composite using procedure LA-265-101. The mean results were $74.9 \mu \mathrm{g} / \mathrm{g}$ for the liquid and $99.7 \mu \mathrm{g} / \mathrm{g}$ for the solid.

B2.1.3.17 Total Inorganic Carbon/Total Organic Carbon (TIC/TOC). TIC/TOC by persulfate/coulometry analyses were performed on the core composite samples. The maximum results for TIC were $1,155 \mu \mathrm{g} / \mathrm{mL}(775.7 \mu \mathrm{g} / \mathrm{g})$ for the liquid and $5,770 \mu \mathrm{g} / \mathrm{g}$ for the solid. None of the results exceeded the TOC notification limit of $30,000 \mu \mathrm{g} / \mathrm{g}$. The mean results for TOC are $3,045 \mu \mathrm{g} / \mathrm{mL}(2,045 \mu \mathrm{g} / \mathrm{g})$ for the liquid and $2,440 \mu \mathrm{g} / \mathrm{g}$ for the solid. 


\section{B2.2 VAPOR PHASE MEASUREMENT}

During September 1996, tank dome space gas samples were taken from tank 241-AN-103. These measurements supported the safety screening DQO (Dukelow et al. 1995). The vapor phase measurements were taken $20 \mathrm{ft}$ below risers $12 \mathrm{~A}$ and $21 \mathrm{~A}$ in the dome space of the tank and results were obtained in the field (that is, no gas sample was sent to the laboratory for analysis). The average results of the vapor phase measurements are provided in Table B2-66.

\section{B2.3 HISTORICAL SAMPLES}

Tank 241-AN-103 was the receiver tank for the 242-A Evaporator during the 86-2 Campaign. The results from a February 10, 1986 sludge sample are shown in Table B2-67. This sludge sample was taken prior to filling the tank with double-shell slurry to characterize the sludge heel. It was "pea-green" with about 5 percent supernatant, 85 percent settled solids, and 10 percent foam. The sample was separated by filtration and each phase was analyzed.

Several evaporator slurry samples were taken during the 86-2 evaporator campaign before waste was sent to tank 241-AN-103. The main constituents of samples taken are shown in Table B2-68.

Following the 86-2 campaign tank 241-AN-103 was push core sampled during December 1986. Eighteen segments were recovered. A composite of the push core sample was sent to Pacific Northwest Laboratory for chemical analysis (Toste 1987). The main constituents are listed in Table B2-69. Radionuclide measurements are listed in Table B2-70. An organic analysis was also completed and physical property measurements were obtained. These results are discussed and described in Toste (1987). Results of dissolution studies performed on the push core sample are reported in Prignano (1988).

\section{PUSH CORE SAMPLES}

Table B2-5. Tank 241-AN-103 Analytical Results: Total Alpha (Alpha Rad). (3 sheets)

\begin{tabular}{|c|c|c|c|c|c|}
\hline Sample & $\begin{array}{l}\text { Sranple } \\
\text { Yrocation }\end{array}$ & Samie & previlil & Buplicate & Menu \\
\hline Wiguas & 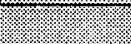 & 2. & 14. Thin & . & 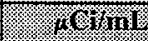 \\
\hline S96T005539 & $166: 3$ & Drainable liquid & $<0.00579$ & $<0.00506$ & $<0.00542$ \\
\hline \$96T005540 & 166: 4 & Drainable liquid & $<0.00361$ & $<0.00506$ & $<0.00433$ \\
\hline S96T005599 & 166: 6 & Drainable liquid & $<0.0202$ & $<0.012$ & $<0.0161$ \\
\hline S96T005816 & 166: 7 & Drainable liquid & $<0.012$ & $<0.023$ & $<0.0175$ \\
\hline
\end{tabular}


Table B2-5. Tank 241-AN-103 Analytical Results: Total Alpha (Alpha Rad). (3 sheets)

\begin{tabular}{|c|c|c|c|c|c|}
\hline samplo & 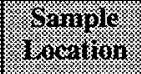 & Mandele & (1) & 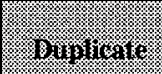 & Hen \\
\hline \$96T005817 & 166: 8 & Drainable liquid & $<0.012$ & $<0.012$ & $<0.012$ \\
\hline S96T005541 & $166: 9$ & Drainable liquid & 0.0039 & $<0.00479$ & $<0.00434^{\mathrm{QC:e}}$ \\
\hline S96T005566 & 166: 10 & Drainable liquid & $<0.00431$ & $<0.00579$ & $<0.00505$ \\
\hline S96T005600 & 166: 11 & Drainable liquid & $<0.0174$ & $<0.023$ & $<0.0202$ \\
\hline \$96T005601 & 166: 12 & Drainable liquid & $<0.0468$ & 0.18 & $<0.113^{\mathrm{QC:e}}$ \\
\hline S96T005861 & $167: 2$ & Drainable liquid & $<0.0804$ & $<0.0535$ & $<0.0669$ \\
\hline S96T005673 & 167: 3 & Drainable liquid & $<0.0224$ & $<0.0195$ & $<0.0209$ \\
\hline S96T005420 & 167: 5 & Drainable liquid & $<0.182$ & $<0.165$ & $<0.173$ \\
\hline S96T005421 & 167: 6 & Drainable liquid & $<0.0514$ & $<0.0514$ & $<0.0514$ \\
\hline S96T005683 & $167: 7$ & Drainable liquid & 0.0397 & 0.0369 & 0.0383 \\
\hline S96T005732 & $167: 8$ & Drainable liquid & $<0.0378$ & 0.034 & $<0.0359$ \\
\hline S96T005862 & 167:9 & Drainable liquid & $<0.0737$ & 0.0514 & $<0.0625^{\mathrm{QC}: \varepsilon}$ \\
\hline S96T005650 & 167: 11 & Drainable liquid & $<0.0169$ & $<0.016$ & $<0.0164$ \\
\hline S96T005991 & Core 166 & $\begin{array}{l}\text { Liquid } \\
\text { composite }\end{array}$ & $<0.00653$ & $<0.00653^{\circ}$ & $<0.00653$ \\
\hline \multicolumn{3}{|l|}{ 60115\% } & 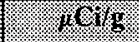 & (7) & wo \\
\hline S96T005608 & 166: 1 & Lower half & $<0.011$ & $<0.00926$ & $<0.01013$ \\
\hline S96T005568 & 166: 4 & Lower half & $<0.00837$ & 0.00764 & $<0.00800^{\mathrm{QC}: c}$ \\
\hline S96T005611 & 166: 6 & Lower half & $<0.00612$ & $<0.00961$ & $<0.00786$ \\
\hline S96T005835 & 166: 7 & Lower half & 0.00656 & $<0.00508$ & $<0.00582^{\mathrm{QC}: c, f}$ \\
\hline \$96T005836 & 166: 8 & Lower half & 0.0048 & $<0.00658$ & $<0.00569^{Q \mathrm{C}: c, \mathrm{e}}$ \\
\hline S96T005574 & 166: 9 & Lower half & $<0.00781$ & $<0.00868$ & $<0.008245^{\mathrm{QC}: \mathrm{f}}$ \\
\hline S96T005569 & 166: 10 & Lower half & $<0.00938$ & $<0.00649$ & $<0.00793$ \\
\hline \$96T005612 & $166: 11$ & Lower half & $<0.00881$ & $<0.0061$ & $<0.00745$ \\
\hline \$96T005613 & $166: 12$ & Lower half & $<0.00917$ & $<0.00482$ & $<0.00699$ \\
\hline S96T005720 & 166: 13 & Lower half & 0.0175 & $<0.0344$ & $<0.0259^{Q \mathrm{C}: c}$ \\
\hline \$96T005408 & $166: 15$ & Lower half & $<0.0121$ & $<0.0131$ & $<0.0126^{\mathrm{QC:c}}$ \\
\hline S96T005614 & $166: 16$ & Lower half & $<0.00423$ & 0.00724 & $<0.00573^{\mathrm{QC:e}}$ \\
\hline \$96T005409 & $166: 17$ & Lower half & $<0.0111$ & $<0.0129$ & $<0.012^{Q \mathrm{QC}: \mathrm{c}, \mathrm{f}}$ \\
\hline S96T005721 & 166: 18 & Lower half & 0.0429 & 0.0156 & $0.0292^{\mathrm{QC}: \mathrm{e}, \mathrm{f}}$ \\
\hline
\end{tabular}


Table B2-5. Tank 241-AN-103 Analytical Results: Total Alpha (Alpha Rad). (3 sheets)

\begin{tabular}{|c|c|c|c|c|c|}
\hline Whapic & 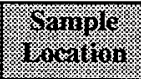 & foring & 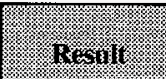 & B) & (1) \\
\hline S96T005838 & 166: 19 & Lower half & 0.023 & 0.0151 & $0.0190^{\text {QC:e }}$ \\
\hline S96T005687 & 167: 1 & Lower half & $<0.0279$ & $<0.0346$ & $<0.03125$ \\
\hline \$96T005855 & 167:2 & Lower half & $<0.00755$ & 0.00605 & $<0.0068^{\mathrm{QC}: \mathrm{e}}$ \\
\hline S96T005688 & $167: 3$ & Lower half & $<0.0131$ & $<0.00927$ & $<0.0112$ \\
\hline S96T005689 & $167: 4$ & Lower half & $<0.00755$ & $<0.00768$ & $<0.00761$ \\
\hline S96T005436 & $167: 5$ & Lower half & $<0.136$ & $<0.12$ & $<0.128^{\mathrm{QC:c}}$ \\
\hline S96T005448 & 167: 6 & Lower half & $<0.0906$ & $<0.0736$ & $<0.0821$ \\
\hline S96T005690 & $167: 7$ & Lower half & $<6.710 \mathrm{E}-04$ & $<0.00149$ & $<0.00108$ \\
\hline S96T005736 & $167: 8$ & Lower half & $<0.00582$ & $<0.00347$ & $<0.00464$ \\
\hline S96T005856 & 167:9 & Lower half & $<0.0114$ & $<0.00961$ & $<0.0105$ \\
\hline S96T005691 & 167: 11 & Lower half & $<0.00648$ & $<0.00208$ & $<0.00428$ \\
\hline S96T005449 & $167: 12$ & Lower half & 0.0283 & 0.0346 & $0.0314^{\mathrm{QC}: f}$ \\
\hline S96T005437 & 167: 14 & Lower half & $<0.0181$ & $<0.0214$ & $<0.0197^{\mathrm{QC}: \mathrm{f}}$ \\
\hline S96T005692 & 167: 15 & Lower half & $<0.011$ & $<0.0131$ & $<0.0120$ \\
\hline S96T005497 & 167: 17 & Lower half & $<0.0168$ & $<0.011$ & $<0.0139$ \\
\hline S96T005986 & Core 166 & Solid composite & $<0.00224$ & $<0.00257$ & $<0.0024^{Q C: c}$ \\
\hline
\end{tabular}

Table B2-6. Tank 241-AN-103 Analytical Results: Total Beta.

\begin{tabular}{|c|c|c|c|c|c|}
\hline Shample & 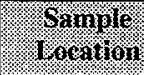 & \% & pesulu & Buploris & (1) \\
\hline Hourds & & (3) & \% 6111 & W.1\% & \% W WIII \\
\hline S96T005991 & Core 166 & $\begin{array}{l}\text { Liquid } \\
\text { composite }\end{array}$ & 626 & 618 & 622 \\
\hline \multicolumn{3}{|c|}{ Solios . Iission } & (x) & (1) & wory \\
\hline S96T005986 & Core 166 & Solid composite & 31.9 & 27.2 & 29.55 \\
\hline
\end{tabular}


Table B2-7. Tank 241-AN-103 Analytical Results: Strontium-89/90 (Sr).

\begin{tabular}{|c|c|c|c|c|c|}
\hline Moningle & 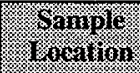 & (6) & Ressull & Bintiente & Mora \\
\hline Waing & & & 6. W11 & 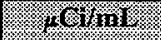 & (6) \\
\hline S97T000020 & Core 166 & Liquid composite & 0.0208 & 0.0208 & $0.0208^{\mathrm{QC:f}}$ \\
\hline \multicolumn{3}{|c|}{ WHids rishon } & 40 & (x) & (1) \\
\hline \$96T005986 & Core 166 & Solid composite & 2.58 & 2.74 & $2.66^{\mathrm{QC}: \mathrm{f}}$ \\
\hline
\end{tabular}

Table B2-8. Tank 241-AN-103 Analytical Results: Americium-241 (GEA).

\begin{tabular}{|c|c|c|c|c|c|}
\hline Gamplo & Waingle & Somingin & pesint & mivelueare & Mern \\
\hline mginas & $\sqrt{3}$ & & 政 & \%. & 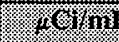 \\
\hline S96T005991 & Core 166 & Liquid composite & $<1.517$ & $<1.51$ & $<1.513$ \\
\hline \multicolumn{3}{|c|}{ 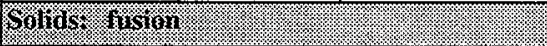 } & 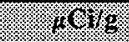 & 4\% & w 19 \\
\hline S96T005986 & Core 166 & Solid composite & $<1.041$ & $<0.951$ & $<0.996$ \\
\hline
\end{tabular}

Table B2-9. Tank 241-AN-103 Analytical Results: Cesium-137 (GEA).

\begin{tabular}{|c|c|c|c|c|c|}
\hline Winolo & Salingle & 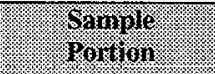 & (nesilu & Sholioare & Menng \\
\hline rigurs & & & .64mI & (3) & mo. \\
\hline S96T005991 & Core 166 & Liquid composite & 739 & 738 & $738^{\circ}$ \\
\hline \multicolumn{3}{|c|}{ 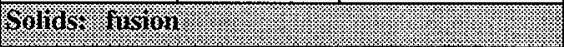 } & 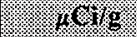 & $10 \%$ & 策 \\
\hline S96T005986 & Core 166 & Solid composite & 297 & 251 & 274 \\
\hline
\end{tabular}

Table B2-10. Tank 241-AN-103 Analytical Results: Cobalt-60 (GEA).

\begin{tabular}{|c|c|c|c|c|c|}
\hline Mample & Somalnotion & Womple & Resilf & OHallonte & Werin \\
\hline \multicolumn{2}{|c|}{ Wuins } & $\sqrt{1}$ & (1) 1211 & KamI & matim \\
\hline S96T005991 & Core 166 & Liquid composite & $<0.0184$ & $<0.0206$ & $<0.0195$ \\
\hline Solitis & riosion & & $401 \%$ & 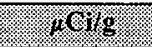 & 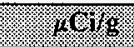 \\
\hline S96T005986 & Core 166 & Solid composite & $<0.0141$ & $<0.0108$ & $<0.0124$ \\
\hline
\end{tabular}


Table B2-11. Tank 241-AN-103 Analytical Results: Europium-154 (GEA).

\begin{tabular}{|c|c|c|c|c|c|}
\hline Hon & Wample & 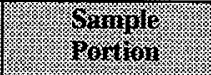 & Pasinit & YYolion. & (18 \\
\hline Sharis. & & 3. & (6) & 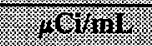 & 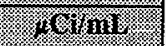 \\
\hline S96T005991 & Core 166 & Liquid composite & $<0.156$ & $<0.138$ & $<0.147$ \\
\hline \multicolumn{3}{|c|}{ OHIIV WISTH } & 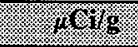 & 46 & 40 \\
\hline S96T005986 & Core 166 & Solid composite & $<0.0676$ & $<0.0597$ & $<0.0636$ \\
\hline
\end{tabular}

Table B2-12. Tank 241-AN-103 Analytical Results: Europium-155 (GEA).

\begin{tabular}{|c|c|c|c|c|c|}
\hline Sample & Shample & Sor & Resuly & 8uplicate & 1) \\
\hline rinuils. & & & How14 & 14\% & (rivin \\
\hline S96T005991 & Core 166 & Liquid composite & $<0.576$ & $<0.577$ & $<0.577$ \\
\hline \multicolumn{3}{|c|}{ Solds hispor } & (1) & . & (1) \\
\hline S96T005986 & Core 166 & Solid composite & $<0.398$ & $<0.363$ & $<0.381$ \\
\hline
\end{tabular}

Table B2-13. Tank 241-AN-103 Analytical Results: Iodine-129 (I129).

\begin{tabular}{|c|c|c|c|c|c|}
\hline Sample & Sarupor & Goungle & issiutu & onjibrice & Meati \\
\hline Mainas & & & 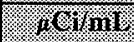 & \% & \% Minl \\
\hline S97T000020 & Core 166 & Liquid composite & $3.940 \mathrm{E}-04$ & $3.640 \mathrm{E}-04$ & $3.790 \mathrm{E}-04$ \\
\hline \multicolumn{2}{|c|}{ Solids watritingst } & & arivg & 46 & \% \\
\hline S97T000023 & Core 166 & Solid composite & $<0.0034$ & 0.00201 & $<0.00270^{0 c: e}$ \\
\hline
\end{tabular}

Table B2-14. Tank 241-AN-103 Analytical Results: Technetium-99 (Tc).

\begin{tabular}{|c|c|c|c|c|c|}
\hline Shimber & Somple & Tortion & mesull & Yupineate & Hean \\
\hline Mriniris & א. & & \% & 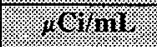 & y. Win \\
\hline S97T000020 & Core 166 & Liquid composite & $1.790 \mathrm{E}-04$ & $1.560 \mathrm{E}-04$ & $1.67 \mathrm{E}-04$ \\
\hline \multicolumn{3}{|c|}{ Solids ristou } & 10 1) & 10.69. & ( $11 \%$ \\
\hline S96T005986 & Core 166 & Solid composite & 0.156 & 0.133 & 0.144 \\
\hline
\end{tabular}


HNF-SD-WM-ER-702 Rev. 0

Table B2-15. Tank 241-AN-103 Analytical Results: Americium-241 (Am241).

\begin{tabular}{|c|c|c|c|c|c|}
\hline STinpic & Sampole & Sinuple & aesint & Burlig. & Mern \\
\hline Wivinis: & & 4 & 46 Mnil & (1) & 169.1113 \\
\hline S96T005992 & Core 166 & Liquid composite & $<0.00617$ & $<0.00526$ & $<0.005715$ \\
\hline \multicolumn{2}{|c|}{ Wollows grow } & & 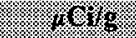 & 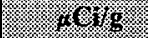 & $10101 \mathrm{~s}$ \\
\hline S96T005986 & Core 166 & Solid composite & $<0.00347$ & 0.00359 & $<0.00353$ \\
\hline
\end{tabular}

Table B2-16. Tank 241-AN-103 Analytical Results: Curium-243/244.

\begin{tabular}{|c|c|c|c|c|c|}
\hline $\begin{array}{l}\text { Crmple } \\
\text { Nuniber }\end{array}$ & Sarnolo & $\begin{array}{l}\text { Saminge } \\
\text { Portion }\end{array}$ & Irsuin & Duplicate & (1)an \\
\hline Miguids & 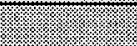 & (1: & (C) & 14. TIII & $1401911 \%$ \\
\hline S96T005992 & Core 166 & Liquid composite & $<0.00617$ & $<0.00526$ & $<0.005715$ \\
\hline \multicolumn{3}{|c|}{ Sollids minion } & 40ig & NeV8 & 16/ \\
\hline S96T005986 & Core 166 & Solid composite & $<0.00347$ & $<0.00299$ & $<0.00323$ \\
\hline
\end{tabular}

Table B2-17. Tank 241-AN-103 Analytical Results: Plutonium-239/240 (Pu239/240).

\begin{tabular}{|c|c|c|c|c|c|}
\hline $\begin{array}{l}\text { Sarivie } \\
\text { Numiner }\end{array}$ & Sample & Samivile. & Resill: & Bupticat & Mena \\
\hline Solios: & 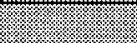 & 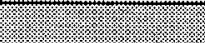 & m ब1, & $1.01 \mathrm{~g}$ & $101 \mathrm{~g}$ \\
\hline S96T005986 & Core 166 & Solid composite & $<0.00173$ & $<0.00181$ & $<0.00177$ \\
\hline Mgurds: & & & KOImL & \% & \#Cim \\
\hline S96T005992 & Core 166 & Liquid composite & $<0.00352$ & $<0.00382$ & $<0.00367$ \\
\hline
\end{tabular}

Table B2-18. Tank 241-AN-103 Analytical Results: Tritium (Liquid Scintillation).

\begin{tabular}{|c|c|c|c|c|c|}
\hline $\begin{array}{l}\text { Sample } \\
\text { Numier }\end{array}$ & Sanple. & Sarnollo: & Resulit. & Buplicate & Wear \\
\hline \multicolumn{3}{|c|}{ Solits: Water digest: } & 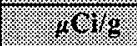 & 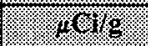 & rovg \\
\hline S97T000023 & Core 166 & Solid composite & 0.00247 & 0.00321 & $0.00284^{\mathrm{QC}: c}$ \\
\hline Wojus & & & Fon & 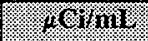 & Herini: \\
\hline S97T000388 & Core 166 & Liquid composite & $7.850 \mathrm{E}-04$ & 0.00235 & $0.001567^{\mathrm{DC}: \mathrm{c}}$ \\
\hline
\end{tabular}


HNF-SD-WM-ER-702 Rev. 0

Table B2-19. Tank 241-AN-103 Analytical Results: Percent Water (TGA). (3 sheets)

\begin{tabular}{|c|c|c|c|c|c|}
\hline Manple & 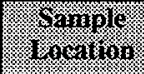 & 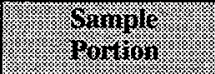 & (3) pesilu & 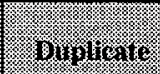 & 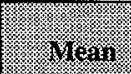 \\
\hline MuMd & (3. & 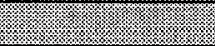 & S: & \% & 89 \\
\hline S96T005539 & $166: 3$ & Drainable liquid & 48.67 & 48.6 & 48.6 \\
\hline S96T005540 & 166: 4 & Drainable liquid & 48.52 & 47.86 & 48.2 \\
\hline S96T005599 & 166: 6 & Drainable liquid & 49.28 & 49.3 & 49.3 \\
\hline S96T005816 & $166: 7$ & Drainable liquid & 49.3 & 49.0 & 49.1 \\
\hline S96T005817 & $166: 8$ & Drainable liquid & 49.4 & 49.0 & 49.2 \\
\hline S96T005541 & 166: 9 & Drainable liquid & 48.5 & 48.5 & 48.5 \\
\hline S96T005566 & $166: 10$ & Drainable liquid & 48.2 & 48.1 & 48.1 \\
\hline S96T005600 & 166: 11 & Drainable liquid & 48.8 & 48.8 & 48.8 \\
\hline S96T005601 & 166: 12 & Drainable liquid & 49.7 & 49.4 & 49.6 \\
\hline S96T005861 & 167: 2 & Drainable liquid & 49.3 & 49.0 & 49.2 \\
\hline S96T005673 & $167: 3$ & Drainable liquid & 48.3 & 48.3 & 48.3 \\
\hline S96T005420 & $167: 5$ & Drainable liquid & 50.1 & 50.2 & 50.1 \\
\hline S96T005421 & $167: 6$ & Drainable liquid & 49.9 & 49.9 & 49.9 \\
\hline S96T005683' & $167: 7$ & Drainable liquid & 48.8 & 48.0 & 48.4 \\
\hline S96T0057321 & $167: 8$ & Drainable liquid & 48.5 & $\overline{48.3}$ & 48.4 \\
\hline \$96T005862 & 167: 9 & Drainable liquid & 49.4 & 49.3 & 49.3 \\
\hline S96T005650 & 167: 11 & Drainable liquid & 49.2 & 49.1 & 49.2 \\
\hline S96T0059901 & Core 166 & Liquid composite & 48.5 & 48.4 & 48.4 \\
\hline \multicolumn{3}{|l|}{ Solis } & \% & $1 \%$ & \% \\
\hline S96T005578 & 166: 1 & Lower half & 28.8 & 35.8 & 32.3 \\
\hline \$96T005542 & 166: 4 & Lower half & 32.9 & 24.7 & 28.8 \\
\hline S96T0055791 & 166: 6 & Lower half & 21.5 & 30.7 & $26.1^{\mathrm{QC}: \mathrm{c}}$ \\
\hline S96T005820 & 166: 7 & Lower half & 15.4 & 19.7 & 17.5 \\
\hline S96T005821 & 166: 8 & Lower half & 19.21 & 17.55 & 18.4 \\
\hline S96T005543 & 166: 9 & Lower half & 43.8 & 44.8 & 44.3 \\
\hline S96T005564 & 166: 10 & Lower half & 27.3 & 38.7 & $33.0^{\mathrm{QC:c}}$ \\
\hline S96T005580 & 166: 11 & Lower half & 22.5 & 20.2 & 21.4 \\
\hline S96T005581 & 166: 12 & Lower half & 24.6 & 30.4 & 27.5 \\
\hline
\end{tabular}


Table B2-19. Tank 241-AN-103 Analytical Results: Percent Water (TGA). (3 sheets)

\begin{tabular}{|c|c|c|c|c|c|}
\hline Shriphe & Sample & 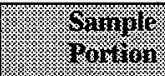 & . & Sopingty & yentin \\
\hline wolids (v) & & & 8 & 2. & 8 \\
\hline S96T005636 & \multirow[t]{2}{*}{$166: 13$} & Upper half & 41.4 & 46.6 & 44.0 \\
\hline S96T005637 & & Lower half & 34.0 & 45.4 & 39.7 \\
\hline S96T005398 & \multirow[t]{2}{*}{$166: 15$} & Upper half & 33.7 & 33.7 & 33.7 \\
\hline S96T005399 & & Lower half & 33.5 & 29.8 & 31.6 \\
\hline S96T005582 & \multirow[t]{3}{*}{$166: 16$} & Upper half & 22.8 & 43.4 & $33.1^{\mathrm{QC} \cdot \mathrm{c}}$ \\
\hline S96T005582.1 & & Upper half & 30.9 & 27.1 & 29.0 \\
\hline S96T005583 & & Lower half & 38.1 & 37.3 & 37.7 \\
\hline S96T005400 & \multirow[t]{2}{*}{ 166: 17} & Upper half & 31.1 & 33.1 & 32.1 \\
\hline S96T005401 & & Lower half & 30.0 & 30.1 & 30.1 \\
\hline S96T005638 & \multirow[t]{2}{*}{ 166: 18} & Upper half & 30.7 & 30.3 & 30.5 \\
\hline \$96T005639 & & Lower half & 38.7 & 40.1 & 39.4 \\
\hline S96T005822 & \multirow[t]{2}{*}{$166: 19$} & Upper half & 39.8 & 40.1 & 40.0 \\
\hline S96T005823 & & Lower half & 41.3 & 42.1 & 41.7 \\
\hline S96T005667 & \multirow[t]{2}{*}{ 167: 1} & Upper half & 32.8 & 32.8 & 32.8 \\
\hline S96T005668 & & Lower half & 35.8 & 35.8 & 35.8 \\
\hline S96T005851' & $167: 2$ & Lower half & 38.1 & 39.8 & 38.9 \\
\hline S96T0056691 & $167: 3$ & Lower half & 33.3 & 42.0 & 37.6 \\
\hline S96T005675 & \multirow[t]{2}{*}{$167: 4$} & Lower half & 29.5 & 18.0 & $23.7^{\mathrm{QC:c}}$ \\
\hline S96T005675.1 & & Lower half & 24.3 & 20.8 & 22.6 \\
\hline S96T005424 1 & $167: 5$ & Lower half & 32.0 & 29.1 & 30.5 \\
\hline S96T005425 & $167: 6$ & Lower half & 39.7 & 32.5 & 36.1 \\
\hline S96T005685 & \multirow[t]{2}{*}{$167: 7$} & Lower half & 49.6 & 18.9 & $34.2^{Q \mathrm{QC:c}}$ \\
\hline S96T005685.1 & & Lower half & 18.1 & 18.5 & 18.3 \\
\hline S96T005734 & $167: 8$ & Lower half & 14.3 & 16.1 & 15.185 \\
\hline S96T005852 & 167: 9 & Lower half & 19.8 & 19.65 & 19.2 \\
\hline S96T005646 & \multirow[t]{2}{*}{$167: 11$} & Upper half & 45.9 & 45.8 & 45.9 \\
\hline S96T005647 & & Lower half & 46.3 & 46.8 & 46.6 \\
\hline S96T005432 & \multirow[t]{2}{*}{ 167: 12} & Upper half & 45.8 & 41.6 & 43.7 \\
\hline S96T005426 & & Lower half & 38.3 & 36.7 & 37.5 \\
\hline
\end{tabular}




\begin{tabular}{|c|c|c|c|}
\hline$L I D$ & p!̣b!̣ әqqu!̣ıa & II : L9I & 0S9S00L96S \\
\hline$s+t$ & pinb!̣ ə̣qeuịeıa & $8: \angle 9 I$ & $\tau E \angle S 00 L 96 \mathrm{~S}$ \\
\hline$I \nabla t$ & punb!̣ әqqụeı & $L: \angle 9 I$ & E89S00L96S \\
\hline$\varepsilon * t \nabla$ & p!nb!̣ әтqeựeıd & $\varepsilon: \angle 9 I$ & EL9S00L96S \\
\hline$I \cdot S t$ & 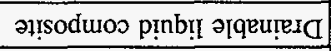 & 991 2103 & $066 S 00 \mathrm{LL} 6 \mathrm{~S}$ \\
\hline $0^{\circ} 9 z$ & fleप дәмот - sp!IOS & s:L9I & $\triangleright Z \downarrow \varsigma 00 L 96 \mathrm{~S}$ \\
\hline$S Z I$ & કlРЧ IәмOT - sp!IOS & $\nabla: \angle 9 I$ & 1 SL9S00L96S \\
\hline$L 62$ & 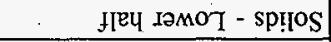 & $\varepsilon: \angle 9 I$ & $699 \$ 00 L 96 S$ \\
\hline$\angle \nabla \mathcal{E}$ & Ileप دәмот - splios & $\tau: \angle 9 I$ & IS8S00J96S \\
\hline$L \triangleright T$ & Irey Iədd $\Omega$ - sprros & $\angle I: \angle 9 I$ & $26 t 500 \mathrm{LL6S}$ \\
\hline $8: L Z$ & J[вu Iədd & $8 \mathrm{I}: 99 \mathrm{I}$ & 8E9S00L96S \\
\hline $56 z$ & I[eप ıədd $\Omega$ - sp!Ios & LI:99I & $00 \star S 00 L 96 \mathrm{~S}$ \\
\hline $6.0 z$ & IIPU IәмOT - spIIOS & $9: 99 \mathfrak{l}$ & $6 L S S 00 L L 6 S$ \\
\hline 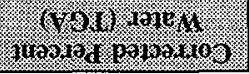 & 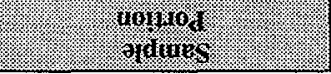 & 100960 & orguring \\
\hline
\end{tabular}

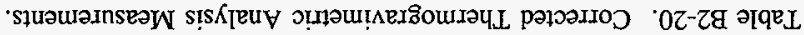

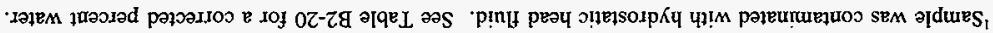

\begin{tabular}{|c|c|c|c|c|c|}
\hline $0^{\circ} \varepsilon \varepsilon$ & $I^{\prime} \mathcal{E}$ & $0^{\circ} \mathcal{E E}$ & วt!soduos p!̣OS & 99I วI0D & $786 \mathrm{SO0L}$ L6S \\
\hline $9^{\circ} \mathrm{SE}$ & $0^{\circ} \varepsilon \varepsilon$ & $\tau \cdot 8 \varepsilon$ & J[ЕЧ IәMOT] & \multirow[b]{2}{*}{$\angle I: \angle 9 I$} & $\varepsilon 67 \$ 00 L 96 S$ \\
\hline $8^{\circ} \varepsilon 2$ & $s^{\circ}+z$ & $0 \cdot \mathcal{E Z}$ & J[Eч $\operatorname{rodd}_{\Omega}$ & & ${ }_{\mathrm{I}}^{2} 6 \mathrm{tS00 \textrm {L } 9 6 \mathrm { S }}$ \\
\hline $9^{\circ} 92$ & $\tau: L Z$ & 0.92 & IГеप IәмоТ & \multirow[b]{2}{*}{ SI : $\angle 9 I$} & 9\$9S00L96S \\
\hline$L^{\circ} \mathcal{E} \mathcal{Z}$ & $\varepsilon+\mathcal{Z}$ & $\tau \cdot \mathcal{E}$ & y[eч Iədd $\Omega$ & & S\$9S00L96S \\
\hline $8^{\circ}+2$ & 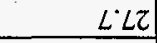 & $0.2 z$ & J[Eप Iәмо ] & \multirow[b]{2}{*}{$\nabla I: \angle 9 I$} & LZtS00J96S \\
\hline$\nabla^{\circ} \mathrm{OE}$ & $\nabla 0 \varepsilon$ & $\varsigma 0 \varepsilon$ & J[eप Iədd $\Omega$ & & EEtS00L96S \\
\hline . & . & 8.6 & & & Wrom solos \\
\hline ren Kr. & 678011169 & 2018 & olow & moromos & Than \\
\hline
\end{tabular}

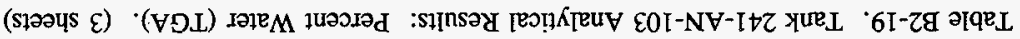


Table B2-21. Tank 241-AN-103 Analytical Results: Exotherm (Differential Scanning Calorimetry, Dry). (2 sheets)

\begin{tabular}{|c|c|c|c|c|c|}
\hline Sanile & 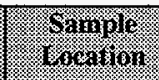 & Samilu & iresill & Buplicate & riten \\
\hline Solids. & (2) & 3 & 18 & $1 / 3$ & 㴗, \\
\hline S96T005578 & 166: 1 & Lower half & 67.8 & 83.0 & 75.4 \\
\hline S96T005543 & $166: 9$ & Lower half & 0 & 91.9 & $45.95^{\mathrm{QC}: 0}$ \\
\hline S96T005580 & 166: 11 & Lower half & 2.16 & 0 & $1.08^{\mathrm{QC}: \mathrm{O}}$ \\
\hline S96T005581 & 166: 12 & Lower half & 26.8 & 29.5 & 28.15 \\
\hline S96T005636 & 166: 13 & Upper half & 0 & 44.3 & $22.15^{\mathrm{QC:c}}$ \\
\hline S96T005398 & \multirow[t]{2}{*}{$166: 15$} & Upper half & 8.0 & 57.8 & $32.9^{\mathrm{QC:c}}$ \\
\hline S96T005399 & & Lower half & 74.1 & 30.1 & $52.1^{\mathrm{QC}: \mathrm{c}}$ \\
\hline S96T005582. & $166: 16$ & Upper half & 66.9 & 50.3 & 58.6 \\
\hline S96T005583 & & Lower half & 82.0 & 66.0 & 74.0 \\
\hline S96T005400 & \multirow[t]{2}{*}{$166: 17$} & Upper half & 38.9 & 30.2 & 34.55 \\
\hline S96T005401 & & Lower half & 32.7 & 53.5 & $43.1^{\mathrm{QC}: \mathrm{c}}$ \\
\hline S96T005639 & 166: 18 & Lower half & 13.2 & 20.3 & $16.75^{\mathrm{QC}: c}$ \\
\hline S96T005822 & \multirow[t]{2}{*}{ 166: 19} & Upper half & 0 & 0 & 0 \\
\hline S96T005823 & & Lower half & 52.0 & 26.6 & $39.3^{\mathrm{QC:c}}$ \\
\hline S96T005984 & Core 166 & Core composite & 15.4 & 38.5 & $26.95^{\mathrm{QC}: \mathrm{e}}$ \\
\hline S96T005668 & 167:1 & Lower half & 16.4 & 16.3 & 16.35 \\
\hline S96T005851 & $167: 2$ & Lower half & 152.0 & 83.7 & $118.1^{\mathrm{QC}: \mathrm{c}}$ \\
\hline S96T005669 & $167: 3$ & Lower half & 82.4 & 45.5 & $63.95^{\mathrm{QC}: c}$ \\
\hline S96T005685 & $167: 7$ & Lower half & 145.0 & 34.0 & $89.5^{\mathrm{QC}: \mathrm{c}}$ \\
\hline S96T005852 & $167: 9$ & Lower half & 13.5 & 0 & $6.75^{\mathrm{QC}: \mathrm{e}}$ \\
\hline S96T005646 & \multirow[t]{2}{*}{$167: 11$} & Upper half & 12.2 & 6.84 & $9.52^{\mathrm{QC}: \mathrm{c}}$ \\
\hline S96T005647 & & Lower half & 8.05 & 15.9 & $11.98^{\mathrm{QC}: \mathrm{e}}$ \\
\hline S96T005432 & $167: 12$ & Upper half & 7.64 & 78.2 & $42.92^{\mathrm{QC}: c}$ \\
\hline S96T005433 & $167: 14$ & Upper half & 46.3 & 32.5 & $39.4^{\mathrm{QCe}}$ \\
\hline S96T005655 & 167: 15 & Upper half & 40.8 & 106.0 & $73.4^{\mathrm{QC}: \mathrm{e}}$ \\
\hline
\end{tabular}


Table B2-21. Tank 241-AN-103 Analytical Results: Exotherm (Differential Scanning Calorimetry, Dry). (2 sheets)

\begin{tabular}{|c|c|c|c|c|c|}
\hline 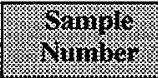 & $\begin{array}{l}\text { Tample } \\
\text { Herivon }\end{array}$ & Molnou & Resnit & 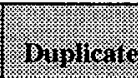 & Vlerin \\
\hline How & ( & (x) & 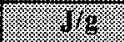 & \% & 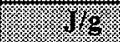 \\
\hline \$96T005539 & 166: 3 & Drainable liquid & 53.4 & 0 & $26.7^{\mathrm{QC:e}}$ \\
\hline S96T005816 & 166: 7 & Drainable liquid & 142.0 & 118.0 & 130.0 \\
\hline S96T005817 & 166: 8 & Drainable liquid & 39.6 & 44.7 & 42.15 \\
\hline S96T005541. & 166: 9 & Drainable liquid & 35.5 & 0 & $17.75^{\mathrm{QC:e}}$ \\
\hline S96T005566 & $166: 10$ & Drainable liquid & 195.0 & 107.0 & $151.0^{\mathrm{QC}: \mathrm{e}}$ \\
\hline S96T005990 & Core 166 & $\begin{array}{l}\text { Drainable liquid } \\
\text { composite }\end{array}$ & 171.0 & 231.0 & $201.0^{\mathrm{QC}: \mathrm{e}}$ \\
\hline S96T005861 & 167: 2 & Drainable liquid & 193.0 & 268.0 & $230.5^{\mathrm{QC:c}}$ \\
\hline S96T005421 & $167: 6$ & Drainable liquid & 0 & 55.7 & $27.85^{\mathrm{QC}: \mathrm{s}}$ \\
\hline S96T005683 & 167: 7 & Drainable liquid & 0 & 172.0 & $86.0^{\mathrm{QC}: \mathrm{c}}$ \\
\hline S96T005862 & 167: 9 & Drainable liquid & 185.0 & 229.0 & $207.0^{\mathrm{QC}: \mathrm{c}}$ \\
\hline$\$ 96 \mathrm{~T} 005650$ & 167: 11 & Drainable liquid & 41.3 & 106.0 & $73.65^{\mathrm{QC:e}}$ \\
\hline
\end{tabular}

Table B2-22. Tank 241-AN-103 Analytical Results: Bulk density. (2 sheets)

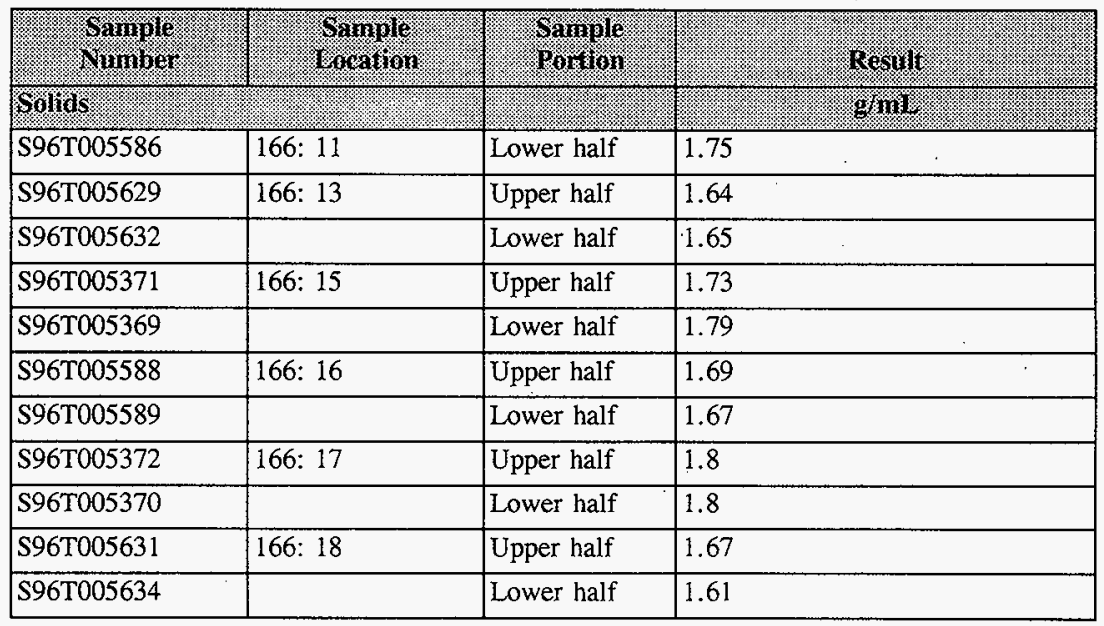


Table B2-22. Tank 241-AN-103 Analytical Results: Bulk density. (2 sheets)

\begin{tabular}{|c|c|c|c|c|}
\hline 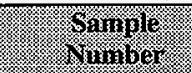 & . 597 gle & 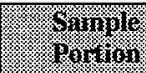 & & 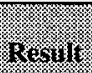 \\
\hline of Hos & (1: & & & g6inim \\
\hline S96T005812 & 166: 19 & Upper half & 1.59 & \\
\hline S96T005813 & & Lower half & 1.6 & \\
\hline S96T005660 & 167: 1 & Upper half & 1.68 & \\
\hline S96T005661 & . & Lower half & 1.72 & \\
\hline S96T005847 & $167: 2$ & Lower half & 1.59 & \\
\hline S96T005374 & $167: 5$ & Lower half & 1.83 & \\
\hline S96T005375 & $167: 6$ & Lower half & 1.8 & \\
\hline S96T005644 & 167: 11 & Upper half & 1.69 & \\
\hline S96T005645 & & Lower half & 1.67 & \\
\hline S96T005378 & 167: 12 & Upper half & 1.7 & \\
\hline \$96T005376 & & Lower half & 1.69 & \\
\hline S96T005379 & $167: 14$ & Upper half & 1.88 & \\
\hline S96T005377 & & Lower half & 1.93 & \\
\hline S96T005653 & $167: 15$ & Upper half & 1.92 & \\
\hline \$96T005654 & & Lower half & 1.88 & \\
\hline S96T005490 & $167: 17$ & Upper half & 1.71 & \\
\hline S96T005491. & & Lower half & 1.7 & \\
\hline S96T005983 & Core 166 & $\begin{array}{l}\text { Solid } \\
\text { composite }\end{array}$ & 1.69 & \\
\hline
\end{tabular}


-sonjen asaul

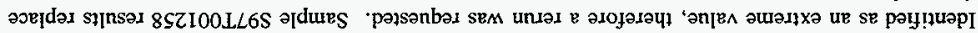

\begin{tabular}{|c|c|c|c|c|c|}
\hline $67^{\circ} \mathrm{I}$ & $6 t^{\circ} I$ & $6 t^{\circ} \mathrm{I}$ & əl!̣soduos p!nbry & 991 ə10 & E66S00L96S \\
\hline $87^{\circ} \mathrm{I}$ & $0 S^{\circ} \mathrm{T}$ & $t t^{\circ} I$ & 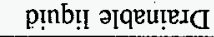 & II $: \angle 9 I$ & 0S9S00L96S \\
\hline$\angle t^{\circ} I$ & $\angle \forall^{\circ} I$ & $\angle t^{\circ} I$ & pịnb!̣ әІqеu!̣ıa & $6: \angle 9 I$ & z98S00L96S \\
\hline$\angle D^{\circ} I$ & $\angle t^{\circ} I$ & $\angle t^{\circ} I$ & pinbụ શqeuresa & $8: \angle 9 I$ & ZELSO0L96S \\
\hline $9 t^{\circ} \mathrm{I}$ & $97^{\circ} I$ & $s_{t}{ }^{*}$ & pinby әqеu!eda & $L: \angle 9 I$ & E89S00L96S \\
\hline$\angle \nabla^{\circ} I$ & $\angle D^{\circ} I$ & $9 t^{\circ} \mathrm{I}$ & \multirow[b]{2}{*}{ p!nb!̣ әрqeutedવ } & \multirow[b]{2}{*}{$9: \angle 91$} & 8SZI00LL6S \\
\hline${ }_{1} Z L Z$ & ${ }_{\mathrm{I}} L L Z Z$ & ${ }_{1} \angle 9^{\circ} \mathrm{Z}$ & & & IZtS00L96S \\
\hline $0 t^{\circ} \mathrm{I}$ & $0 \nabla^{*} \mathrm{I}$ & $00^{\circ} \mathrm{I}$ & 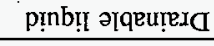 & $\$: \angle 9 I$ & 0Z†S00L96S \\
\hline$E S^{*} I$ & $E S^{\circ} I$ & $t s^{\circ} I$ & 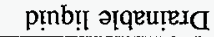 & $\varepsilon: L 9 I$ & EL9S00L96S \\
\hline $6 S t^{\circ} \mathrm{I}$ & $9 t^{\circ} I$ & $97^{\circ} \mathrm{I}$ & pṭb!̣ әlqeuresa & $\tau: \angle 9 I$ & I98S00L96S \\
\hline$s \not s t^{\circ} I$ & $\nabla t^{\circ} I$ & $\angle D I$ & p!nbị әฺqeuteıa & ZI :99I & I09\$00L96S \\
\hline$S S 6 \mathcal{E}^{*} \mathrm{I}$ & $6 \mathcal{E}^{\circ} \mathrm{I}$ & $0 D^{\circ} 1$ & pụnbỵ əॄqeụera & II :99I & 009\$00L96S \\
\hline$\varepsilon \nabla^{\circ} I$ & $\nabla \varepsilon^{-} I$ & IS:I & 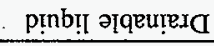 & or :99I & 995S00L96S \\
\hline$\nabla t^{\circ} I$ & $\Delta t^{\circ} I$ & $S t^{\circ} I$ & pı̣nb!̣ әІqеu!̣ı & $6: 99 \mathrm{I}$ & ItSSO0L96S \\
\hline$\angle D^{\circ} I$ & $9 t^{\circ} \mathrm{I}$ & $\angle D^{\circ} I$ & pṭb!l әqqeuresa & $8: 99 \mathrm{I}$ & LI8S00L96S \\
\hline $9 t^{\circ} 1$ & $9 t^{\circ} \mathrm{I}$ & $9 t^{\circ} \mathrm{I}$ & pı̣b!l әІqеuाe.d & $L: 991$ & 9I8S00L96S \\
\hline $80^{\circ} \mathrm{I}$ & $87^{\circ} \mathrm{I}$ & $80^{\circ} \mathrm{I}$ & 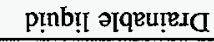 & $9: 991$ & $66 \$ 500 . \mathrm{L} 96 \mathrm{~S}$ \\
\hline $90^{\circ} \mathrm{I}$ & $9 t^{\circ} \mathrm{I}$ & $90^{\circ} \mathrm{I}$ & p!̣b!̣ әlqeu!̣eı & $t: 991$ & 0tSS00L96S \\
\hline$L D^{\circ} I$ & $L t^{\circ} I$ & $9 t^{\circ} I$ & pṭb!̣ әlqeutes & $\mathcal{\varepsilon}: 99 \mathrm{I}$ & 6ESS00L96S \\
\hline s. & 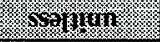 & S5ompun & & & syolog \\
\hline (1) & 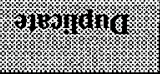 & \% & \%olor & 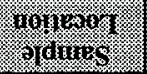 & 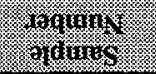 \\
\hline
\end{tabular}

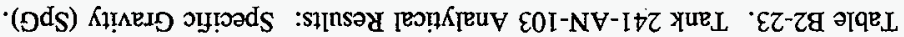


HNF-SD-WM-ER-702 Rev. 0

Table B2-24. Tank 241-AN-103 Analytical Results: Aluminum (ICP). (4 sheets)

\begin{tabular}{|c|c|c|c|c|c|}
\hline $\begin{array}{l}\text { Srinole } \\
\text { Minibey }\end{array}$ & $\begin{array}{l}\text { Sample } \\
\text { Wonition }\end{array}$ & 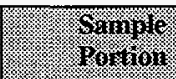 & masum & 多年, & . \\
\hline \multicolumn{3}{|c|}{ 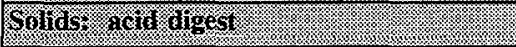 } & 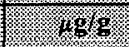 & (1) & (3) \\
\hline S96T005609 & 166: 1 & Lower half & 92,000 & 81,000 & 86,500 \\
\hline S96T005570 & 166: 4 & Lower half & 7,060 & 7,780 & $7,420^{\mathrm{QC}: c}$ \\
\hline S96T005615 & 166: 6 & Lower half & 8,670 & 8,630 & 8,650 \\
\hline S96T005839 & 166: 7 & Lower half & 7,190 & 7,360 & 7,280 \\
\hline S96T005840 & $166: 8$ & Lower half & 7,290 & 7,270 & $7,280^{\mathrm{QC:A}}$ \\
\hline S96T005575 & $166: 9$ & Lower half & 14,200 & 12,300 & 13,200 \\
\hline S96T005571 & 166: 10 & Lower half & 10,400 & 10,400 & 10,400 \\
\hline S96T005616 & 166: 11 & Lower half & 7,300 & 6,600 & 6,950 \\
\hline S96T005617 & $166: 12$ & Lower half & 6,700 & 7,120 & 6,910 \\
\hline S96T005716 & \multirow[t]{2}{*}{$166: 13$} & Upper half & 52,700 & 56,600 & 54,600 \\
\hline S96T005722 & & Lower half & 55,900 & 59,900 & 57,900 \\
\hline S96T005416 & \multirow[t]{2}{*}{ 166: 15} & Upper half & 68,900 & 73,200 & 71,000 \\
\hline S97T000459 & & Lower half & 67,700 & 78,500 & $73,100^{\mathrm{QC:d}}$ \\
\hline S96T005624 & \multirow[t]{2}{*}{$166: 16$} & Upper half & 83,700 & 81,800 & 82,700 \\
\hline$\$ 96 \mathrm{~T} 005618$ & & Lower half & 75,900 & 76,700 & 76,300 \\
\hline S96T005417 & \multirow[t]{2}{*}{$166: 17$} & Upper half & 88,800 & 91,700 & 90,200 \\
\hline \$96T005411 & & Lower half & 75,600 & 65,500 & $70,500^{\mathrm{QC}: c}$ \\
\hline S96T005717 & \multirow[t]{2}{*}{ 166: 18} & Upper half & 33,400 & 33,700 & 33,500 \\
\hline S96T005723 & & Lower half & 21,700 & 22,700 & 22,200 \\
\hline S96T00584l & \multirow[t]{2}{*}{$166: 19$} & Upper half & 26,000 & 25,500 & $25,700^{\mathrm{QC:a}}$ \\
\hline S96T005842 & & Lower half & 23,400 & 23,200 & $23,300^{\mathrm{QC:}: \mathrm{s}}$ \\
\hline S96T005708 & \multirow[t]{2}{*}{ 167: 1} & Upper half & 55,300 & 84,700 & $70,000^{\mathrm{QC}: \mathrm{e}}$ \\
\hline S96T005693 & & Lower half & 74,100 & 78,800 & 76,400 \\
\hline S96T005857 & $167: 2$ & Lower half & 57,500 & 57,700 & 57,600 \\
\hline S96T005694 & $167: 3$ & Lower half & 12,800 & 8,820 & $10,800^{\mathrm{QC}: c}$ \\
\hline S96T005695 & $167: 4$ & Lower half & 11,400 & 12,600 & 12,000 \\
\hline S96T005438 & $167: 5$ & Lower half & 15,200 & 14,200 & $14,700^{\mathrm{QC:c}}$ \\
\hline S96T005450 & 167: 6 & Lower half & 12,700 & 13,200 & 12,900 \\
\hline S96T005696 & $167: 7$ & Lower half & 6,930 & 7,490 & 7,210 \\
\hline
\end{tabular}




\begin{tabular}{|c|c|c|c|c|c|}
\hline $000^{\prime} \varepsilon \varepsilon$ & $006^{\circ} \mathrm{zE}$ & $00 Z^{\prime} \varepsilon \varepsilon$ & 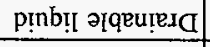 & $\angle: \angle 9 I$ & E89S00L96S \\
\hline $00 S^{6} I E$ & $00 t^{4} I \varepsilon$ & $009^{6} I \mathcal{E}$ & p!̣bỊ ә[qeurerc] & $9: \angle 9 \mathrm{I}$ & IZ† $\$ 00 \mathrm{~L} 96 \mathrm{~S}$ \\
\hline $\mathrm{p}: \supset 0009^{\prime} \tau \varepsilon$ & $00 L^{\prime} Z \varepsilon$ & $00 s^{\prime} z \varepsilon$ & p!̣b!̣ ә[qeu!esa & $S: \angle 9 I$ & $0 Z \triangleright 500 L 96 S$ \\
\hline $00 I^{\prime} 6 z$ & $006^{\circ} 8 z$ & $00 t^{\circ} 62$ & pịnb!r ə[qeu!exa & $\varepsilon: \angle 9 I$ & EL9S00L96S \\
\hline $00 t^{6} Z \varepsilon$ & $008^{\circ} z \mathcal{E}$ & $00 I^{\prime} z \varepsilon$ & 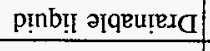 & $Z: \angle 9 I$ & [98S00L96S \\
\hline $0000 z^{\prime} \varepsilon \varepsilon$ & $00 Z^{\prime} \varepsilon \varepsilon$ & $00 z^{\prime} \varepsilon \mathcal{E}$ & pınb!l әrqвuгe. & ZI:99I & I09\$00L96S \\
\hline $00 z^{\prime} I \varepsilon$ & $009^{\circ} 0 \varepsilon$ & $008^{\prime} I \varepsilon$ & p!nb!̣ ә[qeựera & II :99I & 009S00L96S \\
\hline$O O \mathcal{E}^{\prime} 0 \mathcal{E}$ & $00 T^{\prime} 0 \mathcal{E}$ & $009^{6} 0 \mathcal{E}$ & pịb!̣ ә[qeutera] & $01: 99 \mathrm{I}$ & 99SS00L96S \\
\hline$: 0000 Z^{\prime} I \varepsilon$ & $006^{\circ} 0 \varepsilon$ & $00 S^{6} I \varepsilon$ & p!̣b!l әтqeựeIa & $6: 991$ & ItSS00L96S \\
\hline $00 \mathcal{E}^{\prime} \mathrm{ZE}$ & $00 L^{\prime} \varepsilon \mathcal{E}$ & $000^{\circ} \mathrm{IE}$ & pınb!̣ əlqeurexa & $8: 99 \mathrm{I}$ & LI8S00L96S \\
\hline$p=>0000^{\circ} \mathcal{E E}$ & $\cos ^{6} \varepsilon \varepsilon$ & $00 S^{\prime} Z \mathcal{E}$ & p!̣b!̣ әqqeu!̣eI & $L: 99 \mathrm{I}$ & 9 I8S00L96S \\
\hline$: 0000 L^{\prime} \tau \varepsilon$ & $00 L^{6} I \mathcal{E}$ & $00 L^{\prime} \varepsilon \varepsilon$ & pı̣b!̣া әтqeurera & $9: 99$ I & 66\$\$00L96S \\
\hline $006^{\prime} \subseteq \mathcal{Z}$ & $008^{\prime}+z$ & $000^{\circ} \angle Z$ & pinbur ગqqutera & $\nabla: 991$ & $0 t S \$ 00 \mathrm{I} 96 \mathrm{~S}$ \\
\hline $00 L ' 6 Z$ & $00 \mathcal{E}^{\prime} 8 \mathcal{Z}$ & $00 Z^{6} I \varepsilon$ & p!̣b!̣ ગ̨qeureIa & $\mathcal{E}: 99 \mathrm{I}$ & $6 \varepsilon \varsigma \$ 00 L 96 S$ \\
\hline geren & (1401\% & mangh & & & spror \\
\hline $00 Z^{\prime} 8 \varepsilon$ & $00 \mathcal{E}^{\prime} 6 \varepsilon$ & $00 I^{\prime} \angle E$ & әฺ!soduos p![OS & 99I ә10ว & 09t000LL6S \\
\hline $00 \mathcal{E}^{6}$ II & $006^{\prime}$ II & $008^{\circ} 0 \mathrm{~T}$ & fleप IәMO I & \multirow[b]{2}{*}{$\angle I: \angle 9 I$} & $66 t S 00 L 96 \mathrm{~S}$ \\
\hline $00 t^{6} 9 I$ & $00 Z^{6} 9 \mathrm{I}$ & $009^{\circ} 9 \mathrm{I}$ & 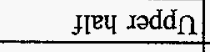 & & $86+500 L 96 S$ \\
\hline $90+370^{\circ} I$ & $S 0+\exists S 0^{\circ} \mathrm{I}$ & $S 0+\exists E 0^{\circ} \mathrm{I}$ & I[Eบ IәMOT] & \multirow[b]{2}{*}{ SI : $\angle 9 I$} & 869 S00L96S \\
\hline $009^{\prime} \angle L$ & $006^{\circ} \angle L$ & $00 \varepsilon^{\prime} \angle L$ & fley rədd $\Omega$ & & OILSO0L96S \\
\hline$p: 0000 S^{6} 16$ & $002^{4} 86$ & $006^{\prime}+8$ & fleY Iәмо7] & \multirow[b]{2}{*}{$\nabla I: \angle 9 I$} & $6 \mathcal{G}+\mathrm{S} 00 \mathrm{~L}$. $96 \mathrm{~S}$ \\
\hline $008^{6} \varepsilon 8$ & $00 t^{\circ} 9 L$ & $00 z^{\prime} \div 6$ & J[Еप $\left.\mathbf{1 ə d} \mathrm{d}_{\Omega}\right]$ & & $S t \nabla S 00 L 96 S$ \\
\hline $006^{\circ} \angle 9$ & $00 S^{\prime} 99$ & $00 \mathcal{E}^{\prime} 69$ & FІеЧ IәMOT & \multirow[b]{2}{*}{$Z \mathrm{I}: \angle 9 \mathrm{I}$} & IStSO0L96S \\
\hline $00 \mathcal{E}^{\prime}[9$ & $00 z^{4} 29$ & $00 \varsigma^{\prime} 09$ & I[eप $\operatorname{sadd}_{\Omega}$ & & $\nabla t t S 00 \mathrm{~J} 96 \mathrm{~S}$ \\
\hline $00 L^{\circ} 9 S$ & $00 \mathcal{E}^{\prime} \nabla \varsigma$ & $00 Z^{6} 6 s$ & J[Еप ІәMOT] & \multirow[b]{2}{*}{ II : $\angle 9$ I } & L69S00L96S \\
\hline $00 s^{\prime} i s$ & $008^{\prime}$ IS & $00 Z^{6}$ IS & J[Еч Iədd $_{\Omega}$ & & $60 \angle S 00 L 96 \mathrm{~S}$ \\
\hline $006^{\circ} \mathrm{L}$ & $086^{6} \mathrm{~L}$ & $0 \varepsilon 8^{\circ} \angle$ & ЈГеप ІәмоТ & $6: \angle 9 \mathrm{I}$ & $8 \$ 8 \$ 00196 S$ \\
\hline $08 L^{*} L$ & $082^{\circ} L$ & $082^{\prime} 8$ & IIRU IəMOT & $8: \angle 9 I$ & LELSO0L96S \\
\hline 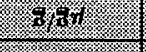 & \%gr: & 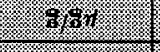 & \multicolumn{3}{|c|}{ r. } \\
\hline 10.6 & $9 \% 610110$ & $\sin 1$ & 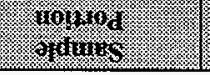 & 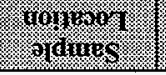 & momenting \\
\hline
\end{tabular}

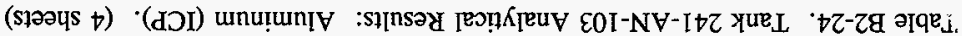


Table B2-24. Tank 241-AN-103 Analytical Results: Aluminum (ICP). (4 sheets)

\begin{tabular}{|c|c|c|c|c|c|}
\hline 6) & Saripie & gongon & rosting & (bin) & (6) \\
\hline \multicolumn{2}{|c|}{ 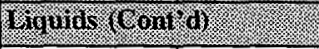 } & & . & W. & $14 \mathrm{or} / 11 \%$ \\
\hline S96T005732 & $167: 8$ & Drainable liquid & 33,100 & 32,600 & 32,800 \\
\hline S96T005862 & 167: 9 & Drainable liquid & 32,300 & 32,600 & 32,400 \\
\hline \$96T005650 & 167: 11 & Drainable liquid & 31,600 & 31,700 & 31,600 \\
\hline \$96T005991 & Core 166 & Liquid composite & 32,000 & 32,200 & $32,100^{\circ \mathrm{CC}: c}$ \\
\hline \multicolumn{2}{|c|}{ 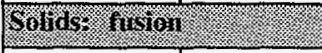 } & & (1) & 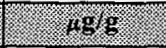 & f \\
\hline$\$ 96 \mathrm{~T} 005608$ & 166:1 & Lower half & 94,200 & 95,600 & 94,900 \\
\hline S96T005568 & 166: 4 & Lower half & 8,550 & 9,220 & $8, \overline{880}$ \\
\hline$\$ 96 \mathrm{~T} 005611$ & $166: 6$ & Lower half & 8,740 & 7,610 & 8,170 \\
\hline S96T005835 & $166: 7$ & Lower half & 9,560 & 9,560 & 9,560 \\
\hline S96T005836 & 166: 8 & Lower half & 6,920 & 5,390 & $6,150^{\mathrm{QC:c}}$ \\
\hline \$96T005574 & $166: 9$ & Lower half & 15,100 & 15,100 & 15,100 \\
\hline S96T005569 & $166: 10$ & Lower half & 10,000 & 8,560 & 9,280 \\
\hline S96T005612 & $166: 11$ & Lower half & 6,410 & 6,550 & 6,480 \\
\hline \$96T005613 & 166: 12 & Lower half & 6,370 & 4,960 & $5,660^{\mathrm{Qc}: e}$ \\
\hline S96T005714 & \multirow[t]{2}{*}{$166: 13$} & Upper half & 71,400 & 71,000 & 71,200 \\
\hline S96T005720 & & Lower half & 76,700 & 76,700 & 76,700 \\
\hline$\$ 96 \mathrm{~T} 005414$ & \multirow[t]{2}{*}{$166: 15$} & Upper half & 21,200 & 20,300 & 20,700 \\
\hline S96T005408 & & Lower half & 23,700 & 23,500 & 23,600 \\
\hline \$96T005623 & \multirow[t]{2}{*}{$166: 16$} & Upper half & $1.05 E+05$ & $1.02 E+05$ & $1.03 E+05$ \\
\hline S96T005614 & & Lower half & 72,400 & 73,300 & 72,800 \\
\hline \$96T005415 & \multirow[t]{2}{*}{$166: 17$} & Upper half & 27,500 & 28,400 & 27,900 \\
\hline$\$ 96 T 005409$ & & Lower half & 86,800 & 87,000 & 86,900 \\
\hline S96T005715 & \multirow[t]{2}{*}{$166: 18$} & Upper half & 43,800 & 45,700 & 44,700 \\
\hline S96T005721 & & Lower half & 31,000 & 29,400 & 30,200 \\
\hline \$96T005837 & \multirow[t]{2}{*}{$166: 19$} & Upper half & 23,500 & 23,600 & 23,500 \\
\hline S96T005838 & & Lower half & 23,900 & 24,100 & 24,000 \\
\hline S96T005705 & \multirow[t]{2}{*}{$167: 1$} & Upper half & $1.02 \mathrm{E}+05$ & $1.04 \mathrm{E}+05$ & $1.03 \mathrm{E}+05$ \\
\hline S96T005687 & & Lower half & 75,400 & 63,900 & 69,600 \\
\hline S96T005855 & $167: 2$ & Lower half & 74,600 & 73,200 & 73,900 \\
\hline
\end{tabular}




\begin{tabular}{|c|c|c|c|c|c|}
\hline $200056^{\circ} \mathrm{II}$ & $006^{6} \mathrm{EI}$ & $000^{\circ} 0 \mathrm{I}$ & fley Iamó I & & $\angle 67 \$ 00 L 96 \mathrm{~S}$ \\
\hline $000 \mathcal{S S}^{6} \varepsilon Z$ & $00 t^{\circ} \angle 2$ & $00 \angle 6 \mathrm{I}$ & JIEบ Iədd & $\angle I: \angle 9 I$ & $96+S 00 L 96 \mathrm{~S}$ \\
\hline$S O+\exists \varepsilon Z^{\prime} I$ & $S 0+B S Z \cdot I$ & $\mathrm{~S}_{0}+\mathrm{GLZZ}^{\prime} \mathrm{I}$ & fЕц Іәмо & \multirow[b]{2}{*}{$\xi I: \angle 9 I$} & Z69500L96S \\
\hline$S O+\exists Z 0^{\circ} \mathrm{I}$ & $\mathrm{SO}+\mathrm{GE} \mathrm{EO}^{\circ} \mathrm{I}$ & SO+BIO'I & Jleu Iədd $\Omega$ & & $\angle 0 \angle S 00 L 96 \mathrm{~S}$ \\
\hline $00 L^{\prime}+6$ & $00 S^{\prime} 96$ & $000^{\circ} \varepsilon 6$ & न Jеद ғәмот & \multirow[b]{2}{*}{ tr : $\angle 9 I$} & $\angle \mathcal{L}$ SOOL $96 \mathrm{~S}$ \\
\hline $00 \varepsilon^{\prime} 98$ & $006^{\circ} \angle 8$ & $008^{\prime}+8$ & Ijey raddn & & EttS00L96S \\
\hline $009^{\prime} 89$ & $009^{\circ} 69$ & $00 L^{\circ} \angle 9$ & J[Еप ІәмоТ & \multirow[b]{2}{*}{ ZI : L9I } & $6 t+500196 \mathrm{~S}$ \\
\hline $000^{6}+8$ & $006^{\prime}$ โ8 & $002^{4} 98$ & fley ıədd $\Omega$ & & $\tau \nabla t \subseteq 00196 \mathrm{~S}$ \\
\hline $008^{\circ} \mathrm{IL}$ & $009^{\circ} Z L$ & OOI'IL & J[еप Іәмот] & \multirow[b]{2}{*}{ I I : $\angle 9 \mathrm{I}$} & I69S00L96S \\
\hline $00 s^{6} z 9$ & $009^{\prime} \varepsilon 9$ & $005^{6}[9$ & fाеप ıәdd $\Omega$ & & $90 L S 00 L 96 \mathrm{~S}$ \\
\hline $00 Z^{6} 0 \mathrm{I}$ & $00 t^{\circ} 0 \mathrm{I}$ & $00 I^{\circ} 0 I$ & I[RY IәMOT & $6: \angle 9 I$ & $9 \$ 8 \$ 00 L 96 \mathrm{~S}$ \\
\hline $00 t^{\prime} 8$ & $000^{\circ} 8$ & $008^{\prime} 8$ & I[еप دәмот & $8: \angle 9 I$ & 9ELS00.L96S \\
\hline $0<8^{\circ} 8$ & $0+9^{6} 8$ & OII'6 & J[Еप Іәмот & $L: \angle 9 I$ & $069 \$ 00 \mathrm{~L} 96 \mathrm{~S}$ \\
\hline $008^{6}+I$ & $000^{\circ}+1$ & $00 L ' \mathrm{SI}$ & I[Eบ . Iәмот & $9: \angle 9 \mathrm{~T}$ & $8 t t S 00 L 96 \mathrm{~S}$ \\
\hline $009^{\prime} \varepsilon I$ & $009^{\circ} \mathrm{ZL}$ & $009^{\prime} \rightarrow I$ & IГеप Iәмот & s:L9I & $9 \varepsilon \nrightarrow S 00 L 96 \mathrm{~S}$ \\
\hline $066^{6} 8$ & $0\left[\varepsilon^{\prime} 6\right.$ & $089^{\prime} 8$ & f[Eप دәмOT & $\downarrow: \angle 9 I$ & $689 \mathrm{~S} 00 \mathrm{I} 96 \mathrm{~S}$ \\
\hline $0 \varepsilon C^{6} 8$ & $018^{\circ} \mathrm{L}$ & $099^{\prime} 8$ & I[Rप IәMOT & $\varepsilon: \angle 9 I$ & $889500 L 96 \mathrm{~S}$ \\
\hline (3) & $6.6 \%$ & $6 \%$ & 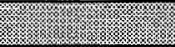 & \multicolumn{2}{|c|}{ 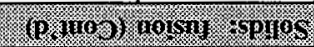 } \\
\hline 1961) & 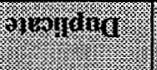 & 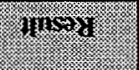 & $\frac{106 \%}{40 \%}$ & 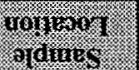 & 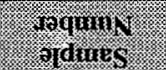 \\
\hline
\end{tabular}

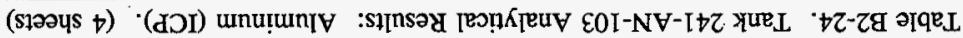


Table B2-25. Tank 241-AN-103 Analytical Results: Antimony (ICP). (4 sheets)

\begin{tabular}{|c|c|c|c|c|c|}
\hline Hampor & 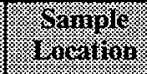 & 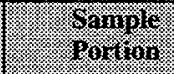 & \%om & S1,pingre & 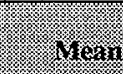 \\
\hline \multicolumn{3}{|c|}{ 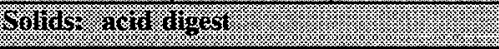 } & (3) & 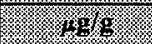 & (xis \\
\hline S96T005609 & 166: 1 & Lower half & $<88.6$ & $<90.5$ & $<89.55$ \\
\hline S96T005570 & 166: 4 & Lower half & $<108$ & $<108$ & $<108$ \\
\hline S96T005615 & $166: 6$ & Lower half & $<82.9$ & $<82.9$ & $<82.9$ \\
\hline S96T005839 & 166:7 & Lower half & $<121$ & $<123$ & $<122$ \\
\hline S96T005840 & $166: 8$ & Lower half & $<118$ & $<118$ & $<118$ \\
\hline \$96T005575 & 166: 9 & Lower half & $<117$ & $<119$ & $<118$ \\
\hline S96T005571 & $166: 10$ & Lower half & $<92.1$ & $<86.7$ & $<89.4$ \\
\hline S96T005616 & 166: 11 & Lower half & $<86.8$ & $<81.6$ & $<84.2$ \\
\hline S96T005617. & $166: 12$ & Lower half & $<84.6$ & $<84.4$ & $<84.5$ \\
\hline S96T005716 & \multirow[t]{2}{*}{ 166: 13} & Upper half & $<116$ & $<118$ & $<117$ \\
\hline S96T005722 & & Lower half & $<123$. & $<124$ & $<123.5$ \\
\hline S96T005416 & \multirow[t]{2}{*}{$166: 15$} & Upper half & $<123$ & $<114$ & $<118.5$ \\
\hline S97T000459 & & Lower half & $<34.1$ & $<33.4$ & $<33.75$ \\
\hline S96T005624 & \multirow[t]{2}{*}{ 166: 16} & Upper half & $<126$ & $<129$ & $<127.5$ \\
\hline \$96T005618 & & Lower half & $<85.1$ & $<87.3$ & $<86.2$ \\
\hline S96T005417 & \multirow[t]{2}{*}{$166: 17$} & Upper half & $<135$ & $<123$ & $<129$ \\
\hline S96T005411 & & Lower half & $<114$ & $<106$ & $<110$ \\
\hline S96T005717 & \multirow[t]{2}{*}{$166: 18$} & Upper half & $<125$ & $<121$ & $<123$ \\
\hline S96T005723 & & Lower half & $<121$ & $<119$ & $<120$ \\
\hline S96T005841 & \multirow[t]{2}{*}{ 166: 19} & Upper half & $<116$ & $<118$ & $<117$ \\
\hline S96T005842 & & Lower half & $<107$ & $<118$ & $<112.5$ \\
\hline S96T005708 & \multirow[t]{2}{*}{ 167: 1} & Upper half & $<108$ & $<123$ & $<115.5$ \\
\hline \$96T005693 & & Lower half & $<133$ & $<105$ & $<119$ \\
\hline S96T005857 & 167: 2 & Lower half & $<100$ & $<104$ & $<102$ \\
\hline S96T005694 & 167:3 & Lower half & $<108$ & $<112$ & $<110$ \\
\hline S96T005695 & 167: 4 & Lower half & $<119$ & $<123$ & $<121$ \\
\hline S96T005438 & $167: 5$ & Lower half & $<31$ & $<33.1$ & $<32.05$ \\
\hline S96T005450 & $167: 6$ & Lower half & $<33.2$ & $<31.3$ & $<32.25$ \\
\hline S96T005696 & $167: 7$ & Lower half & $<115$ & $<113$ & $<114$ \\
\hline
\end{tabular}


Table B2-25. Tank 241-AN-103 Analytical Results: Antimony (ICP). (4 sheets)

\begin{tabular}{|c|c|c|c|c|c|}
\hline Hominger & 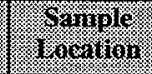 & 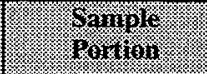 & (2) Iesult & Buplicinte. & 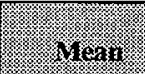 \\
\hline Sollids a ch & gess & & (1) & (1) & 40 \\
\hline \$96T005737 & 167: 8 & Lower half & $<117$ & $<120$ & $<118.5$ \\
\hline S96T005858 & 167: 9 & Lower half & $<125$ & $<105$ & $<115$ \\
\hline S96T005709 & \multirow[t]{2}{*}{ 167: 11} & Upper half & $<108$ & $<112$ & $<110$ \\
\hline S96T005697 & & Lower half & $<119$ & $<113$ & $<116$ \\
\hline S96T005444 & \multirow[t]{2}{*}{$167: 12$} & Upper half & $<33.2$ & $<32.7$ & $<32.95$ \\
\hline \$96T005451 & & Lower half & $<34.1$ & $<35$ & $<34.55$ \\
\hline S96T005445 & \multirow[t]{2}{*}{$167: 14$} & Upper half & $<31.7$ & $<33$ & $<32.35$ \\
\hline S96T005439 & & Lower half & $<34.1$ & $<35$ & $<34.55$ \\
\hline \$96T005710 & \multirow[t]{2}{*}{$167: 15$} & Upper half & $<112$ & $<107$ & $<109.5$ \\
\hline S96T005698 & & Lower half & $<109$ & $<113$ & $<111$ \\
\hline S96T005498 & \multirow[t]{2}{*}{$167: 17$} & Upper half & $<110$ & $<117$ & $<113.5$ \\
\hline S96T005499 & & Lower half & $<107$ & $<116$ & $<111.5$ \\
\hline S97T000460 & Core 166 & Solid composite & $<33.8$ & $<33$ & $<33.4$ \\
\hline \multicolumn{2}{|l|}{ Wouras } & (3. & (1) & Hogning & . \\
\hline S96T005539 & 166: 3 & Drainable liquid & $<24.1$ & $<24.1$ & $<24: 1$ \\
\hline S96T005540 & $166: 4$ & Drainable liquid & $<24.1$ & $<24.1$ & $<24.1$ \\
\hline S96T005599 & 166: 6 & Drainable liquid & $<36.1$ & $<36.1$ & $<36.1$ \\
\hline S96T005816 & $166: 7$ & Drainable liquid & $<36.1$ & $<36.1$ & $<36.1$ \\
\hline S96T005817 & $166: 8$ & Drainable liquid & $<36.1$ & $<36.1$ & $<36.1$ \\
\hline S96T005541 & 166: 9 & Drainable liquid & $<36.1$ & $<36.1$ & $<36.1$ \\
\hline S96T005566 & $166: 10$ & Drainable liquid & $<24.1$ & $<24.1$ & $<24.1$ \\
\hline S96T005600 & 166: 11 & Drainable liquid & $<36.1$ & $<36.1$ & $<36.1$ \\
\hline S96T005601 & 166: 12 & Drainable liquid & $<36.1$ & $<36.1$ & $<36.1$ \\
\hline S96T005861 & $167: 2$ & Drainable liquid & $<36.1$ & $<36.1$ & $<36.1$ \\
\hline S96T005673 & $167: 3$ & Drainable liquid & $<36.1$ & $<36.1$ & $<36.1$ \\
\hline S96T005420 & $167: 5$ & Drainable liquid & $<36.1$ & $<36.1$ & $<36.1$ \\
\hline S96T005421 & 167: 6 & Drainable liquid & $<36.1$ & $<36.1$ & $<36.1$ \\
\hline S96T005683 & 167: 7 & Drainable liquid & $<36.1$ & $<36.1$ & $<36.1$ \\
\hline
\end{tabular}


Table B2-25. Tank 241-AN-103 Analytical Results: Antimony (ICP). (4 sheets)

\begin{tabular}{|c|c|c|c|c|c|}
\hline \% & (9andie & ( & iresint & 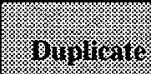 & 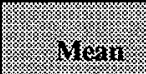 \\
\hline \multicolumn{3}{|c|}{ Malios Gons } & 累 & 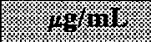 & 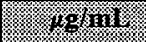 \\
\hline S96T005732 & 167: 8 & Drainable liquid & $<36.1$ & $<36.1$ & $<36.1$ \\
\hline S96T005862 & 167: 9 & Drainable liquid & $<36.1$ & $<36.1$ & $<36.1$ \\
\hline S96T005650 & 167: 11 & Drainable liquid & $<36.1$ & $<36.1$ & $<36.1$ \\
\hline S96T005991 & Core 166 & Liquid composite & $<72.1$ & $<72.1$ & $<72.1$ \\
\hline \multicolumn{3}{|c|}{ Hollag grow } & m & 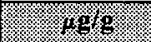 & 498 \\
\hline S96T005608 & 166: 1 & Lower half & $<1,260$ & $<1,240$ & $<1,250$ \\
\hline S96T005568 & 166: 4 & Lower half & $<1,360$ & $<1,330$ & $<1,345$ \\
\hline \$96T005611 & 166: 6 & Lower half & $<1,260$ & $<1,280$ & $<1,270$ \\
\hline S96T005835 & 166: 7 & Lower half & $<1,260$ & $<1,270$ & $<1,265$ \\
\hline \$96T005836 & 166: 8 & Lower half & $<1,180$ & $<1,230$ & $<1,205$ \\
\hline \$96T005574 & $166: 9$ & Lower half & $<1,080$ & 1,240 & $<1,160$ \\
\hline S96T005569 & 166: 10 & Lower half & $<1,250$ & $<1,240$ & $<1,245$ \\
\hline S96T005612 & $166: 11$ & Lower half & $<1,180$ & $<1,220$ & $<1,200$ \\
\hline \$96T005613 & 166: 12 & Lower half & $<1,230$ & $<1,210$ & $<1,220$ \\
\hline S96T005714 & \multirow[t]{2}{*}{$166: 13$} & Upper half & 1,420 & $<1,270$ & $<1,345$ \\
\hline S96T005720 & & Lower half & $<1,220$ & $<1,210$ & $<1,215$ \\
\hline S96T005414 & \multirow[t]{2}{*}{ 166: 15} & Upper half & $<274$ & $<280$ & $<277$ \\
\hline S96T005408 & & Lower half & $<288$ & $<278$ & $<283$ \\
\hline S96T005623 & \multirow[t]{2}{*}{$166: 16$} & Upper half & $<1,310$ & 1,620 & $<1,465^{\mathrm{QC:e}}$ \\
\hline S96T005614 & & Lower half & $<1,230$ & $<1,200$ & $<1,215$ \\
\hline S96T005415 & \multirow[t]{2}{*}{ 166: 17} & Upper half & $<314$ & $<317$ & $<315.5$ \\
\hline \$96T005409 & & Lower half & $<1,270$ & $<1,250$ & $<1,260$ \\
\hline \$96T005715 & \multirow[t]{2}{*}{ 166: 18} & Upper half & $<1,210$ & $<1,230$ & $<1,220$ \\
\hline S96T005721 & & Lower half & $<1,220$ & $<1,190$ & $<1,205$ \\
\hline S96T005837 & \multirow[t]{2}{*}{ 166: 19} & Upper half & $<1,240$ & $<1,260$ & $<1,250$ \\
\hline S96T005838 & & Lower half & $<1,170$ & $<1,180$ & $<1,175$ \\
\hline S96T005705 & \multirow[t]{2}{*}{ 167: 1} & Upper half & $<1,190$ & $<1,200$ & $<1,195$ \\
\hline \$96T005687 & & Lower half & $<1,250$ & $<1,250$ & $<1,250$ \\
\hline S96T005855 & 167: 2 & Lower half & $<1,210$ & $<1,190$ & $<1,200$ \\
\hline
\end{tabular}


Table B2-25. Tank 241-AN-103 Analytical Results: Antimony (ICP). (4 sheets)

\begin{tabular}{|c|c|c|c|c|c|}
\hline THinger & 椾 & Wur & Resulu & Burgligine & Hean \\
\hline \multicolumn{3}{|c|}{ 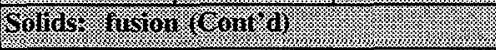 } & (3) & (1) & (1) \\
\hline S96T005688 & $167: 3$ & Lower half & $<1,350$ & $<1,280$ & $<1,315$ \\
\hline S96T005689 & 167: 4 & Lower half & $<1,260$ & $<1,270$ & $<1,265$ \\
\hline S96T005436 & 167:5 & Lower half & 2,120 & 3,120 & $2,620^{\text {QC: }}$ \\
\hline S96T005448 & $167: 6$ & Lower half & $<1,260$ & 1,850 & $<1,555^{\mathrm{QC}: \mathrm{e}}$ \\
\hline S96T005690 & $167: 7$ & Lower half & 1,630 & 1,610 & 1,620 \\
\hline S96T005736 & 167: 8 & Lower half & 1,490 & $<1,120$ & $<1,305^{\text {QC:e }}$ \\
\hline S96T005856 & 167: 9 & Lower half & $<1,100$ & $<1,270$ & $<1,185$ \\
\hline S96T005706 & \multirow[t]{2}{*}{ 167: 11} & Upper half & 1,440 & $<1,330$ & $<1,385$ \\
\hline S96T005691 & & Lower half & 1,630 & $<1,100$ & $<1,365^{\mathrm{QC}: \mathrm{C}}$ \\
\hline S96T005442 & \multirow[t]{2}{*}{$167: 12$} & Upper half & 1,450 & 2,780 & $2,115^{\mathrm{QC}: \mathrm{e}}$ \\
\hline \$96T005449 & & Lower half & $<1,190$ & $<1,210$ & $<1,200$ \\
\hline S96T005443 & \multirow[t]{2}{*}{$167: 14$} & Upper half & $<1,320$ & $<1,270$ & $<1,295$ \\
\hline S96T005437 & & Lower half & $<1,230$ & $<1,220$ & $<1,225$ \\
\hline S96T005707 & \multirow[t]{2}{*}{ 167: 15} & Upper half & 1,620 & $<1,100$ & $<1,360^{\mathrm{QC:c}}$ \\
\hline S96T005692 & & Lower half & $<1,100$ & $<1,310$ & $<1,205$ \\
\hline S96T005496 & \multirow[t]{2}{*}{$167: 17$} & Upper half & $<1,270$ & $<1,260$ & $<1,265$ \\
\hline S96T005497 & & Lower half & $<1,260$ & $<1,260$ & $<1,260$ \\
\hline
\end{tabular}


Table B2-26. Tank 241-AN-103 Analytical Results: Beryllium (ICP). (4 sheets)

\begin{tabular}{|c|c|c|c|c|c|}
\hline 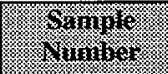 & 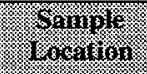 & (9) & hesint & OMingur & (19.1\% \\
\hline \multicolumn{2}{|c|}{ 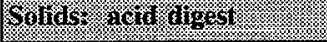 } & & (1) $12 \%$ & (2) & 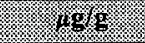 \\
\hline S96T005609 & 166: 1 & Lower half & $<7.38$ & $<7.54$ & $<7.46$ \\
\hline S96T005570 & $166: 4$ & Lower half & $<8.97$ & $<8.97$ & $<8.97$ \\
\hline S96T005615 & 166: 6 & Lower half & $<6.91$ & $<6.91$ & $<6.91$ \\
\hline S96T005839 & $166: 7$ & Lower half & $<10.1$ & $<10.2$ & $<10.15$ \\
\hline S96T005840 & 166: 8 & Lower half & $<9.84$ & $<9.83$ & $<9.835^{\mathrm{QC:a}}$ \\
\hline S96T005575 & 166: 9 & Lower half & $<9.78$ & $<9.94$ & $<9.86$ \\
\hline S96T005571 & 166: 10 & Lower half & $<7.67$ & $<7.23$ & $<7.45$ \\
\hline S96T005616 & 166: 11 & Lower half & $<7.23$ & $<6.8$ & $<7.015$ \\
\hline S96T005617 & 166: 12 & Lower half & $<7.05$ & $<7.04$ & $<7.045$ \\
\hline \$96T005716 & \multirow[t]{2}{*}{$166: 13$} & Upper half & $<9.69$ & $<9.81$ & $<9.75$ \\
\hline S96T005722 & & Lower half & $<10.3$ & $<10.3$ & $<10.3$ \\
\hline S96T005416 & \multirow[t]{2}{*}{ 166: 15} & Upper half & 12.6 & $<9.52$ & $<11.06^{\mathrm{QC}: \mathrm{e}}$ \\
\hline S97T000459 & & Lower half & $<2.84$ & $<2.78$ & $<2.81$ \\
\hline S96T005624 & \multirow[t]{2}{*}{ 166: 16} & Upper half & $<10.5$ & $<10.8$ & $<10.65$ \\
\hline \$96T005618 & & Lower half & $<7.09$ & $<7.28$ & $<7.185$ \\
\hline S96T005417 & \multirow[t]{2}{*}{ 166: 17} & Upper half & $<11.3$ & $<10.2$ & $<10.75$ \\
\hline S96T005411 & & Lower half & $<9.51$ & $<8.8$ & $<9.155$ \\
\hline S96T005717 & \multirow[t]{2}{*}{ 166: 18} & Upper half & $<10.4$ & $<10.1$ & $<10.25$ \\
\hline S96T005723 & & Lower half & $<10.1$ & $<9.9$ & $<10$ \\
\hline S96T005841 & \multirow[t]{2}{*}{ 166: 19} & Upper half & $<9.63$ & $<9.81$ & $<9.72^{\mathrm{QC:a}}$ \\
\hline S96T005842 & & Lower half & $<8.9$ & $<9.81$ & $<9.355^{\mathrm{QC}: \mathrm{a}}$ \\
\hline S96T005708 & \multirow[t]{2}{*}{ 167: 1} & Upper half & $<8.96$ & $<10.3$ & $<9.63$ \\
\hline S96T005693 & & Lower half & $<11.1$ & $<8.77$ & $<9.935$ \\
\hline \$96T005857 & $167: 2$ & Lower half & $<8.34$ & $<8.69$ & $<8.515$ \\
\hline S96T005694 & $167: 3$ & Lower half & $<8.99$ & $<9.36$ & $<9.175$ \\
\hline S96T005695 & $167: 4$ & Lower half & $<9.9$ & $<10.2$ & $<10.05$ \\
\hline S96T005438 & $167: 5$ & Lower half & $<2.58$ & $<2.75$ & $<2.665$ \\
\hline \$96T005450 & 167:6 & Lower half & $<2.77$ & $<2.61$ & $<2.69$ \\
\hline S96T005696 & 167: 7 & Lower half & $<9.6$ & $<9.4$ & $<9.5$ \\
\hline
\end{tabular}




\begin{tabular}{|c|c|c|c|c|c|}
\hline$\varepsilon>$ & $\varepsilon>$ & $\varepsilon>$ & ptnbṛ әqеu!exd & $L: \angle 9 I$ & E89S00L96S \\
\hline$\varepsilon>$ & $\varepsilon>$ & $\varepsilon>$ & pṭnb!̣ əlqeuteda & $9: \mathrm{LgI}$ & $I Z \nrightarrow S 00 L 96 S$ \\
\hline$\varepsilon>$ & $\varepsilon>$ & $\varepsilon>$ & ptnbṭ әqqeụexa & $\subseteq: \angle 9 I$ & $0 Z \nabla S 00 L 96 S$ \\
\hline$\varepsilon>$ & $\varepsilon>$ & $\varepsilon>$ & pụnbṛ әqеuा̣ex & $\varepsilon: \angle 9 I$ & EL9S00L96S \\
\hline$\varepsilon>$ & $\varepsilon>$ & $\varepsilon>$ & pụnb!̣ ә әqеuाe.r & $z: \angle 9 I$ & โ98S00L96S \\
\hline$\varepsilon>$ & $\varepsilon>$ & $\varepsilon>$ & pụnbụ әqеuाpia & ZI :99I & L09S00L96S \\
\hline$\varepsilon>$ & $\varepsilon>$ & $\varepsilon>$ & 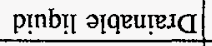 & I I :99I & $009500 \mathrm{~L} 96 \mathrm{~S}$ \\
\hline$z>$ & $z>$ & $\tau>$ & p!nb!̣ әqвu!̣exa & 0I:99I & $99 S \mathrm{~S} 00 \mathrm{~L} 96 \mathrm{~S}$ \\
\hline$\varepsilon>$ & $\varepsilon>$ & $\varepsilon>$ & p!nb!̣ әqеụexa & $6: 991$ & Itss $00 \mathrm{~L} 96 \mathrm{~S}$ \\
\hline$\varepsilon>$ & $\varepsilon>$ & $\varepsilon>$ & 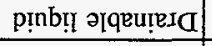 & $8: 991$ & LI8S00L96S \\
\hline$\varepsilon>$ & $\varepsilon>$ & $\varepsilon>$ & ptnbỵ әqqeuteia & $L: 99 I$ & $918500 \mathrm{~L} 96 \mathrm{~S}$ \\
\hline$\varepsilon>$ & $\varepsilon>$ & $\varepsilon>$ & p!nb!l әाqeuाexa & $9: 99 \mathrm{I}$ & $66 \mathrm{SS} 00 \mathrm{~L} 96 \mathrm{~S}$ \\
\hline$z>$ & $z>$ & $\tau>$ & p!nb!l әqRu!̣es & $\nabla: 991$ & $0 t S S 00 L 96 \mathrm{~S}$ \\
\hline$z>$ & $z>$ & $\tau>$ & pinb!̣ әqеu!̣ı & $\varepsilon: 991$ & $6 \varepsilon S S 00 L 96 S$ \\
\hline mingl= & Muyor & gringy) & (2.3. & (2):- & spanting \\
\hline$\$ 8 L \cdot \tau>$ & $S L \cdot Z>$ & $28 \cdot \tau>$ & əu!soduros pryos & 991 วrоว & $09+000 \mathrm{~L} \angle 6 \mathrm{~S}$ \\
\hline $82 \cdot 6>$ & $\$ 9^{\circ} 6>$ & $16.8>$ & Ғ[еप دәмоТ] & \multirow[b]{2}{*}{$\angle I: \angle 9 I$} & $66+S 00 \mathrm{~L} 96 \mathrm{~S}$ \\
\hline$s_{t} \cdot 6>$ & $\nabla L \cdot 6>$ & $91 \cdot 6>$ & Fाеप $x \partial d d \Omega$ & & $86+S 00 \mathrm{~L} 96 \mathrm{~S}$ \\
\hline $\operatorname{sgz} 2^{\circ} 6>$ & $7.6>$ & II $6>$ & ylеч دәмо & \multirow[b]{2}{*}{ SI :L9I } & $869 \subseteq 00 \mathrm{~L} 96 \mathrm{~S}$ \\
\hline SEI $6>$ & $56.8>$ & $2 \varepsilon 6>$ & j[Еધ دədd $\Omega$ & & OI $\angle S 00 L 96 \mathrm{~S}$ \\
\hline $88^{\circ} \tau>$ & $26 \cdot \tau>$ & $18 \cdot \tau>$ & I[EY IOMO]] & \multirow{2}{*}{ tI : $\angle 9 I$} & $6 \varepsilon+500 L 96 S$ \\
\hline$S 69^{\circ} z>$ & $S L \cdot Z>$ & $\$ 9 \cdot z>$ & frey radd $\Omega$ & & SttSO0L96S \\
\hline $88^{\circ} z>$ & $26^{\circ} \mathrm{z}>$ & $\triangleright 8^{\circ} \tau>$ & IIеч Іәмо Т & \multirow[b]{2}{*}{$2 \mathrm{II}: \mathrm{LI}$} & IStS00L96S \\
\hline$s \oplus L \cdot Z>$ & $\varepsilon L \cdot \tau>$ & $9 L \cdot \tau>$ & frey & & $t t+S 00 L 96 \mathrm{~S}$ \\
\hline$\$ 99^{\circ} 6>$ & $I t^{\circ} 6>$ & $26.6>$ & І[еч IәмO & \multirow[b]{2}{*}{ II : $\angle 9 I$} & $\angle 69 S 00 \perp 96 \mathrm{~S}$ \\
\hline s9. $6>$ & $\varepsilon \varepsilon \cdot 6>$ & $6>$ & Ғाеप xәdd $\Omega$ & & $60 \angle S 00 . L 96 \mathrm{~S}$ \\
\hline$S L S^{*} 6>$ & $S L \cdot 8>$ & $t^{\circ} 0 \mathrm{I}>$ & Ireप гэMOT & $6: \angle 9 I$ & $8 S 8 S 00 \mathrm{~L} 96 \mathrm{~S}$ \\
\hline $68^{\circ} 6>$ & $66^{\circ} 6>$ & $6 L 6>$ & J[ЕЧ دәмOТ] & $8: \angle 9 \mathrm{I}$ & $\angle E L S 00 L 96 S$ \\
\hline $3.4 \%$ & is & & & \multicolumn{2}{|c|}{ 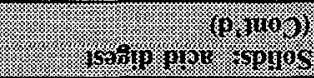 } \\
\hline 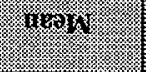 & $29309 \mathrm{dmar}$ & 3100 & nofurad & 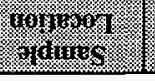 & . \\
\hline
\end{tabular}

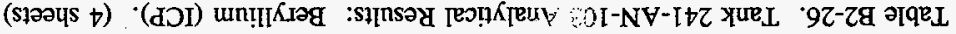


Table B2-26. Tank 241-AN-103 Analytical Results: Beryllium (ICP). (4 sheets)

\begin{tabular}{|c|c|c|c|c|c|}
\hline 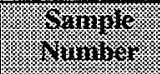 & 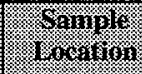 & Marion & histim & Wutuma & \% \\
\hline \multicolumn{2}{|c|}{ 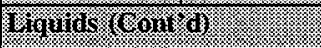 } & $\sqrt{3 .}$ & 848 min & 3. & 1) \\
\hline S96T005732 & 167:8 & Drainable liquid & $<3$ & $<3$ & $<3$ \\
\hline S96T005862 & $167: 9$ & Drainable liquid & $<3$ & $<3$ & $<3$ \\
\hline S96T005650 & 167: 11 & Drainable liquid & $<3$ & $<3$ & $<3$ \\
\hline S96T005991 & Core 166 & Liquid composite & $<6$ & $<6$ & $<6$ \\
\hline \multicolumn{2}{|c|}{ 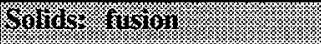 } & r. & xys & (4) & $8 \%$ \\
\hline \$96T005608 & 166: 1 & Lower half & $<105$ & $<103$ & $<104$ \\
\hline S96T005568 & 166: 4 & Lower half & $<113$ & $<111$ & $<112$ \\
\hline S96T005611 & $166: 6$ & Lower half & $<105$ & $<107$ & $<106$ \\
\hline S96T005835 & $166: 7$ & Lower half & $<105$ & $<106$ & $<105.5$ \\
\hline S96T005836 & 166: 8 & Lower half & $<98.1$ & $<103$ & $<100.55$ \\
\hline S96T005574 & 166: 9 & Lower half & $<89.6$ & $<97$ & $<93.3$ \\
\hline \$96T005569 & $166: 10$ & Lower half & $<105$ & $<103$ & $<104$ \\
\hline S96T005612 & $166: 11$ & Lower half & $<98.7$ & $<102$ & $<100.35$ \\
\hline S96T005613 & $166: 12$ & Lower half & $<103$ & $<101$ & $<102$ \\
\hline S96T005714 & \multirow[t]{2}{*}{$166: 13$} & Upper half & $<101$ & $<105$ & $<103$ \\
\hline S96T005720 & & Lower half & $<101$ & $<101$ & $<101$ \\
\hline S96T005414 & \multirow[t]{2}{*}{$166: 15$} & Upper half & $<22.8$ & $<23.3$ & $<23.05$ \\
\hline S96T005408 & & Lower half & $<24$ & $<23.2$ & $<23.6$ \\
\hline S96T005623 & \multirow[t]{2}{*}{ 166: 16} & Upper half & $<109$ & $<105$ & $<107$ \\
\hline S96T005614 & & Lower half & $<102$ & $<100$ & $<101$ \\
\hline S96T005415 & \multirow[t]{2}{*}{$166: 17$} & Upper half & $<26.2$ & $<26.4$ & $<26.3$ \\
\hline S96T005409 & & Lower half & $<106$ & $<104$ & $<105$ \\
\hline \$96T005715 & \multirow[t]{2}{*}{$166: 18$} & Upper half & $<101$ & $<103$ & $<102$ \\
\hline S96T005721 & & Lower half & $<101$ & $<99.4$ & $<100.2$ \\
\hline S96T005837 & \multirow[t]{2}{*}{$166: 19$} & Upper half & $<104$ & $<105$ & $<104.5$ \\
\hline S96T005838 & & Lower half & $<97.8$ & $<98.2$ & $<98$ \\
\hline S96T005705 & \multirow[t]{2}{*}{ 167: 1} & Upper half & $<99.4$ & $<100$ & $<99.7$ \\
\hline S96T005687 & & Lower half & $<104$ & $<104$ & $<104$ \\
\hline
\end{tabular}


HNF-SD-WM-ER-702 Rev. 0

Table B2-26. Tank 241-AN-103 Analytical Results: Beryllium (ICP). (4 sheets)

\begin{tabular}{|c|c|c|c|c|c|}
\hline 6rimie & (6) & Saming & 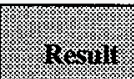 & Hollowe & s. \\
\hline \multicolumn{3}{|c|}{ 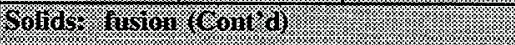 } & 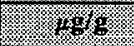 & 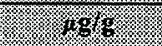 & 63 \\
\hline S96T005855 & $167: 2$ & Lower half & $<101$ & $<98.8$ & $<99.9$ \\
\hline S96T005688. & $167: 3$ & Lower half & $<112$ & $<107$ & $<109.5$ \\
\hline S96T005689 & $167: 4$ & Lower half & $<105$ & $<106$ & $<105.5$ \\
\hline S96T005436 & $167: 5$ & Lower half & $<93.1$ & $<93.9$ & $<93.5$ \\
\hline S96T005448 & $167: 6$ & Lower half & $<105$ & $<102$ & $<103.5$ \\
\hline S96T005690 & 167: 7 & Lower half & $<98.2$ & $<109$ & $<103.6$ \\
\hline S96T005736 & $167: 8$ & Lower half & $<111$ & $<93.7$ & $<102.35$ \\
\hline S96T005856 & $167: 9$ & Lower half & $<92$ & $<105$ & $<98.5$ \\
\hline \$96T005706 & \multirow[t]{2}{*}{$167: 11$} & Upper half & $<93.3$ & $<111$ & $<102.15$ \\
\hline S96T005691 & & Lower half & $<92.5$ & $<92.1$ & $<92.3$ \\
\hline S96T005442 & \multirow[t]{2}{*}{ 167: 12} & Upper half & $<108$ & $<96.9$ & $<102.45$ \\
\hline S96T005449 & & Lower half & $<98.8$ & $<101$ & $<99.9$ \\
\hline S96T005443 & \multirow[t]{2}{*}{$167: 14$} & Upper half & $<110$ & $<105$ & $<107.5$ \\
\hline S96T005437 & & Lower half & $<103$ & $<101$ & $<102$ \\
\hline S96T005707 & \multirow[t]{2}{*}{$167: 15$} & Upper half & $<107$ & $<91.9$ & $<99.45$ \\
\hline S96T005692 & & Lower half & $<91.6$ & $<109$ & $<100.3$ \\
\hline S96T005496 & \multirow[t]{2}{*}{$167: 17$} & Upper half & $<106$ & $<105$ & $<105.5$ \\
\hline S96T005497 & & Lower half & $<105$ & $<105$ & $<105$ \\
\hline
\end{tabular}


Table B2-27. Tank 241-AN-103 Analytical Results: Boron (ICP). (4 sheets)

\begin{tabular}{|c|c|c|c|c|c|}
\hline Shample & Sampie & $\begin{array}{l}\text { Sample } \\
\text { sortion. }\end{array}$ & ressulis: & Bringirate & Merr: \\
\hline \multicolumn{2}{|c|}{ Sutras acri 4 igess } & & 14868 & (4) & 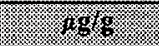 \\
\hline S96T005609 & 166: 1 & Lower half & 107 & 114 & 110.5 \\
\hline S96T005570 & 166: 4 & Lower half & 124 & 109 & 116.5 \\
\hline S96T005615 & 166: 6 & Lower half & 85.9 & 91.5 & 88.7 \\
\hline S96T005839 & 166: 7 & Lower half & 138 & 121 & 129.5 \\
\hline S96T005840 & $166: 8$ & Lower half & 131 & 157 & 144 \\
\hline S96T005575 & 166: 9 & Lower half & 162 & 168 & 165 \\
\hline S96T005571 & $166: 10$ & Lower half & 109 & 96.2 & 102.6 \\
\hline S96T005616 & 166: 11 & Lower half & 86.9 & 93.4 & 90.15 \\
\hline S96T005617 & 166: 12 & Lower half & 99.6 & 90 & 94.8 \\
\hline S96T005716 & \multirow[t]{2}{*}{ 166: 13} & Upper half & 182 & 176 & 179 \\
\hline S96T005722 & & Lower half & 162 & 163 & 162.5 \\
\hline S96T005416 & \multirow[t]{2}{*}{ 166: 15} & Upper half & 194 & 148 & $171^{\mathrm{QC}: \mathrm{c}}$ \\
\hline S97T000459 & & Lower half & 137 & 153 & 145 \\
\hline S96T005624 & \multirow[t]{2}{*}{$166: 16$} & Upper half & 177 & 198 & 187.5 \\
\hline S96T005618 & & Lower half & 102 & 115 & 108.5 \\
\hline S96T005417 & \multirow[t]{2}{*}{$166: 17$} & Upper half & 152 & 169 & 160.5 \\
\hline S96T005411 & & Lower half & 120 & 122 & 121 \\
\hline S96T005717 & \multirow[t]{2}{*}{$166: 18$} & Upper half & 153 & 175 & 164 \\
\hline S96T005723 & & Lower half & 153 & 153 & 153 \\
\hline S96T005841 & \multirow[t]{2}{*}{$166: 19$} & Upper half & $<96.3$ & $<98.1$ & $<97.2$ \\
\hline S96T005842 & & Lower half & $<89$ & 138 & $<113.5^{\mathrm{QC:e}}$ \\
\hline S96T005708 & \multirow[t]{2}{*}{$167: 1$} & Upper half & 117 & 134 & 125.5 \\
\hline S96T005693 & & Lower half & 152 & 140 & 146 \\
\hline S96T005857 & 167:2 & Lower half & 134 & $<86.9$ & $<110.45^{\mathrm{QC}: e}$ \\
\hline S96T005694 & $167: 3$ & Lower half & 109 & 115 & 112 \\
\hline S96T005695 & $167: 4$ & Lower half & 118 & 128 & 123 \\
\hline S96T005438 & $167: 5$ & Lower half & 131 & 143 & 137 \\
\hline S96T005450 & $167: 6$ & Lower half & 141 & 146 & 143.5 \\
\hline S96T005696 & $167: 7$ & Lower half & 154 & 142 & 148 \\
\hline
\end{tabular}


HNF-SD-WM-ER-702 Rev. 0

Table B2-27. Tank 241-AN-103 Analytical Results: Boron (ICP). (4 sheets)

\begin{tabular}{|c|c|c|c|c|c|}
\hline Shanglo & 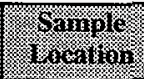 & 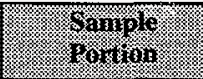 & Pesinilin & 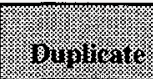 & $\log 1$ \\
\hline (8) & Hest & & . & 3. & $\frac{1}{16}$ \\
\hline S96T005737 & 167: 8 & Lower half & 146 & 153 & 149.5 \\
\hline S96T005858 & 167: 9 & Lower half & 126 & 133 & 129.5 \\
\hline S96T005709 & \multirow[t]{2}{*}{ 167: 11} & Upper half & 147 & 176 & 161.5 \\
\hline S96T005697 & & Lower half & 157 & 143 & 150 \\
\hline S96T005444 & \multirow{2}{*}{ 167: $\begin{array}{c}12 \\
\cdot\end{array}$} & Upper half & 136 & 132 & 134 \\
\hline \$96T005451 & & Lower half & 204 & 141 & $172.5^{\mathrm{QC}: \mathrm{c}}$ \\
\hline S96T005445 & \multirow[t]{2}{*}{$167: 14$} & Upper half & 153 & 178 & 165.5 \\
\hline S96T005439 & & Lower half & 121 & 36 & $78.5^{\mathrm{QC}: c}$ \\
\hline S96T005710 & \multirow[t]{2}{*}{$167: 15$} & Upper half & 128 & 155 & 141.5 \\
\hline S96T005698 & & Lower half & 158 & 157 & 157.5 \\
\hline S96T005498 & \multirow[t]{2}{*}{$167: 17$} & Upper half & 121 & 140 & 130.5 \\
\hline S96T005499 & & Lower half & 124 & 124 & 124 \\
\hline S97T000460 & Core 166 & Solid composite & 132 & 118 & 125 \\
\hline MMUs & 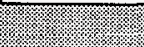 & & \%/mI & 16 & (3) \\
\hline S96T005539 & 166: 3 & Drainable liquid & 63.6 & 62.3 & 62.95 \\
\hline S96T005540 & $166: 4$ & Drainable liquid & 53.8 & 46 & 49.9 \\
\hline S96T005599 & $166: 6$ & Drainable liquid & 70.3 & 65.2 & 67.75 \\
\hline S96T005816 & 166: 7 & Drainable liquid & 66.1 & 69.7 & 67.9 \\
\hline \$96T005817 & $166: 8$ & Drainable liquid & 68.8 & 73.8 & 71.3 \\
\hline S96T005541 & 166: 9 & Drainable liquid & 66.2 & 62.1 & 64.15 \\
\hline S96T005566 & 166: 10 & Drainable liquid & 69.5 & 63 & 66.25 \\
\hline S96T005600 & $166: 11$ & Drainable liquid & 68.3 & 64.9 & 66.6 \\
\hline S96T005601 & $166: 12$ & Drainable liquid & 66 & 67.8 & 66.9 \\
\hline S96T005861 & $167: 2$ & Drainable liquid & 68.8 & 71.4 & 70.1 \\
\hline \$96T005673 & $167: 3$ & Drainable liquid & 56.4 & 56.4 & 56.4 \\
\hline S96T005420 & $167: 5$ & Drainable liquid & 68.9 & 68.9 & 68.9 \\
\hline S96T005421 & $167: 6$ & Drainable liquid & 62.3 & 62.3 & 62.3 \\
\hline S96T005683 & $167: 7$ & Drainable liquid & 62.8 & 67.2 & 65 \\
\hline
\end{tabular}




\begin{tabular}{|c|c|c|c|c|c|}
\hline $666>$ & $886>$ & $0\left[0^{6} I>\right.$ & $\pm[$ Еप Іәмот & $z: \angle 9 I$ & SS8S00L96S \\
\hline $0+0^{\circ} \mathrm{I}>$ & $0 \pm 0^{\circ} \mathrm{I}>$ & $0+0^{6} \mathrm{I}>$ & ІІЕЧ IәмоT & \multirow[b]{2}{*}{ I :L9I } & L89S00L96S \\
\hline$L 66>$ & $000^{6} \mathrm{I}>$ & $\nabla 66>$ & Jleप Iədd $\Omega$ & & S0LS00L96S \\
\hline $086>$ & $286>$ & $8 \angle 6>$ & fleप دәмо I & \multirow[b]{2}{*}{$61: 99 \mathrm{I}$} & 8E8S00L96S \\
\hline$S 70^{\circ} I>$ & $0 S 0^{\circ} \mathrm{I}>$ & $070^{\circ} \mathrm{I}>$ & J[eप sədd $\Omega$ & & LE8S00L96S \\
\hline $200^{6} 1>$ & $t 66>$ & $010^{\circ} \mathrm{I}>$ & J[Еч Іәмо7] & \multirow[b]{2}{*}{ 8I:99I } & IZLS00L96S \\
\hline $020^{\circ} \mathrm{I}>$ & $0 \mathcal{E} 0^{6} \mathrm{I}>$ & $010^{\circ}[>$ & J[Еप Іәdd $\Omega$ & & SILS00L96S \\
\hline $0 S 0^{6} \mathrm{I}>$ & $0+0^{6} 1>$ & $090^{6}$ I > & Ilएप دәмо & \multirow[b]{2}{*}{ LI:99I } & $60+500 L 96 \mathrm{~S}$ \\
\hline$\varepsilon 9 z>$ & $+9 z>$ & $z 9 z>$ & f[eч เәdd & & SIDS00L96S \\
\hline 010'I> & $000^{\circ} \mathrm{I}>$ & $020^{\circ} I>$ & Ғ[ЕЧ Іәмот & \multirow[b]{2}{*}{ 9I :99I } & $\nabla[9 S 00 L 96 \mathrm{~S}$ \\
\hline $0 \angle 0^{6} I>$ & $00^{6} 1>$ & $060^{\circ} I>$ & Irеप Iәdd $\Omega$ & & EZ9S00L96S \\
\hline $9 \varepsilon z>$ & $\tau \varepsilon Z>$ & $0 \neq z>$ & JIEY IәMOT & \multirow[b]{2}{*}{ SI :99I } & $80 t S 00 \mathrm{~L} 96 \mathrm{~S}$ \\
\hline$\varsigma^{\circ} 0 \varepsilon z>$ & $\varepsilon \mathcal{E}>$ & $8 z \tau>$ & fleu Iədd & & $t[t S 00196 \mathrm{~S}$ \\
\hline $010^{6}[>$ & $0\left[0^{6} \mathrm{I}>\right.$ & $010^{6} I>$ & $f\left[{ }^{2}\right.$ प IəMOT] & \multirow[b]{2}{*}{$\mathcal{E}$ I:99I } & $0 Z \angle S 00 L 96 \mathrm{~S}$ \\
\hline $0 \varepsilon 0^{\circ} I>$ & $0 S 0^{\prime} \mathrm{I}>$ & OIO'I> & fleध ıədd $\Omega$ & & tILS00L96S \\
\hline $020^{\circ} I>$ & $010^{6} \mathrm{I}>$ & OEO'I> & J[Еप IәMOT & ZI:991 & El9S00LL6S \\
\hline$S^{\cdot} \varepsilon 00^{6} I>$ & $020^{\circ} I>$ & $\angle 86>$ & J[ЕЧ دәмот & II:99I & ZI9S00L96S \\
\hline Ot0.1> & OEO'I $>$ & $0 S 0^{\prime}[>$ & I[एบ IวMOT & $01: 99 \mathrm{I}$ & 69SSO0L96S \\
\hline$\varepsilon \mathcal{E} 6>$ & $0<6>$ & $968>$ & $\pm[$ І Іәмот & $6: 991$ & TLSS00L96S \\
\hline $\mathcal{S}^{\cdot} S 00^{\prime} Y>$ & $0 \varepsilon 0^{6} I>$ & $186>$ & J[Еप IәMOT] & $8: 991$ & 988S00L96S \\
\hline$S S 0^{6} \mathrm{I}>$ & $090^{\circ} \mathrm{I}>$ & $0 S 0^{\circ} \mathrm{I}>$ & IाЕप IәмоТ & $L: 99 I$ & SE8S00L96S \\
\hline $090^{\circ} I>$ & $0 \angle 0^{\circ} \mathrm{I}>$ & $0 S 0^{\circ} \mathrm{I}>$ & गеЕप ІәмоТ & $9: 991$ & I I9S00L96S \\
\hline OZI'I $>$ & OI I'I $>$ & $0 \mathcal{E} I^{\prime} I>$ & I[Eบ دәMOT & $\triangleright: 99 I$ & $89 \subseteq \$ 00 L 96 \mathrm{~S}$ \\
\hline $0+0^{\circ} I>$ & OEO $0^{6} \mathrm{P}$ & $0 S 0^{6} \mathrm{I}>$ & Iएटप IәMOT & I :99I & $809 \$ 00 L 96 \mathrm{~S}$ \\
\hline ingin & 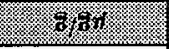 & 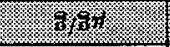 & & \multicolumn{2}{|c|}{ (10) } \\
\hline I.09> & I.09> & {$[09>$} & ә!!soduoo p!nb!̣ & $99[$ วI0ว & I66S00L96S \\
\hline$s \cdot t s$ & $6 \circ 5$ & $I \not S$ & pịbụ әqqeu!̣era & II :L9I & 0S9S00L96S \\
\hline$\nabla 0 L$ & $I \cdot I L$ & $L 69$ & 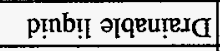 & $6: \angle 9 I$ & Z98500L96S \\
\hline$z \cdot 29$ & $I^{\circ} \mathcal{E} 9$ & $\varepsilon \cdot 19$ & pı̣nby ə̣qeu!̣ı & $8: \angle 91$ & ZELS00L96S \\
\hline 110.7\% & 14rom & 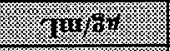 & & \multicolumn{2}{|c|}{ ( } \\
\hline Wog & 9.8. & 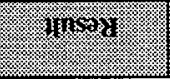 & 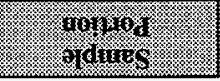 & morom & 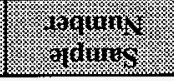 \\
\hline
\end{tabular}

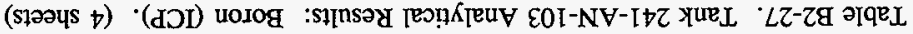




\begin{tabular}{|c|c|c|c|c|c|}
\hline OSOCI $I>$ & $0 S 0^{\prime}[>$ & $0 S 0^{\circ} I>$ & Iرеч гамоТ & \multirow[b]{2}{*}{$\angle I: \angle 9 I$} & $\angle 6 t S 00 \mathrm{~L} 96 \mathrm{~S}$ \\
\hline$S S 0^{\circ}[>$ & $0 S 0^{\circ}[>$ & $090^{\circ} I>$ & J[вu redd $\Omega$ & & $96 t S 00 L 96 S$ \\
\hline$\varepsilon 00^{\prime} I>$ & $060^{4} \mathrm{I}>$ & $9[6>$ & ILEY IOMO & \multirow[b]{2}{*}{$\subseteq I: \angle 9 I$} & $269500 \mathrm{~L} 96 \mathrm{~S}$ \\
\hline$s+666>$ & $616>$ & $0 \angle 0^{\circ} I>$ & 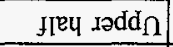 & & $\angle 0 L S 00 L 96 \mathrm{~S}$ \\
\hline $020^{\prime} 1>$ & $010^{6} 1>$ & $0 E 0^{\circ} I>$ & Іlеч гамо & \multirow[b]{2}{*}{$D I: \angle 9 I$} & $\angle E t S 00 \perp 96 S$ \\
\hline$\subseteq \angle 0^{6} I>$ & OSOCI $I>$ & $00 I^{\prime} I>$ & I[एप & & EttS00L96S \\
\hline $666>$ & OI0'I $1>$ & $886>$ & fтеप гәмот & \multirow[b]{2}{*}{$Z I: \angle 9 I$} & $60 t 500 \mathrm{~L} 96 \mathrm{~S}$ \\
\hline$S^{\circ}+20^{\prime} I>$ & $696>$ & $080^{\circ} \mathrm{I}>$ & 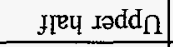 & & $\tau t+S 00 \perp 96 \mathrm{~S}$ \\
\hline$\varepsilon 26>$ & $126>$ & $\$ 26>$ & IlЕY Іәмо Т & \multirow[b]{2}{*}{$I I: \angle 9 I$} & I69500L96S \\
\hline$\xi^{\circ} I 20^{\prime} I>$ & OII'I & $\varepsilon \varepsilon 6>$ & эгеч ১әdd $\Omega$ & & $90 \angle S 00 \mathrm{~L} 96 \mathrm{~S}$ \\
\hline$\$ 86>$ & OSO $I>$ & $026>$ & ІІеЧ ІәмоТ] & $6: \angle 9 I$ & $9 \$ 8 S 00 \mathrm{~L} 96 \mathrm{~S}$ \\
\hline$\varsigma^{\prime} \varepsilon Z 0^{\prime} I>$ & $L \varepsilon 6>$ & OII'I> & ҒГеч ІәмоТ & $8: \angle 9 I$ & $9 \varepsilon \angle S 00 L 96 \mathrm{~S}$ \\
\hline $9 \varepsilon 0^{6} \mathrm{I}>$ & $060^{6} \mathrm{I}>$ & $286>$ & Ғ[ец Іәмо $]$ & $\angle: L 9 I$ & $069 S 00 L 96 \mathrm{~S}$ \\
\hline $\mathcal{S E O}^{\prime} I>$ & $0 Z 0^{6} I>$ & $0 S 0^{\circ}[>$ & I[ЕY ХәМОТ] & $9: \angle 9 I$ & $8 \nabla \nabla S 00 L 96 S$ \\
\hline SE6> & $6 \varepsilon 6>$ & IE6> & ҒाЕप ІәмОТ & $\varsigma: \angle 9 I$ & $9 \varepsilon+S 00 L 96 S$ \\
\hline $\mathcal{S S O}^{6} I>$. & $090^{\circ} 1>$ & $0 S 0^{\circ} I>$ & I[Еप ІәмО & $\rightarrow: \angle 9 I$ & $689500 \mathrm{~L} 96 \mathrm{~S}$ \\
\hline$S_{60^{\prime}} \mathrm{I}>$ & $0 \angle 0^{6} I>$ & OZI'I $I>$ & Іाеч дәмоТ & $\varepsilon: \angle 9 \mathrm{I}$ & $889500 \mathrm{~L} 96 \mathrm{~S}$ \\
\hline 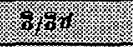 & 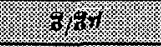 & $67 \% 1=$ & 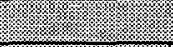 & \multicolumn{2}{|c|}{ 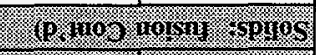 } \\
\hline 1980, & 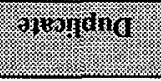 & 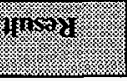 & gropingir & 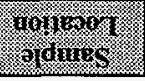 & $\begin{array}{l}\text { Lquinis } \\
\text { Jidings }\end{array}$ \\
\hline
\end{tabular}

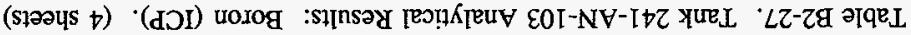


Table B2-28. Tank 241-AN-103 Analytical Results: Cadmium (ICP). (4 sheets)

\begin{tabular}{|c|c|c|c|c|c|}
\hline Maringer & 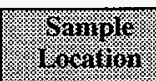 & (1) & tresin: & rion & Month: \\
\hline \multicolumn{2}{|c|}{ 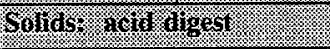 } & (1) & 4919 & 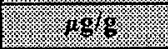 & (3. \\
\hline S96T005609 & 166: 1 & Lower half & 16.1 & 12.9 & $14.5^{\mathrm{QC}: t}$ \\
\hline S96T005570 & 166: 4 & Lower half & $<8.97$ & $<8.97$ & $<8.97$ \\
\hline S96T005615 & 166: 6 & Lower half & $<6.91$ & $<6.91$ & $<6.91$ \\
\hline S96T005839 & $166: 7$ & Lower half & $<10.1$ & $<10.2$ & $<10.15$ \\
\hline S96T005840 & $166: 8$ & Lower half & $<9.84$ & $<9.83$ & $<9.835^{\text {QC: }}$ \\
\hline S96T005575 & 166: 9 & Lower half & 9.89 & $<9.94$ & $<9.915$ \\
\hline S96T005571 & 166: 10 & Lower half & $<7.67$ & $<7.23$ & $<7.45$ \\
\hline S96T005616 & 166: 11 & Lower half & $<7.23$ & $<6.8$ & $<7.015$ \\
\hline S96T005617 & $166: 12$ & Lower half & $<7.05$ & $<7.04$ & $<7.045$ \\
\hline S96T005716 & \multirow[t]{2}{*}{ 166: 13} & Upper half & 13.9 & 10.1 & $12^{\mathrm{QC:c}}$ \\
\hline S96T005722 & & Lower half & $<10.3$ & $<10.3$ & $<10.3$ \\
\hline S96T005416 & \multirow[t]{2}{*}{$166: 15$} & Upper half & 19.9 & $<9.52$ & $<14.71^{\mathrm{QC:c}}$ \\
\hline S97T000459 & & Lower half & 6.3 & 6.88 & 6.59 \\
\hline S96T005624 & \multirow[t]{2}{*}{ 166: 16} & Upper half & 11.2 & $<10.8$ & $<11$ \\
\hline S96T005618 & & Lower half & 8.98 & 8.42 & 8.7 \\
\hline S96T005417 & \multirow[t]{2}{*}{$166: 17$} & Upper half & 11.4 & $<10.2$ & $<10.8$ \\
\hline S96T005411 & & Lower half & $<9.51$ & 10.7 & $<10.105$ \\
\hline S96T005717 & \multirow[t]{2}{*}{ 166: 18} & Upper half & 13.9 & 12.4 & 13.15 \\
\hline S96T005723 & & Lower half & 12.3 & $<9.9$ & $<11.1^{\mathrm{QC:e}}$ \\
\hline S96T005841 & \multirow[t]{2}{*}{$166: 19$} & Upper half & $<9.63$ & $<9.81$ & $<9.72^{\mathrm{QC}: \mathrm{a}}$ \\
\hline S96T005842 & & Lower half & 10.1 & $<9.81$ & $<9.955^{\mathrm{QC:a}}$ \\
\hline S96T005708 & \multirow[t]{2}{*}{$167: 1$} & Upper half & $<8.96$ & $<10.3$ & $<9.63$ \\
\hline S96T005693 & & Lower half & $<11.1$ & 10.1 & $<10.6$ \\
\hline S96T005857 & $167: 2$ & Lower half & $<8.34$ & 8.97 & $<8.655$ \\
\hline S96T005694 & $167: 3$ & Lower half & $<8.99$ & $<9.36$ & $<9.175$ \\
\hline S96T005695 & 167: 4 & Lower half & $<9.9$ & $<10.2$ & $<10.05$ \\
\hline S96T005438 & $167: 5$ & Lower half & $<2.58$ & $<2.75$ & $<2.665^{\mathrm{QC:a}}$ \\
\hline$\$ 96 T 005450$ & $167: 6$ & Lower half & $<2.77$ & $<2.61$ & $<2.69^{0 \mathrm{C:a}}$ \\
\hline S96T005696 & 167:7 & Lower half & $<9.6$ & $<9.4$ & $<9.5$ \\
\hline
\end{tabular}


HNF-SD-WM-ER-702 Rev, 0

Table B2-28. Tank 241-AN-103 Analytical Results: Cadmium (ICP). (4 sheets)

\begin{tabular}{|c|c|c|c|c|c|}
\hline Mramol & 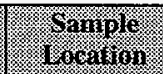 & 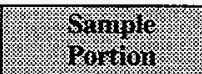 & Rasinu & (3) & Won \\
\hline \multicolumn{3}{|c|}{ 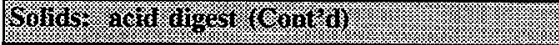 } & 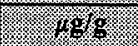 & (3) & 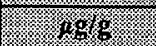 \\
\hline S96T005737 & 167:8 & Lower half & $<9.79$ & $<9.99$ & $<9.89$ \\
\hline S96T005858 & 167: 9 & Lower half & $<10.4$ & $<8.75$ & $<9.575$ \\
\hline \$96T005709 & \multirow[t]{2}{*}{ 167: 11} & Upper half & $<9$ & $<9.33$ & $<9.165$ \\
\hline S96T005697 & & Lower half & 10.1 & $<9.41$ & $<9.755$ \\
\hline S96T005444 & \multirow[t]{2}{*}{$167: 12$} & Upper half & 5.46 & 5.79 & $5.625^{\mathrm{QC}: \mathrm{a}}$ \\
\hline \$96T005451 & & Lower half & 5.92 & 6.16 & $6.04^{\mathrm{QC:a}}$ \\
\hline S96T005445 & \multirow[t]{2}{*}{ 167: 14} & Upper half & 5.54 & 4.57 & $5.055^{\mathrm{QC:a}}$ \\
\hline S96T005439 & & Lower half & 5.18 & 5.88 & $5.53^{\mathrm{QC:a}}$ \\
\hline S96T005710 & \multirow[t]{2}{*}{$167: 15$} & Upper half & $<9.32$ & $<8.95$ & $<9.135$ \\
\hline S96T005698 & & Lower half & $<9.11$ & $<9.4$ & $<9.255$ \\
\hline S96T005498 & \multirow[t]{2}{*}{ 167: 17} & Upper half & $<9.16$ & $<9.74$ & $<9.45$ \\
\hline S96T005499 & & Lower half & $<8.91$ & 10.5 & $<9.705$ \\
\hline S97T000460 & Core 166 & Solid composite & 3.95 & 4.39 & 4.17 \\
\hline \multicolumn{3}{|l|}{ Vorins. } & 筮 & \% & 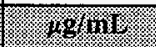 \\
\hline S96T005539 & $166: 3$ & Drainable liquid & 4.44 & 4.13 & 4.285 \\
\hline S96T005540 & 166: 4 & Drainable liquid & 4.31 & $<2$ & $<3.155^{\mathrm{QC}: \mathrm{c}}$ \\
\hline S96T005599 & 166: 6 & Drainable liquid & $4: 73$ & 4.84 & 4.785 \\
\hline S96T005816 & 166: 7 & Drainable liquid & 4.19 & 5.1 & 4.645 \\
\hline S96T005817 & $166: 8$ & Drainable liquid & 5.45 & 4.67 & 5.06 \\
\hline S96T005541 & 166: 9 & Drainable liquid & 4.15 & 4.03 & 4.09 \\
\hline S96T005566 & $166: 10$ & Drainable liquid & 4.29 & 4.03 & 4.16 \\
\hline$\$ 96 \mathrm{~T} 005600$ & 166: 11 & Drainable liquid & 4.94 & 4.22 & 4.58 \\
\hline S96T005601 & $166: 12$ & Drainable liquid & 4.88 & 4.39 & 4.635 \\
\hline S96T005861 & 167: 2 & Drainable liquid & 4.46 & 3.66 & 4.06 \\
\hline S96T005673 & $167: 3$ & Drainable liquid & 3.72 & 4.37 & 4.045 \\
\hline S96T005420 & 167: 5 & Drainable liquid & 5.49 & 4.23 & $4.86^{\mathrm{QCic}}$ \\
\hline S96T005421 & $167: 6$ & Drainable liquid & 5.03 & 4.23 & 4.63 \\
\hline \$96T005683 & $167: 7$ & Drainable liquid & 4.6 & 5.09 & 4.845 \\
\hline S96T005732 & 167:8 & Drainable liquid & 4.67 & 4.83 & 4.75 \\
\hline
\end{tabular}


Table B2-28. Tank 241-AN-103 Analytical Results: Cadmium (ICP). (4 sheets)

\begin{tabular}{|c|c|c|c|c|c|}
\hline 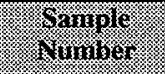 & 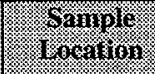 & chamgle & 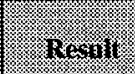 & moring & wing \\
\hline \multicolumn{2}{|c|}{ 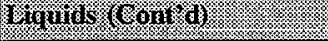 } & 19. & (3g) & .0 .1111 & (3) \\
\hline S96T005862 & 167: 9 & Drainable liquid & 4.78 & 4.87 & 4.825 \\
\hline S96T005650 & 167: 11 & Drainable liquid & $<3$ & 3.37 & $<3.185$ \\
\hline S96T005991 & Core 166 & Liquid composite & $<6$ & $<6$ & $<6$ \\
\hline \multicolumn{2}{|c|}{ Hollide fingion } & & 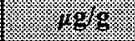 & 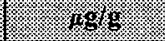 & (2., 1548 \\
\hline S96T005608 & 166: 1 & Lower half & $<105$ & $<103$ & $<104$ \\
\hline S96T005568 & 166: 4 & Lower half & $<113$ & $<111$ & $<112$ \\
\hline \$96T005611 & $166: 6$ & Lower half & $<105$ & $<107$ & $<106$ \\
\hline \$96T005835 & $166: 7$ & Lower half & $<105$ & $<106$ & $<105.5$ \\
\hline S96T005836 & $166: 8$ & Lower half & $<98.1$ & $<103$ & $<100.55$ \\
\hline S96T005574 & 166: 9 & Lower half & $<89.6$ & $<97$ & $<93.3$ \\
\hline S96T005569 & $166: 10$ & Lower half & $<105$ & $<103$ & $<104$ \\
\hline S96T005612 & 166: 11 & Lower half & $<98.7$ & $<102$ & $<100.35$ \\
\hline S96T005613 & 166: 12 & Lower half & $<103$ & $<101$ & $<102$ \\
\hline S96T005714 & \multirow[t]{2}{*}{$166: 13$} & Upper half & $<101$ & $<105$ & $<103$ \\
\hline S96T005720 & & Lower half & $<101$ & $<101$ & $<101$ \\
\hline S96T005414 & \multirow[t]{2}{*}{$166: 15$} & Upper half & $<22.8$ & $<23.3$ & $<23.05$ \\
\hline S96T005408 & & Lower half & $<24$ & $<23.2$ & $<23.6$ \\
\hline S96T005623 & \multirow[t]{2}{*}{$166: 16$} & Upper half & $<109$ & $<105$ & $<107$ \\
\hline S96T005614 & & Lower half & $<102$ & $<100$ & $<101$ \\
\hline S96T005415 & \multirow[t]{2}{*}{$166: 17$} & Upper half & $<26.2$ & $<26.4$ & $<26.3$ \\
\hline S96T005409 & & Lower half & $<106$ & $<104$ & $<105$ \\
\hline S96T005715 & \multirow[t]{2}{*}{$166: 18$} & Upper half & $<101$ & $<103$ & $<102$ \\
\hline S96T005721 & & Lower half & $<101$ & $<99.4$ & $<100.2$ \\
\hline S96T005837 & \multirow[t]{2}{*}{ 166: 19} & Upper half & $<104$ & $<105$ & $<104.5$ \\
\hline S96T005838 & & Lower half & $<97.8$ & $<98.2$ & $<98$ \\
\hline S96T005705 & \multirow[t]{2}{*}{ 167: 1} & Upper half & $<99.4$ & $<100$ & $<99.7$ \\
\hline \$96T005687 & & Lower half & $<104$ & $<104$ & $<104$ \\
\hline S96T005855 & $167: 2$ & Lower half & $<101$ & $<98.8$ & $<99.9$ \\
\hline S96T005688 & $167: 3$ & Lower half & $<112$ & $<107$ & $<109.5$ \\
\hline
\end{tabular}




\begin{tabular}{|c|c|c|c|c|c|}
\hline$\leqq 0 I>$ & SOI $>$ & SOI $>$ & эГеप Iәмо $\mathrm{T}$ & & L6tSO0L96S \\
\hline $\mathrm{s} S 0 I>$ & $\mathrm{SOI}>$ & $90 I>$ & IlEY Iədd $\Omega$ & $\angle I: \angle 9 I$ & $96+500 \mathrm{~L} 96 \mathrm{~S}$ \\
\hline$\varepsilon 00 I>$ & $60 \mathrm{I}>$ & $9 \cdot[6>$ & ҒІЕЧ ІамоТ & \multirow[b]{2}{*}{$S I: \angle 9 I$} & z69S00L96S \\
\hline$s t \cdot 66>$ & $6.16>$ & $\angle O I>$ & fley Jədd $\Omega$ & & $\angle 0 \angle S 00 L 96 \mathrm{~S}$ \\
\hline $\mathrm{ZOI}>$ & IOI $>$ & EOI $>$ & Ғ[ЕЧ ІәмоТ & \multirow[b]{2}{*}{$\nabla I: \angle 9 I$} & $\angle E+S 00 L 96 S$ \\
\hline$S \angle O I>$ & SOI $>$ & OII $>$ & j[eप rədd $\Omega$ & & $\varepsilon+t S 00 L 96 \mathrm{~S}$ \\
\hline $6.66>$ & [0I $>$ & $8.86>$ & ІІеч Іәмот & \multirow[b]{2}{*}{ ZI :L9I } & $6 \triangleright t 500 \mathrm{~L} 96 \mathrm{~S}$ \\
\hline St 20 I > & $696>$ & $80 I>$ & J[eप Iədd $_{\Omega}$ & & $2 \downarrow t S 00 L 96 \mathrm{~S}$ \\
\hline$\varepsilon 26>$ & $I^{\circ} 26>$ & $s 26>$ & f[Еप ІәMOT] & \multirow[b]{2}{*}{ II : L9I } & I69S00L96S \\
\hline $\mathrm{SI}^{\prime} \mathrm{Z} O \mathrm{I}>$ & III $>$ & $\mathcal{E} \varepsilon 6>$ & IIE4 Iədd $_{\Omega}$ & & $90 L S 00 L 96 \mathrm{~S}$ \\
\hline$S^{\circ} 86>$ & SOI $>$ & $z 6>$ & IโЕप IәMOT & $6: \angle 9 I$ & $9 S 8 \mathrm{~S} 00 \mathrm{~L} 96 \mathrm{~S}$ \\
\hline $\mathfrak{S E} Z 0 \mathrm{I}>$ & $L^{*} \varepsilon 6>$ & III $>$ & ІІеч дәмот & $8: \angle 9 I$ & $9 \mathcal{E} L S 00 L 96 \mathrm{~S}$ \\
\hline $9^{\circ} \mathrm{EOI}>$ & $60 \mathrm{I}>$ & $z \cdot 86>$ & भाЕप Іәмот & $L: \angle 9 I$ & 069S00L96S \\
\hline $\mathrm{S}^{\cdot} \mathcal{E O I}>$ & $20 \mathrm{I}>$ & SOI $>$ & J[Eप IәMOT] & $9: \angle 9 I$ & $8 t 7$ S00L96S \\
\hline $\mathcal{S}^{\cdot} \mathcal{E} 6>$ & $6^{\circ} \varepsilon 6>$ & {$\left[{ }^{*} \varepsilon 6>\right.$} & J[еप دәмот] & $s: \angle 9 I$ & 9EtS00L96S \\
\hline $\mathrm{S}^{*} \mathrm{SOI}>$ & $90 I>$ & SOI $>$ & I[Еч гәмот & $\dagger: \angle 9 I$ & $689500 L 96 \mathrm{~S}$ \\
\hline (4) & (.) & (6) & & \multicolumn{2}{|c|}{ 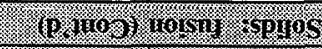 } \\
\hline (19) & 196196 & (1) & molng & \%ownor & Monghn \\
\hline
\end{tabular}

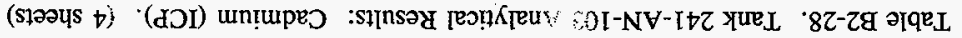


Table B2-29. Tank 241-AN-103 Analytical Results: Calcium (ICP). (4 sheets)

\begin{tabular}{|c|c|c|c|c|c|}
\hline ongup & 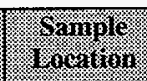 & 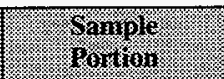 & 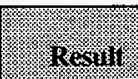 & ouminitic & 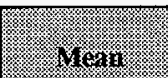 \\
\hline \multicolumn{2}{|c|}{ 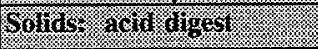 } & (1) & 1. & minge & 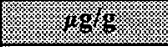 \\
\hline$\$ 967005609$ & $166: 1$ & Lower half & $<148^{\circ}$ & $<151$ & $<149.5$ \\
\hline \$96T005570 & $166: 4$ & Lower half & $<179$ & $<179$ & $<179$ \\
\hline S96T005615 & 166: 6 & Lower half & $<138$ & $<138$ & $<138$ \\
\hline S96T005839 & $166: 7$ & Lower half & $<202$ & $<205$ & $<203.5$ \\
\hline S96T005840 & $166: 8$ & Lower half & $<197$ & $<197$ & $<197^{\mathrm{QC:a}}$ \\
\hline S96T005575 & $166: 9$ & Lower half & $<196$ & $<199$ & $<197.5$ \\
\hline S96T005571 & $166: 10$ & Lower half & $<153$ & $<145$ & $<149$ \\
\hline S96T005616 & 166: 11 & Lower half & $<145$ & $<136$ & $<140.5$ \\
\hline S96T005617 & 166: 12 & Lower half & $<141$ & $<141$ & $<141$ \\
\hline \$96T005716 & \multirow[t]{2}{*}{$166: \cdot 13$} & Upper half & $<194$ & $<196$ & $<195$ \\
\hline S96T005722 & & Lower half & $<206$ & $<206$ & $<206$ \\
\hline S96T005416 & \multirow[t]{2}{*}{$166: 15$} & Upper half & $<204$ & $<190$ & $<197$ \\
\hline S97T000459 & & Lower half & $<56.9$ & $<55.7$ & $<56.3$ \\
\hline \$96T005624 & \multirow[t]{2}{*}{$166: 16$} & Upper half & $<210$ & $<215$ & $<212.5$ \\
\hline \$96T005618 & & Lower half & $<142$ & $<146$ & $<144$ \\
\hline \$96T005417 & \multirow[t]{2}{*}{ 166: 17} & Upper half & $<225$ & $<205$ & $<215$ \\
\hline S96T005411 & & Lower half & $<190$ & $<176$ & $<183$ \\
\hline \$96T005717 & \multirow[t]{2}{*}{$166: 18$} & Upper half & $<208$ & $<202$ & $<205$ \\
\hline S96T005723 & & Lower half & 290 & 207 & $248.5^{\mathrm{QC}: \mathrm{c}}$ \\
\hline S96T005841 & \multirow[t]{2}{*}{ 166: 19} & Upper half & $<193$ & 197 & $<195^{Q C: a}$ \\
\hline S96T005842 & & Lower half & 383 & 227 & $305^{\mathrm{QC:a,c}}$ \\
\hline S96T005708 & \multirow[t]{2}{*}{ 167: 1} & Upper half & $<179$ & $<205$ & $<192$ \\
\hline S96T005693 & & Lower half & $<222$ & $<175$ & $<198.5^{\mathrm{QC}: \mathrm{c}}$ \\
\hline S96T005857 & $167: 2$ & Lower half & $<167$ & $<174$ & $<170.5$ \\
\hline S96T005694. & $167: 3$ & Lower half & $<180$ & $<187$ & $<183.5$ \\
\hline S96T005695 & 167: 4 & Lower haif & $<198$ & $<204$ & $<201$ \\
\hline \$96T005438 & $167: 5$ & Lower half & $<51.7$ & $<55.1$ & $<53.4$ \\
\hline $\mathrm{S} 96 \mathrm{~T} 005450$ & $167: 6$ & Lower half & $<55.3$ & $<52.2$ & $<53.75$ \\
\hline S96T005696 & $167: 7$ & Lower half & $<192$ & $<188$ & $<190$ \\
\hline
\end{tabular}




\begin{tabular}{|c|c|c|c|c|c|}
\hline I.09> & $109>$ & {$[\cdot 09>$} & pinbụl ə[qeutexa & $8: \angle 9 I$ & ZELS00L96S \\
\hline I.09> & $109>$ & {$[.09>$} & 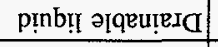 & $\angle: \angle 9 I$ & E89S00L96S \\
\hline$I^{\circ} 09>$ & {$[\cdot 09>$} & {$[\cdot 09>$} & p!nb!l əIqeuresa & $9: \angle 9 I$ & $I Z † S 00 L 96 S$ \\
\hline I.09> & $1.09>$ & {$[\cdot 09>$} & 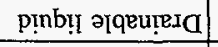 & $s: \angle 9 I$ & $02 t 500 \mathrm{~L} 96 \mathrm{~S}$ \\
\hline$[\cdot 09>$ & I.09> & I.09> & p!nbtr әрqеu!̣esa & $\varepsilon: \angle 9 T$ & $\varepsilon \angle 9 S 00 \mathrm{~L} 96 \mathrm{~S}$ \\
\hline $1.09>$ & $I .09>$ & {$[.09>$} & p!nb!̣া ə[qeu!̣eI & $2: \angle 9 I$ & I98S00L96S \\
\hline$[\cdot 09>$ & $I .09>$ & {$[09>$} & pinb!̣ गqeureIa & ZI :99I & I09S00L96S \\
\hline I.09> & {$[09>$} & I.09> & punb!̣ गlqeu!exa] & I I :99I & 009S00L96S \\
\hline $100 t>$ & $I 0 t>$ & I.Op $>$ & 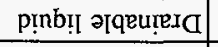 & $0 \mathrm{I}: 99 \mathrm{I}$ & 99 SS00L96S \\
\hline$I \cdot 09>$ & $\mathrm{I} \cdot 09>$ & I09> & p!nb!̣ ә[qеu!̣eI & $6: 99 \mathrm{I}$ & It\$S00I96S \\
\hline$[\cdot 09>$ & I.09> & {$[.09>$} & 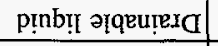 & $8: 99 \mathrm{I}$ & $\angle 18 S 00 L 96 S$ \\
\hline$[\cdot 09>$ & {$[\cdot 09>$} & {$[.09>$} & 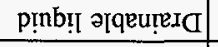 & $L: 99 \mathrm{I}$ & 9I8S00L96S \\
\hline I.09> & I.09> & {$[\cdot 09>$} & pinb!̣ әтqeureIa & $9: 991$ & $66 \varsigma \$ 00 \perp 96 \mathrm{~S}$ \\
\hline I.0t> & $I \cdot 0 t>$ & {$\left[I^{\circ} 0 t>\right.$} & pinb!̣ әрqеuाexd & $\triangleright: 991$ & $0 t S S 00 \mathrm{~L} 96 \mathrm{~S}$ \\
\hline $100>$ & $100>$ & {$[0 t>$} & p!nb!l әqqeutes] & $\varepsilon: 99$ I & $6 \varepsilon S 500 \mathrm{~L} 96 \mathrm{~S}$ \\
\hline 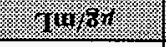 & Irivg bit: & (19:9\% & 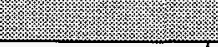 & 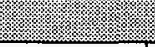 & Sprinbro \\
\hline$L \cdot S S>$ & $I^{\prime} s s>$ & $\varepsilon \cdot 9 \varsigma>$ & әૃ!̨soduros p!̣os & 99[ 2100 & $09+000 \mathrm{~L} \angle 6 \mathrm{~S}$ \\
\hline$\varsigma^{\prime} \mathrm{S}_{8 \mathrm{I}}>$ & $\varepsilon 6 \mathrm{I}>$ & $8 L I>$ & Jाец гәмоТ & & $66 t S 00 \mathrm{~L} 96 \mathrm{~S}$ \\
\hline $68 I>$ & $\subseteq 6 \mathrm{I}>$ & $\varepsilon 8 \mathrm{I}>$ & fleч $\operatorname{radd}_{\Omega}$ & LI: $\angle 9 I$ & $86 t S 00 \mathrm{~L} 96 \mathrm{~S}$ \\
\hline$\$ 8 I>$ & $88 I>$ & $28 I>$ & J[EY IOMOT] & & $869500 \mathrm{~L} 96 \mathrm{~S}$ \\
\hline s.z8I> & $6 L I>$ & $98 \mathrm{I}>$ & JleY rədd $\Omega$ & SI :L9I & $0 I \angle S 00 . \mathrm{L} 96 \mathrm{~S}$ \\
\hline $9 \cdot \angle S>$ & $t^{\circ} 8 \varsigma>$ & $8^{\circ} 9 \varsigma>$ & ДІЕч دәмО人 & & $6 \varepsilon+500 \mathrm{~L} 96 \mathrm{~S}$ \\
\hline $2: 00 \Omega 6^{\circ} \varepsilon L>$ & $I^{\prime} \$ 6$ & $8^{\circ} 25>$ & fleY Iədd $\Omega$ & $t I: \angle 9 I$ & SttS00L96S \\
\hline $9^{\circ} L S>$ & $78 S>$ & $8.95>$ & गгеч & & IStS00L96S \\
\hline $6 . t s>$ & $s+s>$ & $\varepsilon \subseteq \varsigma>$ & frey Iədd $\Lambda$ & $Z I: \angle 9 I$ & $t t t s 00 . \mathrm{L} 96 \mathrm{~S}$ \\
\hline$\varepsilon 6 \mathrm{I}>$ & $88 I>$ & $86 I>$ & JEY JəMOT & & $\angle 69 S 00 \mathrm{~L} 96 \mathrm{~S}$ \\
\hline $\mathcal{S}^{\prime} \mathcal{E} \mathrm{I}>$ & $\angle 8 I>$ & $08 \mathrm{I}>$ & કॉЕ૫ гədd $\Omega$ & II :L9I & $60 \angle S 00 \mathrm{~L} 96 \mathrm{~S}$ \\
\hline S.I6I > & $S L I>$ & $80 z>$ & дाеप дәмот & $6: \angle 9 \mathrm{I}$ & $8 S 8 \subseteq 00 L 96 \mathrm{~S}$ \\
\hline $86 I>$ & $00 \tau>$ & $96 I>$ & f[еч ІәмО & $8: \angle 9 I$ & $\angle E L S 00 L \mathrm{~L} 96$ \\
\hline ojoy & \%ori & int & $\sqrt{3}$ & - samis & 1908 \\
\hline$\sqrt{4}+\sqrt{2}$ & giesingar & 11950\% & moghinger & forverons & 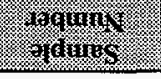 \\
\hline
\end{tabular}

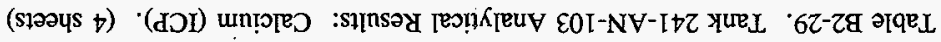


Table B2-29. Tank 241-AN-103 Analytical Results: Calcium (ICP). (4 sheets)

\begin{tabular}{|c|c|c|c|c|c|}
\hline Sumplat & Saminge & Sample & Revilit & 3ilinisura & nerar \\
\hline \multicolumn{3}{|c|}{ Tiguins (contit) } & 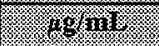 & $2.786 \mathrm{min!}$ & (n) \\
\hline S96T005862 & 167: 9 & Drainable liquid & $<60.1$ & $<60.1$ & $<60.1$ \\
\hline S96T005650 & $167: \cdot 11$ & Drainable liquid & $<60.1$ & $<60.1$ & $<60.1$ \\
\hline S96T005991 & Core 166 & Liquid composite & $<120$ & $<120$ & $<120$ \\
\hline \multicolumn{3}{|c|}{ 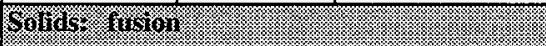 } & 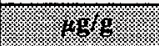 & 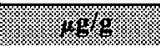 & (3. \\
\hline S96T005608 & 166: 1 & Lower half & $<2,100$ & $<2,060$ & $<2,080$ \\
\hline S96T005568 & 166: 4 & Lower half & $<2,270$ & $<2,220$ & $<2,245$ \\
\hline S96T005611 & $166: 6$ & Lower half & $<2,100$ & $<2,140$ & $<2,120$ \\
\hline S96T005835 & 166: 7 & Lower half & $<2,100$ & $<2,120$ & $<2,110$ \\
\hline S96T005836 & 166: 8 & Lower half & $<1,960$ & $<2,060$ & $<2,010$ \\
\hline S96T005574 & $166: 9$ & Lower half & $<1,790$ & $<1,940$ & $<1,865$ \\
\hline S96T005569 & $166: 10$ & Lower half & $<2,090$ & $<2,070$ & $<2,080$ \\
\hline S96T005612 & 166: 11 & Lower half & $<1,970$ & $<2,040$ & $<2,005$ \\
\hline S96T005613 & $166: 12$ & Lower half & $<2,050$ & $<2,020$ & $<2,035$ \\
\hline S96T005714 & \multirow[t]{2}{*}{$166: 13$} & Upper half & $<2,010$ & $<2,110$ & $<2,060$ \\
\hline S96T005720 & & Lower half & $<2,030$ & $<2,010$ & $<2,020$ \\
\hline S96T005414 & \multirow[t]{2}{*}{ 166: 15} & Upper half & $<456$ & $<466$ & $<461$ \\
\hline S96T005408 & & Lower half & $<480$ & $<463$ & $<471.5$ \\
\hline S96T005623 & \multirow[t]{2}{*}{ 166: 16} & Upper half & $<2,180$ & $<2,100$ & $<2,140$ \\
\hline S96T005614 & & Lower half & $<2,040$ & $<2,010$ & $<2,025$ \\
\hline S96T005415 & \multirow[t]{2}{*}{$166: 17$} & Upper half & $<523$ & $<528$ & $<525.5$ \\
\hline S96T005409 & & Lower half & $<2,110$ & $<2,080$ & $<2,095$ \\
\hline S96T005715 & \multirow[t]{2}{*}{$166: 18$} & Upper half & $<2,010$ & $<2,060$ & $<2,035$ \\
\hline S96T005721 & & Lower half & $<2,030$ & $<1,990$ & $<2,010$ \\
\hline S96T005837 & \multirow[t]{2}{*}{$166: 19$} & Upper half & $<2,070$ & $<2,100$ & $<2,085$ \\
\hline S96T005838 & & Lower half & $<1,960$ & $<1,960$ & $<1,960$ \\
\hline S96T005705 & \multirow[t]{2}{*}{$167: 1$} & Upper half & $<1,990$ & $<2,000$ & $<1,995$ \\
\hline S96T005687 & & Lower half & $<2,080$ & $<2,090$ & $<2,085$ \\
\hline S96T005855 & $167: 2$ & Lower half & $<2,020$ & $<1,980$ & $<2,000$ \\
\hline S96T005688 & $167: 3$ & Lower half & $<2,250$ & $<2,130$ & $<2,190$ \\
\hline
\end{tabular}


Table B2-29. Tank 241-AN-103 Analytical Results: Calcium (ICP). (4 sheets)

\begin{tabular}{|c|c|c|c|c|c|}
\hline Tamplof & \% & for & ingentin & \%) & Vyin \\
\hline \multicolumn{3}{|c|}{ 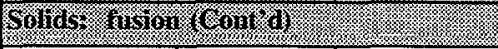 } & (3.68 & (3. & (m) \\
\hline $96 \mathrm{~T} 005689$ & 167: 4 & Lower half & $<2,100$ & $<2,110$ & $<2,105$ \\
\hline S96T005436 & $167: 5$ & Lower half & $<1,860$ & $<1,880$ & $<1,870$ \\
\hline S96T005448 & 167: 6 & Lower half & $<2,110$ & $<2,040$ & $<2,075$ \\
\hline S96T005690 & 167: 7 & Lower half & $<1,960$ & $<2,180$ & $<2,070$ \\
\hline \$96T005736 & $167: 8$ & Lower half & $<2,210$ & $<1,870$ & $<2,040$ \\
\hline S96T005856 & 167: 9 & Lower half & $<1,840$ & $<2,110$ & $<1,975$ \\
\hline S96T005706 & \multirow[t]{2}{*}{ 167: 11} & Upper half & $<1,870$ & $<2,220$ & $<2,045$ \\
\hline S96T005691 & & Lower half & $<1,850$ & $<1,840$ & $<1,845$ \\
\hline S96T005442 & \multirow[t]{2}{*}{ 167: 12} & Upper half. & $<2,160$ & $<1,940$ & $<2,050$ \\
\hline S96T005449 & & Lower half & $<1,980$ & $<2,020$ & $<2,000$ \\
\hline S96T005443 & \multirow[t]{2}{*}{ 167: 14} & Upper half & $<2,200$ & $<2,110$ & $<2,155$ \\
\hline S96T005437 & & Lower half & $<2,050$ & $<2,030$ & $<2,040$ \\
\hline S96T005707 & \multirow[t]{2}{*}{$167: 15$} & Upper half & $<2,130$ & $<1,840$ & $<1,985$ \\
\hline S96T005692 & & Lower half & $<1,830$ & $<2,180$ & $<2,005$ \\
\hline \$96T005496 & \multirow[t]{2}{*}{$167: 17$} & Upper half & $<2,120$ & $<2,100$ & $<2,110$ \\
\hline S96T005497 & & Lower half & $<2,100$ & $<2,110$ & $<2,105$ \\
\hline
\end{tabular}


Table B2-30. Tank 241-AN-103 Analytical Results: Chromium (ICP). (4 sheets)

\begin{tabular}{|c|c|c|c|c|c|}
\hline S & 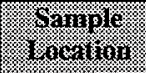 & 6\%or & gersinil & ounticate & (3) \\
\hline \multicolumn{2}{|c|}{ 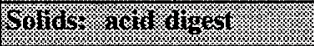 } & & 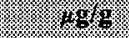 & . & . \\
\hline S96T005609 & 166: 1 & Lower half & 882 & 771 & 826.5 \\
\hline S96T005570 & 166: 4 & Lower half & 133 & 143 & 138 \\
\hline S96T005615 & 166: 6 & Lower half & 169 & 167 & 168 \\
\hline S96T005839 & 166: 7 & Lower half & 138 & 145 & 141.5 \\
\hline S96T005840 & 166: 8 & Lower half & 132 & 131 & $131.5^{\mathrm{QC:a}}$ \\
\hline S96T005575 & $166: 9$ & Lower half & 273 & 227 & 250 \\
\hline S96T005571 & $166: 10$ & Lower half & 200 & 193 & 196.5 \\
\hline S96T005616 & 166: 11 & Lower half & 139 & 123 & 131 \\
\hline S96T005617 & 166: 12 & Lower half & 126 & 133 & 129.5 \\
\hline S96T005716 & \multirow[t]{2}{*}{ 166: 13} & Upper half & 618 & 609 & 613.5 \\
\hline \$96T005722 & & Lower half & 576 & 642 & 609 \\
\hline \$96T005416 & \multirow[t]{2}{*}{ 166: 15} & Upper half & 646 & 672 & 659 \\
\hline S97T000459 & & Lower half & 600 & 715 & 657.5 \\
\hline S96T005624 & \multirow[t]{2}{*}{ 166: 16} & Upper half & 625 & 604 & 614.5 \\
\hline S96T005618 & & Lower half & 708 & 705 & 706.5 \\
\hline S96T005417 & \multirow[t]{2}{*}{$166: 17$} & Upper half & 455 & 458 & 456.5 \\
\hline S96T005411 & & Lower half & 431 & 450 & 440.5 \\
\hline S96T005717 & \multirow[t]{2}{*}{ 166: 18} & Upper half & 642 & 662 & 652 \\
\hline \$96T005723 & & Lower half & 456 & 421 & 438.5 \\
\hline S96T005841 & \multirow[t]{2}{*}{ 166: 19} & Upper half & 301 & 313 & $307^{\mathrm{QC}: \mathrm{a}}$ \\
\hline S96T005842 & & Lower half & 317 & 330 & $323.5^{Q C: A}$ \\
\hline S96T005708 & \multirow[t]{2}{*}{$167: 1$} & Upper half & 532 & 829 & $680.5^{\mathrm{QC}: c}$ \\
\hline S96T005693 & & Lower half & 640 & 691 & 665.5 \\
\hline S96T005857 & $167: 2$ & Lower half & 513 & 597 & $555^{\mathrm{QC}: \mathrm{a}}$ \\
\hline S96T005694 & $167: 3$ & Lower half & 225 & 148 & $186.5^{\mathrm{QC}: \mathrm{c}}$ \\
\hline \$96T005695 & $167: 4$ & Lower half & 210 & 242 & 226 \\
\hline S96T005438 & $167: 5$ & Lower half & 289 & 271 & $280^{\mathrm{QC:a}}$ \\
\hline S96T005450 & $167: 6$ & Lower half & 245 & 257 & $251^{\mathrm{CC:a}}$ \\
\hline S96T005696 & $167: 7$ & Lower half & 138 & 149 & 143.5 \\
\hline
\end{tabular}


Table B2-30. Tank 241-AN-103 Analytical Results: Chromium (ICP). (4 sheets)

\begin{tabular}{|c|c|c|c|c|c|}
\hline (4) & \% & 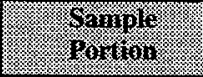 & Hosin & $(5)$ & $\%=a n$ \\
\hline Hoing a ding & 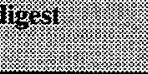 & & fles & & 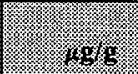 \\
\hline S96T005737 & $167: 8$ & Lower half & 166 & 167 & 166.5 \\
\hline \$96T005858 & 167: 9 & Lower half & 136 & 148 & $142^{\mathrm{QC:a}}$ \\
\hline S96T005709 & \multirow[t]{2}{*}{$167: 11$} & Upper half & 564 & 573 & 568.5 \\
\hline S96T005697 & & Lower half & 667 & 647 & 657 \\
\hline S96T005444 & \multirow[t]{2}{*}{ 167: 12} & Upper half & 622 & 642 & $632^{\mathrm{QC:a}}$ \\
\hline S96T005451 & & Lower half & 654 & 635 & $644.5^{\mathrm{QC:a}}$ \\
\hline S96T005445 & \multirow[t]{2}{*}{$167: 14$} & Upper half & 398 & 343 & $370.5^{\mathrm{QC}: \mathrm{a}}$ \\
\hline S96T005439 & & Lower half. & 379 & 475 & $427^{\mathrm{QC:a,c}}$ \\
\hline S96T005710 & \multirow[t]{2}{*}{$167: 15$} & Upper half & 413 & 411 & 412 \\
\hline S96T005698 & & Lower half & 352 & 351 & 351.5 \\
\hline S96T005498 & \multirow[t]{2}{*}{$167: 17$} & Upper half & 475 & 453 & 464 \\
\hline \$96T005499. & & Lower half & 695 & 573 & 634 \\
\hline S97T000460 & Core 166 & Solid composite & 423 & 453 & 438 \\
\hline \multicolumn{2}{|c|}{ oviglids } & (3) & \% 1914 & 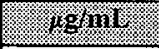 & 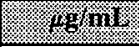 \\
\hline S96T005539 & 166: 3 & Drainable liquid & 583 & 570 & 576.5 \\
\hline S96T005540 & 166: 4 & Drainable liquid & 535 & 511 & 523 \\
\hline S96T005599 & 166: 6 & Drainable liquid & 635 & 614 & 624.5 \\
\hline S96T005816 & 166: 7 & Drainable liquid & 617 & 638 & 627.5 \\
\hline S96T005817 & 166: 8 & Drainable liquid & 607 & 644 & 625.5 \\
\hline S96T005541 & 166: 9 & Drainable liquid & 598 & 590 & 594 \\
\hline S96T005566 & 166: 10 & Drainable liquid & 606 & 594 & 600 \\
\hline S96T005600 & 166: 11 & Drainable liquid & 621 & 608 & 614.5 \\
\hline \$96T005601 & 166: 12 & Drainable liquid & 611 & 612 & 611.5 \\
\hline \$96T005861 & 167: 2 & Drainable liquid & 569 & 576 & 572.5 \\
\hline \$96T005673 & 167: 3 & Drainable liquid & 576 & 566 & 571 \\
\hline S96T005420 & 167: 5 & Drainable liquid & 656 & 621 & 638.5 \\
\hline S96T005421 & 167: 6 & Drainable liquid & 619 & 614 & 616.5 \\
\hline S96T005683 & 167: 7 & Drainable liquid & 608 & 608 & 608 \\
\hline
\end{tabular}


Table B2-30. Tank 241-AN-103 Analytical Results: Chromium (ICP). (4 sheets)

\begin{tabular}{|c|c|c|c|c|c|}
\hline Waingle & sanginge & Samples & 204sin & 8ubingate & 1/ 64 \\
\hline \multicolumn{2}{|c|}{ 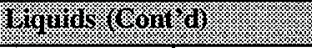 } & & 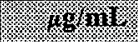 & 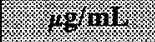 & $(2.49 \mathrm{mII}$ \\
\hline $96 \mathrm{~T} 005732$ & 167: 8 & Drainable liquid & 609 & 598 & 603.5 \\
\hline S96T005862 & 167: 9 & Drainable liquid & 617 & 633 & 625 \\
\hline S96T005650 & 167: 11 & Drainable liquid & 427 & 429 & 428 \\
\hline S96T005991 & Core 166 & Liquid composite & 553 & 567 & 560 \\
\hline \multicolumn{2}{|l|}{ 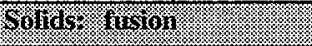 } & & 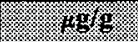 & 8449 & 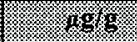 \\
\hline S96T005608 & 166: 1 & Lower half & 999 & 913 & 956 \\
\hline \$96T005568 & 166: 4 & Lower half & $<227$ & $<222$ & $<224.5$ \\
\hline S96T005611 & 166: 6 & Lower half & $<210$ & $<214$ & $<212$ \\
\hline S96T005835 & 166: 7 & Lower half & $<210$ & 219 & $<214.5$ \\
\hline S96T005836 & 166: 8 & Lower half & $<196$ & $<206$ & $<201$ \\
\hline S96T005574 & 166: 9 & Lower half & 238 & 329 & $283.5^{\mathrm{QC}: \mathrm{c}}$ \\
\hline S96T005569 & 166: 10 & Lower half & $<209$ & $<207$ & $<208$ \\
\hline S96T005612 & 166: 11 & Lower half & $<197$ & $<204$ & $<200.5$ \\
\hline S96T005613 & 166: 12 & Lower half & $<205$ & $<202$ & $<203.5$ \\
\hline S96T005714 & \multirow[t]{2}{*}{$166: 13$} & Upper half & 869 & 757 & 813 \\
\hline S96T005720 & & Lower half & 818 & 916 & 867 \\
\hline S96T005414 & \multirow[t]{2}{*}{ 166: 15} & Upper half & 205 & 207 & 206 \\
\hline S96T005408 & & Lower half & 208 & 196 & 202 \\
\hline S96T005623 & \multirow[t]{2}{*}{$166: 16$} & Upper half & 947 & 819 & 883 \\
\hline S96T005614 & & Lower half & 787 & 771 & 779 \\
\hline S96T005415 & \multirow[t]{2}{*}{$166: 17$} & Upper half & 128 & 144 & 136 \\
\hline S96T005409 & & Lower half & 552 & 656 & 604 \\
\hline S96T005715 & \multirow[t]{2}{*}{$166: 18$} & Upper half & 857 & 898 & 877.5 \\
\hline S96T005721 & & Lower half & 540 & 530 & 535 \\
\hline S96T005837 & \multirow[t]{2}{*}{$166: 19$} & Upper half & 303 & 307 & 305 \\
\hline S96T005838 & & Lower half & 321 & 343 & 332 \\
\hline S96T005705 & \multirow[t]{2}{*}{ 167: 1} & Upper half & 1,050 & 1,080 & 1,065 \\
\hline \$96T005687 & & Lower half & 835 & 762 & 798.5 \\
\hline \$96T005855 & 167: 2 & Lower half & 740 & 743 & 741.5 \\
\hline
\end{tabular}




\begin{tabular}{|c|c|c|c|c|c|}
\hline$t \varepsilon 9$ & LL9 & $16 S$ & Ғ[ЕЧ & & $\angle 6 \Rightarrow S 00 L 96 S$ \\
\hline $0: 00769$ & $\mathrm{I} 8 \mathrm{~L}$ & $\angle 09$ & JाЕч Iədd $\Omega$ & $\angle \mathrm{I}: \angle 9 \mathrm{I}$ & $96+S 00 L 96 \mathrm{~S}$ \\
\hline$\varsigma^{-9} 98 p$ & SES & $8 \varepsilon t$ & I[एบ IOMOT & \multirow[b]{2}{*}{ SI:L9I } & $269 \mathrm{~S} 00 \mathrm{~L} 96 \mathrm{~S}$ \\
\hline$S \angle 6 S$ & $96 \mathrm{~S}$ & $66 \mathrm{~S}$ & yleu xədd $\Omega$ & & $\angle 0 L S 00 L 96 \mathrm{~S}$ \\
\hline$\varepsilon z S$ & LZS & $6 \mathrm{IS}$ & I[Еप IәMOT & \multirow[b]{2}{*}{$\nabla I: \angle 9 I$} & $\angle E \pitchfork S 00 L 96 \mathrm{~S}$ \\
\hline $5.09 t$ & $99 t$ & sSt & I[खч Iədd $\Omega$ & & $\varepsilon \nabla t 500 \mathrm{~L} 96 \mathrm{~S}$ \\
\hline $589 L$ & $\varepsilon 6 L$ & $t t L$ & J[EY IәMOT & \multirow[b]{2}{*}{ ZI : $\angle 9 I$} & $6 t+\$ 00 L 96 S$ \\
\hline S.IIO"I & $\varepsilon \angle 6$ & $0 S 0^{\circ} \mathrm{I}$ & 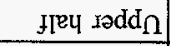 & & Z††S00L96S \\
\hline 096 & $\nabla<6$ & 906 & IIEU IәMOT & \multirow[b]{2}{*}{ II $: \angle 9 I$} & I69\$00L96S \\
\hline$S^{\circ} \triangleright \varepsilon 8$ & $9 z 8$ & $\varepsilon \neq 8$ & $y^{\mathrm{E}} \mathrm{I} \mathrm{Idd}_{\Omega}$ & & $90 \angle S 00 L 96 \mathrm{~S}$ \\
\hline$S^{\circ} \angle 6 I>$ & IIZ $>$ & $781>$ & IlЕપ IәMOT & $6: \angle 9 I$ & 9S8S00L96S \\
\hline $0: 009 \nabla Z$ & $6 \mathrm{IZ}$ & $\varepsilon L \mathcal{Z}$ & J[Eप دәмоT & $8: \angle 9 I$ & $9 \varepsilon \angle S 00 L 96 S$ \\
\hline$\varsigma^{*} I t z>$ & $8 I Z>$ & $\$ 92$ & I]Еप IәMOT & $\angle: \angle 9 I$ & 069S00L96S \\
\hline$: 00 Z \varepsilon E$ & $\angle L Z$ & $\angle 8 \mathcal{E}$ & IIEY IәMOT & $9: \angle 9 I$ & $8 t t 500 L 96 S$ \\
\hline $0 \neq \varepsilon$ & $8 Z \mathcal{E}$ & $\tau \varsigma \varepsilon$ & I[Еप IәMOT & $\varsigma: \angle 9 I$ & $9 \mathcal{E}+500 \mathrm{~L} 96 \mathrm{~S}$ \\
\hline$\subseteq I Z>$ & IIZ> & $6 \mathrm{IZ}$ & I[еप IәмоТ & $t: \angle 9 \mathrm{I}$ & 689 S00.L96 \\
\hline$z z z>$ & $6 \mathrm{IZ}$ & szz> & ІІец Іәмо Т & $\mathcal{E}: \angle 9 I$ & $889 S 00 L 96 \mathrm{~S}$ \\
\hline \% & 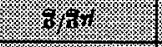 & \% & & \multicolumn{2}{|c|}{ 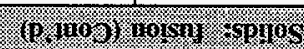 } \\
\hline 9 & 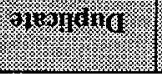 & Honsos & ongros & 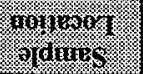 & 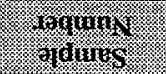 \\
\hline
\end{tabular}

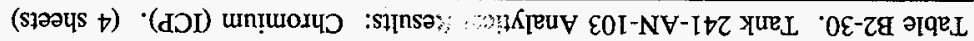


HNF-SD-WM-ER-702 Rev. 0

Table B2-31. Tank 241-AN-103 Analytical Results: Cobalt (ICP). (4 sheets)

\begin{tabular}{|c|c|c|c|c|c|}
\hline Simple & Somprition. & Sample & result: & Bvplienty. & $11 \cdot 9 x$ \\
\hline \multicolumn{2}{|c|}{ 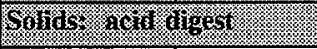 } & 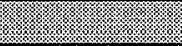 & 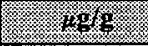 & 3.96 & 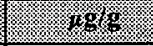 \\
\hline S96T005609 & 166: 1 & Lower half & 52.6 & 49.9 & 51.25 \\
\hline S96T005570 & 166: 4 & Lower half & 57.4 & 61 & 59.2 \\
\hline S96T005615 & $166: 6$ & Lower half & 67 & 66.5 & 66.75 \\
\hline S96T005839 & 166: 7 & Lower half & 67.7 & 59.9 & 63.8 \\
\hline \$96T005840 & $166: 8$ & Lower half & 68.2 & 48.3 & $58.25^{\mathrm{QC:a,e}}$ \\
\hline S96T005575 & $166: 9$ & Lower half & $<39.1$ & 66.6 & $<52.85^{Q \mathrm{QC:c}}$ \\
\hline S96T005571 & $166: 10$ & Lower half & 54.8 & 60.4 & 57.6 \\
\hline S96T005616 & 166: 11 & Lower half & 56.9 & 50.9 & 53.9 \\
\hline S96T005617 & $166: 12$ & Lower half & 61.8 & 67.1 & 64.45 \\
\hline S96T005716 & \multirow{2}{*}{$166: 13$} & Upper half & 45.3 & 48.2 & 46.75 \\
\hline S96T005722 & & Lower half & 64.2 & 51 & $57.6^{\mathrm{OC}: \mathrm{c}}$ \\
\hline S96T005416 & \multirow[t]{2}{*}{$166: 15$} & Upper half & 77 & 64.8 & 70.9 \\
\hline S97T000459 & & Lower half & $<11.4$ & $<11.1$ & $<11.25$ \\
\hline S96T005624 & \multirow[t]{2}{*}{$166: 16$} & Upper half & 82.7 & 71.4 & 77.05 \\
\hline S96T005618 & & Lower half & 67.7 & 62.1 & 64.9 \\
\hline S96T005417 & \multirow[t]{2}{*}{ 166: 17} & Upper half & 49.5 & 49.7 & 49.6 \\
\hline S96T005411 & & Lower half & 55.5 & 76.5 & $66^{\mathrm{QC:e}}$ \\
\hline S96T005717 & \multirow[t]{2}{*}{$166: 18$} & Upper half & 48.1 & 75.5 & $61.8^{\mathrm{QC:e}}$ \\
\hline S96T005723 & & Lower half & 50.9 & 46.7 & 48.8 \\
\hline S96T005841 & \multirow[t]{2}{*}{ 166: 19} & Upper half & 52.3 & 60.1 & $56.2^{\mathrm{QC:aa}}$ \\
\hline S96T005842 & & Lower half & 52.7 & 43.5 & $48.1^{\text {QC:a }}$ \\
\hline S96T005708 & \multirow[t]{2}{*}{ 167: 1} & Upper half & 49.8 & $<41.1$ & $<45.45$ \\
\hline S96T005693 & & Lower half & 67 & 56.5 & 61.75 \\
\hline S96T005857 & $167: 2$ & Lower half & 65.6 & 54.8 & 60.2 \\
\hline S96T005694 & $167: 3$ & Lower half & $<35.9$ & 45.9 & $<40.9^{\mathrm{QC}: \mathrm{e}}$ \\
\hline S96T005695 & 167: 4 & Lower half & 60.1 & 57.6 & 58.85 \\
\hline S96T005438 & $167: 5$ & Lower half & $<10.3$ & $<11$ & $<10.65^{\mathrm{QC:a}}$ \\
\hline S96T005450 & 167: 6 & Lower half & $<11.1$ & $<10.4$ & $<10.75^{\mathrm{QC}: \mathrm{a}}$ \\
\hline S96T005696 & 167: 7 & Lower half & $<38.4$ & $<37.6$ & $<38$ \\
\hline
\end{tabular}


Table B2-31. Tank 241-AN-1R Analytical Results: Cobalt (ICP). (4 sheets)

\begin{tabular}{|c|c|c|c|c|c|}
\hline Samples & osimples. & $\begin{array}{l}\text { Sample } \\
\text { rom sivor. }\end{array}$ & resulit) & (14)ilicate. & 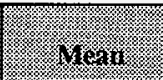 \\
\hline 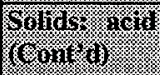 & hest & (1) & $\operatorname{lng} g$ & (3) & 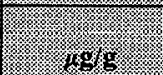 \\
\hline S96T005737 & 167: 8 & Lower half & 64.8 & 57.2 & 61 \\
\hline S96T005858 & $167: 9$ & Lower half & 45.1 & 46.2 & 45.65 \\
\hline S96T005709 & \multirow[t]{2}{*}{$167: 11$} & Upper half & 45.8 & 55.7 & 50.75 \\
\hline S96T005697 & & Lower half & 62.3 & 51.6 & 56.95 \\
\hline S96T005444 & \multirow[t]{2}{*}{$167: 12$} & Upper half & $<11.1$ & $<10.9$ & $<11^{\mathrm{QC}: \mathrm{a}}$ \\
\hline S96T005451 & & Lower half & $<11.4$ & $<11.7$ & $<11.55^{\mathrm{QC:a}}$ \\
\hline S96T005445 & \multirow[t]{2}{*}{ 167: 14} & Upper half & $<10.6$ & $<11$ & $<10.8^{\mathrm{QC}: \mathrm{a}}$ \\
\hline S96T005439 & & Lower half & $<11.4$ & $<11.7$ & $<11.55^{\mathrm{QC:a}}$ \\
\hline S96T005710 & \multirow[t]{2}{*}{$167: 15$} & Upper half & 64.3 & 43.5 & $53.9^{\mathrm{OC}: c}$ \\
\hline S96T005698 & & Lower half & 43.9 & 49.3 & 46.6 \\
\hline S96T005498 & \multirow[t]{2}{*}{$167: 17$} & Upper half & 68.5 & $<38.9$ & $<53.7^{\mathrm{QC}: e}$ \\
\hline S96T005499 & & Lower half & $<35.7$ & 45.2 & $<40.45^{\text {QC:e }}$ \\
\hline S97T000460 & Core 166 & Solid composite & $<11.3$ & $<11$ & $<11.15$ \\
\hline Wrgurds & (2) & (2) & . & 1967nil & 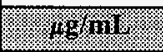 \\
\hline S96T005539 & $166: 3$ & Drainable liquid & $<8.02$ & $<8.02$ & $<8.02$ \\
\hline S96T005540 & $166: 4$ & Drainable liquid & $<8.02$ & $<8.02$ & $<8.02$ \\
\hline S96T005599. & $166: 6$ & Drainable liquid & $<12$ & $<12$ & $<12$ \\
\hline S96T005816 & 166: 7 & Drainable liquid & $<12$ & $<12$ & $<12$ \\
\hline S96T005817 & 166: 8 & Drainable liquid & $<12$ & $<12$ & $<12$ \\
\hline S96T005541 & 166: 9 & Drainable liquid & $<12$ & $<12$ & $<12$ \\
\hline S96T005566 & 166: 10 & Drainable liquid & $<8.02$ & $<8.02$ & $<8.02$ \\
\hline S96T005600 & $166: 11$ & Drainable liquid & $<12$ & $<12$ & $<12$ \\
\hline S96T005601 & $166: 12$ & Drainable liquid & $<12$ & $<12$ & $<12$ \\
\hline S96T005861 & 167: 2 & Drainable liquid & $<12$ & $<12$ & $<12$ \\
\hline S96T005673 & $167: 3$ & Drainable liquid & $<12$ & $<12$ & $<12$ \\
\hline S96T005420 & $167: 5$ & Drainable liquid & $<12$ & $<12$ & $<12$ \\
\hline S96T005421 & 167: 6 & Drainable liquid & $<12$ & $<12$ & $<12$ \\
\hline S96T005683 & 167: 7 & Drainable liquid & $<12$ & $<12$ & $<12$ \\
\hline
\end{tabular}


Table B2-31. Tank 241-AN-103 Analytical Results: Cobalt (ICP). (4 sheets)

\begin{tabular}{|c|c|c|c|c|c|}
\hline $\begin{array}{l}\text { Sampile } \\
\text { Jiminiter }\end{array}$ & Sarraple & $\begin{array}{l}\text { Saming } \\
\text { Portion }\end{array}$ & resistis & Bumlicite & Meri: \\
\hline \multicolumn{3}{|c|}{ 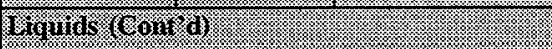 } & 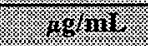 & ivinit & 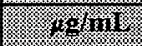 \\
\hline S96T005732. & $167: 8$ & Drainable liquid & $<12$ & $<12$ & $<12$ \\
\hline S96T005862 & $167: 9$ & Drainable liquid & $<12$ & $<12$ & $<12$ \\
\hline S96T005650 & 167: 11 & Drainable liquid & $<12$ & $<12$ & $<12$ \\
\hline S96T005991 & Core 166 & Liquid composite & 55.1 & 66.6 & 60.85 \\
\hline \multicolumn{3}{|c|}{ Sorthe furion } & (x) & 18. & (3) \\
\hline S96T005608 & 166: 1 & Lower half & $<420$ & $<413$ & $<416.5$ \\
\hline S96T005568 & 166: 4 & Lower half & $<453$ & $<445$ & $<449$ \\
\hline S96T005611 & $166: 6$ & Lower half & $<419$ & $<428$ & $<423.5$ \\
\hline S96T005835 & $166: 7$ & Lower half & $<421$ & $<425$ & $<423$ \\
\hline S96T005836 & $166: 8$ & Lower half & $<392$ & $<411$ & $<401.5$ \\
\hline S96T005574 & $166: 9$ & Lower half & $<358$ & 402 & $<380$ \\
\hline S96T005569 & 166: 10 & Lower half & $<418$ & $<414$ & $<416$ \\
\hline S96T005612 & $166: 11$ & Lower half & $<395$ & $<407$ & $<401$ \\
\hline S96T005613 & $166: 12$ & Lower half & $<411$ & $<405$ & $<408$ \\
\hline S96T005714 & \multirow[t]{2}{*}{ 166: 13} & Upper half & $<403$ & $<422$ & $<412.5$ \\
\hline S96T005720 & & Lower half & $<405$ & $<403$ & $<404$ \\
\hline S96T005414 & \multirow[t]{2}{*}{$166: 15$} & Upper half & $<91.3$ & $<93.2$ & $<92.25$ \\
\hline S96T005408 & & Lower half & $<96$ & $<92.7$ & $<94.35$ \\
\hline S96T005623 & \multirow[t]{2}{*}{$166: 16$} & Upper half & $<436$ & $<420$ & $<428$ \\
\hline S96T005614 & & Lower half & $<409$ & $<402$ & $<405.5$ \\
\hline S96T005415 & \multirow[t]{2}{*}{ 166: 17} & Upper half & $<105$ & $<106$ & $<105.5$ \\
\hline S96T005409 & & Lower half & $<422$ & 453 & $<437.5$ \\
\hline S96T005715 & \multirow[t]{2}{*}{ 166: 18} & Upper half & $<403$ & $<411$ & $<407$ \\
\hline S96T005721 & & Lower half & $<405$ & $<398$ & $<401.5$ \\
\hline S96T005837 & \multirow[t]{2}{*}{$166: 19$} & Upper half & $<414$ & $<420$ & $<417$ \\
\hline S96T005838 & & Lower half & $<391$ & $<393$ & $<392$ \\
\hline S96T005705 & \multirow[t]{2}{*}{$167: 1$} & Upper half & $<398$ & $<401$ & $<399.5$ \\
\hline S96T005687 & & Lower half & $<416$ & $<417$ & $<416.5$ \\
\hline S96T005855 & $167: 2$ & Lower half & $<404$ & $<395$ & $<399.5$ \\
\hline
\end{tabular}


HNF-SD-WM-ER-702 Rev. 0

Table B2-31. Tank 241-AN-103 Analytical Results: Cobalt (ICP). (4 sheets)

\begin{tabular}{|c|c|c|c|c|c|}
\hline $\begin{array}{l}\text { Sample } \\
\text { Niminger }\end{array}$ & Sompro & $\begin{array}{l}\text { Sample } \\
\text { roftion }\end{array}$ & Resiu & Buplicate & $14 \mathrm{n}$ \\
\hline \multicolumn{3}{|c|}{ 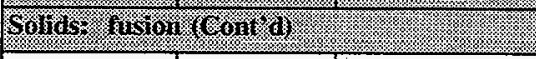 } & 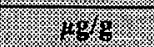 & $\sqrt{14} 986$ & 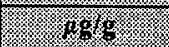 \\
\hline S96T005688 & $167: 3$ & Lower half & $<450$ & $<426$ & $<438$ \\
\hline S96T005689 & $167: 4$ & Lower half & $<420$ & $<422$ & $<421$ \\
\hline S96T005436 & $167: 5$ & Lower half & $<372$ & $<376$ & $<374$ \\
\hline S96T005448 & $167: 6$ & Lower half & $<421$ & $<409$ & $<415$ \\
\hline S96T005690 & 167:7 & Lower half & $<393$ & $<436$ & $<414.5$ \\
\hline S96T005736 & $167: 8$ & Lower half & $<442$ & $<375$ & $<408.5$ \\
\hline S96T005856 & $167: 9$ & Lower half & $<368$ & $<422$ & $<395$ \\
\hline S96T005706 & \multirow[t]{2}{*}{$167: 11$} & Upper half & $<373$ & $<444$ & $<408.5$ \\
\hline S96T005691 & & Lower half & $<370$ & 434 & $<402$ \\
\hline S96T005442 & \multirow[t]{2}{*}{ 167: 12} & Upper half & $<432$ & $<388$ & $<410$ \\
\hline S96T005449 & & Lower half & $<395$ & $<404$ & $<399.5$ \\
\hline S96T005443 & \multirow[t]{2}{*}{$167: 14$} & Upper half & $<441$ & $<422$ & $<431.5$ \\
\hline S96T005437 & & Lower half & $<411$ & $<406$ & $<408.5$ \\
\hline S96T005707 & \multirow[t]{2}{*}{$167: 15$} & Upper half & $<427$ & $<368$ & $<397.5$ \\
\hline S96T005692 & & Lower half & $<366$ & $<435$ & $<400.5$ \\
\hline S96T005496 & \multirow[t]{2}{*}{ 167: 17} & Upper half & $<424$ & $<419$ & $<421.5$ \\
\hline S96T005497 & & Lower half & $<420$ & $<421$ & $<420.5$ \\
\hline
\end{tabular}


Table B2-32. Tank 241-AN-103 Analytical Results: Copper (ICP). (4 sheets)

\begin{tabular}{|c|c|c|c|c|c|}
\hline \%月, & Wariole & $f_{1}$ & Resilil & 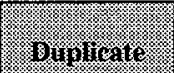 & 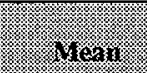 \\
\hline \multicolumn{2}{|c|}{ 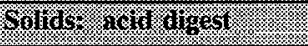 } & 1/. & (6) & 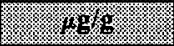 & (ng \\
\hline S96T005609 & 166: 1 & Lower half & $<14.8$ & $<15.1$ & $<14.95$ \\
\hline S96T005570 & 166: 4 & Lower half & $<17.9$ & $<17.9$ & $<17.9$ \\
\hline S96T005615 & $166: 6$ & Lower half & $<13.8$ & $<13.8$ & $<13.8$ \\
\hline S96T005839 & 166: 7 & Lower half & $<20.2$ & $<20.5$ & $<20.35$ \\
\hline S96T005840 & 166: 8 & Lower half & $<19.7$ & $<19.7$ & $<19.7^{\mathrm{QC: \textrm {a }}}$ \\
\hline S96T005575 & 166: 9 & Lower half & $<19.6$ & $<19.9$ & $<19.75$ \\
\hline S96T005571 & 166: 10 & Lower half & $<15.3$ & $<14.5$ & $<14.9$ \\
\hline S96T005616 & 166: 11 & Lower half & $<14.5$ & $<13.6$ & $<14.05$ \\
\hline S96T005617 & 166: 12 & Lower half & $<14.1$ & $<14.1$ & $<14.1$ \\
\hline \$96T005716 & \multirow[t]{2}{*}{$166: 13$} & Upper half & $<19.4$ & $<19.6$ & $<19.5$ \\
\hline S96T005722 & & Lower half & $<20.6$ & $<20.6$ & $<20.6$ \\
\hline S96T005416 & \multirow[t]{2}{*}{$166: 15$} & Upper half & $<20.4$ & $<19$ & $<19.7$ \\
\hline S97T000459 & & Lower half & 6.94 & $<5.57$ & $<6.255^{\mathrm{QC}: \mathrm{a}, \mathrm{e}}$ \\
\hline S96T005624 & \multirow[t]{2}{*}{$166: 16$} & Upper half & $<21$ & $<21.5$ & $<21.25$ \\
\hline S96T005618 & & Lower half & $<14.2$ & $<14.6$ & $<14.4$ \\
\hline S96T005417 & \multirow[t]{2}{*}{ 166: 17} & Upper half & $<22.5$ & $<20.5$ & $<21.5$ \\
\hline S96T005411 & & Lower half & $<19$ & $<17.6$ & $<18.3$ \\
\hline S96T005717 & \multirow[t]{2}{*}{$166: 18$} & Upper half & $<20.8$ & $<20.2$ & $<20.5$ \\
\hline \$96T005723 & & Lower half & $<20.2$ & $<19.8$ & $<20$ \\
\hline S96T005841 & \multirow[t]{2}{*}{$166: 19$} & Upper half & $<19.3$ & $<19.6$ & $<19.45^{\mathrm{QC}: \mathrm{a}}$ \\
\hline S96T005842 & & Lower half & $<17.8$ & $<19.6$ & $<18.7^{\mathrm{QC:a}}$ \\
\hline S96T005708 & \multirow[t]{2}{*}{ 167: 1} & Ujpper half & $<17.9$ & $<20.5$ & $<19.2$ \\
\hline S96T005693 & & Lower half & $<22.2$ & $<17.5$ & $<19.85$ \\
\hline S96T005857 & $167: 2$ & Lower half & $<16.7$ & $<17.4$ & $<17.05$ \\
\hline S96T005694 & $167: 3$ & Lower haif & $<18$ & $<18.7$ & $<18.35$ \\
\hline S96T005695 & $167: 4$ & Lower half & $<19.8$ & $<20.4$ & $<20.1$ \\
\hline S96T005438 & $167: 5$ & Lower half & $<5.17$ & $<5.51$ & $<5.34^{\mathrm{QC:a}}$ \\
\hline S96T005450 & 167:6 & Lower half & $<5.53$ & $<5.22$ & $<5.375^{\mathrm{QC}: \mathrm{a}}$ \\
\hline S96T005696 & 167: 7 & Lower half & $<19.2$ & $<18.8$ & $<19$ \\
\hline
\end{tabular}




\begin{tabular}{|c|c|c|c|c|c|}
\hline$\left[0^{\circ} 9>\right.$ & $10^{\circ} 9>$ & $10^{\circ} 9>$ & pı̣nbỵ ગqeuṭex & $\angle: \angle 9 \mathrm{I}$ & $\varepsilon 89 S 00 L 96 \mathrm{~S}$ \\
\hline $10^{\circ} 9>$ & $10.9>$ & $10^{\circ} 9>$ & pṛnbụ ә & $9: \angle 9 I$ & IZ॰S00L96S \\
\hline $0: 00 \mathrm{SSP}^{\circ} \mathrm{SI}>$ & $10^{\circ} 9>$ & $L S \mathcal{L}$ & p!nb!l әrqeu!̣esa & $\subseteq: \angle 9 \mathrm{I}$ & 0ZtS00L96S \\
\hline $10 \cdot 9>$ & $10^{\circ} 9>$ & $10^{\circ} 9>$ & p!nb!ı әqqeute. & $\varepsilon: \angle 9 \mathrm{I}$ & $\varepsilon \angle 9 S 00 \mathrm{~L} 96 \mathrm{~S}$ \\
\hline$\left[0^{\circ} 9>\right.$ & $10^{\circ} 9>$ & $10^{\circ} 9>$ & pı̣nbț әIqвuțerવ & $2: \angle 9 I$ & I98S00L96S \\
\hline $10^{\circ} 9>$ & $10^{\circ} 9>$ & $10^{\circ} 9>$ & pı̣nb!̣ әңqeuṭeI & ZI :99I & I09\$00L96S \\
\hline $10^{\circ} 9>$ & $10^{\circ} 9>$ & {$\left[0^{\circ} 9>\right.$} & pı̣b!̣ गqueuteIa & II :99 I & 009\$00L96S \\
\hline $10^{\circ} t>$ & $10^{\circ} \nabla>$ & $10^{\circ} \neq>$ & p!̣nb!̣ ગ̨q̨eutera & OI :99T & $99 \$ \$ 00 L 96 S$ \\
\hline $10^{\circ} 9>$ & $10^{\circ} 9>$ & $10^{\circ} 9>$ & 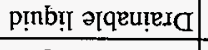 & $6: 99 \mathrm{I}$ & It\$SO0L.96S \\
\hline$: 00 \mathrm{SOI} 8>$ & $10^{\circ} 9>$ & $\tau \circ \mathrm{I}$ & p!̣b!̣ गqеu!eIa & $8: 99$ I & $\angle I 8 \$ 00 I 96 S$ \\
\hline $10^{\circ} 9>$ & $10^{\circ} 9>$ & $10^{\circ} 9>$ & 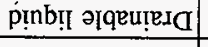 & $\angle: 99 I$ & 918S00L96S \\
\hline $10^{\circ} 9>$ & $10^{\circ} 9>$ & $10^{\circ} 9>$ & pı̣b!̣ әтqeụ̣eId & $9: 991$ & 66\$S00L96S \\
\hline $10^{\circ} t>$ & $10^{\circ} \circ>$ & {$\left[0^{\circ} \rightarrow>\right.$} & pịnb!r गqeurera & $\triangleright: 99 I$ & $07 \varsigma \subseteq 00 x 96 \mathrm{~S}$ \\
\hline $10^{\circ}+>$ & $10^{\circ} t>$ & $10^{\circ} t>$ & pı̣b! श̣qeurexa & $\varepsilon: 99 \mathrm{I}$ & 6ES\$00L96S \\
\hline 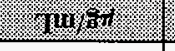 & $410 \%$ & 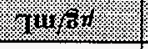 & & & (2) \\
\hline$: 00 \angle S^{\circ} S>$ & $I S^{\circ} S>$ & $\varepsilon 9^{\circ} s>$ & əৈ! & 9912100 & $09+000 \mathrm{~L} L 6 \mathrm{~S}$ \\
\hline $\mathrm{SS}^{\circ} 8 \mathrm{I}>$ & $\mathcal{E} \cdot 6 I>$ & $8 \angle I>$ & fleપ دәмот & \multirow[b]{2}{*}{$\angle I: \angle 9 I$} & $66+500 L 96 S$ \\
\hline $6^{\circ} 8 \mathrm{I}>$ & $S^{\circ} 6 \mathrm{I}>$ & $\varepsilon^{\circ} 8 I>$ & IFEप Iədd $\Omega$ & & $86 t S 00 L 96 \mathrm{~S}$ \\
\hline$S^{\circ} 8 \mathrm{I}>$ & $8.8 I>$ & $2.81>$ & f[ЕЧ IכMOT] & \multirow[b]{2}{*}{$S \mathrm{I}: \angle 9 \mathrm{I}$} & $869 S 00 L 96 \mathrm{~S}$ \\
\hline$S Z \cdot 8 \mathrm{I}>$ & $6^{\circ} \angle I>$ & $98 I>$ & J[eप $\lrcorner ә d d_{\Omega}$ & & 0LLS00L96S \\
\hline в:00\$0\$ 6 & OI & {$[0.6$} & Jएบ ІәмоT & \multirow[b]{2}{*}{$t[: \angle 9 !$} & 6EtS00L96S \\
\hline $00 \Omega \angle 8^{\circ} L$ & $79^{\circ} L$ & II 8 & $\pm[$ Іец ıәdd $\Omega$ & & $s_{t t S 00 L 96 S}$ \\
\hline$: 00 \$ 58^{\circ} \varsigma>$ & $78^{\circ} s>$ & $\angle 8^{\circ} \mathrm{S}$ & I[ЕU IәMOT] & \multirow[b]{2}{*}{ ZI : $\angle 9 \mathrm{I}$} & IStS00L96S \\
\hline 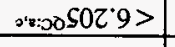 & $s s^{\circ} s>$ & $96^{\circ} 9$ & I[Eบ Iədd $\cap$ & & $t t \nabla S 00 L 196 S$ \\
\hline $\mathcal{E} 6 \mathrm{I}>$ & $8 \cdot 8 I>$ & $8^{\circ} 6 I>$ & J[Eप IOMOT & \multirow[b]{2}{*}{ I I : $\angle 9 I$} & L69S00L96S \\
\hline$S E^{\circ} 8 \mathrm{I}>$ & $L 8 I>$ & $81>$ & fley Iədd $\Omega$ & & 60LS00LI96S \\
\hline$S I^{\circ} 6 I>$ & $S \angle I>$ & $8^{\circ} 0 z>$ & Ғ[ЕЧ Јәмот] & $6: \angle 9 I$ & 8S8S00L96S \\
\hline $86 I>$ & $0 z>$ & $9^{\circ} 6 \mathrm{I}>$ & J[eप IəmoT] & $8: \angle 9 I$ & $\angle E \angle S 00 L 96 \mathrm{~S}$ \\
\hline 6 & byon & $64011=$ & & \multicolumn{2}{|c|}{ 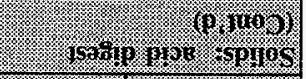 } \\
\hline mox & 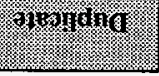 & 169 & 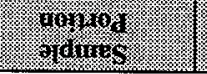 & 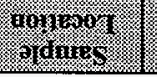 & inguring \\
\hline
\end{tabular}

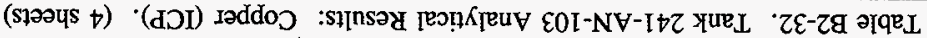


Table B2-32. Tank 241-AN-103 Analytical Results: Copper (ICP). (4 sheets)

\begin{tabular}{|c|c|c|c|c|c|}
\hline Sampere & \%ample & 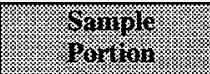 & Resulis: & Hum & (2) \\
\hline \multicolumn{2}{|c|}{ 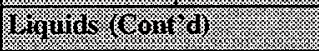 } & & (1961) & 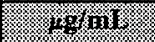 & \% \\
\hline S96T005732 & $167: 8$ & Drainable liquid & $<6.01$ & $<6.01$ & $<6.01$ \\
\hline S96T005862 & 167:9 & Drainable liquid & $<6.01$ & $<6.01$ & $<6.01$ \\
\hline S96T005650 & 167: 11 & Drainable liquid & $<6.01$ & $<6.01$ & $<6.01$ \\
\hline \$96T005991 & Core 166 & Liquid composite & $<12$ & $<12$ & $<12$ \\
\hline \multicolumn{2}{|c|}{ 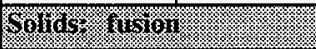 } & & 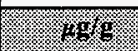 & 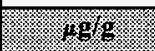 & (3) \\
\hline S96T005608 & 166: 1 & Lower half & $<210$ & $<206$ & $<208$ \\
\hline S96T005568 & 166: 4 & Lower half & $<227$ & $<222$ & $<224.5$ \\
\hline S96T005611 & $166: 6$ & Lower half & $<210$ & $<214$ & $<212$ \\
\hline S96T005835 & 166: 7 & Lower half & $<210$ & $<212$ & $<211$ \\
\hline S96T005836 & $166: 8$ & Lower half & $<196$ & $<206$ & $<201$ \\
\hline \$96T005574 & $166: 9$ & Lower half & $<179$ & 208 & $<193.5$ \\
\hline S96T005569 & 166: 10 & Lower half & $<209$ & $<207$ & $<208$ \\
\hline S96T005612 & 166: 11 & Lower half & $<197$ & $<204$ & $<200.5$ \\
\hline \$96T005613 & $166: 12$ & Lower half & $<205$ & $<202$ & $<203.5$ \\
\hline S96T005714 & \multirow[t]{2}{*}{$166: 13$} & Upper half & $<201$ & $<211$ & $<206$ \\
\hline S96T005720 & & Lower half & $<203$ & $<201$ & $<202$ \\
\hline S96T005414 & \multirow[t]{2}{*}{$166: 15$} & Upper half & $<45.6$ & $<46.6$ & $<46.1$ \\
\hline \$96T005408 & & Lower half & $<48$ & $<46.3$ & $<47.15$ \\
\hline S96T005623 & \multirow[t]{2}{*}{ 166: 16} & Upper half & $<218$ & $<210$ & $<214$ \\
\hline S96T005614 & & Lower half & $<204$ & $<201$ & $<202.5$ \\
\hline S96T005415 & \multirow[t]{2}{*}{ 166: 17} & Upper half & $<52.3$ & $<52.8$ & $<52.55$ \\
\hline \$96T005409 & & Lower half & $<211$ & $<208$ & $<209.5$ \\
\hline S96T005715 & \multirow[t]{2}{*}{$166: 18$} & Upper half & $<201$ & $<206$ & $<203.5$ \\
\hline S96T005721 & & Lower half & $<203$ & $<199$ & $<201$ \\
\hline \$96T005837 & \multirow[t]{2}{*}{$166: 19$} & Upper half & $<207$ & $<210$ & $<208.5$ \\
\hline S96T005838 & & Lower half & $<196$ & $<196$ & $<196$ \\
\hline S96T005705 & \multirow[t]{2}{*}{ 167:1 } & Upper half & $<199$ & $<200$ & $<199.5$ \\
\hline S96T005687 & & Lower half & $<208$ & $<209$ & $<208.5$ \\
\hline \$96T005855 & $167: 2$ & Lower half & $<202$ & $<198$ & $<200$. \\
\hline
\end{tabular}




\begin{tabular}{|c|c|c|c|c|c|}
\hline s.olz> & IIZ> & $0 I \tau>$ & 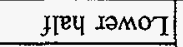 & & $\angle 6+S 00 \mathrm{~L} 96 \mathrm{~S}$ \\
\hline IIZ> & $0[z>$ & $Z I Z>$ & frey sadd $\Omega$ & LI :L9I & $96 t S 00 . L 96 S$ \\
\hline$s: 00 z>$ & $8 I Z>$ & $\varepsilon 8 \mathrm{I}>$ & Іец Іәмо Т] & \multirow[b]{2}{*}{ SI : $\angle 9 \mathrm{I}$} & 269500L96S \\
\hline$s .86 I>$ & $78 \mathrm{I}>$ & $\varepsilon I \tau>$ & $f f^{\mathrm{e}}$ Iədd $\Omega$ & & $\angle 0 \angle S 00 L 96 \mathrm{~S}$ \\
\hline toz> & $\varepsilon 0 z>$ & soz> & Ј[еप دәмо Т] & \multirow[b]{2}{*}{$\nabla I: \angle 9 I$} & $\angle E t S 00 L 96 S$ \\
\hline ş'sIZ> & IIZ> & $0 z z>$ & fley Iədd $\Omega$ & & $\varepsilon t t \$ 00 L 96 \mathrm{~S}$ \\
\hline $00 z>$ & $202>$ & $86 \mathrm{I}>$ & Ј[Еч РәМОТ] & \multirow[b]{2}{*}{ ZI :L9I } & $6+t S 00 \mathrm{~L} 96 \mathrm{~S}$ \\
\hline soz> & $\pm 6 I>$ & $9 I Z>$ & fley xədd $\Omega$ & & $200 S 00 L 96 \mathrm{~S}$ \\
\hline$\varsigma^{\prime \prime} \triangleright 8 I>$ & $+8 I>$ & $\$ 8 I>$ & j[еप дәмо & \multirow[b]{2}{*}{ II :L9I } & I69\$00L96S \\
\hline$\varsigma^{\prime}+0 z>$ & $z z z>$ & $\angle 8 I>$ & f[EY Iədd $\Omega$ & & $90 \angle S 00 \mathrm{~L} 96 \mathrm{~S}$ \\
\hline$\varsigma \angle 6 I>$ & IIZ> & $+8 I>$ & „Геप гәмоТ] & $6: \angle 9 I$ & $9 \$ 8500 \mathrm{~L} 96 \mathrm{~S}$ \\
\hline$t 0 z>$ & $\angle 8 I>$ & IZZ> & Ітеч гәмот & $8: \angle 9 I$ & $9 \varepsilon \angle S 00 \mathrm{~L} 96 \mathrm{~S}$ \\
\hline LOZ> & $8 I Z>$ & $96 I>$ & झाеप дәмо Т & $\angle: \angle 9 I$ & 069500L96S \\
\hline$\underline{s} \angle 0 z>$ & $\pm 0 z>$ & IIZ> & J[Еप $\triangle ә M O T$ & $9: \angle 9 I$ & $8 \mathrm{ttS} 00 \mathrm{~L} 96 \mathrm{~S}$ \\
\hline$\angle 8 I>$ & $881>$ & $981>$ & 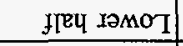 & $\varsigma: \angle 9 I$ & $9 \varepsilon+500 \mathrm{~L} 96 \mathrm{~S}$ \\
\hline s.oIz> & IIZ> & $0\lfloor\tau>$ & IГеप дәмОТ & $t: \angle 9 I$ & $689500 \mathrm{~L} 96 \mathrm{~S}$ \\
\hline $6 \mathrm{IZ}>$ & $\varepsilon \llbracket Z>$ & $s Z z>$ & I[एप & $\varepsilon: \angle 9$ I & I889\$00L96S \\
\hline 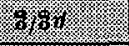 & 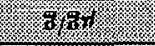 & $4,8=$ & $8>$ & \multicolumn{2}{|c|}{ 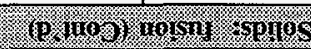 } \\
\hline 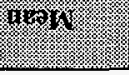 & onegnonge & 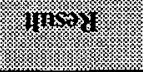 & 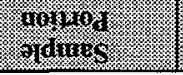 & $\begin{array}{l}\text { momingis } \\
\text { ardines }\end{array}$ & $\begin{array}{l}\text { iqgumst } \\
\text { aimins }\end{array}$ \\
\hline
\end{tabular}

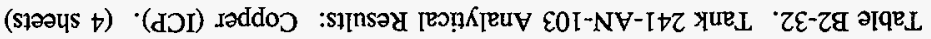


Table B2-33. Tank 241-AN-103 Analytical Results: Iron (ICP). (4 sheets)

\begin{tabular}{|c|c|c|c|c|c|}
\hline samplo & shomp & f & gresull & buyingar. & 40.11 \\
\hline \multicolumn{3}{|c|}{ 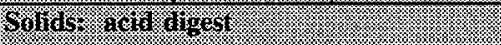 } & 48: & 58 & 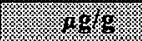 \\
\hline S96T005609 & 166: 1 & Lower half & $<73.8$ & $<75.4$ & $<74.6$ \\
\hline S96T005570 & 166: 4 & Lower half & $<89.7$ & $<89.7$ & $<89.7$ \\
\hline S96T005615 & $166: 6$ & Lower half & $<69.1$ & $<69.1$ & $<69.1$ \\
\hline S96T005839 & 166: 7 & Lower half & $<101$ & $<102$ & $<101.5$ \\
\hline S96T005840 & $166: 8$ & Lower half & $<98.4$ & $<98.3$ & $<98.35^{\mathrm{QC}: \mathrm{a}}$ \\
\hline \$96T005575 & $166: 9$ & Lower half & $<97.8$ & $<99.4$ & $<98.6$ \\
\hline S96T005571 & 166: 10 & Lower half & $<76.7$ & $<72.3$ & $<74.5$ \\
\hline S96T005616 & $166: 11$ & Lower haif & $<72.3$ & $<68$ & $<70.15$ \\
\hline S96T005617 & 166: 12 & Lower half & $<70.5$ & $<70.4$ & $<70.45$ \\
\hline S96T005716 & \multirow[t]{2}{*}{ 166: 13} & Upper half & $<96.9$ & $<98.1$ & $<97.5$ \\
\hline S96T005722 & & Lower half & $<103$ & $<103$ & $<103$ \\
\hline S96T005416 & \multirow[t]{2}{*}{$166: 15$} & Upper half & $<102$ & $<95.2$ & $<98.6$ \\
\hline S97T000459 & & Lower half & 34.5 & 41.5 & $38^{\mathrm{QC:a}}$ \\
\hline S96T005624 & \multirow[t]{2}{*}{$166: 16$} & Upper half & $<105$ & $<108$ & $<106.5$ \\
\hline S96T005618 & & Lower half & $<70.9$ & $<72.8$ & $<71.85$ \\
\hline S96T005417 & \multirow[t]{2}{*}{$166: 17$} & Upper half & $<113$ & $<102$ & $<107.5$ \\
\hline S96T005411 & & Lower half & $<95.1$ & $<88$ & $<91.55$ \\
\hline \$96T005717 & \multirow[t]{2}{*}{$166: 18$} & Upper half & 234 & 240 & 237 \\
\hline S96T005723 & & Lower half & $<101$ & $<99$ & $<100$ \\
\hline S96T005841 & \multirow[t]{2}{*}{$166: 19$} & Upper half & $<96.3$ & $<98.1$ & $<97.2^{\mathrm{QC}: \mathrm{a}}$ \\
\hline S96T005842 & & Lower half & $<89$ & $<98.1$ & $<93.55^{Q \mathrm{C}: \mathrm{a}}$ \\
\hline S96T005708 & \multirow[t]{2}{*}{ 167: 1} & Upper half & 1,600 & 428 & $1,014^{\mathrm{QC}: \mathrm{c}}$ \\
\hline S96T005693 & & Lower half & $<111$ & $<87.7$ & $<99.35$ \\
\hline S96T005857 & 167: 2 & Lower half & $<83.4$ & $<86.9$ & $<85.15$ \\
\hline S96T005694 & $167: 3$ & Lower half & $<89.9$ & $<93.6$ & $<91.75$ \\
\hline S96T005695 & 167: 4 & Lower half & $<99$ & $<102$ & $<100.5$ \\
\hline \$96T005438 & $167: 5$ & Lower half & $<25.8$ & $<27.5$ & $<26.65^{\text {QC:a }}$ \\
\hline \$96T005450 & $167: 6$ & Lower half & 29.8 & 32.1 & $30.95^{\mathrm{QCa}}$ \\
\hline S96T005696 & 167: 7 & Lower half & $<96$ & $<94$ & $<95$ \\
\hline
\end{tabular}


Table B2-33. Tank 241-AN-103 Analytical Results: Iron (ICP). (4 sheets)

\begin{tabular}{|c|c|c|c|c|c|}
\hline $\begin{array}{l}\text { Sample } \\
\text { Suminer }\end{array}$ & S Samples & $\begin{array}{l}\text { Shrome } \\
\text { portion }\end{array}$ & Resinin & Biminate & 16. \\
\hline \multicolumn{3}{|c|}{ 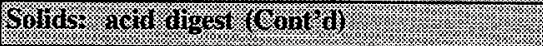 } & (3.8\% & $\sqrt{1.3968}$ & (3) \\
\hline S96T005737 & $167: 8$ & Lower half & $<97.9$ & 116 & $<106.95$ \\
\hline S96T005858 & $167: 9$ & Lower half & $<104$ & $<87.5$ & $<95.75$ \\
\hline S96T005709 & \multirow[t]{2}{*}{ 167: 11} & Upper half & $<90$ & $<93.3$ & $<91.65$ \\
\hline S96T005697 & & Lower half & $<99.2$ & $<94.1$ & $<96.65$ \\
\hline S96T005444 & \multirow[t]{2}{*}{$167: 12$} & Upper half & 39.5 & 49.2 & $44.35^{\mathrm{QC:a}, \mathrm{c}}$ \\
\hline S96T005451 & & Lower half & 39.1 & 38.3 & $38.7^{\mathrm{QC}: \mathrm{a}}$ \\
\hline S96T005445 & \multirow[t]{2}{*}{$167: 14$} & Upper half & 41.3 & 44.1 & $42.7^{\mathrm{OC:a}}$ \\
\hline S96T005439 & & Lower half & 379 & 546 & $462.5^{\propto \mathrm{C}: \mathrm{a}, \mathrm{e}}$ \\
\hline S96T005710 & \multirow[t]{2}{*}{$167: 15$} & Upper half & 352 & 446 & $399^{\mathrm{QC}: \mathrm{c}}$ \\
\hline S96T005698 & & Lower half & $<91.1$ & $<94$ & $<92.55$ \\
\hline S96T005498 & \multirow[t]{2}{*}{ 167: 17} & Upper half & 542 & 444 & 493 \\
\hline S96T005499 & & Lower half & $<89.1$ & $<96.5$ & $<92.8$ \\
\hline S97T000460 & Core 166 & Solid composite & 48.6 & 48.4 & $48.5^{\mathrm{QC}: \mathrm{a}}$ \\
\hline Miquitis & \multicolumn{2}{|c|}{ 1 } & 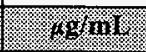 & 1) & . \\
\hline S96T005539 & $166: 3$ & Drainable liquid & $<20.1$ & $<20.1$ & $<20.1$ \\
\hline S96T005540 & 166: 4 & Drainable liquid & $<20.1$ & $<20.1$ & $<20.1$ \\
\hline S96T005599 & 166: 6 & Drainable liquid & $<30.1$ & $<30.1$ & $<30.1$ \\
\hline S96T005816 & 166: 7 & Drainable liquid & $<30.1$ & $<30.1$ & $<30.1$ \\
\hline S96T005817 & $166: 8$ & Drainable liquid & $<30.1$ & $<30.1$ & $<30.1$ \\
\hline S96T005541 & $166: 9$ & Drainable liquid & $<30.1$ & $<30.1$ & $<30.1$ \\
\hline S96T005566 & 166: 10 & Drainable liquid & $<20.1$ & $<20.1$ & $<20.1$ \\
\hline S96T005600 & $166: 11$ & Drainable liquid & $<30.1$ & $<30.1$ & $<30.1$ \\
\hline S96T005601 & $166: 12$ & Drainable liquid & $<30.1$ & $<30.1$ & $<30.1$ \\
\hline S96T005861 & $167: 2$ & \begin{tabular}{|l|} 
Drainable liquid \\
\end{tabular} & $<30.1$ & $<30.1$ & $<30.1$ \\
\hline \$96T005673 & $167: 3$ & Drainable liquid & $<30.1$ & $<30.1$ & $<30.1$ \\
\hline S96T005420 & $167: 5$ & Drainable liquid & 52.9 & $<30.1$ & $<41.5^{\mathrm{QC:e}}$ \\
\hline S96T005421 & 167: 6 & Drainable liquid & $<30.1$ & $<30.1$ & $<30.1$ \\
\hline S96T005683 & $167: 7$ & Drainable liquid & $<30.1$ & $<30.1$ & $<30.1$ \\
\hline
\end{tabular}


Table B2-33. Tank 241-AN-103 Analytical Results: Iron (ICP). (4 sheets)

\begin{tabular}{|c|c|c|c|c|c|}
\hline 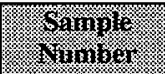 & 9ample & 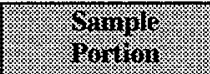 & mesing & a) & (3) \\
\hline \multicolumn{2}{|c|}{ 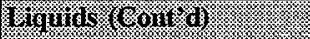 } & 13 & (6) & (3) & (3) \\
\hline S96T005732 & 167:8 & Drainable liquid & $<30.1$ & $<30.1$ & $<30.1$ \\
\hline S96T005862 & $167: 9$ & Drainable liquid & $<30.1$ & $<30.1$ & $<30.1$ \\
\hline S96T005650 & 167: 11 & Drainable liquid & $<30.1$ & $<30.1$ & $<30.1$ \\
\hline S96T005991 & Core 166 & Liquid composite & $<60.1$ & $<60.1$ & $<60.1$ \\
\hline \multicolumn{2}{|c|}{ SWHAs H HOH } & & 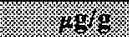 & (1) & (1) \\
\hline S96T005608 & 166: 1 & Lower half & $<1,050$ & $<1,030$ & $<1,040$ \\
\hline S96T005568 & $166: 4$ & Lower half & $<1,130$ & $<1,110$ & $<1,120$ \\
\hline S96T005611 & 166: 6 & Lower half & $<1,050$ & $<1,070$ & $<1,060$ \\
\hline S96T005835 & 166: 7 & Lower half & $<1,050$ & $<1,060$ & $<1,055$ \\
\hline S96T005836 & 166: 8 & Lower half & $<981$ & $<1,030$ & $<1,005.5$ \\
\hline S96T005574 & 166: 9 & Lower half & $<896$ & $<970$ & $<933$ \\
\hline S96T005569 & 166: 10 & Lower half & $<1,050$ & $<1,030$ & $<1,040$ \\
\hline S96T005612 & 166: 11 & Lower half & $<987$ & $<1,020$ & $<1,003.5$ \\
\hline S96T005613 & $166: 12$ & Lower half & $<1,030$ & $<1,010$ & $<1,020$ \\
\hline S96T005714 & \multirow[t]{2}{*}{$166: 13$} & Upper half & $<1,010$ & $<1,050$ & $<1,030$ \\
\hline S96T005720 & & Lower half & $<1,010$ & $<1,010$ & $<1,010$ \\
\hline S96T005414 & \multirow[t]{2}{*}{$166: 15$} & Upper half & $<228$ & $<233$ & $<230.5$ \\
\hline S96T005408 & & Lower half & $<240$ & $<232$ & $<236$ \\
\hline S96T005623 & \multirow[t]{2}{*}{ 166: 16} & Upper half & $<1,090$ & $<1,050$ & $<1,070$ \\
\hline S96T005614 & & Lower half & $<1,020$ & $<1,000$ & $<1,010$ \\
\hline S96T005415 & \multirow[t]{2}{*}{$166: 17$} & Upper half & $<262$ & $<264$ & $<263$ \\
\hline S96T005409 & & Lower half & $<1,060$ & $<1,040$ & $<1,050$ \\
\hline S96T005715 & \multirow[t]{2}{*}{$166: 18$} & Upper half & $<1,010$ & $<1,030$ & $<1,020$ \\
\hline S96T005721 & & Lower half & $<1,010$ & $<994$ & $<1,002$ \\
\hline S96T005837 & \multirow[t]{2}{*}{$166: 19$} & Upper half & $<1,040$ & $<1,050$ & $<1,045$ \\
\hline S96T005838 & & Lower half & $<978$ & $<982$ & $<980$ \\
\hline S96T005705 & \multirow[t]{2}{*}{ 167: 1} & Upper half & $<994$ & $<1,000$ & $<997$ \\
\hline S96T005687 & & Lower half & $<1,040$ & $<1,040$ & $<1,040$ \\
\hline S96T005855 & 167: 2 & Lower half & $<1,010$ & $<988$ & $<999$ \\
\hline
\end{tabular}




\begin{tabular}{|c|c|c|c|c|c|}
\hline $0 S 0^{\circ} \mathrm{I}>$ & $0 S 0^{\prime} I>$ & OSO $0^{\circ} \mathrm{I}>$ & Ј[ЕЧ гәмоТ] & & L6tS00L96S \\
\hline$\triangle 000 E t^{6} I>$ & $0 S 0^{6} \mathrm{I}>$ & $018^{6} \mathrm{I}$ & Jleप $x$ & $\angle I: \angle 9 I$ & $96 t S 00 L 96 S$ \\
\hline$\varepsilon 00^{\prime} \mid>$ & $060^{6} I>$ & $916>$ & f[Еบ ІอмоT & \multirow[b]{2}{*}{$S I: \angle 9 I$} & 269S00L96S \\
\hline$s^{*}+66>$ & $6 \mathrm{I} 6>$ & $0 \angle 0^{\circ} \mid>$ & fleप Jədd $\Omega$ & & $\angle 0 L 500 L 96 \mathrm{~S}$ \\
\hline $020^{6} \mathrm{I}>$ & OLO'I $>$ & $0 \varepsilon 0^{\circ} \mathrm{I}>$ & I[एप IәMOT & \multirow[b]{2}{*}{$D I: \angle 9 I$} & $\angle E \nrightarrow S 00 L 96 \mathrm{~S}$ \\
\hline SLO'I> & $0 S 0^{\circ} \mathrm{I}>$ & $00 I^{\prime} \mathrm{I}>$ & J[Еч.$ә d d_{\Omega}$ & & EttS00L96S \\
\hline $666>$ & OLO'I $>$ & $886>$ & IIEU IәMOT & \multirow[b]{2}{*}{ ZI: L9I } & $6 t t S 00 L 96 \mathrm{~S}$ \\
\hline$S^{\circ}+20^{\circ} I>$ & $696>$ & $080^{6}$ I $>$ & 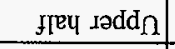 & & ZttS00L96S \\
\hline$\varepsilon 26>$ & $I 26>$ & $\$ 26>$ & А[ЕЧ ІәмоТ] & \multirow[b]{2}{*}{ II : $\angle 9 I$} & I69S00L96S \\
\hline$S^{*} I Z 0^{6} I>$ & OII'I > & $\varepsilon E 6>$ & $f\left[E_{4}\right.$ Iədd $\Omega$ & & $90 L S 00 L 96 \mathrm{~S}$ \\
\hline$\$ 86>$ & $0 S 0^{\circ} I>$ & $0 z 6>$ & Іाеप гәмот & $6: \angle 9 I$ & $9 \$ 8 S 00 L 96 \mathrm{~S}$ \\
\hline$S^{*} E Z O^{6} I>$ & $\angle E 6>$ & OII'I $>$ & IIEप Iәмот & $8: \angle 9 I$ & $9 \varepsilon L S 00 L 96 S$ \\
\hline $9 \mathcal{E} 0^{\circ} \mathrm{I}>$ & $060^{\circ}$ I $>$ & $286>$ & IIEप IәMOT & $L: \angle 9 \mathrm{I}$ & 069S00196S \\
\hline$\subseteq E O^{\prime} I>$ & $0706 \mathrm{I}>$ & $0 S 0^{6} I>$ & J[ЕЧ ЈәмоТ] & $9: \angle 9 I$ & $8 t t 500 \mathrm{~L} 96 \mathrm{~S}$ \\
\hline $\mathfrak{S E 6 >}$ & $6 \mathcal{E} 6>$ & IE6> & J[ЕЧ IәмоT & $\varsigma: \angle 9 I$ & $9 \mathcal{E} \supset S 00 \mathrm{~L} 96 \mathrm{~S}$ \\
\hline $\mathrm{SSO}^{6} \mathrm{I} \mathrm{I}$ & $090^{\circ} \mathrm{I}>$ & $0 S 0^{\circ} \mathrm{I}>$ & J[еप Iәмот & $\nabla: \angle 9 \mathrm{I}$ & $689 \mathrm{~S} 00 \mathrm{~L} 96 \mathrm{~S}$ \\
\hline S60'I > & $0 \angle 0^{6} I>$ & OzI'I $>$ & I[Eप دәмоT & $\varepsilon: \angle 9 I$ & $889500 \mathrm{~L} 96 \mathrm{~S}$ \\
\hline 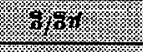 & 9. & 6\% & & \multicolumn{2}{|c|}{ 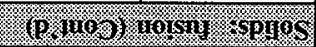 } \\
\hline 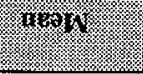 & 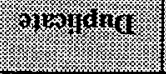 & 119010 & monion & 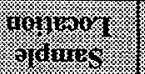 & 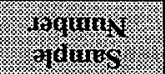 \\
\hline
\end{tabular}

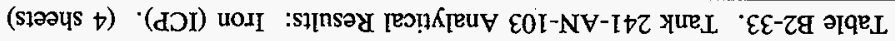


Table B2-34. Tank 241-AN-103 Analytical Results: Lanthanum (ICP). (4 sheets)

\begin{tabular}{|c|c|c|c|c|c|}
\hline Hono & Sampic & \% & iseniti & oyingro: & (3) \\
\hline \multicolumn{3}{|c|}{ 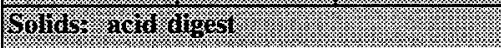 } & tgign & 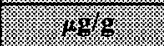 & ing \\
\hline S96T005609 & 166: 1 & Lower half & $<73.8$ & $<75.4$ & $<74.6$ \\
\hline S96T005570 & $166: 4$ & Lower half & $<89.7$ & $<89.7$ & $<89.7$ \\
\hline \$96T005615 & 166: 6 & Lower half & $<69.1$ & $<69.1$ & $<69.1$ \\
\hline \$96T005839 & 166: 7 & Lower half & $<101$ & $<102$ & $<101.5$ \\
\hline S96T005840 & 166: 8 & Lower half & $<98.4$ & $<98.3$ & $<98.35^{\text {QC:a }}$ \\
\hline S96T005575 & 166: 9 & Lower half & $<97.8$ & $<99.4$ & $<98.6$ \\
\hline S96T005571 & 166: 10 & Lower half & $<76.7$ & $<72.3$ & $<74.5$ \\
\hline S96T005616 & 166: 11 & Lower half & $<72.3$ & $<68$ & $<70.15$ \\
\hline S96T005617 & 166: 12 & Lower half & $<70.5$ & $<70.4$ & $<70.45$ \\
\hline S96T005716 & \multirow[t]{2}{*}{$166: 13$} & Upper half & $<96.9$ & $<98.1$ & $<97.5$ \\
\hline S96T005722 & & Lower half & $<103$ & $<103$ & $<103$ \\
\hline S96T005416 & \multirow[t]{2}{*}{$166: 15$} & Upper half & $<102$ & $<95.2$ & $<98.6$ \\
\hline S97T000459 & & Lower half & $<28.4$ & $<27.8$ & $<28.1$ \\
\hline S96T005624 & \multirow[t]{2}{*}{$166: 16$} & Upper half & $<105$ & $<108$ & $<106.5$ \\
\hline S96T005618 & & Lower half & $<70.9$ & $<72.8$ & $<71.85$ \\
\hline S96T005417 & \multirow[t]{2}{*}{ 166: 17} & Upper half & $<113$ & $<102$ & $<107.5$ \\
\hline \$96T005411 & & Lower half & $<95.1$ & $<88$ & $<91.55$ \\
\hline \$96T005717 & \multirow[t]{2}{*}{ 166: 18} & Upper half & $<104$ & $<101$ & $<102.5$ \\
\hline S96T005723 & & Lower half & $<101$ & $<99$ & $<100$ \\
\hline S96T005841 & \multirow[t]{2}{*}{ 166: 19} & Upper half & $<96.3$ & $<98.1$ & $<97.2^{\mathrm{QC:}: \mathrm{a}}$ \\
\hline S96T005842 & & Lower half & $<89$ & $<98.1$ & $<93.55^{\mathrm{QC}: \mathrm{a}}$ \\
\hline \$96T005708 & \multirow[t]{2}{*}{$167: 1$} & Upper half & $<89.6$ & $<103$ & $<96.3$ \\
\hline S96T005693 & & Lower haif & $<111$ & $<87.7$ & $<99.35^{\mathrm{QC}: \mathrm{c}}$ \\
\hline S96T005857 & $167: 2$ & Lower half & $<83.4$ & $<86.9$ & $<85.15$ \\
\hline S96T005694 & $167: 3$ & Lower half & $<89.9$ & $<93.6$ & $<91.75$ \\
\hline S96T005695 & $167: 4$ & Lower half & $<99$ & $<102$ & $<100.5$ \\
\hline \$96T005438 & $167: 5$ & Lower half & $<25.8$ & $<27.5$ & $<26.65$ \\
\hline S96T005450 & $167: 6$ & Lower half & $<27.7$ & $<26.1$ & $<26.9$ \\
\hline S96T005696 & $167: 7$ & Lower half & $<96$ & $<94$ & $<95$ \\
\hline
\end{tabular}




\begin{tabular}{|c|c|c|c|c|c|}
\hline$[\cdot 0 \varepsilon>$ & {$[\cdot 0 \mathcal{E}>$} & {$[0 \varepsilon>$} & 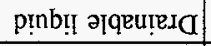 & $\angle: \angle 9 I$ & E89S00L96S \\
\hline$[\cdot 0 \varepsilon>$ & {$[\circ 0 \varepsilon>$} & {$[\cdot 0 \mathcal{E}>$} & pinb!n әqquụexa & $9: \angle 91$ & IZtS00L96S \\
\hline$I^{\circ} O \varepsilon>$ & {$[\cdot 0 \varepsilon>$} & $I \cdot 0 \varepsilon>$ & pṭb!̣ әlqeurela & $\varsigma: \angle 9 I$ & $0 z+500 \mathrm{~L} 96 \mathrm{~S}$ \\
\hline I. $0 \mathcal{E}>$ & {$[0 \varepsilon>$} & $I 0 \mathcal{}>$ & 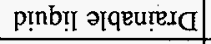 & $\varepsilon:\llcorner 9 I$ & EL9S00L96S \\
\hline I. $0 \varepsilon>$ & {$[\cdot 0 \varepsilon>$} & I $0 \varepsilon>$ & p!̣nb!̣ әqeug̣exa & $\tau: \angle 9 I$ & I98S00L96S \\
\hline$I^{\circ} 0 \varepsilon>$ & $I^{\circ} O \mathcal{E}>$ & $I 0 \mathcal{E}>$ & 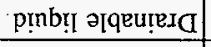 & ZI :99I & I09S00L96S \\
\hline$[\cdot 0 \varepsilon>$ & $I^{\circ} 0 \mathcal{E}>$ & $I^{\circ} 0 \mathcal{E}>$ & pịb!̣ әIqeựera & II :99I & 009S00L96S \\
\hline$[\cdot 0 z>$ & $1 \cdot 0 z>$ & $I^{\circ} 0 z>$ & pịb!l әqqu!esa & 0I :99I & 99SS00L96S \\
\hline$I^{\circ} 0 \varepsilon>$ & {$[0 \mathcal{E}>$} & {$[0 \mathcal{} 0 \mathcal{1}$} & p!̣nb!̣ ə]q̨eu!̣exa & $6: 991$ & ItSS00L96S \\
\hline$[\circ O E>$ & $\mathfrak{I} 0 \mathcal{E}>$ & $I^{\circ} 0 \mathcal{E}>$ & pụnb!l ə̣qqeu!esa & $8: 99$ I & LI8S00L96S \\
\hline I.OE $>$ & {$[\cdot 0 \varepsilon>$} & $I^{\circ} O \mathcal{E}>$ & p!̣b!l əॄqeu!eIC| & L:99I & 9โ8\$00L96S \\
\hline$I^{\circ} 0 \varepsilon>$ & {$[\cdot 0 \mathcal{E}>$} & $I 0 \mathcal{E}>$ & p!nb!̣ әqеu!eI & $9: 99 \mathrm{I}$ & $66 \$ \$ 00 L 96 \mathrm{~S}$ \\
\hline I.0z> & $I^{\circ} 0 z>$ & $1 \cdot 0 z>$ & p!̣b!̣ ә[qеurexa & $\nabla: 99 \Upsilon$ & $07 \$ S 00 L 96 S$ \\
\hline$[\cdot 0 z>$ & $I 0 Z>$ & {$[\cdot 0 z>$} & 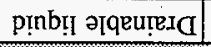 & $\mathcal{E}: 99 \mathrm{I}$ & 6ESS00L96S \\
\hline 1mort & yomon & maring: & & & spmor \\
\hline $98^{\circ} \angle Z>$ & $\underline{S} L Z>$ & $\tau \cdot 8 z>$ & әा!soduos prios & $99 \mathrm{I}$ ว10อ & $09+000 \mathrm{~L} L 6 \mathrm{~S}$ \\
\hline $8^{\prime} 26>$ & $596>$ & I' $68>$ & flष् ІәмоТ & \multirow[b]{2}{*}{$\angle I: \angle 9 I$} & $66 t S 00 \mathrm{~L} 96 \mathrm{~S}$ \\
\hline$s+6>$ & $\nabla L 6>$ & $9^{\circ}[6>$ & „[еप ıәdd & & $86 t 500 \mathrm{~L} 96 \mathrm{~S}$ \\
\hline ss:26> & $\nabla 6>$ & {$[\cdot 16>$} & IГеप Іәмот & \multirow[b]{2}{*}{$S[: \angle 9 I$} & $869 S 00 น 96 \mathrm{~S}$ \\
\hline$S \varepsilon \cdot 16>$ & $\varsigma 68>$ & $\tau \cdot \varepsilon 6>$ & Ileप ıədd $\cap$ & & OLLS00L96S \\
\hline $8 \cdot 87>$ & $\tau \cdot 6 z>$ & $7 \cdot 82>$ & I[Еप IәмOT & \multirow[b]{2}{*}{$D I: \angle 9 I$} & $6 \mathcal{E t S 0 0 L 9 6 S}$ \\
\hline $56.97>$ & $S: L Z>$ & $\nabla \cdot 9 z>$ & fleप Iәdd $\Omega$ & & SttS00L96S \\
\hline $8 \cdot 8 z>$ & $z \cdot 6 z>$ & $t^{\circ} 8 \tau>$ & J[Еч Іәмот & \multirow[b]{2}{*}{ ZI : $\angle 9 T$} & IStS00L96S \\
\hline St $\angle Z>$ & $\varepsilon \angle Z>$ & $9^{\circ} L z>$ & fleप Iədd & & $t \nabla t 500196 \mathrm{~S}$ \\
\hline $59.96>$ & $I * 6>$ & $z \cdot 66>$ & J[еप Іәмот] & \multirow[b]{2}{*}{ II : $\angle 9$ I } & L69S00L96S \\
\hline $59^{\circ} 16>$ & $\mathcal{E} \mathscr{E} 6>$ & $06>$ & I[Eप Iədd & & 60LS00L96S \\
\hline$S L \cdot S 6>$ & $\varsigma \angle 8>$ & $70 I>$ & J[Еч дәмоT & $6: \angle 9 I$ & 858500 L96S \\
\hline $686>$ & $6.66>$ & $6^{\circ} L 6>$ & І[Еप IәMOT. & $8: L 9 I$ & $\angle E L S 00 L 96 S$ \\
\hline 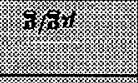 & 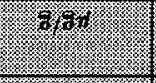 & (n) & & \multicolumn{2}{|c|}{ 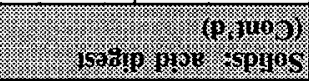 } \\
\hline 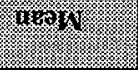 & 910010196 & 1) & 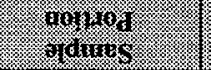 & Thom & thingurat \\
\hline
\end{tabular}

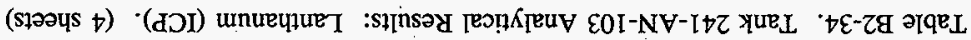


Table B2-34. Tank 241-AN-103 Analytical Results: Lanthanum (ICP). (4 sheets)

\begin{tabular}{|c|c|c|c|c|c|}
\hline 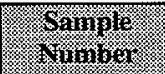 & \% & Somple & 3) & 9) & (1) \\
\hline \multicolumn{3}{|c|}{ 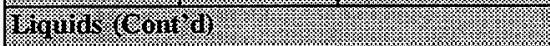 } & 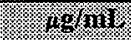 & (46) & 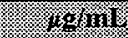 \\
\hline S96T005732 & $167: 8$ & Drainable liquid & $<30.1$ & $<30.1$ & $<30.1$ \\
\hline S96T005862 & 167: 9 & Drainable liquid & $<30.1$ & $<30.1$ & $<30.1$ \\
\hline S96T005650 & $167: 11$ & Drainable liquid & $<30.1$ & $<30.1$ & $<30.1$ \\
\hline S96T005991 & Core 166 & Liquid composite & $<60.1$ & $<60.1$ & $<60.1$ \\
\hline \multicolumn{2}{|c|}{ 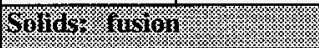 } & (3) & 15\% & (1) & (1) \\
\hline S96T005608 & 166: 1 & Lower half & $<1,050$ & $<1,030$ & $<1,040$ \\
\hline S96T005568 & $166: 4$ & Lower half & $<1,130$ & $<1,110$ & $<1,120$ \\
\hline S96T005611 & $166: 6$ & Lower half & $<1,050$ & $<1,070$ & $<1,060$ \\
\hline S96T005835 & 166: 7 & Lower half & $<1,050$ & $<1,060$ & $<1,055$ \\
\hline S96T005836 & 166: 8 & Lower half & $<981$ & $<1,030$ & $<1,005.5$ \\
\hline \$96T005574 & $166: 9$ & Lower half & $<896$ & $<970$ & $<933$ \\
\hline S96T005569 & $166: 10$ & Lower half & $<1,050$ & $<1,030$ & $<1,040$ \\
\hline \$96T005612 & 166: 11 & Lower half & $<987$ & $<1,020$ & $<1,003.5$ \\
\hline S96T005613 & $166: 12$ & Lower half & $<1,030$ & $<1,010$ & $<1,020$ \\
\hline S96T005714 & \multirow[t]{2}{*}{$166: 13$} & Upper half & $<1,010$ & $<1,050$ & $<1,030$ \\
\hline S96T005720 & & Lower half & $<1,010$ & $<1,010$ & $<1,010$ \\
\hline \$96T005414 & \multirow[t]{2}{*}{ 166: 15} & Upper half & $<228$ & $<233$ & $<230.5$ \\
\hline S96T005408 & & Lower half & $<240$ & $<232$ & $<236$ \\
\hline S96T005623 & \multirow[t]{2}{*}{ 166: 16} & Upper half & $<1,090$ & $<1,050$ & $<1,070$ \\
\hline \$96T005614 & & Lower half & $<1,020$ & $<1,000$ & $<1,010$ \\
\hline S96T005415 & \multirow[t]{2}{*}{ 166: 17} & Upper half & $<262$ & $<264$ & $<263$ \\
\hline S96T005409 & & Lower half & $<1,060$ & $<1,040$ & $<1,050$ \\
\hline \$96T005715 & \multirow[t]{2}{*}{ 166: 18} & Upper half & $<1,010$ & $<1,030$ & $<1,020$ \\
\hline S96T005721 & & Lower half & $<1,010$ & $<994$ & $<1,002$ \\
\hline S96T005837 & \multirow[t]{2}{*}{$166: 19$} & Upper half & $<1,040$ & $<1,050$ & $<1,045$ \\
\hline S96T005838 & & Lower half & $<978$ & $<982$ & $<980$ \\
\hline \$96T005705 & \multirow[t]{2}{*}{ 167: 1} & Upper half & $<994$ & $<1,000$ & $<997$ \\
\hline \$96T005687 & & Lower half & $<1,040$ & $<1,040$. & $<1,040$ \\
\hline S96T005855 & $167: 2$ & Lower half & $<1,010$ & $<988$ & $<999$ \\
\hline
\end{tabular}


HNF-SD-WM-ER-702 Rev. 0

Table B2-34. Tank 241-AN-103 Analyticai Results: Lanthanum (ICP). (4 sheets)

\begin{tabular}{|c|c|c|c|c|c|}
\hline Samper & I Sample? & Saripla. & ingsit. & Oolpiticite & $4 \operatorname{lin}$ \\
\hline \multicolumn{3}{|c|}{ Solids: fision Conitil: } & (2): & (3) & 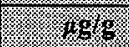 \\
\hline S96T005688 & 167: 3 & Lower half & $<1,120$ & $<1,070$ & $<1,095$ \\
\hline S96T005689 & $167: 4$ & Lower half & $<1,050$ & $<1,060$ & $<1,055$ \\
\hline S96T005436 & $167: 5$ & Lower half & $<931$ & $<939$ & $<935$ \\
\hline S96T005448 & $167: 6$ & Lower half & $<1,050$ & $<1,020$ & $<1,035$ \\
\hline S96T005690 & $167: 7$ & Lower half & $<982$ & $<1,090$ & $<1,036$ \\
\hline S96T005736 & $167: 8$ & Lower half & $<1,110$ & $<937$ & $<1,023.5$ \\
\hline S96T005856 & 167: 9 & Lower half & $<920$ & $<1,050$ & $<985$ \\
\hline S96T005706 & \multirow[t]{2}{*}{$167: 11$} & Upper half & $<933$ & $<1,110$ & $<1,021.5$ \\
\hline S96T005691 & & Lower half & $<925$ & $<921$ & $<923$ \\
\hline S96T005442 & \multirow[t]{2}{*}{$167: 12$} & Upper half & $<1,080$ & $<969$ & $<1,024.5$ \\
\hline S96T005449 & & Lower half & $<988$ & $<1,010$ & $<999$ \\
\hline S96T005443 & \multirow[t]{2}{*}{ 167: 14} & Upper half & $<1,100$ & $<1,050$ & $<1,075$ \\
\hline S96T005437 & & Lower half & $<1,030$ & $<1,010$ & $<1,020$ \\
\hline S96T005707 & \multirow[t]{2}{*}{ 167: 15} & Upper half & $<1,070$ & $<919$ & $<994.5$ \\
\hline S96T005692 & & Lower half & $<916$ & $<1,090$ & $<1,003$ \\
\hline S96T005496 & \multirow[t]{2}{*}{$167: 17$} & Upper half & $<1,060$ & $<1,050$ & $<1,055$ \\
\hline S96T005497 & & Lower half & $<1,050$ & $<1,050$ & $<1,050$ \\
\hline
\end{tabular}


Table B2-35. Tank 241-AN-103 Analytical Results: Lead (ICP). (4 sheets)

\begin{tabular}{|c|c|c|c|c|c|}
\hline simples & Sampres & 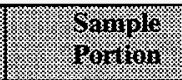 & Rerintis & Buntizotie & 1. Wean \\
\hline \multicolumn{2}{|c|}{ Solits narr Gigest } & & $8 \mathrm{~s} 40$ & . 896 & $4=10 \mathrm{~g} 8 \mathrm{~s}$ \\
\hline S96T005609. & 166: 1 & Lower half & $<148$ & $<151$ & $<149.5$ \\
\hline S96T005570 & $166: 4$ & Lower half & $<179$ & $<179$ & $<179$ \\
\hline S96T005615 & 166: 6 & Lower half & $<138$ & $<138$ & $<138$ \\
\hline S96T005839 & 166: 7 & Lower half & $<202$ & $<205$ & $<203.5$ \\
\hline S96T005840 & $166: 8$ & Lower half & $<197$ & $<197$ & $<197^{\text {QC:a }}$ \\
\hline S96T005575 & $166: 9$ & Lower half & $<196$ & $<199$ & $<197.5$ \\
\hline S96T005571 & $166: 10$ & Lower half & $<153$ & $<145$ & $<149$ \\
\hline S96T005616 & 166: 11 & Lower half & $<145$ & $<136$ & $<140.5$ \\
\hline S96T005617 & $166: 12$ & Lower half & $<141$ & $<141$ & $<141$ \\
\hline S96T005716 & \multirow{2}{*}{ 166: 13} & Upper half & $<194$ & $<196$ & $<195$ \\
\hline S96T005722 & & Lower half & $<206$ & $<206$ & $<206$ \\
\hline S96T005416 & \multirow[t]{2}{*}{$166: 15$} & Upper half & $<204$ & $<190$ & $<197$ \\
\hline S97T000459 & & Lower half & $<56.9$ & $<55.7$ & $<56.3$ \\
\hline S96T005624 & \multirow[t]{2}{*}{ 166: 16} & Upper half & $<210$ & $<215$ & $<212.5$ \\
\hline S96T005618 & & Lower half & $<142$ & $<146$ & $<144$ \\
\hline S96T005417 & \multirow[t]{2}{*}{$166: 17$} & Upper half & $<225$ & $<205$ & $<215$ \\
\hline S96T005411 & & Lower half & $<190$ & $<176$ & $<183$ \\
\hline S96T005717 & \multirow[t]{2}{*}{$166: 18$} & Upper half & $<208$ & $<202$ & $<205$ \\
\hline S96T005723 & & Lower half & $<202$ & $<198$ & $<200$ \\
\hline S96T005841 & \multirow[t]{2}{*}{ 166: 19} & Upper half & $<193$ & 1,180 & $<686.5^{\mathrm{QC}: \mathrm{a}, \mathrm{c}}$ \\
\hline S96T005842 & & Lower half & $<178$ & $<196$ & $<187^{\mathrm{QC}: a}$ \\
\hline S96T005708 & \multirow[t]{2}{*}{$167: 1$} & Upper half & $<179$ & $<205$ & $<192$ \\
\hline \begin{tabular}{|l|} 
S96T005693 \\
\end{tabular} & & Lower half & $<222$ & $<175$ & $<198.5^{\mathrm{QC:C}}$ \\
\hline S96T005857 & $167: 2$ & Lower half & $<167$ & $<174$ & $<170.5^{\mathrm{QC}: \mathrm{a}}$ \\
\hline S96T005694 & $167: 3$ & Lower half & $<180$ & $<187$ & $<183.5$ \\
\hline S96T005695 & $167: 4$ & Lower half & $<198$ & $<204$ & $<201$ \\
\hline S96T005438 & $167: 5$ & Lower half & 60.9 & 63.2 & $62.05^{\mathrm{QC}: \mathrm{a}}$ \\
\hline S96T005450 & $167: 6$ & Lower half & $<55.3$ & 62.2 & $<58.75^{\mathrm{QC}: a}$ \\
\hline
\end{tabular}


Table B2-35. Tank 241-AN-103 Analytical Results: Lead (ICP). (4 sheets)

\begin{tabular}{|c|c|c|c|c|c|}
\hline 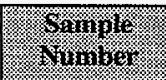 & Samplo & $\begin{array}{l}\text { Sarripie } \\
\text { Pertion }\end{array}$ & Rirsuite & 3ullicart & 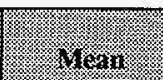 \\
\hline \multicolumn{2}{|c|}{ Solids: adil aygest: } & & $\sqrt{1} \cdot 1.96$ & (1) & $\sqrt{1.085}$ \\
\hline S96T005696 & $167: 7$ & Lower half & $<192$ & $<188$ & $<190$ \\
\hline S96T005737 & $167: 8$ & Lower half & $<196$ & $<200$ & $<198$ \\
\hline S96T005858 & $167: 9$ & Lower half & $<208$ & $<175$ & $<191.5^{\mathrm{QC:a}}$ \\
\hline S96T005709 & \multirow[t]{2}{*}{ 167: 11} & Upper half & $<180$ & 232 & $<206^{\text {QC:c }}$ \\
\hline S96T005697 & & Lower half & $<198$ & $<188$ & $<193$ \\
\hline S96T005444 & \multirow[t]{2}{*}{$167: 12$} & Upper half & 63.6 & 68.9 & $66.25^{\mathrm{QC:} a}$ \\
\hline S96T005451 & & Lower half & $<56.8$ & $<58.4$ & $<57.6^{\text {QC:a }}$ \\
\hline S96T005445 & \multirow[t]{2}{*}{$167: 14$} & Upper half & $<52.8$ & $<55.1$ & $<53.95^{\mathrm{QC}: a}$ \\
\hline S96T005439 & & Lower half & $<56.8$ & $<58.4$ & $<57.6^{\mathrm{QC:a}}$ \\
\hline S96T005710 & \multirow[t]{2}{*}{$167: 15$} & Upper half & $<186$ & $<179$ & $<182.5$ \\
\hline S96T005698 & & Lower half & $<182$ & $<188$ & $<185$ \\
\hline S96T005498 & \multirow[t]{2}{*}{$167: 17$} & Upper half & $<183$ & $<195$ & $<189$ \\
\hline S96T005499 & & Lower half & $<178$ & $<193$ & $<185.5$ \\
\hline S97T000460 & Core 166 & Solid composite & $<56.3$ & $<55.1$ & $<55.7$ \\
\hline \multicolumn{2}{|l|}{ Hojurds } & & Verin & $18 \mathrm{grm} \mathrm{mi}$ & . \\
\hline S96T005539 & $166: 3$ & Drainable liquid & 141 & 133 & 137 \\
\hline S96T005540 & $166: 4$ & Drainable liquid & 136 & 140 & 138 \\
\hline S96T005599 & $166: 6$ & Drainable liquid & 165 & 160 & 162.5 \\
\hline S96T005816 & $166: 7$ & Drainable liquid & 128 & 140 & 134 \\
\hline S96T005817 & $166: 8$ & Drainable liquid & 130 & 152 & 141 \\
\hline S96T005541 & $166: 9$ & Drainable liquid & 138 & 130 & 134 \\
\hline S96T005566 & 166: 10 & Drainable liquid & 147 & 157 & 152 \\
\hline S96T005600 & 166: 11 & Drainable liquid & 184 & 149 & $166.5^{\mathrm{QC}: \mathrm{c}}$ \\
\hline S96T005601 & $166: 12$ & Drainable liquid & 142 & 146 & 144 \\
\hline S96T005861 & $167: 2$ & Drainable liquid & 132 & 128 & 130 \\
\hline S96T005673 & $167: 3$ & Drainable liquid & 139 & 147 & 143 \\
\hline S96T005420 & $167: 5$ & Drainable liquid & 131 & 144 & 137.5 \\
\hline S96T005421 & $167: 6$ & Drainable liquid & 142 & 145 & 143.5 \\
\hline
\end{tabular}


Table B2-35. Tank 241-AN-103 Analytical Results: Lead (ICP). (4 sheets)

\begin{tabular}{|c|c|c|c|c|c|}
\hline $\begin{array}{l}\text { Sample: } \\
\text { Nimingt: }\end{array}$ & Sompiler & $\begin{array}{l}\text { Sample } \\
\text { Sortion }\end{array}$ & gesinit. & bintilate & (1) \\
\hline \multicolumn{3}{|c|}{ 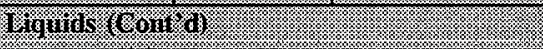 } & (igginil: & $48 \mathrm{~g} \cdot 316$ & 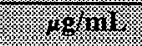 \\
\hline S96T005683 & 167: 7 & Drainable liquid & 135 & 142 & 138.5 \\
\hline S96T005732 & 167: 8 & Drainable liquid & 143 & 141 & 142 \\
\hline S96T005862 & $167: 9$ & Drainable liquid & 136 & 142 & 139 \\
\hline S96T005650 & 167: 11 & Drainable liquid & 143 & 139 & 141 \\
\hline S96T005991 & Core 166 & Liquid composite & 156 & $<120$ & $<138^{\mathrm{QC:e}}$ \\
\hline \multicolumn{3}{|c|}{ Solliter wition } & 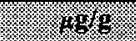 & 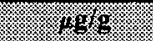 & 1998 \\
\hline S96T005608 & $166: 1$ & Lower half & $<2,100$ & $<2,060$ & $<2,080$ \\
\hline S96T005568 & 166: 4 & Lower half & $<2,270$ & $<2,220$. & $<2,245$ \\
\hline S96T005611 & 166: 6 & Lower half & $<2,100$ & $<2,140$ & $<2,120$ \\
\hline S96T005835 & 166: 7 & Lower half & $<2,100$ & $<2,120$ & $<2,110$ \\
\hline S96T005836 & $166: 8$ & Lower half & $<1,960$ & $<2,060$ & $<2,010$ \\
\hline \$96T005574 & $166: 9$ & Lower half & $<1,790$ & $<1,940$ & $<1,865$ \\
\hline S96T005569 & 166: 10 & Lower half & $<2,090$ & $<2,070$ & $<2,080$ \\
\hline S96T005612 & 166: 11 & Lower half & $<1,970$ & $<2,040$ & $<2,005$ \\
\hline S96T005613 & 166: 12 & Lower half & $<2,050$ & $<2,020$ & $<2,035$ \\
\hline S96T005714 & \multirow[t]{2}{*}{ 166: 13} & Upper half & $<2,010$ & $<2,110$ & $<2,060$ \\
\hline S96T005720 & & Lower half & $<2,030$ & $<2,010$ & $<2,020$ \\
\hline S96T005414 & \multirow[t]{2}{*}{$166: 15$} & Upper half & $<456$ & $<466$ & $<461$ \\
\hline S96T005408 & & Lower half & $<480$ & $<463$ & $<471.5$ \\
\hline S96T005623 & \multirow[t]{2}{*}{$166: 16$} & Upper half & $<2,180$ & $<2,100$ & $<2,140$ \\
\hline S96T005614 & & Lower half & $<2,040$ & $<2,010$ & $<2,025$ \\
\hline S96T005415 & \multirow[t]{2}{*}{ 166: 17} & Upper half & $<523$ & $<528$ & $<525.5$ \\
\hline S96T005409 & & Lower half & $<2,110$ & $<2,080$ & $<2,095$ \\
\hline S96T005715 & \multirow[t]{2}{*}{$166: 18$} & Upper half & $<2,010$ & $<2,060$ & $<2,035$ \\
\hline S96T005721 & & Lower half & $<2,030$ & $<1,990$ & $<2,010$ \\
\hline S96T005837 & \multirow[t]{2}{*}{$166: 19$} & Upper half & $<2,070$ & $<2,100$ & $<2,085$ \\
\hline S96T005838 & & Lower half & $<1,960$ & $<1,960$ & $<1,960$ \\
\hline S961005705 & \multirow[t]{2}{*}{$167: 1$} & Upper half & $<1,990$ & 2,880 & $<2,435^{\mathrm{QC}: \mathrm{e}}$ \\
\hline S96T005687 & & Lower half & $<2,080$ & $<2,090$ & $<2,085$ \\
\hline
\end{tabular}


Table B2-35. Tank 241-AN-103 Analytical Results: Lead. (ICP). (4 sheets)

\begin{tabular}{|c|c|c|c|c|c|}
\hline $\begin{array}{l}\text { Sample } \\
\text { Thiminer }\end{array}$ & Sample & Samiple & Resuils & bivinicate & Wear \\
\hline \multicolumn{3}{|c|}{ 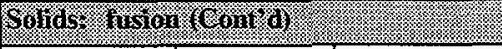 } & (1989) & 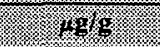 & 48 \\
\hline S96T005855 & $167: 2$ & Lower half & $<2,020$ & $<1,980$ & $<2,000$ \\
\hline \$96T005688 & $167: 3$ & Lower half & $<2,250$ & $<2,130$ & $<2,190$ \\
\hline S96T005689 & $167: 4$ & Lower half & $<2,100$ & $<2,110$ & $<2,105$ \\
\hline S96T005436 & $167: 5$ & Lower half & $<1,860$ & $<1,880$ & $<1,870$ \\
\hline S96T005448 & $167: 6$ & Lower half & $<2,110$ & $<2,040$ & $<2,075$ \\
\hline S96T005690 & 167: 7 & Lower half & $<1,960$ & $<2,180$ & $<2,070$ \\
\hline S96T005736 & $167: 8$ & Lower half & $<2,210$ & $<1,870$ & $<2,040$ \\
\hline S96T005856 & $167: 9$ & Lower half & $<1,840$ & $<2,110$ & $<1,975$ \\
\hline S96T005706 & \multirow[t]{2}{*}{$167: 11$} & Upper half & $<1,870$ & $<2,220$ & $<2,045$ \\
\hline S96T005691 & & Lower half & $<1,850$ & $<1,840$ & $<1,845$ \\
\hline S96T005442 & \multirow[t]{2}{*}{$167: 12$} & Upper half & $<2,160$ & $<1,940^{\circ}$ & $<2,050$ \\
\hline S96T005449 & & Lower half & $<1,980$ & $<2,020$ & $<2,000$ \\
\hline S96T005443 & \multirow[t]{2}{*}{$167: 14$} & Upper half & $<2,200$ & $<2,110$ & $<2,155$ \\
\hline S96T005437 & & Lower half & $<2,050$ & $<2,030$ & $<2,040$ \\
\hline S96T005707 & \multirow[t]{2}{*}{$167: 15$} & Upper half & $<2,130$ & $<1,840$ & $<1,985$ \\
\hline S96T005692 & & Lower half & $<1,830$ & $<2,180$ & $<2,005$ \\
\hline S96T005496 & \multirow[t]{2}{*}{$167: 17$} & Upper half & $<2,120$ & $<2,100$ & $<2,110$ \\
\hline S96T005497 & & Lower half & $<2,100$ & $<2,110$ & $<2,105$ \\
\hline
\end{tabular}




\begin{tabular}{|c|c|c|c|c|c|}
\hline$S I L Z$ & $\angle Z$ & $\varepsilon L Z$ & IIEप Хәмот & $9: \angle 9 I$ & $0 S t S 00 \mathrm{~L} 96 \mathrm{~S}$ \\
\hline$\supset: \supset 0 \mathcal{E} \subseteq \mathcal{E}$ & 562 & I It & I[एप ऽәмот & $S: \angle 9 I$ & $8 \mathcal{E}+500 \mathrm{~L} 96 \mathrm{~S}$ \\
\hline $9^{\circ} \mathrm{ss}$ & $\nabla 6 S$ & 8.15 & Iец ләмот] & $\downarrow: \angle 9 I$ & \$69\$00L96S \\
\hline $0: 00 \varepsilon 9 s$ & $t^{\circ} S t$ & $z \cdot<9$ & 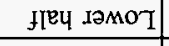 & $\varepsilon: \angle 9 I$ & $\nabla 69 \$ 00 L 96 \mathrm{~S}$ \\
\hline SLOI & sOI & $01 \mathrm{I}$ & Ј[Еप Іәмот & $\tau: \angle 9 I$ & LS8S00L96S \\
\hline$\$ 8.6 \mathrm{I}>$ & $S L I>$ & $\tau \cdot z z>$ & Ј[ЕЧ ІәмоТ] & \multirow[b]{2}{*}{$I: \angle 9 \mathrm{I}$} & E69\$00L96S \\
\hline$z \cdot 6 \mathrm{I}>$ & $s^{\circ} 0 z>$ & $6 \angle \mathrm{L}>$ & $y^{\mathrm{EU}} \mathrm{I \partial d \textrm {d }} \Omega$ & & $80 \angle S 00 L 96 \mathrm{~S}$ \\
\hline$: 00 \angle 8 I>$ & $9.61>$ & $8^{\circ} \mathrm{LI}>$ & $f[\mathrm{CL}$ IOMOT] & \multirow[b]{2}{*}{$6 \mathrm{I}: 99 \mathrm{I}$} & $Z \downarrow 8500 \mathrm{~L} 96 \mathrm{~S}$ \\
\hline :00St $6 I>$ & $9 \cdot 61>$ & $\varepsilon \cdot 6 I>$ & Ileप Iədd $\Omega$ & & It8S00J96S \\
\hline $0 z>$ & $8.6 I>$ & $z \circ 0 z>$ & 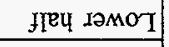 & \multirow[b]{2}{*}{ 8I:99I } & EZLSO0L96S \\
\hline 5962 & 162 & $z 0 \mathcal{E}$ & Jleप Iədd $\Omega$ & & LILS00L96S \\
\hline$\varepsilon^{\prime} 8 I>$ & $9^{\circ} L I>$ & $6 I>$ & Iеप Іәмот & \multirow[b]{2}{*}{ LI :991 } & I It $500 \mathrm{~L} 96 \mathrm{~S}$ \\
\hline$s^{\prime} \mid z>$ & $s^{\circ} 0 z>$ & $s \cdot z z>$ & 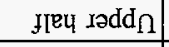 & & $L I t S 00 L 96 S$ \\
\hline$\forall \bullet I>$ & $9^{\circ}+[>$ & $Z \cdot \nabla I>$ & І[еч дәмот & \multirow[b]{2}{*}{$9 \mathrm{I}: 99 \mathrm{I}$} & $819500 \mathrm{~J} 96 \mathrm{~S}$ \\
\hline$s Z \cdot I Z>$ & $S^{\prime} I Z>$ & $I Z>$ & 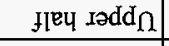 & & $\triangleright 29 \$ 00196 \mathrm{~S}$ \\
\hline$\varepsilon 9^{\circ} \varsigma>$ & $\angle S S>$ & $69^{\circ} \mathrm{S}>$ & f[Рч Іәмот] & \multirow[b]{2}{*}{ SI :99I } & $6 S+000 \mathrm{~L} L 6 \mathrm{~S}$ \\
\hline $0: 008^{\circ} \varepsilon Z$ & $9.6 \mathrm{I}$ & 82 & freप ıədd $\cap$ & & 9 ItS00L96S \\
\hline $9^{\circ} 0 z>$ & $9^{\circ} 0 z>$ & $9^{\circ} 0 z>$ & Ғ[Еप IәMOT & \multirow[b]{2}{*}{ EI :99I } & $Z Z L S 00 L 96 \mathrm{~S}$ \\
\hline $56 \mathrm{I}>$ & $96 \mathrm{I}>$ & $\nabla 6 I>$ & Ј[Еч Iədd $\Omega$ & & $9[\angle 500 L 96 \mathrm{~S}$ \\
\hline$[+I>$ & $I \nabla I>$ & $I \triangleright I>$ & fएप Іәмот & ZI :99I & LI9S00L96S \\
\hline $50^{\circ} \nabla \mathrm{I}>$ & $9^{\circ} \varepsilon \mathrm{I}>$ & $S \nabla I>$ & ІाРप IәMOT & I I :99I & 9I9S00L96S \\
\hline $6^{\circ}+[>$ & $S+D I>$ & $\varepsilon S I>$ & IIPU IวMOT & OI :99I & ILSSO0L96S \\
\hline$S L \cdot 6 I>$ & $6.6 \mathrm{I}>$ & $9^{\circ} 6 \mathrm{I}>$ & fाеप Іәмот & $6: 991$ & SLSS00L96S \\
\hline $8: 00<6 I>$ & $L 6 \mathrm{I}>$ & $L 6 I>$ & IIEบ Iәмот & $8: 99 I$ & $0+8500 \mathrm{~L} 96 \mathrm{~S}$ \\
\hline$\subseteq \mathcal{E} 0 Z>$ & $\varsigma^{\prime} 0 z>$ & $z \cdot 0 z>$ & Ifखप IวMOT & $L: 99 \mathrm{I}$ & 6E8S00L96S \\
\hline $8 \mathrm{I}$ & $2 \cdot 8 \mathrm{I}$ & $8^{\circ} \mathrm{LI}$ & I[एU IәMOT & $9: 99 \mathrm{I}$ & SI9S00L96S \\
\hline $6 \angle I>$ & $6 \angle I>$ & $6 \angle I>$ & $\exists[E 4$ IOMOT] & $\downarrow: 99 \mathrm{I}$ & 0LSS00L96S \\
\hline$S 6^{\circ}+\mathrm{I}>$ & $I \cdot S I>$ & $8 \div[>$ & flеч Іәмо & I :99I] & $609 S 00 L 96 S$ \\
\hline \% & 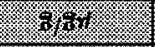 & (6) & & \multicolumn{2}{|c|}{ 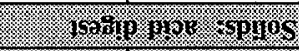 } \\
\hline 16\% & 19019618 & $1196 \%$ & Whomo & Mongrom & Horingry \\
\hline
\end{tabular}

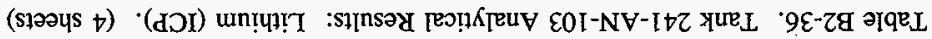




\begin{tabular}{|c|c|c|c|c|c|}
\hline \$S.6I & $\mathcal{E} 6 \mathrm{I}$ & $86 I$ & pụb!̣ əॄqeurera & $9: \angle 9 \mathrm{I}$ & IZtS00L96S \\
\hline$I L I$ & $6^{\circ} 9 \mathrm{I}$ & $\varepsilon L I$ & p!nbII ગ̨qeuteIণ & $S: \angle 9 I$ & $0 Z+500 L 96 \mathrm{~S}$ \\
\hline$\$ 9^{\circ} \mathrm{LI}$ & $S * I$ & $8 \angle I$ & p!nb!̣ ə[qeuाesa & $\varepsilon: \angle 9 I$ & EL9S00L96S \\
\hline$\nabla^{\circ} L I$ & $I^{\circ} \angle \mathrm{I}$ & $L L I$ & p!nb!̣ әqॄu!̣eı & $Z: \angle 9 \mathrm{I}$ & $198 \mathrm{~S} 00 \mathrm{~L} 96 \mathrm{~S}$ \\
\hline 81 & 81 & $8 \mathrm{I}$ & pinbu I әqеuाexa & ZI :99I & I09500L96S \\
\hline$\angle 6 \mathrm{I}$ & $9 \cdot 6 \mathrm{I}$ & $8.6 \mathrm{I}$ & ptnb!̣ әqеuțeI & I I :99I & 009500L96S \\
\hline $90^{\circ} \mathrm{LI}$ & 6.91 & $z<I$ & pinb!r әৃqeurexa & 0I:99I & 99SS00L $96 \mathrm{~S}$ \\
\hline $98^{\circ}+\mathrm{I}$ & $\subseteq \mathrm{I}$ & $L D I$ & 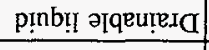 & $6: 991$ & ItSS00L96S \\
\hline $8 \cdot \pi I$ & $\varepsilon \varepsilon I$ & $\varepsilon \cdot Z I$ & p!nb!̣ әqqeụeıa & $8: 991$ & LI8S00L96S \\
\hline$S L L^{\circ} \mathrm{II}$ & $\varepsilon \mathrm{E}$ & $\varsigma^{*} Z \mathrm{II}$ & 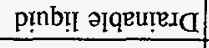 & $L: 99 I$ & $918 \mathrm{~S} 00 \mathrm{~L} 96 \mathrm{~S}$ \\
\hline$\$ 9^{\circ} 0 \varepsilon$ & $z \cdot 0 \mathcal{E}$ & I'IE & pinbri әqquuाera & $9: 991$ & 66S\$00L96S \\
\hline ssol & $6.0 \mathrm{I}$ & 201 & ptnbri әqеu!era & $t: 991$ & $07 \$ S 00196 \mathrm{~S}$ \\
\hline$S L L I$ & $9 \angle I$ & $6 \angle I$ & 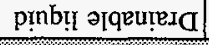 & $\mathcal{E}: 99 \mathrm{I}$ & 6ESS00L96S \\
\hline (3) & 10.6\% & (2) & & & (2) \\
\hline$\varepsilon \neq \tau$ & 92 & $9^{\circ} z 2$ & ว!!soduos p!̣os & 991 210ग & $09+000 L \angle 6 S$ \\
\hline SE:06 & $\tau \cdot 96$ & $\varsigma^{*}+8$ & F[ЕЧ $\triangle 2 M O T$ & \multirow[b]{2}{*}{$\angle I: \angle 9 I$} & $66+\mathrm{S} 00 \mathrm{~L} 96 \mathrm{~S}$ \\
\hline ZIS & $80 \mathrm{~s}$ & $9 \mathrm{IS}$ & Jॉएц Iədd $_{\Omega}$ & & $86+500 \mathrm{~L} 96 \mathrm{~S}$ \\
\hline $\mathrm{s}^{\circ} 8 \mathrm{I}>$ & $8 \cdot 8 I>$ & $\tau \cdot 8 I>$ & f[ЕЧ IәмоТ & \multirow[b]{2}{*}{$S I: \angle 9 I$} & $869 S 00 \mathrm{~L} 96 \mathrm{~S}$ \\
\hline$\varsigma 0<I$ & $\nabla L I$ & $\angle 9 \mathrm{I}$ & J[एч & & OILSOOL96S \\
\hline $569 \varepsilon$ & 100 & $8^{\circ} \varepsilon \varepsilon$ & Jleप IәMOT & \multirow[b]{2}{*}{$\nabla I: \angle 9 I$} & $6 \mathcal{6 \$ 0 0 0 L 9 6 S}$ \\
\hline $\mathfrak{S} 6 \mathcal{E}^{\circ} \mathfrak{S}>$ & $I S S>$ & $8 \tau^{\circ} \varsigma>$ & $f[E 4$ rədd $\Omega$ & & Stt\$00L96S \\
\hline $9 L^{\circ} \mathrm{S}>$ & $58 \cdot s>$ & $89^{\circ} \mathrm{S}>$ & Fеप Іәмот & \multirow[b]{2}{*}{ ZI : $\angle 9 I$} & IStS00L96S \\
\hline$\varepsilon \cdot I$ & Z'II & $D^{\circ} I I$ & $\mathrm{~J}[\mathrm{Y}$. & & $D t 7 \$ 00 \mathrm{~L} 96 \mathrm{~S}$ \\
\hline$\varepsilon 6 I>$ & $8.8 I>$ & $8^{\circ} 6 I>$ & I"Р4 IәMOT & \multirow[b]{2}{*}{ II $: \angle 9 \mathrm{I}$} & L69S00196S \\
\hline SE $8 I>$ & $\angle 8 I>$ & $8 I>$ & I[एч Iədd $\Omega$ & & $60 \angle S 00 L 96 \mathrm{~S}$ \\
\hline$S I 6 I>$ & $S L I>$ & $8^{\circ} 0 z>$ & Іाеप & $6: \angle 9 I$ & $8 \$ 8 \$ 00 L 96 S$ \\
\hline$L \nabla \varepsilon$ & $8^{\circ} 9 \mathcal{E}$ & $9^{\circ} 2 \varepsilon$ & I[ЕЧ ХәMOT] & $8: \angle 9 \mathrm{I}$ & $\angle E L S 00 L 96 \mathrm{~S}$ \\
\hline$\nabla^{\circ} \varepsilon \tau$ & $9^{*}+\tau$ & $\tau \cdot z z$ & \lrcorner$[8, \perp ә M O T]$ & $L: \angle 9 I$ & 969500L96S \\
\hline (6) & (n) & \% & & \multicolumn{2}{|c|}{ (5) } \\
\hline 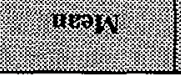 & 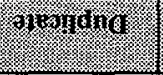 & 119. & 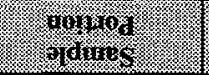 & Promo & Mglingur \\
\hline
\end{tabular}

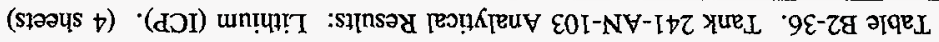


HNF-SD-WM-ER-702 Rev, 0

Table B2-36. Tank 241-AN-103 Analytical Results: Lithium (ICP). (4 sheets)

\begin{tabular}{|c|c|c|c|c|c|}
\hline Hinger & Sompiction & 策anple & Rssurn & oringer & ligingin \\
\hline \multicolumn{3}{|c|}{ 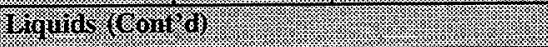 } & 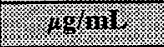 & 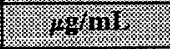 & (2) \\
\hline \$96T005683 & $167: 7$ & Drainable liquid & 40.1 & 40.1 & 40.1 \\
\hline S96T005732 & $167: 8$ & Drainable liquid & 27.3 & 26.6 & 26.95 \\
\hline S96T005862 & 167:9 & Drainable liquid & 27.9 & 29.1 & 28.5 \\
\hline S96T005650 & $167: 11$ & Drainable liquid & 62.7 & 63.1 & 62.9 \\
\hline S96T005991 & Core 166 & Liquid composite & 15.3 & 15 & 15.15 \\
\hline \multicolumn{2}{|c|}{ Solid } & & 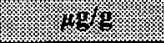 & $14+9$ & 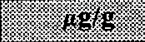 \\
\hline S96T005608 & 166: 1 & Lower half & $<210$ & $<206$ & $<208$ \\
\hline \$96T005568 & 166: 4 & Lower half & $<227$ & $<222$ & $<224.5$ \\
\hline S96T005611 & 166: 6 & Lower half & $<210$ & $<214$ & $<212$ \\
\hline S96T005835 & 166: 7 & Lower half & $<210$ & $<212$ & $<211$ \\
\hline S96T005836 & 166: 8 & Lower half & $<196$ & $<206$ & $<201$ \\
\hline S96T005574 & 166: 9 & Lower half & $<179$ & $<194$ & $<186.5$ \\
\hline S96T005569 & $166: 10$ & Lower half & $<209$ & $<207$ & $<208$ \\
\hline S96T005612 & 166: 11 & Lower half & $<197$ & $<204$ & $<200.5$ \\
\hline S96T005613 & $166: 12$ & Lower half & $<205$ & $<202$ & $<203.5$ \\
\hline S96T005714 & \multirow[t]{2}{*}{$166: 13$} & Upper half & $<201$ & $<211$ & $<206$ \\
\hline \$96T005720 & & Lower half & $<203$ & $<201$ & $<202$ \\
\hline S96T005414 & \multirow[t]{2}{*}{$166: 15$} & Upper half & $<45.6$ & $<46.6$ & $<46.1$ \\
\hline S96T005408 & & Lower half & $<48$ & $<46.3$ & $<47.15$ \\
\hline \$96T005623 & \multirow[t]{2}{*}{$166: 16$} & Upper half & $<218$ & $<210$ & $<214$ \\
\hline S96T005614 & & Lower half & $<204$ & $<201$ & $<202.5$ \\
\hline S96T005415 & \multirow[t]{2}{*}{$166: 17$} & Upper half & $<52.3$ & $<52.8$ & $<52.55$ \\
\hline S96T005409 & & Lower half & $<211$ & $<208$ & $<209.5$ \\
\hline S96T005715 & \multirow[t]{2}{*}{$166: 18$} & Upper half & 377 & 391 & 384 \\
\hline S96T005721 & & Lower half & $<203$ & $<199$ & $<201$ \\
\hline S96T005837 & \multirow[t]{2}{*}{ 166: 19} & Upper half & $<207$ & $<210$ & $<208.5$ \\
\hline S96T005838 & & Lower half & $<196$ & $<196$ & $<196$ \\
\hline S96T005705 & \multirow[t]{2}{*}{ 167: 1} & Upper half & $<199$ & $<200$ & $<199.5$ \\
\hline S96T005687 & & Lower half & $<208$ & $<209$ & $<208.5$ \\
\hline
\end{tabular}




\begin{tabular}{|c|c|c|c|c|c|}
\hline$S^{\circ} 0 I Z>$ & II $Z>$ & $0 ! z>$ & ЈГЕЧ ІәмоТ] & & $\angle 6 \$ S 00 L 96 S$ \\
\hline $0: 00 \leqq L L$ & EE8 & $\angle 6 S$ & Ifeप Iədd & $\angle I: \angle 9 I$ & $96 t \$ 00 L 96 \mathrm{~S}$ \\
\hline$s 00 z>$ & $8 I Z>$ & $\varepsilon 8 \mathrm{I}>$ & flЕY Iәмот & \multirow[b]{2}{*}{ SI : $\angle 9 I$} & Z69500L96S \\
\hline 5292 & $6 S \mathcal{Z}$ & 992 & I[एप Jədd $\Omega$ & & $\angle 0 \angle 500 L 96 \mathrm{~S}$ \\
\hline$t 0 z>$ & $\varepsilon 0 z>$ & soz $>$ & IfЕY IәмоT & \multirow[b]{2}{*}{$D I: \angle 9 I$} & LEtS00.L96S \\
\hline$s^{\prime} s I Z>$ & IIZ> & $0 z z>$ & Jleप $x \partial d d_{n}$ & & Et†S00L96S \\
\hline $00 z>$ & $z 0 z>$ & $86 I>$ & Ileu دәмот & \multirow[b]{2}{*}{$2 I: \angle 9 I$} & $6 t t \$ 00 L 96 \mathrm{~S}$ \\
\hline $\mathrm{s} 0 \tau>$ & $76 \mathrm{I}>$ & 9 IZ> & fleप̣ ıədd $\Omega$ & & $\tau \succsim t S 00 L 96 \mathrm{~S}$ \\
\hline$\varsigma^{\circ}+8 \mathrm{I}>$ & $\$ 8 \mathrm{I}>$ & $\$ 8 I>$ & IEеч хәмот & \multirow[b]{2}{*}{ II :L9I } & I69500L96S \\
\hline$s^{*}+0 z>$ & $z z z>$ & $\angle 8 I>$ & Ilשप Iәdd & & 90LS00L96S \\
\hline$S \angle 6 I>$ & $I I Z>$ & $78 I>$ & I[Eบ IәмоT & $6: \angle 91$ & $9 S 8 S 00 L 96 \mathrm{~S}$ \\
\hline$t 0 z>$ & $\angle 8 I>$ & $I Z Z>$ & IlЕप IәмоT & $8: \angle 9 I$ & $9 \mathcal{E} L 500 L 96 \mathrm{~S}$ \\
\hline$\angle 0 Z>$ & $8 I Z>$ & $96 I>$ & fleप دәмо̆ & $\angle: \angle 9 I$ & 069S00L96S \\
\hline$s^{\circ} \angle 0 z>$ & $t 0 z>$ & $I I Z>$ & flEY IәмOT & $9: \angle 9 I$ & $8 \pitchfork t 500 L 96 \mathrm{~S}$ \\
\hline$\angle 8 I>$ & $88 \mathrm{I}>$ & $98 \mathrm{I}>$ & І[ЕЧ .Іәмо̆Т] & $s: \angle 9 I$ & $9 \mathcal{E} \downarrow$ S00L96S \\
\hline $5012>$ & IIZ> & $0 I z>$ & I[ЕЧ IәмоТ & $t: \angle 9 I$ & $689500 \mathrm{~L} 96 \mathrm{~S}$ \\
\hline $6 \mathrm{I} Z>$ & $\varepsilon I Z>$ & $\$ z Z>$ & ІІеप Іәмот & $\varepsilon: \angle 9 I$ & $889500 L 96 \mathrm{~S}$ \\
\hline $00 z>$ & $86 I>$ & $z 0 z>$ & J[EY دәMOT & $z: \angle 9 I$ & \$S8S00L96S \\
\hline (8) & 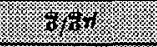 & $4 \%$ & & & $351 \%$ sporos \\
\hline \%or & \% & 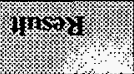 & 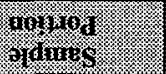 & 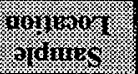 & 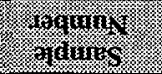 \\
\hline
\end{tabular}

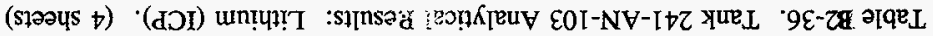


Table B2-37. Tank 241-AN-103 Analytical Results: Manganese (ICP). (4 sheets)

\begin{tabular}{|c|c|c|c|c|c|}
\hline 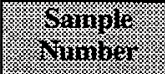 & \%oran & 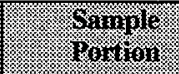 & Whanl & Buplirate & ing \\
\hline \multicolumn{3}{|c|}{ 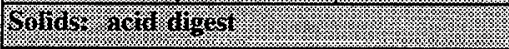 } & (1) & (1) & 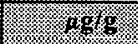 \\
\hline S96T005609 & 166: 1 & Lower half & $<14.8$ & $<15.1$ & $<14.95$ \\
\hline S96T005570 & $166: 4$ & Lower half & $<17.9$ & $<17.9$ & $<17.9$ \\
\hline S96T005615 & 166: 6 & Lower half & $<13.8$ & $<13.8$ & $<13.8$ \\
\hline S96T005839 & 166: 7 & Lower half & $<20.2$ & $<20.5$ & $<20.35$ \\
\hline S96T005840 & 166: 8 & Lower half & $<19.7$ & $<19.7$ & $<19.7^{\mathrm{QC:a}}$ \\
\hline S96T005575 & 166: 9 & Lower haif & $<19.6$ & $<19.9$ & $<19.75$ \\
\hline S96T005571 & $166: 10$ & Lower half & $<15.3$ & $<14.5$ & $<14.9$ \\
\hline S96T005616 & 166: 11 & Lower half & $<14.5$ & $<13.6$ & $<14.05$ \\
\hline S96T005617 & 166: 12 & Lower half & $<14.1$ & $<14.1$ & $<14.1$ \\
\hline S96T005716 & \multirow[t]{2}{*}{ 166: 13} & Upper half & $<19.4$ & $<19.6$ & $<19.5$ \\
\hline S96T005722 & & Lower half & $<20.6$ & $<20.6$ & $<20.6$ \\
\hline S96T005416 & \multirow[t]{2}{*}{ 166: 15} & Upper half & $<20.4$ & $<19$ & $<19.7$ \\
\hline \$97T000459 & & Lower half & $<5.69$ & 6.79 & $<6.24$ \\
\hline S96T005624 & \multirow[t]{2}{*}{$166: 16$} & Upper half & $<21$ & $<21.5$ & $<21.25$ \\
\hline \$96T005618 & & Lower half & $<14.2$ & $<14.6$ & $<14.4$ \\
\hline S96T005417 & \multirow[t]{2}{*}{ 166: 17} & Upper half & $<22.5$ & $<20.5$ & $<21.5$ \\
\hline$\$ 96 \mathrm{~T} 005411$ & & Lower half & $<19$ & $<17.6$ & $<18.3$ \\
\hline S96T005717 & \multirow[t]{2}{*}{ 166: 18} & Upper haif & $<20.8$ & $<20.2$ & $<20.5$ \\
\hline S96T005723 & & Lower half & $<20.2$ & $<19.8$ & $<20$ \\
\hline S96T005841 & \multirow[t]{2}{*}{ 166: 19} & Upper half & $<19.3$ & $<19.6$ & $<19.45^{Q \mathrm{C}: \mathrm{a}}$ \\
\hline \$96T005842 & & Lower half & 20.4 & 22 & $21.2^{\mathrm{QC} \cdot \mathrm{a}}$ \\
\hline S96T005708 & \multirow[t]{2}{*}{ 167: 1} & Upper half & $<17.9$ & $<20.5$ & $<19.2$ \\
\hline S96T005693 & & Lower half & $<22.2$ & $<17.5$ & $<19.85^{\text {QC:e }}$ \\
\hline S96T005857 & 167: 2 & Lower half & $<16.7$ & $<17.4$ & $<17.05$ \\
\hline S96T005694 & 167: 3 & Lower half & $<18$ & $<18.7$ & $<18.35$ \\
\hline \$96T005695 & 167:4 & Lower half & $<19.8$ & $<20.4$ & $<20.1$ \\
\hline \$96T005438 & 167: 5 & Lower half & $<5.17$ & $<5.51$ & $<5.34^{\mathrm{QC:a}}$ \\
\hline S96T005450 & 167: 6 & Lower half & $<5.53$ & $<5.22$ & $<5.375^{Q \mathrm{C}: a}$ \\
\hline S96T005696 & $167: 7$ & Lower half & $<19.2$ & $<18.8$ & $<19$ \\
\hline
\end{tabular}




\begin{tabular}{|c|c|c|c|c|c|}
\hline $10.9>$ & $10^{\circ} 9>$ & $10^{\circ} 9>$ & pinbị ə[qeuţera & $\angle: \angle 9 I$ & E89S00L96S \\
\hline $10^{\circ} 9>$ & $10^{\circ} 9>$ & $10^{\circ} 9>$ & pinbri әүqеuाeı & $9: \angle 9 \mathrm{I}$ & IZtS00.L96S \\
\hline $10^{\circ} 9>$ & $10^{\circ} 9>$ & $10^{\circ} 9>$ & pinbu ә әquuexa & $S: L 9 I$ & $0 Z \$ S 00 L 96 \mathrm{~S}$ \\
\hline $10^{\circ} 9>$ & $10.9>$ & $10^{\circ} 9>$ & pinb!̣ ərqeuịera & $\varepsilon: \angle 9 l$ & EL9S00L96S \\
\hline $10^{\circ} 9>$ & $10^{\circ} 9>$ & $10^{\circ} 9>$ & pı̣nb!l ə[qeutera & $z: \angle 9 \mathrm{I}$ & $198500 \perp 96 \mathrm{~S}$ \\
\hline$\left[0^{\circ} 9>\right.$ & $10^{\circ} 9>$ & $10^{\circ} 9>$ & p!nb! I әүqeụesa & ZI:99I & I09S00196S \\
\hline $10^{\circ} 9>$ & $10^{\circ} 9>$ & $10^{\circ} 9>$ & 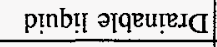 & II :99I & $009 \$ 00 L 96 S$ \\
\hline $10^{\circ} t>$ & $10^{\circ} t>$ & $10^{\circ} t>$ & pı̣nbụ əlqeurexa & 0I:99I & 99SS00L96S \\
\hline $10^{\circ} 9>$ & $10^{\circ} 9>$ & $10^{\circ} 9>$ & pı̣nb!l ə[qeụes] & $6: 99 \mathrm{I}$ & Itऽ500L96S \\
\hline $10^{\circ} 9>$ & $10^{\circ} 9>$ & $10^{\circ} 9>$ & p!nb!l әтqeu!esa & $8: 99 I$ & $\angle I 8 S 00 L 96 \mathrm{~S}$ \\
\hline $10^{\circ} 9>$ & $10.9>$ & $10^{\circ} 9>$ & prinb! I әqueureı & $L: 99 I$ & $918500 L 96 \mathrm{~S}$ \\
\hline $10^{\circ} 9>$ & $10^{\circ} 9>$ & $10^{\circ} 9>$ & 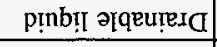 & $9: 99 \mathrm{I}$ & $66 \$ 500 \mathrm{~L} 96 \mathrm{~S}$ \\
\hline $10^{\circ} \downarrow>$ & $10^{\circ} \nabla>$ & $10^{\circ} t>$ & 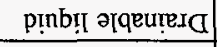 & $\nabla: 99 \mathrm{I}$ & $0 t \$ \$ 00196 \mathrm{~S}$ \\
\hline $10^{\circ} \nabla>$ & $10^{\circ} t>$ & $10^{\circ} \bullet>$ & pinbỵ I ગquutex & $\varepsilon: 99 \mathrm{I}$ & $6 \& S S 00 \mathrm{~L} 96 \mathrm{~S}$ \\
\hline Mangin: & fing & (m) & (1) & 3. & \%pror \\
\hline$\angle S^{\prime} S>$ & IS S & $\varepsilon 9^{\circ} S>$ & วı!soduos plyos & 99โ ว10ग & $09+000 \mathrm{~L} L 6 \mathrm{~S}$ \\
\hline SS.8I > & $\varepsilon 6 I>$ & $8 \angle I>$ & Ileप Іәмот & \multirow[b]{2}{*}{$\angle I: \angle 9 I$} & $66+S 00 \perp 96 \mathrm{~S}$ \\
\hline $6.8 I>$ & $5 \cdot 6 I>$ & $\varepsilon \cdot 8 I>$ & J[eप & & $86 t S 00 L 96 \mathrm{~S}$ \\
\hline $5: 8 \mathrm{I}>$ & $8.8 I>$ & $\tau: 8 I>$ & IlЕЧ IәMOT & \multirow[b]{2}{*}{$\$ \mathrm{I}: \angle 9 \mathrm{I}$} & 869S00L96S \\
\hline$S Z \cdot 8 I>$ & $6 \cdot L I>$ & $9.8 I>$ & Jleप $\operatorname{ləd}_{\Omega}$ & & $0 L \angle S 00 L 96 \mathrm{~S}$ \\
\hline $0: 0056 Z^{\circ} \mathrm{LL}$ & $\mathcal{S E} 8$ & $\nabla Z^{\circ} 9$ & fleप Іәмот & \multirow[b]{2}{*}{ tDI: $\angle 9 I$} & $6 \mathcal{E}+\mathrm{S} 00 \perp 96 \mathrm{~S}$ \\
\hline ::00 $S 6 \varepsilon^{\circ} \mathrm{S}>$ & $1 S S>$ & $8 \pi \cdot s>$ & J[eप Iədd & & SttS00J96S \\
\hline ::009LS $>$ & $78^{\circ} s>$ & $89^{\circ} \subseteq>$ & flеप IәMOT & \multirow[b]{2}{*}{ ZI :L9I } & IStS00L96S \\
\hline$: 006 t^{\circ} \varsigma>$ & $s t^{*} s>$ & $\varepsilon \mathcal{S} S>$ & Iley wodd $\Omega$ & & $t t t S 00 L 96 \mathrm{~S}$ \\
\hline$\varepsilon 6 I>$ & $8 \cdot 8 I>$ & $8 \cdot 6 I>$ & Ileप Iәмот & \multirow[b]{2}{*}{ II :L9I } & L69S00LI96S \\
\hline SE:8I > & $L 8 I>$ & $8 \mathrm{I}>$ & fleप Iədd $_{\Omega}$ & & $60 \angle S 00 L 96 \mathrm{~S}$ \\
\hline SI 6 I > & $S L I>$ & $80 z>$ & f[EЧ Iәмот & $6: \angle 9 I$ & $8 \subseteq 8 \$ 00 L 96 \mathrm{~S}$ \\
\hline $8.6 I>$ & $0 z>$ & $96 I>$ & IIEY IOMOT & $8: \angle 9 I$ & LELSO0L $96 \mathrm{~S}$ \\
\hline (5) & (1) & (4) & & \multicolumn{2}{|c|}{ (57\%) } \\
\hline 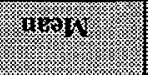 & (2) & 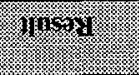 & S. & mogrong & 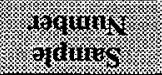 \\
\hline
\end{tabular}

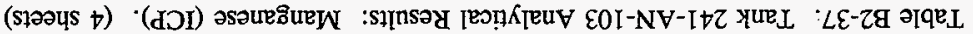


Table B2-37. Tank 241-AN-103 Analytical Results: Manganese (ICP). (4 sheets)

\begin{tabular}{|c|c|c|c|c|c|}
\hline $\begin{array}{l}\text { Sompe } \\
\text { Vinner }\end{array}$ & Symple. & $\begin{array}{l}\text { Simule } \\
\text { Promon }\end{array}$ & Rritur & $\log \log (x)$ & $\sqrt{1,41}$ \\
\hline Wrinis & (3) & (2) & 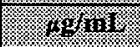 & (2. & (18.6 $\mathrm{mL}$ \\
\hline S96T005732 & $167: 8$ & Drainable liquid & $<6.01$ & $<6.01$ & $<6.01$ \\
\hline S96T005862 & 167: 9 & Drainable liquid & $<6.01$ & $<6.01$ & $<6.01$ \\
\hline S96T005650 & 167: 11 & Drainable liquid & $<6.01$ & $<6.01$ & $<6.01$ \\
\hline S96T005991 & Core 166 & Liquid composite & $<12$ & $<12$ & $<12$ \\
\hline Soldu - Vuriol & l- & 2.3 & . & (1) & $1=1.85$ \\
\hline S96T005608 & 166: 1 & Lower half & $<210$ & $<206$ & $<208$ \\
\hline S96T005568 & $166: 4$ & Lower half & 459 & 515 & 487 \\
\hline S96T005611 & $166: 6$ & Lower half & $<210$ & $<214$ & $<212$ \\
\hline S96T005835 & $166: 7$ & Lower half & $<210$ & $<212$ & $<211$ \\
\hline S96T005836 & $166: 8$ & Lower half & $<196$ & $<206$ & $<201$ \\
\hline S96T005574 & $166: 9$ & Lower half & 1,910 & 1,900 & 1,905 \\
\hline S96T005569 & 166: 10 & Lower half & $<209$ & $<207$ & $<208$ \\
\hline S96T005612 & 166: 11 & Lower half & $<197$ & $<204$ & $<200.5$ \\
\hline S96T005613 & $166: 12$ & Lower half & $<205$ & $<202$ & $<203.5$ \\
\hline S967005714 & \multirow[t]{2}{*}{$166: 13$} & Upper half & $<201$ & $<211$ & $<206$ \\
\hline S96T005720 & & Lower half & $<203$ & 386 & $<294.5^{\mathrm{QC}: \mathrm{e}}$ \\
\hline S96T005414 & \multirow[t]{2}{*}{$166: 15$} & Upper half & 72 & 67.1 & 69.55 \\
\hline S96T005408 & & Lower half & 107 & $<46.3$ & $<76.65^{\text {QC:e }}$ \\
\hline S96T005623 & \multirow[t]{2}{*}{ 166: 16} & Upper half & $<218$ & $<210$ & $<214$ \\
\hline S96T005614 & & Lower half & $<204$ & $<201$ & $<202.5$ \\
\hline S96T005415 & \multirow[t]{2}{*}{$166: 17$} & Upper half & 236 & $<52.8$ & $<144.4^{\mathrm{QC:c}}$ \\
\hline S96T005409 & & Lower half & $<211$ & $<208$ & $<209.5$ \\
\hline S96T005715 & \multirow[t]{2}{*}{$166: 18$} & Upper half & $<201$ & $<206$ & $<203.5$ \\
\hline S96T005721 & & Lower half & $<203$ & $<199$ & $<201$ \\
\hline S96T005837 & \multirow[t]{2}{*}{$166: 19$} & Upper half & $<207$ & $<210$ & $<208.5$ \\
\hline S96T005838 & & Lower half & $<196$ & $<196$ & $<196$ \\
\hline S96T005705 & \multirow[t]{2}{*}{$167: 1$} & Upper half & $<199$ & $<200$ & $<199.5$ \\
\hline S96T005687 & & Lower half & $<208$ & $<209$ & $<208.5$ \\
\hline S96T005855 & $167: 2$ & Lower half & $<202$ & $<198$ & $<200$ \\
\hline
\end{tabular}




\begin{tabular}{|c|c|c|c|c|c|}
\hline soIz> & IIZ> & 0 Iz> & IएЕप دәмоТ] & \multirow[b]{2}{*}{$\angle I: \angle 9 I$} & $\angle 6+S 00 \mathrm{~L} 96 \mathrm{~S}$ \\
\hline IIZ> & $0 I Z>$ & $z\lfloor z>$ & fleप ১ədd $\Omega$ & & $96 \pm S 00 \mathrm{~L} 96 \mathrm{~S}$ \\
\hline$\varsigma 00 z>$ & $8 I Z>$ & $\varepsilon 8 I>$ & Ғ[еप АәмоТ & \multirow[b]{2}{*}{ SI :L9I } & $269500 \mathrm{~L} 96 \mathrm{~S}$ \\
\hline S.86I > & $78 \mathrm{I}>$ & $\varepsilon I Z>$ & „ґеч $\operatorname{lədd}_{\Omega}$ & & $\angle 0 L S 00 L 96 \mathrm{~S}$ \\
\hline toz> & $\varepsilon 0 z>$ & soz> & Jгч دәмо & \multirow[b]{2}{*}{$\nabla I: \angle 9 I$} & $\angle E t S 00 \perp 96 S$ \\
\hline SSIZ> & IIZ> & $0 z z>$ & IIEY Iədd $\Omega$ & & $\varepsilon t t S 00 \perp 96 \mathrm{~S}$ \\
\hline $00 z>$ & $z 0 z>$ & $86 I>$ & IГеप ХəMOT & \multirow[b]{2}{*}{ ZI :L9I } & $6 t t s 00196 \mathrm{~S}$ \\
\hline soz> & $56 I>$ & $9 I Z>$ & J[еч Jədd $\Omega$ & & $z_{t t 500 \mathrm{~L} 96 \mathrm{~S}}$ \\
\hline$\varsigma^{\circ} \downarrow 8 I>$ & $t 8 \mathrm{I}>$ & $\mathrm{S} 8 \mathrm{I}>$ & ILE IәMOT] & \multirow[b]{2}{*}{ II :L9I } & I69S00L96S \\
\hline s.toz> & $z z z>$ & $\angle 8 I\rangle$ & Jॉey Iadd $_{\Omega}$ & & $90 \angle S 00 L 96 \mathrm{~S}$ \\
\hline$\varsigma^{\circ} \angle 6 I>$ & IIZ> & $78 I>$ & Јाष्प Іәмо Т & $6: \angle 9 I$ & $958500 \mathrm{~L} 96 \mathrm{~S}$ \\
\hline toz> & $\angle 8 I>$ & IZZ> & 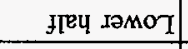 & $8: \angle 9 I$ & $9 \varepsilon \angle S 00: 196 \mathrm{~S}$ \\
\hline$\angle O Z>$ & $812>$ & $96 \mathrm{I}>$ & І[еч دәмот & $\angle: \angle 9 I$ & $069 S 00 L 96 \mathrm{~S}$ \\
\hline$s^{\prime} \angle 0 z>$ & t0z> & III $>$ & Јгеч хәмо Т & $9: \angle 9 I$ & $8 t t S 00 \mathrm{~L} 96 \mathrm{~S}$ \\
\hline$\angle 8 \mathrm{I}\rangle$ & $881>$ & $98 \mathrm{I}>$ & IEY ІәмоТ & $S: \angle 9 I$ & $9 \varepsilon+S 00 \mathrm{~L} 96 \mathrm{~S}$ \\
\hline$s^{*} 0 \mathrm{I} z>$ & IIZ> & $0 I z>$ & Іाएч гәмо & $\nabla: \angle 9 I$ & $689 S 00 L 96 \mathrm{~S}$ \\
\hline $6 I Z>$ & $\varepsilon I Z>$ & $s z z>$ & 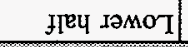 & $\varepsilon: \angle 9 I$ & 889 S00.L96S \\
\hline 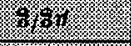 & (3) 40 & $8 \%, \%$ & 4 & \multicolumn{2}{|c|}{ 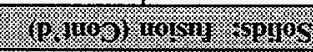 } \\
\hline movn & 3130741169 & $11159+1$ & aringe & 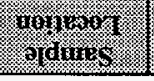 & $\begin{array}{l}\text { Irqurny: } \\
\text { adrins }\end{array}$ \\
\hline
\end{tabular}

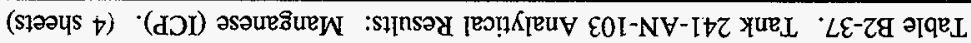


Table B2-38. Tank 241-AN-103 Analytical Results: Molybdenum (ICP). (4 sheets)

\begin{tabular}{|c|c|c|c|c|c|}
\hline 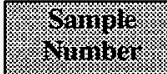 & gample & aring & gosing & 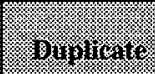 & $(6)$ \\
\hline \multicolumn{2}{|c|}{ 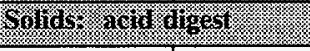 } & (ly & (1) & 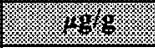 & (4) \\
\hline S96T005609 & 166: 1 & Lower half & $<73.8$ & $<75.4$ & $<74.6$ \\
\hline S96T005570 & $166: 4$ & Lower half & $<89.7$ & $<89.7$ & $<89.7$ \\
\hline S96T005615 & 166: 6 & Lower half & $<69.1$ & $<69.1$ & $<69.1$ \\
\hline S96T005839 & $166: 7$ & Lower half & $<101$ & $<102$ & $<101.5$ \\
\hline S96T005840 & $166: 8$ & Lower half & $<98.4$ & $<98.3$ & $<98.35$ \\
\hline S96T005575 & 166: 9 & Lower half & $<97.8$ & $<99.4$ & $<98.6$ \\
\hline S96T005571 & $166: 10$ & Lower half & $<76.7$ & $<72.3$ & $<74.5$ \\
\hline S96T005616 & $166: 11$ & Lower half & $<72.3$ & $<68$ & $<70.15$ \\
\hline \$96T005617 & 166: 12 & Lower half & $<70.5$ & $<70.4$ & $<70.45$ \\
\hline S96T005716 & \multirow[t]{2}{*}{$166: 13$} & Upper half & $<96.9$ & $<98.1$ & $<97.5$ \\
\hline \$96T005722 & & Lower half & $<103$ & $<103$ & $<103$ \\
\hline S96T005416 & \multirow[t]{2}{*}{$166: 15$} & Upper half & $<102$ & $<95.2$ & $<98.6$ \\
\hline S97T000459 & & Lower half & 35.7 & 44.5 & $40.1^{\text {QC:e }}$ \\
\hline S96T005624 & \multirow[t]{2}{*}{ 166: 16} & Upper half & $<105$ & $<108$ & $<106.5$ \\
\hline \$96T005618 & & Lower half & $<70.9$ & $<72.8$ & $<71.85$ \\
\hline S96T005417 & \multirow[t]{2}{*}{$166: 17$} & Upper half & $<113$ & $<102$ & $<107.5$ \\
\hline S96T005411 & & Lower half & $<95.1$ & $<88$ & $<91.55$ \\
\hline \$96T005717 & \multirow[t]{2}{*}{$166: 18$} & Upper half & $<104$ & $<101$ & $<102.5$ \\
\hline S96T005723 & & Lower half & $<101$ & $<99$ & $<100$ \\
\hline S96T005841 & \multirow[t]{2}{*}{$166: 19$} & Upper half & $<96.3$ & $<98.1$ & $<97.2$ \\
\hline S96T005842 & & Lower half & $<89$ & $<98.1$ & $<93.55$ \\
\hline \$96T005708 & \multirow[t]{2}{*}{ 167: 1} & Upper half & $<89.6$ & $<103$ & $<96.3$ \\
\hline S96T005693 & & Lower half & $<111$ & $<87.7$ & $<99.35$ \\
\hline S96T005857 & $167: 2$ & Lower half & $<83.4$ & $<86.9$ & $<85.15$ \\
\hline \$96T005694 & $167: 3$ & Lower half & $<89.9$ & $<93.6$ & $<91.75$ \\
\hline S96T005695 & 167: 4 & Lower half & $<99$ & $<102$ & $<100.5$ \\
\hline S96T005438 & $167: 5$ & Lower half & 53 & 51.3 & 52.15 \\
\hline S96T005450 & 167: 6 & Lower half & 44.5 & 47.5 & 46 \\
\hline S96T005696 & $167: 7$ & Lower half & $<96$ & $<94$ & $<95$ \\
\hline
\end{tabular}


HNF-SD-WM-ER-702 Rev. 0

Table B2-38. Tank 241-AN-103 Analytical Results: Molybdenum (ICP). (4 sheets)

\begin{tabular}{|c|c|c|c|c|c|}
\hline \%110\% & WHaphe & $f_{2}$ & Resull & oumigrit & (1) \\
\hline 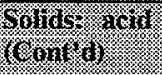 & 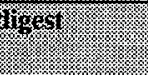 & (1) & 40 & mp. & (4) \\
\hline S96T005737 & 167: 8 & Lower half & $<97.9$ & $<99.9$ & $<98.9$ \\
\hline S96T005858 & 167: 9 & Lower half & $<104$ & $<87.5$ & $<95.75$ \\
\hline S96T005709 & 167: 11 & Upper half & $<90$ & $<93.3$ & $<91.65$ \\
\hline S96T005697 & & Lower half & $<99.2$ & $<94.1$ & $<96.65$ \\
\hline S96T005444 & 167: 12 & Upper half & 56.3 & 53.7 & 55 \\
\hline S96T005451 & & Lower half & 46.4 & 46 & 46.2 \\
\hline S96T005445 & 167: 14 & Upper half & 33.1 & 28.3 & 30.7 \\
\hline S96T005439 & & Lower half & $<28.4$ & 29.2 & $<28.8$ \\
\hline S96T005710 & 167: 15 & Upper half & $<93.2$ & $<89.5$ & $<91.35$ \\
\hline S96T005698 & & Lower half & $<91.1$ & $<94$ & $<92.55$ \\
\hline S96T005498 & $167: 17$ & Upper half & $<91.6$ & $<97.4$ & $<94.5$ \\
\hline S96T005499 & & Lower half & $<89.1$ & $<96.5$ & $<92.8$ \\
\hline S97T000460 & Core 166 & Solid composite & 42 & 43.3 & 42.65 \\
\hline Froundo & & & \%gring & (1) & 10981 \\
\hline S96T005539 & $166: 3$ & Drainable liquid & 117 & 112 & 114.5 \\
\hline \$96T005540 & 166: 4 & Drainable liquid & 107 & 102 & 104.5 \\
\hline S96T005599 & $166: 6$ & Drainable liquid & 125 & 117 & 121 \\
\hline S96T005816 & 166: 7 & Drainable liquid & 117 & 121 & 119 \\
\hline S96T005817 & $166: 8$ & Drainable liquid & 111 & 123 & 117 \\
\hline S96T005541 & 166: 9 & Drainable liquid & 116 & 114 & 115 \\
\hline S96T005566 & 166: 10 & Drainable liquid & 119 & 113 & 116 \\
\hline S96T005600 & $166: 11$ & Drainable liquid & 118 & 116 & 117 \\
\hline S96T005601 & $166: 12$ & Drainable liquid & 118 & 117 & 117.5 \\
\hline \$96T005861 & 167: 2 & Drainable liquid & 112 & 116 & 114 \\
\hline \$96T005673 & $167: 3$ & Drainable liquid & 110 & 111 & 110.5 \\
\hline S96T005420 & $167: 5$ & Drainable liquid & 116 & 118 & 117 \\
\hline \$96T005421 & $167: 6$ & Drainable liquid & 117 & 116 & 116.5 \\
\hline S96T005683 & $167: 7$ & Drainable liquid & 117 & 119 & 118 \\
\hline
\end{tabular}


HNF-SD-WM-ER-702 Rev. 0

Table B2-38. Tank 241-AN-103 Analytical Results: Molybdenum (ICP). (4 sheets)

\begin{tabular}{|c|c|c|c|c|c|}
\hline Whane & 9.7mple & (6) & resuil & 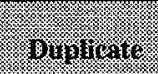 & (1) \\
\hline \multicolumn{3}{|c|}{ 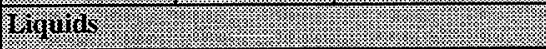 } & (3) & (4) & \% \\
\hline S96T005732 & 167: 8 & Drainable liquid & 117 & 115 & 116 \\
\hline S96T005862 & 167: 9 & Drainable liquid & 117 & 120 & 118.5 \\
\hline S96T005650 & 167: 11 & Drainable liquid & 98.9 & 101 & 99.95 \\
\hline S96T005991 & Core 166 & Liquid composite & 109 & 109 & 109 \\
\hline \multicolumn{3}{|c|}{ WWiling } & 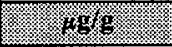 & 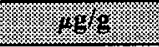 & (3) \\
\hline S96T005608 & 166: 1 & Lower half & $<1,050$ & $<1,030$ & $<1,040$ \\
\hline S96T005568 & 166: 4 & Lower half & $<1,130$ & $<1,110$ & $<1,120$ \\
\hline S96T005611 & 166: 6 & Lower half & $<1,050$ & $<1,070$ & $<1,060$ \\
\hline S96T005835 & 166: 7 & Lower half & $<1,050$ & $<1,060$ & $<1,055$ \\
\hline S96T005836 & 166: 8 & Lower half & $<981$ & $<1,030$ & $<1,005.5$ \\
\hline S96T005574 & $166: 9$ & Lower half & $<896$ & $<970$ & $<933$ \\
\hline S96T005569 & $166: 10$ & Lower half & $<1,050$ & $<1,030$ & $<1,040$ \\
\hline S96T005612 & 166: 11 & Lower half & $<987$ & $<1,020$ & $<1,003.5$ \\
\hline S96T005613 & $166: 12$ & Lower half & $<1,030$ & $<1,010$ & $<1,020$ \\
\hline S96T005714 & \multirow[t]{2}{*}{$166: 13$} & Upper half & $<1,010$ & $<1,050$ & $<1,030$ \\
\hline S96T005720 & & Lower half & $<1,010$ & $<1,010$ & $<1,010$ \\
\hline S96T005414 & \multirow[t]{2}{*}{$166: 15$} & Upper half & $<228$ & $<233$ & $<230.5$ \\
\hline \$96T005408 & & Lower half & $<240$ & $<232$ & $<236$ \\
\hline S96T005623 & \multirow[t]{2}{*}{$166: 16$} & Upper half & $<1,090$ & $<1,050$ & $<1,070$ \\
\hline S96T005614 & & Lower half & $<1,020$ & $<1,000$ & $<1,010$ \\
\hline S96T005415 & \multirow[t]{2}{*}{$166: 17$} & Upper half & $<262$ & $<264$ & $<263$ \\
\hline S96T005409 & & Lower half & $<1,060$ & $<1,040$ & $<1,050$ \\
\hline S96T005715 & \multirow[t]{2}{*}{ 166: 18} & Upper half & $<1,010$ & $<1,030$ & $<1,020$ \\
\hline S96T005721 & & Lower half & $<1,010$ & $<994$ & $<1,002$ \\
\hline S96T005837 & \multirow[t]{2}{*}{ 166: 19} & Upper half & $<1,040$ & $<1,050$ & $<1,045$ \\
\hline S96T005838 & & Lower half & $<978$ & $<982$ & $<980$ \\
\hline S96T005705 & \multirow[t]{2}{*}{ 167: 1} & Upper half & $<994$ & $<1,000$ & $<997$ \\
\hline S96T005687 & & Lower half & $<1,040$ & $<1,040$ & $<1,040$ \\
\hline S96T005855 & 167:2 & Lower half & $<1,010$ & $<988$ & $<999$ \\
\hline
\end{tabular}


HNF-SD-WM-ER-702 Rev. 0

Table B2-38. Tank 241-AN-103 Analytical Results: Molybdenum (ICP). (4 sheets)

\begin{tabular}{|c|c|c|c|c|c|}
\hline Hamol & Orampior & 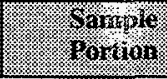 & Reting & ginourate. & 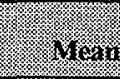 \\
\hline \multicolumn{2}{|c|}{ 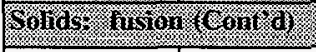 } & ry & . & 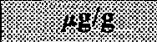 & 40 \\
\hline S96T005688 & 167: 3 & Lower half & $<1,120$ & $<1,070$ & $<1,095$ \\
\hline S96T005689 & 167: 4 & Lower half & $<1,050$ & $<1,060$ & $<1,055$ \\
\hline S96T005436 & 167: 5 & Lower half & $<931$ & $<939$ & $<935$ \\
\hline \$96T005448 & $167: 6$ & Lower half & $<1,050$ & $<1,020$ & $<1,035$ \\
\hline \$96T005690 & $167: 7$ & Lower half & $<982$ & $<1,090$ & $<1,036$ \\
\hline S96T005736 & 167: 8 & Lower half & $<1,110$ & $<937$ & $<1,023.5$ \\
\hline S96T005856 & $167: 9$ & Lower half & $<920$ & $<1,050$ & $<985$ \\
\hline S96T005706 & \multirow[t]{2}{*}{ 167: 11} & Upper half & $<933$ & $<1,110$ & $<1,021.5$ \\
\hline \$96T005691 & & Lower half & $<925$ & $<921$ & $<923$ \\
\hline S96T005442 & \multirow[t]{2}{*}{$167: 12$} & Upper half & $<1,080$ & $<969$ & $<1,024.5$ \\
\hline S96T005449 & & Lower half & $<988$ & $<1,010$ & $<999$ \\
\hline S96T005443 & \multirow[t]{2}{*}{$167: 14$} & Upper half & $<1,100$ & $<1,050$ & $<1,075$ \\
\hline S96T005437 & & Lower half & $<1,030$ & $<1,010$ & $<1,020$ \\
\hline S96T005707 & \multirow[t]{2}{*}{$167: 15$} & Upper half & $<1,070$ & $<919$ & $<994.5$ \\
\hline \$96T005692 & & Lower half & $<916$ & $<1,090$ & $<1,003$ \\
\hline S96T005496 & \multirow[t]{2}{*}{ 167: 17} & Upper half & $<1,060$ & $<1,050$ & $<1,055$ \\
\hline S96T005497 & & Lower half & $<1,050$ & $<1,050$ & $<1,050$ \\
\hline
\end{tabular}


Table B2-39. Tank 241-AN-103 Analytical Results: Nickel (ICP). (4 sheets)

\begin{tabular}{|c|c|c|c|c|c|}
\hline oringor & 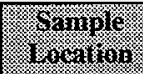 & \%ape & 180411 & 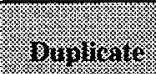 & in \\
\hline \multicolumn{2}{|c|}{ Sinds } & & . & $4 x$ & 85 \\
\hline S96T005609 & 166: 1 & Lower half & 44.6 & 33.2 & $38.9^{\mathrm{eC}: \mathrm{e}}$ \\
\hline S96T005570 & 166: 4 & Lower half & $<35.9$ & $<35.9$ & $<35.9$ \\
\hline S96T005615 & $166: 6$ & Lower half & $<27.6$ & $<27.6$ & $<27.6$ \\
\hline S96T005839 & 166: 7 & Lower half & $<40.5$ & $<41$ & $<40.75$ \\
\hline S96T005840 & 166: 8 & Lower half & $<39.4$ & $<39.3$ & $<39.35^{\mathrm{QC}: \mathrm{a}}$ \\
\hline S96T005575 & 166: 9 & Lower half & $<39.1$ & $<39.8$ & $<39.45$ \\
\hline S96T005571 & $166: 10$ & Lower half & $<30.7$ & $<28.9$ & $<29.8$ \\
\hline S96T005616 & 166:11 & Lower half & $<28.9$ & $<27.2$ & $<28.05$ \\
\hline S96T005617 & $166: 12$ & Lower half & $<28.2$ & $<28.1$ & $<28.15$ \\
\hline S96T005716 & \multirow[t]{2}{*}{ 166: 13} & Upper half & $<38.8$ & $<39.2$ & $<39$ \\
\hline S96T005722 & & Lower half & $<41.1$ & $<41.3$ & $<41.2$ \\
\hline S96T005416 & \multirow[t]{2}{*}{$166: 15$} & Upper half & $<40.8$ & $<38.1$ & $<39.45$. \\
\hline S97T000459 & & Lower half & 18.9 & 28.5 & $23.7^{\mathrm{QC}: c}$ \\
\hline S96T005624 & \multirow[t]{2}{*}{$166: 16$} & Upper half & $<41.9$ & $<43$ & $<42.45$ \\
\hline S96T005618 & & Lower half & 29.2 & 32.8 & 31 \\
\hline S96T005417 & \multirow[t]{2}{*}{$166: 17$} & Upper half & $<45.1$ & $<40.9$ & $<43$ \\
\hline S96T005411 & & Lower half & $<38.1$ & $<35.2$ & $<36.65$ \\
\hline S96T005717 & \multirow[t]{2}{*}{$166: 18$} & Upper half & $<41.7$ & $<40.4$ & $<41.05$ \\
\hline S96T005723 & & Lower half & $<40.5$ & $<39.6$ & $<40.05$ \\
\hline S96T005841 & \multirow[t]{2}{*}{ 166: 19} & Upper half & $<38.5$ & $<39.2$ & $<38.85^{\mathrm{QC:a}}$ \\
\hline S96T005842 & & Lower half & $<35.6$ & $<39.2$ & $<37.4^{\mathrm{QC:a}}$ \\
\hline S96T005708 & \multirow[t]{2}{*}{ 167: 1} & Upper half & $<35.8$ & $<41.1$ & $<38.45$ \\
\hline \$96T005693 & & Lower half & $<44.4$ & $<35.1$ & $<39.75^{\text {QC:c }}$ \\
\hline S96T005857 & 167:2 & Lower half & $<33.4$ & $<34.8$ & $<34.1$ \\
\hline S96T005694 & $167: 3$ & Lower half & $<35.9$ & $<37.4$ & $<36.65$ \\
\hline S96T005695 & 167: 4 & Lower half & $<39.6$ & $<40.8$ & $<40.2$ \\
\hline S96T005438 & $167: 5$ & Lower half & $<10.3$ & $<11$ & $<10.65^{\mathrm{QC:a}}$ \\
\hline \$96T005450 & 167: 6 & Lower half & $<11.1$ & $<10.4$ & $<10.75^{\mathrm{QC}: \mathrm{a}}$ \\
\hline S96T005696 & $167: 7$ & Lower half & $<38.4$ & $<37.6$ & $<38$ \\
\hline
\end{tabular}


Table B2-39. Tank 241-AN-103 Analytical Results: Nickel (ICP). (4 sheets)

\begin{tabular}{|c|c|c|c|c|c|}
\hline $\begin{array}{l}\text { Sample } \\
\text { Tinilot }\end{array}$ & S.morarle & $\begin{array}{l}\text { Siarpla } \\
\text { rofrion }\end{array}$ & ressint & Divilicar & 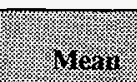 \\
\hline 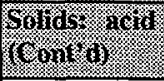 & figes: & $\sqrt{4}$ & 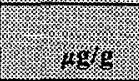 & - & 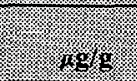 \\
\hline S96T005737 & 167: 8 & Lower half & $<39.2$ & $<40$ & $<39.6$ \\
\hline S96T005858 & $167: 9$ & Lower half & $<41.6$ & $<35$ & $<38.3$ \\
\hline S96T005709 & \multirow[t]{2}{*}{$167: 11$} & Upper half & $<36$ & $<37.3$ & $<36.65$ \\
\hline S96T005697 & & Lower half & $<39.7$ & $<37.6$ & $<38.65$ \\
\hline S96T005444 & \multirow[t]{2}{*}{$167: 12$} & Upper half & 17 & 17.9 & $17.45^{\mathrm{QC:a}}$ \\
\hline S96T005451 & & Lower half & 23.8 & 18.1 & $20.95^{\mathrm{QC:a,c}}$ \\
\hline S96T005445 & \multirow[t]{2}{*}{ 167: 14} & Upper half & 13.8 & 12 & $12.9^{\text {oC:a }}$ \\
\hline S96T005439 & & Lower half & 18.6 & 19.9 & $19.25^{\mathrm{QCa}}$ \\
\hline S96T005710 & \multirow[t]{2}{*}{$167: 15$} & Upper half & $<37.3$ & $<35.8$ & $<36.55$ \\
\hline S96T005698 & & Lower half & $<36.4$ & $<37.6$ & $<37$ \\
\hline S96T005498 & \multirow[t]{2}{*}{$167: 17$} & Upper half & $<36.6$ & $<38.9$ & $<37.75$ \\
\hline S96T005499 & & Lower half & $<35.7$ & $<38.6$ & $<37.15$ \\
\hline S97T000460 & Core 166 & Solid composite & $<11.3$ & $<11$ & $<11.15$ \\
\hline Igrivgs & \multicolumn{2}{|l|}{ (1) } & 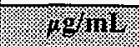 & 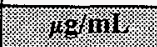 & . $.16 \mathrm{~g} / \mathrm{mi}$ \\
\hline S96T005539 & $166: 3$ & Drainable liquid & $<8.02$ & $<8.02$ & $<8.02$ \\
\hline S96T005540 & $166: 4$ & Drainable liquid & $<8.02$ & $<8.02$ & $<8.02$ \\
\hline S96T005599 & 166: 6 & Drainable liquid & $<12$ & $<12$ & $<12$ \\
\hline S96T005816 & 166: 7 & Drainable liquid & $<12$ & $<12$ & $<12$ \\
\hline S96T005817 & $166: 8$ & Drainable liquid & $<12$ & $<12$ & $<12$ \\
\hline S96T005541 & $166: 9$ & Drainable liquid & $<12$ & $<12$ & $<12$ \\
\hline S96T005566 & $166: 10$ & Drainable liquid & 8.06 & $<8.02$ & $<8.04$ \\
\hline S96T005600 & 166: 11 & Drainable liquid & $<12$ & $<12$ & $<12$ \\
\hline S96T005601 & $166: 12$ & Drainable liquid & $<12$ & $<12$ & $<12$ \\
\hline S96T005861 & $167: 2$ & Drainable liquid & $<12$ & $<12$ & $<12$ \\
\hline S96T005673 & $167: 3$ & Drainable liquid & $<12$ & $<12$ & $<12$ \\
\hline S96T005420 & $167: 5$ & Drainable liquid & 12.3 & $<12$ & $<12.15$ \\
\hline S96T005421 & $167: 6$ & Drainable liquid & $<12$ & $<12$ & $<12$ \\
\hline S96T005683 & 167: 7 & Drainable liquid & $<12$ & $<12$ & $<12$ \\
\hline
\end{tabular}


HNF-SD-WM-ER-702 Rev. 0

Table B2-39. Tank 241-AN-103 Analytical Results: Nickel (ICP). (4 sheets)

\begin{tabular}{|c|c|c|c|c|c|}
\hline 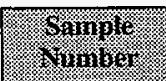 & 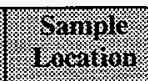 & 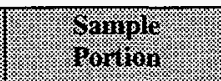 & (n) & 8)inticise & $\sqrt{1}$ \\
\hline \multicolumn{3}{|c|}{ 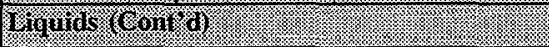 } & (Y) & Prom & 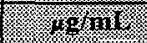 \\
\hline S96T005732 & $167: 8$ & Drainable liquid & $<12$ & $<12$ & $<12$ \\
\hline S96T005862 & 167:9 & Drainable liquid & $<12$ & $<12$ & $<12$ \\
\hline \$96T005650 & 167: 11 & Drainable liquid & $<12$ & $<12$ & $<12$ \\
\hline S96T005991 & Core 166 & Liquid composite & $<24$ & $<24$ & $<24$ \\
\hline \multicolumn{3}{|c|}{ Siniog $110510 \%$} & 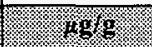 & (1) & (x) \\
\hline S96T005608 & 166: 1 & Lower half & 525 & 1,420 & $972.5^{\mathrm{QC}: \mathrm{c}}$ \\
\hline S96T005568 & 166: 4 & Lower half & $1.880 \mathrm{E}+05$ & $2.130 \mathrm{E}+05$ & $2.005 \mathrm{E}+05$ \\
\hline \$96T005611 & $166: 6$ & Lower half & 1,390 & 754 & $1,072^{\mathrm{QC}: c}$ \\
\hline S96T005835 & 166: 7 & Lower half & 2,810 & 4,010 & $3,410^{\mathrm{QC}: \mathrm{e}}$ \\
\hline S96T005836 & $166: 8$ & Lower half & 30,900 & 15,200 & $23,050^{\mathrm{QC}: \mathrm{c}}$ \\
\hline S96T005574 & 166: 9 & Lower half & $7.610 \mathrm{E}+05$ & $7.650 \mathrm{E}+05$ & $7.630 \mathrm{E}+05$ \\
\hline S96T005569 & 166: 10 & Lower half & 1,450 & 530 & $990^{\mathrm{QC}: \mathrm{c}}$ \\
\hline S96T005612 & 166: 11 & Lower half & 1,160 & 1,010 & 1,085 \\
\hline S96T005613 & 166: 12 & Lower half & $<411$ & $<405$ & $<408$ \\
\hline S96T005714 & \multirow[t]{2}{*}{$166: 13$} & Upper half & 5,620 & 12,000 & $8,810^{\text {QC:e }}$ \\
\hline S96T005720 & & Lower half & 4,000 & $1.630 \mathrm{E}+05$ & $83,500^{\mathrm{QC}: c}$ \\
\hline \$96T005414 & \multirow[t]{2}{*}{$166: 15$} & Upper half & 27,400 & 25,700 & 26,550 \\
\hline S96T005408 & & Lower half & 43,300 & 5,630 & $24,465^{\mathrm{QC}: \mathrm{e}}$ \\
\hline S96T005623 & \multirow[t]{2}{*}{$166: 16$} & Upper half & 3,480 & 3,190 & 3,335 \\
\hline S96T005614 & & Lower half & 1,450 & 1,450 & 1,450 \\
\hline S96T005415 & \multirow[t]{2}{*}{$166: 17$} & Upper half & 96,600 & 2,010 & $49,305^{\mathrm{QC:c}}$ \\
\hline S96T005409 & & Lower half & 1,910 & 76,100 & $39,005^{\mathrm{QC}: c}$ \\
\hline S96T005715 & \multirow[t]{2}{*}{$166: 18$} & Upper half & 2,270 & 3,420 & $2,845^{\mathrm{QC}: \mathrm{c}}$ \\
\hline S96T005721 & & Lower half & 1,670 & 2,250 & $1,960^{\text {QC:e }}$ \\
\hline S96T005837 & \multirow[t]{2}{*}{$166: 19$} & Upper half & 11,400 & 25,100 & $18,250^{\mathrm{QC}: \mathrm{c}}$ \\
\hline S96T005838 & & Lower half & 1,310 & 1,980 & $1,645^{\mathrm{QC}: c}$ \\
\hline \$96T005705 & \multirow[t]{2}{*}{ 167: 1} & Upper half & 3,560 & 1,110 & $2,335^{Q C: c}$ \\
\hline S96T005687 & & Lower half & 1,710 & 1,040 & $1,375^{\mathrm{QC}: c}$ \\
\hline S96T005855 & $167: 2$ & Lower half & 523 & 635 & 579 \\
\hline
\end{tabular}


punoq jaddn

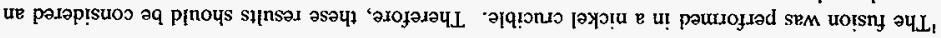

\begin{tabular}{|c|c|c|c|c|c|}
\hline $0 Z \varepsilon^{6} I$ & $09 Z^{\prime} I$ & $08 \mathcal{E}^{*} \mathrm{~T}$ & 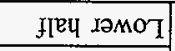 & & L6tS00L96S \\
\hline $0: 0 S^{*} \varepsilon 08$ & $\angle 8 \mathrm{~S}$ & $020^{\circ} \mathrm{I}$ & $f[\varepsilon \Psi$ Iədd $\Omega$ & $\angle I: \angle 9 I$ & $96+500 \mathrm{~L} 96 \mathrm{~S}$ \\
\hline ::00SI0'Z & $0 \angle S^{4} \mathrm{I}$ & $09 t^{\prime} z$ & JొЕप ІәMOT & \multirow[b]{2}{*}{ SI :L9I } & Z69S00L96S \\
\hline $00 \mathrm{SII} I^{\prime} \mathrm{Z}$ & $0+0^{\circ} \mathrm{I}$ & $06 I^{\prime} \varepsilon$ & Ғाеप Iəddn & & LOLSO0L96S \\
\hline ::00906 & $0 \angle Z^{*} \mathrm{I}$ & $z \downarrow s$ & I[Еप IOMOT] & \multirow[b]{2}{*}{$\nabla I: \angle 9 I$} & $\angle \mathcal{E}$ S00L96S \\
\hline a:0o $S^{\circ} I E I^{\prime} I$ & $\varepsilon \subseteq 8$ & $01 t^{6} \mathrm{I}$ & આाеप ıәdd & & $\mathcal{E} \triangleright \triangleright S 00 \mathrm{~L} 96 \mathrm{~S}$ \\
\hline $2: 00 \neq \varepsilon 9$ & $2 \neq 8$ & $92 t$ & fleप Iәмот & \multirow[b]{2}{*}{ ZI $: \angle 9 I$} & $6 \downarrow t S 00 L 96 \mathrm{~S}$ \\
\hline $099^{\prime} \mathrm{I}$ & $06 \mathrm{~S}^{6} \mathrm{I}$ & $0 \mathcal{E} L^{\prime} Y$ & $f[\varepsilon y$ ıədd $\Omega$ & & 乙† \$00L96S \\
\hline $0: 00 \mathcal{S}^{\circ} \nabla \mathcal{E}$ & $\nabla \varepsilon \nabla$ & SE9 & Iाएч хәмот & \multirow[b]{2}{*}{ II $: \angle 9 I$} & I69S00L96S \\
\hline$S I L ' I$ & $088^{6} \mathrm{I}$ & $0 S S^{\prime} I$ & I[EY Iədd $\Omega$ & & $90 \angle 500 \mathrm{~L} 96 \mathrm{~S}$ \\
\hline$: 00 S^{\circ} 08 t>$ & $\tau z \downarrow>$ & $6 \varepsilon \subseteq$ & 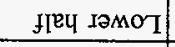 & $6: \angle 9 \mathrm{I}$ & 9S8\$00L96S \\
\hline $\operatorname{szS}^{6} \mathrm{I}$ & $0 \varepsilon \nabla^{\circ} \mathrm{I}$ & $029^{\circ} \mathrm{I}$ & Jеप дәмот & $8: \angle 9 I$ & $9 \varepsilon \angle S 00 L 96 S$ \\
\hline $0: 00 S^{*} \subseteq 60>$ & $86 \mathrm{~S}$ & $\varepsilon 6 \varepsilon>$ & J[Еप IOMOT] & $\angle: \angle 9 I$ & 069 S00I96S \\
\hline SIt> & $60 t>$ & $I Z\rangle>$ & Неч дәмот & $9: \angle 9 I$ & $8 t t S 00 L 96 S$ \\
\hline$: 00 S^{\circ} \mathcal{E} \angle 9$ & 896 & $68 \mathcal{E}$ & I[еप хәмот & $S: \angle 9 I$ & $9 \mathcal{E} \downarrow 500 L 96 \mathrm{~S}$ \\
\hline s־ \pm 29 & 599 & $\$ 8 \subseteq$ & I[ЕЧ ЈәМОТ] & $\downarrow: \angle 9 I$ & 689ऽ00L96S \\
\hline 2:00 $S^{\circ} 9 S 9$ & ZE8 & I8t & J[Еч Іәмот] & $\varepsilon: \angle 9 I$ & 889 S00L96S \\
\hline gig & (3) & (5) & r & \multicolumn{2}{|c|}{ 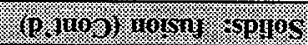 } \\
\hline 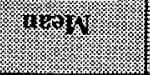 & gesnging & 195. & Hownory & Howor & 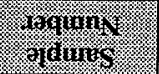 \\
\hline
\end{tabular}

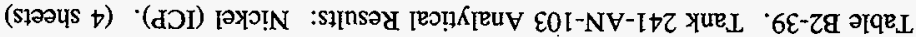


Table B2-40. Tank 241-AN-103 Analytical Results: Phosphorous (ICP). (4 sheets)

\begin{tabular}{|c|c|c|c|c|c|}
\hline Straniler & Manto & ormorion & pestith & Boylmar & 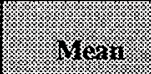 \\
\hline \multicolumn{2}{|c|}{ 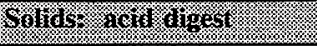 } & א. & 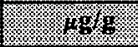 & \% & (n) \\
\hline S96T005609 & 166: 1 & Lower half & 716 & 383 & $549.5^{\mathrm{QC}: \mathrm{c}}$ \\
\hline S96T005570 & 166: 4 & Lower half & 1,370 & 917 & $1,143.5^{\mathrm{QC}: c, e}$ \\
\hline \$96T005615 & 166: 6 & Lower half & 1,340 & 1,310 & 1,325 \\
\hline S96T005839 & $166: 7$ & Lower half & 1,900 & 1,900 & 1,900 \\
\hline S96T005840 & 166: 8 & Lower half & 1,200 & 1,190 & 1,195 \\
\hline S96T005575 & $166: 9$ & Lower half & 2,230 & 1,910 & 2,070 \\
\hline S96T005571 & 166: 10 & Lower half & 1,790 & 1,750 & 1,770 \\
\hline \$96T005616 & $166: 11$ & Lower half & 1,700 & 1,550 & 1,625 \\
\hline S96T005617 & $166: 12$ & Lower half & 1,400 & 1,620 & 1,510 \\
\hline S96T005716 & \multirow[t]{2}{*}{$166: 13$} & Upper half & 570 & 527 & 548.5 \\
\hline \$96T005722 & & Lower half & 499 & 554 & 526.5 \\
\hline \$96T005416 & \multirow[t]{2}{*}{$166: 15$} & Upper half & 442 & 480 & 461 \\
\hline S97T000459 & & Lower half & 397 & 456 & 426.5 \\
\hline S96T005624 & \multirow[t]{2}{*}{$166: 16$} & Upper half & 449 & 441 & 445 \\
\hline S96T005618 & & Lower half & 505 & 490 & 497.5 \\
\hline S96T00S417 & \multirow[t]{2}{*}{$166: 17$} & Upper half & $<451$ & 465 & $<458$ \\
\hline \$96T005411 & & Lower half & 477 & 449 & $463^{Q C: c}$ \\
\hline S96T005717 & \multirow[t]{2}{*}{$166: 18$} & Upper half & 594 & 2,120 & $1,357^{\mathrm{Qc}: \mathrm{e}}$ \\
\hline S96T005723 & & Lower half & 3,010 & 1,220 & $2,115^{\mathrm{QC}: e}$ \\
\hline S96T005841 & \multirow[t]{2}{*}{ 166: 19} & Upper half & 1,080 & 1,140 & 1,110 \\
\hline S96T005842 & & Lower half & 1,200 & 1,280 & 1,240 \\
\hline S96T005708 & \multirow[t]{2}{*}{$167: 1$} & Upper half & 446 & 463 & 454.5 \\
\hline S96T005693 & & Lower half & 742 & 426 & $584^{\mathrm{QC:c}}$ \\
\hline S96T005857 & $167: 2$ & Lower half & 950 & 951 & 950.5 \\
\hline S96T005694 & 167: 3 & Lower half & 1,750 & 1,620 & 1,685 \\
\hline S96T005695 & $167: 4$ & Lower half & 2,010 & 2,200 & 2,105 \\
\hline S96T005438 & $167: 5$ & Lower half & 2,120 & 1,700 & $1,910^{\mathrm{QC:c,c}}$ \\
\hline S96T005450 & $167: 6$ & Lower half & 1,370 & 1,530 & 1,450 \\
\hline S96T005696 & 167: 7 & Lower half & 1,590 & 1,480 & 1,535 \\
\hline
\end{tabular}


HNF-SD-WM-ER-702 Rev. 0

Table B2-40. Tank 241-AN-103 Analytical Results: Phosphorous (ICP). (4 sheets)

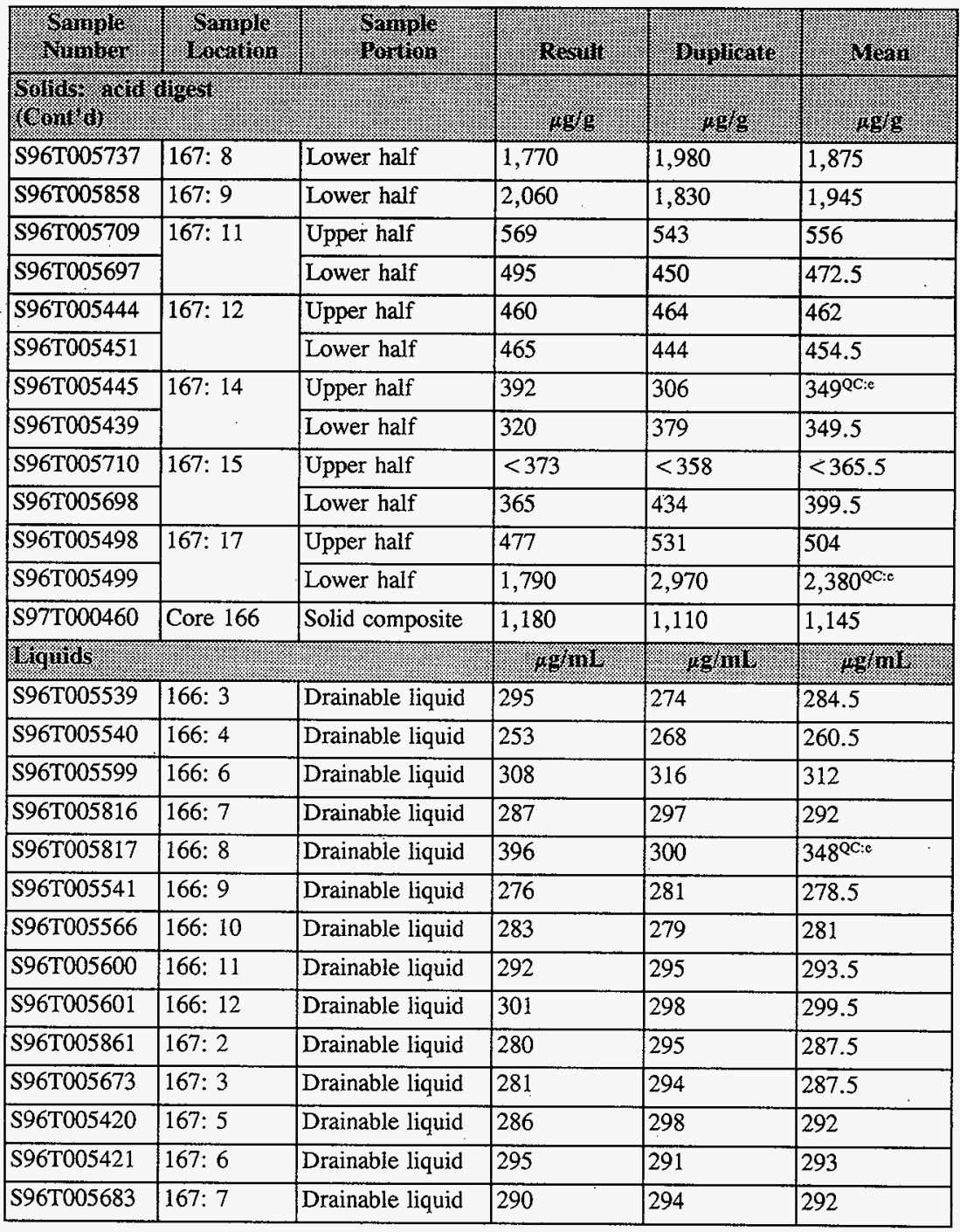


Table B2-40. Tank 241-AN-103 Analytical Results: Phosphorous (ICP). (4 sheets)

\begin{tabular}{|c|c|c|c|c|c|}
\hline 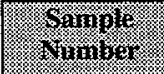 & hande & 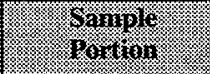 & ressint & 3ropirarie & 6yan \\
\hline \multicolumn{3}{|c|}{ 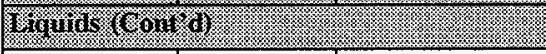 } & xgring & (6) & (2) S111 \\
\hline S96T005732 & $167: 8$ & Drainable liquid & 308 & 284 & 296 \\
\hline S96T005862 & $167: 9$ & Drainable liquid & 292 & 295 & 293.5 \\
\hline S96T005650 & 167: 11 & Drainable liquid & 278 & 279 & 278.5 \\
\hline S96T005991 & Core 166 & Liquid composite & 266 & 282 & 274 \\
\hline \multicolumn{2}{|l|}{ Solnos $145 \%$} & & . & (x) & 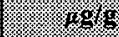 \\
\hline S96T005608 & 166: 1 & Lower half & $<4,200$ & $<4,130$ & $<4,165$ \\
\hline S96T005568 & 166: 4 & Lower half & $<4,530$ & $<4,450$ & $<4,490$ \\
\hline S96T005611 & 166: 6 & Lower half & $<4,190$ & $<4,280$ & $<4,235$ \\
\hline S96T005835 & 166: 7 & Lower half & $<4,210$ & $<4,250$ & $<4,230$ \\
\hline S96T005836 & 166: 8 & Lower half & $<3,920$ & $<4,110$ & $<4,015$ \\
\hline S96T005574 & 166: 9 & Lower half & $<3,580$ & $<3,880$ & $<3,730$ \\
\hline S96T005569 & 166: 10 & Lower half & $<4,180$ & $<4,140$ & $<4,160$ \\
\hline S96T005612 & 166: 11 & Lower half & $<3,950$ & $<4,070$ & $<4,010$ \\
\hline S96T005613 & 166: 12 & Lower half & $<4,110$ & $<4,050$ & $<4,080$ \\
\hline S96T005714 & \multirow[t]{2}{*}{ 166: 13} & Upper half & $<4,030$ & $<4,220$ & $<4,125$ \\
\hline S96T005720 & & Lower half & $<4,050$ & $<4,030$ & $<4,040$ \\
\hline S96T005414 & \multirow[t]{2}{*}{$166: 15$} & Upper half & $<913$ & $<932$ & $<922.5$ \\
\hline S96T005408 & & Lower half & $<960$ & $<927$ & $<943.5$ \\
\hline S96T005623 & \multirow[t]{2}{*}{ 166: 16} & Upper half & $<4,360$ & $<4,200$ & $<4,280$ \\
\hline \$961005614 & & Lower half & $<4,090$ & $<4,020$ & $<4,055$ \\
\hline S96T005415 & \multirow[t]{2}{*}{ 166: 17} & Upper half & $<1,050$ & $<1,060$ & $<1,055$ \\
\hline S96T005409 & & Lower half & $<4,220$ & $<4,150$ & $<4,185$ \\
\hline S96T005715 & \multirow[t]{2}{*}{ 166: 18} & Upper half & $<4,030$ & $<4,110$ & $<4,070$ \\
\hline S96T005721 & & Lower half & $<4,050$ & $<3,980$ & $<4,015$ \\
\hline S96T005837 & \multirow[t]{2}{*}{ 166: 19} & Upper half & $<4,140$ & $<4,200$ & $<4,170$ \\
\hline \$96T005838 & & Lower half & $<3,910$ & $<3,930$ & $<3,920$ \\
\hline S96T005705 & \multirow[t]{2}{*}{ 167: 1} & Upper half & $<3,980$ & $<4,010$ & $<3,995$ \\
\hline S96T005687 & & Lower half & $<4,160$ & $<4,170$ & $<4,165$ \\
\hline S96T005855 & $167: 2$ & Lower half & $<4,040$ & $<3,950$ & $<3,995$ \\
\hline
\end{tabular}




\begin{tabular}{|c|c|c|c|c|c|}
\hline $00008^{6} L>$ & $00 t^{6} \mathrm{II}$ & $00 z^{6} t>$ & J[EU IOMOT] & & $\angle 6+S 00 L 96 \mathrm{~S}$ \\
\hline$s I Z^{6} \downarrow>$ & $06 I^{6} t>>$ & $0 \nabla z^{6} \downarrow>$ & J[eY radd & $\angle I: \angle 9 I$ & $967 \$ 00 L 96 \mathrm{~S}$ \\
\hline$S 00^{6} t>$ & $0 S \varepsilon^{6} \nabla>$ & $099^{6} \varepsilon>$ & 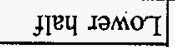 & \multirow[b]{2}{*}{ SI: $\angle 9 \mathrm{I}$} & z69S00L96S \\
\hline$S L 6^{\mathrm{C}} \varepsilon>$ & $089^{\circ} \varepsilon>$ & $0<z^{6} \nabla>$ & fleप rəddn & & $\angle 0 L S 00 L 96 \mathrm{~S}$ \\
\hline $580^{\circ} t>$ & $090^{\circ} t>$ & OII't> & Ғ[ЕЧ АәмоТ] & \multirow[b]{2}{*}{$\nabla[: \angle 9 I]$} & LEtS00L $96 \mathrm{~S}$ \\
\hline$S I \varepsilon^{\prime} \downarrow>$ & $0 z Z^{‘} \bullet>$ & $0 \pi t^{6} t>$ & 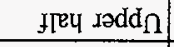 & & $\varepsilon \neq t S 00 L 96 S$ \\
\hline$\$ 66^{\circ} \mathcal{E}>$ & $0+0^{6} t>$ & $056^{\circ} \mathcal{E}>$ & IЕч Іәмот & \multirow[b]{2}{*}{$2 \mathrm{I} \cdot \angle 9 \mathrm{I}$} & $6 t t S 00 L 96 S$ \\
\hline $00 I^{\prime} \downarrow>$ & $088^{6} \varepsilon>$ & $0 z \varepsilon^{6} \phi>$ & 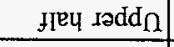 & & $z t 5 \$ 00 L 96 \mathrm{~S}$ \\
\hline $069^{6} \varepsilon>$ & $089^{\prime} \varepsilon>$ & $00 L^{\prime} \dot{E}>$ & I[EY IOMOT & \multirow[b]{2}{*}{$\mathrm{I}: \angle 9 \mathrm{I}$} & I69S00J96S \\
\hline $580^{\circ} t>$ & $0 t t^{6} \nabla>$ & $O \varepsilon L^{\prime} \varepsilon>$ & fleધ دədd $\Omega$ & & $90 \angle S 00 L 96 S$ \\
\hline $0 S 6^{\circ} \varepsilon>$ & $022^{\prime} t>$ & $089^{\circ} \mathcal{E}>$ & I[ЕЧ Іәмот & $6: \angle 9 \mathrm{I}$ & 9\$8S00L96S \\
\hline$\$ 80^{\circ} \triangleright>$ & $0 S L^{\prime} \varepsilon>$ & $0 Z \nabla^{6} \triangleright>$ & J[Еप Іәмот & $8: \angle 9 I$ & $9 \mathcal{E} L S 00 L 96 \mathrm{~S}$ \\
\hline$\underline{s}+I^{\prime} t>$ & $09 \mathcal{E}^{6} \nabla>$ & $0 \mathcal{E} 6^{\circ} \mathcal{E}>$ & flец Іәмо & $\angle: \angle 9 \mathrm{I}$ & 069\$00L96S \\
\hline $0 S I^{\prime} \nabla>$ & $060^{6} t>$ & $0 \operatorname{IZ} Z^{6} t>$ & Ғ[еЧ Іәмот & $9: \mathrm{LgI}$ & $8 t t S 00 L 96 \mathrm{~S}$ \\
\hline $0 t L^{\prime} \varepsilon>$ & $09 L^{\prime} E>$ & $0 Z L^{\prime} \varepsilon>$ & Ғ[Еप IәMOТ] & $s: \angle 9 I$ & $9 \varepsilon+S 00 L 96 S$ \\
\hline $0 I \tau^{\prime} \downarrow>$ & $0 z z^{\prime} \triangleright>$ & $00 \tau^{4} t>$ & Ilе4 дәмот & $t: \angle 9 \mathrm{I}$ & 689 S00L96S \\
\hline $08 \mathcal{E}^{6} \downarrow>$ & $09 z^{\prime} t>$ & $00 S^{\prime} \nabla>$ & I[एบ Іәмот] & $\varepsilon: \angle 9 I$ & $889500 \mathrm{~L} 96 \mathrm{~S}$ \\
\hline (1) & 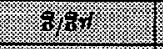 & (3) & & \multicolumn{2}{|c|}{ 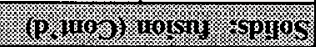 } \\
\hline 10 & 916916190 & 3isory & 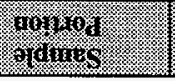 & 3\%19) & 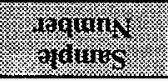 \\
\hline
\end{tabular}

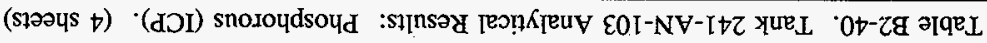


Table B2-41. Tank 241-AN-103 Analytical Results: Potassium (ICP). (3 sheets)

\begin{tabular}{|c|c|c|c|c|c|}
\hline 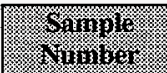 & Mampies & 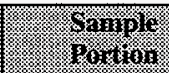 & 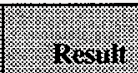 & 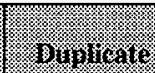 & (1) \\
\hline \multicolumn{2}{|c|}{ 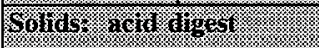 } & & 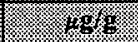 & 40 & (3) \\
\hline S96T005609 & $166: 1$ & Lower half & 6,610 & 5,830 & 6,220 \\
\hline \$96T005570 & 166: 4 & Lower half & 3,960 & 4,230 & 4,095 \\
\hline S96T005615 & $166: 6$ & Lower half & 4,770 & 4,880 & 4,825 \\
\hline S96T005839 & 166: 7 & Lower half & 3,990 & 4,320 & 4,155 \\
\hline S96T005840 & $166: 8$ & Lower half & 4,100 & 4,040 & $4,070^{\mathrm{QC:a}}$ \\
\hline S96T005575 & 166: 9 & Lower half & 7,680 & 6,790 & 7,235 \\
\hline \$96T005571 & 166: 10 & Lower half & 5,750 & 5,630 & 5,690 \\
\hline S96T005616 & 166: 1 & Lower half & 4,410 & 3,870 & 4,140 \\
\hline S96T005617 & 166: 2 & Lower half & 3,960 & 4,240 & 4,100 \\
\hline S96T005716 & \multirow{2}{*}{ 166: 3} & Upper half & 8,970 & 9,070 & 9,020 \\
\hline S96T005722 & & Lower half & 7,270 & 9,080 & $8,175^{\mathrm{QC:c}}$ \\
\hline S96T005416 & \multirow[t]{2}{*}{ 166: 5} & Upper half & 6,740 & 7,420 & 7,080 \\
\hline S97T000459 & & Lower half & 5,740 & 6,670 & $6,205^{Q \mathrm{C}: \mathrm{d}}$ \\
\hline S96T005624 & \multirow[t]{2}{*}{ 166: 6} & Upper half & 6,680 & 6,960 & 6,820 \\
\hline S96T005618 & & Lower half & 7,060 & 6,960 & 7,010 \\
\hline S96T005417 & \multirow[t]{2}{*}{ 166: 7} & Upper half & 5,590 & 5,560 & 5,575 \\
\hline S96T005411 & & Lower half & 5,000 & 5,530 & 5,265 \\
\hline \$96T005717 & \multirow[t]{2}{*}{ 166: 8} & Upper half & 5,730 & 5,740 & 5,735 \\
\hline \$96T005723 & & Lower half & 5,670 & 5,880 & 5,775 \\
\hline S96T005841 & \multirow[t]{2}{*}{$166: 9$} & Upper half & 5,990 & 6,090 & $6,040^{\mathrm{QC:a}}$ \\
\hline \$96T005842 & & Lower half & 5,680 & 5,630 & $5,655^{\mathrm{QC:a}}$ \\
\hline S96T005708 & \multirow[t]{2}{*}{ 167: 1} & Upper half & 4,590 & 6,330 & $5,460^{\mathrm{QC:c}}$ \\
\hline S96T005693 & & Lower half & 6,600 & 6,770 & 6,685 \\
\hline \$96T005857 & $167: 2$ & Lower half & 6,140 & 6,360 & 6,250 \\
\hline S96T005694 & 167: 3 & Lower half & 6,350 & 4,300 & $5,325^{\mathrm{QC:e}}$ \\
\hline \$96T005695 & $167: 4$ & Lower half & 5,770 & 6,360 & 6,065 \\
\hline S96T005438 & $167: 5$ & Lower half & 8,770 & 8,210 & $8,490^{Q C a a, c}$ \\
\hline S96T005450 & $167: 6$ & Lower half & 7,290 & 7,660 & $7,475^{\mathrm{QC:a}}$ \\
\hline S96T005696 & $167: 7$ & Lower half & 4,060 & 4,090 & 4,075 \\
\hline
\end{tabular}




\begin{tabular}{|c|c|c|c|c|c|}
\hline $00 L^{\prime} 8 \mathrm{I}$ & $00 S^{\prime} 8 \mathrm{I}$ & $006^{\circ} 8 \mathrm{I}$ & p̣nb!̣ әqеuाexa & $\angle: \angle 9 I$ & E89S00L96S \\
\hline $00 z^{\prime} \angle I$ & $00 Z^{6} \angle \mathrm{I}$ & $00 \tau^{\prime} \angle I$ & p!nbII әqеurexa & $9: \angle 9 \mathrm{I}$ & IZ†S00L96S \\
\hline $\mathrm{p}: 000 \mathrm{OSZ} z^{\prime} 81$ & $00 t^{6} 8 I$ & $00 I^{\prime} 8 \mathrm{I}$ & 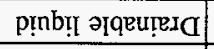 & $\varsigma: \angle 9 I$ & $0 Z+S 00 L 96 S$ \\
\hline $009^{\circ} 9 \mathrm{I}$ & $009^{6} 9 \mathrm{I}$ & $009^{\circ} 9 \mathrm{I}$ & prnnbụ әqeurex & $\varepsilon: \angle 9 l$ & EL9S00L96S \\
\hline OSt'LI & $00 L^{\prime} L I$ & $00 z^{6} \angle \mathrm{I}$ & pinb! әq әрu!exa & $\tau: \angle 9 \mathrm{I}$ & [98SO0L96S \\
\hline $0: 000566^{\circ} 81$ & $000^{\prime} 61$ & $006^{\prime} 8 \mathrm{I}$ & pụb!̣ әрqеu!esa & $z: 991$ & 109500L96S \\
\hline $00 L^{\circ} \mathrm{LI}$ & $00 S^{\prime} L I$ & $006^{\circ} \angle I$ & 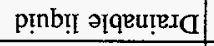 & I :991 & $009500 \mathrm{~L} 96 \mathrm{~S}$ \\
\hline $0 S^{\prime} \angle I$ & $00 \varepsilon^{\prime} \angle I$ & $008^{\circ} \mathrm{LI}$ & 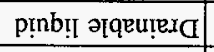 & OI: $: 99 \mathrm{I}$ & $99 S S 00 \mathrm{~L} 96 \mathrm{~S}$ \\
\hline$\triangle: 000 S Z^{\prime} \angle I$ & $00 I^{\prime} \angle I$ & $00 t^{6} \angle I$ & p̣̣nb!̣ әqqeurexa & $6: 99 \mathrm{I}$ & ItSS00L96S \\
\hline $00 L^{\prime} \angle I$ & $00 E^{\prime} 8 \mathrm{I}$ & $00 I^{\circ} L I$ & 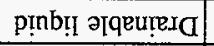 & $8: 99 \mathrm{I}$ & LI8S00L96S \\
\hline$p: 2000 \varepsilon^{\prime} 8 \mathrm{~L}$ & $009^{\prime} 8 \mathrm{I}$ & $000^{\prime} 8 \mathrm{I}$ & p̣nbț ə[qвuाexa & $L: 99 \mathrm{I}$ & 9I8S00.L96S \\
\hline $0: 0000 L^{\prime} 8 \mathrm{I}$ & $00 t^{\prime} 8 \mathrm{I}$ & $000^{\prime} 6 \mathrm{I}$ & p!nḅ̣l әqquurexa & $9: 99$ I & $66 \$ 500 \mathrm{~L} 96 \mathrm{~S}$ \\
\hline $006^{6}+I$ & $00 z^{6}+1$ & $009^{\circ} \mathrm{SI}$ & p̣nbț әqqeutesd & $\nabla: 99 \mathrm{I}$ & $0+S \$ 00 L 96 \mathrm{~S}$ \\
\hline$O S L^{\prime} L I$ & $009^{\circ} \mathrm{LI}$ & $006^{\circ} \angle I$ & pinb!I әqвuाera & $\varepsilon: 99 \mathrm{I}$ & 6ES\$00L96S \\
\hline Mator: & (14) & rayoi: & 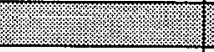 & 4 & spriby \\
\hline $0+s^{\circ} 9$ & $008^{\prime} 9$ & $08 \tau^{\prime} 9$ & วң!soduos p!̣os & 99 I ә10ว & $09+000 \mathrm{LL6S}$ \\
\hline $0 \mathrm{~L} z^{\circ} \mathrm{s}$ & $0 S S^{\prime} s$ & $0 \angle 8^{\circ} \mathrm{t}$ & Уге дәмо I & \multirow[b]{2}{*}{$\angle: \angle 9 I$} & $66+S 00 \mathrm{~L} 96 \mathrm{~S}$ \\
\hline$s z 6^{\circ} \mathrm{t}$ & $0 \pm 8^{6} t$ & $010^{\circ} \mathrm{s}$ & $\mathrm{J}[\mathrm{EY}$ גədd $\Omega$ & & $86+S 00 \mathrm{~L} 96 \mathrm{~S}$ \\
\hline $0 \varepsilon \varepsilon^{\prime} s$ & $0+9^{\circ} \mathrm{s}$ & $020^{\circ} \mathrm{s}$ & J[एч Iәмо $\mathrm{T}$ & \multirow[b]{2}{*}{$\varsigma: \angle 9 \mathrm{I}$} & $869 \subseteq 00 \mathrm{~L} 96 \mathrm{~S}$ \\
\hline $0+8^{6} t$ & $0 \pm 8^{\circ}$ & $0+8^{\circ} t$ & 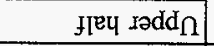 & & $0 I \angle S 00 L 96 S$ \\
\hline$x=000<t^{\circ} t$ & $0 \varepsilon 8^{\prime} \mathrm{D}$ & oil't & I[еप ХOMOT] & \multirow[b]{2}{*}{$\nabla: \angle 9 I$} & $6 \varepsilon+S 00 \mathrm{~L} 96 \mathrm{~S}$ \\
\hline $\mathrm{k}: 00090^{\circ} \mathrm{s}$ & $099 \div$ & $09 t^{\prime} s$ & fleप Iədd $\Omega$ & & SttS00.L96S \\
\hline : $: 00 \Omega \varepsilon^{\prime} L$ & $09 \varepsilon^{\prime} L$ & $00 \varepsilon^{\circ} L$ & У[еч гәмо Т] & \multirow[b]{2}{*}{$Z: \angle 9 I$} & IStS00L96S \\
\hline : $: 00 \mathrm{~S}^{\circ} L^{\prime} 8$ & OE9'8 & $08 L^{\prime} 8$ & Irey radd $\mathrm{\Omega}$ & & $t t t S 00 \mathrm{~L} 96 \mathrm{~S}$ \\
\hline $010^{6} 8$ & $069^{\circ} \mathrm{L}$ & $0 \varepsilon \varepsilon^{\prime} 8$ & Ғтеч Х्моТ & \multirow[b]{2}{*}{$\mathrm{I}: \angle 9 \mathrm{I}$} & L69S00L96S \\
\hline $000^{\circ} 6$ & $0 \angle \varepsilon^{\prime} 6$ & $0 \varepsilon 9^{\circ} 8$ & freч Iədd & & $60 \angle 500 L 96 \mathrm{~S}$ \\
\hline$s \varepsilon \tau^{\prime} t$ & $0 z z^{\circ} t$ & $0 \operatorname{sz}^{6} t$ & I[Еч ІәмоТ] & $6: \angle 9 I$ & $8 \$ 8 S 00 L 96 \mathrm{~S}$ \\
\hline$S 6 I^{*} t$ & $086^{\circ} \varepsilon$ & $0\left[t^{c} t\right.$ & Ғ[еч Іәмо Т & $8: \angle 9 I$ & $\angle E L S 00.196 S$ \\
\hline 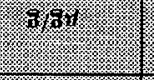 & 7. & (2) & & \multicolumn{2}{|c|}{ 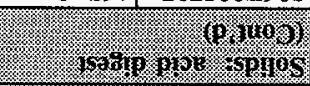 } \\
\hline neral & 2) & Iyasyod: & $\begin{array}{l}\text { roy.jugd } \\
\text { odrues }\end{array}$ & $\begin{array}{l}\text { orgurou } \\
\text { ordines }\end{array}$ & $\begin{array}{l}\text { wainur } \\
\text { Winins }\end{array}$ \\
\hline
\end{tabular}

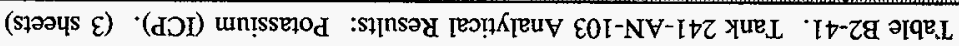




\begin{tabular}{|c|c|c|c|c|c|}
\hline $0 S Z^{\prime} \angle I$ & $00 \varepsilon^{\prime} \angle I$ & $00 Z^{\prime} L I$ & əl!soduos p!̣nb!T & $99 \mathrm{I}$ วI0 & I66S00L96S \\
\hline $0 S \mathcal{E}^{\prime} \mathrm{SI}$ & $00 Z^{\prime} \mathrm{sI}$ & $00 S^{\prime} S I$ & p!̣nb!̣t əqueuteda & $I: \angle 9 I$ & 0\$9500L96S \\
\hline $00 L^{\prime} \angle I$ & $008^{\circ} \mathrm{LI}$ & $009^{\circ} \angle I$ & 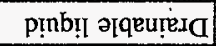 & $6: \angle 9 \mathrm{I}$ & 298ऽ00L96S \\
\hline $0 \$ 9^{\circ} 8 I$ & $00 S^{\prime} 8 \mathrm{I}$ & $008^{\circ} 81$ & 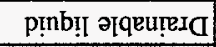 & $8: L 9 T$ & ZELS00.L96S \\
\hline Xingis: & mor \% & gurgin: & & 10 & nof sunning \\
\hline arean & 893890160 & & moror & mojorous & $\begin{array}{l}\text { aggumy } \\
\text { ajtines }\end{array}$ \\
\hline
\end{tabular}

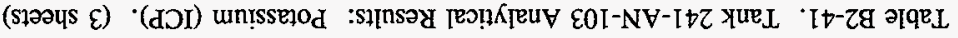


Table B2-42. Tank 241-AN-103 Analytical Results: Selenium (ICP). (4 sheets)

\begin{tabular}{|c|c|c|c|c|c|}
\hline 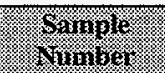 & Saringle & 3.7\% & Resing & 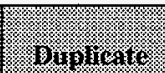 & minan \\
\hline \multicolumn{2}{|c|}{ 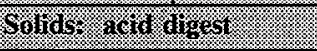 } & 新 & . & (19) & (6. \\
\hline S96T005609 & 166: 1 & Lower half & 171 & $<151$ & $<161^{\mathrm{QC:a}}$ \\
\hline S96T005570 & 166: 4 & Lower half & $<179$ & $<179$ & $<179$ \\
\hline S96T005615 & $166: 6$ & Lower half & $<138$ & $<138$ & $<138^{\mathrm{QC:a}}$ \\
\hline S96T005839 & 166: 7 & Lower half & $<202$ & $<205$ & $<203.5^{\mathrm{QC}: \mathrm{a}}$ \\
\hline S96T005840 & $166: 8$ & Lower half & $<197$ & 216 & $<206.5^{\text {QC:a }}$ \\
\hline$\$ 96 \mathrm{~T} 005575$ & 166: 9 & Lower half & $<196$ & $<199$ & $<197.5$ \\
\hline \$96T005571 & $166: 10$ & Lower half & $<153$ & $<145$ & $<149^{\mathrm{QC:a}}$ \\
\hline S96T005616 & $166: 1$ & Lower half & $<145$ & $<136$ & $<140.5^{\mathrm{QC}: \mathrm{a}}$ \\
\hline S96T005617 & $166: 2$ & Lower half & $<141$ & $<141$ & $<141^{\text {QC:a }}$ \\
\hline \$96T005716 & \multirow[t]{2}{*}{ 166: 3} & Upper half & $<194$ & $<196$ & $<195^{\mathrm{QC}: \mathrm{a}}$ \\
\hline S96T005722 & & Lower half & $<206$ & $<206$ & $<206^{\mathrm{QC}: \mathrm{a}}$ \\
\hline$\$ 96 T 005416$ & \multirow[t]{2}{*}{$166: 5$} & Upper half & $<204$ & $<190$ & $<197$ \\
\hline S97T000459 & & Lower half & $<56.9$ & $<55.7$ & $<56.3^{\mathrm{QC}: \mathrm{a}}$ \\
\hline S96T005624 & \multirow[t]{2}{*}{$166: 6$} & Upper half & $<210$ & $<215$ & $<212.5^{\mathrm{QC:a}}$ \\
\hline S96T005618 & & Lower half & $<142$ & $<146$ & $<144^{\mathrm{QC}: \mathrm{a}}$ \\
\hline S96T005417 & \multirow[t]{2}{*}{ 166: 7} & Upper half & $<225$ & $<205$ & $<215$ \\
\hline S96T005411 & & Lower half & $<190$ & $<176$ & $<183$ \\
\hline S96T005717 & \multirow[t]{2}{*}{$166: 8$} & Upper half & $<208$ & $<202$ & $<205^{\mathrm{QC}: a}$ \\
\hline \$96T005723 & & Lower half & $<202$ & $<198$ & $<200^{\mathrm{QC}: \mathrm{a}}$ \\
\hline S96T005841 & \multirow[t]{2}{*}{$166: 9$} & Upper half & $<193$ & $<196$ & $<194.5^{\mathrm{QC:a}}$ \\
\hline \$96T005842 & & Lower half & $<178$ & 265 & $<221.5^{\mathrm{QC:a,e}}$ \\
\hline S96T005708 & \multirow[t]{2}{*}{ 167: 1} & Upper half & 251 & 268 & 259.5 \\
\hline S96T005693 & & Lower half & 357 & 203 & $280^{\mathrm{QC}: \mathrm{e}}$ \\
\hline S96T005857 & $167: 2$ & Lower half & 250 & 337 & $293.5^{\text {QC:e }}$ \\
\hline S96T005694 & $167: 3$ & Lower half & $<180$ & $<187$ & $<183.5$ \\
\hline S96T005695 & $167: 4$ & Lower half & $<198$ & $<204$ & $<201$ \\
\hline S96T005438 & $167: 5$ & Lower half & $<51.7$ & $<55.1$ & $<53.4^{\mathrm{QC:a}}$ \\
\hline S96T005450 & 167: 6 & Lower half & $<55.3$ & $<52.2$ & $<53.75^{\mathrm{QC}: \mathrm{a}}$ \\
\hline S96T005696 & $167: 7$ & Lower half & $<192$ & $<188$ & $<190^{\mathrm{QC}: \mathrm{a}}$ \\
\hline
\end{tabular}


Table B2-42. Tank 241-AN-103 Analytical Results: Selenium (ICP). (4 sheets)

\begin{tabular}{|c|c|c|c|c|c|}
\hline Mainore & Shorition & 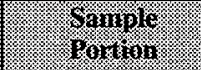 & resing & S) 1nitinge & Won \\
\hline 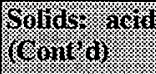 & Husest & & $6.69 \mathrm{gg}$ & 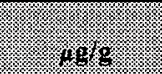 & (1) \\
\hline S96T005737 & 167: 8 & Lower half & $<196$ & $<200$ & $<198^{\mathrm{QC}: \mathrm{a}}$ \\
\hline S96T005858 & 167: 9 & Lower half & 246 & 176 & $211^{\mathrm{QC}: \mathrm{e}}$ \\
\hline S96T005709 & \multirow[t]{2}{*}{ 167: 1} & Upper half & $<180$ & $<187$ & $<183.5^{\mathrm{QC:a}}$ \\
\hline S96T005697 & & Lower half & $<198$ & $<188$ & $<193^{\mathrm{QC:a}}$ \\
\hline S96T005444 & \multirow[t]{2}{*}{ 167: 2} & Upper half & $<55.3$ & $<54.5$ & $<54.9^{\mathrm{QC}: \mathrm{a}}$ \\
\hline \$96T005451 & & Lower half & $<56.8$ & $<58.4$ & $<57.6^{\mathrm{QC:a}}$ \\
\hline S96T005445 & \multirow[t]{2}{*}{ 167: 4} & Upper half & $<52.8$ & $<55.1$ & $<53.95^{\mathrm{QC}: \mathrm{a}}$ \\
\hline S96T005439 & & Lower half & $<56.8$ & $<58.4$ & $<57.6^{\mathrm{QC:a}}$ \\
\hline \$96T005710 & \multirow[t]{2}{*}{$167: 5$} & Upper half & $<186$ & $<179$ & $<182.5^{\mathrm{QC}: \mathrm{a}}$ \\
\hline S96T005698 & & Lower half & $<182$ & $<188$ & $<185^{\mathrm{QC:a}}$ \\
\hline S96T005498 & \multirow[t]{2}{*}{ 167: 7} & Upper half & $<183$ & 215 & $<199$ \\
\hline S96T005499 & & Lower half & 275 & 239 & 257 \\
\hline S97T000460 & Core 166 & Solid composite & $<56.3$ & $<55.1$ & $<55.7^{\mathrm{QC}: \mathrm{a}}$ \\
\hline grimis & & & 19.6\% & 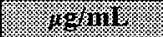 & . \\
\hline \$96T005539 & 166: 3 & Drainable liquid & $<40.1$ & $<40.1$ & $<40.1$ \\
\hline S96T005540 & 166: 4 & Drainable liquid & $<40.1$ & 56.2 & $<48.15^{\mathrm{QC}: c}$ \\
\hline S96T005599 & 166: 6 & Drainable liquid & $<60.1$ & $<60.1$ & $<60.1$ \\
\hline S96T005816 & 166: 7 & Drainable liquid & $<60.1$ & $<60.1$ & $<60.1$ \\
\hline S96T005817 & 166: 8 & Drainable liquid & $<60.1$ & $<60.1$ & $<60.1$ \\
\hline S96T005541 & 166: 9 & Drainable liquid & $<60.1$ & $<60.1$ & $<60.1$ \\
\hline S96T005566 & 166: 10 & Drainable liquid & $<40.1$ & $<40.1$ & $<40.1$ \\
\hline S96T005600 & 166: 1 & Drainable liquid & $<60.1$ & $<60.1$ & $<60.1$ \\
\hline \$96T005601 & 166: 2 & Drainable liquid & $<60.1$ & $<60.1$ & $<60.1$ \\
\hline S96T005861 & 167: 2 & Drainable liquid & $<60.1$ & $<60.1$ & $<60.1$ \\
\hline S96T005673 & 167: 3 & Drainable liquid & $<60.1$ & $<60.1$ & $<60.1$ \\
\hline S96T005420 & 167:5 & Drainable liquid & $<60.1$ & $<60.1$ & $<60.1$ \\
\hline S96T005421 & 167:6 & Drainable liquid & $<60.1$ & $<60.1$ & $<60.1$ \\
\hline S96T005683 & $167: 7$ & Drainable liquid & $<60.1$ & $<60.1$ & $<60.1$ \\
\hline
\end{tabular}


Table B2-42. Tank 241-AN-103 Analytical Results: Selenium (ICP). (4 sheets)

\begin{tabular}{|c|c|c|c|c|c|}
\hline 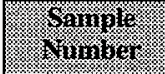 & \%omplo & f & R & 9.14) & (4) \\
\hline \multicolumn{3}{|c|}{ Mojidis rom } & . & (3) & \% \\
\hline \$96T005732 & $167: 8$ & Drainable liquid & $<60.1$ & $<60.1$ & $<60.1$ \\
\hline S96T005862 & 167: 9 & Drainable liquid & $<60.1$ & $<60.1$ & $<60.1$ \\
\hline S96T005650 & 167: 1 & Drainable liquid & $<60.1$ & $<60.1$ & $<60.1$ \\
\hline \$96T005991 & Core 166 & Liquid composite & 126 & $<120$ & $<123$ \\
\hline \multicolumn{3}{|c|}{ 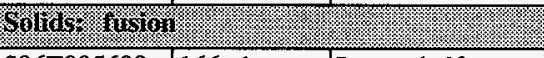 } & 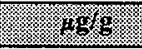 & 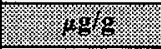 & (1) \\
\hline S96T005608 & 166: 1 & Lower half & $<2,100$ & $<2,060$ & $<2,080$ \\
\hline S96T005568 & 166: 4 & Lower half & $<2,270$ & $<2,220$ & $<2,245$ \\
\hline \$96T005611 & 166: 6 & Lower half & $<2,100$ & $<2,140$ & $<2,120$ \\
\hline S96T005835 & 166: 7 & Lower half. & $<2,100$ & $<2,120$ & $<2,110$ \\
\hline S96T005836 & 166: 8 & Lower half & $<1,960$ & $<2,060$ & $<2,010$ \\
\hline S96T005574 & 166: 9 & Lower half & $<1,790$ & $<1,940$ & $<1,865$ \\
\hline S96T005569 & $166: 10$ & Lower half & $<2,090$ & $<2,070$ & $<2,080$ \\
\hline \$96T005612 & 166: 1 & Lower half & $<1,970$ & $<2,040$ & $<2,005$ \\
\hline S96T005613 & 166: 2 & Lower half & $<2,050$ & $<2,020$ & $<2,035$ \\
\hline S96T005714 & \multirow[t]{2}{*}{$166: 3$} & Upper half & $<2,010$ & $<2,110$ & $<2,060$. \\
\hline S96T005720 & & Lower half & $<2,030$ & $<2,010$ & $<2,020$ \\
\hline \$96T005414 & \multirow[t]{2}{*}{$166: 5$} & Upper half & $<456$ & $<466$ & $<461$ \\
\hline S96T005408 & & Lower half & $<480$ & $<463$ & $<471.5$ \\
\hline \$96T005623 & \multirow[t]{2}{*}{ 166: 6} & Upper half & $<2,180$ & $<2,100$ & $<2,140$ \\
\hline S96T005614 & & Lower half & $<2,040$ & $<2,010$ & $<2,025$ \\
\hline S96T005415 & \multirow[t]{2}{*}{$166: 7$} & Upper half & $<523$ & $<528$ & $<525.5$ \\
\hline S96T005409 & & Lower half & $<2,110$ & $<2,080$ & $<2,095$ \\
\hline S96T005715 & \multirow[t]{2}{*}{$166: 8$} & Upper half & $<2,010$ & $<2,060$ & $<2,035$ \\
\hline S96T005721 & & Lower half & $<2,030$ & $<1,990$ & $<2,010$ \\
\hline S96T005837 & \multirow[t]{2}{*}{$166: 9$} & Upper half & $<2,070$ & $<2,100$ & $<2,085$ \\
\hline S96T005838 & & Lower half & $<1,960$ & $<1,960$ & $<1,960$ \\
\hline \$96T005705 & \multirow[t]{2}{*}{ 167: 1} & Upper half & $<1,990$ & $<2,000$ & $<1,995$ \\
\hline S96T005687 & & Lower half & $<2,080$ & $<2,090$ & $<2,085$ \\
\hline S96T005855 & 167:2 & Lower half & $<2,020$ & $<1,980$ & $<2,000$ \\
\hline
\end{tabular}




\begin{tabular}{|c|c|c|c|c|c|}
\hline $\operatorname{sol} I^{\prime} z>$ & $0\left[I^{6} Z>\right.$ & $00 I^{\prime} z>$ & Jley دәмо & & $\angle 67 S 00 \mathrm{~L} 96 \mathrm{~S}$ \\
\hline $0 I^{\prime} z>$ & $00 I^{6} z>$ & $0 Z I^{\prime} z>$ & Ipeu xədd & $\angle: \angle 9 \mathrm{I}$ & 967 S00L $96 \mathrm{~S}$ \\
\hline $500^{\circ} z>$ & $08 I^{\prime} z>$ & $0 \varepsilon 8^{\prime} \mathrm{I}>$ & IEบ Гәмот & \multirow[b]{2}{*}{$S: \angle 9 I$} & Z69S00L96S \\
\hline $586^{6} \mathrm{I}>$ & $0+8^{\circ} I>$ & $0 \varepsilon I^{\prime} Z>$ & न[еप xәdd & & $\angle 0 \angle S 00 L 96 \mathrm{~S}$ \\
\hline $0+0^{6} z>$ & $0 \varepsilon 0^{\circ} \tau>$ & $0 s 0^{\prime} \tau>$ & $\mathrm{I}^{\varepsilon \mathrm{Y}}$ IวMOT & \multirow[b]{2}{*}{$\nabla: \angle 9 \mathrm{I}$} & $\angle E t S 00 L 96 S$ \\
\hline$s I^{\prime} Z>$ & oll'z> & $00 z^{\prime} z>$ & Itey Iədd $\Omega$ & & EttS00L96S \\
\hline $000^{\prime} z>$ & $0 z 0^{6} z>$ & $086^{6} \mathrm{I}>$ & J[eц دәмот] & \multirow[b]{2}{*}{$\tau: \angle 9 I$} & $6 t+500 \mathrm{~L} 96 \mathrm{~S}$ \\
\hline $0 s 0^{\circ} z>$ & $076^{6} \mathrm{I}>$ & $09 r^{6} z>$ & Jॉeप دәdd & & Z†tS00L96S \\
\hline$S t 8^{\circ} \mathrm{I}>$ & $078^{\prime} \mathrm{I}>$ & $0 S 8^{\circ} \mathrm{I}>$ & IfЕप IәMOT & \multirow[b]{2}{*}{$1: \angle 9 I$} & I69S00L96S \\
\hline$s t 0^{\prime} z>$ & $0 z z^{\prime} z>$ & $0 \angle 8^{6} \mathrm{I}>$ & flEบ Iədd $\Omega$ & & $90 \angle S 00 L 96 S$ \\
\hline SL6'I $I>$ & OII'Z> & $0+8^{6} \mathrm{I}>$ & ذ[ЕЧ Іәмот & $6: \angle 9 I$ & $9 \$ 8 \$ 00 L 96 S$ \\
\hline $0+00^{\circ} z>$ & $0 \angle 8^{+} I>$ & $01 z^{6} z>$ & 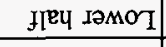 & $8: \angle 9 I$ & $9 \& L S 00 L 96 S$ \\
\hline $0 \angle 0^{\circ} z>$ & $08 I^{\prime} z>$ & $096^{6} I>$ & $f{ }^{84}$ Iәмот & $\angle: \angle 9 I$ & 069\$00L96S \\
\hline$S \angle 0^{\prime} Z>$ & $0+0^{\circ} z>$ & 0 II' $Z>$ & I[eप Iәмо T & $9: \angle 9 I$ & $87 t 500 \mathrm{~L} 96 \mathrm{~S}$ \\
\hline $0 \angle 8^{4} I>$ & $088^{4} \mathrm{I}>$ & $098^{6} \mathrm{I}>$ & fleप Іәмо T & $S: \angle 9 T$ & $9 \mathcal{E} \supset S 00 \mathrm{~L} 96 \mathrm{~S}$ \\
\hline $\mathrm{sOI}^{+} z>$ & $0 I^{\prime} Z>$ & $00 I^{\prime} Z>$ & Ileप IәMOT & $\pm: \angle 9 I$ & 689S00L96S \\
\hline $06 I^{\prime} z>$ & $0 E I^{-6} \tau>$ & $0 S Z^{6} Z>$ & Ireप IәмоT & $\varepsilon \cdot: \angle 9 I$ & $889900 \mathrm{~L} 96 \mathrm{~S}$ \\
\hline 3. & 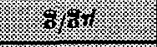 & (4) & & \multicolumn{2}{|c|}{ 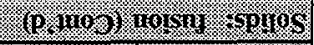 } \\
\hline 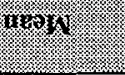 & Horugrog & Hist 8 & tom & 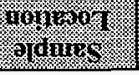 & molutinge \\
\hline
\end{tabular}

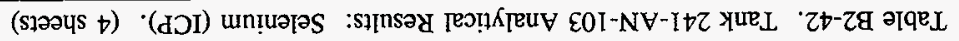


HNF-SD-WM-ER-702 Rev. 0

Table B2-43. Tank 241-AN-103 Analytical Results: Silicon (ICP). (4 sheets)

\begin{tabular}{|c|c|c|c|c|c|}
\hline (6) & 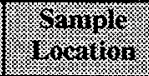 & 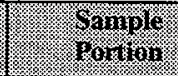 & Resull. & Holingit & wealin \\
\hline \multicolumn{2}{|c|}{ 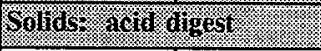 } & & (1) & 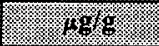 & (3) \\
\hline S96T005609 & 166: 1 & Lower half & 642 & 651 & $646.5^{\text {QC:b }}$ \\
\hline S96T005570 & 166: 4 & Lower half & 104 & 178 & $141^{\mathrm{QC:a,e}}$ \\
\hline S96T005615 & $166: 6$ & Lower half & 297 & 310 & $303.5^{\text {QC:b }}$ \\
\hline S96T005839 & 166: 7 & Lower half & 1,000 & 1,040 & $1,020^{\mathrm{QC}: \mathrm{b}}$ \\
\hline S96T005840 & $166: 8$ & Lower half & 348 & 589 & $468.5^{\mathrm{QC}: b, e}$ \\
\hline S96T005575 & $166: 9$ & Lower half & 199 & 211 & $205^{Q C: a}$ \\
\hline \$96T005571 & $166: 10$ & Lower half & 380 & 363 & $371.5^{Q C: b}$ \\
\hline \$96T005616 & $166: 1$ & Lower half & 316 & 366 & $341^{\mathrm{QC:b}}$ \\
\hline S96T005617 & $166: 2$ & Lower half & 317 & 367 & $342^{\text {C: }: b}$ \\
\hline S96T005716 & \multirow[t]{2}{*}{$166: 3$} & Upper half & 691 & 594 & $642.5^{\mathrm{QC}: \mathrm{b}}$ \\
\hline S96T00S722 & & Lower half & 618 & 665 & $641.5^{\mathrm{QC}: \mathrm{b}}$ \\
\hline S96T005416 & \multirow[t]{2}{*}{$166: 5$} & Upper half & 307 & 306 & $306.5^{\mathrm{QC}: \mathrm{a}}$ \\
\hline S97T000459 & & Lower half & 785 & 999 & $892^{\mathrm{Qc}: b, c}$ \\
\hline S96T005624 & \multirow[t]{2}{*}{ 166: 6} & Upper half & 659 & 639 & $649^{Q C: b}$ \\
\hline S96T005618 & & Lower half & 469 & 361 & $415^{\mathrm{QC}: b, \mathrm{c}}$ \\
\hline S96T005417 & \multirow[t]{2}{*}{$166: 7$} & Upper half & 321 & 385 & $353^{Q C: a}$ \\
\hline S96T005411 & & Lower half & 327 & 296 & $311.5^{\mathrm{QC:a,c}}$ \\
\hline \$96T005717 & \multirow[t]{2}{*}{ 166: 8} & Upper half & 563 & 632 & $597.5^{\text {QC:b }}$ \\
\hline S96T005723 & & Lower half & 604 & 587 & $595.5^{0 C: b}$ \\
\hline S96T005841 & \multirow[t]{2}{*}{ 166: 9} & Upper half & 204 & 217 & $210.5^{\mathrm{QC}: \mathrm{b}}$ \\
\hline S96T005842 & & Lower half & 451 & 624 & $537.5^{\mathrm{QC:b,c}}$ \\
\hline S96T005708 & \multirow[t]{2}{*}{ 167: 1} & Upper half & 375 & 672 & $523.5^{\mathrm{QC:b,e}}$ \\
\hline S96T005693 & & Lower half & 656 & 746 & $701^{\mathrm{QC}: \mathrm{b}}$ \\
\hline S96T005857 & 167: 2 & Lower half & 466 & 629 & $547.5^{\alpha \mathrm{C}: b, c}$ \\
\hline S96T005694 & $167: 3$ & Lower half & 553 & 403 & $478^{\mathrm{QC}: \mathrm{b}, \mathrm{c}}$ \\
\hline S96T005695 & 167: 4 & Lower half & 301 & 518 & $409.5^{\mathrm{Qc}: b, c}$ \\
\hline S96T005438 & 167: 5 & Lower half & 666 & 486 & $576^{\text {QC:b.c }}$ \\
\hline S96T005450 & 167: 6 & Lower half & 407 & 654 & $530.5^{\mathrm{QC}: b, c}$ \\
\hline S96T005696 & 167: 7 & Lower half & 617 & 555 & $586^{\mathrm{QC}: b}$ \\
\hline
\end{tabular}


Table B2-43. Tank 241-AN-103 Analytical Results: Silicon (ICP). (4 sheets)

\begin{tabular}{|c|c|c|c|c|c|}
\hline $\begin{array}{l}\text { Saringer } \\
\text { tronobr: }\end{array}$ & $\begin{array}{l}\text { Sample } \\
\text { sostitum }\end{array}$ & 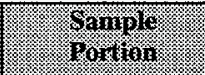 & Resili: & Bupticats & (1) \\
\hline \multicolumn{3}{|c|}{$\begin{array}{l}\text { Solias: aent agest } \\
\text { (Cont al }\end{array}$} & $=148$. & $(8 \mathrm{~g}$ & (3) \\
\hline S96T005737 & 167: 8 & Lower half & 756 & 683 & $719.5^{\mathrm{eC}: \mathrm{b}}$ \\
\hline S96T005858 & 167: 9 & Lower half & 258 & 318 & $288^{\mathrm{QC}: b, c}$ \\
\hline S96T005709 & \multirow[t]{2}{*}{ 167: 1} & Upper half & 442 & 553 & $497.5^{\text {QC:b,c }}$ \\
\hline S96T005697 & & Lower half & 553 & 630 & $591.5^{\mathrm{QC}: \mathrm{b}}$ \\
\hline S96T005444 & \multirow[t]{2}{*}{$167: 2$} & Upper half & 496 & 534 & $515^{\propto \mathrm{QC}: \mathrm{b}}$ \\
\hline S96T005451 & & Lower half & 682 & 854 & $768^{\mathrm{QC}: b, c}$ \\
\hline S96T005445 & \multirow[t]{2}{*}{$167: 4$} & Upper half & 630 & 630 & $630^{\mathrm{QC}: \mathrm{b}}$ \\
\hline S96T005439 & & Lower half & 551 & 407 & $479^{\mathrm{QC}: \mathrm{b}, \mathrm{c}}$ \\
\hline S96T005710 & \multirow[t]{2}{*}{$167: 5$} & Upper half & 657 & 667 & $662^{\mathrm{QC}: \mathrm{b}}$ \\
\hline S96T005698 & & Lower half & 693 & 766 & $729.5^{Q C: b}$ \\
\hline S96T005498 & \multirow[t]{2}{*}{$167: 7$} & Upper half & 400 & 533 & $466.5^{\mathrm{QC:b,c}}$ \\
\hline S96T005499 & & Lower half & 463 & 413 & $438^{Q C: b}$ \\
\hline S97T000460 & Core 166 & Solid composite & 839 & 837 & $838^{\mathrm{QC}: 6}$ \\
\hline Wiguins: & \multicolumn{2}{|c|}{ (3) } & (6) & 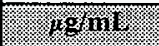 & 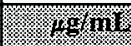 \\
\hline S96T005539 & 166: 3 & Drainable liquid & 241 & 227 & 234 \\
\hline S96T005540 & 166: 4 & Drainable liquid & 241 & 237 & 239 \\
\hline S96T005599 & $166: 6$ & Drainable liquid & 253 & 234 & 243.5 \\
\hline S96T005816 & 166: 7 & Drainable liquid & 330 & 355 & $342.5^{\mathrm{QC:d}}$ \\
\hline S96T005817 & $166: 8$ & Drainable liquid & 282 & 296 & 289 \\
\hline S96T005541 & $166: 9$ & Drainable liquid & 218 & 216 & 217 \\
\hline S96T005566 & 166: 10 & Drainable liquid & 231 & 222 & 226.5 \\
\hline S96T005600 & $166: 1$ & Drainable liquid & 243 & 243 & 243 \\
\hline S96T005601 & $166: 2$ & Drainable liquid & 280 & 281 & 280.5 \\
\hline S96T005861 & $167: 2$ & Drainable liquid & $329^{\circ}$ & 331 & 330 \\
\hline S96T005673 & $167: 3$ & Drainable liquid & 217 & 214 & 215.5 \\
\hline S96T005420 & $167: 5$ & Drainable liquid & 247 & 243 & 245 \\
\hline S96T005421 & $167: 6$ & Drainable liquid & 234 & 231 & 232.5 \\
\hline S96T005683 & 167: 7 & Drainable liquid & 235 & 235 & 235 \\
\hline
\end{tabular}


Table B2-43. Tank 241-AN-103 Analytical Results: Silicon (ICP). (4 sheets)

\begin{tabular}{|c|c|c|c|c|c|}
\hline Simple & Sample & $\begin{array}{l}\text { Saniplo } \\
\text { zortion }\end{array}$ & Ressinif: & Bupirate & II) \\
\hline \multicolumn{2}{|c|}{ 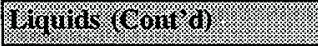 } & ; & 496111 & / & (1) Ginil \\
\hline S96T005732 & $167: 8$ & Drainable liquid & 213 & 209 & 211 \\
\hline S96T005862 & $167: 9$ & Drainable liquid & 242 & 254 & 248 \\
\hline S96T005650 & $167: 1$ & Drainable liquid & 218 & 225 & 221.5 \\
\hline S96T005991 & Core 166 & Liquid composite & 190 & 191 & 190.5 \\
\hline \multicolumn{2}{|c|}{ Solide fursion } & & tysis: & (6) & 1085 \\
\hline S96T005608 & 166: 1 & Lower half & 1,500 & 1,520 & 1,510 \\
\hline S96T005568 & 166: 4 & Lower half & $<1,130$ & $<1,110$ & $<1,120$ \\
\hline S96T005611 & $166: 6$ & Lower half & $<1,050$ & $<1,070$ & $<1,060$ \\
\hline S96T005835 & $166: 7$ & Lower half & 2,610 & 2,160 & 2,385 \\
\hline S96T005836 & $166: 8$ & Lower half & $<981$ & $<1,030$ & $<1,005.5$ \\
\hline S96T005574 & 166: 9 & Lower half & 1,460 & 1,160 & $1,310^{\mathrm{QC}: \mathrm{e}}$ \\
\hline S96T005569 & $166: 10$ & Lower half & 1,220 & 1,060 & 1,140 \\
\hline S96T005612 & $166: 1$ & Lower half & 1,090 & $<1,020$ & $<1,055$ \\
\hline S96T005613 & $166: 2$ & Lower half & 1,040 & 1,080 & 1,060 \\
\hline S96T005714 & \multirow[t]{2}{*}{$166: 3$} & Upper half & $<1,010$ & $<1,050$ & $<1,030$ \\
\hline S96T005720 & & Lower half & $<1,010$ & $<1,010$ & $<1,010$ \\
\hline S96T005414 & \multirow[t]{2}{*}{$166: 5$} & Upper half & $<228$ & $<233$ & $<230.5$ \\
\hline S96T005408 & & Lower half & 279 & 232 & 255.5 \\
\hline S96T005623 & \multirow[t]{2}{*}{$166: 6$} & Upper half & $<1,090$ & $<1,050$ & $<1,070$ \\
\hline S96T005614 & & Lower half & 1,410 & $<1,000$ & $<1,205^{\mathrm{Q}: c}$ \\
\hline S96T005415 & \multirow[t]{2}{*}{$166: 7$} & Upper half & 276 & $<264$ & $<270$ \\
\hline S96T005409 & & Lower haif & $<1,060$ & $<1,040$ & $<1,050$ \\
\hline S96T005715 & \multirow[t]{2}{*}{$166: 8$} & Upper half & $<1,010$ & $<1,030$ & $<1,020$ \\
\hline S96T005721 & & Lower half & 1,070 & $<994$ & $<1,032$ \\
\hline S96T005837 & \multirow[t]{2}{*}{$166: 9$} & Upper half & $<1,040$ & $<1,050$ & $<1,045$ \\
\hline S96T005838 & & Lower half & $<978$ & 988 & $<983$ \\
\hline S96T005705 & \multirow[t]{2}{*}{$167: 1$} & Upper half & $<994$ & $<1,000$ & $<997$ \\
\hline S96T005687 & & Lower half & 1,250 & $<1,040$ & $<1,145$ \\
\hline S96T005855 & $167: 2$ & Lower half & 1,150 & 1,050 & 1,100 \\
\hline
\end{tabular}




\begin{tabular}{|c|c|c|c|c|c|}
\hline $0 S 0^{4} I>$ & $0 S 0^{\prime} I>$ & $0 S 0^{\circ} \mathrm{I}>$ & IГЕप ГРМОТ & & $\angle 6 t S 00 L 96 S$ \\
\hline SEI I I > & $0\left[Z^{6} \mathrm{I}\right.$ & $090^{6} \mathrm{I}>$ & İЕध Iədd $\Omega$ & $\angle: \angle 9 I$ & $96 t S 00 \mathrm{~L} 96 \mathrm{~S}$ \\
\hline$\varepsilon 00^{\circ} I>$ & $060^{\prime} I>$ & $916>$ & J[Еप IәMOT & \multirow[b]{2}{*}{$S: \angle 9 I$} & z69\$00L96S \\
\hline$s+66>$ & $6 \mathrm{I} 6>$ & $0 \angle 0^{6} I>$ & 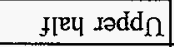 & & LOLSO0L96S \\
\hline $020^{\circ} \mathrm{I}>$ & $010^{\prime} \mathrm{I}>$ & $0 \varepsilon 0^{6} Y>$ & J[Еप Іәмот & \multirow[b]{2}{*}{$\downarrow: \angle 9 \mathrm{I}$} & LEtS00L96S \\
\hline SLO'I $>$ & $0 S 0^{\circ} I>$ & $00 I^{\prime \prime} I>$ & ક[вц raddח & & $\varepsilon t+500 L 96 \mathrm{~S}$ \\
\hline $590^{\circ} \mathrm{I}>$ & $0+I^{6} I$ & $886>$ & J[Еप دәмо I & \multirow[b]{2}{*}{$z: \angle 9 \mathrm{I}$} & $6 t+\$ 00 \mathrm{~L} 96 \mathrm{~S}$ \\
\hline$\varsigma^{\prime} t 20^{\prime} I>$ & $696>$ & $080^{6} I>$ & fleu Iədd $\Omega$ & & $z t t S 00 L 96 \mathrm{~S}$ \\
\hline$\varepsilon 26>$ & $126>$ & $\$ 26>$ & Јाеч ләмот & \multirow[b]{2}{*}{$I: \angle 9 I$} & I69S00L96S \\
\hline$S^{*}\left[20^{\circ} \mathrm{I}>\right.$ & OII'I> & $\varepsilon \varepsilon 6>$ & Ifeч Iədd $\Omega$ & & $90 \angle S 00 L 96 \mathrm{~S}$ \\
\hline$\$ 86>$ & $0 S 0^{\circ} \mathrm{I}>$ & $026>$ & IIEप دәмоT & $6: \angle 9 I$ & $958500 \mathrm{~L} 96 \mathrm{~S}$ \\
\hline $0 \mathcal{E} \mathcal{E}^{6} \mathrm{I}$ & $0 L Z^{6} \mathrm{I}$ & $06 \varepsilon^{\prime} I$ & IIEप دәмоТ & $8: \angle 9 I$ & 9ELSO0L96S \\
\hline $9 \varepsilon 0^{\circ} \mathrm{I}>$ & $060^{\circ} \mathrm{I}>$ & $286>$ & fleप دәмо I & $\angle: \angle 9 I$ & $069500196 \mathrm{~S}$ \\
\hline $0+00^{\circ} I>$ & $020^{\circ} \mathrm{I}>$ & $090^{\circ} \mathrm{I}$ & J[Еप Јәмот & $9: \angle 9 I$ & $8 \nabla t S 00 L 96 \mathrm{~S}$ \\
\hline$\subseteq \& 6>$ & $6 \varepsilon 6>$ & I\&6> & 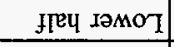 & $\$: \angle 9 I$ & $9 \varepsilon+500 \mathrm{~L} 96 \mathrm{~S}$ \\
\hline$S 60^{\prime} I>$ & $090^{\prime} I>$ & $0 \varepsilon I^{*} I$ & J[Eप IəMOT & $\downarrow: \angle 9 \mathrm{I}$ & $689500 \mathrm{~L} 96 \mathrm{~S}$ \\
\hline OIE'I & $08 \mathcal{E}^{\prime} \mathrm{I}$ & $0 \Delta Z^{\prime} I$ & $\exists[\mathrm{EY}$ Іәмот] & $\varepsilon: \angle 9 I$ & 889 S00L96S \\
\hline 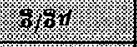 & (6) & (5) & & \multicolumn{2}{|c|}{ 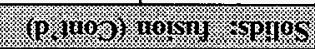 } \\
\hline 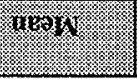 & (1) & 11980d & 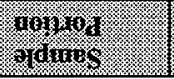 & oroushom & rogum \\
\hline
\end{tabular}

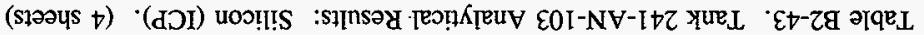


Table B2-44. Tank 241-AN-103 Analytical Results: Silver (ICP). (4 sheets)

\begin{tabular}{|c|c|c|c|c|c|}
\hline Singer & 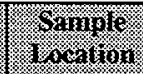 & 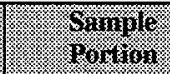 & hesiug & 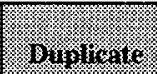 & 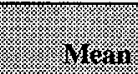 \\
\hline \multicolumn{2}{|c|}{ 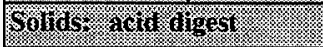 } & (18. & . & . & 45 \\
\hline \$96T005609 & 166:1 & Lower half & $<14.8$ & $<15.1$ & $<14.95$ \\
\hline S96T005570 & $166: 4$ & Lower half & $<17.9$ & $<17.9$ & $<17.9^{Q C: a, c}$ \\
\hline S96T005615 & $166: 6$ & Lower half & $<13.8$ & $<13.8$ & $<13.8$ \\
\hline S96T005839 & 166: 7 & Lower half & $<20.2$ & $<20.5$ & $<20.35^{\text {QC:a }}$ \\
\hline S96T005840 & 166: 8 & Lower half & $<19.7$ & $<19.7$ & $<19.7^{\mathrm{QC:a}}$ \\
\hline S96T005575 & 166: 9 & Lower half & 24.7 & $<19.9$ & $<22.3^{Q C: a, e}$ \\
\hline S96T005571 & $166: 10$ & Lower half & $<15.3$ & $<14.5$ & $<14.9$ \\
\hline S96T005616 & $166: 1$ & Lower half & $<14.5$ & $<13.6$ & $<14.05$ \\
\hline S96T005617 & $166: 2$ & Lower half & $<14.1$ & $<14.1$ & $<14.1$ \\
\hline S96T005716 & \multirow[t]{2}{*}{$166: 3$} & Upper half & $<19.4$ & $<19.6$ & $<19.5^{\mathrm{QC:a}}$ \\
\hline S96T005722 & & Lower half & $<20.6$ & $<20.6$ & $<20.6^{\mathrm{QC:a}}$ \\
\hline S96T005416 & \multirow[t]{2}{*}{ 166: 5} & Upper half & 49.6 & 24.6 & $37.1^{\text {QC:a,c }}$ \\
\hline S97T000459 & & Lower half & 13.6 & 15.2 & $14.4^{\mathrm{QCa} a}$ \\
\hline S96T005624 & \multirow[t]{2}{*}{ 166: 6} & Upper half & $<21$ & $<21.5$ & $<21.25^{\mathrm{QC:a}}$ \\
\hline S96T005618 & & Lower half & $<14.2$ & $<14.6$ & $<14.4$ \\
\hline S96T005417 & \multirow[t]{2}{*}{$166: 7$} & Upper half & 29.1 & $<20.5$ & $<24.8^{\mathrm{QC}: \mathrm{a}, \mathrm{e}}$ \\
\hline S96T005411 & & Lower half & $<19$ & $<17.6$ & $<18.3^{\mathrm{QC}: \mathrm{a}, \mathrm{c}}$ \\
\hline S96T005717 & \multirow[t]{2}{*}{ 166: 8} & Upper half & $<20.8$ & $<20.2$ & $<20.5^{Q C: a}$ \\
\hline S96T005723 & & Lower half & $<20.2$ & $<19.8$ & $<20^{\mathrm{QC}: a}$ \\
\hline S96T005841 & \multirow[t]{2}{*}{ 166: 9} & Upper half & $<19.3$ & $<19.6$ & $<19.45^{\mathrm{QC}: \mathrm{a}}$ \\
\hline S96T005842 & & Lower half & $<17.8$ & $<19.6$ & $<18.7^{\mathrm{QC:a}}$ \\
\hline S96T005708 & \multirow[t]{2}{*}{$167: 1$} & Upper half & $<17.9$ & $<20.5$ & $<19.2$ \\
\hline S96T005693 & & Lower half & $<22.2$ & $<17.5$ & $<19.85$ \\
\hline S96T005857 & 167: 2 & Lower half & $<16.7$ & $<17.4$ & $<17.05$ \\
\hline S96T005694 & $167: 3$ & Lower half & $<18$ & $<18.7$ & $<18.35$ \\
\hline S96T005695 & 167: 4 & Lower half & $<19.8$ & $<20.4$ & $<20.1$ \\
\hline S96T005438 & $167: 5$ & Lower half & 14.9 & 14.5 & $14.7^{\mathrm{QC:a,c}}$ \\
\hline S96T005450 & 167: 6 & Lower half & 15.7 & 15.2 & $15.45^{\mathrm{QC}: \mathrm{a}}$ \\
\hline S96T005696 & $167: 7$ & Lower half & $<19.2$ & $<18.8$ & $<19$ \\
\hline
\end{tabular}


HNF-SD-WM-ER-702 Rev. 0

Table B2-44. Tank 241-AN-103 Analytical Results: Silver (ICP). (4 sheets)

\begin{tabular}{|c|c|c|c|c|c|}
\hline Sample & $\begin{array}{l}\text { Sampole } \\
\text { U c caritor }\end{array}$ & $\begin{array}{l}\text { Sample } \\
\text { Tomtion }\end{array}$ & Resuli & Buylicate: & (1) Wean \\
\hline S96T005737 & $167: 8$ & Lower half & $<19.6$ & $<20$ & $<19.8$ \\
\hline S96T005858 & 167: 9 & Lower half & $<20.8$ & $<17.5$ & $<19.15$ \\
\hline S96T005709 & \multirow[t]{2}{*}{$167: 1$} & Upper half & $<18$ & $<18.7$ & $<18.35$ \\
\hline S96T005697 & & Lower half & $<19.8$ & $<18.8$ & $<19.3$ \\
\hline S96T005444 & \multirow[t]{2}{*}{ 167: 2} & Upper half & 17.5 & 16 & $16.75^{\mathrm{QC:a}}$ \\
\hline S96T005451 & & Lower half & 14.3 & 14.2 & $14.25^{\mathrm{QC}: a}$ \\
\hline S96T005445 & \multirow[t]{2}{*}{$167: 4$} & Upper half & 15.4 & 15.7 & $15.55^{\mathrm{QC:a}}$ \\
\hline S96T005439 & & Lower half & 15.8 & 15.5 & $15.65^{\mathrm{QC}: a}$ \\
\hline S96T005710 & \multirow[t]{2}{*}{$167: 5$} & Upper half & $<18.6$ & $<17.9$ & $<18.25$ \\
\hline S96T005698 & & Lower half & $<18.2$ & $<18.8$ & $<18.5$ \\
\hline S96T005498 & \multirow[t]{2}{*}{ 167: 7} & Upper half & $<18.3$ & $<19.5$ & $<18.9$ \\
\hline S96T005499 & & Lower half & $<17.8$ & $<19.3$ & $<18.55$ \\
\hline S97T000460 & Core 166 & Solid composite & 16.4 & 15.7 & $16.05^{\mathrm{QC:a}}$ \\
\hline \multicolumn{2}{|l|}{ Woguds } & & . $19 \mathrm{gmill}$ & 148, & 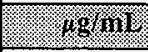 \\
\hline S96T005539 & $166: 3$ & Drainable liquid & 19.6 & 18.8 & 19.2 \\
\hline S96T005540 & $166: 4$ & Drainable liquid & 15.5 & 8.91 & $12.205^{\mathrm{QC}: \mathrm{e}}$ \\
\hline S96T005599 & $166: 6$ & Drainable liquid & 20 & 18.6 & 19.3 \\
\hline S96T005816 & 166: 7 & Drainable liquid & 18.6 & 19.6 & 19.1 \\
\hline S96T005817 & 166: 8 & Drainable liquid & 18.2 & 20.2 & 19.2 \\
\hline S96T005541 & 166: 9 & Drainable liquid & 18.9 & 18.9 & 18.9 \\
\hline S96T005566 & 166: 10 & Drainable liquid & 20.5 & 18.6 & 19.55 \\
\hline S96T005600 & 166: 1 & Drainable liquid & 20.2 & 18.7 & 19.45 \\
\hline S96T005601 & 166: 2 & Drainable liquid & 19.9 & 20.3 & 20.1 \\
\hline S96T005861 & $167: 2$ & Drainable liquid & 18.9 & 19 & 18.95 \\
\hline S96T005673 & $167: 3$ & Drainable liquid & 16.4 & 17.4 & 16.9 \\
\hline S96T005420 & 167: 5 & Drainable liquid & 19 & 19.7 & 19.35 \\
\hline S96T005421 & 167: 6 & \begin{tabular}{|l|} 
Drainable liquid \\
\end{tabular} & 17.9 & 17.2 & 17.55 \\
\hline S96T005683 & 167: 7 & \begin{tabular}{|l|} 
Drainable liquid \\
\end{tabular} & 19.7 & 21.1 & 20.4 \\
\hline S96T005732 & $167: 8$ & Drainable liquid & 19.5 & 20.3 & 19.9 \\
\hline S96T005862 & $167: 9$ & Drainable liquid & 19.5 & 19.3 & 19.4 \\
\hline
\end{tabular}


Table B2-44. Tank 241-AN-103 Analytical Results: Silver (ICP). (4 sheets)

\begin{tabular}{|c|c|c|c|c|c|}
\hline Sainglo & 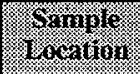 & 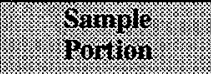 & Rerull: & 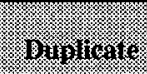 & yean \\
\hline S96T005650 & 167: 1 & Drainable liquid & 17.6 & 16.6 & 17.1 \\
\hline S96T005991 & Core 166 & Liquid composite & $<12$ & $<12$ & $<12$ \\
\hline 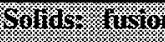 & & & (2) & 49 & 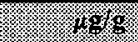 \\
\hline S96T005608 & 166: 1 & Lower half & $<210$ & $<206$ & $<208$ \\
\hline S96T005568 & 166: 4 & Lower half & $<227$ & $<222$ & $<224.5$ \\
\hline S96T005611 & 166: 6 & Lower half & $<210$ & $<214$ & $<212$ \\
\hline S96T005835 & 166: 7 & Lower half & $<210$ & $<212$ & $<211$ \\
\hline S96T005836 & $166: 8$ & Lower half & $<196$ & $<206$ & $<201$ \\
\hline S96T005574 & 166: 9 & Lower half & $<179$ & $<194$ & $<186.5$ \\
\hline S967005569 & 166: 10 & Lower half & $<209$ & $<207$ & $<208$ \\
\hline S96T005612 & 166: 1 & Lower half & $<197$ & $<204$ & $<200.5$ \\
\hline S96T005613 & $166: 2$ & Lower half & $<205$ & $<202$ & $<203.5$ \\
\hline S96T005714 & \multirow[t]{2}{*}{$166: 3$} & Upper half & $<201$ & $<211$ & $<206$ \\
\hline S96T005720 & & Lower half & $<203$ & $<201$ & $<202$ \\
\hline S96T005414 & \multirow[t]{2}{*}{ 166: 5} & Upper half & $<45.6$ & $<46.6$ & $<46.1$ \\
\hline S96T005408 & & Lower half & $<48$ & $<46.3$ & $<47.15^{\mathrm{QC}: \mathrm{c}}$ \\
\hline S96T005623 & \multirow[t]{2}{*}{ 166: 6} & Upper half & $<218$ & $<210$ & $<214^{\mathrm{QC}: c}$ \\
\hline S96T005614 & & Lower half & $<204$ & $<201$ & $<202.5$ \\
\hline \$96T005415 & \multirow[t]{2}{*}{$166: 7$} & Upper half & $<52.3$ & $<52.8$ & $<52.55$ \\
\hline$\$ 96 \mathrm{~T} 005409$ & & Lower half & $<211$ & $<208$ & $<209.5$ \\
\hline S96T005715 & \multirow[t]{2}{*}{$166: 8$} & Upper half & $<201$ & $<206$ & $<203.5$ \\
\hline S96T005721 & & Lower half & $<203$ & $<199$ & $<201$ \\
\hline S96T005837 & \multirow[t]{2}{*}{ 166: 9} & Upper half & $<207$ & $<210$ & $<208.5$ \\
\hline S96T005838 & & Lower half & $<196$ & $<196$ & $<196$ \\
\hline S96T005705 & \multirow[t]{2}{*}{$167: 1$} & Upper half & $<199$ & $<200$ & $<199.5$ \\
\hline S96T005687 & & Lower half & $<208$ & $<209$ & $<208.5$ \\
\hline S96T005855 & 167: 2 & Lower half & $<202$ & $<198$ & $<200$ \\
\hline S96T005688 & 167: 3 & Lower half & $<225$ & $<213$ & $<219$ \\
\hline S96T005689 & 167: 4 & Lower half & $<210$ & $<211$ & $<210.5$ \\
\hline S96T005436 & $167: 5$ & Lower half & $<16$ & $<188$ & $<187$ \\
\hline
\end{tabular}




\begin{tabular}{|c|c|c|c|c|c|}
\hline s.0Iz> & IIZ> & orz> & ІІеч Іәмо Т & & $\angle 6+S 00 L 96 S$ \\
\hline IIZ> & $0 I Z>$ & $z I Z>$ & fley Iədd $\Omega$ & $L: \angle 9 I$ & $96 t s 00 L 96 \mathrm{~S}$ \\
\hline s.00z> & $8[\tau>$ & $\varepsilon 8 I>$ & Јाеч дәмо & \multirow[b]{2}{*}{$\subseteq: \angle 9 I$} & $269 S 00.196 \mathrm{~S}$ \\
\hline ؟.86I > & $581>$ & $\varepsilon I Z>$ & fley rədd $\Pi$ & & $\angle 0 \angle S 00 L 96 \mathrm{~S}$ \\
\hline$t 0 z>$ & $\varepsilon 0 z>$ & soz> & 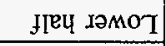 & \multirow[b]{2}{*}{$t: \angle 9 I$} & $\angle \varepsilon+S 00 \mathrm{~L} 96 \mathrm{~S}$ \\
\hline S'SIZ> & IIZ> & $0 z z>$ & fle્ xәdd & & $\varepsilon t t S 00 L 96 \mathrm{~S}$ \\
\hline $00 z>$ & $20 z>$ & $86 I>$ & Iाएч Іәмо & \multirow[b]{2}{*}{$\tau: \angle 9 I$} & $6 t+500 \mathrm{~L} 96 \mathrm{~S}$ \\
\hline soz> & $D 6 I>$ & $9[2>$ & 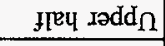 & & $2 t+500 L 96 \mathrm{~S}$ \\
\hline$S^{*}+8 I>$ & $\pm 8 I>$ & $581>$ & IॉЕप ІәмОТ & \multirow[b]{2}{*}{$I: \angle 9 I$} & $169500 \mathrm{~L} 96 \mathrm{~S}$ \\
\hline$s+t 0 z>$ & $z z z>$ & $\angle 8 I>$ & ғтеч radd & & $90 \angle S 00 L 96 \mathrm{~S}$ \\
\hline$S^{\circ} \angle 6 I>$ & IIZ> & $581>$ & 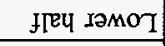 & $6: \angle 9 I$ & $9 \$ 8 \mathrm{~S} 00 \mathrm{~L} 96 \mathrm{~S}$ \\
\hline$t 0 z>$ & $\angle 8 I>$ & IZZ> & Ј[ец ІәмоТ & $8: \angle 9 I$ & $9 \varepsilon \angle S 00 L 96 \mathrm{~S}$ \\
\hline$\angle 0 z>$ & $8 I \tau>$ & $96 \mathrm{I}>$ & fГеч Іәмо Т & $L: \angle 9 I$ & $069500 L 96 \mathrm{~S}$ \\
\hline$s^{\prime} \angle O Z>$ & toz> & IIZ> & fґеч Іәмот & $9: \angle 9 I$ & $8+t S 00 L 96 S$ \\
\hline $4001 \%$ & opognd & 31 soros: & $\begin{array}{l}\text { roprigy } \\
\text { 2irmes. }\end{array}$ & noulerol & 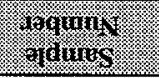 \\
\hline
\end{tabular}

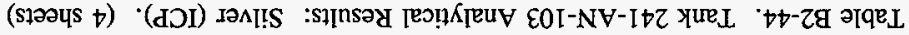


Table B2-45. Tank 241-AN-103 Analytical Results: Sodium (ICP). (4 sheets)

\begin{tabular}{|c|c|c|c|c|c|}
\hline Mamaris & S.mple & Gonoly & Ko & Bimplicante & Marin \\
\hline Sondur arof & aystis & (1. & 1396 & 40 & 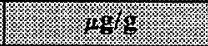 \\
\hline S96T005609 & 166: 1 & Lower half & $1.910 \mathrm{E}+05$ & $1.860 \mathrm{E}+05$ & $1.885 \mathrm{E}+05$ \\
\hline S96T005570 & 166: 4 & Lower half & $2.440 \mathrm{E}+05$ & $2.410 \mathrm{E}+05$ & $2.425 \mathrm{E}+05^{\mathrm{QC}: \mathrm{b}, \mathrm{c}}$ \\
\hline \$96T005615 & 166: 6 & Lower haif & $2.410 \mathrm{E}+05$ & $2.390 E+05$ & $2.400 \mathrm{E}+05$ \\
\hline S96T005839 & 166: 7 & Lower half & $2.280 \mathrm{E}+05$ & $2.360 \mathrm{E}+05$ & $2.320 \mathrm{E}+05$ \\
\hline S96T005840 & 166: 8 & Lower half & $2.470 \mathrm{E}+05$ & $2.530 \mathrm{E}+05$ & $2.500 \mathrm{E}+05$ \\
\hline S96T005575 & 166: 9 & Lower half & $2.160 \mathrm{E}+05$ & $2.320 E+05$ & $2.240 \mathrm{E}+05^{\mathrm{QC}: \mathrm{b}}$ \\
\hline S96T005571 & 166: 10 & Lower half & $2.310 \mathrm{E}+05$ & $2.320 \mathrm{E}+05$ & $2.315 \mathrm{E}+05$ \\
\hline S96T005616 & 166: 1 & Lower half & $2.410 \mathrm{E}+05$ & $2.500 \mathrm{E}+05$ & $2.455 \mathrm{E}+05$ \\
\hline S96T005617 & 166: 2 & Lower half & $2.400 \mathrm{E}+05$ & $2.410 E+05$ & $2.405 \mathrm{E}+05$ \\
\hline S96T005716 & \multirow[t]{2}{*}{$166: 3$} & Upper half & $1.930 \mathrm{E}+05$ & $2.040 \mathrm{E}+05$ & $1.985 \mathrm{E}+05$ \\
\hline S96T005722 & & Lower half & $1.800 \mathrm{E}+05$ & $1.950 \mathrm{E}+05$ & $1.875 E+05$ \\
\hline S96T005416 & \multirow[t]{2}{*}{$166: 5$} & Upper half & $2.080 \mathrm{E}+05$ & $1.980 \mathrm{E}+05$ & $2.030 \mathrm{E}+05^{\mathrm{QC}: \mathrm{b}}$ \\
\hline S97T000459 & & Lower half & $1.920 E+05$ & $2.100 \mathrm{E}+05$ & $2.010 \mathrm{E}+05^{\mathrm{QC}: \mathrm{d}}$ \\
\hline S96T005624 & \multirow[t]{2}{*}{$166: 6$} & Upper half & $2.120 \mathrm{E}+05$ & $2.060 \mathrm{E}+05$ & $2.090 \mathrm{E}+05$ \\
\hline S96T005618 & & Lower half & $2.040 \mathrm{E}+05$ & $2.080 \mathrm{E}+05$ & $2.060 \mathrm{E}+05$ \\
\hline S96T005417 & \multirow[t]{2}{*}{$166: 7$} & Upper half & $2.100 \mathrm{E}+05$ & $2.160 \mathrm{E}+05$ & $2.130 \mathrm{E}+05^{\mathrm{QC}: \mathrm{b}}$ \\
\hline S96T005411 & & Lower half & $2.150 \mathrm{E}+05$ & $2.190 \mathrm{E}+05$ & $2.170 \mathrm{E}+05^{\mathrm{Qc}: \mathrm{b}, \mathrm{c}}$ \\
\hline \$96T005717 & \multirow[t]{2}{*}{ 166: 8} & Upper half & $1.960 \mathrm{E}+05$ & $2.090 \mathrm{E}+05$ & $2.025 E+05$ \\
\hline S96T005723 & & Lower half & $2.120 \mathrm{E}+05$ & $2.040 \mathrm{E}+05$ & $2.080 \mathrm{E}+05$ \\
\hline S96T005841 & \multirow[t]{2}{*}{ 166: 9} & Upper half & $2.060 \mathrm{E}+05$ & $2.050 \mathrm{E}+05$ & $2.055 \mathrm{E}+05$ \\
\hline S96T005842 & & Lower half & $1.980 \mathrm{E}+05$ & $2.040 \mathrm{E}+05$ & $2.010 \mathrm{E}+05$ \\
\hline S96T005708 & \multirow[t]{2}{*}{ 167: 1} & Upper half & $2.590 \mathrm{E}+05$ & $2.190 \mathrm{E}+05$ & $2.390 \mathrm{E}+05$ \\
\hline S96T005693 & & Lower half & $2.070 \mathrm{E}+05$ & $2.090 \mathrm{E}+05$ & $2.080 E+05$ \\
\hline S96T005857 & 167: 2 & Lower half & $1.990 \mathrm{E}+05$ & $2.010 \mathrm{E}+05$ & $2.000 \mathrm{E}+05$ \\
\hline S96T005694 & 167: 3 & Lower half & $2.330 \mathrm{E}+05$ & $2.510 \mathrm{E}+05$ & $2.420 \mathrm{E}+05$ \\
\hline S96T005695 & $167: 4$ & Lower half & $2.230 \mathrm{E}+05$ & $2.220 \mathrm{E}+05$ & $2.225 \mathrm{E}+05$ \\
\hline S96T005438 & 167: 5 & Lower half & $2.120 \mathrm{E}+05$ & $2.110 \mathrm{E}+05$ & $2.115 \mathrm{E}+05^{\mathrm{QC:d}}$ \\
\hline S96T005450 & 167: 6 & Lower half & $2.220 \mathrm{E}+05$ & $2.150 \mathrm{E}+05$ & $2.185 \mathrm{E}+05$ \\
\hline S96T005696 & 167: 7 & Lower half & $2.480 \mathrm{E}+05$ & $2.440 \mathrm{E}+05$ & $2.460 \mathrm{E}+05$ \\
\hline
\end{tabular}


Table B2-45. Tank 241-AN-103 Analytical Results: Sodium (ICP). (4 sheets)

\begin{tabular}{|c|c|c|c|c|c|}
\hline Numiner & Saringe & oramplo & fosing & Hown & yeriv \\
\hline \multicolumn{2}{|c|}{ Hollds quid ingest } & & 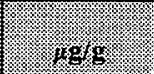 & 4opgr & 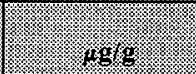 \\
\hline S96T005737 & 167: 8 & Lower half & $2.350 \mathrm{E}+05$ & $2.360 \mathrm{E}+05$ & $2.355 \mathrm{E}+05$ \\
\hline S96T005858 & 167:9 & Lower half & $2.550 \mathrm{E}+05$ & $2.480 \mathrm{E}+05$ & $2.515 \mathrm{E}+05$ \\
\hline S96T005709 & \multirow[t]{2}{*}{ 167: 1} & Upper half & $1.970 \mathrm{E}+05$ & $1.880 \mathrm{E}+05$ & $1.925 \mathrm{E}+05$ \\
\hline S96T005697 & & Lower half & $2.000 E+05$ & $2.000 \mathrm{E}+05$ & $2.000 \mathrm{E}+05$ \\
\hline S96T005444 & \multirow[t]{2}{*}{ 167: 2} & Upper half & $2.050 \mathrm{E}+05$ & $2.040 \mathrm{E}+05$ & $2.045 \mathrm{E}+05$ \\
\hline S96T005451 & & Lower half & $2.080 \mathrm{E}+05$ & $2.060 \mathrm{E}+05$ & $2.070 \mathrm{E}+05$ \\
\hline S96T005445 & \multirow[t]{2}{*}{$167: 4$} & Upper half & $2.190 \mathrm{E}+05$ & $2.230 \mathrm{E}+05$ & $2.210 \mathrm{E}+05$ \\
\hline S96T005439 & & Lower half & $2.230 \mathrm{E}+05$ & $2.190 \mathrm{E}+05$ & $2.210 \mathrm{E}+05^{\alpha \mathrm{C:d}}$ \\
\hline S96T005710 & \multirow[t]{2}{*}{$167: 5$} & Upper half & $2.130 E+05$ & $2.150 \mathrm{E}+05$ & $2.140 \mathrm{E}+05$ \\
\hline S96T005698 & & Lower half & $2.150 \mathrm{E}+05$ & $2.180 E+05$ & $2.165 \mathrm{E}+05$ \\
\hline \$96T005498 & \multirow[t]{2}{*}{ 167:7 } & Upper half & $2.280 \mathrm{E}+05$ & $2.340 \mathrm{E}+05$ & $2.310 \mathrm{E}+05$ \\
\hline \$96T005499 & & Lower half & $2.440 \mathrm{E}+05$ & $2.270 \mathrm{E}+05$ & $2.355 E+05$ \\
\hline S97T000460 & Core 166 & Solid composite & $2.200 \mathrm{E}+05$ & $2.130 \mathrm{E}+05$ & $2.165 E+05$ \\
\hline manirs & & & 6 & girit & ${ }_{1}$ \\
\hline S96T005539 & $166: 3$ & Drainable liquid & $2.670 \mathrm{E}+05$ & $2.620 \mathrm{E}+05$ & $2.645 E+05$ \\
\hline S96T005540 & $166: 4$ & Drainable liquid & $2.240 \mathrm{E}+05$ & $2.010 \mathrm{E}+05$ & $2.125 \mathrm{E}+05$ \\
\hline S96T005599 & $166: 6$ & Drainable liquid & $2.740 \mathrm{E}+05$ & $2.610 E+05$ & $2.675 \mathrm{E}+05^{\mathrm{QC}: \mathrm{c}}$ \\
\hline S96T005816 & 166: 7 & Drainable liquid & $2.610 \mathrm{E}+05$ & $2.690 \mathrm{E}+05$ & $2.650 \mathrm{E}+05^{\mathrm{QC:d}}$ \\
\hline \$96T005817 & $166: 8$ & Drainable liquid & $2.490 \mathrm{E}+05$ & $2.700 \mathrm{E}+05$ & $2.595 \mathrm{E}+05$ \\
\hline S96T005541 & 166: 9 & Drainable liquid & $2.560 \mathrm{E}+05$ & $2.500 \mathrm{E}+05$ & $2.530 \mathrm{E}+05^{\mathrm{QC:c}}$ \\
\hline \$96T005566 & 166: 10 & Drainable liquid & $2.570 \mathrm{E}+05$ & $2.490 \mathrm{E}+05$ & $2.530 \mathrm{E}+05$ \\
\hline S96T005600 & 166: 1 & Drainable liquid & $2.640 \mathrm{E}+05$ & $2.560 \mathrm{E}+05$ & $2.600 \mathrm{E}+05$ \\
\hline S96T005601 & 166: 2 & Drainable liquid & $2.700 \mathrm{E}+05$ & $2.700 \mathrm{E}+05$ & $2.700 \mathrm{E}+05^{\mathrm{QC}: c}$ \\
\hline S96T005861 & 167: 2 & Drainable liquid & $2.600 \mathrm{E}+05$ & $2.640 \mathrm{E}+05$ & $2.620 \mathrm{E}+05$ \\
\hline S96T005673 & 167: 3 & Drainable liquid & $2.420 \mathrm{E}+05$ & $2.400 \mathrm{E}+05$ & $2.410 \mathrm{E}+05$ \\
\hline S96T005420 & 167: 5 & Drainable liquid & $2.590 \mathrm{E}+05$ & $2.630 \mathrm{E}+05$ & $2.610 \mathrm{E}+05^{\mathrm{QC}: \mathrm{d}}$ \\
\hline S96T005421 & 167: 6 & Drainable liquid & $2.450 \mathrm{E}+05$ & $2.430 \mathrm{E}+05$ & $2.440 \mathrm{E}+05$ \\
\hline S96T005683 & $167: 7$ & Drainable liquid & $2.770 \mathrm{E}+05$ & $2.730 \mathrm{E}+05$ & $2.750 \mathrm{E}+05$ \\
\hline
\end{tabular}


HNF-SD-WM-ER-702 Rev. 0

Table B2-45. Tank 241-AN-103 Analytical Results: Sodium (ICP). (4 sheets)

\begin{tabular}{|c|c|c|c|c|c|}
\hline Minger & 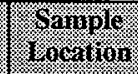 & Ganule & Ficsuls & Duulicate & 1) 16 \\
\hline \multicolumn{3}{|c|}{ 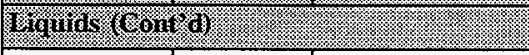 } & (2) & 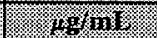 & (x) \\
\hline S96T005732 & $167: 8$ & Drainable liquid & $2.710 \mathrm{E}+05$ & $2.670 \mathrm{E}+05$ & $2.690 \mathrm{E}+05$ \\
\hline S96T005862 & 167: 9 & Drainable liquid & $2.600 \mathrm{E}+05$ & $2.630 \mathrm{E}+05$ & $2.615 \mathrm{E}+05$ \\
\hline \$96T005650 & 167: 1 & Drainable liquid & $2.330 \mathrm{E}+05$ & $2.340 \mathrm{E}+05$ & $2.335 \mathrm{E}+05$ \\
\hline S96T005991 & Core 166 & Liquid composite & $2.630 \mathrm{E}+05$ & $2.620 \mathrm{E}+05$ & $2.625 \mathrm{E}+05^{\mathrm{QC}: \mathrm{c}}$ \\
\hline \multicolumn{2}{|c|}{ 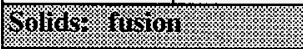 } & & vis & wg & ng \\
\hline S96T005608 & 166: 1 & Lower half & $2.300 \mathrm{E}+05$ & $2.500 \mathrm{E}+05$ & $2.400 \mathrm{E}+05$ \\
\hline S96T005568 & 166: 4 & Lower half & $3.830 \mathrm{E}+05$ & $3.430 \mathrm{E}+05$ & $3.630 \mathrm{E}+05^{\mathrm{QC}: c}$ \\
\hline S96T005611 & 166: 6 & Lower half & $2.900 \mathrm{E}+05$ & $2.980 \mathrm{E}+05$ & $2.940 \mathrm{E}+05$ \\
\hline S96T005835 & 166: 7 & Lower half & $3.660 \mathrm{E}+05$ & $3.590 \mathrm{E}+05$ & $3.625 E+05$ \\
\hline S96T005836 & 166: 8 & Lower half & $2.730 \mathrm{E}+05$ & $2.780 \mathrm{E}+05$ & $2.755 \mathrm{E}+05^{\mathrm{QC}: c}$ \\
\hline S96T005574 & 166: 9 & Lower half & $3.330 \mathrm{E}+05$ & $3: 410 \mathrm{E}+05$ & $3.370 \mathrm{E}+05$ \\
\hline S96T005569 & $166: 10$ & Lower half & $2.860 \mathrm{E}+05$ & $2.820 \mathrm{E}+05$ & $2.840 \mathrm{E}+05^{\mathrm{QC}: \mathrm{d}}$ \\
\hline S96T005612 & 166: 1 & Lower half & $2.720 \mathrm{E}+05$ & $2.760 \mathrm{E}+05$ & $2.740 \mathrm{E}+05$ \\
\hline S96T005613 & 166: 2 & Lower half & $2.620 \mathrm{E}+05$ & $1.760 E+05$ & $2.190 \mathrm{E}+05^{\mathrm{QC}: e}$ \\
\hline S96T005714 & \multirow[t]{2}{*}{ 166: 3} & Upper half & $2.980 \mathrm{E}+05$ & $2.990 \mathrm{E}+05$ & $2.985 \mathrm{E}+05$ \\
\hline S96T005720 & & Lower half & $2.990 \mathrm{E}+05$ & $2.850 \mathrm{E}+05$ & $2.920 \mathrm{E}+05$ \\
\hline \$96T005414 & \multirow[t]{2}{*}{$166: 5$} & Upper half & 77,400 & 78,300 & 77,850 \\
\hline \$96T005408 & & Lower half & 75,400 & 78,000 & $76,700^{\mathrm{QC}: c}$ \\
\hline S96T005623 & \multirow[t]{2}{*}{$166: 6$} & Upper half & $3.070 \mathrm{E}+05$ & $3.030 \mathrm{E}+05$ & $3.050 \mathrm{E}+05$ \\
\hline S96T005614 & & Lower half & $2.460 E+05$ & $2.440 \mathrm{E}+05$ & $2.450 \mathrm{E}+05$ \\
\hline S96T005415 & \multirow[t]{2}{*}{ 166: 7} & Upper half & 84,300 & 82,300 & 83,300 \\
\hline S96T005409 & & Lower half & $3.130 \mathrm{E}+05$ & $3.140 \mathrm{E}+05$ & $3.135 \mathrm{E}+05^{\mathrm{QC:d}}$ \\
\hline S96T005715 & \multirow[t]{2}{*}{$166: 8$} & Upper half & $3.040 \mathrm{E}+05$ & $3.110 \mathrm{E}+05$ & $3.075 \mathrm{E}+05$ \\
\hline S96T005721 & & Lower half & $3.150 \mathrm{E}+05$ & $3.070 \mathrm{E}+05$ & $3.110 \mathrm{E}+05^{\mathrm{QC}: \mathrm{c}}$ \\
\hline \$96T005837 & \multirow[t]{2}{*}{ 166: 9} & Upper half & $2.580 \mathrm{E}+05$ & $2.460 \mathrm{E}+05$ & $2.520 \mathrm{E}+05$ \\
\hline S96T005838 & & Lower half & $2.450 E+05$ & $2.440 \mathrm{E}+05$ & $2.445 \mathrm{E}+05$ \\
\hline S96T005705 & \multirow[t]{2}{*}{$167: 1$} & Upper half & $2.930 \mathrm{E}+05$ & $3.000 \mathrm{E}+05$ & $2.965 \mathrm{E}+05$ \\
\hline S96T005687 & & Lower half & $2.250 \mathrm{E}+05$ & $2.500 \mathrm{E}+05$ & $2.375 \mathrm{E}+05^{\mathrm{QC:d}}$ \\
\hline S96T005855 & 167: 2 & Lower half & $3.090 \mathrm{E}+05$ & $2.970 E+05$ & $3.030 \mathrm{E}+05$ \\
\hline
\end{tabular}


HNF-SD-WM-ER-702 Rev. 0

Table B2-45. Tank 241-AN-103 Analytical Results: Sodium (ICP). (4 sheets)

\begin{tabular}{|c|c|c|c|c|c|}
\hline Sinple & Samifie. & $\begin{array}{l}\text { Sinume } \\
\text { Towition }\end{array}$ & Resint: & Durblatire & (2.3. $11=11$. \\
\hline \multicolumn{2}{|c|}{ Sollds - fision (contid) } & & 8.189 .8 & 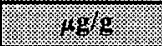 & (4) \\
\hline S96T005688 & $167: 3$ & Lower half & $2.580 \mathrm{E}+05$ & $2.610 \mathrm{E}+05$ & $2.595 \mathrm{E}+05$ \\
\hline S96T005689 & $167: 4$ & Lower half & $2.570 \mathrm{E}+05$ & $2.640 \mathrm{E}+05$ & $2.605 \mathrm{E}+05$ \\
\hline S96T005436 & $167: 5$ & Lower half & $3.240 \mathrm{E}+05$ & $3.180 \mathrm{E}+05$ & $3.210 \mathrm{E}+05$ \\
\hline S96T005448 & $167: 6$ & Lower half & $3.140 \mathrm{E}+05$ & $3.260 \mathrm{E}+05$ & $3.200 \mathrm{E}+05$ \\
\hline S96T005690 & 167: 7 & Lower half & $3.300 \mathrm{E}+05$ & $3.690 \mathrm{E}+05$ & $3.495 \mathrm{E}+05$ \\
\hline S96T005736 & $167: 8$ & Lower half & $3.580 \mathrm{E}+05$ & $3.050 \mathrm{E}+05$ & $3.315 \mathrm{E}+05$ \\
\hline S96T005856 & $167: 9$ & Lower half & $3.640 \mathrm{E}+05$ & $3.480 \mathrm{E}+05$ & $3.560 \mathrm{E}+05$ \\
\hline S96T005706 & \multirow[t]{2}{*}{ 167: 1} & Upper half & $2.870 \mathrm{E}+05$ & $3.000 \mathrm{E}+05$ & $2.935 E+05$ \\
\hline S96T005691 & & Lower half & $2.890 \mathrm{E}+05$ & $2.790 \mathrm{E}+05$ & $2.840 \mathrm{E}+05$ \\
\hline S96T005442 & \multirow[t]{2}{*}{$167: 2$} & Upper half & $2.990 \mathrm{E}+05$ & $2.920 \mathrm{E}+05$ & $2.955 \mathrm{E}+05$ \\
\hline S96T005449 & & Lower half & $2.450 \mathrm{E}+05$ & $2.380 \mathrm{E}+05$ & $2.415 E+05$ \\
\hline S96T005443 & \multirow[t]{2}{*}{ 167: 4} & Upper half & $2.510 \mathrm{E}+05$ & $2.570 \mathrm{E}+05$ & $2.540 \mathrm{E}+05$ \\
\hline S96T005437 & & Lower half & $2.470 \mathrm{E}+05$ & $2.460 \mathrm{E}+05$ & $2.465 \mathrm{E}+05^{\mathrm{QC}: \mathrm{c}}$ \\
\hline S96T005707 & \multirow[t]{2}{*}{$167: 5$} & Upper half & $3.240 \mathrm{E}+05$ & $3.070 \mathrm{E}+05$ & $3.155 \mathrm{E}+05$ \\
\hline S96T005692 & & Lower half & $3.170 \mathrm{E}+05$ & $3.100 \mathrm{E}+05$ & $3.135 \mathrm{E}+05$ \\
\hline S96T005496 & \multirow[t]{2}{*}{$167: 7$} & Upper half & $3.200 \mathrm{E}+05$ & $4.380 \mathrm{E}+05$ & $3.790 \mathrm{E}+05^{\mathrm{QC}: \mathrm{c}}$ \\
\hline S96T005497 & & Lower half & $3.330 \mathrm{E}+05$ & $3.120 \mathrm{E}+05$ & $3.225 \mathrm{E}+05$ \\
\hline
\end{tabular}




\begin{tabular}{|c|c|c|c|c|c|}
\hline $06 \mathrm{I}>$ & $88 I>$ & $26 \mathrm{I}>$ & f[еч Іәмо ] & $L: \angle 9 I$ & 969S00L $96 \mathrm{~S}$ \\
\hline ะ:0006I & $26 \mathrm{I}$ & 881 & f[Еप & $9: \angle 9 \mathrm{I}$ & 0StS00L96S \\
\hline ::00s'zZz & $9 I Z$ & 622 & ІІеप ІәмоТ & $S: \angle 9 \mathrm{I}$ & $8 \mathcal{b} \rightarrow 00 \mathrm{~L} 96 \mathrm{~S}$ \\
\hline I0Z> & $t 0 z>$ & $86 I>$ & fГеч Iәмо & $\nabla: \angle 9 I$ & S69S00196S \\
\hline$S^{\cdot} E 8 I>$ & $\angle 8 I>$ & $08 \mathrm{I}>$ & IlРY хәмот & $\varepsilon: \angle 9 \mathrm{I}$ & $769 S 00 \mathrm{~L} 96 \mathrm{~S}$ \\
\hline $2 \downarrow 0^{\circ} \mathrm{I}$ & OzI'I & 796 & Jleप Іәмот & $\tau: \angle 9 I$ & LS8S00L96S \\
\hline 09 I'I & $0 I Z^{6} \mathrm{I}$ & OII' I & IIеบ دәмо I & \multirow[b]{2}{*}{$I: \angle 9 I$} & E69S00L96S \\
\hline $0: 00 S^{\circ} 06 L$ & 126 & 099 & કાеч Iədd $\Omega$ & & $80 \angle S 00 L 96 \mathrm{~S}$ \\
\hline $\operatorname{SSS}^{6} I$ & $0 \varepsilon 9^{\circ} \mathrm{I}$ & $08 t^{\prime} \mathrm{I}$ & I[Еप ІәмоТ] & \multirow[b]{2}{*}{$6: 99 \mathrm{I}$} & $\tau \neq 8 \mathrm{~S} 00 \mathrm{~L} 96 \mathrm{~S}$ \\
\hline$s t \nabla^{\circ} \mathrm{I}$ & $06 t^{\circ} \mathrm{I}$ & $00 t^{\circ} \mathrm{I}$ & Јॉец цәdd $\Omega$ & & It $8500196 \mathrm{~S}$ \\
\hline $500^{\prime} z$ & $016^{6} \mathrm{I}$ & $001^{\prime} z$ & IF" גәмоТ & \multirow[b]{2}{*}{$8: 99$ I } & EZLS00I96S \\
\hline $085^{\prime} \mathrm{I}$ & $029^{\circ} \mathrm{I}$ & $0 t S^{\prime} I$ & $\pm[$ [eч xәdd $\Omega$ & & $\angle I L S 00 L 96 S$ \\
\hline$: 000 S L^{\prime} \mathrm{I}$ & $0 E 6^{6} \mathrm{I}$ & $0 \angle S^{\prime} I$ & J[Eप IәMOT & \multirow[b]{2}{*}{$L: 99 \mathrm{I}$} & I t $\$$ S00L96S \\
\hline S6I'I & $0 \operatorname{Li} 1$ & $08 I^{\prime} \mathrm{I}$ & 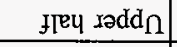 & & LItS00L96S \\
\hline$S S \angle 6$ & $8 \angle 6$ & $\varepsilon L 6$ & J[एц ләмот & \multirow[b]{2}{*}{$9: 99 \mathrm{I}$} & $819 \$ 00 L 96 \mathrm{~S}$ \\
\hline $070^{6} \mathrm{I}$ & $090^{6} \mathrm{I}$ & $020^{\circ} \mathrm{T}$ & $f^{\mathbb{E}}$ เәdd $\Omega$ & & t29500L96S \\
\hline$S^{\circ} 6 \varepsilon 0^{\circ} \mathrm{I}$ & $0 t I^{\prime} \mathrm{I}$ & $6 \varepsilon 6$ & ذГеЧ ऽәмоТ & \multirow[b]{2}{*}{ s:99I } & $6 S+000 L L 6 S$ \\
\hline s.s26 & $z \varepsilon 6$ & $6 \mathrm{I} 6$ & f[Eบ Iadd & & $9 I t S 00 L 96 S$ \\
\hline$I \varepsilon L$ & $09 L$ & $20 L$ & J[EU IәMот & \multirow[b]{2}{*}{$\varepsilon: 99 \mathrm{I}$} & ZZLSO0L96S \\
\hline $58 \angle 8$ & I06 & $9 \$ 8$ & fley Iədd & & $9 \mathrm{ILSO0L}$ L6S \\
\hline$I t I>$ & ItI $>$ & $I t I>$ & I[EY دәмоT] & $z: 99 \mathrm{I}$ & $\angle I 9 S 00 L 96 \mathrm{~S}$ \\
\hline$\varsigma 00 I>$ & $9 \varepsilon I>$ & $\mathrm{StI}>$ & ІІеч ІәмоТ & I:99I & 919S00L96S \\
\hline s.991 & $z \angle I$ & I9I & I[еप I2MOT] & OI :99I & ILSS00L96S \\
\hline$\varsigma 6 I Z>$ & $66 \mathrm{I}>$ & $0 \leftarrow z$ & म[Еप دәмоТ] & $6: 99 I$ & SLSS00L96S \\
\hline$\angle 6 I>$ & $\angle 6 \mathrm{I}>$ & $\angle 6 I>$ & IIEY IOMOT & $8: 99 \mathrm{I}$ & $0+8 \mathrm{~S} 00 \mathrm{~L} 96 \mathrm{~S}$ \\
\hline$s \mathcal{E O Z}>$ & $\underline{S_{0 Z}>}$ & $z 0 z>$ & I[एप IәMOT & $L: 991$ & $6 \mathcal{E S} \mathrm{S} 00 \mathrm{~L} 96 \mathrm{~S}$ \\
\hline $8 \mathcal{E I}>$ & $8 E I>$ & $8 E I>$ & I[Eบ IOMOT] & $9: 991$ & SI9S00L96S \\
\hline $6 L I>$ & $6 L I>$ & $6 \angle I>$ & Ғ[Еप IәMOT] & $t: 99 \mathrm{I}$ & 0LSSO0L $96 \mathrm{~S}$ \\
\hline$s+66$ & 268 & $\angle 66$ & I[एप IәMOT] & [ :99I & $609 \$ 00 L 96 \mathrm{~S}$ \\
\hline 6301 & \% & 济 & & \multicolumn{2}{|c|}{ 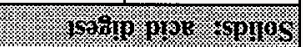 } \\
\hline 1001 & 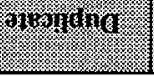 & (1) & . & rowes of & 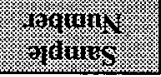 \\
\hline
\end{tabular}

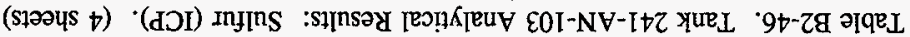


Table B2-46. Tank 241-AN-103 Analytical Results: Sulfur (ICP). (4 sheets)

\begin{tabular}{|c|c|c|c|c|c|}
\hline Squmple: & Srample & Sarmie & Resurit & oyininite & $4 \times 2 n$ \\
\hline \multicolumn{2}{|c|}{ 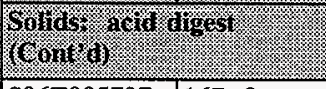 } & (3): & $\sqrt{196}$ & (1) & 10 \\
\hline S96T005737 & 167: 8 & Lower half & $<196$ & $<200$ & $<198$ \\
\hline S96T005858 & $167: 9$ & Lower half & $<208$ & $<175$ & $<191.5$ \\
\hline S96T005709 & \multirow[t]{2}{*}{ 167: 1} & Upper half & 645 & 645 & 645 \\
\hline S96T005697 & & Lower half & 774 & 691 & 732.5 \\
\hline S96T005444 & \multirow[t]{2}{*}{$167: 2$} & Upper half & 789 & 809 & $799^{\mathrm{QC:a}}$ \\
\hline S96T005451 & & Lower half & 979 & 862 & $920.5^{\mathrm{QC}: \mathrm{a}}$ \\
\hline S96T005445 & \multirow[t]{2}{*}{$167: 4$} & Upper half & 805 & 687 & $746^{\mathrm{QC}: a}$ \\
\hline S96T005439 & & Lower half & 635 & 761 & $698^{\mathrm{QC}: a}$ \\
\hline S96T005710 & \multirow[t]{2}{*}{$167: 5$} & Upper half & 788 & 798 & 793 \\
\hline S96T005698 & & Lower half & 1,010 & 1,030 & 1,020 \\
\hline S96T005498 & \multirow[t]{2}{*}{$167: 7$} & \begin{tabular}{|l} 
Upper half \\
\end{tabular} & 1,120 & 1,090 & 1,105 \\
\hline S96T005499 & & \begin{tabular}{|l|} 
Lower half \\
\end{tabular} & 1,640 & 1,630 & 1,635 \\
\hline S97T000460 & Core 166 & Solid composite & 809 & 847 & 828 \\
\hline \multirow{2}{*}{\multicolumn{3}{|c|}{\begin{tabular}{l|l|l} 
Miguids & \\
S96T005539 & $166: 3$ & Drainable liquid
\end{tabular}}} & pgmin & 10.931 & 4 \\
\hline & & & 425 & 307 & $366^{\mathrm{eC}: \mathrm{e}}$ \\
\hline S96T005540 & $166: 4$ & Drainable liquid & 352 & 292 & 322 \\
\hline S96T005599 & $166: 6$ & Drainable liquid & 490 & 418 & 454 \\
\hline S96T005816 & $166: 7$ & Drainable liquid & 477 & 482 & 479.5 \\
\hline S96T005817 & $166: 8$ & Drainable liquid & 466 & 489 & 477.5 \\
\hline S96T005541 & $166: 9$ & Drainable liquid & 485 & 486 & 485.5 \\
\hline S96T005566 & 166: 10 & Drainable liquid & 372 & 383 & 377.5 \\
\hline S96T005600 & $166: 1$ & Drainable liquid & 433 & 395 & 414 \\
\hline S96T005601 & $166: 2$ & Drainable liquid & 466 & 451 & 458.5 \\
\hline S96T005861 & 167: 2 & Drainable liquid & 464 & 480 & 472 \\
\hline S96T005673 & $167: 3$ & Drainable liquid & 429 & 400 & 414.5 \\
\hline S96T005420 & $167: 5$ & Drainable liquid & 475 & 455 & 465 \\
\hline S96T005421 & 167: 6 & Drainable liquid & 463 & 456 & 459.5 \\
\hline S96T005683 & $167: 7$ & Drainable liquid & 469 & 465 & 467 \\
\hline
\end{tabular}


Table B2-46. Tank 241-AN-103 Analytical Results: Sulfur (ICP). (4 sheets)

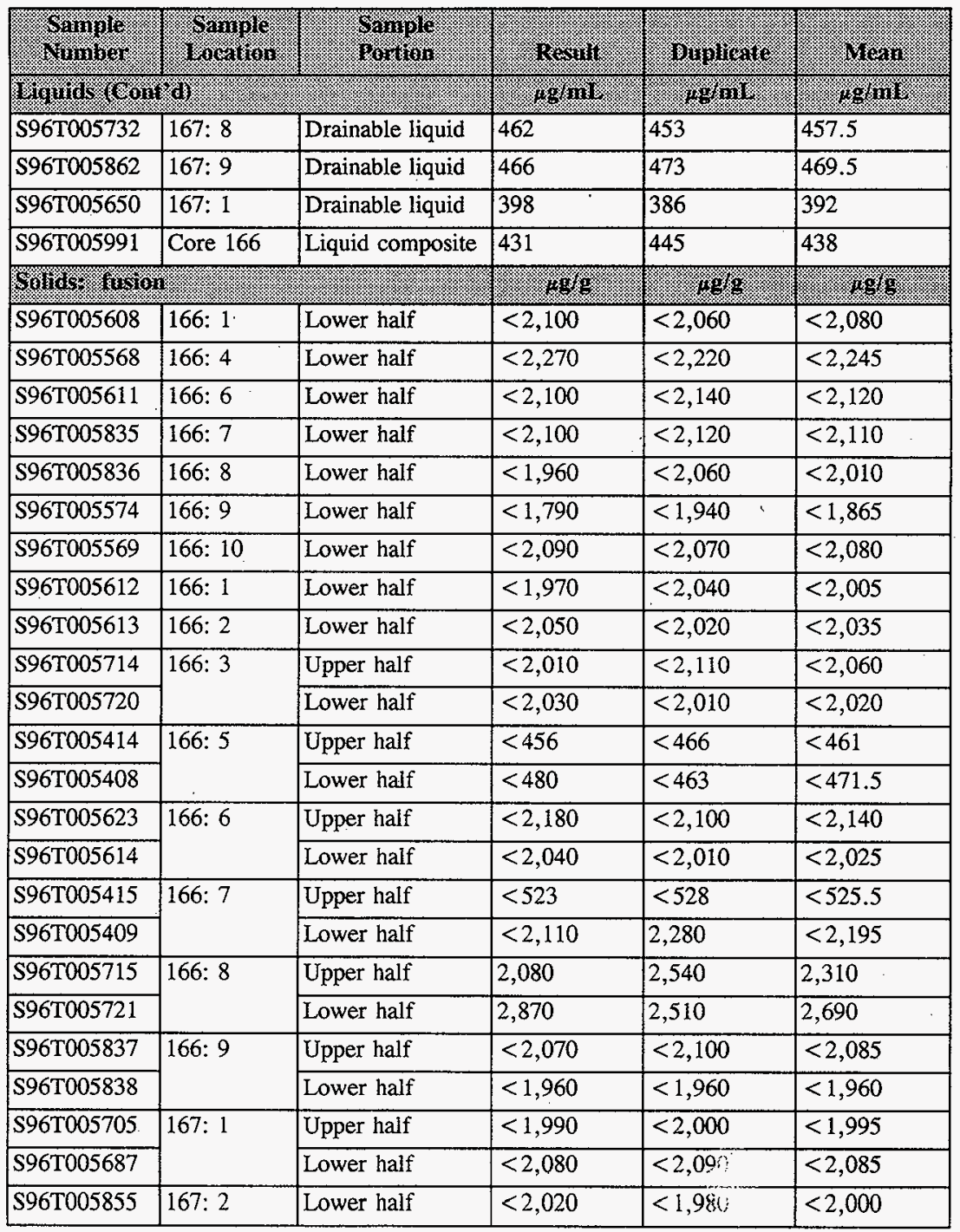




\begin{tabular}{|c|c|c|c|c|c|}
\hline SOI' $Z>$ & OII'Z> & $00 I^{\prime} z>$ & Ғтец дәмот & & $\angle 6 t S 00 \mathrm{~L} 96 \mathrm{~S}$ \\
\hline oII'Z> & $00 I^{\prime} z>$ & $0 Z I^{\prime} Z>$ & freч ıədd $\Omega$ & $\angle: \angle 9 I$ & $96+500 \mathrm{~L} 96 \mathrm{~S}$ \\
\hline$S 00^{\circ} \mathrm{r}>$ & $08 I^{\prime} \tau>$ & $0 E 8^{6} i>$ & ҒГеप ІәмоТ & \multirow[b]{2}{*}{$S: \angle 9 I$} & Z69S00L96S \\
\hline S86 I $>$ & $0+8^{\prime} 1>$ & $0 \varepsilon I^{\prime} Z>$ & JIEч Iədd $\Omega$ & & LOLSO0L96S \\
\hline $0+0^{\circ} \mathrm{z}>$ & $0 \varepsilon 0^{\prime} z>$ & $0 \mathrm{SO}^{\prime} \mathrm{z}>$ & Јгеप ЈәмO ] & \multirow[b]{2}{*}{$\nabla: \angle 9 l$} & $\angle E+S 00 \mathrm{~L} 96 \mathrm{~S}$ \\
\hline SSI' $Z>$ & ofI'Z> & $00 z^{6} z>$ & 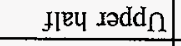 & & $\varepsilon t+S 00196 \mathrm{~S}$ \\
\hline $000 \% \tau>$ & $020^{\circ} z>$ & $086^{6} 1>$ & I[еप دәмот & \multirow[b]{2}{*}{$z: \angle 9 I$} & $6 t+500196 \mathrm{~S}$ \\
\hline OSO $2>$ & $006^{\circ} \mathrm{I}>$ & $09 I^{\prime} z>$ & fleप Iadd $\Omega$ & & $\tau t+S 00 L 96 S$ \\
\hline$S+8^{\circ} i>$ & $0+8^{6} 1>$ & $0 S 8^{6} \mathrm{I}>$ & Iец دәмоТ] & \multirow[b]{2}{*}{$I: \angle 9 I$} & I69S00L96S \\
\hline$s+0^{\circ} z>$ & $0 z z^{\prime} z>$ & $0<8^{\prime} \mid>$ & IfEY $\mathrm{I} ә \mathrm{dd} \Omega$ & & $90 \angle S 00 L 96 \mathrm{~S}$ \\
\hline$S \angle 6^{\prime} \backslash>$ & 0II'Z> & $0+8^{\circ} I>$ & ҒЕप гәмоТ & $6: \mathrm{LgI}$ & $9 S 8 \mathrm{~S} 00 \mathrm{~L} 96 \mathrm{~S}$ \\
\hline $0+0^{\circ} r>$ & $0<8^{6} I>$ & $0 โ Z^{\prime} Z>$ & IRQ & $8: \angle 9 I$ & $9 \varepsilon \angle S 00 L 96 \mathrm{~S}$ \\
\hline $0 \angle 0^{\circ} \mathrm{Z}>$ & $08 I^{\prime} Z>$ & $096^{6} \mid>$ & Ғाеч دәмот & $L: \angle 9 \mathrm{I}$ & $069500 \mathrm{~L} 96 \mathrm{~S}$ \\
\hline SLO' $\mathrm{Z}>$ & $0+0^{\circ} z>$ & OII'Z> & म[EY IəMOT & $9: \mathrm{LII}$ & $87 t 500 L 96 S$ \\
\hline $0<8^{\circ} 1>$ & $088^{6} \mathrm{I}>$ & $098^{\circ} \mathrm{I}>$ & fгец دәмо & $\varsigma: \angle 9 \mathrm{I}$ & $9 \varepsilon+S 00 \mathrm{~L} 96 \mathrm{~S}$ \\
\hline$S_{0} I^{\prime} Z>$ & OII'Z> & $00 I^{\prime} \tau>$ & ңтеч дәмо & $\forall: \angle 9 \mathrm{I}$ & $689500 \mathrm{~L} 96 \mathrm{~S}$ \\
\hline $06 I^{\prime} z>$ & $0 E I^{\prime} Z>$ & $0 \subseteq Z^{\prime} Z>$ & I[RY ІәмоТ] & $\varepsilon: \angle 9 \mathrm{I}$ & $889500 \perp 96 \mathrm{~S}$ \\
\hline 8.18 8 . & ( & 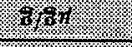 & & \multicolumn{2}{|c|}{ 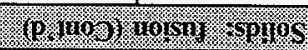 } \\
\hline trantis & 9uermand & 17098 & 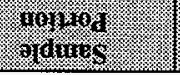 & 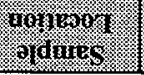 & 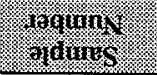 \\
\hline
\end{tabular}

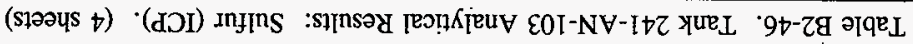


Table B2-47. Tank 241-AN-103 Analytical Results: Titanium (ICP). (4 sheets)

\begin{tabular}{|c|c|c|c|c|c|}
\hline Samples: & Sample & $\begin{array}{l}\text { Sample: } \\
\text { riontion: }\end{array}$ & Reraili & Buplienter & Mrean \\
\hline \multicolumn{2}{|c|}{ Sollde aridigest } & 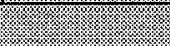 & 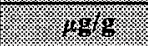 & 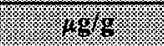 & 1.899 \\
\hline S96T005609 & $166: 1$ & Lower half & $<14.8$ & $<15.1$ & $<14.95^{\text {QC:a }}$ \\
\hline S96T005570 & $166: 4$ & Lower half & $<17.9$ & $<17.9$ & $<17.9^{\mathrm{QC}: \mathrm{c}}$ \\
\hline S96T005615 & $166: 6$ & Lower half & $<13.8$ & $<13.8$ & $<13.8^{\mathrm{QC}: \mathrm{a}}$ \\
\hline S96T005839 & 166: 7 & Lower half & $<20.2$ & $<20.5$ & $<20.35^{\mathrm{QC}: \mathrm{a}}$ \\
\hline S96T005840 & $166: 8$ & Lower half & $<19.7$ & $<19.7$ & $<19.7^{\mathrm{QC}: \mathrm{a}}$ \\
\hline S96T005575 & 166: 9 & Lower half & $<19.6$ & $<19.9$ & $<19.75$ \\
\hline S96T005571 & $166: 10$ & Lower half & $<15.3$ & $<14.5$ & $<14.9^{\mathrm{QC}: \mathrm{a}}$ \\
\hline S96T005616 & $166: 1$ & Lower half & $<14.5$ & $<13.6$ & $<14.05^{\mathrm{QC}: \mathrm{a}}$ \\
\hline S96T005617 & $166: 2$ & Lower half & $<14.1$ & $<14.1$ & $<14.1^{\mathrm{QC:a}}$ \\
\hline S96T005716 & \multirow[t]{2}{*}{$166: 3$} & Upper half & $<19.4$ & $<19.6$ & $<19.5^{\mathrm{QC:A}}$ \\
\hline S96T005722 & & Lower half & $<20.6$ & $<20.6$ & $<20.6^{\mathrm{QC}: \mathrm{a}}$ \\
\hline S96T005416 & \multirow[t]{2}{*}{$166: 5$} & Upper half & $<20.4$ & $<19$ & $<19.7$ \\
\hline S97T000459 & & Lower half & $<5.69$ & $<5.57$ & $<5.63^{\text {QC:a }}$ \\
\hline S96T005624 & \multirow[t]{2}{*}{ 166: 6} & Upper half & $<21$ & $<21.5$ & $<21.25^{\mathrm{QC:}: \mathrm{a}}$ \\
\hline S96T005618 & & Lower half & $<14.2$ & $<14.6$ & $<14.4^{\mathrm{QC}: a}$ \\
\hline S96T005417 & \multirow[t]{2}{*}{$166: 7$} & Upper half & $<22.5$ & $<20.5$ & $<21.5$ \\
\hline S96T005411 & & Lower half & $<19$ & $<17.6$ & $<18.3$ \\
\hline S96T005717 & \multirow[t]{2}{*}{$166: 8$} & Upper half & $<20.8$ & $<20.2$ & $<20.5^{\mathrm{QC}: \mathrm{a}}$ \\
\hline S96T005723 & & Lower half & $<20.2$ & $<19.8$ & $<20^{\mathrm{QC}: \mathrm{a}}$ \\
\hline S96T005841 & \multirow[t]{2}{*}{$166: 9$} & Upper half & $<19.3$ & $<19.6$ & $<19.45^{\mathrm{QC}: \mathrm{a}}$ \\
\hline S96T005842 & & Lower half & $<17.8$ & $<19.6$ & $<18.7^{\mathrm{QC:a}}$ \\
\hline S96T005708 & \multirow[t]{2}{*}{$167: 1$} & Upper half & $<17.9$ & $<20.5$ & $<19.2$ \\
\hline S96T005693 & & Lower half & $<22.2$ & $<17.5$ & $<19.85^{\mathrm{QC}: \mathrm{c}}$ \\
\hline S96T005857 & $167: 2$ & Lower half & $<16.7$ & $<17.4$ & $<17.05^{\mathrm{QC:a}}$ \\
\hline S96T005694 & $167: 3$ & Lower half & $<18$ & $<18.7$ & $<18.35$ \\
\hline S96T005695 & $167: 4$ & Lower half & $<19.8$ & $<20.4$ & $<20.1$ \\
\hline S96T005438 & $167: 5$ & Lower half & $<5.17$ & $<5.51$ & $<5.34^{\mathrm{QC}: \mathrm{a}}$ \\
\hline S96T005450 & $167: 6$ & Lower half & $<5.53$ & $<5.22$ & $<5.375^{\mathrm{QC}: \mathrm{a}}$ \\
\hline S96T005696 & $167: 7$ & Lower half & $<19.2$ & $<18.8$ & $<19$ \\
\hline
\end{tabular}


Table B2-47. Tank 241-AN-103 Analytical Results: Titanium (ICP). (4 sheets)

\begin{tabular}{|c|c|c|c|c|c|}
\hline 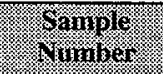 & 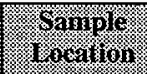 & sample & presilis & Yunlionto & Mran \\
\hline Solud ach & Hest & 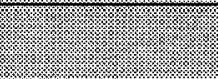 & F. & 1009 & \% \\
\hline S96T005737 & 167: 8 & Lower half & $<19.6$ & $<20$ & $<19.8$ \\
\hline S96T005858 & 167:9 & Lower half & $<20.8$ & $<17.5$ & $<19.15^{\mathrm{QC:a}}$ \\
\hline \$96T005709 & \multirow[t]{2}{*}{ 167: 1} & Upper half & $<18$ & $<18.7$ & $<18.35$ \\
\hline S96T005697 & & Lower half & $<19.8$ & $<18.8$ & $<19.3$ \\
\hline S96T005444. & \multirow[t]{2}{*}{ 167: 2} & Upper half & $<5.53$ & $<5.45$ & $<5.49^{Q \mathrm{C}: \mathrm{a}}$ \\
\hline S96T005451 & & Lower half & $<5.68$ & $<5.84$ & $<5.76^{\mathrm{QC}: \mathrm{a}}$ \\
\hline S96T005445 & \multirow[t]{2}{*}{$167: 4$} & Upper half & $<5.28$ & $<5.51$ & $<5.395^{\mathrm{QC}: \mathrm{a}}$ \\
\hline S96T005439 & & Lower half & $<5.68$ & $<5.84$ & $<5.76^{\mathrm{QC}: \mathrm{a}}$ \\
\hline \$96T005710 & \multirow[t]{2}{*}{$167: 5$} & Upper half & $<18.6$ & $<17.9$ & $<18.25$ \\
\hline S96T005698 & & Lower half & $<18.2$ & $<18.8$ & $<18.5$ \\
\hline S96T005498 & \multirow[t]{2}{*}{$167: 7$} & Upper half & $<18.3$ & $<19.5$ & $<18.9$ \\
\hline S96T005499 & & Lower half & $<17.8$ & $<19.3$ & $<18.55$ \\
\hline S97T000460 & Core 166 & Solid composite & $<5.63$ & $<5.51$ & $<5.57^{\mathrm{QC}: a}$ \\
\hline 19uros & & & $189 \mathrm{IIL}$ & (1) & . \\
\hline S96T005539 & $166: 3$ & Drainable liquid & $<4.01$ & $<4.01$ & $<4.01$ \\
\hline S96T005540 & $166: 4$ & Drainable liquid & $<4.01$ & $<4.01$ & $<4.01$ \\
\hline S96T005599 & 166: 6 & Drainable liquid & $<6.01$ & $<6.01$ & $<6.01$ \\
\hline S96T005816 & 166: 7 & Drainable liquid & $<6.01$ & $<6.01$ & $<6.01$ \\
\hline S96T005817 & $166: 8$ & Drainable liquid & $<6.01$ & $<6.01$ & $<6.01$ \\
\hline S96T005541 & 166: 9 & Drainable liquid & $<6.01$ & $<6.01$ & $<6.01$ \\
\hline S96T005566 & 166: 10 & Drainable liquid & $<4.01$ & $<4.01$ & $<4.01$ \\
\hline S96T005600 & 166: 1 & Drainable liquid & $<6.01$ & $<6.01$ & $<6.01$ \\
\hline S96T005601 & $166: 2$ & Drainable liquid & $<6.01$ & $<6.01$ & $<6.01$ \\
\hline S96T005861 & 167: 2 & Drainable liquid & $<6.01$ & $<6.01$ & $<6.01$ \\
\hline S96T005673 & $167: 3$ & Drainable liquid & $<6.01$ & $<6.01$ & $<6.01$ \\
\hline S96T005420 & $167: 5$ & Drainable liquid & $<6.01$ & $<6.01$ & $<6.01$ \\
\hline S96T005421 & $167: 6$ & Drainable liquid & $<6.01$ & $<6.01$ & $<6.01$ \\
\hline S96T005683 & 167: 7 & Drainable liquid & $<6.01$ & $<6.01$ & $<6.01$ \\
\hline
\end{tabular}


Table B2-47. Tank 241-AN-103 Analytical Results: Titanium (ICP). (4 sheets)

\begin{tabular}{|c|c|c|c|c|c|}
\hline Sample & Sample & $\begin{array}{l}\text { Sample } \\
\text { Yonkor }\end{array}$ & Resilit & Burlulerite & Veri. \\
\hline \multicolumn{2}{|c|}{ 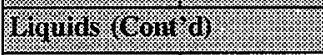 } & (1: & (6ginit & $\log 1011$ & $4.8 \mathrm{~min}$ \\
\hline S96T005732 & 167: 8 & Drainable liquid & $<6.01$ & $<6.01$ & $<6.01$ \\
\hline S96T005862 & $167: 9$ & Drainable liquid & $<6.01$ & $<6.01$ & $<6.01$ \\
\hline S96T005650 & 167: 1 & Drainable liquid & $<6.01$ & $<6.01$ & $<6.01$ \\
\hline S96T005991 & Core 166 & Liquid composite & $<12$ & $<12$ & $<12$ \\
\hline \multicolumn{2}{|l|}{ Holdis firion } & (2) & $4.986=$ & 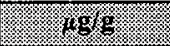 & (3) \\
\hline S96T005608 & 166: 1 & Lower half & $<210$ & $<206$ & $<208$ \\
\hline S96T005568 & $166: 4$ & Lower half & $<227$ & $<222$ & $<224.5$ \\
\hline S96T005611 & $166: 6$ & Lower half & $<210$ & $<214$ & $<212$ \\
\hline S96T005835 & $166: 7$ & Lower half & $<210$ & $<212$ & $<211$ \\
\hline S96T005836 & $166: 8$ & Lower half & $<196$ & $<206$ & $<201$ \\
\hline S96T005574 & $166: 9$ & Lower half & 293 & 261 & 277 \\
\hline S96T005569 & $166: 10$ & Lower half & $<209$ & $<207$ & $<208$ \\
\hline S96T005612 & $166: 1$ & Lower half & $<197$ & $<204$ & $<200.5$ \\
\hline S96T005613 & $166: 2$ & Lower half & $<205$ & $<202$ & $<203.5$ \\
\hline S96T005714 & \multirow[t]{2}{*}{$166: 3$} & Upper half & $<201$ & $<211$ & $<206$ \\
\hline S96T005720 & & Lower half & $<203$ & $<201$ & $<202$ \\
\hline S96T005414 & \multirow[t]{2}{*}{$166: 5$} & Upper half & $<45.6$ & $<46.6$ & $<46.1$ \\
\hline S96T005408 & & Lower half & $<48$ & $<46.3$ & $<47.15$ \\
\hline S96T005623 & \multirow[t]{2}{*}{$166: 6$} & Upper half & $<218$ & $<210$ & $<214$ \\
\hline S96T005614 & & Lower half & $<204$ & $<201$ & $<202.5^{\mathrm{QC}: \mathrm{a}}$ \\
\hline S96T005415 & \multirow[t]{2}{*}{ 166: 7} & Upper half & $<52.3$ & $<52.8$ & $<52.55$ \\
\hline S96T005409 & & Lower half & $<211$ & $<208$ & $<209.5$ \\
\hline S96T005715 & \multirow[t]{2}{*}{ 166: 8} & Upper half & $<201$ & $<206$ & $<203.5$ \\
\hline S96T005721 & & Lower half & $<203$ & $<199$ & $<201$ \\
\hline S96T005837 & \multirow[t]{2}{*}{$166: 9$} & Upper half & $<207$ & $<210$ & $<208.5$ \\
\hline S96T005838 & & Lower half & $<196$ & $<196$ & $<196$ \\
\hline S96T005705 & \multirow[t]{2}{*}{$167: 1$} & Upper half & $<199$ & $<200$ & $<199.5$ \\
\hline S96T005687 & & Lower half & $<208$ & $<209$ & $<208.5$ \\
\hline S96T005855 & $167: 2$ & Lower half & $<202$ & $<198$ & $<200$ \\
\hline
\end{tabular}




\begin{tabular}{|c|c|c|c|c|c|}
\hline s.0IZ> & II $2>$ & $0 \mathrm{IZ}>$ & JाЕप JәMOT & \multirow[b]{2}{*}{$\angle: \angle 9 \mathrm{I}$} & L6tS00L96S \\
\hline IIZ> & $0 I z>$ & zIZ> & fley Lədd $\Omega$ & & $96 t$ S00I96S \\
\hline$s 00 z>$ & $812>$ & $\varepsilon 8 \mathrm{I}>$ & J[Еप ІәмО I & \multirow[b]{2}{*}{$\subseteq: \angle 9 I$} & Z69S00L96S \\
\hline$\varsigma^{\circ} 86 \mathrm{I}>$ & $78 \mathrm{I}>$ & $\varepsilon[z>$ & Jॉeч xadd $\Omega$ & & $\angle 0 L S 00 L 96 \mathrm{~S}$ \\
\hline t0z> & $E 0 z>$ & $s 0 z>$ & JIеप IOMOT & \multirow[b]{2}{*}{$\nabla: \angle 9 I$} & $L \dot{\varepsilon}+S 00 L 96 \mathrm{~S}$ \\
\hline s'sIZ> & III $>$ & $0 z z>$ & fley sədd $\Omega$ & & $\varepsilon+t S 00 L 96 \mathrm{~S}$ \\
\hline $00 z>$ & $z 0 z>$ & $86 I>$ & JાЕч دәмо & \multirow[b]{2}{*}{$\tau: \angle 9 I$} & $6 t t S 00 L 96 S$ \\
\hline$\varsigma_{0 Z>}$ & $76 \mathrm{I}>$ & 9 II > & गृеप Iәdd $\Omega$ & & $z_{t+S 00 L 96 S}$ \\
\hline$S^{\circ}+8 I>$ & $78 I>$ & $S 8 I>$ & IIЕЧ ІәмOТ & \multirow[b]{2}{*}{$I: \angle 9 !$} & I $69500 \mathrm{~L} 96 \mathrm{~S}$ \\
\hline$s+0 z>$ & $z z \tau>$ & $\angle 8 I>$ & Ғтеч Jədd $_{\Omega}$ & & 90LS00L96S \\
\hline$S: \angle 6 I>$ & III > & $\pm 8 \mathrm{I}>$ & Ј[Еч دәмо Т & $6: 29 \mathrm{I}$ & $9 S 8 S 00 \perp 96 S$ \\
\hline$t 0 z>$ & $\angle 8 I>$ & IZZ> & дгеप гәмо Т & $8: \angle 9 I$ & $9 \varepsilon \angle S 00 \perp 96 \mathrm{~S}$ \\
\hline$\angle 0 z>$ & $8 I Z>$ & $961>$ & Iाеप гәмот & $\angle: \angle 9 I$ & 069500ـ96S \\
\hline$\varsigma^{\prime} \angle O Z>$ & toz> & IIZ> & ҒाЕч Іәмо $\mathrm{T}$ & $9: \angle 9 \mathrm{I}$ & $8 t+500 \mathrm{~L} 96 \mathrm{~S}$ \\
\hline$\angle 8 I>$ & $881>$ & $98 I>$ & Ғ[ЕЧ دәмоТ & $s: \angle 9 I$ & $9 \varepsilon t S 00 \mathrm{~L} 96 \mathrm{~S}$ \\
\hline s.oIz> & IIZ> & orz> & fleप دәмот & $\nabla: \angle 9 I$ & $689 S 00 \mathrm{~L} 96 \mathrm{~S}$ \\
\hline $6 I z>$ & $\varepsilon I Z>$ & szZ> & Ітеч гәмол & $\varepsilon: \angle 9 I$ & $889500 L 96 S$ \\
\hline 7. & 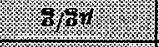 & ingar & & \multicolumn{2}{|c|}{ 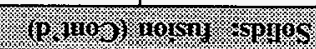 } \\
\hline rovin: & 3imorang & 17w5yd & $\begin{array}{l}\text { molurors } \\
\text { ajgrings }\end{array}$ & rojorong & sogrmon \\
\hline
\end{tabular}

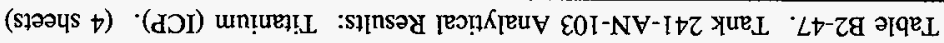


Table B2-48. Tank 241-AN-103 Analytical Results: Zinc (ICP). (4 sheets)

\begin{tabular}{|c|c|c|c|c|c|}
\hline $\begin{array}{l}\text { Sample } \\
\text { Number }\end{array}$ & $\begin{array}{l}\text { Sampie } \\
\text { Sucation }\end{array}$ & Sample & (1) & Buinicare & 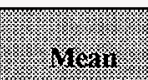 \\
\hline \multicolumn{2}{|c|}{ Sords a dil aygest } & 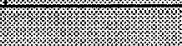 & 16.6. & 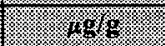 & ) \\
\hline S96T005609 & 166: 1 & Lower half & $<14.8$ & 17.8 & $<16.3$ \\
\hline S96T005570 & 166: 4 & Lower half & $<17.9$ & 20.1 & $<19$ \\
\hline S96T005615 & 166: 6 & Lower half & $<13.8$ & $<13.8$ & $<13.8$ \\
\hline S96T005839 & 166:7 & Lower half & $<20.2$ & $<20.5$ & $<20.35$ \\
\hline S96T005840 & 166: 8 & Lower half & 34.8 & 19.9 & $27.35^{\mathrm{QC:a,c}}$ \\
\hline S96T005575 & 166: 9 & Lower half & 22.1 & 20.1 & 21.1 \\
\hline S96T005571 & 166: 10 & Lower half & 29.4 & $<14.5$ & $<21.95^{\mathrm{QC}: c}$ \\
\hline S96T005616 & 166: 1 & Lower half & $<14.5$ & $<13.6$ & $<14.05$ \\
\hline S96T005617 & 166: 2 & Lower half & $<14.1$ & $<14.1$ & $<14.1$ \\
\hline S96T005716 & \multirow[t]{2}{*}{$166: 3$} & Upper half & $<19.4$ & $<19.6$ & $<19.5$ \\
\hline S96T005722 & & Lower half & $<20.6$ & $<20.6$ & $<20.6$ \\
\hline S96T005416 & \multirow[t]{2}{*}{ 166: 5} & Upper half & 22.7 & 21.3 & 22 \\
\hline S97T000459 & & Lower half & $<5.69$ & $<5.57$ & $<5.63^{\mathrm{QC}: a}$ \\
\hline S96T005624 & \multirow[t]{2}{*}{$166: 6$} & Upper half & $<21$ & 22.8 & $<21.9$ \\
\hline S96T005618 & & Lower half & $<14.2$ & 39.7 & $<26.95^{\mathrm{QC}: \mathrm{C}}$ \\
\hline S96T005417 & \multirow[t]{2}{*}{$166: 7$} & Upper half & 23.2 & $<20.5$ & $<21.85$ \\
\hline S96T005411 & & Lower half & 23.1 & $<17.6$ & $<20.35^{\mathrm{QC}: \mathrm{c}}$ \\
\hline S96T005717 & \multirow[t]{2}{*}{$166: 8$} & Upper half & 68.6 & 70.3 & 69.45 \\
\hline S96T005723 & & Lower half & $<20.2$ & $<19.8$ & $<20$ \\
\hline S96T005841 & \multirow[t]{2}{*}{$166: 9$} & Upper half & 30.5 & 22 & $26.25^{\text {QC:a,e }}$ \\
\hline S96T005842 & & Lower half & 24.7 & 35.3 & $30^{\mathrm{QC:a,c}}$ \\
\hline S96T005708 & \multirow[t]{2}{*}{$167: 1$} & Upper half & 84.1 & 87.1 & 85.6 \\
\hline S96T005693 & & Lower half & 67 & 41.2 & $54.1^{\mathrm{QC:e}}$ \\
\hline S96T005857 & $167: 2$ & Lower half & 29.8 & 27.2 & 28.5 \\
\hline S96T005694 & $167: 3$ & Lower half & 18.9 & 44.8 & $31.85^{\mathrm{QC}: \mathrm{e}}$ \\
\hline S96T005695 & $167: 4$ & Lower half & 65.6 & 31.6 & $48.6^{\mathrm{OC}: \mathrm{e}}$ \\
\hline S96T005438 & $167: 5$ & Lower half & 9.57 & 7.95 & $8.76^{\mathrm{QCa} a}$ \\
\hline S96T005450 & $167: 6$ & Lower half & 7.07 & 8.4 & $7.735^{\mathrm{QC}: \mathrm{a}}$ \\
\hline S96T005696 & $167: 7$ & Lower half & 27.8 & 20.2 & $24^{\mathrm{QC:B}}$ \\
\hline
\end{tabular}




\begin{tabular}{|c|c|c|c|c|c|}
\hline$\varsigma 9$ & $\angle D^{\circ} 9$ & $\varepsilon S^{*} 9$ & p!̣b!̣ı əશqeutesવ & $\angle: \angle 9 I$ & E89S00L96S \\
\hline$S^{\circ} D I$ & $I+1$ & $6 \pm I$ & p!nb!l әрquụexa & $9: \angle 9 I$ & IZtS00L96S \\
\hline LIII & 8.11 & 9.II & pı̣bọা әIquuịeIa & $\mathcal{S : L 9 I}$ & $0 z \triangleright \$ 00 L 96 \mathrm{~S}$ \\
\hline $10^{\circ} 9>$ & $10^{\circ} 9>$ & {$\left[0^{\prime} 9>\right.$} & plnbị ərqeuresa & $\varepsilon: \angle 9 \mathrm{I}$ & $\varepsilon \angle 9 S 00 J 96 \mathrm{~S}$ \\
\hline$E \nabla^{\circ} L$ & $t^{\circ} L$ & $90^{\circ} \mathrm{L}$ & p!̣b!̣ әqеuाexd & $2: \angle 9 I$ & I98S00L96S \\
\hline $10^{\circ} 9>$ & $10^{\circ} 9 ?$ & $10^{\circ} 9>$ & pṭb!̣! ə[qeu!eı & Z:99I & I09S00L96S \\
\hline$s 22^{\circ} 9>$ & $10^{\circ} 9>$ & $\nabla t 9$ & pinby ә әqеurexa & I:991 & $009 \mathrm{~S} 00 \mathrm{~L} 96 \mathrm{~S}$ \\
\hline t.II & $Z^{\prime} \mathrm{II}$ & $9^{\circ}$ II & 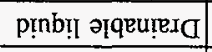 & $01: 991$ & $99 S S 00 \mathrm{~L} 96 \mathrm{~S}$ \\
\hline 88.6 & $95 \cdot 6$ & $20 \mathrm{I}$ & p!nb!̣ ə[qеu!̣eIव & $6: 99 \mathrm{I}$ & $1+5 \$ 00 L 96 \mathrm{~S}$ \\
\hline $9 L L$ & $6 \mathrm{I} 8$ & $\varepsilon \varepsilon\llcorner$ & pịnbỵ әlqeurera & $8: 991$ & LI8S00L96S \\
\hline$\nabla Z L$ & 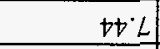 & $70^{\circ} \mathrm{L}$ & pụnb!̣ ә әqеu!eıa & $L: 991$ & 918S00L96S \\
\hline $10^{\circ} 9>$ & {$\left[0^{\circ} 9>\right.$} & $10 \cdot 9>$ & p!nb!l әqеu!esa & $9: 99 \mathrm{I}$ & $66 \mathrm{SS} 00 \mathrm{~L} 96 \mathrm{~S}$ \\
\hline$S 8 \cdot \varepsilon I$ & $\nabla^{\circ} \varepsilon I$ & $\varepsilon * t$ & 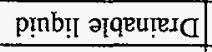 & $\nabla: 991$ & 0†SS00L96S \\
\hline $\mathrm{SSOI}$ & $\varepsilon 0 \mathrm{I}$ & $80 \mathrm{I}$ & pı̣nbỵ ə[quuṭxa & $\varepsilon: 991$ & 6ESS00L96S \\
\hline rinon & martiti & 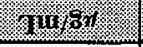 & & & sprition \\
\hline ::00ZI L $L$ & $\nabla 6.9$ & $\varepsilon L$ & əl!soduos pịos & 991 วI0) & $097000 \mathrm{~L} \angle 6 \mathrm{~S}$ \\
\hline$\varepsilon \cdot \tau \varepsilon$ & $9^{\circ} \varepsilon \varepsilon$ & IE & IIRY IәMOT & & $66+500 \mathrm{~L} 96 \mathrm{~S}$ \\
\hline$\$ 62 I$ & $\angle I I$ & $z b I$ & fleu Iədd $\Omega$ & $\angle: \angle 9 I$ & $867 S 00 \mathrm{~L} 96 \mathrm{~S}$ \\
\hline $0: 00 S L^{\prime} E \mathcal{E}$ & $\varepsilon \subseteq$ & $\tau \cdot z t$ & I Iеप् دәмо Т & & $869 S 00 L 96 S$ \\
\hline$I+s$ & 605 & $\varepsilon \angle S$ & fleu rədd $\Omega$ & $s: \angle 9 I$ & OLLS00L96S \\
\hline $8: 002 \cdot 8 \mathrm{I}$ & $\tau \cdot 6 I$ & $Z \angle I$ & IГЕЧ ІәмоТ & & $6 \mathcal{5} \$ 500 \mathrm{~L} 96 \mathrm{~S}$ \\
\hline $8: 0086 \mathrm{~L}$ & 96.5 & OI & Ireप Iodd $\Omega$ & $\downarrow: \angle 9 \mathrm{I}$ & SttS00L96S \\
\hline $8: 0080^{\circ} 9>$ & $78^{\circ} \varsigma>$ & $z \varepsilon^{\circ} 9$ & IEY IOMOT & & IStS00L96S \\
\hline Or: $: 00 S \angle S \cdot 8$ & $\mathrm{II}$ & SI 9 & $\mathfrak{I}[\mathrm{ey}$ Iədd $\Omega$ & $\tau: \angle 9 \mathrm{I}$ & $\nabla t t S 00 L 96 \mathrm{~S}$ \\
\hline $2: 00 L^{\circ} \mathcal{E} t$ & $\varepsilon \cdot 9 \varepsilon$ & I.IS & IГеप ІәмоТ & & L69S00L96S \\
\hline ::00Z'IE & $58 \mathcal{E}$ & $6^{\circ} \varepsilon 2$ & $y^{1 E Y}$ Iədd $\Omega$ & {$[: \angle 9 I$} & 60LS00L96S \\
\hline$=00 S \nabla^{\circ} \nabla \tau>$ & $\mathrm{I} 8 \mathrm{z}$ & $80 z>$ & f[еप دәмо I & $6: \angle 9 I$ & 8S8500L96S \\
\hline$=00 \$ 29 t$ & $\varsigma 99$ & $9 \mathcal{Z}$ & IIRU IOMOT & $8: \angle 9 \mathrm{I}$ & $\angle E \angle S 00 L 96 \mathrm{~S}$ \\
\hline a & (7) & 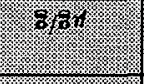 & & 1.7 .5711 & 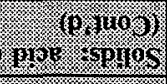 \\
\hline (10.6. & 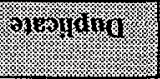 & 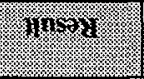 & Mongrog & 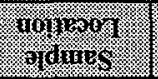 & oghan \\
\hline
\end{tabular}

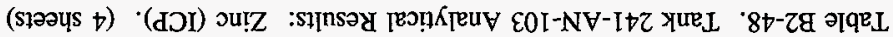


Table B2-48. Tank 241-AN-103 Analytical Results: Zinc (ICP). (4 sheets)

\begin{tabular}{|c|c|c|c|c|c|}
\hline $\begin{array}{l}\text { Samile } \\
\text { Sumbr: }\end{array}$ & $\begin{array}{l}\text { Sample } \\
\text { S }\end{array}$ & Sample & 19esin) & inghicate & Mlain \\
\hline \multicolumn{3}{|c|}{ Yiguiris (Comi d) } & (4) & $14 \mathrm{~g} / \mathrm{mi}$ & S \\
\hline S96T005732 & $167: 8$ & Drainable liquid & 21.7 & 21.1 & 21.4 \\
\hline S96T005862 & $167: 9$ & Drainable liquid & 8.59 & 8.72 & 8.655 \\
\hline S96T005650 & 167: 1 & Drainable liquid & 13.9 & 14 & 13.95 \\
\hline S96T005991 & Core 166 & Liquid composite & $<12$ & $<12$ & $<12$ \\
\hline \multicolumn{3}{|c|}{ Sofids - rivion } & 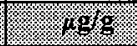 & 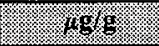 & 14.8g. \\
\hline S96T005608 & 166: 1 & Lower half & $<210$ & $<206$ & $<208$ \\
\hline S96T005568 & 166: 4 & Lower half & 811 & 952 & 881.5 \\
\hline S96T005611 & $166: 6$ & Lower half & $<210$ & $<214$ & $<212$ \\
\hline S96T005835 & 166: 7 & Lower half & $<210$ & $<212$ & $<211$ \\
\hline S96T005836 & $166: 8$ & Lower half & $<196$ & $<206$ & $<201$ \\
\hline S96T005574 & $166: 9$ & Lower half & 3,000 & 2,940 & 2,970 \\
\hline S96T005569 & $166: 10$ & Lower half & $<209$ & $<207$ & $<208$ \\
\hline S96T005612 & $166: 1$ & Lower half & $<197$ & $<204$ & $<200.5$ \\
\hline S96T005613 & 166: 2 & Lower half & $<205$ & $<202$ & $<203.5$ \\
\hline S96T005714 & \multirow[t]{2}{*}{$166: 3$} & Upper half & $<201$ & $<211$ & $<206$ \\
\hline S96T005720 & & Lower half & $<203$ & 658 & $<430.5^{\mathrm{QC}: \mathrm{c}}$ \\
\hline S96T005414 & \multirow[t]{2}{*}{$166: 5$} & Upper half & 177 & 114 & $145.5^{\mathrm{QC}: c}$ \\
\hline S96T005408 & & Lower half & 177 & 46.6 & $111.8^{\mathrm{QC}: \mathrm{c}}$ \\
\hline S96T005623 & \multirow[t]{2}{*}{$166: 6$} & Upper half & $<218$ & $<210$ & $<214$ \\
\hline S96T005614 & & Lower half & $<204$ & $<201$ & $<202.5$ \\
\hline S96T005415 & \multirow[t]{2}{*}{$166: 7$} & Upper half & 369 & $<52.8$ & $<210.9^{\alpha \mathrm{C}: e}$ \\
\hline S96T005409 & & Lower half & $<211$ & 343 & $<277^{\mathrm{QC}: c}$ \\
\hline S96T005715 & \multirow[t]{2}{*}{$166: 8$} & Upper half & $<201$ & $<206$ & $<203.5$ \\
\hline S96T005721 & & Lower half & $<203$ & $<199$ & $<201$ \\
\hline S96T005837 & \multirow[t]{2}{*}{$166: 9$} & Upper half & $<207$ & $<210$ & $<208.5$ \\
\hline S96T005838 & & Lower half & $<196$ & $<196$ & $<196$ \\
\hline S96T005705 & \multirow[t]{2}{*}{$167: 1$} & Upper half & 466 & 209 & $337.5^{\mathrm{QC}: e}$ \\
\hline S96T005687 & & Lower half & $<208$ & $<209$ & $<208.5$ \\
\hline S96T005855 & $167: 2$ & Lower half & $<202$ & $<198$ & $<200$ \\
\hline
\end{tabular}




\begin{tabular}{|c|c|c|c|c|c|}
\hline solz> & IIZ> & $0\lfloor z>$ & fleप $\triangle \mathrm{MMO}_{\mathrm{T}}$ & & $\angle 6 \oplus S 00 L 96 S$ \\
\hline $2000 S^{\circ} \varepsilon 92$ & IEZ & 962 & 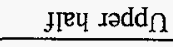 & $\angle: \angle 9 I$ & $96+S 00 \perp 96 \mathrm{~S}$ \\
\hline$s .00 z>$ & $812>$ & $\varepsilon 8 I>$ & Jॉеप ІәмоТ & \multirow[b]{2}{*}{$\subseteq: \angle 9 I$} & $269500 \mathrm{~L} 96 \mathrm{~S}$ \\
\hline I0z> & $68 \mathrm{I}$ & $\varepsilon I Z>$ & J[E् & & $\angle 0 \angle S 00 \perp 96 \mathrm{~S}$ \\
\hline t0Z> & $\varepsilon 0 z>$ & SOZ $>$ & IГеप Iәмо & \multirow[b]{2}{*}{$t: \angle 9 I$} & $\angle E t S 00 \mathrm{~L} 96 \mathrm{~S}$ \\
\hline s.SIZ> & IIZ> & $0 z z>$ & f[еч & & $\varepsilon+t S 00 L 96 S$ \\
\hline $00 z>$ & $z 0 z>$ & $86 I>$ & J[Еप & \multirow[b]{2}{*}{$Z: \angle 9 I$} & $6 t t s 00 \mathrm{~L} 96 \mathrm{~S}$ \\
\hline soz> & $161>$ & $9 I Z>$ & $\mathrm{j} \mathrm{eq}$ цәdd $\Omega$ & & $\tau \downarrow t S 00 L 96 S$ \\
\hline$S^{\prime}+8 I>$ & $t 8 I>$ & $58 \mathrm{I}>$ & IFЕY IәMOT & \multirow[b]{2}{*}{$\mathrm{I}: \angle 9 \mathrm{I}$} & I69S00L96S \\
\hline$s+0 z>$ & $z z z>$ & $\angle 8 I>$ & $\mathrm{f}^{\mathrm{E} Y}$ Iadd $\Omega$ & & $90 \angle S 00 \mathrm{~L} 96 \mathrm{~S}$ \\
\hline$S \angle 6 I>$ & $112>$ & $+8 I>$ & Jеप גәMOT & $6: \angle 9 I$ & 9S8S00L96S \\
\hline$+0 z>$ & $\angle 8 I>$ & $I Z Z>$ & IГеч хәмо & $8: \angle 9 I$ & $9 \mathcal{E} \angle S 00 \perp 96 \mathrm{~S}$ \\
\hline$\angle 0 Z>$ & $8 I Z>$ & $96 I>$ & I[Еप دәмOT & $\angle: \angle 9 \mathrm{I}$ & $069500 \mathrm{I} 96 \mathrm{~S}$ \\
\hline$: 00 \varepsilon 6 Z>$ & $\subseteq L E$ & IIZ> & J[Еप JәMOT & $9: \angle 9 I$ & $8 t+500 . \mathrm{L} 96 \mathrm{~S}$ \\
\hline$s^{\prime} s 0 z>$ & $88 \mathrm{I}>$ & $\varepsilon z Z$ & JГеप دәMOT & $S: \angle 9 \mathrm{I}$ & $9 \varepsilon+S 00 L 96 S$ \\
\hline sorz> & IIZ> & $0 I Z>$ & Јाеч хәмо Т & $\nabla: \angle 9 I$ & $689500 \perp 96 \mathrm{~S}$ \\
\hline $6 I Z>$ & $\varepsilon I Z>$ & $\leq Z Z>$ & IFE JәMOT & $\cdot \varepsilon: \angle 9 I$ & $889500 L 96 \mathrm{~S}$ \\
\hline expyl & 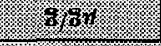 & $8 \%$ & (3) & \multicolumn{2}{|c|}{ 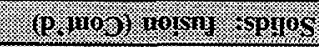 } \\
\hline $\operatorname{lng} 1$ & Olfougrar & Mrove? & $\begin{array}{l}\text { movingar } \\
\text { rarros }\end{array}$ & 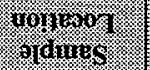 & rovininus \\
\hline
\end{tabular}

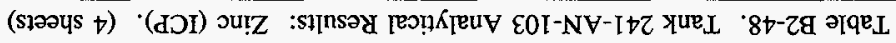


Table B2-49. Tank 241-AN-103 Analytical Results: Zirconium (ICP). (4 sheets)

\begin{tabular}{|c|c|c|c|c|c|}
\hline 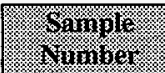 & Symple & 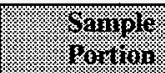 & (2) pesinin & 8joling & ) \\
\hline \multicolumn{3}{|c|}{ 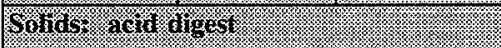 } & , w & \% & 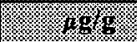 \\
\hline S96T005609 & $166: 1$ & Lower half & $<14.8$ & $<15.1$ & $<14.95$ \\
\hline S96T005570 & $166: 4$ & Lower half & $<17.9$ & $<17.9$ & $<17.9$ \\
\hline S96T005615 & $166: 6$ & Lower half & $<13.8$ & $<13.8$ & $<13.8$ \\
\hline S96T005839 & 166: 7 & Lower half & $<20.2$ & $<20.5$ & $<20.35$ \\
\hline S96T005840 & 166: 8 & Lower half & $<19.7$ & $<19.7$ & $<19.7$ \\
\hline S96T005575 & 166: 9 & Lower half & $<19.6$ & $<19.9$ & $<19.75$ \\
\hline \$96T005571 & $166: 10$ & Lower half & $<15.3$ & $<14.5$ & $<14.9$ \\
\hline S96T005616 & $166: 1$ & Lower half & $<14.5$ & $<13.6$ & $<14.05$ \\
\hline S96T005617 & 166: 2 & Lower half & $<14.1$ & $<14.1$ & $<14.1$ \\
\hline \$96T005716 & \multirow{2}{*}{ 166: 3} & Upper half & $<19.4$ & $<19.6$ & $<19.5$ \\
\hline \$96T005722 & & Lower half & $<20.6$ & $<20.6$ & $<20.6$ \\
\hline S96T005416 & \multirow[t]{2}{*}{$166: 5$} & Upper half & $<20.4$ & $<19$ & $<19.7$ \\
\hline S97T000459 & & Lower half & $<5.69$ & $<5.57$ & $<5.63$ \\
\hline S96T005624 & \multirow[t]{2}{*}{ 166: 6} & Upper half & $<21$ & $<21.5$ & $<21.25$ \\
\hline S96T005618 & & Lower half & $<14.2$ & $<14.6$ & $<14.4$ \\
\hline \$96T005417 & \multirow[t]{2}{*}{$166: 7$} & Upper half & $<22.5$ & $<20.5$ & $<21.5$ \\
\hline S96T005411 & & Lower half & $<19$ & $<17.6$ & $<18.3^{\mathrm{QC}: \mathrm{c}}$ \\
\hline S96T005717 & \multirow[t]{2}{*}{$166: 8$} & Upper half & $<20.8$ & $<20.2$ & $<20.5$ \\
\hline S96T005723 & & Lower half & $<20.2$ & $<19.8$ & $<20$ \\
\hline S96T005841 & \multirow[t]{2}{*}{$166: 9$} & Upper half & $<19.3$ & $<19.6$ & $<19.45$ \\
\hline S96T005842 & & Lower half & $<17.8$ & $<19.6$ & $<18.7$ \\
\hline S96T005708 & \multirow[t]{2}{*}{ 167: 1} & Upper half & $<17.9$ & $<20.5$ & $<19.2$ \\
\hline S96T005693 & & Lower half & $<22.2$ & $<17.5$ & $<19.85^{\mathrm{QC}: \mathrm{e}}$ \\
\hline S96T005857 & 167: 2 & Lower half & $<16.7$ & $<17.4$ & $<17.05$ \\
\hline S96T005694 & $167: 3$ & Lower half & $<18$ & $<18.7$ & $<18.35$ \\
\hline S96T005695 & $167: 4$ & Lower half & $<19.8$ & $<20.4$ & $<20.1$ \\
\hline S96T005438 & $167: 5$ & Lower half & $<5.17$ & $<5.51$ & $<5.34$ \\
\hline S96T005450 & $167: 6$ & Lower half & $<5.53$ & $<5.22$ & $<5.375$ \\
\hline S96T005696 & $167: 7$ & Lower half & $<19.2$ & $<18.8$ & $<19$ \\
\hline
\end{tabular}


Table B2-49. Tank 241-AN-103 Analytical Results: Zirconium (ICP). (4 sheets)

\begin{tabular}{|c|c|c|c|c|c|}
\hline Sumple & $\begin{array}{l}\text { Samples } \\
\text { Iogrition }\end{array}$ & Somple & ingin & Buplicate & V. \\
\hline 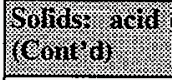 & Mgesi & & 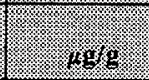 & $=0$ & 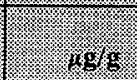 \\
\hline S96T005737 & 167:8 & Lower half & $<19.6$ & $<20$ & $<19.8$ \\
\hline S96T005858 & $167: 9$ & Lower half & $<20.8$ & $<17.5$ & $<19.15$ \\
\hline S96T005709 & \multirow[t]{2}{*}{ 167: 1} & Upper half & $<18$ & $<18.7$ & $<18.35$ \\
\hline S96T005697 & & Lower half & $<19.8$ & $<18.8$ & $<19.3$ \\
\hline S96T005444 & \multirow[t]{2}{*}{ 167: 2} & Upper half & $<5.53$ & $<5.45$ & $<5.49$ \\
\hline S96T005451 & & Lower half & $<5.68$ & $<5.84$ & $<5.76$ \\
\hline S96T005445 & \multirow[t]{2}{*}{$167: 4$} & Upper half & $<5.28$ & $<5.51$ & $<5.395$ \\
\hline S96T005439 & & Lower half & $<5.68$ & $<5.84$ & $<5.76$ \\
\hline S96T005710 & \multirow[t]{2}{*}{$167: 5$} & Upper half & $<18.6$ & $<17.9$ & $<18.25$ \\
\hline S96T005698 & & Lower half & $<18.2$ & $<18.8$ & $<18.5$ \\
\hline S96T005498 & \multirow[t]{2}{*}{$167: 7$} & Upper half & $<18.3$ & $<19.5$ & $<18.9$ \\
\hline S96T005499 & & Lower half & $<17.8$ & $<19.3$ & $<18.55$ \\
\hline S97T000460 & Core 166 & Solid composite & $<5.63$ & $<5.51$ & $<5.57$ \\
\hline Migunde & (1) & (2) & 1.96 ind & (1) & Fin \\
\hline S96T005539 & $166: 3$ & Drainable liquid & 6.11 & 6.55 & 6.33 \\
\hline S96T005540 & 166: 4 & Drainable liquid & 8.4 & 12.2 & $10.3^{\mathrm{QC:c}}$ \\
\hline S96T005599 & $166: 6$ & Drainable liquid & 7.21 & 8.82 & $8.015^{\mathrm{QC}: \mathrm{e}}$ \\
\hline S96T005816 & 166: 7 & \begin{tabular}{|l|} 
Drainable liquid \\
\end{tabular} & 6.34 & 6.38 & 6.36 \\
\hline S96T005817 & 166: 8 & Drainable liquid & 6.09 & 6.63 & 6.36 \\
\hline S96T005541 & 166: 9 & Drainable liquid & 7.06 & 7.39 & 7.225 \\
\hline S96T005566 & 166: 10 & Drainable liquid & 7.81 & 7.85 & 7.83 \\
\hline S96T005600 & $166: 1$ & Drainable liquid & 9.11 & 9.79 & 9.45 \\
\hline S96T005601 & $166: 2$ & Drainable liquid & $<6.01$ & $<6.01$ & $<6.01$ \\
\hline S96T005861 & $167: 2$ & Drainable liquid & 6.36 & 7.24 & 6.8 \\
\hline S96T005673 & $167: 3$ & Drainable liquid & 11.7 & 11.8 & 11.75 \\
\hline S96T005420 & 167: 5 & Drainable liquid & $<6.01$ & $<6.01$ & $<6.01$ \\
\hline S96T005421 & 167: 6 & Drainable liquid & 8.13 & 8.13 & 8.13 \\
\hline S96T005683 & $167: 7$ & Drainable liquid & $<6.01$ & $<6.01$ & $<6.01$ \\
\hline
\end{tabular}


Table B2-49. Tank 241-AN-103 Analytical Results: Zirconium (ICP). (4 sheets)

\begin{tabular}{|c|c|c|c|c|c|}
\hline $\begin{array}{l}\text { Sample } \\
\text { Trumber }\end{array}$ & $\int_{\text {Sanuple }}$ & Singles & Rresulit & Bunficate & M. \\
\hline \multicolumn{2}{|c|}{ 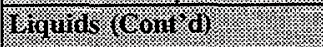 } & ': & (6\%) & $186.131:$ & . 1.9611 \\
\hline S96T005732 & $167: 8$ & Drainable liquid & $<6.01$ & $<6.01$ & $<6.01$ \\
\hline S96T005862 & $167: 9$ & Drainable liquid & 6.72 & 6.49 & 6.605 \\
\hline S96T005650 & $167: 1$ & Drainable liquid & 10 & 10.5 & 10.25 \\
\hline S96T005991 & Core 166 & Liquid composite & $<12$ & $<12$ & $<12$ \\
\hline \multicolumn{2}{|c|}{ Soldos frision) } & & 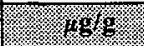 & 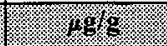 & (1) 196 \\
\hline S96T005608 & 166: 1 & Lower half & $<210$ & $<206$ & $<208$ \\
\hline S96T005568 & $166: 4$ & Lower half & $<227$ & $<222$ & $<224.5$ \\
\hline S96T005611 & $166: 6$ & Lower half & $<210$ & $<214$ & $<212$ \\
\hline S96T005835 & $166: 7$ & Lower half & $<210$ & $<212$ & $<211$ \\
\hline S96T005836 & $166: 8$ & Lower half & $<\overline{196}$ & $<206$ & $<201$ \\
\hline S96T005574 & $166: 9$ & Lower half & $<179$ & $<194$ & $<186.5$ \\
\hline S96T005569 & 166: 10 & Lower half & $<209$ & $<207$ & $<208$ \\
\hline S96T005612 & $166: 1$ & Lower half & $<197$ & $<204$ & $<200.5$ \\
\hline S96T005613 & $166: 2$ & Lower half & $<205$ & $<202$ & $<203.5$ \\
\hline S96T005714 & \multirow[t]{2}{*}{$166: 3$} & Upper half & $<201$ & $<211$ & $<206$ \\
\hline S96T005720 & & Lower half & $<203$ & $<201$ & $<202$ \\
\hline S96T005414 & \multirow[t]{2}{*}{$166: 5$} & Upper half & $<45.6$ & $<46.6$ & $<46.1$ \\
\hline S96T005408 & & Lower half & $<48$ & $<46.3$ & $<47.15$ \\
\hline S96T005623 & \multirow[t]{2}{*}{$166: 6$} & Upper half & $<218$ & $<210$ & $<214$ \\
\hline S96T005614 & & Lower half & $<204$ & $<201$ & $<202.5$ \\
\hline S96T005415 & \multirow[t]{2}{*}{$166: 7$} & Upper half & $<52.3$ & $<52.8$ & $<52.55$ \\
\hline S96T005409 & & Lower half & $<211$ & $<208$ & $<209.5$ \\
\hline S96T005715 & \multirow[t]{2}{*}{$166: 8$} & Upper half & $<201$ & $<206$ & $<203.5$ \\
\hline S96T005721 & & Lower half & $<203$ & $<199$ & $<201$ \\
\hline S96T005837 & \multirow[t]{2}{*}{$166: 9$} & Upper half & $<207$ & $<210$ & $<208.5$ \\
\hline S96T005838 & & Lower half & $<196$ & $<196$ & $<196$ \\
\hline S96T005705 & \multirow[t]{2}{*}{ 167: 1} & Upper half & $<199$ & $<200$ & $<199.5$ \\
\hline S96T005687 & & Lower half & $<208$ & $<209$ & $<208.5$ \\
\hline S96T005855 & $167: 2$ & Lower half & $<202$ & $<198$ & $<200$ \\
\hline
\end{tabular}




\begin{tabular}{|c|c|c|c|c|c|}
\hline$S O I Z>$ & IIZ> & $0\lfloor Z>$ & I'ец ІәмоТ & \multirow[b]{4}{*}{$\varsigma: \angle 9 I$} & $L 6 \pm S 00 L 96 S$ \\
\hline IIZ> & $0 I \tau>$ & $z I Z>$ & 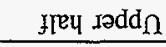 & & $96+S 00 L 96 \mathrm{~S}$ \\
\hline$\varsigma^{\circ} 00 z>$ & $8 I 2>$ & E8I > & JlЕप Іәмот & & $269 \$ 00 \mathrm{~L} 96 \mathrm{~S}$ \\
\hline$\$ 86 I>$ & $581>$ & $\varepsilon I Z>$ & Jеप Iədd $_{\Omega}$ & & $\angle 0 L S 00 \mathrm{~L} 96 \mathrm{~S}$ \\
\hline$\dashv 0 z>$ & $\varepsilon 0 Z>$ & $s 0 z>$ & IГЕЧ IOMOT & \multirow[b]{2}{*}{$\nabla: \angle 9 \mathrm{I}$} & $\angle E \nrightarrow S 00 L 96 \mathrm{~S}$ \\
\hline$\varsigma S I Z>$ & IIZ> & $0 z z>$ & Ileq Iədd $\Omega$ & & $\varepsilon \nabla t \$ 00 L 96 S$ \\
\hline $002>$ & $z 0 z>$ & $86 I>$ & IIEY IOMOT & \multirow[b]{2}{*}{$\tau: \angle 9 \mathrm{I}$} & $6 \nabla t 500 \mathrm{~L} 96 \mathrm{~S}$ \\
\hline soz $>$ & $761>$ & $912>$ & fleप Iədd $_{\Omega}$ & & Ztb500L96S \\
\hline$S^{\circ}+8 \mathrm{I}>$ & $581>$ & $58 I>$ & freu дамот & \multirow[b]{2}{*}{$: \angle 9 I$} & I69\$00L96S \\
\hline$s+0 z>$ & $z z z>$ & $\angle 8 I>$ & 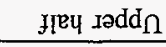 & & $90 \angle 500 L 96 \mathrm{~S}$ \\
\hline$\underline{s} \angle 61>$ & IIZ> & $+8 I>$ & Ileप IәMOT & $6: \angle 9 \mathrm{I}$ & 9\$8S00L96S \\
\hline$+0 z>$ & $\angle 8 I>$ & $12 z>$ & IIEY IәMOT & $8: \angle 9 I$ & 9ELS00L96S \\
\hline$\angle O Z>$ & $81 Z>$ & $96 \mathrm{I}>$ & I[еч Iaмот & $\angle: \angle 9 I$ & 069S00L96S \\
\hline$S \angle O Z>$ & toz> & IIZ> & дГеप дамот & $9: \angle 9 \mathrm{I}$ & $8 t t S 00 L 96 S$ \\
\hline$\angle 8 \mathrm{I}>$ & $88 \mathrm{I}>$ & $98 I>$ & Iाеप IәMO & $\varsigma: \angle 9 I$ & $9 \varepsilon \downarrow S 00 \mathrm{~L} 96 \mathrm{~S}$ \\
\hline solz> & $I I Z>$ & $0 I Z>$ & f[RY IәMOT & $\nabla: \angle 9 I$ & $689 S 00 L 96 \mathrm{~S}$ \\
\hline $6 \mathrm{IZ}>$ & $\varepsilon I Z>$ & $\mathfrak{s z z}>$ & J[Еप Iәмот & $\varepsilon: \angle 9 \mathrm{I}$ & $889500 L 96 \mathrm{~S}$ \\
\hline (6) & (6) & 0.7871 & & \multicolumn{2}{|c|}{ 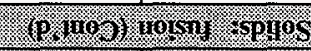 } \\
\hline (16) & 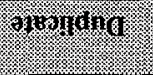 & Wid & \%omor & oromory & aldint \\
\hline
\end{tabular}

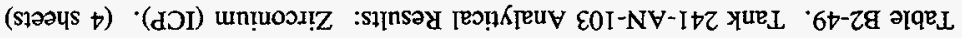


Table B2-50. Tank 241-AN-103 Inductively Coupled Plasma Results with Less Than Value.

\begin{tabular}{|l|l|}
\hline Arsenic & Manim \\
\hline Barium & $<2270$ \\
\hline Bismuth & $<1130$ \\
\hline Cerium & $<2270$ \\
\hline Magnesium & $<2270$ \\
\hline Neodymium & $<2270$ \\
\hline Samarium & $<2270$ \\
\hline Strontium & $<2270$ \\
\hline Thallium & $<227$ \\
\hline Total Uranium & $<4530$ \\
\hline Vanadium & $<11,300$ \\
\hline
\end{tabular}




\begin{tabular}{|c|c|c|c|c|c|}
\hline$s+\angle 6 t>$ & $025>$ & $6+\angle D>$ & Jाвц دәмоТ & $6: \angle 9 I$ & 098S00L96S \\
\hline$\angle 006>>$ & $\angle \varepsilon 6>$ & $\nabla+766>$ & „ાеч Іәмо Т & $8: \angle 9 I$ & $8 \varepsilon \angle S 00 \mathrm{~L} 96 \mathrm{~S}$ \\
\hline $9\left\lfloor z^{\prime}\right\rfloor>$ & $0 \angle Z^{\prime} I>$ & $29 I^{\prime} I>$ & Jleप дәмо & $\angle: \angle 9 I$ & $20 L S 00 \mathrm{~L} 96 \mathrm{~S}$ \\
\hline$\varepsilon \angle S 6>$ & $296>$ & $9 \cdot 256>$ & fтеप Іәмот & $9: \angle 9 \mathrm{I}$ & $z S t S 00 L 96 \mathrm{~S}$ \\
\hline $\mathrm{s}^{\circ} \mathrm{SsO}^{\circ} \mathrm{I}$ & 886 & EZI'I & Эाеप ІәMOT & $\mathrm{s: \angle 9I}$ & $0 t+500.496 \mathrm{~S}$ \\
\hline$\varsigma^{*} \varepsilon L \sigma^{*} I$ & $0 \angle 0^{\circ} \mathrm{Z}$ & $\angle L 8^{\prime} \mathrm{I}$ & дाвч $\triangle ә м о 7$ & $\nabla: \angle 9 I$ & IOLS00L96S \\
\hline $0+6^{\circ} \mathrm{I}$ & $096^{\circ} \mathrm{I}$ & $026^{\circ} \mathrm{I}$ & ҒાЕч & $\varepsilon: \angle 9 \mathrm{I}$ & $00 \angle 500 \mathrm{~L} 96 \mathrm{~S}$ \\
\hline S'III'I & $00 T^{\prime} \mathrm{I}$ & $\varepsilon Z I^{\prime} I$ & Ғ[ЕЧ Іәмо I & $z: \angle 9 \mathrm{I}$ & $6 \mathrm{~S} 8500 \mathrm{~L} 96 \mathrm{~S}$ \\
\hline $5089^{\circ} 1>$ & $0+9^{4} \mathrm{I}>$ & $I Z L^{\prime} \mid>$ & Јाец دәмо I & \multirow[b]{2}{*}{$: \angle 9 \mathrm{I}$} & $669500 \mathrm{~L} 96 \mathrm{~S}$ \\
\hline S99'I $\mathrm{I}$ & $00 L^{\prime} I>$ & $0 \varepsilon 9^{i} I>$ & 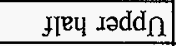 & & ILLS00L96S \\
\hline$\therefore: 00 S 8^{\circ} 9 t t$ & ZIS & $L^{\circ}\lfloor 8 \mathcal{E}$ & Јाеप IәмOT & \multirow[b]{2}{*}{$6: 99 \mathrm{I}$} & $9+8 S 00 L 96 \mathrm{~S}$ \\
\hline $0: 00 S I^{\prime} 66 \varepsilon$ & $0 s t$ & $\varepsilon \cdot 8 \nabla \varepsilon$ & Jॉeप Iədd $\Lambda$ & & St8S00L96S \\
\hline$s S^{\circ} t\langle t\rangle$ & $0<t>$ & $1 \cdot 6 \angle t>$ & fru $\triangle 2 M O T$ & \multirow[b]{2}{*}{$8: 99$} & SZLS00L96S \\
\hline St $\angle E 9$ & $\$ 29$ & 6059 & 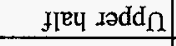 & & 6ILS00L96S \\
\hline$\xi \sqcup \angle 0^{\circ} I>$ & $02 I^{\prime} I>$ & $620^{6} 1>$ & I[Еप دәмоТ] & \multirow[b]{2}{*}{$\angle: 99 \mathrm{I}$} & $\varepsilon 1+S 00 . \mathrm{L} 96 \mathrm{~S}$ \\
\hline $\mathrm{S} 8^{\circ} \mathrm{S}+9$ & 829 & $L \cdot \varepsilon 99$ & freu rədd $\Lambda$ & & $6 \mathrm{ItSO0L96S}$ \\
\hline $920^{\circ} I>$ & $0 \varepsilon 0^{6}[>$ & $220^{\circ} 1>$ & נГеч & \multirow[b]{2}{*}{$9: 99 \mathrm{I}$} & $229500 \mathrm{~L} 96 \mathrm{~S}$ \\
\hline$S I^{\circ} 9 Z 6>$ & $\$ 26>$ & $\varepsilon \angle 26>$ & дॄеч Iədd $_{\Lambda}$ & & \$29\$00L96S \\
\hline S.IIO'I & $000^{\circ} \mathrm{I}>$ & $\varepsilon 20^{6} \mathrm{I}>$ & 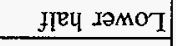 & \multirow[b]{2}{*}{ S:99I } & $2 I+500196 \mathrm{~S}$ \\
\hline $59 \angle 89$ & 299 & $\varepsilon^{*} \varepsilon I L$ & $y_{[\mathrm{eY}} \operatorname{I\partial dd}_{\Omega}$ & & $8 \mathfrak{l}+S 00 L 96 S$ \\
\hline$z \cdot 20 s>$ & $\angle O S$ & $t^{\circ} \angle 6 t>$ & J[ЕY دәмо & \multirow[b]{2}{*}{$\varepsilon: 99 \mathrm{I}$} & $+Z L S 00 L 96 \mathrm{~S}$ \\
\hline$S^{\prime} 060^{\prime}[>$ & $00 I^{\prime} \mid>$ & $180^{\circ} \mathrm{I}>$ & $f[\varepsilon y$ 工әdd $\Omega$ & & 8I $\angle S 00 L 96 \mathrm{~S}$ \\
\hline$s^{\circ} \cos z>$ & OEs'z> & $180^{\prime} \mathrm{Z}>$ & J[Еप دәмо $]$ & $\tau: 99 \mathrm{I}$ & Iz9S00L96S \\
\hline$s^{*}+8 t^{\prime} z>$ & $08 t^{\circ} Z>$ & $68 t^{\circ} \tau>$ & УГеч دәмоТ & I :99I & $029 S 00 L 96 \mathrm{~S}$ \\
\hline$S^{\prime} 90 I^{\prime}\lfloor>$ & $060^{\circ} \mathrm{I}>$ & $\varepsilon Z I^{\prime} I>$ & J[еч гәмо & OI:991 & $\varepsilon L S S 00 \mathrm{~L} 96 \mathrm{~S}$ \\
\hline$S S^{\circ} I L 6>$ & $9 \pm 6>$ & I $\angle 66>$ & Јтеч гәмо Т & $6: 991$ & $9 L S S 00 L 96 \mathrm{~S}$ \\
\hline$\varepsilon 10^{6} I>$ & $010^{6} \mathrm{I}>$ & 910'1> & J[Еप دәMO] & $8: 991$ & $7+8 S 00 \mathrm{~L} 96 \mathrm{~S}$ \\
\hline S6 I66> & $986>$ & $6 \cdot 266>$ & JाЕЧ & $L: 991$ & $\varepsilon+8500 L 96 \mathrm{~S}$ \\
\hline 6ZI'I & $0 \varepsilon I^{\prime} I>$ & $82 I^{\prime} I>$ & गाЕч دәMOT & $9: 99 I$ & $619500 \mathrm{~L} 96 \mathrm{~S}$ \\
\hline $\mathrm{S}^{\circ} 8 S 0^{\circ} \mathrm{I}>$ & OE0'I $I>$ & $\angle 80^{6} \mathrm{I}>$ & У[Еप & $\downarrow: 991$ & ZLSS00L96S \\
\hline$S^{\prime} 260^{\prime} I>$ & $080^{\circ} I>$ & SOI'I > & IfEY IOMOT & I:99I & $019500 \mathrm{~L} 96 \mathrm{~S}$ \\
\hline 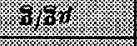 & \% 611 & opit & & \multicolumn{2}{|c|}{ 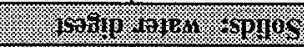 } \\
\hline trour & pimonging & (115\%) & moriris. & 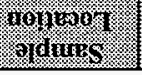 & $\begin{array}{l}\text { Iogring } \\
\text { surmes }\end{array}$ \\
\hline
\end{tabular}

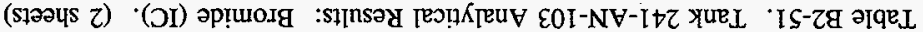


Table B2-51. Tank 241-AN-103 Analytical Results: Bromide (IC). (2 sheets)

\begin{tabular}{|c|c|c|c|c|c|}
\hline Sainple & trampigy & $\begin{array}{l}\text { Sample } \\
\text { Sortigir }\end{array}$ & incsili & 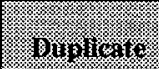 & (16) \\
\hline Sollas wart & drigest & 2.: & ? & $\sqrt{4} \sqrt{8}$ & (l. \\
\hline S96T005712 & \multirow[t]{2}{*}{ 167: 1} & Upper half & $<970.3$ & $<1,010$ & $<990.15$ \\
\hline S96T005703 & & Lower half & $<514.4$ & $<495$ & $<504.7$ \\
\hline S96T005446 & \multirow[t]{2}{*}{$167: 2$} & Upper half & $<494.1$ & $<518$ & $<506.05$ \\
\hline S96T005453 & & Lower half & $<506.4$ & $<508$ & $<507.2$ \\
\hline S96T005447 & \multirow[t]{2}{*}{$167: 4$} & Upper half & $<485.8$ & $<470$ & $<477.9$ \\
\hline S96T005441 & & Lower half & $<1,178$ & $<1,180$ & $<1,179$ \\
\hline S96T005713 & \multirow[t]{2}{*}{$167: 5$} & Upper half & $<980.5$ & $<961$ & $<970.75$ \\
\hline S96T005704 & & Lower half & $<1,015$ & $<990$ & $<1,002.5$ \\
\hline S96T005500 & \multirow[t]{2}{*}{$167: 7$} & Upper half & $<1,694$ & 1,820 & $<1,757$ \\
\hline S96T005501 & & Lower half & $<1,712$ & $<1,630$ & $<1,671$ \\
\hline S97T000023 & Core 166 & Solid composite & 491.4 & 402 & 446.7 \\
\hline Srouds & . & 10 & (2) & 10.96110 & 4. \\
\hline S96T005539 & $166: 3$ & Drainable liquid & $<1,275$ & $<1,280$ & $<1,277.5$ \\
\hline S96T005540 & $166: 4$ & Drainable liquid & $<1,275$ & $<1,280$ & $<1,277.5$ \\
\hline S96T005599 & $166: 6$ & Drainable liquid & 971.2 & 743 & $857.1^{\mathrm{QC:c}}$ \\
\hline S96T005816 & $166: 7$ & Drainable liquid & 795.1 & 788 & 791.55 \\
\hline S96T005817 & $166: 8$ & Drainable liquid & 764.4 & 751 & 757.7 \\
\hline S96T005541 & $166: 9$ & Drainable liquid & 408.7 & 419 & 413.85 \\
\hline S96T005566 & 166: 10 & Drainable liquid & 436.2 & 435 & 435.6 \\
\hline S96T005600 & 166: 1 & \begin{tabular}{|l|} 
Drainable liquid \\
\end{tabular} & 992.2 & 976 & 984.1 \\
\hline S96T005601 & 166: 2 & Drainable liquid & 808.4 & 710 & 759.2 \\
\hline S96T005861 & $167: 2$ & Drainable liquid & 1,081 & 1,090 & $1,085.5$ \\
\hline S96T005673 & $167: 3 \cdots$ & Drainable liquid & 1,902 & 1,890 & 1,896 \\
\hline S96T005420 & 167: 5 & Drainable liquid & 931.9 & 944 & 937.95 \\
\hline S96T005421 & 167: 6 & Drainable liquid & 768.3 & 750 & 759.15 \\
\hline S96T005683 & 167: 7 & \begin{tabular}{|l} 
Drainable liquid \\
\end{tabular} & 1,947 & 1,910 & $1,928.5$ \\
\hline S96T005732 & 167: 8 & Drainable liquid & 1,805 & 1,790 & $1,79.7 .5$ \\
\hline S96T005862 & 167:9 & Drainable liquid & 910.7 & 901 & 905.85 \\
\hline S96T005650 & 167: 1 & Drainable liquid & 3,273 & 3,270 & $3,271.5$ \\
\hline S96T005991 & Core 166 & Liquid composite & 1,567 & 1,590 & $1,578.5$ \\
\hline
\end{tabular}


Table B2-52. Tank 241-AN-103 Analytical Results: Chloride (IC). (3 sheets)

\begin{tabular}{|c|c|c|c|c|c|}
\hline $\begin{array}{l}\text { Sample } \\
\text { Muninge }\end{array}$ & Sample: & $\begin{array}{l}\text { Sample } \\
\text { orrion }\end{array}$ & resulit & Ouplicite & nesu \\
\hline \multicolumn{2}{|c|}{ 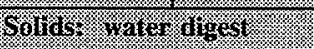 } & ⿸厂冫: & $\sqrt{1689}$ & l: 1898 & 1.68 \\
\hline S96T005610 & $166: 1$ & Lower half & 2,695 & 3,580 & $3,137.5^{\mathrm{QC}: \mathrm{c}}$ \\
\hline S96T005572 & 166: 4 & Lower half & 2,748 & 3,120 & $2,934^{\mathrm{QC}: \mathrm{c}}$ \\
\hline S96T005619 & 166: 6 & Lower half & 2,908 & 2,800 & 2,854 \\
\hline S96T005843 & $166: 7$ & Lower half & 2,369 & 2,020 & $2,194.5$ \\
\hline S96T005844 & $166: 8$ & Lower half & 1,987 & 2,310 & $2,148.5$ \\
\hline S96T005576 & 166: 9 & Lower half & 3,751 & 3,970 & $3,860.5$ \\
\hline S96T005573 & 166: 10 & Lower half & 2,742 & 2,990 & 2,866 \\
\hline S96T005620 & 166: 1 & Lower half & 2,265 & 2,150 & $2,207.5$ \\
\hline S96T005621 & $166: 2$ & Lower half & 2,383 & 2,770 & $2,576.5$ \\
\hline S96T005718 & \multirow[t]{2}{*}{$166: 3$} & Upper half & 5,228 & 5,640 & 5,434 \\
\hline S96T005724 & & Lower half & 5,365 & 4,850 & $5,107.5$ \\
\hline S96T005418 & \multirow[t]{2}{*}{$166: 5$} & Upper half & 4,102 & 3,660 & 3,881 \\
\hline S96T005412 & & Lower half & 4,136 & 3,330 & $3,733^{\mathrm{QC}: \mathrm{e}}$ \\
\hline S96T005625 & \multirow[t]{2}{*}{$166: 6$} & Upper half & 3,726 & 4,000 & 3,863 \\
\hline S96T005622 & & Lower half & 4,118 & 3,950 & 4,034 \\
\hline S96T005419 & \multirow[t]{2}{*}{$166: 7$} & Upper half & 3,042 & 3,120 & $3,081^{\mathrm{QC}: d}$ \\
\hline S96T005413 & & Lower half & 3,644 & 3,450 & 3,547 \\
\hline S96T005719 & \multirow[t]{2}{*}{$166: 8$} & Upper half & 4,070 & 3,820 & 3,945 \\
\hline S96T005725 & & Lower half & 4,356 & 4,140 & 4,248 \\
\hline S96T005845 & \multirow[t]{2}{*}{$166: 9$} & Upper half & 3,741 & 4,370 & $4,055.5$ \\
\hline S96T005846 & & Lower half & 3,986 & 4,210 & 4,098 \\
\hline S96T005711 & \multirow[t]{2}{*}{$167: 1$} & Upper half & 3,789 & 4,060 & $3,924.5^{\mathrm{QC}: \mathrm{d}}$ \\
\hline S96T005699 & & Lower half & 3,760 & 3,640 & 3,700 \\
\hline S96T005859 & $167: 2$ & Lower half & 3,016 & 3,130 & 3,073 \\
\hline S96T005700 & $167: 3$ & Lower half & 4,150 & 4,120 & 4,135 \\
\hline S96T005701 & 167: 4 & Lower half & 4,577 & 4,520 & $4,548.5$ \\
\hline S96T005440 & $167: 5$ & Lower half & 4,757 & 4,020 & $4,388.5$ \\
\hline S96T005452 & 167: 6 & Lower half & 3,833 & 4,350 & $4,091.5$ \\
\hline S96T005702 & $167: 7$ & Lower half & 1,959 & 1,950 & $1,954.5$ \\
\hline
\end{tabular}


Table B2-52. Tank 241-AN-103 Analytical Results: Chloride (IC). (3 sheets)

\begin{tabular}{|c|c|c|c|c|c|}
\hline Shimple & Sample & 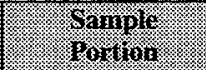 & Pesilil & 8utherit & Whan \\
\hline molug & rang & & xy & 18g & (1) \\
\hline S96T005738 & $167: 8$ & Lower half & 2,363 & 1,760 & $2,061.5^{\mathrm{QC}: \mathrm{c}}$ \\
\hline S96T005860 & 167:9 & Lower half & 1,156 & 1,110 & 1,133 \\
\hline S96T005712 & \multirow[t]{2}{*}{ 167: 1} & Upper half & 6,416 & 6,640 & 6,528 \\
\hline S96T005703 & & Lower half & 5,194 & 5,040 & 5,117 \\
\hline S96T005446 & \multirow[t]{2}{*}{ 167: 2} & Upper half & 5,077 & 4,840 & $4,958.5$ \\
\hline S96T005453 & & Lower half & 2,633 & 2,520 & $2,576.5$ \\
\hline S96T005447 & \multirow[t]{2}{*}{ 167:4 } & Upper half & 1,322 & 1,540 & 1,431 \\
\hline S96T005441 & & Lower half & 2,322 & 2,480 & 2,401 \\
\hline S96T005713 & \multirow[t]{2}{*}{ 167: 5} & Upper half & 2,994 & 2,790 & 2,892 \\
\hline S96T005704 & & Lower half & 6,010 & 5,930 & 5,970 \\
\hline S96T005500 & \multirow[t]{2}{*}{$167: 7$} & Upper half & 2,852 & 2,970 & $2,911^{\mathrm{QC}: \mathrm{d}}$ \\
\hline S96T005501 & & Lower half & 4,195 & 4,130 & $4,162.5^{\mathrm{QC}: \mathrm{d}}$ \\
\hline S97T000023 & Core 166 & Solid composite & 3,784 & 3,680 & 3,732 \\
\hline 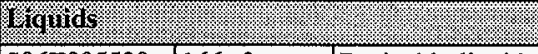 & \multicolumn{2}{|c|}{ (4) } & 19gom & 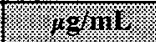 & 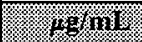 \\
\hline S96T005539 & 166: 3 & Drainable liquid & 12,490 & 12,200 & 12,345 \\
\hline S96T005540 & 166: 4 & Drainable liquid & 11,380 & 11,300 & 11,340 \\
\hline S96T005599 & $166: 6$ & Drainable liquid & 7,574 & 8,080 & 7,827 \\
\hline S96T005816 & 166: 7 & Drainable liquid & 11,450 & 11,400 & 11,425 \\
\hline S96T005817 & 166: 8 & Drainable liquid & 8,432 & 8,250 & 8,341 \\
\hline S96T005541 & 166: 9 & Drainable liquid & 11,790 & 11,500 & 11,645 \\
\hline S96T005566 & 166: 10 & Drainable liquid & 11,050 & 11,000 & 11,025 \\
\hline S96T005600 & $166: 1$ & Drainable liquid & 6,598 & 6,930 & 6,764 \\
\hline S96T005601 & $166: 2$ & Drainable liquid & 5,448 & 7,830 & $6,639^{\mathrm{QC}: \mathrm{e}}$ \\
\hline S96T005861 & $167: 2$ & Drainable liquid & 10,170 & 10,200 & 10,185 \\
\hline S96T005673 & $167: 3$ & Drainable liquid & 10,970 & 11,400 & 11,185 \\
\hline S96T005420 & $167: 5$ & Drainable liquid & 9,865 & 10,000 & $9,932.5$ \\
\hline S96T005421 & $167: 6$ & Drainable liquid & 11,040 & 10,900 & 10,970 \\
\hline \$96T005683 & 167:7 & Drainable liquid & 11,220 & 11,100 & 11,160 \\
\hline
\end{tabular}


HNF-SD-WM-ER-702 Rev. 0

Table B2-52. Tank 241-AN-103 Analytical Results: Chloride (IC). (3 sheets)

\begin{tabular}{|c|c|c|c|c|c|}
\hline Sample & Sarnple & $\begin{array}{l}\text { Samile: } \\
\text { fornivil }\end{array}$ & Restill: & 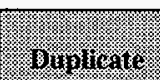 & Vean \\
\hline \multicolumn{2}{|c|}{ 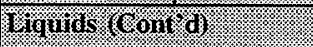 } & : & 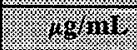 & $139 \mathrm{mil}$ & (2) \\
\hline S96T005732 & $167: 8$ & Drainable liquid & 12,470 & 12,700 & 12,585 \\
\hline S96T005862 & $167: 9$ & Drainable liquid & 9,378 & 9,580 & 9,479 \\
\hline S96T005650 & 167: 1 & Drainable liquid & 10,360 & 10,500 & 10,430 \\
\hline S96T005991 & Core 166 & Liquid composite & 9,770 & 10,000 & 9,885 \\
\hline
\end{tabular}


HNF-SD-WM-ER-702 Rev. 0

Table B2-53. Tank 241-AN-103 Analytical Results: Fluoride (IC). (3 sheets)

\begin{tabular}{|c|c|c|c|c|c|}
\hline $\begin{array}{l}\text { Shanple } \\
\text { Vininimer }\end{array}$ & 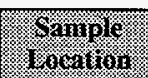 & $\begin{array}{l}\text { Simple } \\
\text { porrin }\end{array}$ & Restill & Bullivaro & (2) \\
\hline \multicolumn{3}{|c|}{ Golids water algest, } & (6) & 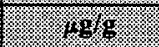 & 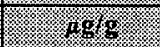 \\
\hline S96T005610 & $166: 1$ & Lower half & 692.2 & 836 & 764.1 \\
\hline S96T005572 & 166: 4 & Lower half & 422.9 & 624 & $523.45^{\mathrm{QC:c}}$ \\
\hline S96T005619 & $166: 6$ & Lower half & 589.4 & 483 & 536.2 \\
\hline S96T005843 & 166: 7 & Lower half & 528.2 & 548 & 538.1 \\
\hline S96T005844 & $166: 8$ & Lower half & 313.4 & 406 & $359.7^{\mathrm{QC:c}}$ \\
\hline S96T005576 & $166: 9$ & Lower half & 809.7 & 900 & 854.85 \\
\hline S96T005573 & 166: 10 & Lower half & 423.4 & 705 & $564.2^{\mathrm{QC}: \mathrm{C}}$ \\
\hline S96T005620 & 166: 1 & Lower half & 781.5 & 952 & 866.75 \\
\hline S96T005621 & 166: 2 & Lower half & 1,014 & 1,210 & 1,112 \\
\hline S96T005718 & \multirow[t]{2}{*}{$166: 3$} & Upper half & 646.4 & 649 & 647.7 \\
\hline S96T005724 & & Lower half & 715.6 & 351 & $533.3^{\mathrm{QC}: c}$ \\
\hline S96T005418 & \multirow[t]{2}{*}{$166: 5$} & Upper half & 602.1 & 543 & 572.55 \\
\hline S96T005412 & & Lower half & 823.6 & 702 & 762.8 \\
\hline S96T005625 & \multirow[t]{2}{*}{ 166: 6} & Upper half & 632.5 & 606 & 619.25 \\
\hline S96T005622 & & Lower half & 499.2 & 497 & 498.1 \\
\hline S96T005419 & \multirow[t]{2}{*}{$166: 7$} & Upper half & 645.2 & 753 & 699.1 \\
\hline S96T005413 & & Lower half & 679.2 & 676 & 677.6 \\
\hline S96T005719 & \multirow[t]{2}{*}{$166: 8$} & Upper half & 1,295 & 989 & $1,142^{\mathrm{QC}: \mathrm{c}}$ \\
\hline S96T005725 & & Lower half & 1,527 & 1,550 & $1,538.5$ \\
\hline S96T005845 & \multirow[t]{2}{*}{$166: 9$} & Upper half & 880.9 & 902 & 891.45 \\
\hline S96T005846 & & Lower half & 1,159 & 1,040 & $1,099.5^{\mathrm{QC}: \mathrm{e}}$ \\
\hline S96T005711 & \multirow[t]{2}{*}{$167: 1$} & Upper half & 911 & 961 & $936^{\mathrm{QC:d}}$ \\
\hline S96T005699 & & Lower half & 964.6 & 892 & 928.3 \\
\hline S96T005859 & $167: 2$ & Lower half & 434.8 & 423 & 428.9 \\
\hline S96T005700 & $167: 3$ & Lower half & 850 & 786 & 818 \\
\hline S96T005701 & $167: 4$ & Lower half & 659.8 & 987 & $823.4^{\mathrm{QC}: \mathrm{e}}$ \\
\hline S96T005440 & $167: 5$ & Lower half & 1,932 & 1,710 & 1,821 \\
\hline S96T005452 & $167: 6$ & Lower half & 1,703 & 637 & $1,170^{\mathrm{QC}: \mathrm{c}}$ \\
\hline S96T005702 & $167: 7$ & Lower half & 840.3 & 916 & 878.15 \\
\hline
\end{tabular}


Table B2-53. Tank 241-AN-103 Analytical Results: Fluoride (IC). (3 sheets)

\begin{tabular}{|c|c|c|c|c|c|}
\hline $\begin{array}{l}\text { Sample: } \\
\text { Nrinber: }\end{array}$ & $\begin{array}{l}\text { Sample } \\
\text { Horition }\end{array}$ & $\begin{array}{l}\text { Sample } \\
\text { Iromtion }\end{array}$ & insin & 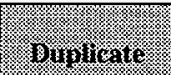 & 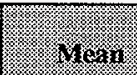 \\
\hline \multicolumn{3}{|c|}{ 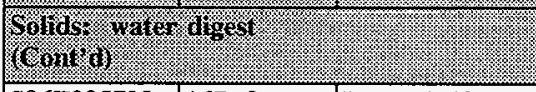 } & 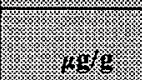 & . & . \\
\hline S96T005738 & $167: 8$ & Lower half & 1,107 & 1,000 & $1,053.5$ \\
\hline S96T005860 & $167: 9$ & Lower half & 670.4 & 621 & 645.7 \\
\hline S96T005712 & \multirow[t]{2}{*}{ 167: 1} & Upper half & 719.4 & 728 & 723.7 \\
\hline S96T005703 & & Lower half & 293 & 282 & 287.5 \\
\hline S96T005446 & \multirow[t]{2}{*}{$167: 2$} & Upper half & 372.3 & 373 & 372.65 \\
\hline S96T005453 & & Lower half & 386.4 & 367 & 376.7 \\
\hline S96T005447 & \multirow[t]{2}{*}{ 167: 4} & Upper half & 269.7 & 268 & 268.85 \\
\hline S96T005441 & & Lower half & 286.9 & 315 & 300.95 \\
\hline S96T005713 & \multirow[t]{2}{*}{$167: 5$} & Upper half & 405.8 & 1,270 & $837.9^{\mathrm{CC:0}}$ \\
\hline S96T005704 & & Lower half & 703.8 & 663 & 683.4 \\
\hline S96T005500 & \multirow[t]{2}{*}{$167: 7$} & Upper half & 1,897 & 1,790 & $1,843.5$ \\
\hline S96T005501 & & Lower half & 4,114 & 3,980 & $4,047^{\mathrm{QC}: \mathrm{d}}$ \\
\hline S97T000023 & Core 166 & Solid composite & 3,925 & 4,350 & $4,137.5$ \\
\hline Signids: & $\frac{1}{18}$ & 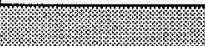 & 4grinl & (1) & I. \\
\hline S96T005539 & $166: 3$ & Drainable liquid & 572.4 & 538 & 555.2 \\
\hline S96T005540 & 166: 4 & Drainable liquid & 634.3 & 633 & 633.65 \\
\hline S96T005599 & 166: 6 & Drainable liquid & 409.1 & 391 & 400.05 \\
\hline S96T005816 & $166: 7$ & Drainable liquid & 491.5 & 539 & 515.25 \\
\hline S96T005817 & $166: 8$ & Drainable liquid & 389.3 & 355 & 372.15 \\
\hline S96T005541 & 166: 9 & Drainable liquid & 653 & 639 & 646 \\
\hline S96T005566 & 166: 10 & Drainable liquid & 674.1 & 610 & 642.05 \\
\hline S96T005600 & 166: 1 & Drainable liquid & 440.6 & 443 & 441.8 \\
\hline S96T005601 & $166: 2$ & Drainable liquid & 426.8 & 447 & 436.9 \\
\hline S96T005861 & 167: 2 & Drainable liquid & 461 & 424 & 442.5 \\
\hline S96T005673 & $167: 3$ & Drainable liquid & 646.6 & 619 & 632.8 \\
\hline S96T005420 & $167: 5$ & Drainable liquid & 708.4 & 702 & 705.2 \\
\hline S96T005421 & $167: 6$ & Drainable liquid & 801.5 & 826 & 813.75 \\
\hline S96T005683 & $167: 7$ & Drainable liquid & 713.1 & 660 & 686.55 \\
\hline
\end{tabular}




\begin{tabular}{|c|c|c|c|c|c|}
\hline I.965 & 209 & $z^{\circ} 06 \mathrm{~s}$ & əl!nsoduros p!nb!̣ & 991 2100 & I66S00L96S \\
\hline$S L \angle t 9$ & LE9 & $\varsigma 859$ & pṭnb!̣l әqвu!̣eda & $\mathrm{I}: \angle 9 \mathrm{I}$ & 0S9S00L96S \\
\hline$\$ 8 . \angle 6 \mathcal{E}$ & $\varepsilon 6 \varepsilon$ & L.ZOt & pṭnb!̣l әqeuṭesd & $6: \angle 9 I$ & 298S00.L96S \\
\hline IIL & $80 L$ & $t I L$ & pụnb!̣ әрqеuाraa & $8: \angle 9 I$ & zELS00L96S \\
\hline gurtis & Iniph & (13) & 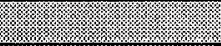 & 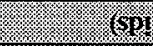 & (3). $1 /$ spinbor \\
\hline 1.2. & $77=319196$ & IIrody & 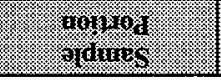 & $\begin{array}{l}\text { montrol } \\
\text { adrarrs }\end{array}$ & vajuin: \\
\hline
\end{tabular}

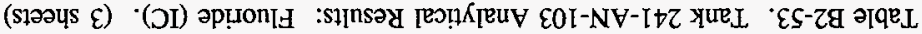


Table B2-54. Tank 241-AN-103 Analytical Results: Formate (IC). (3 sheets)

\begin{tabular}{|c|c|c|c|c|c|}
\hline Whingl & 94anple & Shar & mosulu & Stopturate & $\log$ \\
\hline \multicolumn{2}{|c|}{ Woidg } & \%: & 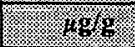 & *6. & (1) \\
\hline S96T005610. & 166: 1 & Lower half & 732.3 & 871 & 801.65 \\
\hline S96T005572 & 166: 4 & Lower half & 531.5 & 439 & 485.25 \\
\hline S96T005619 & 166: 6 & Lower half & 655 & 670 & 662.5 \\
\hline S96T005843 & 166: 7 & Lower half & 508 & 561 & 534.5 \\
\hline S96T005844 & 166: 8 & Lower half & 501.7 & 541 & 521.35 \\
\hline \$96T005576 & 166: 9 & Lower half & $<167.5$ & 590 & $<378.75^{\mathrm{QC:e}}$ \\
\hline S96T005573 & 166: 10 & Lower half & 667.5 & 740 & 703.75 \\
\hline S96T005620 & 166: 1 & Lower half & 603.9 & 561 & 582.45 \\
\hline S96T005621 & $166: 2$ & Lower half & 583.8 & 576 & 579.9 \\
\hline S96T005718 & \multirow[t]{2}{*}{ 166: 3} & Upper half & 1,031 & 1,050 & $1,040.5$ \\
\hline S96T005724 & & Lower half & 940.3 & 1,010 & 975.15 \\
\hline S96T005418 & \multirow[t]{2}{*}{ 166:5 } & Upper half & 799 & 778 & 788.5 \\
\hline S96T005412 & & Lower half & 860.7 & 776 & 818.35 \\
\hline \$96T005625 & \multirow[t]{2}{*}{$166: 6$} & Upper half & 812.9 & 838 & 825.45 \\
\hline S96T005622 & & Lower half & 906.1 & 946 & 926.05 \\
\hline S96T005419 & \multirow[t]{2}{*}{ 166: 7} & Upper half & 818.7 & $<89.5$ & $<454.1^{\mathrm{QC}: c}$ \\
\hline S96T005413 & & Lower half & 825.2 & 883 & 854.1 \\
\hline S96T005719 & \multirow[t]{2}{*}{ 166: 8} & Upper half & 885.4 & 838 & 861.7 \\
\hline S96T005725 & & Lower half & 769.5 & 797 & 783.25 \\
\hline S96T005845 & \multirow[t]{2}{*}{$166: 9$} & Upper half & 713.1 & 826 & 769.55 \\
\hline S96T005846 & & Lower half & 842.6 & 941 & 891.8 \\
\hline S96T005711 & \multirow[t]{2}{*}{ 167: 1} & Upper half & 842.7 & 873 & 857.85 \\
\hline S96T005699 & & Lower half & 818.6 & 921 & 869.8 \\
\hline S96T005859 & $167: 2$ & Lower half & 708.8 & 745 & 726.9 \\
\hline S96T005700 & $167: 3$ & Lower half & 776.2 & 796 & 786.1 \\
\hline S96T005701 & 167: 4 & Lower half & 822.8 & 838 & 830.4 \\
\hline S96T005440 & $167: 5$ & Lower half & 760.7 & 686 & 723.35 \\
\hline S96T005452 & $167: 6$ & Lower half & 624.3 & 665 & 644.65 \\
\hline S96T005702 & 167:7 & Lower half & $<769.7$ & $<838$ & $<803.85$ \\
\hline
\end{tabular}




\begin{tabular}{|c|c|c|c|c|c|}
\hline$\varsigma^{\prime}\left[9 \mathcal{E}^{\prime} \mathcal{E}\right.$ & $08 \mathcal{E}^{\star} \varepsilon$ & $\varepsilon \supsetneqq \varepsilon^{6} \varepsilon$ & pı̣b!! әqеu!exa & $\angle: \angle 9 I$ & $\varepsilon 89 \subseteq 00 J 96 \mathrm{~S}$ \\
\hline S'6IL'I & $0+L^{\prime} !$ & $669^{6} I$ & pụbII әүqeureIa & $9: \angle 9 I$ & $1 Z \triangleright S 00 L 96 S$ \\
\hline$S^{\circ}+0 L^{\prime} \mathrm{I}$ & $O Z L^{\prime} \mathrm{I}$ & $689^{\circ} \mathrm{I}$ & pinbII arqeurera & $\mathcal{S}: \angle 9 I$ & 0ZtS00L96S \\
\hline$s^{\circ} Z \angle S^{\prime} I$ & $06 S^{\prime} \mathrm{I}$ & $\operatorname{sSS}^{\prime} I$ & pụnby ərqeureıd & $\varepsilon: \angle 9 I$ & EL9S00L96S \\
\hline$s^{\prime}+s 6^{6} \nabla$ & $068^{\circ} t$ & $610^{\circ} \mathrm{s}$ & p!nbị əॄqeu!̣eıd & $z: 291$ & I98\$00L96S \\
\hline$S^{\prime}\left[L L^{6} \mathrm{I}\right.$ & OLL'I & $\varepsilon L L^{\prime} I$ & pinb!̣ əүqeụex & $2: 99 \mathrm{I}$ & I09500L96S \\
\hline$\tau \varepsilon L^{\prime} t$ & $09 L^{\prime} D$ & $t 0 L^{\prime} \nabla$ & pinb!l әqвu!̣ı & I :99 I & $009 \$ 00 \mathrm{~L} 96 \mathrm{~S}$ \\
\hline $999^{\prime} \mathrm{I}$ & $0 \angle S^{\prime} I$ & $29 L^{\prime} \mathrm{I}$ & p!nb!̣ ә[qеure. & or :99I & 99SS00L96S \\
\hline$\angle I 0^{\circ} I$ & 286 & $250^{\circ} \mathrm{I}$ & pinbri әrqвựerc & $6: 991$ & ItSS00L96S \\
\hline$s^{\prime} z o z^{\prime} z$ & $0 I Z^{\prime} z$ & $\$ 6 I^{\prime} z$ & pụb!ı әqеuाeIa & $8: 99 \mathrm{I}$ & LI8S00L96S \\
\hline$\$ 80^{\circ} \mathrm{Z}$ & $0 z z^{\prime} z$ & $0 S 6^{\prime} I$ & 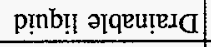 & $L: 99 I$ & 918500L96S \\
\hline$S^{*} \angle 8 S^{6} \mathrm{I}$ & $009 \div$ & $S \angle S^{\prime} I$ & pịnbụ ə[qRụteIa & $9: 991$ & $66 \$ \$ 00 L 96 \mathrm{~S}$ \\
\hline $0: 00 S \mathcal{E} t^{6} 8$ & $09 t^{\prime} s$ & OIt II & p!nb!ı әๆqeuteı & $t: 99 \mathrm{I}$ & E9I000LL6S \\
\hline$S^{*} L S S^{\prime} I$ & $0 \varepsilon S^{6} I$ & S8S I & p!nb! I әqeựera & $\mathcal{E}: 99 \mathrm{I}$ & $6 \varepsilon \$ 500 L 96 \mathrm{~S}$ \\
\hline 棌, & 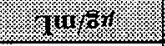 & 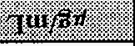 & & & sporbut \\
\hline $88 Z^{6} \mathrm{I}$ & $00 \mathcal{E}^{\prime} \mathrm{I}$ & $9 \angle Z^{\prime} I$ & I & 99I ว10د & $\varepsilon Z 0000 L \angle 6 S$ \\
\hline :०ठS'I\&6 & 064 & $\varepsilon \angle 0^{\circ} \mathrm{I}$ & I[ЕU IวMOT & & IOSS00L96S \\
\hline $8^{\circ} \mathcal{E S 8}$ & 908 & $9 \cdot 106$ & 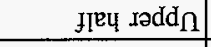 & $\angle: \angle 9 I$ & $00 \$ \$ 00 \mathrm{~L} 96 \mathrm{~S}$ \\
\hline$\Im \angle S L$ & $\mathrm{IOL}$ & tI8 & Jleप Iәмо I & & $70 \angle 500 L 96 \mathrm{~S}$ \\
\hline $58+0 L$ & $\tau I L$ & $\angle \angle 69$ & fley xədid & $\varsigma: \angle 9 I$ & E[LS00L96S \\
\hline$s s^{\circ} \nabla 2 s$ & 195 & 1.880 & मॉЕЧ Іәмот & & $I t \nabla \$ 00196 \mathrm{~S}$ \\
\hline $8 \cdot 205$ & $6 \varepsilon S$ & $9.99 t$ & Jfeप $\mathrm{s} \partial \mathrm{dd} \cap$ & $\nabla: \angle 9 I$ & $\angle \supset t S 00 L 96 \mathrm{~S}$ \\
\hline $9 \div S L$ & $\varepsilon \nabla L$ & $2 \cdot 99 L$ & f[Еप دәMOT & & $\mathcal{E S}$ S00L96S \\
\hline $\mathrm{SI}^{\prime} 8 \mathcal{E} L$ & $9 \nabla L$ & $\mathcal{E}^{\circ} O \mathcal{E} L$ & fleч sədd $_{\Omega}$ & $\tau: \angle 9 I$ & $9 t 7$ S00L96S \\
\hline $0: 09^{\circ} 8 S^{6} S^{6} I>$ & $06 z^{\prime} z$ & $z \cdot L 28>$ & I[Еप IจMOT & & EOLSO0L96S \\
\hline$\tau \cdot 878$ & 088 & t.918 & fIRY rədd $\cap$ & $\mathrm{I}: \angle 9 \mathrm{I}$ & ZILS00L96S \\
\hline$\therefore s+62 s$ & $\angle \nabla S$ & $6^{\circ}$ IIS & Iㅌ⿻ Іәмот & $6: \angle 9 I$ & 098S00L96S \\
\hline$z: 20 s$ & $S L \downarrow$ & 7625 & I[EY IәMOT] & $8: \angle 9 I$ & 8ELS00L96S \\
\hline (6) & totong & 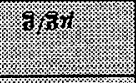 & & . & 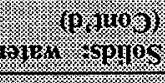 \\
\hline 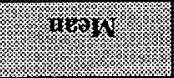 & 917) & 1196\% & movos & 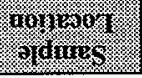 & arings \\
\hline
\end{tabular}

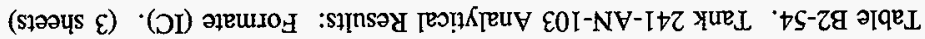




\begin{tabular}{|c|c|c|c|c|c|}
\hline $629^{\circ} \mathrm{I}$ & $O S L^{\prime} I$ & $80 S^{6} I$ & ə१!soduos p!nb! & 99I ว10D & I66S00L96S \\
\hline$S^{\prime} 99 t^{\prime} I$ & $09 t^{6} \mathrm{I}$ & $\varepsilon \angle \nabla^{\circ} I$ & p!nby әрqu!̣eı & $\mathrm{I}: \angle 9 \mathrm{I}$ & 0\$9S00L96S \\
\hline SOZ'0I & $0 t L ' 6$ & $0 \angle 9^{\circ} 0 \mathrm{I}$ & p!̣b!̣ әqеuाera & $6: \angle 9 I$ & z98S00L96S \\
\hline$\nabla t 0^{\circ} \nabla$ & $0 \triangleright 8^{\prime} \varepsilon$ & $8 \pitchfork Z^{6} t$ & pinb! I әqеu!eıd & $8: \angle 9 I$ & ZELS00L96S \\
\hline 1106\% & (m) & 3or & 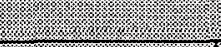 & 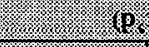 & $102)^{2} 31111 \%$ \\
\hline 19\% & 4) & $\operatorname{lng} 2$ & 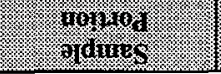 & 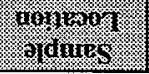 & Olominge \\
\hline
\end{tabular}

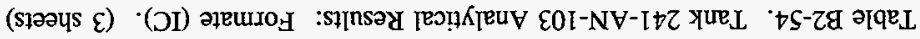


Table B2-55. Tank 241-AN-103 Analytical Results: Nitrate (IC). (3 sheets)

\begin{tabular}{|c|c|c|c|c|c|}
\hline 9uingl & 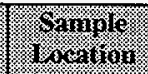 & 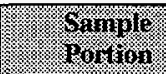 & Resulu & Buplitate & 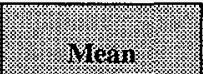 \\
\hline Sollas wat w & difyest & & $18 \%$ & $x y$ & . \\
\hline S96T005610 & 166: 1 & Lower half & $2.413 E+05$ & 79,000 & $1.602 \mathrm{E}+05^{\mathrm{QC:c}}$ \\
\hline S96T005572 & 166: 4 & Lower half & $3.015 \mathrm{E}+05$ & $2.930 \mathrm{E}+05$ & $2.973 \mathrm{E}+05^{\mathrm{QC}: c}$ \\
\hline S96T005619 & $166: 6$ & Lower half & $3.019 E+05$ & $2.830 \mathrm{E}+05$ & $2.925 \mathrm{E}+05$ \\
\hline S96T005843 & 166: 7 & Lower half & $3.147 \mathrm{E}+05$ & $3.210 \mathrm{E}+05$ & $3.179 \mathrm{E}+05$ \\
\hline S96T005844 & 166: 8 & Lower half & $3.290 \mathrm{E}+05$ & $2.840 \mathrm{E}+05$ & $3.065 \mathrm{E}+05$ \\
\hline S96T005576 & 166: 9 & Lower half & $2.332 \mathrm{E}+05$ & $2.330 \mathrm{E}+05$ & $2.331 \mathrm{E}+05$ \\
\hline S96T005573 & 166: 10 & Lower half & $2.564 \mathrm{E}+05$ & $2.250 \mathrm{E}+05$ & $2.407 \mathrm{E}+05$ \\
\hline S96T005620 & 166: 1 & Lower half & $2.954 \mathrm{E}+05$ & $3.280 \mathrm{E}+05$ & $3.117 \mathrm{E}+05$ \\
\hline S96T005621 & 166: 2 & Lower half & $3.030 \mathrm{E}+05$ & $2.900 \mathrm{E}+05$ & $2.965 \mathrm{E}+05$ \\
\hline S96T005718 & \multirow[t]{2}{*}{ 166: 3} & Upper half & 87,830 & 88,900 & 88,365 \\
\hline \$96T005724 & & Lower half & 94,600 & 90,100 & 92,350 \\
\hline S96T005418 & \multirow[t]{2}{*}{ 166: 5} & Upper half & $1.432 \mathrm{E}+05$ & $1.330 \mathrm{E}+05$ & $1.381 \mathrm{E}+05$ \\
\hline S96T005412 & & Lower half & $1.374 \mathrm{E}+05$ & $1.700 \mathrm{E}+05$ & $1.537 \mathrm{E}+05^{\mathrm{QC}: \mathrm{c}}$ \\
\hline S96T005625 & \multirow[t]{2}{*}{ 166: 6} & Upper half & $1.306 \mathrm{E}+05$ & $1.160 \mathrm{E}+05$ & $1.233 \mathrm{E}+05$ \\
\hline S96T005622 & & Lower half & $1.252 \mathrm{E}+05$ & $1.430 \mathrm{E}+05$ & $1.341 \mathrm{E}+05$ \\
\hline \$96T005419 & \multirow[t]{2}{*}{$166: 7$} & Upper half & $1.782 \mathrm{E}+05$ & $1.190 \mathrm{E}+05$ & $1.486 \mathrm{E}+05^{\mathrm{QC}: \mathrm{d}, \mathrm{e}}$ \\
\hline S96T005413 & & Lower half & $1.380 \mathrm{E}+05$ & $1.380 \mathrm{E}+05$ & $1.380 \mathrm{E}+05$ \\
\hline S96T005719 & \multirow[t]{2}{*}{$166: 8$} & Upper half & $1.895 \mathrm{E}+05$ & $2.080 \mathrm{E}+05$ & $1.988 \mathrm{E}+05$ \\
\hline S96T005725 & & Lower half & $1.282 \mathrm{E}+05$ & $1.240 \mathrm{E}+05$ & $1.261 E+05$ \\
\hline S96T005845 & \multirow[t]{2}{*}{ 166: 9} & Upper half & 85,490 & 93,000 & 89,245 \\
\hline S96T005846 & & Lower haif & 89,340 & 99,800 & 94,570 \\
\hline \$96T005711 & \multirow[t]{2}{*}{$167: 1$} & Upper half & $1.566 \mathrm{E}+05$ & $1.610 \mathrm{E}+05$ & $1.588 \mathrm{E}+05^{\mathrm{QC:d}}$ \\
\hline S96T005699 & & Lower half & $1.450 \mathrm{E}+05$ & $1.390 E+05$ & $1.420 \mathrm{E}+05$ \\
\hline S96T005859 & $167: 2$ & Lower half & $1.174 \mathrm{E}+05$ & $1.240 \mathrm{E}+05$ & $1.207 \mathrm{E}+05$ \\
\hline \$96T005700 & 167: 3 & Lower half & $2.796 \mathrm{E}+05$ & $2.790 \mathrm{E}+05$ & $2.793 E+05$ \\
\hline S96T005701 & $167: 4$ & Lower half & $2.168 \mathrm{E}+05$ & $3.000 \mathrm{E}+05$ & $2.584 \mathrm{E}+05^{\mathrm{QC:c}}$ \\
\hline S96T005440 & $167: 5$ & Lower half & $1.877 \mathrm{E}+05$ & $2.040 E+05$ & $1.959 \mathrm{E}+05$ \\
\hline S96T005452 & $167: 6$ & Lower half & $2.738 \mathrm{E}+05$ & $2.410 \mathrm{E}+05$ & $2.574 \mathrm{E}+05^{\mathrm{QC:c}}$ \\
\hline S96T005702 & $167: 7$ & Lower half & $3.721 \mathrm{E}+05$ & $3.510 E+05$ & $3.616 \mathrm{E}+05^{\mathrm{QC}: \mathrm{c}}$ \\
\hline
\end{tabular}


Table B2-55. Tank 241-AN-103 Analytical Results: Nitrate (IC). (3 sheets)

\begin{tabular}{|c|c|c|c|c|c|}
\hline Mamplo & \%ow & 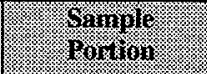 & Resiut & Binilicatic & . \\
\hline \multicolumn{2}{|c|}{ 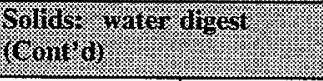 } & & (19\% & $\%$ & 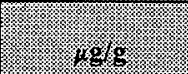 \\
\hline S96T005738 & 167: 8 & Lower half & $3.120 \mathrm{E}+05$ & $3.380 \mathrm{E}+05$ & $3.250 \mathrm{E}+05^{\mathrm{QC}: \mathrm{c}}$ \\
\hline S96T005860 & 167: 9 & Lower half & $1.344 \mathrm{E}+05$ & $1.310 \mathrm{E}+05$ & $1.327 \mathrm{E}+05$ \\
\hline S96T005712 & \multirow[t]{2}{*}{ 167: 1} & Upper half & $1.132 \mathrm{E}+05$ & $1.210 \mathrm{E}+05$ & $1.171 \mathrm{E}+05$ \\
\hline S96T005703 & & Lower half & 98,760 & $1.030 \mathrm{E}+05$ & $1.009 \mathrm{E}+05$ \\
\hline S96T005446 & \multirow[t]{2}{*}{ 167: 2} & Upper half & 87,030 & 98,900 & 92,965 \\
\hline$\$ 967005453$ & & Lower half & 59,040 & 51,100 & 55,070 \\
\hline S96T005447 & \multirow[t]{2}{*}{ 167: 4} & Upper half & $1.438 \mathrm{E}+05$ & 93,100 & $1.185 \mathrm{E}+05^{\mathrm{QC}: \mathrm{c}}$ \\
\hline S96T005441 & & Lower half & $3.099 \mathrm{E}+05$ & $2.730 \mathrm{E}+05$ & $2.915 \mathrm{E}+05$ \\
\hline S96T005713 & \multirow[t]{2}{*}{$167: 5$} & Upper half & $2.336 \mathrm{E}+05$ & $2.440 E+05$ & $2.388 \mathrm{E}+05$ \\
\hline \$96T005704 & & Lower half & $1.010 \mathrm{E}+05$ & 99,400 & $1.002 \mathrm{E}+05$ \\
\hline S96T005500 & \multirow[t]{2}{*}{$167: 7$} & Upper half & $4.184 \mathrm{E}+05$ & $4.340 \mathrm{E}+05$ & $4.262 \mathrm{E}+05^{Q C: d}$ \\
\hline S96T005501 & & Lower half & $3.847 \mathrm{E}+05$ & $3.640 E+05$ & $3.744 \mathrm{E}+05^{\mathrm{QC}: d}$ \\
\hline S97T000023 & Core 166 & Solid composite & $1.378 \mathrm{E}+05$ & $1.260 \mathrm{E}+05$ & $1.319 \mathrm{E}+05$ \\
\hline Wuido & (3) & & (69) & rglail & ( \\
\hline S96T005539 & 166: 3 & Drainable liquid & $1.562 E+05$ & $1.600 \mathrm{E}+05$ & $1.581 \mathrm{E}+05$ \\
\hline S96T005540 & 166: 4 & Drainable liquid & $1.359 \mathrm{E}+05$ & $1.370 \mathrm{E}+05$ & $1.365 \mathrm{E}+05$ \\
\hline S96T005599 & 166: 6 & Drainable liquid & $1.273 \mathrm{E}+05$ & $1.290 \mathrm{E}+05$ & $1.282 \mathrm{E}+05$ \\
\hline S96T005816 & 166: 7 & Drainable liquid & $1.344 \mathrm{E}+05$ & $1.320 \mathrm{E}+05$ & $1.332 \mathrm{E}+05$ \\
\hline S96T005817 & 166: 8 & Drainable liquid & 93,990 & 95,200 & 94,595 \\
\hline S96T005541 & 166: 9 & Drainable liquid & $1.402 \mathrm{E}+05$ & $1.410 \mathrm{E}+0 \mathrm{~S}$ & $1.406 \mathrm{E}+05$ \\
\hline S96T005566 & 166: 10 & Drainable liquid & $1.378 \mathrm{E}+05$ & $1.370 \mathrm{E}+05$ & $1.374 \mathrm{E}+05$ \\
\hline S96T005600 & 166: 1 & Drainable liquid & $1.612 \mathrm{E}+05$ & $1.640 \mathrm{E}+05$ & $1.626 \mathrm{E}+05$ \\
\hline S96T005601 & 166: 2 & Drainable liquid & $1.373 \mathrm{E}+05$ & $1.370 \mathrm{E}+05$ & $1.372 \mathrm{E}+05$ \\
\hline S96T005861 & 167: 2 & Drainable liquid & $1.267 \mathrm{E}+05$ & $1.290 \mathrm{E}+05$ & $1.279 \mathrm{E}+05$ \\
\hline S96T005673 & 167: 3 & Drainable liquid & $1.380 \mathrm{E}+05$ & $1.380 \mathrm{E}+05$ & $1.380 \mathrm{E}+05$ \\
\hline S96T005420 & $167: 5$ & Drainable liquid & $1.192 \mathrm{E}+05$ & $1.180 \mathrm{E}+05$ & $1.186 \mathrm{E}+05$ \\
\hline S96T005421 & 167: 6 & Drainable liquid & $1.274 \mathrm{E}+05$ & $1.250 \mathrm{E}+05$ & $1.262 \mathrm{E}+05$ \\
\hline S96T005683 & 167: 7 & Drainable liquid & $1.418 \mathrm{E}+05$ & $1.420 \mathrm{E}+05$ & $1.419 \mathrm{E}+05$ \\
\hline
\end{tabular}




\begin{tabular}{|c|c|c|c|c|c|}
\hline $\mathrm{SO}+\mathrm{B \varepsilon 9I^{ \circ } \mathrm { I }}$ & $S O+\exists 0 L I^{\circ} \mathrm{I}$ & $S 0+B 9 S I \cdot I$ & วł!sodwos p!nb! & 991 วлоว & I $66 \mathrm{~S} 00 \mathrm{~L} 96 \mathrm{~S}$ \\
\hline$S 0+G S Z t^{\circ} I$ & $S 0+Z 0 E t^{\circ} \mathrm{I}$ & $\mathrm{s} 0+\mathrm{B} 6 \mathrm{It} \cdot \mathrm{I}$ & punbu! әqеu!exd & $\mathrm{I}: \angle 9 \mathrm{I}$ & 0S9S00L96S \\
\hline$s_{0}+B \triangleright E I \cdot I$ & SO+EOEI'I & $S 0+B \angle \varepsilon I \cdot I$ & 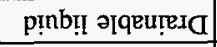 & $6: \mathrm{L9I}$ & Z98S00L96S \\
\hline S0+3209. I & $50+7009^{\circ} \mathrm{I}$ & $50+3809^{\circ} \mathrm{I}$ & 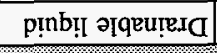 & $8: L 9 I$ & $\tau \varepsilon \angle S 00 L 96 \mathrm{~S}$ \\
\hline 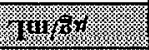 & Trapor & gyophi & & & 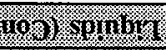 \\
\hline tran & 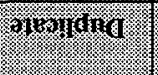 & Insay & romoro: & 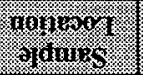 & 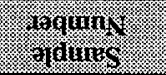 \\
\hline
\end{tabular}

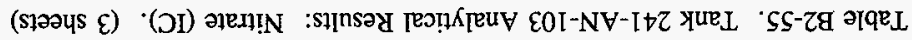


Table B2-56. Tank 241-AN-103 Analytical Results: Nitrite (IC). (3 sheets)

\begin{tabular}{|c|c|c|c|c|c|}
\hline Sample & trompile & $\begin{array}{l}\text { Sample } \\
\text { Torrtion }\end{array}$ & Resint & Hugnigrie & N Ifesin \\
\hline \multicolumn{3}{|c|}{ 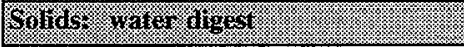 } & 1.968 & 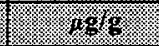 & 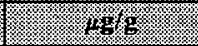 \\
\hline S96T005610 & 166: 1 & Lower half & 48,880 & 65,000 & $56,940^{\mathrm{QC:}: \mathrm{e}}$ \\
\hline S96T005572 & 166: 4 & Lower half & $2.483 \mathrm{E}+05$ & $2.490 \mathrm{E}+05$ & $2.487 \mathrm{E}+05^{\mathrm{QC:c}}$ \\
\hline S96T005619 & 166: 6 & Lower half & $2.430 \mathrm{E}+05$ & $2.650 \mathrm{E}+05$ & $2.540 \mathrm{E}+05$ \\
\hline S96T005843 & 166: 7 & Lower half & $2.774 \mathrm{E}+05$ & $2.900 \mathrm{E}+05$ & $2.837 \mathrm{E}+05$ \\
\hline S96T005844 & 166: 8 & Lower half & $2.700 \mathrm{E}+05$ & $3.120 \mathrm{E}+05$ & $2.910 \mathrm{E}+05$ \\
\hline S96T005576 & $166: 9$ & Lower half & $2.264 E+05$ & $2.030 \mathrm{E}+05$ & 2.147E+05 \\
\hline S96T005573 & 166: 10 & Lower half & $2.996 \mathrm{E}+05$ & $2.860 \mathrm{E}+05$ & $2.928 \mathrm{E}+05$ \\
\hline S96T005620 & $166: 1$ & Lower half & $3.006 \mathrm{E}+05$ & $2.840 \mathrm{E}+05$ & $2.923 \mathrm{E}+05$ \\
\hline S96T005621 & 166: 2 & Lower half & $2.827 \mathrm{E}+05$ & $2.940 \mathrm{E}+05$ & $2.884 \mathrm{E}+05$ \\
\hline S96T005718 & \multirow{2}{*}{$166: 3$} & Upper half & 75,640 & 78,500 & 77,070 \\
\hline S96T005724 & & Lower half & 84,800 & 78,500 & 81,650 \\
\hline S96T005418 & \multirow{2}{*}{$166: 5$} & Upper half & 75,400 & 68,800 & 72,100 \\
\hline S96T005412 & & Lower half & 77,880 & 60,800 & $69,340^{\mathrm{QC}: \mathrm{d}, \mathrm{e}}$ \\
\hline S96T005625 & \multirow[t]{2}{*}{$166: 6$} & Upper half & 61,920 & 65,100 & 63,510 \\
\hline S96T005622 & & Lower half & 78,950 & 74,600 & 76,775 \\
\hline S96T005419 & \multirow[t]{2}{*}{ 166: 7} & Upper half & 61,010 & 61,000 & $61,005^{\mathrm{QC:d}}$ \\
\hline S96T005413 & & Lower half & 56,910 & 55,600 & 56,255 \\
\hline S96T005719 & \multirow[t]{2}{*}{$166: 8$} & Upper haif & 72,860 & 70,800 & 71,830 \\
\hline S96T005725 & & Lower half & 77,980 & 77,300 & 77,640 \\
\hline S96T005845 & \multirow[t]{2}{*}{ 166: 9} & Upper half & 67,640 & 80,100 & $73,870^{\mathrm{QC:d}}$ \\
\hline S96T005846 & & Lower half & 72,200 & 77,900 & $75,050^{\mathrm{QC}: \mathrm{d}}$ \\
\hline S96T005711 & \multirow{2}{*}{ 167: 1} & Upper half & 60,880 & 64,700 & $62,790^{\mathrm{ec:d}}$ \\
\hline S96T005699 & & Lower half & 67,230 & 63,500 & 65,365 \\
\hline S96T005859 & $167: 2$ & Lower half & 93,480 & 92,400 . & 92,940 \\
\hline S96T005700 & $167: 3$ & Lower half & $2.672 \mathrm{E}+05$ & $2.650 \mathrm{E}+05$ & $2.661 \mathrm{E}+05^{\mathrm{QC}: d}$ \\
\hline S96T005701 & 167: 4 & Lower half & $2.162 \mathrm{E}+05$ & $2.850 \mathrm{E}+05$ & $2.506 \mathrm{E}+05^{\mathrm{QC}: \mathrm{d}, \mathrm{e}}$ \\
\hline S96T005440 & $167: 5$ & Lower half & $1.660 \mathrm{E}+05$ & $1.920 \mathrm{E}+05$ & $1.790 \mathrm{E}+05$ \\
\hline S96T005452 & $167: 6$ & Lower half & $1.823 \mathrm{E}+05$ & $1.790 \mathrm{E}+05$ & $1.807 \mathrm{E}+05$ \\
\hline S96T005702 & $167: 7$ & Lower half & $2.459 \mathrm{E}+05$ & $3.140 \mathrm{E}+05$ & $2.800 \mathrm{E}+05^{Q \mathrm{C}: c}$ \\
\hline
\end{tabular}




\begin{tabular}{|c|c|c|c|c|c|}
\hline $50+\exists \varepsilon 89^{\circ} I$ & $50+\exists 069^{\circ} 1$ & $50+39 \angle 9^{\circ} I$ & pinb!̣ әqеuịe & $\angle: \angle 9 I$ & E89S00L96S \\
\hline $50+\exists z 2 z^{\prime} \mathrm{I}$ & SO+ & so+ $\mathrm{B} \downarrow I Z^{\prime} \mathrm{I}$ & 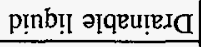 & $9: \angle 9 \mathrm{I}$ & IZtS00L96S \\
\hline$s_{0}+\exists 2 \neq I^{\circ} \mathrm{I}$ & $S_{0}+\underline{G} 0 \mathrm{SI}^{\circ} \mathrm{I}$ & $\varsigma_{0}+\exists \not \varepsilon I \cdot I$ & pinb!̣ ərqeuteı & $s: \angle 9 \mathrm{I}$ & oztS00L96S \\
\hline $\mathrm{SO}+\mathrm{B} 86 \mathrm{~S}^{\circ} \mathrm{I}$ & $50+\exists 019^{\circ} \mathrm{I}$ & SO+ & p!̣b!ı әाqеu!e.Iव & $\varepsilon: \angle 9 I$ & EL9S00L96S \\
\hline$S 0+$ GIEt $I$ & $50+$ TOLt I & so+azst I & pịbụ ә[qeurexa & $z: \angle 9 I$ & I98\$00I96S \\
\hline 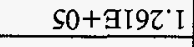 & $50+309 Z^{\circ} \mathrm{I}$ & SO+BZ29 'I & pụbị elquutera & $2: 99 \mathrm{I}$ & I09S00L96S \\
\hline$S 0+\exists I S Z \cdot I$ & $50+9092: I$ & $s_{0}+\exists z \triangleright z \cdot I$ & 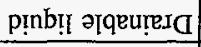 & I :99I & 009S00L96S \\
\hline$\subseteq 0+\exists Z \mathcal{E} \varepsilon^{\prime} \mathrm{I}$ & $50+30 S E^{\circ} \mathrm{I}$ & 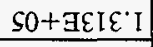 & p!nb!̣ ә[qeutesa & OI:99I & 99SS00L96S \\
\hline $50+\exists \angle \downarrow \varepsilon^{\circ} I$ & $50+30+\varepsilon \cdot I$ & SO+ & pịnb!l әүqeuresa & $6: 99 \mathrm{I}$ & ItSS00L96S \\
\hline $50+3 I S 0^{\circ} I$ & $50+\exists 0 \$ 0^{\circ} \mathrm{I}$ & $\mathrm{SO}+\mathrm{GZ} \mathrm{CS0^{ \circ } \mathrm { I }}$ & p!nb!̣ ə[qeuresa & $8: 99 \mathrm{I}$ & LI8S00L96S \\
\hline $50+30 L 0^{\circ} \mathrm{I}$ & $90+306 t^{\circ} \mathrm{I}$ & $s 0+\exists 0 s t^{\circ}\lceil$ & pịnb!̣ әqqeuteId] & $\angle: 99 \mathrm{I}$ & 9I8S00L96S \\
\hline$S 0+\exists \not \varepsilon t^{\circ} \mathrm{I}$ & $S 0+Z 0 E t^{\circ} \mathrm{I}$ & $\mathrm{SO}+\mathrm{G} 8 \mathcal{E} \mathrm{V}^{\circ} \mathrm{I}$ & p!nb!I әүqеu!eı & $9: 991$ & $66 \$ S 00 L 96 \mathrm{~S}$ \\
\hline SO+Z109\&'I & $S O+\exists O S E$ I & $S_{0}+\exists 69 \varepsilon \cdot \mathrm{I}$ & pı̣b!̣ әтqeu!̣er & $+: 991$ & $0+5500196 S$ \\
\hline$\$ 0+3029^{\circ}[$ & $\mathrm{SO}+\mathrm{G} 06 \mathrm{~S}^{\circ} \mathrm{I}$ & $S 0+70 S 9^{\circ} \mathrm{I}$ & pı̣nbII әтqpureI & $\varepsilon: 99 I$ & 6ESSO0L96S \\
\hline wrot & (1106\% & 19. & & & 9p110) \\
\hline $\mathrm{p}: 0 \mathrm{SO} 9 \varepsilon^{\prime} \varepsilon L$ & $00 L^{6} \varepsilon L$ & $0 \varepsilon 0^{\circ} \mathcal{E} L$ & วt!soduos p!̣os & 991 210ว & \&Z0000LL6S \\
\hline$p: 00 S 08^{4} 9 L$ & $00 t^{\prime} S L$ & $0 I Z^{\prime} 8 L$ & $f[\mathrm{EY}$ IOMO] & & $105 \$ 00196 \mathrm{~S}$ \\
\hline $\mathrm{p}: 00 S \angle \sigma^{\prime} \tau S$ & $00 z^{\circ}+s$ & OSL'IS & Iley sәdd & $\angle: \angle 9 I$ & $00 \$ 500196 \mathrm{~S}$ \\
\hline $\mathrm{SO}+\mathrm{GEE} 0^{\circ} \mathrm{I}$ & SO+GOLO I & $\$ 0+3950^{\circ} \mathrm{I}$ & J[Еप IәMOT & & t0LSOOL96S \\
\hline$p: 00 s z t^{6} 9 s$ & $00 t^{\prime} s s$ & $0 S \nabla^{\circ} \angle S$ & IIEY Iaddn & $S: \angle 9 I$ & ELLSO0L96S \\
\hline$\$ 29^{\circ} \angle t$ & $000^{\prime} 6 t$ & $0 s 2^{\circ} 9 t$ & J[ЕЧ ЈәМОТ & & I $\nabla t S 00 \mathrm{~L} 96 \mathrm{~S}$ \\
\hline $\mathrm{p}=\mathrm{xo} 0 \mathrm{C} \tau^{\prime} 9 z$ & $00 t^{\prime} 82$ & $001^{\prime} \triangleright z$ & IIEบ Iədd $\Omega$ & $\triangleright: \angle 9 I$ & $\angle t D S 00 L 96 S$ \\
\hline $0 S t^{\circ} t \theta$ & $00 S^{\prime} 2 t$ & $00 t^{*} 9 t$ & J[Еप ГАMOT & & EStS00L96S \\
\hline$\$ 90^{\circ} \mathrm{LL}$ & $000^{\prime} 8 L$ & OEI' $9 L$ & IIRU rədd $\Omega$ & $2: \angle 9 \mathrm{I}$ & $9 \not t \$ 00 L 96 \mathrm{~S}$ \\
\hline $0 \varepsilon 0^{\prime} 96$ & $00 t^{\circ} 96$ & $099^{\circ} \$ 6$ & f[Еч IәMOT] & & EOLSO0L $96 \mathrm{~S}$ \\
\hline $\mathrm{p}: 0 \mathrm{SO} 0+\mathrm{GZ}+50^{\circ} \mathrm{I}$ & $\mathrm{SO}+\mathrm{B} 090^{\circ} \mathrm{I}$ & $\mathrm{S} 0+\mathrm{B} \varepsilon z 0^{\circ} \mathrm{I}$ & Iॉeu Iədd $\Omega$ & $\mathrm{I}: \angle 9 \mathrm{I}$ & ZILSOOL96S \\
\hline so+300z'I & $\mathrm{SO}+\underline{B} 09 \mathrm{I}^{\circ} \mathrm{I}$ & S0+B $6 \varepsilon Z^{\circ} \mathrm{I}$ & J[EY IәMOT & $6: \angle 9 I$ & 098S00L96S \\
\hline$S 0+39 \mathcal{E} 6^{\circ} z$ & $S O+\exists 0 Z I^{\circ} \mathcal{E}$ & $S 0+\exists I S L^{\circ} Z$ & f[RY IәMOT] & $8: \angle 9 I$ & $8 E \angle S 00 L 96 S$ \\
\hline $60 \%$ r. & 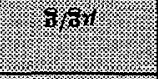 & (6) & & 5. & (3)ing \\
\hline mont & 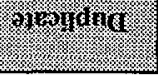 & 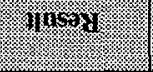 & 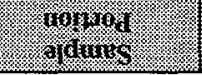 & 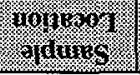 & 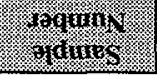 \\
\hline
\end{tabular}

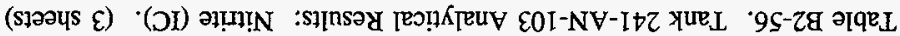




\begin{tabular}{|c|c|c|c|c|c|}
\hline$s_{0}+B+\tau \varepsilon^{\cdot} \mathrm{I}$ & $S_{0}+B 0+\varepsilon^{\prime} I$ & SO+BZ $\angle O E^{\circ} I$ & әlisoduros pinb! $\mathrm{T}$ & 9912100 & I66S00.L96S \\
\hline SO+BSSI I & S0+B09I'I & SO+BOSI I & 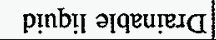 & $\mathrm{I}: \angle 9 \mathrm{I}$ & 0S9S00L96S \\
\hline$s 0+\exists \varepsilon \varepsilon Z^{\prime} I$ & so+BoEz'l & SO+GSEZ:I & pịnb!̣ əquu!̣exd & $6: \angle 9 \mathrm{I}$ & $298 S 00 \mathrm{~L} 96 \mathrm{~S}$ \\
\hline$S 0+79 z L \cdot I$ & SO+GOZL'I & SO+GZEL'I & pụnbị әqqeựesa & $8: \angle 9 I$ & ZELSO0L96S \\
\hline 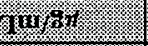 & gury & 1rato: & (2) & 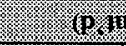 & 10y y sponily \\
\hline 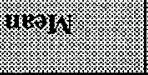 & angigias & 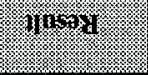 & $\begin{array}{l}\text { mogrod } \\
\text { ariouss }\end{array}$ & moprinod & \\
\hline
\end{tabular}

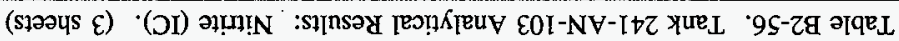


Table B2-57. Tank 241-AN-103 Analytical Results: Phosphate (IC). (3 sheets)

\begin{tabular}{|c|c|c|c|c|c|}
\hline (1) & Shingle & (6) & (n) & 1010) & mean \\
\hline \multicolumn{3}{|c|}{ 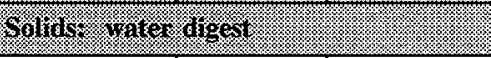 } & $4 \%$ & (1) & \% \\
\hline S96T005610 & 166: 1 & Lower half & $<1,060$ & $<1,040$ & $<1,050$ \\
\hline S96T005572 & 166: 4 & Lower half & 4,776 & 4,720 & 4,748 \\
\hline S96T005619 & 166: 6 & Lower half & 4,955 & 5,680 & $5,317.5$ \\
\hline S96T005843 & 166: 7 & Lower half & 5,784 & 5,970 & 5,877 \\
\hline S96T005844 & 166: 8 & Lower half & 3,729 & 4,240 & $3,984.5$ \\
\hline S96T005576 & 166: 9 & Lower half & 6,127 & 6,900 & $6,513.5$ \\
\hline S96T005573 & 166: 10 & Lower half & 4,588 & 7,560 & $6,074^{\mathrm{QC}: c}$ \\
\hline \$96T005620 & 166: 1 & Lower half & 5,059 & 6,440 & $5,749.5^{Q C: c}$ \\
\hline S96T005621 & 166: 2 & Lower half & 4,490 & 4,420 & 4,455 \\
\hline S96T005718 & \multirow[t]{2}{*}{$166: 3$} & Upper half & 2,441 & 1,810 & $2,125.5^{\mathrm{QC}: \mathrm{e}}$ \\
\hline S96T005724 & & Lower half & 1,503 & 1,430 & $1,466.5$ \\
\hline S96T005418 & \multirow[t]{2}{*}{$166: 5$} & Upper half & 1,277 & 1,130 & $1,203.5$ \\
\hline S96T005412 & & Lower half & 1,715 & 1,510 & $1,612.5$ \\
\hline S96T005625 & \multirow[t]{2}{*}{ 166: 6} & Upper half & 1,530 & 1,540 & 1,535 \\
\hline \$96T005622 & & Lower half & $<981.3$ & 1,410 & $<1,195.65^{\mathrm{QC}: \mathrm{e}}$ \\
\hline S96T005419 & \multirow[t]{2}{*}{$166: 7$} & Upper half & 1,227 & 1,280 & $1,253.5$ \\
\hline S96T005413 & & Lower half & 1,650 & $<1,070$ & $<1,360^{\mathrm{Qc}: \mathrm{e}}$ \\
\hline S96T005719 & \multirow[t]{2}{*}{ 166: 8} & Upper half & 4,301 & 1,610 & $2,955.5^{\mathrm{QC}: \mathrm{c}}$ \\
\hline \$96T005725 & & Lower half & 3,435 & 3,110 & $3,272.5$ \\
\hline \$96T005845 & \multirow[t]{2}{*}{$166: 9$} & Upper half & 2,240 & 2,450 & 2,345 \\
\hline S96T005846 & & Lower half & 3,038 & 2,870 & 2,954 \\
\hline S96T005711 & \multirow[t]{2}{*}{ 167: 1} & Upper half & 2,724 & 2,310 & 2,517 \\
\hline S96T005699 & & Lower half & $<1,653$ & $<1,580$ & $<1,616.5$ \\
\hline \$96T005859 & 167: 2 & Lower half & 2,352 & 2,850 & 2,601 \\
\hline
\end{tabular}


Table B2-57. Tank 241-AN-103 Analytical Results: Phosphate (IC). (3 sheets)

\begin{tabular}{|c|c|c|c|c|c|}
\hline Sample & Sample & Sample & Ifsinit & Buplicane & Vean \\
\hline \multicolumn{3}{|c|}{ 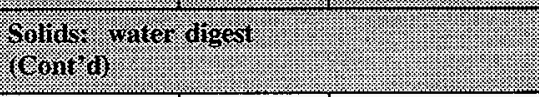 } & 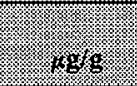 & . & 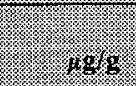 \\
\hline S96T005700 & $167: 3$ & Lower half & 6,471 & 6,230 & $6,350.5$ \\
\hline S96T005701 & 167: 4 & Lower half & 4,285 & 6,290 & $5,287.5^{\mathrm{QC}: e}$ \\
\hline S96T005440 & $167: 5$ & Lower half & 5,611 & 5,500 & $5,555.5$ \\
\hline S96T005452 & 167: 6 & Lower half & 4,914 & 5,730 & 5,322 \\
\hline S96T005702 & 167: 7 & Lower half & 3,307 & 3,880 & $3,593.5$ \\
\hline S96T005738 & $167: 8$ & Lower half & 4,848 & 5,190 & 5,019 \\
\hline S96T005860 & 167: 9 & Lower half & 5,582 & 4,940 & $5 ; 261$ \\
\hline S96T005712 & \multirow{2}{*}{$167: 1$} & Upper half & 1,246 & 1,260 & 1,253 \\
\hline S96T005703 & & Lower half & 1,264 & 1,360 & 1,312 \\
\hline S96T005446 & \multirow[t]{2}{*}{$167: 2$} & Upper half & 1,593 & 1,350 & $1,471.5$ \\
\hline S96T005453 & & Lower half & 827.4 & 827 & 827.2 \\
\hline S96T005447 & \multirow[t]{2}{*}{ 167: 4} & Upper half & $<466.4$ & $<452$ & $<459.2$ \\
\hline S96T005441 & & Lower half & $<1,131$ & $<1,130$ & $<1,130.5$ \\
\hline S96T005713 & \multirow[t]{2}{*}{$167: 5$} & Upper haif & $<941.3$ & $<923$ & $<932.15$ \\
\hline S96T005704 & & Lower half & 1,702 & 1,810 & 1,756 \\
\hline S96T005500 & \multirow[t]{2}{*}{$167: 7$} & Upper half & 3,121 & 1,950 & $2,535.5^{\mathrm{QC}: \mathrm{c}}$ \\
\hline S96T005501 & & Lower half & 15,330 & 14,500 & 14,915 \\
\hline S97T000023 & Core 166 & Solid composite & 3,437 & 3,520 & $3,478.5$ \\
\hline \multicolumn{3}{|l|}{ Erinuts. } & (19glanle. & (2. & 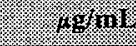 \\
\hline S96T005539 & $166: 3$ & Drainable liquid & $<1,224$ & $<1,220$ & $<1,222$ \\
\hline S96T005540 & 166: 4 & Drainable liquid & $<1,224$ & $<1,220$ & $<1,222$ \\
\hline S96T005599 & $166: 6$ & Drainable liquid & 464.6 & 502 & 483.3 \\
\hline S96T005816 & $166: 7$ & Drainable liquid & $<618.1$ & $<618$ & $<618.05$ \\
\hline
\end{tabular}




\begin{tabular}{|c|c|c|c|c|c|}
\hline$Z Z Z^{6} \mathrm{I}>$ & $0 \operatorname{czt}^{6} I>$ & $\operatorname{tZZ}[I>$ & $\begin{array}{r}\text { entsoduos } \\
\text { pinbi] }\end{array}$ & 9912100 & I66S00I96S \\
\hline$Z Z Z^{6} I>$ & $0 \operatorname{cz}(\mathrm{I}>$ & $\nabla Z Z^{4} I>$ & pinb!!l गॄqeu!̣ıda & I :L9I & 0S9S00L96S \\
\hline $50.819>$ & $819>$ & I' $819>$ & pinbII әтquu!eI & $6: \angle 9 I$ & Z98500L96S \\
\hline $2 Z Z^{6} \mid>$ & $0 z z^{6} 1>$ & $\nabla z \tau^{6} I>$ & p!nb!̣ əqqeu!̣eIa & $8: \angle 9 I$ & ZELS00L96S \\
\hline $2 z Z^{6} \mathrm{I}>$ & $0 z Z^{6} \mathrm{I}>$ & $\triangleright Z Z^{\prime} I>$ & pinbII әqquाeIa & $L: \angle 9 I$ & E89S00L96S \\
\hline ว:00208 & 666 & S09 & pı̣nb!l әqеu!̣. & $9: \angle 9 \mathrm{I}$ & $I Z t S 00 \perp 96 S$ \\
\hline 50.899 & $\angle I 9$ & $I^{\circ} 6 \mathrm{IL}$ & pịnbyl әฺ̣eureıa & $S: \angle 9 I$ & 0ZtS00L96S \\
\hline$Z Z Z^{\prime} I>$ & $0 z Z^{6} \mathrm{I}>$ & $\nabla Z Z^{\prime} I>$ & p!nb!̣ әтqеureı & $\mathcal{E}: \angle 9 I$ & EL9S00L96S \\
\hline $50.819>$ & $8[9>$ & I'8โ9> & pInb!I गॄqeurera & $\tau: \angle 9 I$ & [98S00L96S \\
\hline SSL6S & $88 S$ & $1 \% 209$ & 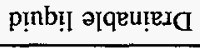 & $\tau: 99 I$ & L09S00L96S \\
\hline $0<9$ & $20 L$ & $8 \varepsilon 9$ & p!̣b!̣া әqеu!̣exa & I :99I & $009500 \mathrm{~L} 96 \mathrm{~S}$ \\
\hline $869 \varepsilon$ & ISE & $9.88 \mathcal{E}$ & pinb!l गqеu!eId & 0I :99 I & 99\$S00L96S \\
\hline$: 00 S I^{\circ} 0 E t$ & $6 S \mathcal{E}$ & $\varepsilon \cdot I 0 S$ & 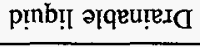 & $6: 99 I$ & ItSSO0L96S \\
\hline S0.8I9> & $8[9>$ & I'8โ9> & 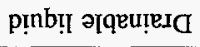 & $8: 99$ I & LI8S00L96S \\
\hline mant & mininy & 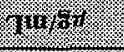 & & \multicolumn{2}{|c|}{ 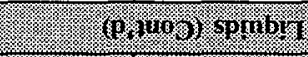 } \\
\hline 117\% & 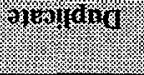 & Sustor & 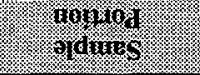 & 1010\% & Hatum \\
\hline
\end{tabular}

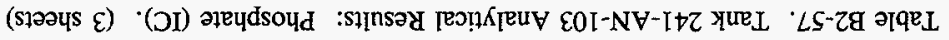


Table B2-58. Tank 241-AN-103 Analytical Results: Sulfate (IC). (3 sheets)

\begin{tabular}{|c|c|c|c|c|c|}
\hline ormole & \%and & Goming & (1) purtil & (1) & Hown \\
\hline Wolds & \% & & r. & 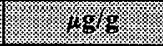 & ris \\
\hline S96T005610 & 166: 1 & Lower half & 2,596 & 3,300 & $2,948^{\mathrm{QC}: c}$ \\
\hline S96T005572 & 166: 4 & Lower half & $<1,200$ & $<1,130$ & $<1,165$ \\
\hline S96T005619 & 166: 6 & Lower half & $<1,245$ & $<1,240$ & $<1,242.5$ \\
\hline S96T005843 & 166: 7 & Lower half & $<1,102$ & $<1,090$ & $<1,096$ \\
\hline S96T005844 & 166: 8 & Lower half & 1,842 & 1,650 & 1,746 \\
\hline S96T005576 & 166: 9 & Lower half & $<1,101$ & $<1,040$ & $<1,070.5$ \\
\hline S96T005573 & $166: 10$ & Lower half & $<1,240$ & $<1,210$ & $<1,225$ \\
\hline \$96T005620 & 166: 1 & Lower half & $<2,748$ & $<2,740$ & $<2,744$ \\
\hline S96T005621 & 166: 2 & Lower half & $<2,739$ & $<2,790$ & $<2,764.5$ \\
\hline S96T005718 & \multirow[t]{2}{*}{ 166: 3} & Upper half & 2,382 & 3,180 & $2,781^{\mathrm{QC}: \mathrm{c}}$ \\
\hline S96T005724 & & Lower half & 1,488 & 2,010 & $1,749^{\mathrm{QC}: \mathrm{c}}$ \\
\hline S96T005418 & \multirow[t]{2}{*}{$166: 5$} & Upper half & 3,155 & 2,820 & $2,987.5$ \\
\hline S96T005412 & & Lower half & 4,370 & 4,020 & 4,195 \\
\hline S96T005625 & \multirow[t]{2}{*}{$166: 6$} & Upper haif & 2,876 & 2,150 & $2,513^{\mathrm{QC}: \mathrm{e}}$ \\
\hline S96T005622 & & Lower half & 1,804 & $<1,130$ & $<1,467^{\mathrm{QC:e}}$ \\
\hline S96T005419 & \multirow[t]{2}{*}{ 166: 7} & Upper half & 3,329 & 4,030 & $3,679.5$ \\
\hline S96T005413 & & Lower half & 5,440 & 5,270 & 5,355 \\
\hline S96T005719 & \multirow[t]{2}{*}{ 166: 8} & Upper half & 5,080 & 4,970 & 5,025 \\
\hline S96T005725 & & Lower half & 6,289 & 6,090 & $6,189.5$ \\
\hline S96T005845 & \multirow[t]{2}{*}{ 166: 9} & Upper half & 4,326 & 4,850 & 4,588 \\
\hline S96T005846 & & Lower half & 5,654 & 5,000 & 5,327 \\
\hline S96T005711 & \multirow[t]{2}{*}{ 167: 1} & Upper half & $<1,799$ & $<1,880$ & $<1,839.5$ \\
\hline S96T005699 & & Lower half & 4,615 & 4,290 & $4,452.5$ \\
\hline S96T005859 & 167: 2 & Lower half & 1,722 & 1,820 & 1,771 \\
\hline S96T005700 & 167: 3 & Lower half & $<1,749$ & $<1,720$ & $<1,734.5$ \\
\hline S96T005701 & 167: 4 & Lower half & $<1,810$ & $<1,830$ & $<1,820$ \\
\hline \$96T005440 & 167: 5 & Lower half & $<1,167$ & $<1,070$ & $<1,118.5$ \\
\hline S96T005452 & 167: 6 & Lower half & $<1,052$ & $<1,060$ & $<1,056$ \\
\hline S96T005702 & 167: 7 & Lower half & $<1,283$ & $<1,400$ & $<1,341.5$ \\
\hline
\end{tabular}


Table B2-58. Tank 241-AN-103 Analytical Results: Sulfate (IC). (3 sheets)

\begin{tabular}{|c|c|c|c|c|c|}
\hline Sample & Samples & $\begin{array}{l}\text { Sample } \\
\text { Romtion }\end{array}$ & Imesili! & Dupliticic & Mran: \\
\hline 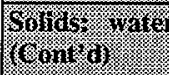 & ingest & & . & . & . \\
\hline S96T005738 & 167: 8 & Lower half & $<1,043$ & $<1,030$ & $<1,036.5$ \\
\hline S96T005860 & $167: 9$ & Lower half & $<524.2$ & $<574$ & $<549.1$ \\
\hline S96T005712 & \multirow[t]{2}{*}{$167: 1$} & Upper half & 2,758 & 3,260 & 3,009 \\
\hline S96T005703 & & Lower half & 991.6 & 1,170 & $1,080.8$ \\
\hline S96T005446 & \multirow[t]{2}{*}{$167: 2$} & Upper half & 1,748 & 1,720 & 1,734 \\
\hline S96T005453 & & Lower half & 1,390 & 1,450 & 1,420 \\
\hline S96T005447 & \multirow[t]{2}{*}{ 167:4 } & Upper half & 1,157 & 1,350 & $1,253.5$ \\
\hline S96T005441 & & Lower half & 2,240 & 2,390 & 2,315 \\
\hline S96T005713 & \multirow[t]{2}{*}{$167: 5$} & Upper half & $<1,082$ & $<1,060$ & $<1,071$ \\
\hline S96T005704 & & Lower half & 2,994 & 2,930 & 2,962 \\
\hline S96T005500 & \multirow[t]{2}{*}{$167: 7$} & Upper half & $<1,870$ & $<1,940$ & $<1,905$ \\
\hline S96T005501 & & Lower half & 5,674 & 5,020 & 5,347 \\
\hline S97T000023 & Core 166 & Solid composite & 5,388 & 5,660 & 5,524 \\
\hline IElgunas & & & $186 \mathrm{mo}$ & (1) & 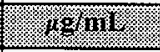 \\
\hline S96T005539 & $166: 3$ & Drainable liquid & 1,896 & 2,160 & 2,028 \\
\hline S96T005540 & $166: 4$ & Drainable liquid & $<1,408$ & $<1,410$ & $<1,409$ \\
\hline S96T005599 & $166: 6$ & Drainable liquid & 471.8 & 469 & 470.4 \\
\hline S96T005816 & $166: 7$ & Drainable liquid & $<710.8$ & $<711$ & $<710.9$ \\
\hline S96T005817 & $166: 8$ & Drainable liquid & $<710.8$ & $<711$ & $<710.9$ \\
\hline S96T005541 & 166: 9 & Drainable liquid & $<292.7$ & $<293$ & $<292.85$ \\
\hline S96T005566 & 166: 10 & \begin{tabular}{|l|} 
Drainable liquid \\
\end{tabular} & $<292.7$ & $<293$ & $<292.85$ \\
\hline S96T005600 & $166: 1$ & Drainable liquid & 535.5 & 560 & 547.75 \\
\hline S96T005601 & $166: 2$ & Drainable liquid & 490.7 & 528 & 509.35 \\
\hline S96T005861 & $167: 2$ & Drainable liquid & 752.9 & 892 & 822.45 \\
\hline S96T005673 & 167: 3 & Drainable liquid & 2,636 & 2,820 & 2,728 \\
\hline S96T005420 & $167: 5$ & Drainable liquid & 800.7 & $<571$ & $<685.85^{\mathrm{QC}: \mathrm{c}}$ \\
\hline S96T005421 & $167: 6$ & \begin{tabular}{|l} 
Drainable liquid \\
\end{tabular} & 912.1 & 820 & 866.05 \\
\hline S96T005683 & $167: 7$ & Drainable liquid & 3,243 & 3,260 & $3,251.5$ \\
\hline
\end{tabular}




\begin{tabular}{|c|c|c|c|c|c|}
\hline $60 t^{6} I>$ & OIt $[>$ & $80 t^{\prime} \mathrm{I}>$ & วn!soduos p!nb!T & 991 ว10ว & I66S00I96S \\
\hline $98 t^{\circ} \varepsilon$ & $009^{6} \varepsilon$ & $Z \angle E^{6} \varepsilon$ & p!̣nb!l әтqeu!̣eIa & $\mathrm{I}: \angle 9 \mathrm{I}$ & 0S9S00L96S \\
\hline$\varepsilon 0 z L>$ & $I I L>$ & $962 L$ & 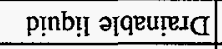 & $6: \angle 9 I$ & Z98\$00L96S \\
\hline $959^{\prime} z$ & $0 I L^{\prime} Z$ & $209^{\circ} z$ & pinby əlqeuresc & $8: \angle 91$ & ZELS00L96S \\
\hline 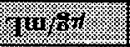 & : & 1 & & $\sqrt{2}$ & 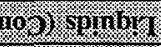 \\
\hline$(1001)$ & Honjoring & $\sqrt{145} \mathrm{~s}$ & erovar & $101416 \%$ & roundiry \\
\hline
\end{tabular}

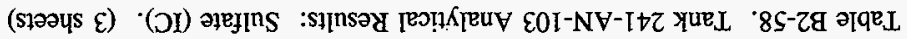


Table B2-59. Tank 241-AN-103 Analytical Results: Acetate (IC). (3 sheets)

\begin{tabular}{|c|c|c|c|c|c|}
\hline Sininger & 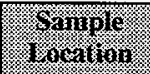 & f & 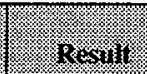 & 9uminger & $\operatorname{los} 1$ \\
\hline \multicolumn{3}{|c|}{ WHIH } & 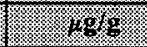 & . 1.19 & 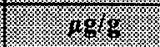 \\
\hline \$96T005610 & 166: 1 & Lower half & 691.6 & 1,400 & $1,045.8^{\mathrm{QC:e}}$ \\
\hline S96T005572 & 166: 4 & Lower half & $<173.9$ & $<164$ & $<168.95$ \\
\hline S96T005619 & 166: 6 & Lower half & 1,141 & 868 & $1,004.5^{\mathrm{QC}: \mathrm{c}}$ \\
\hline S96T005843 & $166: 7$ & Lower half & 303.3 & 484 & $393.65^{\mathrm{QC}: \mathrm{C}}$ \\
\hline S96T005844 & $166: 8$ & Lower half & 698.9 & 791 & 744.95 \\
\hline S96T005576 & $166: 9$ & Lower half & $<159.5$ & 1,210 & $<684.75^{\text {QC:e }}$ \\
\hline S96T005573 & 166: 10 & Lower half & 700.2 & 902 & $801.1^{\mathrm{QC}: \mathrm{c}}$ \\
\hline S96T005620 & 166: 1 & Lower half & 762 & 556 & $659^{Q C: c}$ \\
\hline S96T005621 & $166: 2$ & Lower half & 771.1 & 747 & 759.05 \\
\hline \$96T005718 & \multirow[t]{2}{*}{ 166: 3} & Upper half & 1,971 & 1,760 & $1,865.5$ \\
\hline S96T005724 & & Lower half & 1,777 & 1,790 & $1,783.5$ \\
\hline S96T005418 & \multirow[t]{2}{*}{$166: 5$} & Upper half & 1,394 & $<80.6$ & $<737.3^{\mathrm{QC}: \mathrm{e}}$ \\
\hline S96T005412 & & Lower half & 1,412 & 1,430 & 1,421 \\
\hline S96T005625 & \multirow[t]{2}{*}{ 166: 6} & Upper half & 1,217 & 980 & $1,098.5^{\mathrm{QC}: \mathrm{e}}$ \\
\hline S96T005622 & & Lower half & 1,441 & 1,340 & $1,390.5$ \\
\hline \$96T005419 & \multirow[t]{2}{*}{ 166: 7} & Upper half & 1,509 & 1,470 & $1,489.5$ \\
\hline S96T005413 & & Lower half & 865.1 & 1,270 & $1,067.55^{\mathrm{QC}: \mathrm{e}}$ \\
\hline S96T005719 & \multirow[t]{2}{*}{ 166: 8} & Upper half & 710.1 & 748 & 729.05 \\
\hline S96T005725 & & Lower half & 823 & 829 & 826 \\
\hline S96T005845 & \multirow[t]{2}{*}{ 166: 9} & Upper half & 1,257 & 1,500 & $1,378.5$ \\
\hline S96T005846 & & Lower half & 1,425 & 1,650 & $1,537.5$ \\
\hline S96T005711 & \multirow[t]{2}{*}{$167: 1$} & Upper half & 1,504 & 1,490 & 1,497 \\
\hline S96T005699 & & Lower half & 705.9 & 1,340 & $1,022.95^{\mathrm{QC}: c}$ \\
\hline S96T005859 & 167: 2 & Lower half & 804.9 & 752 & 778.45 \\
\hline$\$ 96 \mathrm{~T} 005700$ & $167: 3$ & Lower half & 1,085 & 1,080 & $1,082.5$ \\
\hline \$96T005701 & $167: 4$ & Lower half & 958.7 & 966 & 962.35 \\
\hline S96T005440 & 167: 5 & Lower half & 1,357 & 2,540 & $1,948.5^{\mathrm{QC}: \mathrm{e}}$ \\
\hline S96T005452 & 167: 6 & Lower half & 1,183 & 1,200 & $1,191.5^{\mathrm{QC}: \mathrm{c}}$ \\
\hline
\end{tabular}


Table B2-59. Tank 241-AN-103 Analytical Results: Acetate (IC). (3 sheets)

\begin{tabular}{|c|c|c|c|c|c|}
\hline $\begin{array}{l}\text { Gample } \\
\text { Namier }\end{array}$ & Sormple & 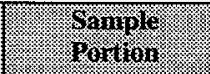 & Iresing & Montingito & Yeain \\
\hline \multicolumn{2}{|c|}{ 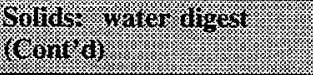 } & & (2) 13.96 & (1) & 10 \\
\hline S96T005702 & $167: 7$ & Lower half & $<733$ & $<798$ & $<765.5$ \\
\hline S96T005738 & 167: 8 & Lower half & 843 & 685 & $764^{\mathrm{OC}: \mathrm{c}, \mathrm{e}}$ \\
\hline S96T005860 & $167: 9$ & Lower half & 634.4 & 443 & $538.7^{\mathrm{CC}: \mathrm{e}}$ \\
\hline S96T005712 & \multirow{2}{*}{$167: 1$} & Upper half & 1,615 & 1,610 & $1,612.5$ \\
\hline S96T005703 & & Lower half & $<787.8$ & $<757$ & $<772.4$ \\
\hline S96T005446 & \multirow{2}{*}{$167: 2$} & Upper half & 1,398 & 1,430 & 1,414 \\
\hline S96T005453 & & Lower half & 1,295 & 1,330 & $1,312.5$ \\
\hline S96T005447 & \multirow[t]{2}{*}{$167: 4$} & Upper half & 630.4 & 792 & $711.2^{\mathrm{QC}: \mathrm{c}}$ \\
\hline S96T005441 & & Lower half & 883.1 & 963 & 923.05 \\
\hline S96T005713 & \multirow[t]{2}{*}{$167: 5$} & Upper half & 1,049 & 615 & $832^{\mathrm{QC}: \mathrm{c}}$ \\
\hline S96T005704 & & Lower half & 694.1 & 669 & 681.55 \\
\hline S96T005500 & \multirow[t]{2}{*}{$167: 7$} & Upper half & 705.5 & 1,310 & $1,007.75^{\mathrm{QC}: \mathrm{c}}$ \\
\hline S96T005501 & & Lower half & 1,053 & $<158$ & $<605.5^{Q C: c}$ \\
\hline S97T000023 & Core 166 & Solid composite ${ }^{\mathrm{l}}$ & 572.8 & 558 & 565.4 \\
\hline Uiguads & (1) & (5) & (1) & (1) & (2) \\
\hline S96T005539 & $166: 3$ & Drainable liquid & 2,122 & 2,300 & 2,211 \\
\hline S97T000163 & $166: 4$ & Drainable liquid & 3,594 & 3,720 & 3,657 \\
\hline S96T005599 & $166: 6$ & Drainable liquid & 3,505 & 3,420 & $3,462.5$ \\
\hline S96T005816 & $166: 7$ & Drainable liquid & 3,876 & 3,940 & 3,908 \\
\hline S96T005817 & $166: 8$ & Drainable liquid & 3,674 & 3,750 & 3,712 \\
\hline S96T005541 & 166: 9 & Drainable liquid & 3,817 & 3,420 & $3,618.5$ \\
\hline S96T005566 & 166: 10 & Drainable liquid & 2,964 & 2,520 & 2,742 \\
\hline S96T005600 & $166: 1$ & Drainable liquid & 10,510 & 10,400 & 10,455 \\
\hline S96T005601 & 166: 2 & Drainable liquid & 3,144 & 3,480 & 3,312 \\
\hline S96T005861 & $167: 2$ & Drainable liquid & 3,458 & 3,570 & 3,514 \\
\hline S96T005673 & $167: 3$ & Drainable liquid & 1,905 & 2,090 & $1,997.5$ \\
\hline S96T005420 & $167: 5$ & Drainable liquid & 2,285 & 2,160 & $2,222.5$ \\
\hline S96T005421 & 167: 6 & Drainable liquid & 2,798 & 2,620 & 2,709 \\
\hline
\end{tabular}


Table B2-59. Tank 241-AN-103 Analytical Results: Acetate (IC). (3 sheets)

\begin{tabular}{|c|c|c|c|c|c|}
\hline Sample & 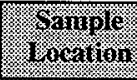 & $\begin{array}{l}\text { Sample: } \\
\text { romtion. }\end{array}$ & Reruil & 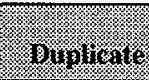 & Mern \\
\hline \multicolumn{2}{|c|}{ Giquinas (couri of } & & 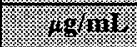 & 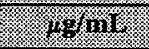 & 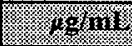 \\
\hline S96T005683 & $167: 7$ & Drainable liquid & 5,587 & 5,660 & $5,623.5$ \\
\hline S96T005732 & $167: 8$ & Drainable liquid & 7,745 & 6,470 & $7,107.5$ \\
\hline S96T005862 & $167: 9$ & Drainable liquid & 3,713 & 3,550 & $3,631.5$ \\
\hline S96T005650 & 167:1 & Drainable liquid & 1,942 & 2,040 & 1,991 \\
\hline S96T005991 & Core 166 & Liquid composite & 2,824 & 2,920 & 2,872 \\
\hline
\end{tabular}

Note:

Composite sample results are lower than most segment samples. This may be because of sample heterogeneity or incomplete homogenization. 
Table B2-60. Tank 241-AN-103 Analytical Results: Oxalate (IC). (3 sheets)

\begin{tabular}{|c|c|c|c|c|c|}
\hline Sangula & Wariple & 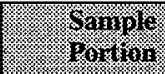 & mesuin: & oulativito & $1 \%$ \\
\hline \multicolumn{3}{|c|}{ 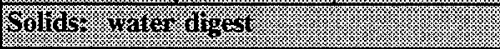 } & 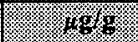 & $8 \%$ & 9 \\
\hline S96T005610 & 166: 1 & Lower half & 7,370 & 9,880 & $8,625^{\mathrm{QC}: \mathrm{c}}$ \\
\hline S96T005572 & 166: 4 & Lower half & $<913$ & $<863$ & $<888$ \\
\hline S96T005619 & 166: 6 & Lower half & $<947.2$ & $<946$ & $<946.6$ \\
\hline S96T005843 & $166: 7$ & Lower half & $<838.2$ & $<828$ & $<833.1$ \\
\hline S96T005844 & 166: 8 & Lower half & $<853.3$ & $<852$ & $<852.65$ \\
\hline S96T005576 & 166: 9 & Lower half & $<837.5$ & $<795$ & $<816.25$ \\
\hline S96T005573 & 166: 10 & Lower half & $<943.5$ & $<920$ & $<931.75$ \\
\hline S96T005620 & $166: 1$ & Lower half & $<2,091$ & $<2,080$ & $<2,085.5$ \\
\hline S96T005621 & 166: 2 & Lower half & $<2,084$ & $<2,120$ & $<2,102$ \\
\hline S96T005718 & \multirow[t]{2}{*}{ 166: 3} & Upper half & 7,132 & 7,110 & 7,121 \\
\hline S96T005724 & & Lower half & 8,680 & 9,070 & 8,875 \\
\hline S96T005418 & \multirow[t]{2}{*}{ 166: 5} & Upper half & 6,634 & 5,730 & 6,182 \\
\hline \$96T005412 & & Lower half & 6,539 & 5,730 & $6,134.5$ \\
\hline S96T005625 & \multirow[t]{2}{*}{ 166: 6} & Upper half & 7,115 & 9,160 & $8,137.5^{Q C: c}$ \\
\hline \$96T005622 & & Lower half & 7,945 & 7,960 & $7,952.5$ \\
\hline S96T005419 & \multirow[t]{2}{*}{ 166: 7} & Upper half & 2,698 & 2,890 & 2,794 \\
\hline S96T005413 & & Lower half & 2,167 & 2,090 & $2,128.5$ \\
\hline S96T005719 & \multirow[t]{2}{*}{ 166: 8} & Upper half & 3,344 & 3,180 & 3,262 \\
\hline S96T005725 & & Lower half & 4,865 & 4,690 & $4,777.5$ \\
\hline S96T005845 & \multirow[t]{2}{*}{$166: 9$} & Upper half & 4,235 & 4,590 & $4,412.5$ \\
\hline \$96T005846 & & Lower half & 5,707 & 4,880 & $5,293.5$ \\
\hline S96T005711 & \multirow[t]{2}{*}{ 167: 1} & Upper half & 10,540 & 10,700 & 10,620 \\
\hline S96T005699 & & Lower half & 5,956 & 6,600 & 6,278 \\
\hline S96T005859 & $167: 2$ & Lower half & 5,801 & 5,940 & $5,870.5$ \\
\hline S96T005700 & $167: 3$ & Lower half & $<1,330$ & $<1,310$ & $<1,320$ \\
\hline S96T005701 & $167: 4$ & Lower half & $<1,377$ & $<1,390$ & $<1,383.5$ \\
\hline S96T005440 & $167: 5$ & Lower half & $<888.2$ & $<815$ & $<851.6$ \\
\hline S96T005452 & 167: 6 & Lower half & $<800.2$ & 884 & $<842.1$ \\
\hline \$96T005702 & 167: 7 & Lower half & $<976.5$ & $<1,060$ & $<1,018.25$ \\
\hline
\end{tabular}


Table B2-60. Tank 241-AN-103 Analytical Results: Oxalate (IC). (3 sheets)

\begin{tabular}{|c|c|c|c|c|c|}
\hline (6) & Sample & Moxkno & \% & $8+40114918$ & $W_{4}$ \\
\hline 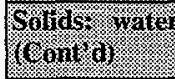 & \%ight & 1. & .6 & (1) & (3) \\
\hline S96T005738 & $167: 8$ & Lower half & $<793.3$ & $<787$ & $<790.15$ \\
\hline S96T005860 & $167: 9$ & Lower half & $<398.9$ & $<437$ & $<417.95$ \\
\hline S96T005712 & 167: 1 & Upper half & 5,722 & 4,830 & 5,276 \\
\hline S96T005703 & & Lower half & 7,187 & 7,950 & $7,568.5$ \\
\hline S96T005446 & 167:2 & Upper half & 9,779 & 9,320 & $9,549.5$ \\
\hline S96T005453 & & Lower half & 4,385 & 4,260 & $4,322.5$ \\
\hline S96T005447 & $167: 4$ & Upper half & 2,588 & 2,810 & 2,699 \\
\hline S96T005441 & & Lower half & 7,210 & 7,970 & 7,590 \\
\hline$\$ 96 T 005713$ & $167: 5$ & Upper half & 2,262 & 1,830 & $2,046^{\mathrm{QC:c}}$ \\
\hline S96T005704 & & Lower half & 5,925 & 5,750 & $5,837.5$ \\
\hline \$96T005500 & 167: 7 & Upper half & 2,942 & 3,150 & $3,046^{\mathrm{QC:d}}$ \\
\hline S96T005501 & & Lower half & 4,552 & 3,910 & 4,231 \\
\hline S97T000023 & Core 166 & Solid composite & 9,574 & 11,200 & 10,387 \\
\hline Wing & & 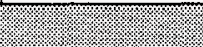 & 1. & 3. & (1) \\
\hline S96T005539 & 166: 3 & Drainable liquid & $<222.7$ & 313 & $<267.85^{\mathrm{QC}: \mathrm{e}}$ \\
\hline S96T005540 & $166: 4$ & Drainable liquid & $<222.7$ & 359 & $<290.85^{\mathrm{QC:c}}$ \\
\hline S96T005599 & 166: 6 & Drainable liquid & 293.3 & 250 & 271.65 \\
\hline S96T005816 & $166: 7$ & Drainable liquid & $<540.9$ & $<541$ & $<540.95$ \\
\hline$\$ 96 \mathrm{~T} 005817$ & $166: 8$ & Drainable liquid & $<540.9$ & $<541$ & $<540.95$ \\
\hline \$96T005541 & 166: 9 & Drainable liquid & 311.9 & 354 & 332.95 \\
\hline S96T005566 & $166: 10$ & Drainable liquid & 364.1 & 383 & 373.55 \\
\hline \$96T005600 & $166: 1$ & Drainable liquid & 302.9 & 273 & 287.95 \\
\hline S96T005601 & $166: 2$ & Drainable liquid & 281.6 & 258 & 269.8 \\
\hline S96T005861 & $167: 2$ & Drainable liquid & $<540.9$ & $<541$ & $<540.95$ \\
\hline S96T005673 & 167: 3 & Drainable liquid & $<1,071$ & $<1,070$ & $<1,070.5$ \\
\hline$\$ 96 \mathrm{~T} 005420$ & $167: 5$ & Drainable liquid & $<434.8$ & $<435$ & $<434.9$ \\
\hline S96T005421 & $167: 6$ & Drainable liquid & $<434.8$ & $<435$ & $<434.9$ \\
\hline S96T005683 & $167: 7$ & Drainable liquid & $<1,071$ & $<1,070$ & $<1,070.5$ \\
\hline
\end{tabular}


Table B2-60. Tank 241-AN-103 Analytical Results: Oxalate (IC). (3 sheets)

\begin{tabular}{|c|c|c|c|c|c|}
\hline $\begin{array}{l}\text { Sampler } \\
\text { unomiser }\end{array}$ & Samingles & Sample & istil & Ouplicate & $n_{\text {gin }}$ \\
\hline \multicolumn{3}{|c|}{ 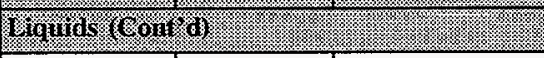 } & ig/me & $4.49101 \%$ & 1686118 \\
\hline S96T005732 & $167: 8$ & Drainable liquid & $<1,071$ & $<1,070$ & $<1,070.5$ \\
\hline S96T005862 & $167: 9$ & Drainable liquid & $<540.9$ & $<541$ & $<540.95$ \\
\hline S96T005650 & 167: 1 & Drainable liquid & $<1,071$ & $<1,070$ & $<1,070.5$ \\
\hline S96T005991 & Core 166 & Liquid composite & $<1,071$ & $<1,070$ & $<1,070.5$ \\
\hline
\end{tabular}

Table B2-61. Tank 241-AN-103 Analytical Results: Total Uranium (U).

\begin{tabular}{|c|c|c|c|c|c|}
\hline $\begin{array}{l}\text { Samile } \\
\text { Niminer }\end{array}$ & $\begin{array}{l}\text { Somple } \\
\text { yocition }\end{array}$ & Sample & Yessuil & Duplicar & Meni: \\
\hline Solitas firso & 1 & & 1.846 & 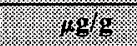 & 1.864 \\
\hline S96T005986 & Core 166 & Solid composite & 46.5 & 43.7 & 45.1 \\
\hline 12igunds & : & & I Ifril & $190 \mathrm{mi}$ & 18.0 .111 \\
\hline S96T005993 & Core 166 & Liquid composite & 1.99 & 1.68 & $1.835^{\mathrm{QC:c,f}}$ \\
\hline
\end{tabular}

Table B2-62. Tank 241-AN-103 Analytical Results: Hydroxide (OH Direct).

\begin{tabular}{|c|c|c|c|c|c|}
\hline $\begin{array}{l}\text { Saringle } \\
\text { Number }\end{array}$ & $\begin{array}{l}\text { Samingle. } \\
\text { Uraction }\end{array}$ & Sarmple & resin: & Buplizate & Mean \\
\hline Miguris & (1) & 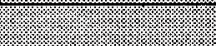 & 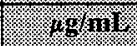 & 796.111. & inguriv \\
\hline S96T005993 & Core 166 & Liquid composite & 96,700 & 99,700 & 98,200 \\
\hline Soldits water & argest & & 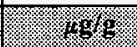 & $169+2$ & 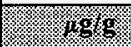 \\
\hline S96T005988 & Core 166 & Solid composite & 29,800 & 36,000 & $32,900^{\circ \mathrm{C}: \mathrm{e}}$ \\
\hline
\end{tabular}


Table B2-63. Tank 241-AN-103 Analytical Results: Hexavalent Chromium $(\mathrm{Cr}+6)$.

\begin{tabular}{|c|c|c|c|c|c|}
\hline 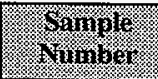 & Samples & 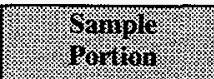 & 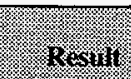 & Bingiterit & 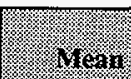 \\
\hline Minion & 3 & (x) & m, & 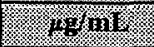 & 49rit \\
\hline S96T005992 & Core 166 & Liquid composite & 113 & 110 & 111.5 \\
\hline Sollis w wain & 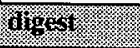 & & 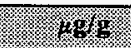 & 459 & 20 \\
\hline S96T005988 & Core 166 & Solid composite & 97.3 & 102 & 99.65 \\
\hline
\end{tabular}

Table B2-64. Tank 241-AN-103 Analytical Results: Total Organic Carbon (TOC).

\begin{tabular}{|c|c|c|c|c|c|}
\hline Nunine? & Sampile & \% & gesuil & Virpingate & 1940 \\
\hline Solids & & & . & (3. & (v. \\
\hline S96T005984 & Core 166 & Solid composite & 2,060 & 2,820 & $2,440^{\mathrm{QC}: c}$ \\
\hline Malinas & & & 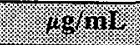 & 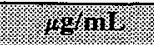 & $14 \mathrm{~g}+\mathrm{mi}$ \\
\hline \$96T005990 & Core 166 & Liquid composite & 3,070 & 3,020 & $3,045^{\mathrm{QC:f}}$ \\
\hline
\end{tabular}

B2-65. Tank 241-AN-103 Analytical Results: Total Inorganic Carbon (TIC)

\begin{tabular}{|c|c|c|c|c|c|}
\hline GMU⿴囗 & \%oung & Saguplo & Iresills & Ploping & Han \\
\hline Sollids r. & V. & & 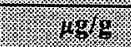 & (3. & hys \\
\hline S96T005984 & Core 166 & Solid composite & 5,640 & 5,900 & $5,770^{\mathrm{QC}: \mathrm{d}}$ \\
\hline Shaviog & & & 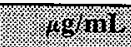 & mation & (1) 1094 \\
\hline S96T005990 & Core 166 & Liquid composite & 1,160 & 1,150 & $1,155^{\mathrm{QC}: \mathrm{f}}$ \\
\hline \$97T001500 & Core 166:7 & Drainable liquid & 2,500 & 2,720 & 2,610 \\
\hline
\end{tabular}


HNF-SD-WM-ER-702 Rev. 0

\section{VAPOR SAMPLES}

Table B2-66. Tank 241-AN-103 Vapor Sampling Results.

\begin{tabular}{|l|l|}
\hline \multicolumn{1}{|c|}{ Meacricnen } \\
\hline Total Organic Carbon & 1 part per million (ppmv) \\
\hline Lower Explosive Limit (LEL) & 0 percent of LEL \\
\hline Oxygen & 20.9 percent \\
\hline Ammonia & $8 \mathrm{ppm}$ \\
\hline
\end{tabular}




\section{HISTORICAL SAMPLES}

Table B2-67. Composition of Sludge Sample R-8190. ${ }^{1,2,3}$ (2 sheets)

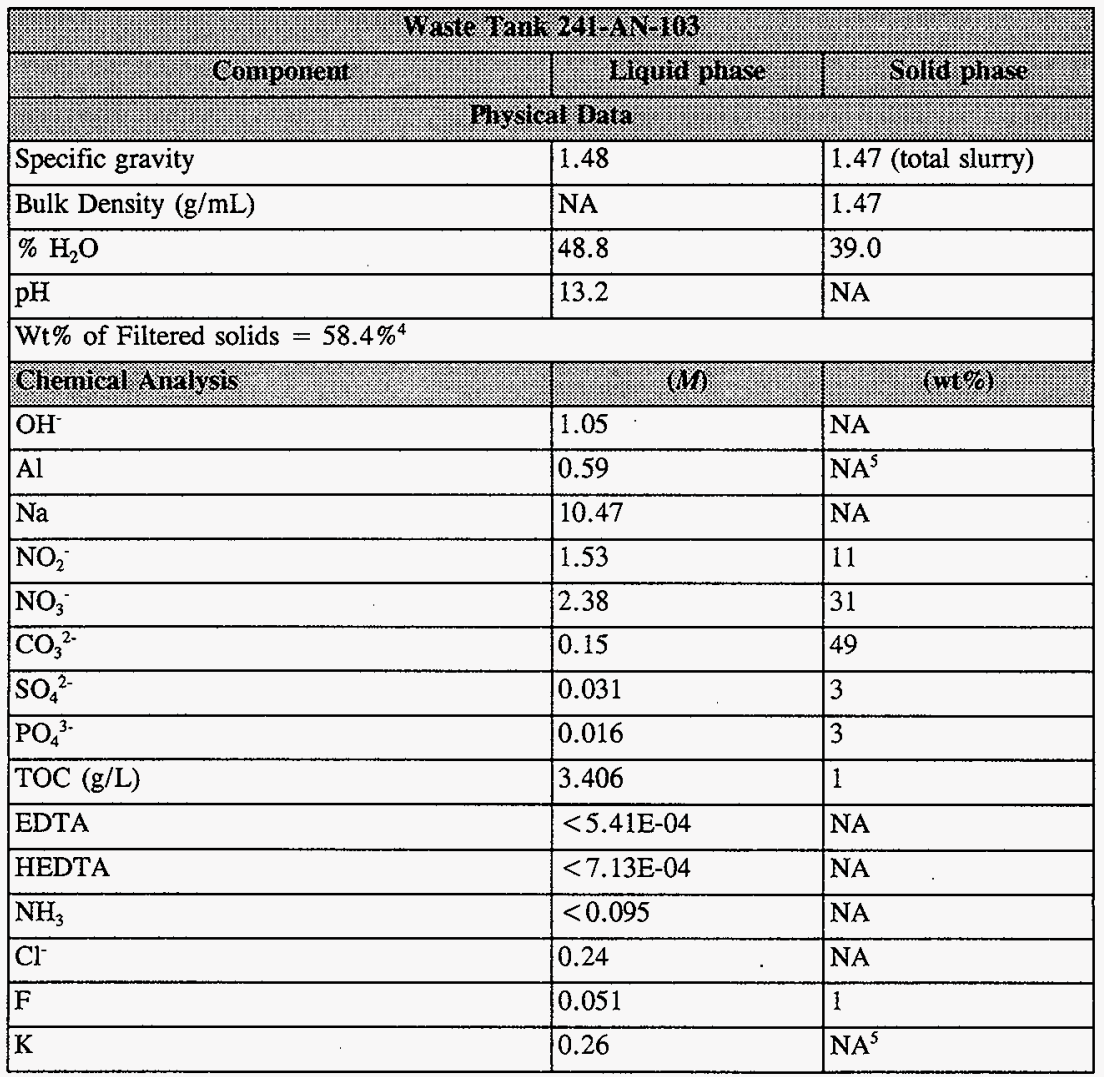




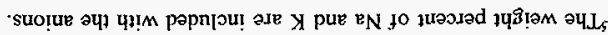

(tusog

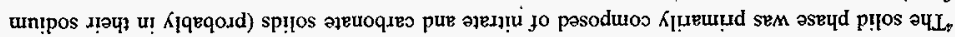

'uo!̣euroju!

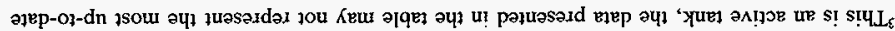

(986I) ssnew

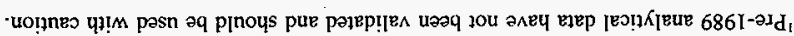

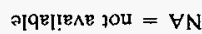

:SOJON

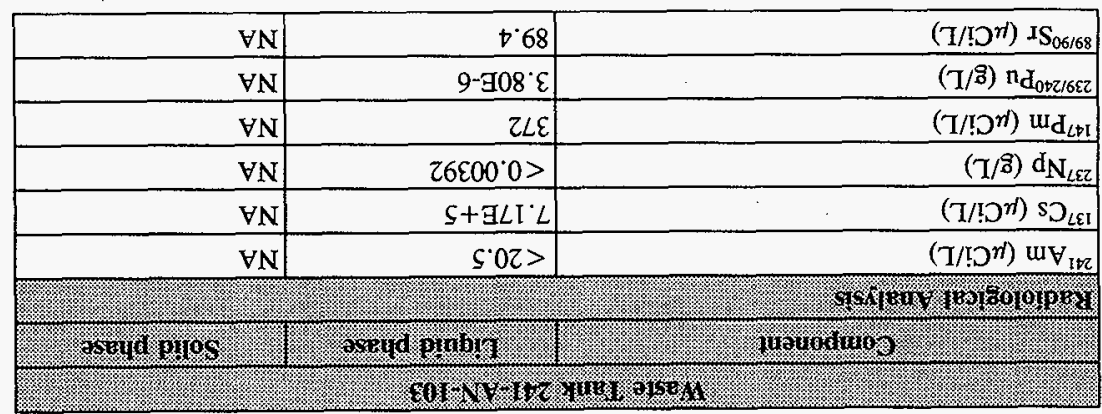

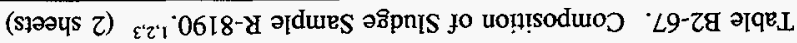


Table B2-68. Main Constituents of Slurry Samples Taken During Campaign 86-2., ${ }^{1,2,3}$

\begin{tabular}{|c|c|c|c|c|c|}
\hline & & Sannile V Vurio & 1. Boro Sning & 6. & \\
\hline Componerir & (1) $811 \%$ & (2) & (1) & (1) 6010 & f rrerage \\
\hline $\mathrm{Al}(\mathrm{M})$ & 2.87 & 2.67 & 2.48 & 2.50 & 2.63 \\
\hline$\overline{\mathrm{OH}}(\mathrm{M})$ & 5.50 & 5.75 & 4.91 & 5.50 & 5.42 \\
\hline $\mathrm{NO}_{2}(\mathrm{M})$ & 3.20 & 3.56 & 3.21 & 2.85 & 3.2 \\
\hline $\mathrm{NO}_{3}(\mathrm{M})$ & 2.54 & 4.08 & 3.20 & 2.81 & 3.16 \\
\hline $\mathrm{CO}_{3}(\mathrm{M})$ & 0.30 & 0.27 & 0.16 & 0.14 & 0.22 \\
\hline $\mathrm{PO}_{4}(\mathrm{M})$ & 0.025 & 0.025 & 0.023 & 0.020 & 0.023 \\
\hline $\bar{F}(\mathrm{M})$ & 0.070 & 0.105 & 0.10 & 0.11 & 0.096 \\
\hline $\mathrm{Cl}(\mathrm{M})$ & 0.29 & 0.99 & 0.22 & 0.28 & 0.45 \\
\hline $\mathrm{K}(\mathrm{M})$ & 0.31 & 0.40 & 0.47 & 0.49 & 0.42 \\
\hline $\mathrm{Na}(\mathrm{M})$ & 16.25 & 14.10 & 15.28 & 15.30 & 15.23 \\
\hline TOC $(g / L)$ & 4.70 & 6.15 & 4.33 & 4.29 & 4.9 \\
\hline${ }^{137} \mathrm{Cs}(\mu \mathrm{Ci} / \mathrm{L})$ & $9.25 \mathrm{E}+05$ & $8.50 \mathrm{E}+05$ & $6.90 \mathrm{E}+05$ & $7.65 \mathrm{E}+05$ & $8.08 \mathrm{E}+05$ \\
\hline $\mathrm{Pm}(\mu \mathrm{Ci} / \mathrm{L})$ & 95.5 & NA & NA & NA & NA \\
\hline${ }^{239 / 240} \mathrm{Pu}(\mathrm{g} / \mathrm{L})$ & $8.35 \mathrm{E}-06$ & $4.10 \mathrm{E}-06$ & $7.20 \mathrm{E}-06$ & $3.58 \mathrm{E}-06$ & $5.8 \mathrm{E}-06$ \\
\hline${ }^{89 / 90} \mathrm{Sr}(\mu \mathrm{Ci} / \mathrm{L})$ & 1310 & 1230 & 715 & 530 & 946 \\
\hline Spec. gravity & NA & 1.79 & 1.51 & 1.54 & 1.61 \\
\hline $\mathrm{H}_{2} \mathrm{O}(\%)$ & 45.1 & 37.4 & 45.5 & 47 & 43.8 \\
\hline $\mathrm{pH}$ & 13.3 & 13.3 & 13.3 & 13.3 & 13.3 \\
\hline
\end{tabular}

Notes:

IPre-1989 analytical data have not been validated and should be used with caution.

${ }^{2}$ Mauss (1986)

${ }^{3}$ This is an active tank, the data presented in the table may not represent the most up-to-date information. 
Table B2-69. Inorganic Analyses of $241-\mathrm{AN}-103$ Core Composite. ${ }^{1.2}$ (2 sheets)

\begin{tabular}{|c|c|c|}
\hline (10) & (minolic/pin) & (1) \\
\hline $\mathrm{Al}$ & 1,670 & 45,000 \\
\hline $\mathrm{Ag}$ & $<0.090$ & $<10$ \\
\hline As & $<1.33$ & $<100$ \\
\hline$\overline{\mathrm{Ba}}$ & $<0.070$ & $<10$ \\
\hline $\mathrm{Be}$ & $<1.11$ & $<10$ \\
\hline $\mathrm{Bi}$ & $<0.480$ & $<100$ \\
\hline $\mathrm{Ca}$ & 2.38 & 95 \\
\hline $\mathrm{Cd}$ & 0.090 & 10 \\
\hline $\mathrm{Cr}$ & 11.7 & 610 \\
\hline $\mathrm{Cu}$ & 0.080 & 5 \\
\hline $\mathrm{Fe}$ & 1.38 & 77 \\
\hline $\mathrm{K}$ & 231 & 9,000 \\
\hline $\mathrm{Mg}$ & 1.04 & 25 \\
\hline $\mathrm{Mn}$ & 0.450 & 25 \\
\hline Mo & 0.520 & $<50$ \\
\hline $\mathrm{Na}$ & 10,300 & 236,000 \\
\hline $\mathrm{Ni}$ & 0.340 & $<20$ \\
\hline $\mathrm{Pb}$ & 0.390 & 80 \\
\hline $\mathrm{Sb}$ & $<0.070$ & $<10$ \\
\hline $\mathrm{Si}$ & 10.4 & 290 \\
\hline $\mathrm{Sn}$ & 0.840 & $<100$ \\
\hline$S$ & $<3.13$ & $<100$ \\
\hline $\mathrm{Ti}$ & 0.210 & $<10$ \\
\hline $\mathrm{U}$ & 0.220 & 53 \\
\hline $\mathrm{V}$ & 1.96 & $<100$ \\
\hline W & 0.870 & 160 \\
\hline $\mathrm{Zr}$ & 0.220 & $<20$ \\
\hline $\mathrm{Zn}$ & 0.770 & 50 \\
\hline $\mathrm{Hg}{ }^{(3)}$ & 0.10 & $20 \pm 1.35$ \\
\hline $\mathrm{NH}_{3}$ & 12.9 & 220 \\
\hline $\mathrm{CO}_{3}^{2-(4)}$ & 92.8 & 5,570 \\
\hline
\end{tabular}


HNF-SD-WM-ER-702 Rev. 0

Table B2-69. Inorganic Analyses of 241-AN-103 Core Composite. ${ }^{1,2}$ (2 sheets)

\begin{tabular}{|l|l|l|}
\hline $\mathrm{CN}^{-(5)}$ & & \\
\hline $\mathrm{Cl}^{-}$ & 0.850 & 22 \\
\hline $\mathrm{F}^{-}$ & 151 & 5,300 \\
\hline $\mathrm{SO}_{4}{ }^{2-}$ & 11.05 & 210 \\
\hline $\mathrm{NO}_{3}{ }^{-}$ & 18.8 & 1,800 \\
\hline $\mathrm{NO}_{2}{ }^{-}$ & 1,180 & 73,000 \\
\hline $\mathrm{PO}_{4}{ }^{3-}$ & 1,630 & 75,000 \\
\hline $\mathrm{OH}^{-}$ & 9.48 & 900 \\
\hline
\end{tabular}

Notes:

'Pre-1989 analytical data have not been validated and should be used with caution.

${ }^{2}$ This is an active tank, the data presented in the table may not represent the most up-to-date information.

${ }^{3}$ Analyzed in replicate.

${ }^{4}$ Computed by difference from carbon analysis: total carbon minus total organic carbon equals inorganic carbon or, at highly basic $\mathrm{pH}, \mathrm{CO}_{3}{ }^{3-}$ concentration.

${ }^{5}$ Total $\mathrm{CN}^{-}$in water soluble portion of waste; free $\mathrm{CN}^{\cdot}$ in same fraction was $2.5 \mu \mathrm{g} / \mathrm{g}(0.100 \mu \mathrm{mole} / \mathrm{g})$. 


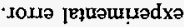

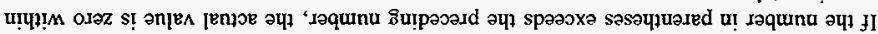

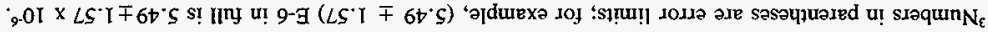

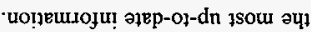

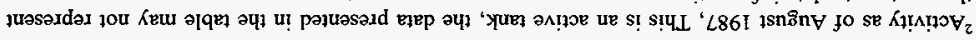

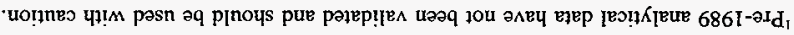

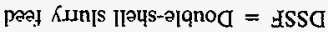

:səION

\begin{tabular}{|c|c|c|}
\hline$s-30^{\circ}+$ & $I-\exists\left(60^{\circ} 0 \mp 68^{\circ} \mathrm{L}\right)$ & Ełag TęoL \\
\hline t-GSII & 9-马 $\left(26^{\circ} \mathrm{I} \mp \mathrm{I} \mathrm{Z}^{\circ} \mathrm{L}\right)$ & RudIV IEOL \\
\hline \multirow[t]{2}{*}{$\tau-\exists 0^{\circ} \varepsilon$} & $L-\exists\left(\neq \varepsilon^{\circ} 0 \mp s 8^{\circ} \varepsilon\right)$ & $u_{S_{t+2}}$ \\
\hline & $9-\exists\left(20^{\circ} 0 \mp t \mathcal{E}^{\circ} \mathcal{\varepsilon}\right)$ & $u_{V_{I D Z}}$ \\
\hline \multirow[t]{6}{*}{. } & $9-\exists\left(\angle 0^{\circ} 0 \mp \nu \mathcal{Z}^{\circ} I\right)$ & $\mathrm{n}_{\mathbf{d}_{0+Z / 6 \varepsilon z}}$ \\
\hline & $\angle-G\left(80^{\circ} 0 \mp \angle 9^{\circ} S\right)$ & $\mathrm{n}_{\mathbf{d}_{8 \varepsilon \tau}}$ \\
\hline & 8- $\exists\left(89^{\circ} I \mp s \varsigma^{\circ} Z\right)$ & $\mathrm{d}_{\mathrm{N}_{L \varepsilon}}$ \\
\hline & 8-G(8E.0干 $\left.\varsigma \varepsilon^{\circ} I\right)$ & $\Omega_{8 \varepsilon z}$ \\
\hline & $0 I^{-}-\exists\left(I 8^{\circ} S \mp I^{\circ} L\right)$ & $\Omega_{\varsigma \varepsilon \tau}$ \\
\hline & $8-3\left(10^{\circ} 0 \mp 59 \mathcal{E}\right)$ & $\Omega_{+\varepsilon \tau}$ \\
\hline \multirow[t]{2}{*}{$\varepsilon-30 I$} & $I-B\left(60^{\circ} 0 \mp s S^{\circ} L\right)$ & $\mathrm{s} \supset_{L \varepsilon \mathrm{I}}$ \\
\hline & $t-g(t I 0 \mp \angle I Z)$ & $s \mathrm{D}_{\text {DEI }}$ \\
\hline \multirow[t]{2}{*}{$s-30.8$} & $L-\exists\left(10^{\circ} I \mp 2 D^{\circ} I^{-}\right)$ & $I_{6 Z I}$ \\
\hline & $s-3\left(9 s^{\circ} 8 \mp 89\right)$ & $n y_{901}$ \\
\hline$\varepsilon-\exists 0^{\circ} \varepsilon$ & $\nabla-\exists\left(0 I^{\circ} 0 \mp 80^{\circ} \mathrm{I}\right)$ & $0 L_{66}$ \\
\hline$t-30^{\circ} z$ & 9-马(SZII $\mp$ & $\mathrm{qN}_{\mathrm{p6}}$ \\
\hline \multirow[t]{2}{*}{$s-30^{\circ} t$} & $z-g\left(s 0^{\circ} 0 \mp t t^{\prime} z\right)$ & ${ }^{1} \mathbf{S}_{06}$ \\
\hline & $s-\exists\left(I \varepsilon^{\circ} \varepsilon \mp t \varepsilon^{\circ} t\right)$ & ${ }^{2} \mathrm{~S}_{6 \mathrm{C}}$ \\
\hline $1-30^{\circ} L$ & $s-g\left(t 0^{\circ} I \mp I L \mathcal{E}\right)$ & $\mathrm{o}_{09}$ \\
\hline $\mathcal{E}-30.8$ & $9-3\left(0 I^{\circ} \triangleright \mp 00^{\circ} t\right)$ & $D_{\mathrm{t} t}$ \\
\hline$z-30^{\circ} t$ & $9-3\left(5.61 \mp 80^{\circ} 8\right)$ & $\mathrm{H}_{\varepsilon}$ \\
\hline 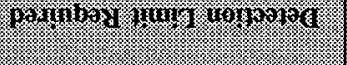 & 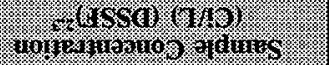 & O191910\%199\% \\
\hline
\end{tabular}




\section{B3.0 ASSESSMENT OF CHARACTERIZATION RESULTS}

The purpose of this chapter is to discuss the overall quality and consistency of the current sampling results for tank $241-\mathrm{AN}-103$, and to present the results of the calculation of an analytical-based inventory.

This section also evaluates sampling and analysis factors that may impact interpretation of the data. These factors are used to assess the overall quality and consistency of the data and to identify any limitations in the use of the data.

\section{B3.1 FIELD OBSERVATIONS}

The safety screening DQO (Dukelow et al. 1995) requires all vertical waste profiles be from two widely spaced risers. The flammable gas DQO (McDuffie and Johnson 1995) requires at least one complete core will be taken. These requirements were fulfilled. Contamination by hydrostatic head fluid resulted in adjusting the weight percent water results in one phase of 12 subsegments and the core composite. The waste recovered in segments with less than full recovery was assumed to be representative of the whole segment.

\section{B3.2 QUALITY CONTROL ASSESSMENT}

The usual quality control assessment includes an evaluation of the appropriate standard recoveries, spike recoveries, duplicate analyses, and blanks that are performed in conjunction with the chemical analyses. All the pertinent quality control tests were conducted on the 1996 push core samples, allowing a full assessment regarding the accuracy and precision of the data. The SAP (Kruger 1996) established the specific criteria for all analytes. Sample and duplicate pairs that had one or more quality control (QC) results outside the specified criteria were identified by footnotes in the data summary tables.

The standard and spike recovery results provide an estimate of the accuracy of the analysis. If a standard or spike recovery is above or below the given criterion, the analytical results may be biased high or low, respectively. The precision is estimated by the relative percent difference (RPD), which is defined as the absolute value of the difference between the primary and duplicate samples, divided by their mean, times one hundred.

RPDs greater than 30 percent were reported for twenty-eight of the sixty-one subsamples submitted for differential scanning calorimetry. The high RPDs can be attributed to the small exotherms and the heterogeneous nature of the samples. No reruns were requested. Thermograms for several subsamples showed small sharp peaks near $200{ }^{\circ} \mathrm{C}$, which indicated a decomposition of a pure compound. The standard recoveries for this analysis were within the required limits. 
RPDs greater than 30 percent were reported for five of the sixty-one subsamples submitted for thermogravimetric analysis. Selected samples were reanalyzed, and the reruns resulted in RPDs of less than 30 percent. Several thermograms showed small sharp peaks. These were the result of instrument vibration and were not used in the calculation of the result. The standard recoveries for this analysis were within the required limits.

A high RPD was reported for ten of the fifty subsamples submitted for total alpha activity. The sample results were near the detection limit which decreased the precision of the analyses. No reruns were requested because of the low alpha activity in the samples.

High RPDs (greater than 20 percent) were reported for the two samples submitted for tritium analyses. The high RPD for the solid core composite was attributed to sample inhomogeneity. No rerun was requested because of the low tritium activity in the samples. The chemist noted the high RPD for the liquid core composite was because of a small amount of ${ }^{137} \mathrm{Cs}$ contamination present on the duplicate aliquot mount. This contamination is unavoidable in samples containing high levels of ${ }^{137} \mathrm{Cs}$. The sample was analyzed three times due to ${ }^{137} \mathrm{Cs}$ contamination, and no further reruns were requested. Sr contamination was found in the field blank, but the level was minimal.

Some of the high RPDs for the IC analytes may be attributable to sample homogeneity problems. Spike recoveries outside of the required range were reported for acetate, $\mathrm{Cl}, \mathrm{Fl}$, $\mathrm{NO}_{2}, \mathrm{NO}_{3}$, and oxalate. The chemist noted that the poor spike recoveries were because of matrix interferences. In particular, spike failures for fluoride were because of organic acid interference, and spike failures for $\mathrm{NO}_{2}$ and $\mathrm{NO}_{3}$ were because of the high concentration of these analytes in the sample with respect to the amount of spike standard added.

Many of the ICP analytes also had one or more QC parameters outside the specified limits. High RPDs (greater than 20 percent) were reported for several analytes (Al, B, Ca, Cr, Fe, $\mathrm{K}, \mathrm{Li}, \mathrm{Na}, \mathrm{Ni}, \mathrm{Si}, \mathrm{Zn}, \mathrm{Zr}$ ) and were attributed to sample inhomogeneity. $\mathrm{Ca}, \mathrm{Fe}, \mathrm{Li}, \mathrm{Ni}$, $\mathrm{Zn}$, and $\mathrm{Zr}$ were detected at or below detection limits. The Ni fusion results are influenced by sample contamination (the fusion is performed in a nickel crucible). Reruns were not requested. Poor spike recoveries for $\mathrm{Al}, \mathrm{K}$, and $\mathrm{Na}$ were attributed to the high concentration of these analytes in the samples with respect to the amount of spike standard added.

Therefore, the matrix spike recovery results for these three analytes should not be used as a means to determine the accuracy of the results.

A high RPD (greater than 20 percent) for TIC/TOC was reported for the core 166 solid core composite and was attributed to sample inhomogeneity. No rerun was requested. A spike recovery outside of the required range was reported for one sample submitted for uranium analysis. The chemist noted the low spike recovery was because of matrix interferences and no rerun was requested. 
In summary, the vast majority of the QC results were within the boundaries specified in the SAPs. The discrepancies mentioned here and footnoted in the data summary tables should not impact either the validity or the use of the data.

\section{B3.3 DATA CONSISTENCY CHECKS}

Comparisons of different analytical methods can help to assess the consistency and quality of the data. Several comparisons were possible with the data set provided by the two push core samples, including a comparison of phosphorous as analyzed by ICP with phosphate as analyzed by IC, and a comparison of weight percent water by TGA with the weight percent water by gravimetry. In addition, mass and charge balances were calculated to help assess the overall data consistency.

\section{B3.3.1 Comparison of Results from Different Analytical Methods}

The following data consistency checks compare the results from two different analytical methods. A close comparison between the two methods strengthens the credibility of both results, whereas a poor comparison brings the reliability of the data into question. All analytical mean results were calculated from tables in Section B3.4, using Table B3-10 for the convective layer, Tables B3-12 and B3-13 for the nonconvective layer, and Table B3-14 for the crust.

The analytical phosphorous mean result as determined by ICP for the crust layer was $667 \mu \mathrm{g} / \mathrm{g}$, which converts to $2,044 \mu \mathrm{g} / \mathrm{g}$ of phosphate. This compared well with the IC phosphate mean result of $1,950 \mu \mathrm{g} / \mathrm{g}$ (Table B3-1). The RPD between these two phosphate results was 4.7 percent. The analytical phosphorus mean result as determined by ICP for the nonconvective layer was $778 \mu \mathrm{g} / \mathrm{g}$, which converts to $2386 \mu \mathrm{g} / \mathrm{g}$ of phosphate. The IC phosphate result was $2500 \mu \mathrm{g} / \mathrm{g}$. The RPD between these two phosphate results is 4.7 percent. A comparison was not made for results from the convective layer because the $\mathrm{PO}_{4}$ measurements were below detection limits.

Table B3-1. Phosphate versus Phosphorus.

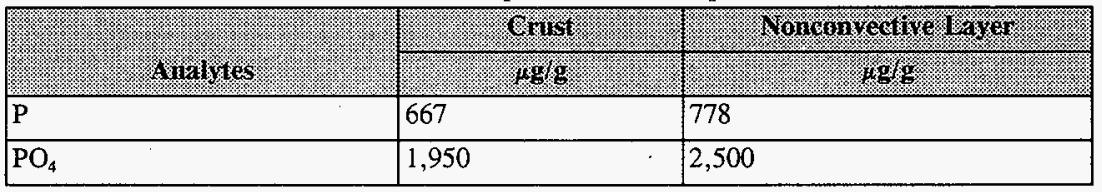




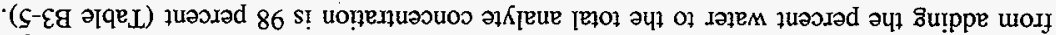

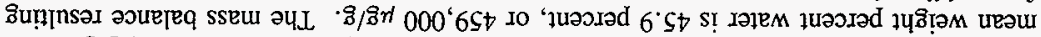
วЧ.L

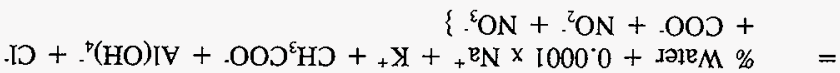

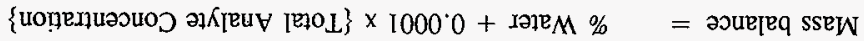

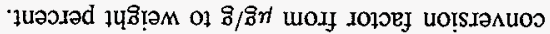

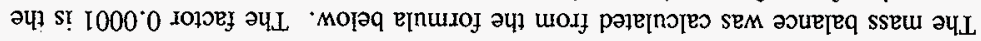

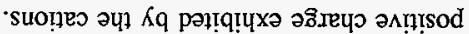

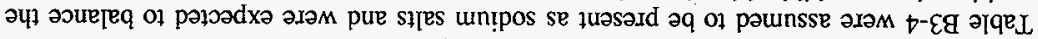

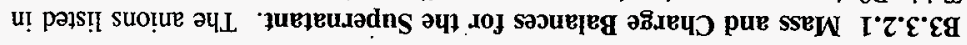

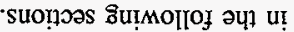

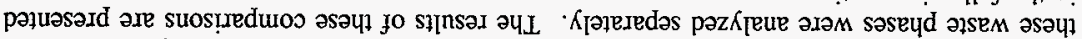

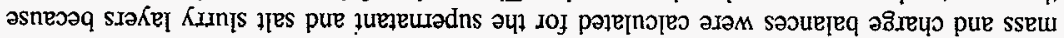

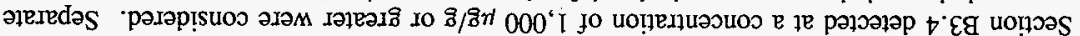

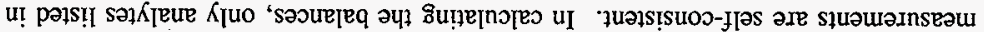

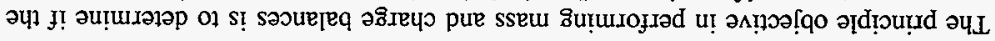

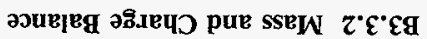

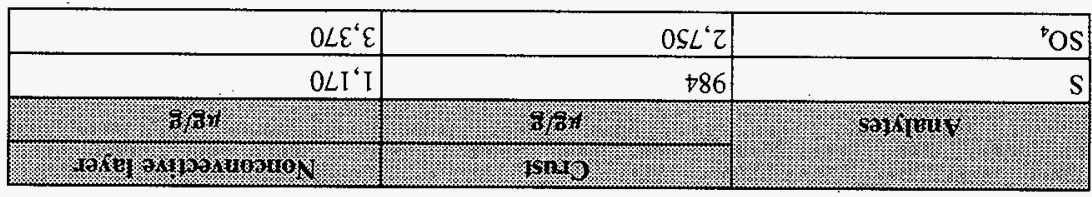

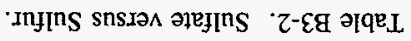

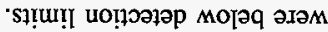

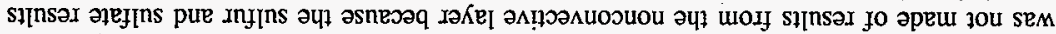

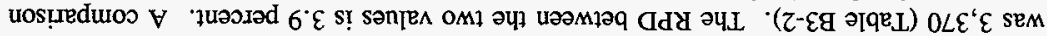

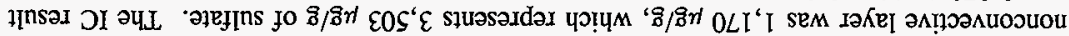

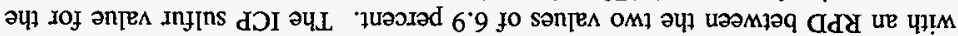

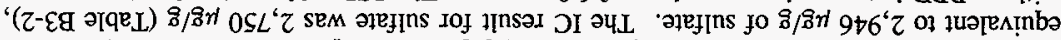

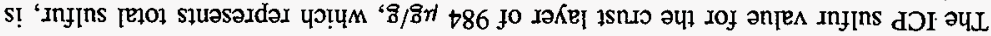


The following equations demonstrate the derivation of total cations and total anions; the charge balance is the ratio of these two values.

$$
\begin{aligned}
\text { Total cations }(\mu \mathrm{eq} / \mathrm{g})= & {\left[\mathrm{K}^{+}\right] / 39+\left[\mathrm{Na}^{+}\right] / 23.0=8,886 \mu \mathrm{eq} / \mathrm{g} } \\
\text { Total anions }(\mu \mathrm{eq} / \mathrm{g})= & {\left[\mathrm{Al}(\mathrm{OH})_{4} / 95\right]+\left[\mathrm{Cl}^{-}\right] / 35.5+\left[\mathrm{COO}^{-}\right] / 44.0+\left[\mathrm{OH}^{-}\right] / 17.0+} \\
& {\left[\mathrm{NO}_{2}^{-}\right] / 46.0+\left[\mathrm{NO}_{3}^{-}\right] / 62.0+\left[\mathrm{CH}_{3} \mathrm{COO}^{-}\right] / 59.0=5,334 \mu \mathrm{eq} / \mathrm{g} }
\end{aligned}
$$

The charge balance obtained by dividing the sum of the positive charge by the sum of the negative charge was 1.7 with a net positive charge of 3,552 microequivalents. Boundary conditions for this system are 1.00 for the charge balance with no net charge remaining. This result indicates some cations and/or anions have not been accounted for. Additional assumptions are needed regarding the species present. .

The simplest assumption is to set the net charge equal to zero. Assuming the free positive charge is balanced by sufficient hydroxide to make the waste neutral $\left(-3,552 \mu \mathrm{eq} / \mathrm{g} \mathrm{OH}^{*}\right)$, and additional $60,391 \mu \mathrm{g} / \mathrm{g}$ of hydroxide is added bringing the total mass to approximately $586,826 \mu \mathrm{g} / \mathrm{g}$ ( 104.6 percent). Table $3-5$ shows the balance totals. This assumption is reasonable given the history of the waste and the uncertainty in the measurements, and it provides sufficient charge to acceptably account for the individual analytes that comprise the solids.

With this assumption and the uncertainty regarding these measurements, the mass and charge

\begin{tabular}{|c|c|c|c|c|}
\hline 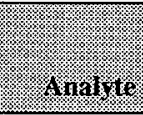 & 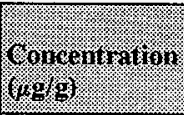 & obsumes & 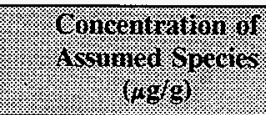 & \%ogs \\
\hline Potassium & 10,700 & $\mathrm{~K}^{+}$ & 10,700 & 274 \\
\hline Sodium & 198,000 & $\mathrm{Na}^{+}$ & 198,000 & 8609 \\
\hline \multicolumn{3}{|l|}{ Total } & 208,700 & 8,880 \\
\hline
\end{tabular}
balance calculations were considered to be consistent.

Table B3-3. Supernatant Cation Mass and Charge Data. 
HNF-SD-WM-ER-702 Rev. 0

Table B3-4. Supernatant Anion Mass and Charge Data.

\begin{tabular}{|c|c|c|c|c|}
\hline (linalste & 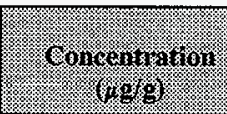 & (1) & 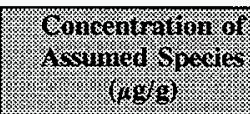 & 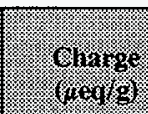 \\
\hline Aluminum & 25,100 & $\mathrm{Al}(\mathrm{OH})_{4}$ & 88,385 & 930 \\
\hline Chloride & 6,130 & $\mathrm{Cl}^{-}$ & 6,130 & 173 \\
\hline Formate & 1,860 & $\mathrm{COO}^{-}$ & 1,860 & 42 \\
\hline Acetate & 2,360 & $\mathrm{CH}_{3} \mathrm{COO}^{-}$ & 2,360 & 40 \\
\hline Nitrate & 109,000 & $\mathrm{NO}_{3}^{-}$ & 109,000 & 1,758 \\
\hline Nitrite & 110,000 & $\mathrm{NO}_{2}{ }^{-}$ & 110,000 & 2,391 \\
\hline \multicolumn{3}{|l|}{ Total } & 317,735 & 5,334 \\
\hline
\end{tabular}

Table B3-5. Mass Balance Totals.

\begin{tabular}{|c|c|c|}
\hline (l) & 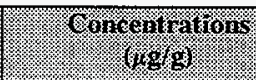 & (liarge \\
\hline Total from Table B3-3 & 208,700 & 8,880 \\
\hline Total from Table B3-4 & 317,735 & $-5,334$ \\
\hline Water percent & 459,000 & 0 \\
\hline Assumed $\mathrm{OH}^{-}$ & 60,400 & $-3,552$ \\
\hline Total & $1,045,835$ & 0 \\
\hline
\end{tabular}

B3.3.2.2 Mass and Charge Balances for the Nonconvective Layer. The positive charges attributed to sodium and potassium were expected to balance the negative charges exhibited by the anions. Sulfur was assumed to be present as sulfate. Phosphate, as determined by IC, is assumed to be completely water soluble and appears only in the anion mass and charge calculations. The concentrations of cationic species in Table B3-6, the anionic species in Table B3-7, and the weight percent water were ultimately used to calculate the mass balance.

The mass balance was calculated from the formula below. The factor 0.0001 is the conversion factor from $\mu \mathrm{g} / \mathrm{g}$ to weight percent.

Mass balance $=\%$ Water $+0.0001 \times\{$ Total Analyte Concentration $\}$

$$
\begin{aligned}
= & \% \text { Water }+0.0001 \times\left\{\mathrm{Al}(\mathrm{OH})_{4}{ }^{+}+\mathrm{Cr}(\mathrm{OH})_{3}+\mathrm{Na}^{+}+\mathrm{K}^{+}+\mathrm{Cl}^{-}\right. \\
& +\mathrm{COO}^{-}+(\mathrm{COO})_{2}{ }^{2-}+\mathrm{CH}_{3} \mathrm{COO}^{-}+\mathrm{F}^{-}+\mathrm{NO}_{2}{ }^{-}+\mathrm{NO}_{3}{ }^{-}+\mathrm{OH}^{-}+ \\
& \left.\mathrm{PO}_{4}{ }^{-3}+\mathrm{SO}_{4}{ }^{-2}\right\}
\end{aligned}
$$


$8 / \mathrm{bart} 0 \angle 0^{\circ} L=8 \nabla /\left[z_{z}^{\circ} \mathrm{OS}\right]+$

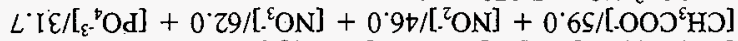

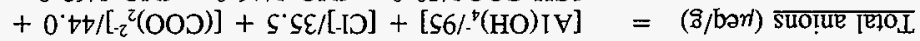

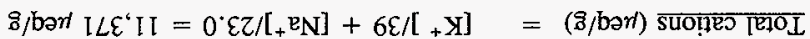

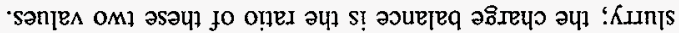

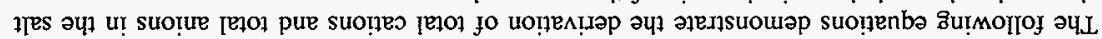

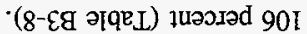

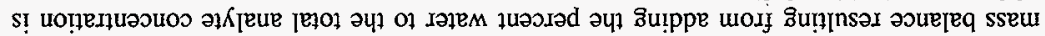

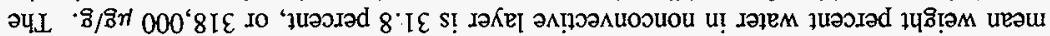

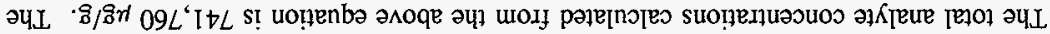

\begin{tabular}{|c|c|c|c|c|}
\hline $0<0^{6} \angle$ & $O S L^{\prime} \angle L t$ & & & [E]OL \\
\hline $0 L$ & $0<\varepsilon^{\prime} \varepsilon$ &.$^{7} \mathrm{OS}$ & $O L E^{\prime} \varepsilon$ & olefins \\
\hline $6 L$ & $00 s^{\prime} z$ & ${ }_{-\varepsilon}^{t} \mathrm{Od}$ & $00 s^{2} z$ & әңеudsoyd \\
\hline LII & $0 t I ' s$ & ${ }_{-2}^{z}($ OOD $)$ & $0 t I^{\prime} s$ & әтерх0 \\
\hline$t \angle t^{\circ} \mathrm{I}$ & $008^{\circ} \angle 9$ & ${ }^{2} \mathrm{ON}$ & $008^{\circ} \angle 9$ & วน!ฺ!N \\
\hline $8+0^{\circ} \varepsilon$ & $000^{\prime} 68 \mathrm{I}$ & ${ }^{E} \mathrm{ON}$ & $000^{\circ} 68 \mathrm{I}$ & วยอก!N \\
\hline $6 \mathrm{I}$ & OZII & $.00 \supset^{\varepsilon} \mathrm{H}$ & OZII & ә1Еाә0V \\
\hline $80 \mathrm{I}$ & $0 z 8^{\circ} \varepsilon$ & 10 & $0 z 8^{\circ} \mathcal{E}$ & วp!ro|นว \\
\hline SSIZ & $000^{\circ} \mathrm{s} 0 \mathrm{Z}$ &. $\mathrm{t}(\mathrm{HO}) \mathrm{IV}$ & $00 z^{\prime} 8 \subseteq$ & unutunniv \\
\hline (6ibain: & 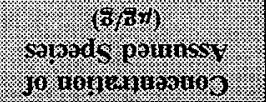 & pormatis & 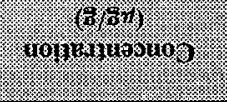 & ampint? \\
\hline
\end{tabular}

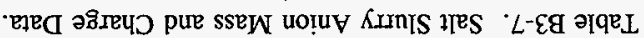

\begin{tabular}{|c|c|c|c|c|}
\hline I $\angle E^{\prime} I I$ & $010^{6}+9 z$ & & & [E]OL \\
\hline LIZ'YI & $000^{\prime} 8 \mathrm{sZ}$ & $+\mathrm{EN}$ & $000^{6} 852$ & un!̣pos \\
\hline$\nabla S I$ & $010^{6} 9$ & $+\mathrm{Y}$ & 0L10'9 & unisseto \\
\hline 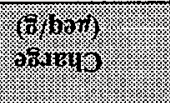 & 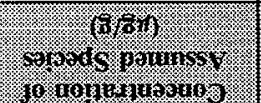 & primants: & 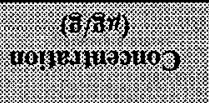 & aming: \\
\hline
\end{tabular}

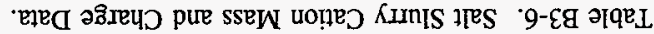




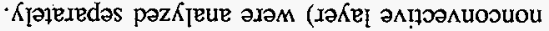

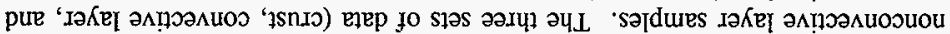

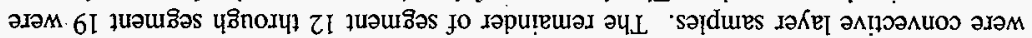

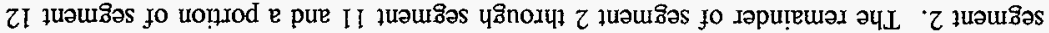

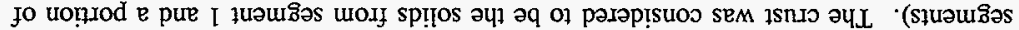

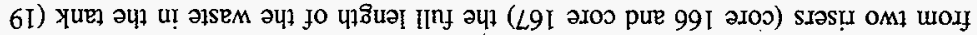

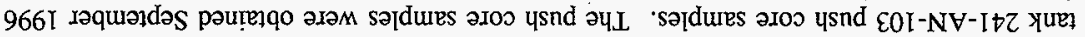

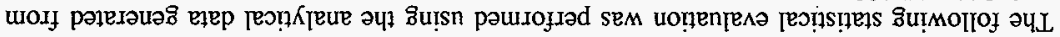

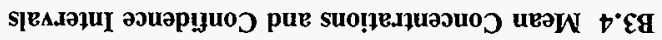

\begin{tabular}{|c|c|c|}
\hline 0 & LLI'ZEI'I I & [E]OL \\
\hline IOEt- & $L I T^{\prime} \varepsilon L$ & .HO pounss \\
\hline 0 & $000^{\prime} 8 I \varepsilon$ & quasiad IәtеM \\
\hline $0 \angle 0^{\circ} \angle-$ & $0 S 0^{\circ} \angle L D$ & 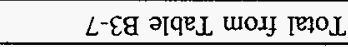 \\
\hline$I L E^{\prime} I I$ & $010^{6} \neq 9 z$ & 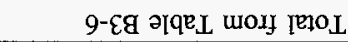 \\
\hline$(8,15)$ \% & 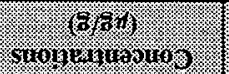 & 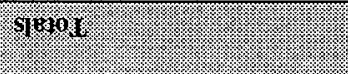 \\
\hline
\end{tabular}

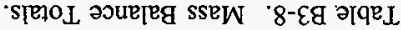

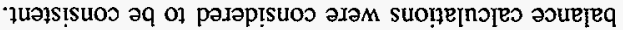

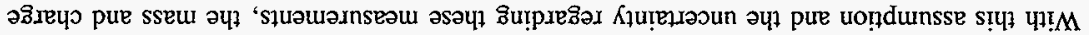

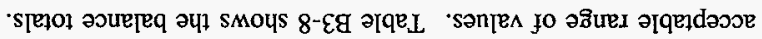

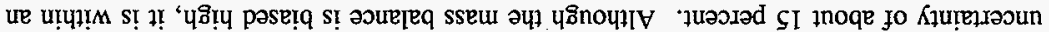

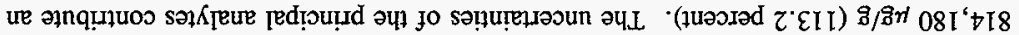
Кәㄹ

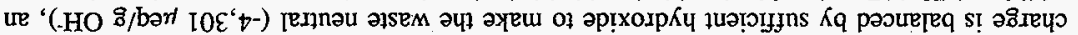

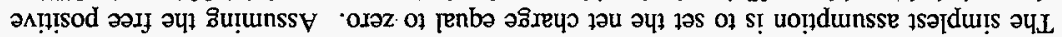

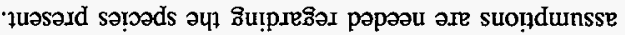

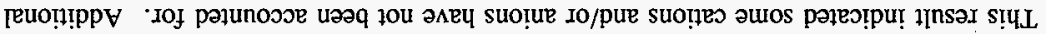

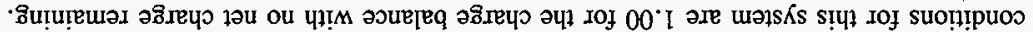

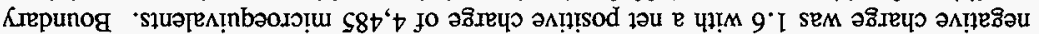

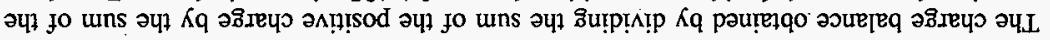


For each data set, a mean concentration and the associated variability. were calculated for each analyte. A two-sided 95 percent confidence interval for the mean concentration was also calculated for each analyte. The confidence interval takes into account the sampling and analytical uncertainties. The upper and lower limits (UL and LL) of a two-sided 95 percent confidence interval for the mean are

$$
\hat{\mu} \pm t_{(d f, 0.05)} \times \hat{\sigma}_{\hat{\mu}}
$$

In these equations, $\hat{\mu}$ is the estimate of the mean concentration, $\hat{\sigma}_{\hat{\mu}}$ is the estimate of the standard deviation of the mean concentration, and $\mathrm{t}_{\mathrm{dff}, 0.025)}$ is the quantile from Student's $\mathrm{t}$ distribution with $d f$ degrees of freedom for a two-sided 95 percent confidence interval. The mean, $\hat{\mu}$, and the standard deviation of the mean, $\hat{\sigma}_{\hat{\mu}}$, were estimated using restricted maximum likelihood estimation (REML) methods.

\section{B3.4.1 Mean Concentrations}

\section{B3.4.1.1 Convective Layer Data}

The statistics in this section were based on analytical data from the 1996 sampling event of tank 241-AN-103. Because of the length of time the convective layer (liquid) samples were stored before being extruded at the laboratory, solids precipitated. The laboratory analyzed both the liquid and solid materials. The following equation was used to provide the analytical concentration of the convective layer material before precipitation. The statistical analysis was performed using (1) the liquid data only and (2) the calculated convective layer data.

$$
\frac{\mu \mathrm{g}}{\mathrm{g}_{\mathrm{cv}}}=\frac{\frac{\mu \mathrm{g}}{\mathrm{mL}}}{\frac{\mathrm{g}}{\mathrm{mL}}} * \mathrm{wtfrac}_{\text {liquid }}+\frac{\mu \mathrm{g}}{\mathrm{g}} * \mathrm{wtfrac}_{\text {solids }}
$$

The 1996 data were statistically evaluated using two different models. The first model used a nested analysis of variance (ANOVA): the data are identified by segment within riser. The second model used one-way ANOVA: the data are identified by one variable (the sample). Analysis of variance techniques were used to estimate the mean and its associated variability for all analytes that had at least 50 percent of the reported data as quantitative values.

For those analytes which had a mixture of both quantitative values and "less than" values, the upper value of the "less than" (for example, 3.5 for $<3.5$ ) was used to represent all "less than" analytical values. This produces a bias of unknown magnitude in both the mean analyte concentration and the variance of the mean; the mean analyte concentration is biased high. The extension ".lt" was added to the analyte name in the tables to distinguish which analyte was statistically analyzed using "less than" values. 
No ANOVA estimates were computed for analytes that had less than 50 percent of the reported data as quantitative values.

The mean concentration estimates for the convective layer (liquid data only), along with the two-sided 95 percent confidence interval for the mean concentration, are given in Table B3-9 (one-way ANOVA) for those analytes with at least 50 percent of the reported data as quantitative values. No difference in the analyte means was observed between the one-way ANOVA and nested ANOVA models, except for $\mathrm{Br}$ and $\mathrm{SO}_{4}$ analytes. This indicates that there was little significant difference in the waste composition for the two risers sampled, and the tank is vertically homogeneous. As a result, except as noted in Tables B3-9 and B3-10, only the results for the one-way ANOVA analysis are presented. The mean concentration estimates for the convective layer (calculated data; liquids and solids), along with the two-sided 95 percent confidence interval for the mean concentration, are given in Table B3-10.

For some of the analytes, the lower limit of the 95 percent confidence interval was a negative value because of the magnitude of the variability. Because the actual concentration of a tank sample cannot be less than zero, the lower limit is reported as zero.

Table B3-9. Summary Statistics: Convective Layer - Liquid Data Only (one-way ANOVA). ${ }^{1}$ (2 sheets)

\begin{tabular}{|c|c|c|c|c|c|c|}
\hline $4104 \%$ & Bnin & H. & 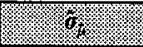 & 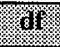 & 4 & 68 \\
\hline$\%$ Water.tga & wt \% & $4.90 \mathrm{E}+01$ & $1.46 \mathrm{E}-01$ & 16 & $4.86 \mathrm{E}+01$ & $4.93 \mathrm{E}+01$ \\
\hline Acetate.ic & $\mu \mathrm{g} / \mathrm{mL}$ & $3.87 E+03$ & $5.17 \mathrm{E}+02$ & 16 & $2.78 \mathrm{E}+03$ & $4.97 E+03$ \\
\hline Ag.icp.d & $\mu \mathrm{g} / \mathrm{mL}$ & $1.86 E+01$ & $4.65 \mathrm{E}-01$ & 16 & $1.76 \mathrm{E}+01$ & $1.96 \mathrm{E}+01$ \\
\hline Al.icp.d & $\mu \mathrm{g} / \mathrm{mL}$ & $3.15 E+04$ & $4.54 \mathrm{E}+02$ & 16 & $3.05 E+04$ & $3.25 E+04$ \\
\hline B.icp.d & $\mu \mathrm{g} / \mathrm{mL}$ & $6.43 E+01$ & $1.43 \mathrm{E}+00$ & 16 & $6.13 E+01$ & $6.74 \mathrm{E}+01$ \\
\hline $\mathrm{Br}$.ic. $\mathrm{lt}^{1}$ & $\mu \mathrm{g} / \mathrm{mL}$ & $1.20 \mathrm{E}+03$ & $3.66 \mathrm{E}+02$ & 1 & $0.00 E+00$ & $5.86 E+03$ \\
\hline Cd.icp.d.lt & $\mu \mathrm{g} / \mathrm{mL}$ & $4.39 E+00$ & 1.35E-01 & 16 & $4.10 \mathrm{E}+00$ & $4.67 E+00$ \\
\hline $\mathrm{Cl}^{-} . \mathrm{ic}$ & $\mu \mathrm{g} / \mathrm{mL}$ & $1.02 E+04$ & $4.40 \mathrm{E}+02$ & 16 & $9.26 \mathrm{E}+03$ & $1.11 \mathrm{E}+04$ \\
\hline Cr.icp.d & $\mu \mathrm{g} / \mathrm{mL}$ & $5.92 \mathrm{E}+02$ & $1.23 \mathrm{E}+01$ & 16 & $5.66 \mathrm{E}+02$ & $6.18 \mathrm{E}+02$ \\
\hline DSC.dry & $J / g$ dry & $5.84 \mathrm{E}+01$ & $1.86 \mathrm{E}+01$ & 16 & $1.90 \mathrm{E}+01$ & $9.78 E+01$ \\
\hline DSC.exo & $\mathrm{J} / \mathrm{g}$ wet & $2.97 \mathrm{E}+01$ & $9.47 E+00$ & 16 & $9.67 \mathrm{E}+00$ & $4.98 \mathrm{E}+01$ \\
\hline $\mathrm{F}^{*}$.ic & $\mu \mathrm{g} / \mathrm{mL}$ & $5.69 \mathrm{E}+02$ & $3.25 \mathrm{E}+01$ & 16 & $5.01 \mathrm{E}+02$ & $6.38 \mathrm{E}+02$ \\
\hline Formate.ic & $\mu \mathrm{g} / \mathrm{mL}$ & $3.18 \mathrm{E}+03$ & $6.35 \mathrm{E}+02$ & 16 & $1.84 \mathrm{E}+03$ & $4.53 E+03$ \\
\hline K.icp.d & $\mu \mathrm{g} / \mathrm{mL}$ & $1.76 \mathrm{E}+04$ & $2.71 E+02$ & 16 & 1.70E+04 & $1.81 \mathrm{E}+04$ \\
\hline Li.icp.d & $\mu \mathrm{g} / \mathrm{mL}$ & $2.26 \mathrm{E}+01$ & $3.10 \mathrm{E}+00$ & 16 & $1.60 \mathrm{E}+01$ & $2.92 \mathrm{E}+01$ \\
\hline
\end{tabular}


Table B3-9. Summary Statistics: Convective Layer - Liquid Data Only (one-way ANOVA). ${ }^{1}$ ( 2 sheets)

\begin{tabular}{|c|c|c|c|c|c|c|}
\hline $\operatorname{lng} 14 \%$ & 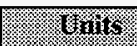 & $\pi$ & 0 & of & Ele? & 61 \\
\hline Mo.icp.d & $\mu \mathrm{g} / \mathrm{mL}$ & $1.15 \mathrm{E}+02$ & $1.29 \mathrm{E}+00$ & 16 & $1.12 \mathrm{E}+02$ & $1.18 \mathrm{E}+02$ \\
\hline $\mathrm{NO}_{2}^{-}$.ic & $\mu \mathrm{g} / \mathrm{mL}$ & $1.37 \mathrm{E}+05$ & $4.81 \mathrm{E}+03$ & 16 & $1.27 \mathrm{E}+05$ & $1.47 \mathrm{E}+05$ \\
\hline $\mathrm{NO}_{3} \cdot \mathrm{ic}$ & $\mu \mathrm{g} / \mathrm{mL}$ & $1.35 \mathrm{E}+05$ & $4.12 \mathrm{E}+03$ & 16 & $1.26 E+05$ & $1.44 \mathrm{E}+05$ \\
\hline Na.icp.d & $\mu \mathrm{g} / \mathrm{mL}$ & $2.56 \mathrm{E}+05$ & $3.79 \mathrm{E}+03$ & 16 & $2.48 \mathrm{E}+05$ & $2.64 \mathrm{E}+05$ \\
\hline P.icp.d & $\mu \mathrm{g} / \mathrm{mL}$ & $2.92 \mathrm{E}+02$ & $4.37 \mathrm{E}+00$ & 16 & $2.83 \mathrm{E}+02$ & $3.02 \mathrm{E}+02$ \\
\hline Pb.icp.d & $\mu \mathrm{g} / \mathrm{mL}$ & $1.43 \mathrm{E}+02$ & $2.33 \mathrm{E}+00$ & 16 & $1.38 \mathrm{E}+02$ & $1.48 \mathrm{E}+02$ \\
\hline S.icp.d & $\mu \mathrm{g} / \mathrm{mL}$ & $4.37 \mathrm{E}+02$ & $1.16 \mathrm{E}+01$ & 16 & $4.13 E+02$ & $4.62 \mathrm{E}+02$ \\
\hline $\mathrm{SO}_{4}{ }^{2-}$.ic. $1 \mathrm{tt}^{1}$ & $\mu \mathrm{g} / \mathrm{mL}$ & $1.33 \mathrm{E}+03$ & $5.63 \mathrm{E}+02$ & 16 & $0.00 \mathrm{E}+00$ & $8.49 E+03$ \\
\hline Si.icp.d & $\mu \mathrm{g} / \mathrm{mL}$ & $2.50 \mathrm{E}+02$ & $9.31 \mathrm{E}+00$ & 16 & $2.30 \mathrm{E}+02$ & $2.70 \mathrm{E}+02$ \\
\hline SpG & $\cdots$ & $1.46 \mathrm{E}+00$ & $7.59 \mathrm{E}-03$ & 16 & $1.44 \mathrm{E}+00$ & $1.47 \mathrm{E}+00$ \\
\hline Zn.icp.d.lt & $\mu \mathrm{g} / \mathrm{mL}$ & $9.95 \mathrm{E}+00$ & $1.02 \mathrm{E}+00$ & 16 & $7.79 \mathrm{E}+00$ & $1.21 \mathrm{E}+01$ \\
\hline Zr.icp.d.lt & $\mu \mathrm{g} / \mathrm{mL}$ & $7.61 \mathrm{E}+00$ & $4.37 \mathrm{E}-01$ & 16 & $6.69 \mathrm{E}+00$ & $8.54 \mathrm{E}+00$ \\
\hline
\end{tabular}

Note:

' $\mathrm{Br}$ and SO4 statistical means were calculated using the nested ANOVA model because variability was observed between the risers for these analytes.

Table B3-10. Summary Statistics: Convective Layer - Calculated.' (one-way ANOVA). (2 sheets)

\begin{tabular}{|c|c|c|c|c|c|c|}
\hline 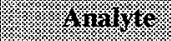 & Inits: & 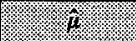 & 0 & If & Y. & WI. \\
\hline \% Water.tga & $\mu \mathrm{g} / \mathrm{g}$ & $4.59 \mathrm{E}+01$ & $4.41 \mathrm{E}-01$ & 17 & $4.49 \mathrm{E}+01$ & $4.68 \mathrm{E}+01$ \\
\hline Acetate.ic.lt & $\mu \mathrm{g} / \mathrm{g}$ & $2.36 \mathrm{E}+03$ & $3.13 \mathrm{E}+02$ & 17 & $1.70 \mathrm{E}+03$ & $3.02 \mathrm{E}+03$ \\
\hline Acetate.ic.nlt & $\mu \mathrm{g} / \mathrm{g}$ & $2.32 \mathrm{E}+03$ & $3.41 \mathrm{E}+02$ & 15 & $1.60 \mathrm{E}+03$ & $3.05 \mathrm{E}+03$ \\
\hline Al.icp.a & $\mu \mathrm{g} / \mathrm{g}$ & $2.49 E+04$ & $2.96 \mathrm{E}+03$ & 17 & $1.87 \mathrm{E}+04$ & $3.12 E+04$ \\
\hline Al.icp.f & $\mu \mathrm{g} / \mathrm{g}$ & $2.51 E+04$ & $3.32 \mathrm{E}+03$ & 17 & $1: 81 \mathrm{E}+04$ & $3.21 \mathrm{E}+04$ \\
\hline B.icp.a.lt & $\mu \mathrm{g} / \mathrm{g}$ & $6.26 \mathrm{E}+01$ & $6.06 \mathrm{E}+00$ & 17 & $4.98 \mathrm{E}+01$ & $7.54 \mathrm{E}+01$ \\
\hline B.icp.a.nlt & $\mu \mathrm{g} / \mathrm{g}$ & $6.29 \mathrm{E}+01$ & $6.11 \mathrm{E}+00$ & 17 & $5.00 \mathrm{E}+01$ & $7.58 \mathrm{E}+01$ \\
\hline $\mathrm{Cl}^{-}$.ic & $\mu \mathrm{g} / \mathrm{g}$ & $6.13 \mathrm{E}+03$ & $3.41 \mathrm{E}+02$ & 17 & $5.41 \mathrm{E}+03$ & $6.85 \mathrm{E}+03$ \\
\hline Cr.icp.a & $\mu \mathrm{g} / \mathrm{g}$ & $4.00 \mathrm{E}+02$ & $1.54 \mathrm{E}+01$ & 17 & $3.67 \mathrm{E}+02$ & $4.32 \mathrm{E}+02$ \\
\hline Cr.icp.f.1t & $\mu \mathrm{g} / \mathrm{g}$ & $4.33 \mathrm{E}+02$ & $2.94 \mathrm{E}+01$ & 17 & $3.71 \mathrm{E}+02$ & $4.95 \mathrm{E}+02$ \\
\hline Cr.icp.f.nlt & $\mu \mathrm{g} / \mathrm{g}$ & $4.64 \mathrm{E}+02$ & $4.74 E+01$ & 10 & $3.58 \mathrm{E}+02$ & $5.70 E+02$ \\
\hline
\end{tabular}


Table B3-10. Summary Statistics: Convective Layer - Calculated. ${ }^{1}$ (one-way ANOVA). (2 sheets)

\begin{tabular}{|c|c|c|c|c|c|c|}
\hline Inalite & 1) & 4 & (3) & If? & 111. & W! \\
\hline F-ic & $\mu \mathrm{g} / \mathrm{g}$ & $4.34 \mathrm{E}+02$ & $2.96 \mathrm{E}+01$ & 17 & $3.71 E+02$ & $4.96 \mathrm{E}+02$ \\
\hline Formate.ic.lt & $\mu \mathrm{g} / \mathrm{g}$ & $1.86 \mathrm{E}+03$ & $3.54 \mathrm{E}+02$ & 17 & $1.11 \mathrm{E}+03$ & $2.60 \mathrm{E}+03$ \\
\hline Formate.ic.nlt & $\mu \mathrm{g} / \mathrm{g}$ & $1.85 \mathrm{E}+03$ & $3.75 \mathrm{E}+02$ & 16 & $1.05 E+03$ & $2.64 \mathrm{E}+03$ \\
\hline K.icp.a & $\mu \mathrm{g} / \mathrm{g}$ & $1.07 \mathrm{E}+04$ & $3.97 \mathrm{E}+02$ & 17 & $9.90 \mathrm{E}+03$ & $1.16 \mathrm{E}+04$ \\
\hline $\mathrm{NO}_{2}$. ic & $\mu \mathrm{g} / \mathrm{g}$ & $1.10 \mathrm{E}+05$ & $5.85 \mathrm{E}+03$ & 17 & $9.76 \mathrm{E}+04$ & $1.22 \mathrm{E}+05$ \\
\hline $\mathrm{NO}_{3}^{-}$.ic & $\mu \mathrm{g} / \mathrm{g}$ & $1.09 \mathrm{E}+05$ & $4.76 \mathrm{E}+03$ & 17 & $9.90 \mathrm{E}+04$ & $1.19 \mathrm{E}+05$ \\
\hline Na.icp.a & $\mu \mathrm{g} / \mathrm{g}$ & $1.82 \mathrm{E}+05$ & $4.60 \mathrm{E}+03$ & 17 & $1.72 \mathrm{E}+05$ & $1.91 \mathrm{E}+05$ \\
\hline Na.icp.f & $\mu \mathrm{g} / \mathrm{g}$ & $1.98 \mathrm{E}+05$ & $5.63 \mathrm{E}+03$ & 17 & $1.86 \mathrm{E}+05$ & $2.10 \mathrm{E}+05$ \\
\hline P.icp.a & $\mu \mathrm{g} / \mathrm{g}$ & $3.83 \mathrm{E}+02$ & $1.85 \mathrm{E}+01$ & 17 & $3.44 \mathrm{E}+02$ & $4.23 \mathrm{E}+02$ \\
\hline Si.icp.a & $\mu \mathrm{g} / \mathrm{g}$ & $2.42 \mathrm{E}+02$ & $2.82 \mathrm{E}+01$ & 17 & $1.83 \mathrm{E}+02$ & $3.02 \mathrm{E}+02$ \\
\hline Zn.icp.a.lt & $\mu \mathrm{g} / \mathrm{g}$ & $9.19 \mathrm{E}+00$ & $9.65 \mathrm{E}-01$ & 17 & $7.16 E+00$ & $1.12 \mathrm{E}+01$ \\
\hline Zn.icp.a.nlt & $\mu \mathrm{g} / \mathrm{g}$ & $1.02 \mathrm{E}+01$ & $1.17 \mathrm{E}+00$ & 13 & $7.72 \mathrm{E}+00$ & $1.28 \mathrm{E}+01$ \\
\hline
\end{tabular}

Note:

' Weighted average of liquid and solid concentrations in the convective layer (see equation in Section B3.4.1)

A liquid composite sample was formed from liquid subsamples from Core 166 segments 3-12 (40 $\mathrm{mL}$ from segments $3,4,6,7,8,9,10,11$, and 12). The arithmetic mean, the associated variability, and a two-sided 95 percent confidence interval for the mean concentration were calculated for each analyte with at least 50 percent of the reported data as quantitative values. The confidence interval takes into account only the analytical uncertainty. The summary statistics are listed in Table B3-11 and Table B3-16. 
HNF-SD-WM-ER-702 Rev. 0

Table B3-11. Summary Statistics: Liquid Composite

(Core 166, segments 3-12).

\begin{tabular}{|c|c|c|c|c|c|c|}
\hline 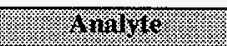 & Srits: & 18 & $\sqrt{1 \times 18}$ & or & II: & 10 \\
\hline$\%$ Water.tga & wt $\%$ & $4.85 \mathrm{E}+01$ & $5.00 \mathrm{E}-02$ & 1 & $4.78 \mathrm{E}+01$ & $4.91 \mathrm{E}+01$ \\
\hline Acetate.ic & $\mu \mathrm{g} / \mathrm{mL}$ & $2.87 \mathrm{E}+03$ & $5.00 \mathrm{E}+01$ & 1 & $2.23 \mathrm{E}+03$ & $3.51 \mathrm{E}+03$ \\
\hline Al.icp.d & $\mu \mathrm{g} / \mathrm{mL}$. & $3.21 \mathrm{E}+04$ & $1.00 \mathrm{E}+02$ & 1 & $3.08 \mathrm{E}+04$ & $3.34 \mathrm{E}+04$ \\
\hline $\mathrm{Br} . \mathrm{ic}$ & $\mu \mathrm{g} / \mathrm{mL}$ & $1.58 \mathrm{E}+03$ & $1.00 \mathrm{E}+01$ & 1 & $1.45 \mathrm{E}+03$ & $\longdiv { 1 . 7 1 \mathrm { E } + 0 3 }$ \\
\hline$\overline{\mathrm{Cl}^{-} . \mathrm{ic}}$ & $\mu \mathrm{g} / \mathrm{mL}$ & $9.89 \mathrm{E}+03$ & $1.15 \mathrm{E}+02$ & 1 & $8.42 \mathrm{E}+03$ & $1.13 \mathrm{E}+04$ \\
\hline Co.icp.d & $\mu \mathrm{g} / \mathrm{mL}$ & $6.09 \mathrm{E}+01$ & $5.75 \mathrm{E}+00$ & 1 & $0.00 \mathrm{E}+00$ & $1.34 \mathrm{E}+02$ \\
\hline $\mathrm{Cr}(\mathrm{VI})$ & $\mu \mathrm{g} / \mathrm{mL}$ & $1.12 \mathrm{E}+02$ & $1.50 \mathrm{E}+00$ & 1 & $9.24 \mathrm{E}+01$ & $1.31 \mathrm{E}+02$ \\
\hline Cr.icp.d & $\mu \mathrm{g} / \mathrm{mL}$ & $5.60 \mathrm{E}+02$ & $7.00 \mathrm{E}+00$ & 1 & $4.71 \mathrm{E}+02$ & $6.49 \mathrm{E}+02$ \\
\hline${ }^{137}$ Cs.gea & $\mu \mathrm{Ci} / \mathrm{mL}$ & $7.39 \mathrm{E}+02$ & $5.00 \mathrm{E}-01$ & 1 & $7.32 \mathrm{E}+02$ & $7.45 \mathrm{E}+02$ \\
\hline DSC.dry & $\mathrm{J} / \mathrm{g}$ dry & $2.01 \mathrm{E}+02$ & $3.00 \mathrm{E}+01$ & 1 & $0.00 \mathrm{E}+00$ & $5.82 \mathrm{E}+02$ \\
\hline DSC.exo & $\mathrm{J} / \mathrm{g}$ wet & $1.03 \mathrm{E}+02$ & $1.56 \mathrm{E}+01$ & 1 & $0.00 \mathrm{E}+00$ & $3.01 \mathrm{E}+02$ \\
\hline $\bar{F}$.ic & $\mu \mathrm{g} / \mathrm{mL}$ & $5.96 \mathrm{E}+02$ & $6.00 \mathrm{E}+00$ & 1 & $5.20 \mathrm{E}+02$ & $6.72 \mathrm{E}+02$ \\
\hline Formate.ic & $\mu \mathrm{g} / \mathrm{mL}$ & $1.63 \mathrm{E}+03$ & $1.20 \mathrm{E}+02$ & 1 & $1.05 \mathrm{E}+02$ & $3.15 \mathrm{E}+03$ \\
\hline${ }^{3} \mathrm{H}$ & $\mu \mathrm{Ci} / \mathrm{mL}$ & $1.57 \mathrm{E}-03$ & $7.83 \mathrm{E}-04$ & 1 & $0.00 \mathrm{E}+00$ & $1.15 \mathrm{E}-02$ \\
\hline${ }^{129} \mathrm{I}$ & $\mu \mathrm{Ci} / \mathrm{mL}$ & $3.79 E-04$ & $1.50 \mathrm{E}-05$ & 1 & $1.88 \mathrm{E}-04$ & $5.70 \mathrm{E}-04$ \\
\hline K.icp.d & $\mu \mathrm{g} / \mathrm{mL}$ & $1.73 \mathrm{E}+04$ & $5.00 \mathrm{E}+01$ & 1 & $1.66 \mathrm{E}+04$ & $1.79 \mathrm{E}+04$ \\
\hline Li.icp.d & $\mu \mathrm{g} / \mathrm{mL}$ & $1.52 \mathrm{E}+01$ & $1.50 \mathrm{E}-01$ & 1 & $1.32 \mathrm{E}+01$ & $1.71 \mathrm{E}+01$ \\
\hline Mo.icp.d & $\mu \mathrm{g} / \mathrm{mL}$ & $1.09 \mathrm{E}+02$ & $0.00 \mathrm{E}+00$ & 1 & $1.09 \mathrm{E}+02$ & $1.09 \mathrm{E}+02$ \\
\hline Na.icp.d & $\mu \mathrm{g} / \mathrm{mL}$ & $2.63 \mathrm{E}+05$ & $5.00 \mathrm{E}+02$ & 1 & $2.56 \mathrm{E}+05$ & $2.69 \mathrm{E}+05$ \\
\hline $\mathrm{NO}_{2}^{-}$. ic & $\mu \mathrm{g} / \mathrm{mL}$ & $1.33 \mathrm{E}+05$ & $1.50 \mathrm{E}+03$ & 1 & $1.13 \mathrm{E}+05$ & $1.52 \mathrm{E}+05$ \\
\hline $\mathrm{NO}_{3} \cdot \mathrm{ic}$ & $\mu \mathrm{g} / \mathrm{mL}$ & $1.17 \mathrm{E}+05$ & $5.00 \mathrm{E}+02$ & 1 & $1.10 \mathrm{E}+05$ & $1.23 \mathrm{E}+05$ \\
\hline $\mathrm{OH}^{-}$ & $\mu \mathrm{g} / \mathrm{mL}$ & $9.82 \mathrm{E}+04$ & $1.50 \mathrm{E}+03$ & 1 & $7.91 \mathrm{E}+04$ & $1.17 \mathrm{E}+05$ \\
\hline P.icp.d & $\mu \mathrm{g} / \mathrm{mL}$ & $2.74 \mathrm{E}+02$ & $8.00 \mathrm{E}+00$ & 1 & $1.72 \mathrm{E}+02$ & $3.76 \mathrm{E}+02$ \\
\hline Pb.icp.d.lt & $\mu \mathrm{g} / \mathrm{mL}$ & $1.38 \mathrm{E}+02$ & $1.80 \mathrm{E}+01$ & 1 & $0.00 \mathrm{E}+00$ & $3.67 \mathrm{E}+02$ \\
\hline S.icp.d & $\mu \mathrm{g} / \mathrm{mL}$ & $4.38 \mathrm{E}+02$ & $7.00 \mathrm{E}+00$ & 1 & $3.49 \mathrm{E}+02$ & $5.27 \mathrm{E}+02$ \\
\hline Se.icp.d.lt & $\mu \mathrm{g} / \mathrm{mL}$ & $1.23 \mathrm{E}+02$ & $3.00 \mathrm{E}+00$ & 1 & $8.49 \mathrm{E}+01$ & $1.61 \mathrm{E}+02$ \\
\hline Si.icp.d & $\mu \mathrm{g} / \mathrm{mL}$ & $1.91 \mathrm{E}+02$ & $5.00 \mathrm{E}-01$ & 1 & $1.84 \mathrm{E}+02$ & $1.97 \mathrm{E}+02$ \\
\hline SpG & --- & $1.49 \mathrm{E}+00$ & $2.00 \mathrm{E}-03$ & 1 & $1.46 \mathrm{E}+00$ & $1.51 \mathrm{E}+00$ \\
\hline${ }^{891 / 80} \mathrm{Sr}$ & $\mu \mathrm{Ci} / \mathrm{mL}$ & $2.08 \mathrm{E}-02$ & $0.00 \mathrm{E}+00$ & 1 & $2.08 \mathrm{E}-02$ & $2.08 \mathrm{E}-02$ \\
\hline${ }^{969} \mathrm{Tc}$ & $\mu \mathrm{Ci} / \mathrm{mL}$ & $1.68 \mathrm{E}-04$ & $1.15 \mathrm{E}-05$ & 1 & $2.14 \mathrm{E}-05$ & $3.14 \mathrm{E}-04$ \\
\hline TIC & $\mu \mathrm{g} / \mathrm{mL}$ & $1.16 \mathrm{E}+03$ & $5.00 \mathrm{E}+00$ & 1 & $1.09 \mathrm{E}+03$ & $1.22 \mathrm{E}+03$ \\
\hline TOC & $\mu \mathrm{g} / \mathrm{mL}$ & $3.05 \mathrm{E}+03$ & $2.50 \mathrm{E}+01$ & 1 & $2.73 \mathrm{E}+03$ & $3.36 \mathrm{E}+03$ \\
\hline Total Beta & $\mu \mathrm{Ci} / \mathrm{mL}$ & $6.22 \mathrm{E}+02$ & $4.00 \mathrm{E}+00$ & 1 & $5.71 E+02$ & $6.73 \mathrm{E}+02$ \\
\hline U.phos & $\mu \mathrm{g} / \mathrm{mL}$ & $1.84 \mathrm{E}+00$ & $1.55 \mathrm{E}-01$ & 1 & $0.00 \mathrm{E}+00$ & $3.80 \mathrm{E}+00$ \\
\hline
\end{tabular}




\section{B3.4.1.2 Nonconvective Layer (Salt Slurry)}

The mean concentration estimates for the nonconvective or slurry layer, along with the two-sided 95 percent confidence interval for the mean concentration, are given in Table B3-18 (nested ANOVA without the riser term) for those analytes with at least 50 percent of the reported data as quantitative values. For some of the analytes, the lower limit of the 95 percent confidence interval was a negative value due to the magnitude of the variability. Because the actual concentration of a tank sample cannot be less than zero, the lower limit is reported as zero.

Table B3-12. Summary Statistics: Nonconvective Layer (Slurry) (nested ANOVA without riser). (2 sheets)

\begin{tabular}{|c|c|c|c|c|c|c|}
\hline rnay ne & Gins: & 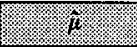 & 6 & (6) & 11: & (6) \\
\hline Acetate.ic.lt & $\mu \mathrm{g} / \mathrm{g}$ & $1.12 \mathrm{E}+03$ & $1.23 \mathrm{E}+02$ & 8 & $8.32 \mathrm{E}+02$ & $1.40 \mathrm{E}+03$ \\
\hline Al.icp.a & $\mu \mathrm{g} / \mathrm{g}$ & $5.92 \mathrm{E}+04$ & $9.92 \mathrm{E}+03$ & 8 & $3.64 \mathrm{E}+04$ & $8.21 E+04$ \\
\hline Al.icp.f & $\mu \mathrm{g} / \mathrm{g}$ & $5.82 \mathrm{E}+04$ & $1.16 \mathrm{E}+04$ & 8 & $3.14 \mathrm{E}+04$ & $8.50 \mathrm{E}+04$ \\
\hline B.icp.a.lt & $\mu \mathrm{g} / \mathrm{g}$ & $1.42 \mathrm{E}+02$ & $7.08 \mathrm{E}+00$ & 8 & $1.26 \mathrm{E}+02$ & $1.59 \mathrm{E}+02$ \\
\hline $\mathrm{Cl}^{-}$:ic & $\mu \mathrm{g} / \mathrm{g}$ & $3.82 \mathrm{E}+03$ & $3.03 \mathrm{E}+02$ & 8 & $3.12 \mathrm{E}+03$ & $4.52 \mathrm{E}+03$ \\
\hline Co.icp.a.lt & $\mu \mathrm{g} / \mathrm{g}$ & $4.87 \mathrm{E}+01$ & $5.42 \mathrm{E}+00$ & 8 & $3.62 \mathrm{E}+01$ & $6.12 \mathrm{E}+01$ \\
\hline Cr.icp.a & $\mu \mathrm{g} / \mathrm{g}$ & $5.08 \mathrm{E}+02$ & $4.22 \mathrm{E}+01$ & 8 & $4.10 \mathrm{E}+02$ & $6.05 E+02$ \\
\hline Cr.icp.f & $\mu \mathrm{g} / \mathrm{g}$ & $5.52 \mathrm{E}+02$ & $7.54 \mathrm{E}+01$ & 8 & $3.78 \mathrm{E}+02$ & $7.26 \mathrm{E}+02$ \\
\hline DSC.dry & $\mathrm{J} / \mathrm{g}$ dry & $2.70 \mathrm{E}+01$ & $6.93 \mathrm{E}+00$ & 8 & $1.10 \mathrm{E}+01$ & $4.30 \mathrm{E}+01$ \\
\hline DSC.exo & $\mathrm{J} / \mathrm{g}$ wet & $1.81 \mathrm{E}+01$ & $4.75 \mathrm{E}+00$ & 8 & $7.19 \mathrm{E}+00$ & $2.91 \mathrm{E}+01$ \\
\hline F.ic & $\mu \mathrm{g} / \mathrm{g}$ & $9.82 \mathrm{E}+02$ & $2.64 \mathrm{E}+02$ & 8 & $3.72 \mathrm{E}+02$ & $1.59 \mathrm{E}+03$ \\
\hline Formate.ic & $\mu \mathrm{g} / \mathrm{g}$ & $7.92 \mathrm{E}+02$ & $4.80 \mathrm{E}+01$ & 8 & $6.82 \mathrm{E}+02$ & $9.03 \mathrm{E}+02$ \\
\hline K.icp:a & $\mu \mathrm{g} / \mathrm{g}$ & $6.01 \mathrm{E}+03$ & $4.02 \mathrm{E}+02$ & 8 & $5.08 \mathrm{E}+03$ & $6.94 \mathrm{E}+03$ \\
\hline $\mathrm{NO}_{2}^{-} . \mathrm{ic}$ & $\mu \mathrm{g} / \mathrm{g}$ & $6.78 \mathrm{E}+04$ & $4.46 \mathrm{E}+03$ & 8 & $5.75 \mathrm{E}+04$ & $7.80 \mathrm{E}+04$ \\
\hline Na.icp.f & $\mu \mathrm{g} / \mathrm{g}$ & $2.58 \mathrm{E}+05$ & $2.70 \mathrm{E}+04$ & 8 & $1.95 \mathrm{E}+05$ & $3.20 \mathrm{E}+05$ \\
\hline Ni.icp.f & $\mu \mathrm{g} / \mathrm{g}$ & $1.50 \mathrm{E}+04$ & $6.27 \mathrm{E}+03$ & 8 & $5.07 \mathrm{E}+02$ & $2.94 \mathrm{E}+04$ \\
\hline Oxalate.ic & $\mu \mathrm{g} / \mathrm{g}$ & $5.14 \mathrm{E}+03$ & $6.44 \mathrm{E}+02$ & 8 & $3.65 \mathrm{E}+03$ & $6.62 \mathrm{E}+03$ \\
\hline P.icp.a.lt & $\mu \mathrm{g} / \mathrm{g}$ & $7.78 \mathrm{E}+02$ & $1.76 \mathrm{E}+02$ & 8 & $3.73 E+02$ & $1.18 \mathrm{E}+03$ \\
\hline P.icp.a.nlt & $\mu \mathrm{g} / \mathrm{g}$ & $7.97 \mathrm{E}+02$ & $1.79 \mathrm{E}+02$ & 8 & $3.84 \mathrm{E}+02$ & $1.21 \mathrm{E}+03$ \\
\hline $\mathrm{PO}_{4}{ }^{3-}$.ic.lt & $\mu \mathrm{g} / \mathrm{g}$ & $2.50 \mathrm{E}+03$ & $8.14 \mathrm{E}+02$ & 8 & $6.23 \mathrm{E}+02$ & $4.38 \mathrm{E}+03$ \\
\hline S.icp.a & $\mu \mathrm{g} / \mathrm{g}$ & $1.17 \mathrm{E}+03$ & $1.23 \mathrm{E}+02$ & 8 & $8.89 E+02$ & $1.46 \mathrm{E}+03$ \\
\hline $\mathrm{SO}_{4}{ }^{2-}$.ic.lt & $\mu \mathrm{g} / \mathrm{g}$ & $3.37 \mathrm{E}+03$ & $4.78 \mathrm{E}+02$ & 8 & $2.27 \mathrm{E}+03$ & $4.47 \mathrm{E}+03$ \\
\hline
\end{tabular}


Table B3-12. Summary Statistics: Nonconvective Layer (Slurry) (nested ANOVA without riser). (2 sheets)

\begin{tabular}{|c|c|c|c|c|c|c|}
\hline dimartit & Uiniss & i: & 6 & ar: & (3) & E1 \\
\hline Si.icp.a & $\mu \mathrm{g} / \mathrm{g}$ & $5.31 E+02$ & $4.07 \mathrm{E}+01$ & 8 & $4.37 \mathrm{E}+02$ & $6.25 \mathrm{E}+02$ \\
\hline Zn.icp.a.lt & $\mu \mathrm{g} / \mathrm{g}$ & $3.22 \mathrm{E}+01$ & $7.19 \mathrm{E}+00$ & 8 & $1.57 \mathrm{E}+01$ & $4.88 \mathrm{E}+01$ \\
\hline Bulk Density & $\mathrm{g} / \mathrm{mL}$ & $1.74 \mathrm{E}+00$ & $3.76 \mathrm{E}-02$ & 8 & $1.65 \mathrm{E}+00$ & $1.82 \mathrm{E}+00$ \\
\hline
\end{tabular}

A slurry composite sample was formed from slurry subsamples from Core 166 segments 1-19 (10 grams from segments $1 \mathrm{LH}, 4 \mathrm{LH}, 6 \mathrm{LH}, 7 \mathrm{LH}, 8 \mathrm{LH}, 9 \mathrm{LH}, 10 \mathrm{LH}, 11 \mathrm{LH}, 12 \mathrm{LH}$, $13 \mathrm{UH}, 13 \mathrm{LH}, 15 \mathrm{UH}, 15 \mathrm{LH}, 16 \mathrm{UH}, 16 \mathrm{LH}, 17 \mathrm{UH}, 17 \mathrm{LH}, 18 \mathrm{UH}, 18 \mathrm{LH}, 19 \mathrm{UH}$, and $19 \mathrm{LH})$. The arithmetic mean, the associated variability, and a two-sided 95 percent confidence interval for the mean concentration were calculated for each analyte with at least 50 percent of the reported data as quantitative values. The confidence interval takes into account only the analytical uncertainty. The summary statistics are listed in Tables B3-13 and B3-14.

Table B3-13. Summary Statistics: Slurry Composite (Core 166, segments 1 to 19 ). (2 sheets)

\begin{tabular}{|c|c|c|c|c|c|c|}
\hline minare & Ginis & $1 \%$ & (5) & (1) & 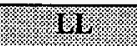 & 11. \\
\hline$\%$ Water.tga & wt\% & $3.30 \mathrm{E}+01$ & $4.00 \mathrm{E}-02$ & 1 & $3.25 \mathrm{E}+01$ & $3.35 \mathrm{E}+01$ \\
\hline Acetate.ic & $\mu \mathrm{g} / \mathrm{g}$ & $5.66 \mathrm{E}+02$ & $7.50 \mathrm{E}+00$ & 1 & $4.70 \mathrm{E}+02$ & $6.61 \mathrm{E}+02$ \\
\hline Ag.icp.a & $\mu \mathrm{g} / \mathrm{g}$ & $1.61 \mathrm{E}+01$ & $3.50 \mathrm{E}-01$ & 1 & $1.16 \mathrm{E}+01$ & $2.05 \mathrm{E}+01$ \\
\hline Al.icp.a & $\mu \mathrm{g} / \mathrm{g}$ & $3.82 \mathrm{E}+04$ & $1.10 \mathrm{E}+03$ & 1 & $0.00 \mathrm{E}+00$ & $5.22 \mathrm{E}+04$ \\
\hline${ }^{241} \mathrm{Am} \cdot \mathrm{aea}^{1}$ & $\mu \mathrm{Ci} / \mathrm{g}$ & $3.53 \mathrm{E}-03$ & $6.00 \mathrm{E}-05$ & 1 & $2.77 \mathrm{E}-03$ & $4.29 \mathrm{E}-03$ \\
\hline B.icp.a & $\mu \mathrm{g} / \mathrm{g}$ & $1.25 \mathrm{E}+02$ & $7.00 \mathrm{E}+00$ & 1 & $3.61 \mathrm{E}+01$ & $2.14 \mathrm{E}+02$ \\
\hline $\mathrm{Br}$.ic & $\mu \mathrm{g} / \mathrm{g}$ & $4.47 \mathrm{E}+02$ & $4.45 \mathrm{E}+01$ & 1 & $0.00 \mathrm{E}+00$ & $1.01 \mathrm{E}+03$ \\
\hline Bulk Density & $\mathrm{g} / \mathrm{mL}$ & 1.69 & NA & 1 & NA & NA \\
\hline Cd.icp.a & $\mu \mathrm{g} / \mathrm{g}$ & $4.17 \mathrm{E}+00$ & $2.20 \mathrm{E}-01$ & 1 & $1.37 \mathrm{E}+00$ & $6.97 \mathrm{E}+00$ \\
\hline $\mathrm{Cl}^{-}$.ic & $\mu \mathrm{g} / \mathrm{g}$ & $3.73 \mathrm{E}+03$ & $5.00 \mathrm{E}+01$ & 1 & $3.09 \mathrm{E}+03$ & $4.37 \mathrm{E}+03$ \\
\hline $\mathrm{Cr}(\mathrm{VI})$ & $\mu \mathrm{g} / \mathrm{g}$ & $9.97 \mathrm{E}+0 \mathrm{I}$ & $2.35 \mathrm{E}+00$ & 1 & $6.98 \mathrm{E}+01$ & $1.30 \mathrm{E}+02$ \\
\hline Cr.icp.a & $\mu \mathrm{g} / \mathrm{g}$ & $4.38 \mathrm{E}+02$ & $1.50 \mathrm{E}+01$ & 1 & $2.47 \mathrm{E}+02$ & $6.29 \mathrm{E}+02$ \\
\hline${ }^{137}$ Cs.gea & $\mu \mathrm{Ci} / \mathrm{g}$ & $2.74 \mathrm{E}+02$ & $2.30 \mathrm{E}+01$ & 1 & $0.00 \mathrm{E}+00$ & $5.66 \mathrm{E}+02$ \\
\hline DSC.dry & $J / g$ dry & $2.70 \mathrm{E}+01$ & $1.16 \mathrm{E}+01$ & 1 & $0.00 \mathrm{E}+00$ & $1.74 \mathrm{E}+02$ \\
\hline DSC.exo & $\mathrm{J} / \mathrm{g}$ wet & $1.81 \mathrm{E}+01$ & $7.75 \mathrm{E}+00$ & 1 & $0.00 \mathrm{E}+00$ & $1.17 \mathrm{E}+02$ \\
\hline
\end{tabular}


Table B3-13. Summary Statistics: Slurry Composite (Core 166, segments 1 to 19). (2 sheets)

\begin{tabular}{|c|c|c|c|c|c|c|}
\hline Wratgro & ryou & 16 & 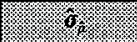 & 6 & 1.1. & 将: \\
\hline F-ic & $\mu \mathrm{g} / \mathrm{g}$ & $4.14 \mathrm{E}+03$ & $2.15 E+02$ & 1 & $1.40 \mathrm{E}+03$ & $6.87 E+03$ \\
\hline Fe.icp.a & $\mu \mathrm{g} / \mathrm{g}$ & $4.85 E+01$ & $1.00 \mathrm{E}-01$ & 1 & $4.72 \mathrm{E}+01$ & $4.98 \mathrm{E}+01$ \\
\hline Formate.ic & $\mu \mathrm{g} / \mathrm{g}$ & $1.29 \mathrm{E}+03$ & $1.00 \mathrm{E}+01$ & 1 & $1.16 \mathrm{E}+03$ & $1.42 \mathrm{E}+03$ \\
\hline${ }^{3} \mathrm{H}$ & $\mu \mathrm{Ci} / \mathrm{g}$ & $2.84 \mathrm{E}-03$ & $3.70 \mathrm{E}-04$ & 1 & $0.00 \mathrm{E}+00$ & $7.54 \mathrm{E}-03$ \\
\hline${ }^{129} \mathrm{I}^{1}$ & $\mu \mathrm{Ci} / \mathrm{g}$ & $2.70 \mathrm{E}-03$ & $7.00 \mathrm{E}-04$ & 1 & $0.00 \mathrm{E}+00$ & $1.16 \mathrm{E}-02$ \\
\hline K.icp.a & $\mu \mathrm{g} / \mathrm{g}$ & $6.54 \mathrm{E}+03$ & $2.60 \mathrm{E}+02$ & 1 & $0.00 \mathrm{E}+00$ & $9.84 \mathrm{E}+03$ \\
\hline Li.icp.a & $\mu \mathrm{g} / \mathrm{g}$ & $2.43 \mathrm{E}+01$ & $1.70 \mathrm{E}+00$ & 1 & $2.70 \mathrm{E}+00$ & $4.59 \mathrm{E}+01$ \\
\hline Mn.icp. $\mathrm{a}^{1}$ & $\mu \mathrm{g} / \mathrm{g}$ & $5.57 E+00$ & $6.00 \mathrm{E}-02$ & 1 & $4.81 \mathrm{E}+00$ & $6.33 E+00$ \\
\hline Mo.icp.a & $\mu \mathrm{g} / \mathrm{g}$ & $4.27 \mathrm{E}+01$ & $6.50 \mathrm{E}-01$ & 1 & $3.44 E+01$ & $5.09 \mathrm{E}+01$ \\
\hline Na.icp.a & $\mu \mathrm{g} / \mathrm{g}$ & $2.17 \mathrm{E}+05$ & $3.50 \mathrm{E}+03$ & 1 & $1.72 \mathrm{E}+05$ & $2.61 E+05$ \\
\hline $\mathrm{NO}_{2}^{-}$.ic & $\mu \mathrm{g} / \mathrm{g}$ & $7.34 \mathrm{E}+04$ & $3.50 \mathrm{E}+02$ & 1 & $6.89 \mathrm{E}+04$ & $7.78 \mathrm{E}+04$ \\
\hline $\mathrm{NO}_{3}^{-} \cdot \mathrm{ic}$ & $\mu \mathrm{g} / \mathrm{g}$ & $1.32 \mathrm{E}+05$ & $6.00 \mathrm{E}+03$ & 1 & $5.58 \mathrm{E}+04$ & $2.08 \mathrm{E}+05$ \\
\hline $\mathrm{OH}^{-}$ & $\mu \mathrm{g} / \mathrm{g}$ & $3.29 E+04$ & $3.10 \mathrm{E}+03$ & 1 & $0.00 \mathrm{E}+00$ & $7.23 \mathrm{E}+04$ \\
\hline Oxalate.ic & $\mu \mathrm{g} / \mathrm{g}$ & $1.04 E+04$ & $8.15 E+02$ & 1 & $2.94 \mathrm{E}+01$ & $2.07 \mathrm{E}+04$ \\
\hline P.icp.a & $\mu \mathrm{g} / \mathrm{g}$ & $1.15 \mathrm{E}+03$ & $3.50 \mathrm{E}+01$ & 1 & $7.00 \mathrm{E}+02$ & $1.59 E+03$ \\
\hline $\mathrm{PO}_{4}^{3-}$.ic & $\mu \mathrm{g} / \mathrm{g}$ & $3.48 \mathrm{E}+03$ & $4.00 \mathrm{E}+01$ & 1 & $2.97 \mathrm{E}+03$ & $3.99 E+03$ \\
\hline S.icp.a & $\mu \mathrm{g} / \mathrm{g}$ & $8.28 \mathrm{E}+02$ & $1.90 \mathrm{E}+01$ & 1 & $0.00 \mathrm{E}+00$ & $1.07 \mathrm{E}+03$ \\
\hline Si.icp.a & $\mu \mathrm{g} / \mathrm{g}$ & $8.38 \mathrm{E}+02$ & $1.00 \mathrm{E}+00$ & 1 & $8.25 E+02$ & $8.51 E+02$ \\
\hline $\mathrm{SO}_{4}{ }^{2-}$.ic & $\mu \mathrm{g} / \mathrm{g}$ & $5.53 \mathrm{E}+03$ & $1.35 \mathrm{E}+02$ & 1 & $3.81 \mathrm{E}+03$ & $7.24 \mathrm{E}+03$ \\
\hline${ }^{89 / 90} \mathrm{Sr}$ & $\mu \mathrm{Ci} / \mathrm{g}$ & $2.66 \mathrm{E}+00$ & $8.00 \mathrm{E}-02$ & 1 & $1.64 \mathrm{E}+00$ & $3.68 \mathrm{E}+00$ \\
\hline${ }^{99} \mathrm{Tc}$ & $\mu \mathrm{Ci} / \mathrm{g}$ & $1.45 \mathrm{E}-01$ & $1.15 \mathrm{E}-02$ & 1 & $0.00 \mathrm{E}+00$ & 2.91E-01 \\
\hline TIC & $\mu \mathrm{g} / \mathrm{g}$ & $5.77 \mathrm{E}+03$ & $1.30 \mathrm{E}+02$ & 1 & $4.12 \mathrm{E}+03$ & $7.42 \mathrm{E}+03$ \\
\hline TOC & $\mu \mathrm{g} / \mathrm{g}$ & $2.44 E+03$ & $3.80 \mathrm{E}+02$ & 1 & $0.00 \mathrm{E}+00$ & $7.27 \mathrm{E}+03$ \\
\hline Total Beta & $\mu \mathrm{Ci} / \mathrm{g}$ & $2.96 \mathrm{E}+01$ & $2.35 \mathrm{E}+00$ & 1 & $0.00 \mathrm{E}+00$ & $5.94 \mathrm{E}+01$ \\
\hline U.phos & $\mu \mathrm{g} / \mathrm{g}$ & $4.51 \mathrm{E}+01$ & $1.40 \mathrm{E}+00$ & 1 & $2.73 \mathrm{E}+01$ & $6.29 \mathrm{E}+01$ \\
\hline
\end{tabular}

Note:

"One of the measurements is a "less than" value. 


\section{B3.4.1.3 Crust Layer}

The mean concentration estimates for the crust layer, along with the two-sided 95 percent confidence interval for the mean concentration, are given in Table B3-14 (nested ANOVA without the riser term) for those analytes with at least 50 percent of the reported data as quantitative values. For the analytes, the lower limit of the 95 percent confidence interval was a negative value due to the magnitude of the variability. Because the actual concentration of a tank sample cannot be less than zero, the lower limit is reported as zero.

Table B3-14. Summary Statistics: Crust Layer (nested ANOVA without the riser term). (2 sheets)

\begin{tabular}{|c|c|c|c|c|c|c|}
\hline Ninglye & UWIs & 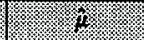 & 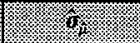 & 61 & W1: & 6 \\
\hline \% Water.tga & wt\% & $3.51 \mathrm{E}+01$ & $1.81 \mathrm{E}+00$ & 2 & $2.73 E+01$ & $4.29 \mathrm{E}+01$ \\
\hline Acetate.ic & $\mu \mathrm{g} / \mathrm{g}$ & $1.09 \mathrm{E}+03$ & $1.49 \mathrm{E}+02$ & 2 & $4.43 E+02$ & $1.73 \mathrm{E}+03$ \\
\hline Al.icp.a & $\mu \mathrm{g} / \mathrm{g}$ & $7.25 \mathrm{E}+04$ & $7.94 \mathrm{E}+03$ & 2 & $3.83 \mathrm{E}+04$ & $1.07 \mathrm{E}+05$ \\
\hline Al.icp.f & $\mu \mathrm{g} / \mathrm{g}$ & $8.54 \mathrm{E}+04$ & $8.06 \mathrm{E}+03$ & 2 & $5.07 \mathrm{E}+04$ & $1.20 \mathrm{E}+05$ \\
\hline B.icp.a.lt & $\mu \mathrm{g} / \mathrm{g}$ & $1.21 \mathrm{E}+02$ & $9.28 \mathrm{E}+00$ & 2 & $8.11 \mathrm{E}+01$ & $1.61 \mathrm{E}+02$ \\
\hline B.icp.a.nlt & $\mu \mathrm{g} / \mathrm{g}$ & $1.29 \mathrm{E}+02$ & $7.77 \mathrm{E}+00$ & 2 & $9.53 \mathrm{E}+01$ & $1.62 \mathrm{E}+02$ \\
\hline Bulk Density & $\mathrm{g} / \mathrm{mL}$ & $1.65 \mathrm{E}+00$ & $5.50 \mathrm{E}-02$ & 1 & $9.48 \mathrm{E}-01$ & $2.35 \mathrm{E}+00$ \\
\hline Cd.icp.a.lt & $\mu \mathrm{g} / \mathrm{g}$ & $1.11 \mathrm{E}+01$ & $1.74 \mathrm{E}+00$ & 2 & $3.61 \mathrm{E}+00$ & $1.85 \mathrm{E}+01$ \\
\hline $\mathrm{Cl}^{-}$.ic & $\mu \mathrm{g} / \mathrm{g}$ & $3.36 \mathrm{E}+03$ & $2.46 \mathrm{E}+02$ & 2 & $2.31 \mathrm{E}+03$ & $4.42 \mathrm{E}+03$ \\
\hline Co.icp.a.lt & $\mu \mathrm{g} / \mathrm{g}$ & $5.47 \mathrm{E}+01$ & $3.85 \mathrm{E}+00$ & 2 & $3.81 \mathrm{E}+01$ & $7.12 \mathrm{E}+01$ \\
\hline Co.icp.a.nlt & $\mu \mathrm{g} / \mathrm{g}$ & $5.64 \mathrm{E}+01$ & $2.98 \mathrm{E}+00$ & 2 & $4.35 \mathrm{E}+01$ & $6.92 \mathrm{E}+01$ \\
\hline Cr.icp.a & $\mu \mathrm{g} / \mathrm{g}$ & $6.84 \mathrm{E}+02$ & $7.40 \mathrm{E}+01$ & 2 & $3.66 \mathrm{E}+02$ & $1.00 \mathrm{E}+03$ \\
\hline Cr.icp.f & $\mu \mathrm{g} / \mathrm{g}$ & $8.90 \mathrm{E}+02$ & $7.38 \mathrm{E}+01$ & 2 & $5.73 \mathrm{E}+02$ & $1.21 \mathrm{E}+03$ \\
\hline Cr.icp.f.lt & $\mu \mathrm{g} / \mathrm{g}$ & $8.90 \mathrm{E}+02$ & $7.38 \mathrm{E}+01$ & 2 & $5.73 \mathrm{E}+02$ & $1.21 \mathrm{E}+03$ \\
\hline Cr.icp.f.nlt & $\mu \mathrm{g} / \mathrm{g}$ & $8.94 \mathrm{E}+02$ & $6.77 \mathrm{E}+01$ & 2 & $6.03 E+02$ & $1.19 \mathrm{E}+03$ \\
\hline DSC.dry & $\mathrm{J} / \mathrm{g}$ dry & $1.13 \mathrm{E}+02$ & $7.67 \mathrm{E}+01$ & 2 & $0.00 \mathrm{E}+00$ & $4.43 \mathrm{E}+02$ \\
\hline DSC.exo & J/g wet & $7.04 \mathrm{E}+01$ & $4.66 \mathrm{E}+01$ & 2 & $0.00 \mathrm{E}+00$ & $2.71 \mathrm{E}+02$ \\
\hline F.ic & $\mu \mathrm{g} / \mathrm{g}$ & $7.09 \mathrm{E}+02$ & $1.48 \mathrm{E}+02$ & 2 & $7.25 \mathrm{E}+01$ & $1.35 \mathrm{E}+03$ \\
\hline Formate.ic & $\dot{\mu g} / \mathrm{g}$ & $8.01 \mathrm{E}+02$ & $4.05 \mathrm{E}+01$ & 2 & $6.27 \mathrm{E}+02$ & $9.75 \mathrm{E}+02$ \\
\hline K.icp.a & $\mu \mathrm{g} / \mathrm{g}$ & $6.15 \mathrm{E}+03$ & $2.54 \mathrm{E}+02$ & 2 & $5.06 \mathrm{E}+03$ & $7.25 \mathrm{E}+03$ \\
\hline $\mathrm{NO}_{2} \cdot$.ic & $\mu \mathrm{g} / \mathrm{g}$ & $7.13 \mathrm{E}+04$ & $1.09 \mathrm{E}+04$ & 2 & $2.42 \mathrm{E}+04$ & $1.18 \mathrm{E}+05$ \\
\hline $\mathrm{NO}_{3} \cdot$. ic & $\mu \mathrm{g} / \mathrm{g}$ & $1.45 \mathrm{E}+05$ & $1.65 \mathrm{E}+04$ & 2 & $7.44 \mathrm{E}+04$ & $2.16 \mathrm{E}+05$ \\
\hline Na.icp.a & $\mu \mathrm{g} / \mathrm{g}$ & $2.08 \mathrm{E}+05$ & $1.14 \mathrm{E}+04$ & 2 & $1.58 \mathrm{E}+05$ & $2.57 \mathrm{E}+05$ \\
\hline
\end{tabular}


Table B3-14. Summary Statistics: Crust Layer (nested ANOVA without the riser term). (2 sheets)

\begin{tabular}{|c|c|c|c|c|c|c|}
\hline 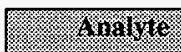 & Writits & ix & . & 6 & III. & (1) \\
\hline Na.icp.f & $\mu \mathrm{g} / \mathrm{g}$ & $2.69 \mathrm{E}+05$ & $1.77 \mathrm{E}+04$ & 2 & $1.93 \mathrm{E}+05$ & $3.45 \mathrm{E}+05$ \\
\hline Ni.icp.f & $\mu \mathrm{g} / \mathrm{g}$ & $1.25 \mathrm{E}+03$ & $4.15 \mathrm{E}+02$ & 2 & $0.00 \mathrm{E}+00$ & $3.03 \mathrm{E}+03$ \\
\hline Oxalate.ic & $\mu \mathrm{g} / \mathrm{g}$ & $7.84 \mathrm{E}+03$ & 1.10E+03 & 2 & $3.11 \mathrm{E}+03$ & $1.26 \mathrm{E}+04$ \\
\hline P.icp.a & $\mu \mathrm{g} / \mathrm{g}$ & $6.67 \mathrm{E}+02$ & $1.37 \mathrm{E}+02$ & 2 & $7.78 \mathrm{E}+01$ & $1.26 \mathrm{E}+03$ \\
\hline $\mathrm{PO}_{4}^{33}$.ic.lt & $\mu \mathrm{g} / \mathrm{g}$ & $1.95 \mathrm{E}+03$ & $3.72 \mathrm{E}+02$ & 2 & $3.43 \mathrm{E}+02$ & $3.55 \mathrm{E}+03$ \\
\hline $\mathrm{PO}_{4}{ }^{3-}$.ic.nit & $\mu \mathrm{g} / \mathrm{g}$ & $2.56 \mathrm{E}+03$ & $1.34 \mathrm{E}+02$ & 1 & $8.52 \mathrm{E}+02$ & $4.26 \mathrm{E}+03$ \\
\hline S.icp.a.lt & $\mu \mathrm{g} / \mathrm{g}$ & $9.84 \mathrm{E}+02$ & $7.82 \mathrm{E}+01$ & 2 & $6.48 \mathrm{E}+02$ & $1.32 \mathrm{E}+03$ \\
\hline S.icp.a.nlt & $\mu \mathrm{g} / \mathrm{g}$ & $9.59 \mathrm{E}+02$ & $6.75 \mathrm{E}+01$ & 2 & $6.68 \mathrm{E}+02$ & $1.25 \mathrm{E}+03$ \\
\hline $\mathrm{SO}_{4}^{2-}$.ic.lt & $\mu \mathrm{Ci} / \mathrm{g}$ & $2.75 \mathrm{E}+03$ & $6.28 \mathrm{E}+02$ & 2 & $5.09 \mathrm{E}+01$ & $5.46 \mathrm{E}+03$ \\
\hline $\mathrm{SO}_{4}{ }^{2-}$.ic.nlt & $\mu \mathrm{Ci} / \mathrm{g}$ & $3.06 \mathrm{E}+03$ & $7.77 \mathrm{E}+02$ & 2 & $0.00 \mathrm{E}+00$ & $6.40 \mathrm{E}+03$ \\
\hline Si.icp.a & $\mu \mathrm{g} / \mathrm{g}$ & $6.05 \mathrm{E}+02$ & $4.29 \mathrm{E}+01$ & 2 & $4.20 \mathrm{E}+02$ & $7.89 \mathrm{E}+02$ \\
\hline Si.icp.f.nlt & $\mu \mathrm{g} / \mathrm{g}$ & $1.29 \mathrm{E}+03$ & $1.21 \mathrm{E}+02$ & 2 & $7.67 \mathrm{E}+02$ & $1.81 E+03$ \\
\hline Zn.icp.a.It & $\mu \mathrm{g} / \mathrm{g}$ & $4.10 \mathrm{E}+01$ & $1.73 \mathrm{E}+01$ & 2 & $0.00 \mathrm{E}+00$ & $1.15 \mathrm{E}+02$ \\
\hline Zn.icp.a.nlt & $\mu \mathrm{g} / \mathrm{g}$ & $4.20 \mathrm{E}+01$ & $1.70 \mathrm{E}+01$ & 2 & $0.00 \mathrm{E}+00$ & $1.15 \mathrm{E}+02$ \\
\hline
\end{tabular}

\section{B3.4.2 Analysis of Variance Model}

A statistical model is needed to account for the spatial and measurement variability in $\hat{\sigma}_{\hat{\mu}}$. This cannot be done using an ordinary standard deviation of the data (Snedecor and Cochran 1980).

\section{B3.4.2.1 Convective Layer}

The data were statistically evaluated using two different models. The first model used a nested analysis of variance. The nested analysis of variance statistical model used to describe the structure of the data is

where

$$
\begin{gathered}
Y_{i j k}=\mu+R_{i}+S_{i j}+A_{i j k}, \\
i=1,2, \ldots, a ; j=1,2, \ldots, b_{i} ; k=1,2, \ldots, n_{i j} ;
\end{gathered}
$$

$$
Y_{i j k}=\begin{aligned}
& \text { concentration from the } k^{\text {th }} \text { analytical result from the } j^{\text {th }} \text { segment } \\
& \text { from the } i^{\text {th }} \text { riser }
\end{aligned}
$$




$\begin{array}{ll}\mu & =\text { the grand mean } \\ \mathrm{R}_{\mathrm{i}} & =\text { the effect of the } \mathrm{i}^{\text {th }} \text { riser } \\ \mathrm{S}_{\mathrm{ij}} & =\quad \text { the effect of the } \mathrm{j}^{\text {th }} \text { segment from the } \mathrm{i}^{\text {th }} \text { riser } \\ \mathrm{A}_{\mathrm{ijk}} & =\quad \text { the effect of the } k^{\text {th }} \text { analytical result from the } \mathrm{j}^{\text {th }} \text { segment from } \\ \mathrm{a} & =\quad \text { the number of risers } \\ b_{\mathrm{i}} & =\quad \text { the number of segments from the } \mathrm{i}^{\text {th }} \text { riser } \\ \mathrm{n}_{\mathrm{ij}} & \mathrm{i}^{\mathrm{th}} \text { riser. }\end{array}$

The variabies $R_{i}$ and $S_{i j}$ are assumed to be a random effects. These variables, as well as $A_{i j k}$, are assumed to be uncorrelated and normally distributed with means zero and variances $\sigma^{2}(\mathrm{R}), \sigma^{2}(\mathrm{~S})$, and $\sigma^{2}(\mathrm{~A})$, respectively. Estimates of $\sigma^{2}(\mathrm{R}), \sigma^{2}(\mathrm{~S})$, and $\sigma^{2}(\mathrm{~A})$ were obtained using Restricted Maximum Likelihood Estimation (REML) techniques. This method applied to variance component estimation is described in Harville (1977). . The results using the REML techniques were obtained using the statistical analysis package S-PLUS (Statistical Sciences, 1993). The $d f$ associated with the standard deviation of the mean (a function of $\sigma^{2}(\mathrm{R}), \sigma^{2}(\mathrm{~S})$, and $\left.\sigma^{2}(\mathrm{~A})\right)$ is the number of risers minus one.

The second model used one-way analysis of variance. The one-way analysis of variance statistical model used to describe the structure of the data is

where

$$
\begin{gathered}
Y_{i j}=\mu+S_{i}+A_{i j}, \\
i=1,2, \ldots, a, j=1,2, \ldots, n_{i},
\end{gathered}
$$

$\begin{array}{ll}Y_{i j} & =\text { concentration from the } j^{\text {th }} \text { analytical result from the } i^{\text {th }} \text { sample } \\ \mu & =\text { the grand mean } \\ S_{i} & =\text { the effect of the } i^{\text {th }} \text { sample } \\ A_{i j} & =\text { the effect of the } j^{\text {th }} \text { analytical result from the } i^{\text {th }} \text { sample } \\ \mathrm{a} & =\text { the number of analytical results from the } i^{\text {th }} \text { sample. }\end{array}$


The variable $S_{i}$ is assumed to be a random effect. This variable, as well as $A_{i j}$, are assumed to be uncorrelated and normally distributed with means zero and variances $\sigma^{2}(\mathbf{S})$ and $\sigma^{2}(\mathrm{~A})$, respectively. Estimates of $\sigma^{2}(\mathrm{~S})$ and $\sigma^{2}(\mathrm{~A})$ were obtained using REML techniques. The results using the REML techniques were obtained using the statistical analysis package S-PLUS (Statistical Sciences, 1993). The $d f$ associated with the standard deviation of the mean (a function of $\sigma^{2}(\mathrm{~S})$ and $\sigma^{2}(\mathrm{~A})$ ) is the number of samples minus one.

\section{B3.4.2.2 Nonconvective Layer (Salt Slurry) and Crust}

The data were statistically evaluated using two different models. The first model used a nested analysis of variance with a riser term. The nested analysis of variance statistical model used to describe the structure of the data is

where

$$
\begin{gathered}
Y_{i j k 1}=\mu+R_{i}+S_{i j}+L_{i j k}+A_{i j k l}, \\
i=1,2, \ldots, a ; j=1,2, \ldots, b_{i} ; k=1,2, \ldots, c_{i j} ; l=1,2, \ldots, n_{i j k} ;
\end{gathered}
$$

$$
\begin{aligned}
& \mathrm{Y}_{\mathrm{ijkl}}=\text { concentration from the } \mathrm{I}^{\mathrm{th}} \text { analytical result from the } \mathrm{k}^{\text {th }} \text { location } \\
& \text { from the } \mathrm{j}^{\text {th }} \text { segment from the } \mathrm{i}^{\text {th }} \text { riser } \\
& \mu \quad=\quad \text { the grand mean } \\
& \mathbf{R}_{\mathbf{i}} \quad=\quad \text { the effect of the } \mathrm{i}^{\text {th }} \text { riser } \\
& S_{i j}=\text { the effect of the } j^{\text {th }} \text { segment from the } i^{\text {th }} \text { riser } \\
& \mathrm{L}_{\mathrm{ijk}} \quad=\quad \text { the effect of the } \mathrm{k}^{\text {th }} \text { location from the } j^{\text {ih }} \text { segment from the } \\
& i^{\text {th }} \text { riser } \\
& \mathrm{A}_{\mathrm{ijkl}}=\quad \text { the effect of the } 1^{\text {th }} \text { analytical result from the } \mathrm{k}^{\text {th }} \text { location from } \\
& \text { the } \mathrm{j}^{\text {th }} \text { segment from the } \mathrm{i}^{\text {th }} \text { riser } \\
& \text { a } \quad=\quad \text { the number of risers } \\
& b_{i}=\text { the number of segments from the } i^{\text {th }} \text { riser } \\
& c_{i j}=\text { the number of locations from the } j^{\text {th }} \text { segment from the } i^{i^{\text {th }}} \text { riser } \\
& n_{i j k}=\quad \text { the number of analytical results from the } k^{\text {th }} \text { location from the } j^{\text {th }} \\
& \text { segment from the } \mathrm{i}^{\text {th }} \text { riser. }
\end{aligned}
$$

The variables $R_{i}, S_{i j}$, and $L_{i j k}$ are assumed to be a random effects. These variables, as well as $A_{i j k 1}$, are assumed to be uncorrelated and normally distributed with means zero and variances $\sigma^{2}(\mathrm{R}), \sigma^{2}(\mathrm{~S}), \sigma^{2}(\mathrm{~L})$, and $\sigma^{2}(\mathrm{~A})$, respectively. Estimates of $\sigma^{2}(\mathrm{R}), \sigma^{2}(\mathrm{~S}), \sigma^{2}(\mathrm{~L})$, and 
$\sigma^{2}(\mathrm{~A})$ were obtained using REML techniques. This method applied to variance component estimation is described in Harville (1977). The results using the REML techniques were obtained using the statistical analysis package S-PLUS (Statistical Sciences, 1993). The $d f$ associated with the standard deviation of the mean (a function of $\sigma^{2}(\mathrm{R}), \sigma^{2}(\mathrm{~S}), \sigma^{2}(\mathrm{~L})$, and $\left.\sigma^{2}(\mathrm{~A})\right)$ is the number of risers minus one.

The second model used nested analysis of variance without the riser term. This nested ANOVA model is

where

$$
\begin{gathered}
\mathrm{Y}_{\mathrm{ijk}}=\mu+\mathrm{S}_{\mathrm{i}}+\mathrm{L}_{\mathrm{ij}}+\mathrm{A}_{\mathrm{ijk}}, \\
\mathrm{i}=1,2, \ldots, \mathrm{a} ; \mathrm{j}=1,2, \ldots, \mathrm{b}_{\mathrm{i}} ; \mathrm{k}=\mathrm{l}, 2, \ldots, \mathrm{n}_{\mathrm{ij}} ;
\end{gathered}
$$

\begin{tabular}{|c|c|c|}
\hline$Y_{i j k}$ & $=$ & $\begin{array}{l}\text { concentration from the } \mathrm{k}^{\text {th }} \text { analytical result from the } \mathrm{j}^{\text {th }} \text { location } \\
\text { from the } \mathrm{i}^{\text {th }} \text { sample }\end{array}$ \\
\hline$\mu$ & $=$ & the grand mean \\
\hline$S_{i}$ & $=$ & the effect of the $i^{\text {th }}$ sample \\
\hline$L_{\mathbf{i j}}$ & $=$ & the effect of the $j^{\text {th }}$ location from the $i^{\text {th }}$ sample \\
\hline $\mathrm{A}_{\mathrm{ijk}}$ & $=$ & $\begin{array}{l}\text { the effect of the } k^{\text {th }} \text { analytical result from the } j^{\text {th }} \text { location from } \\
\text { the } \mathrm{i}^{\text {th }} \text { sample }\end{array}$ \\
\hline a & $=$ & the number of samples \\
\hline$b_{i}$ & $=$ & the number of locations from the $\mathrm{i}^{\text {th }}$ sample \\
\hline$n_{i j}$ & $=$ & $\begin{array}{l}\text { the number of analytical results from the } \mathrm{j}^{\text {th }} \text { location from the } \\
\mathrm{i}^{\text {th }} \text { sample. }\end{array}$ \\
\hline
\end{tabular}

The variables $S_{i}$ and $L_{i j}$ are assumed to be a random effects. These variables, as well as $A_{i j k}$, are assumed to be uncorrelated and normally distributed with means zero and variances $\sigma^{2}(S)$, $\sigma^{2}(\mathrm{~L})$, and $\sigma^{2}(\mathrm{~A})$, respectively. Estimates of $\sigma^{2}(\mathrm{~S}), \sigma^{2}(\mathrm{~L})$, and $\sigma^{2}(\mathrm{~A})$ were obtained using REML techniques. This method applied to variance component estimation is described in Harville (1977). The results using the REML techniques were obtained using the statistical analysis package S-PLUS (Statistical Sciences, 1993). The $d f$ associated with the standard deviation of the mean (a function of $\sigma^{2}(\mathrm{~S}), \sigma^{2}(\mathrm{~L})$, and $\sigma^{2}(\mathrm{~A})$ ) is the number of samples minus one.

Refer to Appendix D for inventory calculations. 


\section{B4.0 APPENDIX B REFERENCES}

Cash, R. J., 1996a, Application of "Flammable Gas Tank Safety Program: Data Requirements for Core Sampling Analysis Developed Through the Data Quality Objective Process" Rev. 2, (internal memorandum 79300-96-028 to A. J. Eberlein, July 12), Westinghouse Hanford Company, Richland, Washington.

Cash, R. J., 1996b, Scope Increase of "Data Quality .Objective to Support Resolution of the Organic Complexant Safety Issue" Rev. 2, (internal memorandum 79300-96-029 to S. J. Eberlein, July 12), Westinghouse Hanford Company, Richland, Washington.

DOE-RL, 1996, Recommendation 93-5 Implementation Plan, DOE/RL-94-0001, Rev. 1, U. S. Department of Energy, Richland, Washington.

Dukelow, G. T., J. W. Hunt, H. Babad, and J. E. Meacham, 1995, Tank Safety Screening Data Quality Objective, WHC-SD-WM-SP-004, Rev. 2, Westinghouse Hanford. Company, Richland, Washington.

Harville, D. A., 1977, "Maximum Likelihood Approaches to Variance Component Estimation and to Related Problems," Journal of the American Statistical Association, Vol. 72, pp. 320-340.

Jones, T. E., and K. D. Wiemers, 1996, Data Requirements for TWRS Privatization Characterization of Potential Low Activity Waste Feed, WHC-SD-WM-SP-023, Rev. 0, Westinghouse Hanford Company, Richland, Washington.

Kruger, A. A., 1996, Tank 241-AN-103 Push Mode Core Sampling and Analysis Plan, WHC-SD-WM-TSAP-105, Rev. 0, Westinghouse Hanford Company, Richland, Washington.

Mauss, B. M., 1986, The 86-2 Evaporator Campaign: Laboratory Results on the Production of Double-Shell Slurry, (internal memorandum \#65453-86-038 to M. G. Kelly, dated March 28), Rockwell Hanford Operation, Richland, Washington.

McDuffie, N. G., and G. D Johnson, 1995, Flammable Gas Tank Safety Program - Data Requirements for Core Sample Analysis Developed Through the Data Quality Objectives (DQO) Process, WHC-SD-WM DQO-004, Rev. 2, Westinghouse Hanford Company, Richland, Washington.

Priguano, A. L., 1988, Dissolution Study of Composite from Tanks 103-AW, (letter 12221-PCL88-060 to Distribution, January 13), Westinghouse Hanford Company, Richland, Washington. 
Snedecor, G. W., and W. G. Cochran, 1980, Statistical Methods, 7th Edition, Iowa State University Press, Ames, Iowa.

Statistical Sciences, Inc., 1993, S-PLUS Reference Manual, Version 3.2, StatSci, a division of MathSoft, Inc., Seattle, Washington.

Steen, F. H., 1997, Tank 241-AN-103, Cores 166 and 167, Analytical Results for the Final Report, HNF-SD-WM-DP-223, Rev. 0, Rust Federal Services of Hanford, Inc., Richland, Washington.

Toste, A. P., 1987, (untitled internal memorandum to W. G. Richmond, dated September 25), Pacific Northwest Laboratory, Richland, Washington. 
HNF-SD-WM-ER-702 Rev. 0

APPENDIX C

STATISTICAL ANALYSIS FOR ISSUE RESOLUTION

C-1 
HNF-SD-WM-ER-702 Rev. 0

This page intentionally left blank. 


\section{APPENDIX C}

\section{STATISTICAL ANALYSIS FOR ISSUE RESOLUTION}

Results of statistical calculations required by the safety screening DQO (Dukelow et al. 1995) are documented in this appendix.

\section{C1.0 STATISTICS FOR SAFETY SCREENING DQO}

The safety screening DQO (Dukelow et al. 1995) defines acceptable decision confidence limits in terms of one-sided 95 percent confidence intervals. In this appendix, one-sided confidence limits supporting the safety screening DQO are calculated for tank 241-AN-103. The data in this section are from the 1996 sampling event for tank 241-AN-103.

Confidence intervals were computed for each sample number from tank 241-AN-103 anaiytical data (Steen 1997). The upper limit (UL) of a one-sided 95 percent confidence interval for the mean is

$$
\hat{\mu}+\mathrm{t}_{(\mathrm{df}, 0.05)} * \hat{\sigma}_{\hat{\mu}} .
$$

In this equation, $\hat{\mu}$ is the arithmetic mean of the data, $\hat{\sigma}_{\hat{\mu}}$ is the estimate of the standard deviation of the mean, and $t_{(d f, 0.05)}$ is the quantile from Student's $t$ distribution with $d f$ degrees of freedom for a one-sided 95 percent confidence interval. For the tank 241-AN-103 data (per sample number), $d f$ equals the number of observations minus one.

The upper limit of the 95 percent confidence interval was calculated for each subsample which had DSC measurements. The upper limit for each sample number is listed in Table C1.1. Each confidence interval can be used to make the following statement. If the upper limit is less than $480 \mathrm{~J} / \mathrm{g}$ dry, then one would reject the null hypothesis that DSC is greater than or equal to $480 \mathrm{~J} / \mathrm{g}$ dry at the 0.05 level of significance. All but one of the upper limits were less than $480 \mathrm{~J} / \mathrm{g}$ dry. The UL for Core 167 , segment 7 , drainable liquid was $629 \mathrm{~J} / \mathrm{g}$. Therefore TOC analysis was required. TOC results were well below notification limits. As a result, the high 95 percent CI value for DSC is not of concern.

The upper limit of the 95 percent confidence interval was calculated for each subsample which had at least 50 percent of the reported total alpha data as quantitative values. The sample numbers and the upper limit of the 95 percent confidence intervals are listed in Table C1.2. Each confidence interval can be used to make the following statement. If the upper limit is less than $61.5 \mu \mathrm{Ci} / \mathrm{mL}$ or $34.9 \mu \mathrm{Ci} / \mathrm{g}$ (conversion used the mean bulk density of $1.76 \mathrm{~g} / \mathrm{mL}$ observed for the sludge subsamples), then one would reject the null hypothesis that total alpha is greater than or equal to $61.5 \mu \mathrm{Ci} / \mathrm{mL}$ (or $34.9 \mu \mathrm{Ci} / \mathrm{g}$ ) at the 0.05 level of 
significance. For all the subsamples with at least 50 percent of the reported Total Alpha data as quantitative values, the upper limits were less than $61.5 \mu \mathrm{Ci} / \mathrm{mL}$ or $34.9 \mu \mathrm{Ci} / \mathrm{g}$. For the subsamples where both analytical results were "less than" values, the maximum "less than" value is orders of magnitude smalier than either $61.5 \mu \mathrm{Ci} / \mathrm{mL}$ or $34.9 \mu \mathrm{Ci} / \mathrm{g}$. Thus, the hypothesis that the Total Alpha results are greater than $61.5 \mu \mathrm{Ci} / \mathrm{mL}$ or $34.9 \mu \mathrm{Ci} / \mathrm{g}$ is rejected for all subsamples, meaning there is no criticality issue.

Table C1-1. Tank 241-AN-103 Differential Scanning Calorimetry Results (J/g dry). (3 sheets)

\begin{tabular}{|c|c|c|c|c|c|c|}
\hline 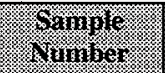 & 16.0. & 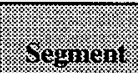 & 180.01501: & $\pi$ & 80 & ing \\
\hline S96T005539 & 166 & 3 & Drainable liquid & $2.67 \mathrm{E}+01$ & $2.67 \mathrm{E}+01$ & $1.95 E+02$ \\
\hline S96T005540 & 166 & 4 & Drainable liquid & $0.00 \mathrm{E}+00$ & $0.00 \mathrm{E}+00$ & $0.00 \mathrm{E}+00$ \\
\hline \$96T005599 & 166 & 6 & Drainable liquid & $0.00 \mathrm{E}+00$ & $0.00 \mathrm{E}+00$ & $0.00 \mathrm{E}+00$ \\
\hline S96T005816 & 166 & 7 & Drainable liquid & $1.30 \mathrm{E}+02$ & $1.20 \mathrm{E}+01$ & $2.06 \mathrm{E}+02$ \\
\hline S96T005817 & 166 & 8 & Drainable liquid & $4.22 \mathrm{E}+01$ & $2.55 \mathrm{E}+00$ & $5.83 \mathrm{E}+01$ \\
\hline S96T005541 & 166 & 9 & Drainable liquid & $1.78 \mathrm{E}+01$ & $1.78 \mathrm{E}+01$ & $1.30 \mathrm{E}+02$ \\
\hline S96T005566 & 166 & 10 & Drainable liquid & $1.51 E+02$ & $4.40 \mathrm{E}+01$ & $4.29 E+02$ \\
\hline S96T005600 & 166 & 11 & Drainable liquid & $0.00 \mathrm{E}+00$ & $0.00 \mathrm{E}+00$ & $0.00 \mathrm{E}+00$ \\
\hline S96T005601 & 166 & 12 & Drainable liquid & $0.00 \mathrm{E}+00$ & $0.00 \mathrm{E}+00$ & $0.00 \mathrm{E}+00$ \\
\hline S96T005861 & 167 & 2 & Drainable liquid & $2.31 \mathrm{E}+02$ & $3.75 \mathrm{E}+01$ & $4.67 \mathrm{E}+02$ \\
\hline S96T005673 & 167 & 3 & Drainable liquid & $0.00 \mathrm{E}+00$ & $0.00 \mathrm{E}+00$ & $0.00 \mathrm{E}+00$ \\
\hline \$96T005420 & 167 & 5 & Drainable liquid & $0.00 \mathrm{E}+00$ & $0.00 \mathrm{E}+00$ & $0.00 \mathrm{E}+00$ \\
\hline \$96T005421 & 167 & 6 & Drainable liquid & $2.79 E+01$ & $2.79 \mathrm{E}+01$ & $2.04 \mathrm{E}+02$ \\
\hline S96T0056831 & 167 & 7 & Drainable liquid & $8.60 \mathrm{E}+01$ & $8.60 \mathrm{E}+01$ & $6.29 \mathrm{E}+02$ \\
\hline S96T005732 & 167 & 8 & Drainable liquid & $0.00 \mathrm{E}+00$ & $0.00 \mathrm{E}+00$ & $0.00 \mathrm{E}+00$ \\
\hline S96T005862 & 167 & 9 & Drainable liquid & $2.07 \mathrm{E}+02$ & $2.20 \mathrm{E}+01$ & $3.46 \mathrm{E}+02$ \\
\hline S96T005650 & 167 & 11 & Drainable liquid & $7.37 \mathrm{E}+01$ & $3.24 \mathrm{E}+01$ & $2.78 \mathrm{E}+02$ \\
\hline \$96T005990 & 166 & Composite & Drainable liquid & $2.01 \mathrm{E}+02$ & $3.00 \mathrm{E}+01$ & $3.90 \mathrm{E}+02$ \\
\hline S96T005578 & 166 & 1 & Lower half & $7.54 \mathrm{E}+01$ & $7.60 \mathrm{E}+00$ & $1.23 \mathrm{E}+02$ \\
\hline S96T005542 & 166 & 4 & Lower half & $0.00 \mathrm{E}+00$ & $0.00 \mathrm{E}+00$ & $0.00 \mathrm{E}+00$ \\
\hline S96T005579 & 166 & 6 & Lower half & $0.00 \mathrm{E}+00$ & $0.00 \mathrm{E}+00$ & $0.00 \mathrm{E}+00$ \\
\hline \$96T005820 & 166 & 7 & Lower half & $0.00 \mathrm{E}+00$ & $0.00 \mathrm{E}+00$ & $0.00 \mathrm{E}+00$ \\
\hline S96T005821 & 166 & 8 & Lower half & $0.00 \mathrm{E}+00$ & $0.00 \mathrm{E}+00$ & $0.00 \mathrm{E}+00$ \\
\hline
\end{tabular}


Table C1-1. Tank 241-AN-103 Differential Scanning Calorimetry Results (J/g dry). ( 3 sheets)

\begin{tabular}{|c|c|c|c|c|c|c|}
\hline Sample & Core & Sygrion & Joration. & t) & 17 & 610.: \\
\hline S96T005543 & 166 & 9 & Lower half & $4.60 \mathrm{E}+01$ & $4.60 \mathrm{E}+01$ & $3.36 \mathrm{E}+02$ \\
\hline S96T005564 & 166 & 10 & Lower half & $0.00 \mathrm{E}+00$ & $0.00 \mathrm{E}+\infty 0$ & $0.00 \mathrm{E}+00$ \\
\hline S96T005580 & 166 & 11 & Lower half & $1.08 \mathrm{E}+00$ & $1.08 \mathrm{E}+00$ & $7.90 \mathrm{E}+00$ \\
\hline S96T005581 & 166 & 12 & Lower half & $2.82 \mathrm{E}+01$ & $1.35 \mathrm{E}+00$ & $3.67 \mathrm{E}+01$ \\
\hline S96T005637 & 166 & 13 & Lower half & $0.00 \mathrm{E}+00$ & $0.00 \mathrm{E}+00$ & $0.00 \mathrm{E}+00$ \\
\hline S96T005636 & 166 & 13 & Upper half & $2.22 \mathrm{E}+01$ & $2.22 \mathrm{E}+01$ & $1.62 \mathrm{E}+02$ \\
\hline S96T005399 & 166 & 15 & Lower half & $5.21 \mathrm{E}+01$ & $2.20 \mathrm{E}+01$ & $1.91 \mathrm{E}+02$ \\
\hline S96T005398 & 166 & 15 & Upper half & $3.29 \mathrm{E}+01$ & $2.49 \mathrm{E}+01$ & $1.90 \mathrm{E}+02$ \\
\hline S96T005583 & 166 & 16 & Lower half & $7.40 \mathrm{E}+01$ & $8.00 \mathrm{E}+00$ & $1.25 \mathrm{E}+02$ \\
\hline S96T005582 & 166 & 16 & Upper half & $5.86 \mathrm{E}+01$ & $8.30 \mathrm{E}+00$ & $1.11 \mathrm{E}+02$ \\
\hline S96T005401 & 166 & 17 & Lower half & $4.31 \mathrm{E}+01$ & $1.04 \mathrm{E}+01$ & $1.09 \mathrm{E}+02$ \\
\hline S96T005400 & 166 & 17 & Upper half & $3.46 \mathrm{E}+01$ & $4.35 \mathrm{E}+00$ & $6.20 \mathrm{E}+01$ \\
\hline S96T005639 & 166 & 18 & Lower half & $1.68 \mathrm{E}+01$ & $3.55 \mathrm{E}+00$ & $3.92 \mathrm{E}+01$ \\
\hline S96T005638 & 166 & 18 & Upper half & $0.00 \mathrm{E}+00$ & $0.00 \mathrm{E}+00$ & $0.00 \mathrm{E}+00$ \\
\hline S96T005823 & 166 & 19 & Lower half & $3.93 \mathrm{E}+01$ & $1.27 \mathrm{E}+01$ & $1.19 \mathrm{E}+02$ \\
\hline S96T005822 & 166 & 19 & Upper half & $0.00 \mathrm{E}+00$ & $0.00 \mathrm{E}+00$ & $0.00 \mathrm{E}+00$ \\
\hline S96T005668 & 167 & 1 & Lower half & $1.64 \mathrm{E}+01$ & $5.00 \mathrm{E}-02$ & $1.67 \mathrm{E}+01$ \\
\hline S96T005667 & 167 & 1 & Upper half & $0.00 \mathrm{E}+00$ & $0.00 \mathrm{E}+00$ & $0.00 \mathrm{E}+00$ \\
\hline S96T005851 & 167 & 2 & Lower half & 152 & 83.7 & \\
\hline S96T005669 & 167 & 3 & Lower half & $6.40 \mathrm{E}+01$ & $1.85 \mathrm{E}+01$ & $1.80 \mathrm{E}+02$ \\
\hline S96T005675 & 167 & 4 & Lower half & $0.00 \mathrm{E}+00$ & $0.00 \mathrm{E}+00$ & $0.00 \mathrm{E}+00$ \\
\hline S96T005424 & 167 & 5 & Lower half & $0.00 \mathrm{E}+00$ & $0.00 \mathrm{E}+00$ & $0.00 \mathrm{E}+00$ \\
\hline S96T005425 & 167 & 6 & Lower half & $0.00 \mathrm{E}+00$ & $0.00 \mathrm{E}+00$ & $0.00 \mathrm{E}+00$ \\
\hline S96T005685 & 167 & 7 & Lower half & $8.95 \mathrm{E}+01$ & $5.55 \mathrm{E}+01$ & $4.40 \mathrm{E}+02$ \\
\hline S96T005734 & 167 & 8 & Lower half & $0.00 \mathrm{E}+00$ & $0.00 \mathrm{E}+00$ & $0.00 \mathrm{E}+00$ \\
\hline S96T005852 & 167 & 9 & Lower half & $6.75 \mathrm{E}+00$ & $6.75 \mathrm{E}+00$ & $4.94 \mathrm{E}+01$ \\
\hline S96T005647 & 167 & 11 & Lower half & $1.20 \mathrm{E}+01$ & $3.93 \mathrm{E}+00$ & $3.68 \mathrm{E}+01$ \\
\hline S96T005646 & 167 & 11 & Upper half & $9.52 \mathrm{E}+00$ & $2.68 \mathrm{E}+00$ & $2.64 \mathrm{E}+01$ \\
\hline S96T005426 & 167 & 12 & Lower half & $0.00 \mathrm{E}+00$ & $0.00 \mathrm{E}+00$ & $0.00 \mathrm{E}+00$ \\
\hline
\end{tabular}




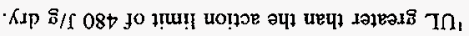

\begin{tabular}{|c|c|c|c|c|c|c|}
\hline $00+300^{\circ} 0$ & $00+300^{\circ} 0$ & $00+300^{\circ} 0$ & flEપ Iəddగ & $\angle I$ & $\angle 9 I$ & $26 t 500 \mathrm{~L} 96 \mathrm{~S}$ \\
\hline $00+\exists 00^{\circ} 0$ & $00+\exists 00^{\circ} 0$ & $00+\exists 00^{\circ} 0$ & $\exists[\mathrm{EY}$ دәмот & $L I$ & $\angle 9 I$ & $\varepsilon 6+$ S00L96S \\
\hline $20+B 6 L^{\circ} \mathrm{Z}$ & $I 0+B 9 Z^{\prime} \mathcal{E}$ & $10+\exists \neq \varepsilon \cdot L$ & $f[E 4$ Iədd $\Omega$ & SI & $\angle 9 I$ & s\$9S00L96S \\
\hline $00+300^{\circ} 0$ & $00+300^{\circ} 0$ & $00+300^{\circ} 0$ & I[Еप دәмот & $S I$ & $\angle 9 \mathrm{I}$ & $9 \$ 9 \$ 00 L 96 \mathrm{~S}$ \\
\hline $10+30 \varepsilon^{\circ} 8$ & $00+306^{\circ} 9$ & $10+376 \cdot \varepsilon$ & fley Iədd $\Omega$ & $t r$ & $\angle 9 I$ & $\varepsilon \mathcal{E} 7500 L 96 \mathrm{~S}$ \\
\hline $00+300^{\circ} 0$ & $00+300^{\circ} 0$ & $00+700^{\circ} 0$ & I[एप Iәмот & $\nabla I$ & $\angle 9 I$ & $L Z \downarrow \$ 00196 \mathrm{~S}$ \\
\hline $20+399 \cdot z$ & $\tau 0+\exists \varepsilon \varsigma^{\prime} \varepsilon$ & $10+362 \cdot t$ & fley Iəddn & ZI & $\angle 9 I$ & zE\$S00.L96S \\
\hline \% & (2.7. & ה. & (1048\%) & $10 \% 11605$ & \% & thans \\
\hline
\end{tabular}

(słәวบุ \&)

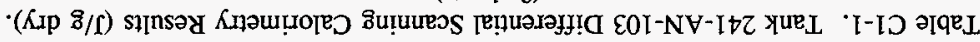


Table C1-2.Tank 241-AN-103 Total Alpha Results. (2 sheets)

\begin{tabular}{|c|c|c|c|c|c|c|}
\hline $\begin{array}{l}\text { Sample } \\
\text { Noningr. }\end{array}$ & 36 & Sogrivient: & $18 a c i n i b u$ & Marrinario & 6. & yin \\
\hline \multicolumn{7}{|c|}{ Wiguif Samples: } \\
\hline S96T005539 & 166 & 3 & Drainable liquid & $<5.79 \mathrm{E}-03$ & $n / a$ & $\mathrm{n} / \mathrm{a}$ \\
\hline S96T005540 & 166 & 4 & Drainable liquid & $<5.06 \mathrm{E}-03$ & $n / a$ & $\mathrm{n} / \mathrm{a}$ \\
\hline S96T005599 & 166 & 6 & Drainable liquid & $<2.02 \mathrm{E}-02$ & $n / a$ & $n / a$ \\
\hline S96T005816 & 166 & 7 & Drainable liquid & $<2.30 \mathrm{E}-02$ & $n / a$ & $\mathrm{n} / \mathrm{a}$ \\
\hline S96T005817 & 166 & 8 & Drainable liquid & $<1.20 \mathrm{E}-02$ & $n / a$ & $n / a$ \\
\hline S96T005541 & 166 & 9 & Drainable liquid & $4.35 \mathrm{E}-03$ & $4.45 \mathrm{E}-03$ & $7.15 \mathrm{E}-03$ \\
\hline S96T005566 & 166 & 10 & Drainable liquid & $<5.79 \mathrm{E}-03$ & $n / a$ & $\mathrm{n} / \mathrm{a}$ \\
\hline S96T005600 & 166 & 11 & Drainabie liquid & $<2.30 \mathrm{E}-02$ & $n / a$ & $n / a$ \\
\hline S96T005601 & 166 & 12 & Drainable liquid & $1.13 \mathrm{E}-01$ & $6.66 \mathrm{E}-02$ & $5.34 \mathrm{E}-01$ \\
\hline S96T005861 & 167 & 2 & Drainable liquid & $<8.04 \mathrm{E}-02$ & $n / a$ & $n / a$ \\
\hline S96T005673 & 167 & 3 & Drainable liquid & $<2.24 \mathrm{E}-02$ & $n / a$ & $\mathrm{n} / \mathrm{a}$ \\
\hline S96T005420 & 167 & 5 & Drainable liquid & $<1.82 \mathrm{E}-01$ & $n / a$ & $n / a$ \\
\hline S96T005421 & 167 & 6 & Drainable liquid & $<5.14 \mathrm{E}-02$ & $n / a$ & $\mathrm{n} / \mathrm{a}$ \\
\hline S96T005683 & 167 & 7 & Drainable liquid & $3.83 \mathrm{E}-02$ & $1.40 \mathrm{E}-03$ & $4.71 \mathrm{E}-02$ \\
\hline S96T005732 & 167 & 8 & Drainable liquid & $3.59 \mathrm{E}-02$ & $1.90 \mathrm{E}-03$ & $4.79 \mathrm{E}-02$ \\
\hline S96T005862 & 167 & 9 & Drainable liquid & $6.26 \mathrm{E}-02$ & $1.12 \mathrm{E}-02$ & $1.33 \mathrm{E}-01$ \\
\hline S96T005650 & 167 & 11 & Drainable liquid & $<1.69 \mathrm{E}-02$ & $n / a$ & $n / a$ \\
\hline S96T005991 & 166 & Composite & Drainable liquid & $<6.53 \mathrm{E}-03$ & $n / a$ & $\mathrm{n} / \mathrm{a}$ \\
\hline \multicolumn{7}{|c|}{ Shudge samiles (1) (Gig) } \\
\hline S96T005608 & 166. & 1 & Lower half & $<1.10 \mathrm{E}-02$ & $n / a$ & $\mathrm{n} / \mathrm{a}$ \\
\hline S96T005568 & 166 & 4 & Lower half & $8.01 \mathrm{E}-03$ & $3.65 \mathrm{E}-04$ & $1.03 \mathrm{E}-02$ \\
\hline S96T005611 & 166 & 6 & Lower half & $<9.61 \mathrm{E}-03$ & $n / a$ & $n / a$ \\
\hline S96T005835 & 166 & 7 & Lower half & $5.82 \mathrm{E}-03$ & $7.40 \mathrm{E}-04$ & $1.05 \mathrm{E}-02$ \\
\hline S96T005836 & 166 & 8 & Lower half & $5.69 \mathrm{E}-03$ & $8.90 \mathrm{E}-04$ & $1.13 \mathrm{E}-02$ \\
\hline S96T005574 & 166 & 9 & Lower half & $<8.68 \mathrm{E}-03$ & $n / a$ & $n / a$ \\
\hline S96T005569 & 166 & 10 & Lower half & $<9.38 \mathrm{E}-03$ & $n / a$ & $\mathrm{n} / \mathrm{a}$ \\
\hline S96T005612 & 166 & 11 & Lower half & $<8.81 \mathrm{E}-03$ & $n / a$ & $n / a$ \\
\hline S96T005613 & 166 & 12 & Lower half & $<9.17 \mathrm{E}-03$ & $n / a$ & $n / a$ \\
\hline
\end{tabular}




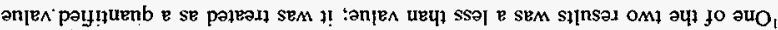
ə[qeor]dde $]$ ON $=\mathrm{v} / \mathrm{u}$

:Se10N

\begin{tabular}{|c|c|c|c|c|c|c|}
\hline $\mathrm{e} / \mathrm{u}$ & $\mathrm{e} / \mathrm{u}$ & $20^{-}-389^{\circ} \mathrm{I}>$ & fleप JPMOT & $\angle I$ & $\angle 9 I$ & $\angle 6+S 00 L 96 S$ \\
\hline $\mathbf{e} / \mathrm{u}$ & $\mathrm{e} / \mathrm{u}$ & 20-ヨIE I > & J[ЕЧ JәMOT & SI & $\angle 9 I$ & Z69S00L96S \\
\hline $\mathrm{e} / \mathrm{u}$ & $\mathrm{e} / \mathrm{u}$ & $20-\exists t I^{\circ} z>$ & J[Еप JәMOT & $\nabla I$ & $\angle 9 I$ & $\angle \mathcal{L} 500 \mathrm{LS6S}$ \\
\hline 20 -GEI $\mathrm{s}$ & $\varepsilon 0^{-} \exists S I^{\circ} \varepsilon$ & $20-\exists \subseteq I^{\circ} \mathcal{E}$ & I[Еч Iәмо T & ZI & $\angle 9 I$ & $6 \$ t 500 \mathrm{~L} 96 \mathrm{~S}$ \\
\hline $\mathrm{e} / \mathrm{u}$ & $\mathrm{e} / \mathrm{u}$ & $\varepsilon 0-38 t^{\circ} 9>$ & ЈГеч хәмот & II & $\angle 9 I$ & I69\$00L96S \\
\hline $\mathrm{e} / \mathrm{u}$ & $\mathrm{E} / \mathrm{u}$ & 20-ב̈॰ I"I > & I[EU IәMOT & 6 & $\angle 9 I$ & 9\$8S00L96S \\
\hline $\mathrm{e} / \mathrm{u}$ & $\mathrm{e} / \mathrm{u}$ & $\varepsilon 0^{-} \exists \tau 8^{\circ} \varsigma>$ & I[Еप IәMOT] & 8 & $\angle 9 I$ & $9 \varepsilon \angle S 00 L 96 S$ \\
\hline $\mathrm{e} / \mathrm{u}$ & $\mathrm{e} / \mathrm{u}$ & $\varepsilon 0^{-}-96 t^{*} I>$ & IIEप دәмоT & $L$ & $\angle 9 I$ & 069S00L96S \\
\hline $\mathrm{e} / \mathrm{u}$ & $\mathrm{e} / \mathrm{u}$ & $20^{-} 390^{\circ} 6>$ & І[ЕЧ ऽәмоТ] & 9 & $\angle 9 I$ & $87 t 500 \mathrm{~L} 96 \mathrm{~S}$ \\
\hline $\mathrm{e} / \mathrm{u}$ & $\mathrm{E} / \mathrm{u}$ & $10-\exists 9 \varepsilon^{*} I>$ & J[Еप Iәмот & $\mathrm{s}$ & $\angle 9 I$ & $9 \varepsilon \diamond \$ 00 \mathrm{~L} 96 \mathrm{~S}$ \\
\hline $\mathrm{e} / \mathrm{u}$ & $\mathrm{e} / \mathrm{u}$ & $\varepsilon 0-389^{\circ} \mathrm{L}>$ & IIEप JәMOT & $t$ & $\angle 91$ & $689 S 00 \mathrm{~L} 96 \mathrm{~S}$ \\
\hline $\mathrm{e} / \mathrm{u}$ & $\mathrm{e} / \mathrm{u}$ & 20-ヨ] $\left[\varepsilon^{*} I>\right.$ & 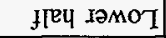 & $\varepsilon$ & $\angle 9 I$ & $889 \$ 00 L 96 S$ \\
\hline $20-3 \mathrm{SI}^{\circ} \mathrm{I}$ & $50-\exists 0 S^{\circ} L$ & E0-908.9 & Ilеy IәMOT & $z$ & $\angle 9 I$ & ${ }_{\mathrm{I}} \mathrm{S} 8 \mathrm{~S} 900 \mathrm{~L} 96 \mathrm{~S}$ \\
\hline $\mathrm{B} / \mathrm{u}$ & $\mathrm{B} / \mathrm{u}$ & $20-\exists 9 t^{\circ} \varepsilon>$ & IГеप дәмо T & I & $\angle 9 I$ & $\angle 89 S 00 \mathrm{~L} 96 \mathrm{~S}$ \\
\hline $20-$ - $00 t^{\circ}+$ & $\varepsilon 0^{-} \exists \subseteq 6^{\circ} \varepsilon$ & $20-316 \cdot 1$ & I[еप Iәмот] & $6 \mathrm{I}$ & $99 I$ & 8E8S00L96S \\
\hline I0-BSI'I & $20-\theta \angle \varepsilon^{\circ} I$ & $\tau 0-\exists \varepsilon 6^{\circ} \tau$ & IlEY دәMOT & $8 \mathrm{I}$ & $99 \mathrm{I}$ & IZLS00L96S \\
\hline $\mathrm{e} / \mathrm{u}$ & $\mathrm{e} / \mathrm{u}$ & $20-962 \cdot I>$ & IIEप دәMOT & $\angle I$ & $99 \mathrm{I}$ & $60 t S 00 \perp 96 \mathrm{~S}$ \\
\hline $20-3251$ & $\mathrm{EO}^{-\mathrm{BI}} \mathrm{IS} \mathrm{I}$ & $\varepsilon 0^{-}-\mathrm{B} t L^{\circ} \mathrm{s}$ & IГЕप 1әмоТ & $9 \mathrm{I}$ & $99 \mathrm{I}$ & ItI9S00L96S \\
\hline $\mathrm{e} / \mathrm{u}$ & $\mathrm{e} / \mathrm{u}$ & 20 -GIE I> & IFEY IOMOT & SI & $99 \mathrm{I}$ & $80 t S 00 L 96 S$ \\
\hline $20-\exists \varepsilon 6^{\circ} L$ & $\mathcal{E} 0-\exists S t^{\circ} 8$ & $z 0-309 \cdot \tau$ & IIеप دәмо & $\mathcal{E} I$ & $99 \mathrm{I}$ & ${ }_{\mathrm{I}} 0 Z L S 00196 \mathrm{~S}$ \\
\hline (r) & & & & \multicolumn{3}{|c|}{ 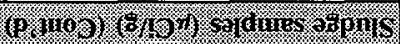 } \\
\hline 祖 & (x) & V) & 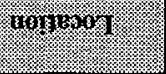 & 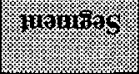 & 6. & P\&ininu \\
\hline
\end{tabular}

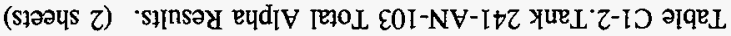




\section{C2.0 APPENDIX C REFERENCES}

Dukelow, G. T., J. W. Hunt, H. Babad, and J. E. Meacham, 1995, Tank Safety Screening Data Quality Objective, WHC-SD-WM-SP-004, Rev. 2, Westinghouse Hanford Company, Richland, Washington.

Steen, F. H., 1997, Tank 241-AN-103, Cores 166 and 167, Analytical Results for the Final Report, HNF-SD-WM-DP-223, Rev. 0, Rust Federal Services of Hanford, Inc. for Fluor Daniel Hanford, Inc., Richland, Washington. 
HNF-SD-WM-ER-702 Rev. 0

This page intentionally left blank. 


\section{APPENDIX D}

EVALUATION TO ESTABLISH BEST-BASIS INVENTORY FOR

DOUBLE-SHELL TANK 241-AN-103 
HNF-SD-WM-ER-702 Rev. 0

This page intentionally left blank. 


\section{APPENDIX D \\ EVALUATION TO ESTABLISH BEST-BASIS INVENTORY FOR DOUBLE-SHELL TANK 241-AN-103}

An effort is underway to provide waste inventory estimates that will serve as standard characterization source terms for the various waste management activities (Hodgson and LeClair 1996). As part of this effort, an evaluation of available information for tank 241-AN-103 was performed, and a best-basis inventory was established. This work, detailed in the following sections, follows the methodology that was established by the standard inventory task.

\section{D1.0 CHEMICAL INFORMATION SOURCES}

Available composition information for the waste in tank 241-AN-103 is as follows:

- Appendix B of this report provides characterization results from the September 1996 core sampling event.

- A feed projection document that supported grout treatment facility studies provides estimates of the waste in tank 241-AN-103 based on a 1986 push core sample. (Hendrickson 1994).

- The HDW model document (Agnew et al. 1997) provides tank content estimates derived from the LANL model, in terms of component concentrations and inventories. A complete list of data sources used in this evaluation is provided at the end of this section.

\section{D2.0 COMPARISON OF COMPONENT INVENTORY VALUES}

There have been no transfers into or out of tank 241-AN-103 since 1986. The HDW model provides composition estimates for the waste in tank 241-AN-103 as of January 1, 1994. Sample-based inventories derived from the September 1996 push core samples are compared to sample based-inventories derived from a 1988 push core sample and inventories generated by the HDW model (Agnew et al. 1997), in Tabies D2-1 and D2-2. A tank volume of $3,615 \mathrm{~kL}$ (955 kgal) was used to generate the sample-based and HDW inventories (Hanlon 1997). The chemical species are reported without charge designation per the best-basis inventory convention. 
The sample-based densities reported in Appendix B are $1.53 \mathrm{~g} / \mathrm{mL}$ for the liquid and $1.76 \mathrm{~g} / \mathrm{mL}$ for the slurry. The slurry density is based on measurements of centrifuged solids; it is not necessarily the same as the density of the slurry in the tank. Gases generated in the tank are trapped in the slurry layer and form gas pockets. Because of this, a lower density than was found in the laboratory is expected for the waste in the tank. In tank 241-AN-103, the retained gas is $6.7 \mathrm{vol} \%$ of the slurry volume (Shekarriz et al. 1996). The mean slurry density was corrected by multiplying by 0.933 ( 1 - vol\% retained gas). The corrected slurry density is $1.64 \mathrm{~g} / \mathrm{mL}$. The HDW model bulk density is $1.60 \mathrm{~g} / \mathrm{mL}$.

The slurry volume, determined from temperature profiles, rheometer and push core sample data is $1,552 \mathrm{~kL}$ (410 kgal) (Stauffer 1997). Based on the extrusion reports, there is a considerable crust layer in tank 241-AN-103. Approximately $35.6 \mathrm{~cm}$ (14 in.) of solids and no drainable liquid was extruded from the first segment of core 167 and $15.2 \mathrm{~cm}(6 \mathrm{in}$.) of solids were found in segment two of core 167 with $190 \mathrm{~mL}$ of drainable liquid. Segment 1 of core 166 was found to contain only $6.4 \mathrm{~cm}$ ( $2.5 \mathrm{in}$.) of solids and no drainable liquid. Segment 2 of core 166 was taken for retained gas analysis; the solid content is not known.

Based on core 167, the crust layer would be $50.8 \mathrm{~cm}$ (20 in.) deep. However, it is evident that salts precipitated from supernatant after samples were removed from the tank and allowed to cool to ambient temperatures. The average temperature in tank 241-AN-103 is $41.1^{\circ} \mathrm{C}\left(106^{\circ} \mathrm{F}\right)$ (Brevick 1997). Samples from both cores that were expected to contain only liquid contained from 1 to 5 inches of solids. It was assumed that $7.6 \mathrm{~cm}(3 \mathrm{in}$.) of the $15.2 \mathrm{~cm}(6 \mathrm{in}$.) of solids in the second segment of core 167 , which contained $15.2 \mathrm{~cm}(6 \mathrm{in}$.) of solids, precipitated after the segment was taken from the tank. The fourteen inches of solids in segment 1 of core 167 are assumed to be crust. The total crust layer is assumed therefore to be $43.2 \mathrm{~cm}$ ( $17 \mathrm{in}$.) or $177 \mathrm{~kL}$ ( $46.7 \mathrm{kgal}$ ) with a density of 1.50 (an assumed density that is slightly lower than the mean liquid density).

The supernatant volume, equal to the total volume minus the slurry and crust volumes, is estimated to be $1,885 \mathrm{~kL}$ (498 kgal) from these observations. The supernatant volume in Hanlon (1997) of 2,063 kL (545 kgal) is expected to be updated to the newer basis in the future. 


\begin{tabular}{|c|c|c|c|c|}
\hline $88^{\circ} \mathrm{I}$ & $00 \nabla^{\prime} \mathrm{II}$ & EIt & $029^{\prime} s>$ & $\Omega$ \\
\hline $0 z z^{\prime} z$ & $002^{\prime} 09$ & $000^{\circ} \mathrm{ZI}$ & YN & $\mathrm{OOJ}$ \\
\hline $\mathrm{YN}$ & 0 & $0^{\circ} \varepsilon t>$ & $z I I>$ & IS \\
\hline $018^{\circ} \mathrm{s}$ & $000^{\circ} \mathrm{s}$ & $00 \varepsilon^{\prime} \angle I$ & $00 z^{6} \varepsilon I$ & tOS se $S$ \\
\hline$\$ 86$ & $0 \varepsilon \varepsilon^{\prime} 8$ & $0 \div 8^{\circ} \mathrm{z}$ & OSE'Z & IS \\
\hline $09 \varepsilon^{\prime} \varepsilon$ & $00 L ' S S$ & $00 \varepsilon^{\prime} I I$ & $008^{\prime} 6$ & tOd se $d$ \\
\hline 092 & 298 & $t 9 t>$ & $\subseteq 6 L>$ & $\mathrm{qd}$ \\
\hline $000^{\circ} \mathrm{ESE}$ & $000^{\circ} \angle L S$ & $000^{\circ} 0 L 2$ & บN & $\mathrm{HO}$ \\
\hline $000^{\circ} 6 L S$ & $90+9 I^{\circ} \mathrm{I}$ & $000^{\circ} \angle 6 \mathrm{~S}$ & $000^{\circ} T L L$ & EON \\
\hline $000^{\circ} 86 t$ & $000^{\circ} 0 \varepsilon \varepsilon$ & $000^{\prime} 8 \mathrm{st}$ & $000^{\circ} \pm 6 t$ & ZON \\
\hline$\angle 8$ & $0 s z^{\prime} z$ & I.98> & $s z Z>$ & !N \\
\hline $90+3 I Z^{\prime} \mathrm{I}$ & $90+38 \mathrm{I}^{\circ} \mathrm{I}$ & $90+\exists Z I^{\circ} I$ & $90+3 \angle Z^{\circ} I$ & $\mathrm{eN}_{\mathrm{N}}$ \\
\hline 0 & $0 \varepsilon 8^{\prime} \mathrm{I}$ & $8^{\circ} \mathrm{zt>}$ & ZII $>$ & $u_{\mathcal{N}}$ \\
\hline 0 & $6 I^{\circ} 9$ & $t 9 t>$ & I I8> & $\mathrm{ET}$ \\
\hline $00 I^{\prime} \mathrm{SS}$ & $00 I^{\prime} 9 z$ & $00 I^{\prime} I S$ & $00 \varepsilon^{6} 9 t$ & $\mathrm{y}$ \\
\hline 85 & $0 \mathrm{I}$ & 0 & 0 & ${ }^{8} \mathrm{H}$ \\
\hline$\varsigma \subseteq \mathcal{L}$ & $0 t t^{\prime} \varepsilon$ & $915>$ & $\nabla L L>$ & $\partial \underline{I}$ \\
\hline $099^{\circ} \mathrm{Z}$ & $00 L^{\prime} Z I$ & $006^{6} \mathrm{II}$ & $098^{\circ} \varepsilon$ & 포 \\
\hline $0 \angle 0^{\circ} \varepsilon$ & $008^{\circ} 0 z$ & $098^{\circ} \mathrm{z}$ & $028^{\prime} \mathrm{z}$ & ID \\
\hline $00 t^{\prime} \tau \varepsilon$ & $000^{\circ} 9 \mathrm{tI}$ & $009^{\prime} \triangleright 8$ & $\quad \mathrm{UN}$ & EOD \\
\hline $008^{\circ} \dagger \varepsilon$ & $006^{\circ} 0 \varepsilon$ & $0 Z I ' 6 z$ & $00 S^{\prime} \angle Z$ & Iอ \\
\hline$\nabla I \varepsilon$ & $018^{\prime} 9$ & $0 \varepsilon t>$ & $00 I^{\prime} 9>$ & ED \\
\hline$\nabla L I$ & $t \angle 8$ & $8 S \mathcal{}>$ & $0 Z I^{\prime} I>$ & !g \\
\hline $000^{\circ} 80 z$ & $000^{\prime} \mathrm{ZSI}$ & $000^{\prime} 18 \mathrm{I}$ & $000^{\circ} 6 \varepsilon z$ & IV \\
\hline 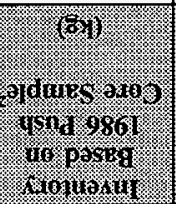 & 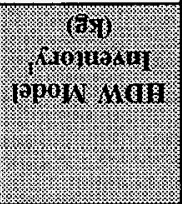 & 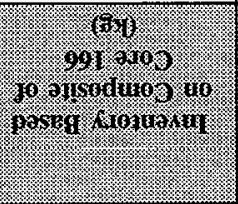 & 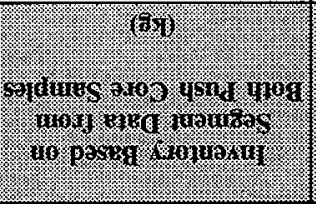 & agrove \\
\hline
\end{tabular}

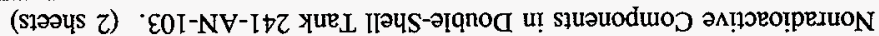

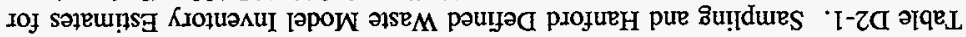


Table D2-1. Sampling and Hanford Defined Waste Model Inventory Estimates for Nonradioactive Components in Double-Shell Tank 241-AN-103. (2 sheets)

\begin{tabular}{|c|c|c|c|c|}
\hline inarie & 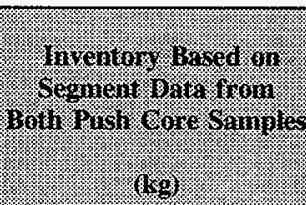 & 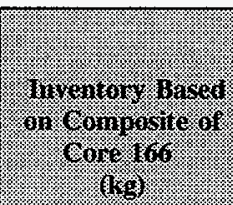 & 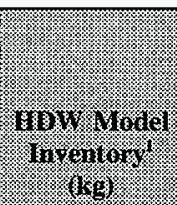 & 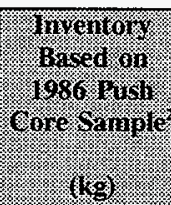 \\
\hline $\mathrm{Zr}$ & $<113$ & $<42.9$ & 566 & 426 \\
\hline$\%$ water & 48 & 41.0 & 32.6 & NR \\
\hline $\begin{array}{l}\text { density, } \\
\text { g/mL }\end{array}$ & 1.54 & 1.62 & 1.60 & 1.6 \\
\hline
\end{tabular}

Notes:

$$
\begin{aligned}
& \text { NR = not reported } \\
& \text { 'Agnew et al. (1997) } \\
& { }^{2} \text { Hendrickson (1994) }
\end{aligned}
$$

Table D2-2. Sampling and Hanford Defined Waste Model Inventory Estimates for

\begin{tabular}{|c|c|c|c|c|}
\hline 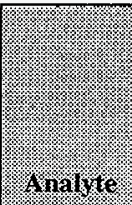 & 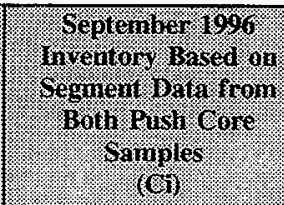 & 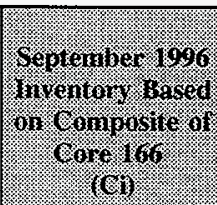 & (l) & 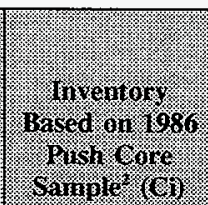 \\
\hline${ }^{3} \mathrm{H}$ & NR & 9.31 & 805 & 8.8 \\
\hline${ }^{14} \mathrm{C}$ & NR & NR & 105 & 7.2 \\
\hline${ }^{59} \mathrm{Ni}$ & NR & NR & 7.58 & NR \\
\hline${ }^{60} \mathrm{Co}$ & NR & $<112$ & 139 & 43.5 \\
\hline${ }^{79} \mathrm{Se}$ & NR & NR & 12.3 & 156 \\
\hline${ }^{90} \mathrm{Sr}$ & NR & 8,030 & 444,000 & 38,000 \\
\hline${ }^{90} \mathrm{Y}$ & NR & 8,030 & 444,000 & NR \\
\hline${ }^{99} \mathrm{Tc}$ & NR & 456 & 784 & 616 \\
\hline${ }^{106} \mathrm{Ru}$ & NR & NR & 0.0533 & 0.627 \\
\hline${ }^{129} I$ & NR & 8.32 & 1.51 & 1.88 \\
\hline${ }^{134} \mathrm{Cs}$ & NR & NR & 189 & 20.8 \\
\hline
\end{tabular}
Radioactive Components in Double-Shell Tank 241-AN-103

(Decayed to January 1, 1994). (2 sheets) 


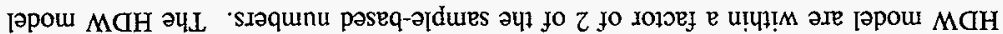

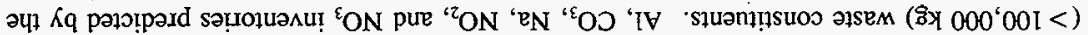

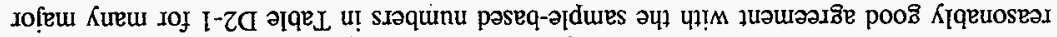

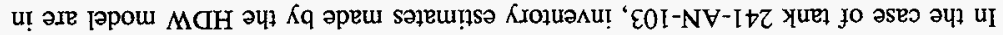

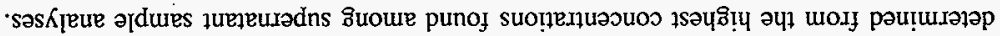

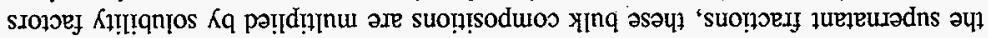

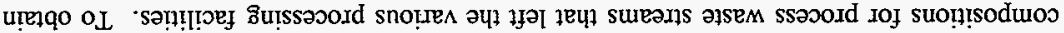

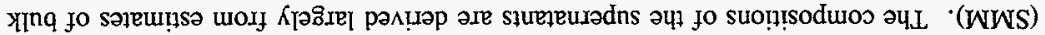

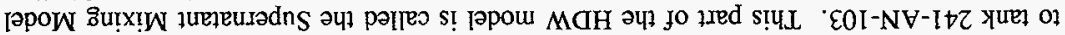

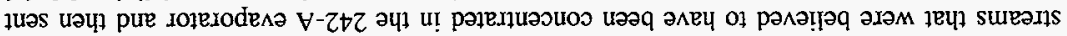

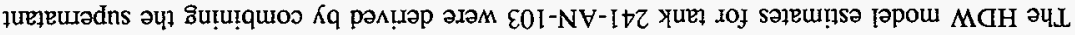

(๖66I) uоsжютриән (L66I) ']e do Mauş ,

:SOION

\begin{tabular}{|c|c|c|c|c|}
\hline $60^{\circ} \mathrm{I}$ & $\mathcal{E 9 8 ^ { \circ }} 0$ & $0^{\circ} \varepsilon \mathrm{I}>$ & $\mathrm{dN}$ & uว \\
\hline ZNN & $\tau \varepsilon 90^{\circ} 0$ & $58^{\circ} \nabla>$ & $\mathrm{dN}$ & $u \supset_{\varepsilon \downarrow z}$ \\
\hline $22 \cdot 8$ & $80 \mathcal{E}$ & $L \cdot I Z>$ & $\mathrm{XN}$ & $\mathrm{wV}_{\mathrm{IPZ}}$ \\
\hline $88^{\circ} 9$ & 782 & $\mathcal{E} Z I$ & $\mathrm{yN}$ & $\mathrm{n}_{\mathbf{d}_{0+2 / 6 \varepsilon Z}}$ \\
\hline $190^{\circ} 0$ & $78^{\circ} \nabla$ & $\mathrm{dN}$ & $\mathrm{ZN}$ & $\Omega_{8 \varepsilon z}$ \\
\hline$I \mathcal{E}^{\circ} \mathcal{E}$ & $9 \cdot Z \mathrm{I}$ & $\mathrm{dN}$ & $\mathrm{HN}$ & $\mathrm{n}_{\mathbf{d}_{8 \varepsilon z}}$ \\
\hline$\triangle Z \angle O^{\circ} O$ & $28^{\circ} 2$ & $0^{\circ} I \varepsilon>$ & $\mathrm{GN}$ & $\mathrm{d}_{\mathbf{N}_{L E Z}}$ \\
\hline $86 \varepsilon 00^{\circ} 0$ & $681^{\circ} 0$ & $\mathbf{Z N}$ & $\mathrm{HN}$ & $\Omega_{s \varepsilon \varepsilon}$ \\
\hline $26 \mathrm{I}^{\circ} 0$ & $\angle 8^{\circ} \mathrm{t}$ & $\mathrm{XN}$ & YN & $\Omega_{t \varepsilon \varepsilon}$ \\
\hline $\mathrm{dN}$ & 166 & $00 \varepsilon^{\prime} \varepsilon>$ & $\mathrm{UN}$ & $\mathrm{n}_{\exists_{S \mathrm{I}}}$ \\
\hline $\mathrm{dN}$ & $00 z^{\prime} z$ & $709>$ & $\mathbf{d N}$ & $\mathrm{n}_{\text {G }}$ \\
\hline $90+$ BII $\mathrm{Z}$ & $000^{\circ} 9 \nabla L$ & $90+36 \mathrm{I}^{\circ} \mathrm{Z}$ & $\mathrm{ZN}$ & $\mathrm{eg}_{\mathbf{w L E L}_{L}}$ \\
\hline $90+322 \cdot 2$ & $000^{\circ} 68 L$ & $90+\exists I \varepsilon z$ & $\mathrm{YN}$ & $s \mathcal{D}_{L \varepsilon !}$ \\
\hline 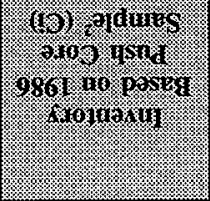 & 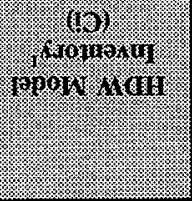 & 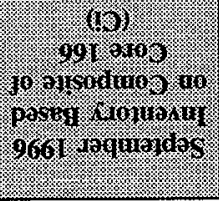 & 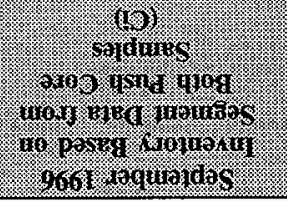 & 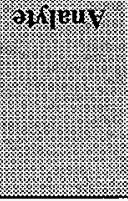 \\
\hline
\end{tabular}

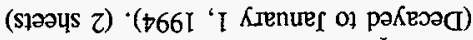

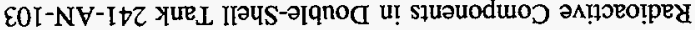

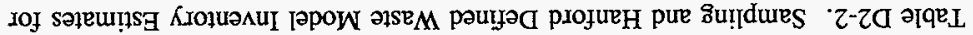


hydroxide estimate is 2.14 times higher than the sample-based result. $\mathrm{Cr}, \mathrm{Fe}$, and $\mathrm{Ni}$ inventory estimates made by the HDW model are higher than the sample-based inventories because the HDW model overestimates the rate of corrosion in process vessels and storage tanks and it overestimates the solubility of these species in the alkaline environment of Hanford Site tank waste. The model estimate for $\mathrm{Ca}$ is much higher than the sample-based result; this is a product of the model overestimating the concentration of $\mathrm{Ca}$ in raw water. The agreement for ${ }^{90} \mathrm{Sr}$ and ${ }^{137} \mathrm{Cs}$ is also not close.

An earlier push core sample taken in 1986 offers more data to make comparisons with. Results of this sampling event are published in Hendrickson (1994) and are shown in column 5 of Table D2-1 $1^{1}$. Less than values were reported as real values in the Hendrickson report. For major components, the agreement between the 1986 push core sample and samples taken more recently is very good (RPDs of less than 30 percent). There is less agreement for minor components, including $\mathrm{F}, \mathrm{PO}_{4}, \mathrm{Si}, \mathrm{SO}_{4}$, and $\mathrm{U}$, and TOC. Pre-1989 sample data has not been validated and should be used with caution. However, the 1986 push core samples suggest that the inventories calcuiated from the 1996 push core sample data are valid.

\section{D3.0 COMPONENT INVENTORY EVALUATION}

In Table D3-1, concentrations for the supernatant, slurry, and crust portions based on the means of the segment data for both cores and the composite of core 166 from Appendix B are shown. The calculated liquid values from the one-way ANOVA (Table B3-9) divided by the density of the convective layer $(1.46 \mathrm{~g} / \mathrm{mL})$ were used to derive the supernatant inventory. The results from the nested ANOVA without the riser term were used to derive the slurry inventory. Core 166 was taken from riser $12 \mathrm{~A}$ and core 167 was taken from riser 21A. Mean concentrations from segment data are based on segments from both cores. Mean concentrations from composite data are based on a composite of Core 166 only.

\footnotetext{
${ }^{1}$ Inventories based on a volume of $3,622 \mathrm{~kL}(957 \mathrm{kgal})$.
} 
Table D3-1. Comparison of September 1996 Push Core Sample Concentrations. ${ }^{1}$ (2 sheets)

\begin{tabular}{|c|c|c|c|c|c|}
\hline $\begin{array}{l}2.0 \\
.\end{array}$ & 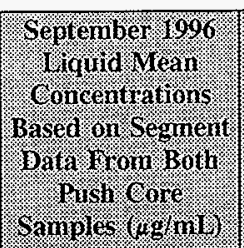 & 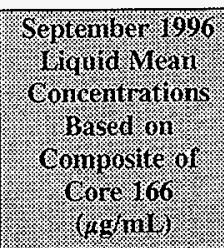 & 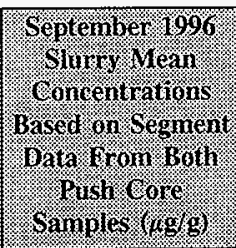 & 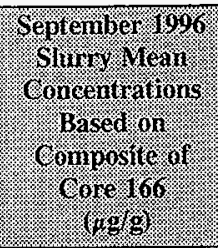 & 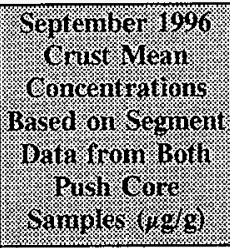 \\
\hline $\mathrm{Al}$ & 36,600 & 32,100 & $58,200=$ & 38,200 & 85,400 \\
\hline $\mathrm{Bi}$ & $<261$ & $<120$ & $<225$ & $<28.2$ & $<222$ \\
\hline$\overline{\mathrm{Ca}}$ & $<261$ & $<120$ & $<2,200$ & $<56.3$ & $<222$ \\
\hline$\overline{\mathrm{Cl}}$ & 8,950 & 9,890 & 3,820 & 3,730 & 3,360 \\
\hline $\mathrm{CO} 3$ & NR & 5,800 & NR & 28,850 & NR \\
\hline $\mathrm{Cr}$ & 632 & 662 & 552 & 538 & 890 \\
\hline $\bar{F}$ & 634 & 596 & 982 & 4,140 & 709 \\
\hline $\mathrm{Fe}$ & $<131$ & $<60.1$ & $<48.5^{2}$ & $<48.5$ & $<1,050$ \\
\hline$\overline{\mathrm{Hg}}$ & NR & $\mathrm{NR}$ & $\mathrm{NR}$ & $\mathrm{NR}$ & $\mathrm{NR}$ \\
\hline$\overline{\mathrm{K}}$ & 15,600 & 17,300 & 6,010 & 6,540 & 6,150 \\
\hline $\mathrm{La}$ & $<131$ & $<60.1$ & $<113$ & $<28.2$ & $<111$ \\
\hline $\mathrm{Mn}$ & $<26.1$ & $<12$ & $<22.5$ & $<5.57$ & $<22.1$ \\
\hline $\mathrm{Na}$ & 289,000 & 263,000 & 258,000 & 217,000 & 269,000 \\
\hline $\mathrm{Ni}$ & $<52.4$ & $<24$ & $<45.1$ & $<11.3$ & $<44.6$ \\
\hline $\mathrm{NO} 2$ & 161,000 & 133,000 & 67,800 & 73,400 & 71,300 \\
\hline NO3 & 159,000 & 117,000 & 171,000 & 132,000 & 145,000 \\
\hline
\end{tabular}




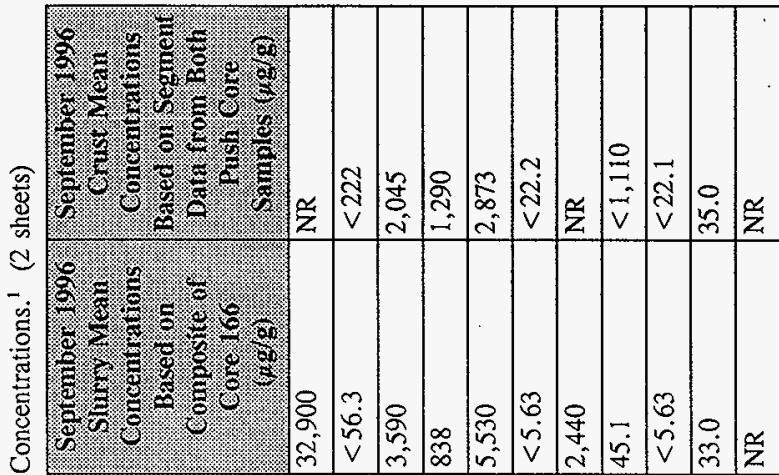

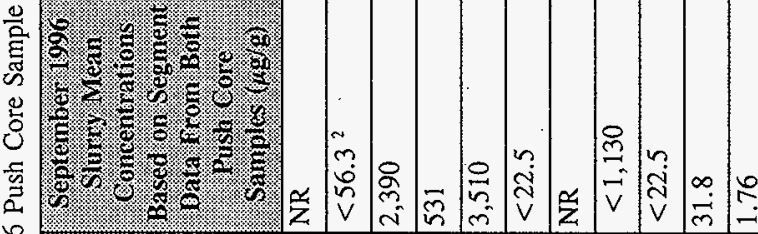

وั

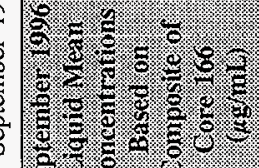

\%

:

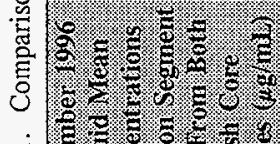

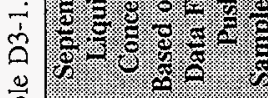

है⿴囗十

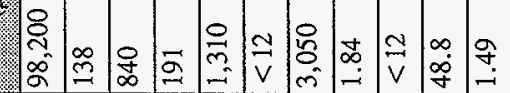

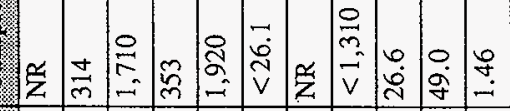

$\frac{3}{8}$

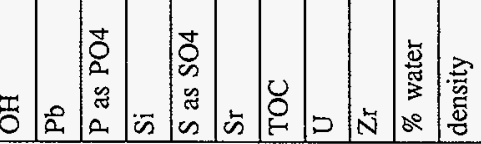

을.

言 五

용

牙 总

蹗

$\frac{\ddot{3}}{\stackrel{3}{0}}$ 
Acid and fusion digests were performed on the individual segments to obtain cation concentrations, but only acid digests were performed on the core composite. Fusion digests with $\mathrm{KOH}$ or $\mathrm{NaOH}$ are usually a more complete method of dissolving solids than acid dissolution. The detection limits from the fusion procedure are up to 10 times higher than the limits set in the acid digestion procedure. Although complete digestion does not always occur with the use of acid, the less-than values reported from this procedure for many analytes are more accurate than the fusion digest less-than values.

The mean concentrations from the segment data are a better source of information than the core composite data. The mean concentrations calculated from the segment data were obtained from a large number of data points as opposed to one, and these segments were treated with the more complete fusion digest as opposed to acid digest. Also, the segment data was taken from two cores as opposed to one. The largest discrepancies between the two sets of data occur for $\mathrm{F}, \mathrm{NO}_{3}$ and $\mathrm{SO}_{4}$. The ratio of the segment inventory to the composite inventory for these three components is $0.33,1.29$, and 0.76 , respectively. The fluoride concentration from the core composite $(4,140 \mu \mathrm{g} / \mathrm{g})$ is at least 3 times greater than all the individual segment analyses for fluoride with the exception of segment 17 of core 167 and is therefore not reliable. The differences for $\mathrm{NO}_{3}$ and $\mathrm{SO}_{4}$ are not significant; the segment-based data is assumed to be more accurate because there were more analyses.

The composite core data contains information on several chemicals not found in the segment-based data or the analysis of the crust. These analytes include $\mathrm{CO}_{3}, \mathrm{OH}$, and TOC. The composite data is also the only source of individual radionuclide concentrations from the 1996 sampling event. The composite concentrations were used in these instances for both the segment-based inventory and the crust layer.

The best-basis inventory is therefore a combination of both segment level data and core composite data. Core composite concentrations are used for components not included in the segment analyses.

Concentrations for the $43.8 \mathrm{~cm}$ (17 in.) of crust are included in Table D3-1 which shows that this material is similar to the slurry below it, except for the higher aluminum concentration in the crust.

\section{D4.0 DEFINE THE BEST-BASIS AND ESTABLISH COMPONENT INVENTORIES}

Inventories based on the September 1996 sampling event should serve as the basis for the best estimate inventory to tank $241-\mathrm{AN}-103$ for the following reasons:

1. The September 1996 sampling event provides the most recent data for the waste.

2. Estimates based on the 1988 core sampling event agree with the September 1996 data. 
3. The HDW model estimates, although in reasonable agreement with the 1996 sampling data, do not check as well with the 1988 data.

Total Hydroxide. Once the best-basis inventories were determined, the hydroxide inventory was calculated by performing a charge balance with the valence of other analytes. In some cases, this approach requires that other analyte (for example, sodium or nitrate) inventories be adjusted to achieve the charge balance. During such adjustments, significant figures are retained. This charge balance approach is consistent with that used by Agnew et al. (1997).

Best-basis inventory estimates for tank 241-AN-103 are presented in Tables D4-1 through D4-8. A tank volume of $3,622 \mathrm{~kL}$ (957 kgal) was used to generate the sample-based and HDW inventories (Hanlon 1997). Radionuclide values are decayed to January 1, 1994.

The inventory values reported in Tables D4-1 and D4-2 are subject to change. Refer to the Tank Characterization Database (TCD) for the most current inventory values.

Best-basis tank inventory values are derived for 46 key radionuclides (as defined in Section 3.1 of Kupfer et al. 1997), all decayed to a common report date of January 1, 1994. Often, waste sample analyses have only reported ${ }^{90} \mathrm{Sr},{ }^{137} \mathrm{Cs},{ }^{239 / 240} \mathrm{Pu}$, and total uranium, or (total beta and total alpha), while other key radionuclides such as ${ }^{60} \mathrm{Co},{ }^{99} \mathrm{Tc},{ }^{129} \mathrm{~T},{ }^{154} \mathrm{Eu}$, ${ }^{155} \mathrm{Eu}$, and ${ }^{241} \mathrm{Am}$, etc., have been infrequently reported. For this reason it has been necessary to derive most of the $46 \mathrm{key}$ radionuclides by computer models. These models estimate radionuclide activity in batches of reactor fuel, account for the split of radionuclides to various separations plant waste streams, and track their movement with tank waste transactions. (These computer models are described in Kupfer et al. 1997, Section 6.1 and in Watrous and Wootan 1997). Model generated values for radionuclides in any of 177 tanks are reported in the Hanford Defined Waste Rev. 4 model results (Agnew et al. 1997). The best-basis value for any one analyte may be either a model result or a sample or engineering assessment-based result, if available. (No attempt has been made to ratio or normalize model results for all 46 radionuclides when values for measured radionuclides disagree with the model.) For a discussion of typical error between model derived values and sample derived values, see Kupfer et al..1997, Section 6.1.10. 


\begin{tabular}{|c|c|c|c|}
\hline & $s$ & $08 t^{\prime} \tau>$ & ${ }^{\text {TVLOI }} \mathrm{n}$ \\
\hline \multirow[t]{6}{*}{ 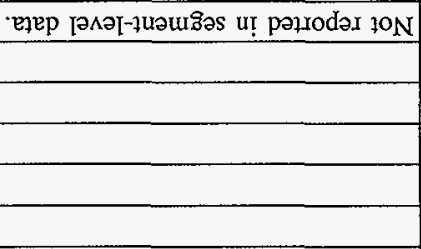 } & $S$ & $O L L^{\prime} S$ & DOL \\
\hline & S & $t \cdot 6 t>$ & IS \\
\hline & $S$ & $O \varepsilon 9^{6} \varepsilon$ & ${ }^{\mathrm{t} O S} \mathrm{se} \mathrm{S}$ \\
\hline & $s$ & 699 & !S \\
\hline & S & $0 \star z^{6} \varepsilon$ & ${ }^{\mathrm{D}} \mathrm{Od} \mathrm{se} \mathrm{d}$ \\
\hline & $\mathrm{S}$ & $\nabla 6 \varsigma>$ & $9 d$ \\
\hline \multirow[t]{11}{*}{ 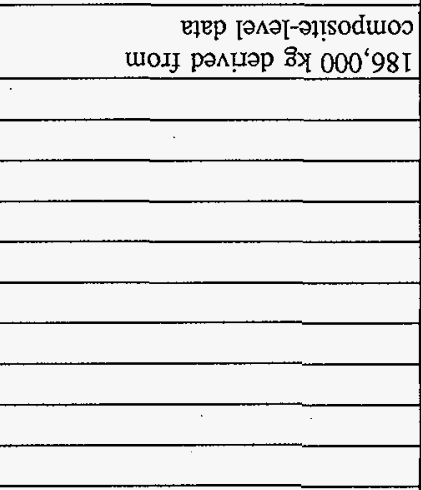 } & D) & $000^{6} \triangleright t \varepsilon$ & $\mathrm{HO}$ \\
\hline & $S$ & $000^{\circ} \mathrm{IOE}$ & ${ }^{\varepsilon} \mathrm{ON}$ \\
\hline & $s$ & $000^{\circ} \pm 0 \varepsilon$ & ${ }^{2} \mathrm{ON}$ \\
\hline & S & $2: 66>$ & !n \\
\hline & $s$ & $000^{\circ} \angle t s$ & EN \\
\hline & $S$ & $+6 t>$ & uN \\
\hline & s) & $8+z>$ & $\mathrm{EI}$ \\
\hline & S & $009^{\circ} 6 z$ & $\mathrm{X}$ \\
\hline & $S$ & $8 \nabla z>$ & $\partial \underline{H}$ \\
\hline & $\mathrm{s}$ & $002^{6} 1$ & $\underline{H}$ \\
\hline & S & $00 z^{\prime} \mathrm{I}$ & ID \\
\hline \multirow[t]{5}{*}{ 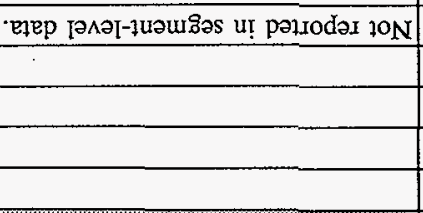 } & $\mathrm{S}$ & $000^{\prime} \mathrm{II}$ & EOO se JII \\
\hline & $s$ & $006^{6} 91$ & โอ \\
\hline & $\mathrm{s}$ & $s 6 t>$ & ED \\
\hline & $\mathrm{s}$ & $\varsigma 6 t>$ & !g \\
\hline & $\mathrm{s}$ & $00 \varepsilon^{\prime} 69$ & IV \\
\hline Imanos & (46:5: & 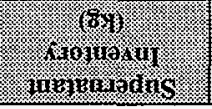 & (3) 1404 \\
\hline
\end{tabular}

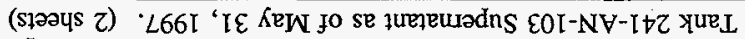

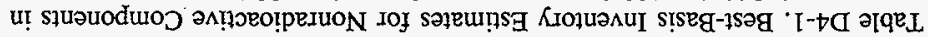


Table D4-1. Best-Basis Inventory Estimates for Nonradioactive Components in Tank 241-AN-103 Supernatant as of May 31, 1997. (2 sheets)

\begin{tabular}{|c|c|c|c|}
\hline (2) & 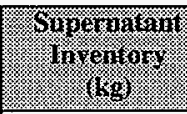 & $65,10.6 \%$. & rominen \\
\hline $\mathrm{Zr}$ & $<50.3$ & $S$ & \\
\hline$\%$ Water & 51.1 & $\mathbf{S}$ & \\
\hline Density, $\mathrm{g} / \mathrm{mL}$ & 1.46 & $\mathrm{~s}$ & \\
\hline
\end{tabular}

\section{Notes:}

Volume basis $=1,885 \mathrm{~kL}$ supernatant $(498 \mathrm{kgal})$

'S = Sample-based, $\mathrm{M}=\mathrm{HDW}$ model-based, $\mathrm{C}=$ Calculated by charge balance; includes oxides as hydroxides, not including $\mathrm{CO}_{3}, \mathrm{NO}_{2}, \mathrm{NO}_{3}, \mathrm{PO}_{4}, \mathrm{SO}_{4}$, and $\mathrm{SiO}_{3}$, and $\mathrm{E}=$ Engineering assessment-based. 
Table D4-2. Best-Basis Inventory Estimates for Radioactive Components in Tank 241-AN-103 Supernatant as of May 31, 1997

(Decayed to January 1, 1994).

\begin{tabular}{|c|c|c|c|}
\hline 4nalute: & 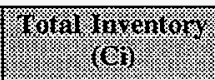 & 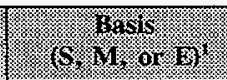 & 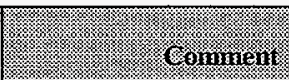 \\
\hline${ }^{60} \mathrm{Co}$ & $<55.7$ & $\mathrm{~S}$ & \\
\hline${ }^{90} \mathrm{Sr}$ & 42.0 & $S$ & \\
\hline${ }^{900} \mathrm{Y}$ & 42.0 & $S$ & Referenced to ${ }^{90} \mathrm{Sr}$. \\
\hline${ }^{99} \mathrm{Tc}$ & 0.318 & $S$ & . \\
\hline${ }^{129} \mathrm{I}$ & 0.717 & $S$ & \\
\hline${ }^{137} \mathrm{Cs}$ & $1.49 E+06$ & $S$ & \\
\hline${ }^{137 m} \mathrm{Ba}$ & $1.41 \mathrm{E}+06$ & $S$ & Referenced to ${ }^{137} \mathrm{Cs}$ \\
\hline${ }^{154} \mathrm{Eu}$ & $<367$ & $S$ & - \\
\hline${ }^{155} \mathrm{Eu}$ & $<1,630$ & $s$ & \\
\hline${ }^{237} \mathrm{~Np}$ & $<0.048$ & $S$ & \\
\hline${ }^{239 / 240} \mathrm{Pu}$ & 7.23 & $S$ & \\
\hline${ }^{241} \mathrm{Am}$ & $<11.7$ & $S$ & \\
\hline${ }^{243} \mathrm{Cm}$ & $<0.52$ & $S$ & . \\
\hline${ }^{244} \mathrm{Cm}$ & $<12.5$ & $S$ & \\
\hline
\end{tabular}

Notes:

Volume basis $=1,885 \mathrm{~kL}$ supernatant $(498 \mathrm{kgal})$

'S = Sample-based, $M=H D W$ model-based, and $E=$ Engineering assessment-based. 
Table D4-3. Best-Basis Inventory Estimates for Nonradioactive Components in Tank 241-AN-103 Salt Slurry as of May 31, 1997. (2 sheets)

\begin{tabular}{|c|c|c|c|}
\hline inatite & 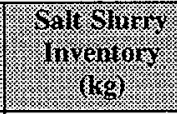 & 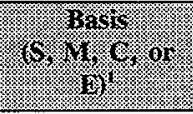 & ( enminat \\
\hline $\mathrm{Al}$ & 148,000 & $\mathrm{~S}$ & \\
\hline $\mathrm{Bi}$ & $<574$ & S & \\
\hline $\mathrm{Ca}$ & $<5,610$ & $S$ & \\
\hline $\mathrm{Cl}$ & 9,740 & $S$ & \\
\hline TIC as $\mathrm{CO}_{3}$ & 73,600 & $S$ & Not reported in segment-level data \\
\hline $\mathrm{Cr}$ & 1,410 & $S$ & \\
\hline F & 2,500 & $S$ & \\
\hline $\mathrm{Fe}$ & $<251$ & $S$ & " \\
\hline $\mathrm{K}$ & 15,300 & $S$ & \\
\hline $\mathrm{La}$ & $<288$ & $\mathrm{~S}$ & \\
\hline $\overrightarrow{\mathrm{Mn}}$ & 57.4 & $S$ & \\
\hline $\mathrm{Na}$ & 658,000 & $S$ & \\
\hline $\mathrm{Ni}$ & 115 & $S$ & \\
\hline $\mathrm{NO}_{2}$ & 173,000 & $S$ & \\
\hline $\mathrm{NO}_{3}$ & 436,000 & $S$ & \\
\hline $\mathrm{OH}$ & 577,000 & C & $\begin{array}{l}83,900 \mathrm{~kg} \text { derived from } \\
\text { composite-level data }\end{array}$ \\
\hline $\mathrm{Pb}$ & $<144$ & $S$ & \\
\hline $\mathrm{PO}_{4}$ & 6,080 & $S$ & \\
\hline$\overline{\mathrm{Si}}$ & 1,350 & $S$ & . \\
\hline $\mathrm{SO}_{4}$ & 8,940 & $S$ & \\
\hline Sr & $<57.4$ & $S$ & : \\
\hline TOC & 6,220 & $S$ & Not reported in segment-level data \\
\hline $\mathrm{U}_{\text {TOTAL }}$ & $<2,880$ & $S$ & Not reported in segment-level data \\
\hline
\end{tabular}


Table D4-3. Best-Basis Inventory Estimates for Nonradioactive Components in Tank 241-AN-103 Salt Slurry as of May 31, 1997. (2 sheets)

\begin{tabular}{|c|c|c|c|}
\hline : & 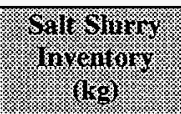 & 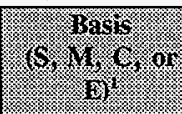 & Cominitur \\
\hline $\mathrm{Zr}$ & $<57.4$ & $S$ & \\
\hline$\%$ Water & 31.8 & $S$ & \\
\hline Density, $\mathrm{g} / \mathrm{mL}$ & 1.62 & $S$ & \\
\hline
\end{tabular}

Notes:

Volume basis $=1,552 \mathrm{~kL}$ supernatant $(410 \mathrm{kgal})$

is = Sample-based, $\mathrm{M}=\mathrm{HDW}$ model-based, $\mathrm{C}=$ Calculated by charge balance; includes oxides as hydroxides, not including $\mathrm{CO}_{3}, \mathrm{NO}_{2}, \mathrm{NO}_{3}, \mathrm{PO}_{4}, \mathrm{SO}_{4}$, and $\mathrm{SiO}_{3}$, and $\mathrm{E}=$ Engineering assessment-based. 
Table D4-4. Best-Basis Inventory Estimates for Radioactive Components in Tank 241-AN-103 Salt Slurry as of May 31, 1997

(Decayed to January 1, 1994).

\begin{tabular}{|c|c|c|c|}
\hline - & 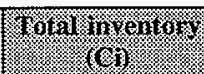 & 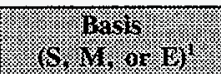 & (cominent. \\
\hline${ }^{3} \mathrm{H}$ & 8.44 & $S$ & \\
\hline${ }^{60} \mathrm{Co}$ & $<51.4$ & $S$ & \\
\hline 90 $\mathrm{Sr}$ & 7,240 & $S$ & \\
\hline${ }^{90} \mathrm{Y}$ & 7,240 & $S$ & Referenced to ${ }^{90} \mathrm{Sr}$ \\
\hline${ }^{99} \mathrm{Tc}$ & 413 & $S$ & \\
\hline${ }^{129} \mathrm{I}$ & 6.89 & $S$ & \\
\hline${ }^{137} \mathrm{Cs}$ & 744,000 & $S$ & \\
\hline${ }^{137 \mathrm{~m}} \mathrm{Ba}$ & 707,000 & $S$ & Referenced to ${ }^{137} \mathrm{Cs}$ \\
\hline${ }^{154} \mathrm{Eu}$ & $<215$ & $S$ & \\
\hline${ }^{155} \mathrm{Eu}$ & $<1,510$ & $S$ & \\
\hline${ }^{237} \mathrm{~Np}$ & $<28.1$ & $S$ & \\
\hline${ }^{239 / 240} \mathrm{Pu}$ & 11.8 & $S$. & \\
\hline${ }^{241} \mathrm{Am}$ & 9.04 . & $S$ & \\
\hline${ }^{243} \mathrm{Cm}$ & $<3.93$ & $\bar{S}$ & \\
\hline${ }^{244} \mathrm{Cm}$ & $<94.3$ & $S$ & \\
\hline
\end{tabular}

Notes:

Volume basis $=1,552 \mathrm{~kL}$ supernatant $(410 \mathrm{kgal})$

's = Sample-based, $M=$ HDW model-based, and $E=$ Engineering assessment-based. 
Table D4-5. Best-Basis Inventory Estimates for Nonradioactive Components in Tank 241-AN-103 Crust as of May 31, 1997. (2 sheets)

\begin{tabular}{|c|c|c|c|}
\hline (1) & 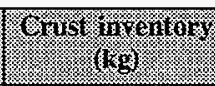 & 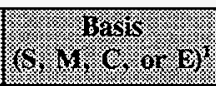 & (3. \\
\hline $\mathrm{Al}$ & 22,700 & $S$ & \\
\hline $\mathrm{Bi}$ & $<58.9$ & $S$ & \\
\hline $\mathrm{Ca}$ & $<58.9$ & $S$ & \\
\hline $\mathrm{Cl}$ & 892 & $S$ & \\
\hline TIC as $\mathrm{CO}_{3}$ & 7,660 & $S$ & $\begin{array}{l}\text { Assumed to be equal to slurry } \\
\text { concentration }\end{array}$ \\
\hline $\mathrm{Cr}$ & 236 & $S$ & ? \\
\hline $\mathrm{F}$ & 188 & $S$ & \\
\hline $\mathrm{Fe}$ & 279 & $S$ & 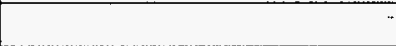 \\
\hline $\mathrm{K}$ & 1,630 & $\mathrm{~S}$ & \\
\hline $\mathrm{La}$ & $<279$ & $S$ & \\
\hline$\overline{\mathrm{Mn}}$ & 5.87 & $S$ & \\
\hline $\mathrm{Na}$ & 71,400 & $\mathrm{~s}$ & \\
\hline $\mathrm{Ni}$ & 11.8 & $S$ & \\
\hline $\mathrm{NO}_{2}$ & 18,900 & $S$ & \\
\hline $\mathrm{NO}_{3}$ & 38,500 & $S$ & \\
\hline$\overline{\mathrm{OH}}$ & 78,100 & C & $\begin{array}{l}8,730 \mathrm{~kg} \text { derived from composite } \\
\text { slurry concentration }\end{array}$ \\
\hline $\mathrm{Pb}$ & $<58.9$ & $S$ & \\
\hline $\mathrm{PO}_{4}$ & 543 & $S$ & \\
\hline $\mathrm{Si}$ & 342 & S & \\
\hline $\mathrm{SO}_{4}$ & 763 & $S$ & \\
\hline $\mathrm{Sr}$ & $<5.89$ & $S$ & \\
\hline TOC & 648 & S & $\begin{array}{l}\text { Assumed to be equal to slurry } \\
\text { concentration }\end{array}$ \\
\hline $\mathrm{U}_{\text {TOTAL }}$ & $<295$ & $S$ & \\
\hline
\end{tabular}




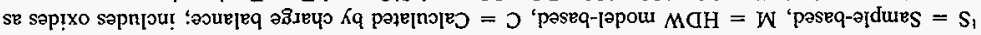

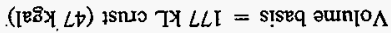

:SOION

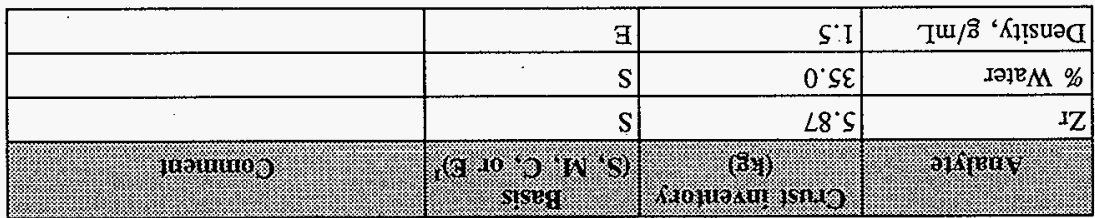

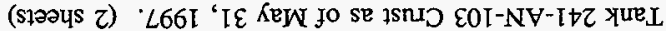

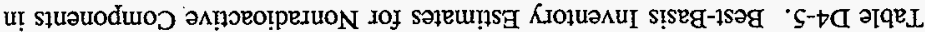


HNF-SD-WM-ER-702 Rev. 0

Table D4-6. Best-Basis Inventory Estimates for Radioactive Components in Tank 241-AN-103 Crust as of May 31, 1997

(Decayed to January 1, 1994).

\begin{tabular}{|c|c|c|c|}
\hline : Analifo: & 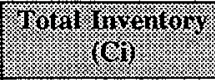 & 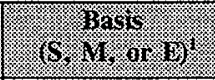 & U Corment \\
\hline${ }^{3} \mathrm{H}$ & 0.878 & $S$ & \\
\hline${ }^{60} \mathrm{Co}$ & $<5.35$ & $S$ & \\
\hline${ }^{90} \mathrm{Sr}$ & 753 & $S$ & \\
\hline${ }^{90} \mathrm{Y}$ & 753 & $s$ & Referenced to ${ }^{90} \mathrm{Sr}$ \\
\hline${ }^{99} \mathrm{TC}$ & 43.0 & $S$ & \\
\hline${ }^{129} \mathrm{I}$ & 0.717 & $S$ & \\
\hline${ }^{137} \mathrm{Cs}$ & 77,400 & $S$ & \\
\hline${ }^{137 \mathrm{~m}} \mathrm{Ba}$ & 73,500 & $S$ & Referenced to ${ }^{137} \mathrm{Cs}$ \\
\hline${ }^{154} \mathrm{Eu}$ & $<22.3$ & $S$ & \\
\hline${ }^{155} \mathrm{Eu}$ & $<157$ & $S$ & \\
\hline${ }^{237} \mathrm{~Np}$ & $<2.92$ & $S$ & \\
\hline${ }^{239 / 240} \mathrm{Pu}$ & 0.480 & $S$ & \\
\hline${ }^{241} \mathrm{Am}$ & 0.941 & $S$ & \\
\hline${ }^{243} \mathrm{Cm}$ & $<0.409$ & $S$ & \\
\hline${ }^{244} \mathrm{Cm}$ & $<9.81$ & $S$ & \\
\hline
\end{tabular}

Notes:

Volume basis $=177 \mathrm{~kL}$ crust $(47 \mathrm{kgal})$

${ }^{1} S=$ Sample-based, $M=$ HDW model-based, and $E=$ Engineering assessment-based. 
Table D4-7. Best-Basis Total Inventory Estimates for Nonradioactive Components in Tank 241-AN-103 as of May 31,1997. (2 sheets)

\begin{tabular}{|c|c|c|c|}
\hline : & 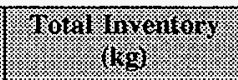 & 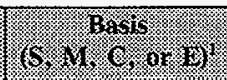 & 1. \\
\hline $\mathrm{Al}$ & 239,000 & $\mathrm{~S}$ & \\
\hline $\mathrm{Bi}$ & $<1,120$ & $S$ & \\
\hline $\mathrm{Ca}$ & $<6,100$ & $s$ & Slurry $=977 \mathrm{~kg}$ \\
\hline $\mathrm{Cl}$ & 27,500 & $\mathrm{~s}$ & \\
\hline TIC as $\mathrm{CO}_{3}$ & 84,600 & $S$ & \\
\hline $\mathrm{Cr}$ & 2,820 & $S$ & \\
\hline $\bar{F}$ & 3,860 & $S$ & \\
\hline $\mathrm{Fe}$ & $<774$ & $\mathrm{~s}$ & \\
\hline $\bar{K}$ & 46,300 & $\mathrm{~s}$ & \\
\hline $\mathrm{La}$ & $<811$ & $S$ & \\
\hline$\overline{\mathrm{Mn}}$ & $<112$ & $\mathrm{~S}$ & \\
\hline $\mathrm{Na}$ & $1.27 \mathrm{E}+06$ & $S$ & \\
\hline $\mathrm{Ni}$ & $<225$ & $s$ & \\
\hline $\mathrm{NO}_{2}$ & 494,000 & $S$ & \\
\hline $\mathrm{NO}_{3}$ & 771,000 & $S$ & \\
\hline $\mathrm{OH}$ & 999,000 & $\mathrm{C}$ & $\begin{array}{l}278,000 \mathrm{~kg} \text { calculated from } \\
\text { sample data }\end{array}$ \\
\hline $\mathrm{Pb}$ & $<795$ & $s$ & \\
\hline $\mathrm{PO}_{4}$ & 9,800 & $S$ & \\
\hline$\overline{\mathrm{Si}}$ & 2,350 & $S$ & \\
\hline $\mathrm{SO}_{4}$ & 13,200 & $S$ & \\
\hline $\mathrm{Sr}$ & $<112$ & $S$ & \\
\hline TOC & 12,000 & $S$ & \\
\hline $\mathrm{U}_{\text {TOTAL }}$ & $<5,620$ & $S$ & \\
\hline
\end{tabular}




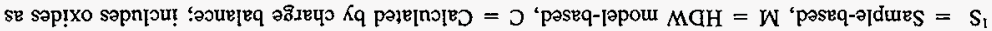

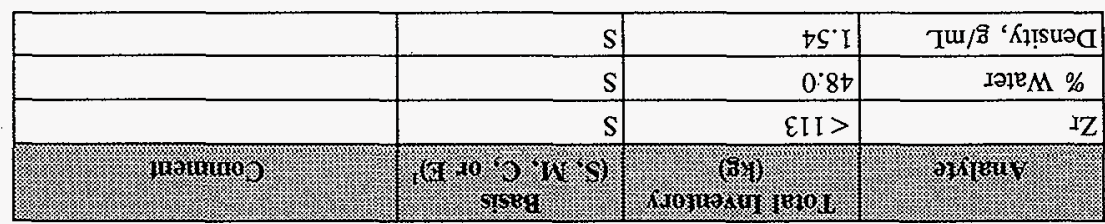

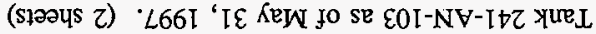

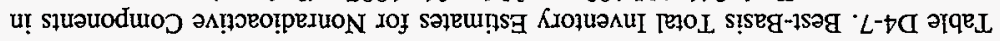


HNF-SD-WM-ER-702 Rev. 0

Table D4-8. Best-Basis Inventory Estimates for Radioactive Components in

Tank 241-AN-103 as of May 31, 1997

(Decayed to January 1, 1994). (2 sheets)

\begin{tabular}{|c|c|c|c|}
\hline - & $10401 / 10$ contory & 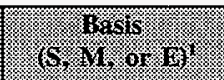 & Corninenit \\
\hline${ }^{3} \mathrm{H}$ & 9.31 & $S$ & Slurry/Crust only \\
\hline${ }^{14} \mathrm{C}$ & 112 & $S$ & \\
\hline${ }^{59} \mathrm{Ni}$ & 7.58 & $\mathbf{M}$ & \\
\hline${ }^{60} \mathrm{Co}$ & $<112$ & $S$ & \\
\hline${ }^{63} \mathrm{Ni}$ & 747 & $\mathrm{M}$ & \\
\hline${ }^{79} \mathrm{Se}$ & 12.3 & $\bar{M}$ & \\
\hline${ }^{90} \mathrm{Sr}$ & 8,030 & $S$ & \\
\hline${ }^{90} \mathrm{Y}$ & 8,030 & $S$ & Referenced to ${ }^{90} \mathrm{Sr}$ \\
\hline${ }^{93} \mathrm{Zr}$ & 59.6 & $\overline{\mathrm{M}}$ & \\
\hline${ }^{93 \mathrm{~m}} \mathrm{Nb}$ & 43.2 & $\overline{\mathrm{M}}$ & \\
\hline${ }^{99} \mathrm{Tc}$ & 456 & $S$ & \\
\hline${ }^{106} \mathrm{Ru}$ & 0.0533 & $\vec{M}$ & \\
\hline${ }^{113 m} \mathrm{Cd}$ & 302 & $M$ & \\
\hline${ }^{125} \mathrm{Sb}$ & 865 & $M$ & \\
\hline${ }^{126} \mathrm{Sn}$ & 18.8 & $\mathrm{M}$ & \\
\hline${ }^{129} \mathrm{I}$ & 8.32 & $S$ & \\
\hline${ }^{134} \mathrm{Cs}$ & 189 & $\mathrm{M}$ & \\
\hline${ }^{137} \mathrm{Cs}$ & $2.31 \mathrm{E}+06$ & $S$ & Referenced to ${ }^{137} \mathrm{Cs}$ \\
\hline${ }^{137 m} \mathrm{Ba}$ & $2.19 \mathrm{E}+06$ & $S$ & . \\
\hline${ }^{151} \mathrm{Sm}$ & 43,600 & $\mathrm{M}$ & \\
\hline${ }^{152} \mathrm{Eu}$ & 15.8 & $\bar{M}$ & . \\
\hline${ }^{154} \mathrm{Eu}$ & $<604$ & $S$ & \\
\hline${ }^{155} \mathrm{Eu}$ & $<3,300$ & $S$ & \\
\hline${ }^{226} \mathrm{Ra}$ & $5.81 \mathrm{E}-04$ & $\mathrm{M}$ & \\
\hline${ }^{227} \mathrm{Ac}$ & 0.00355 & $\mathrm{M}$ & \\
\hline${ }^{228} \mathrm{Ra}$ & 1.05 & $\mathrm{M}$ & \\
\hline${ }^{229} \mathrm{Th}$ & 0.0243 & $\mathrm{M}$ & \\
\hline${ }^{231} \mathrm{~Pa}$ & 0.0143 & $\mathrm{M}$ & \\
\hline${ }^{232} \mathrm{Th}$ & 0.103 & $M$ & \\
\hline
\end{tabular}




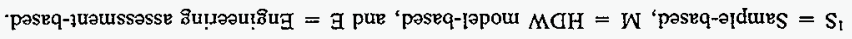

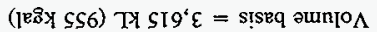

:SР1ON

\begin{tabular}{|c|c|c|c|}
\hline & $\mathrm{s}$ & $0^{\circ} \varepsilon\lceil>$ & $w \supset_{t p z}$ \\
\hline & $\mathrm{S}$ & $s 8^{\circ} \dagger>$ & 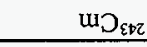 \\
\hline & N & $60200^{\circ} 0$ & 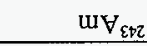 \\
\hline & N & $\angle 6+00^{\circ} 0$ & $\mathrm{n}_{\mathrm{d}_{2 \mathfrak{} 2}}$ \\
\hline & W & $\varepsilon Z 9^{\circ} 0$ & $w \partial_{\tau \uparrow \tau}$ \\
\hline & $\mathrm{W}$ & $090^{\circ} \mathrm{I}$ & $\mathrm{n}_{\mathrm{d}_{i+2}}$ \\
\hline $10+0.6=\sin I S$ & $\mathrm{~s}$ & $\angle \cdot I Z$ & $u_{v_{I t z}}$ \\
\hline & $\mathrm{s}$ & $\varepsilon \cdot 2 I$ & 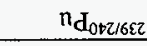 \\
\hline & $\mathrm{W}$ & $78^{\circ} \mathrm{t}$ & $\mathrm{n}_{8 \varepsilon \tau}$ \\
\hline & W & $9 \cdot 2 \mathrm{I}$ & $\mathrm{n}_{\mathrm{d}_{8 \varepsilon z}}$ \\
\hline & $\mathrm{s}$ & $0^{\circ}\lfloor\varepsilon>$ & $\mathrm{d}_{\mathbf{N}_{L \varepsilon Z}}$ \\
\hline & $\mathrm{N}$ & $582 \cdot 0$ & $\Omega_{9 \varepsilon z}$ \\
\hline & $\mathrm{N}$ & $68 \mathrm{I}^{\circ} 0$ & $\mathrm{n}_{S E Z}$ \\
\hline & $\mathrm{W}$ & $\angle 8^{\circ} \mathrm{t}$ & $\Omega_{t \varepsilon \tau}$ \\
\hline & W & $8^{\circ} \varepsilon I$ & $\Omega_{\varepsilon \varepsilon \tau}$ \\
\hline & N & $6 S^{\circ} \varepsilon$ & $\Omega_{z \varepsilon z}$ \\
\hline mirmos & 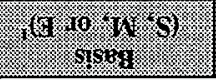 & 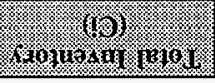 & -214า \\
\hline
\end{tabular}

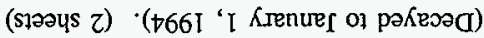

L66I "I $E$ KEW Jo Se EOI-NV-I TZ YireL

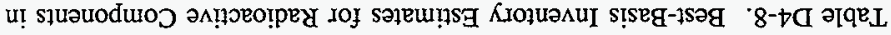




\section{D5.0 REFERENCES}

Agnew, S. F., J. Boyer, R. A. Corbin, T. B. Duran, J. R. FitzPatrick, K. A. Jurgensen, T. P. Ortiz, and B. L. Young, 1997, Hanford Tank Chemical and Radionuclide Inventories: HDW Model Rev. 4, LA-UR-96-3860, Los Alamos National Laboratory, Los Alamos, New Mexico.

Brevick, C. H., 1997, Historical Tank Content Estimate for the Southeast Quadrant of the Hanford 200 Areas, HNF-SD-WM-ER-350, Rev. 1, Fluor Daniel Northwest Inc., Richland, Washington.

Hanlon, B. M., 1997, Waste Tank Summary Report for Month Ending March 31, 1997., WHC-EP-0182-108, Rev. 0, Lockheed Martin Hanford Corp. for Fluor Daniel, Inc., Richland, Washington.

Hendrickson, D. W., 1994, Grout Treatment Facility Waste Feed Projections, WHC-SD-WM-TI-528, Westinghouse Hanford Company, Richland, Washington.

Hodgson, K. M., and M. D. LeClair, 1996, Work Plan for Defining a Standard Inventory Estimate for Wastes Stored in Hanford Site Underground Tanks, WHC-SD-WM-WP-311, Rev. 1, Lockheed Martin Hanford Corporation for Fluor Daniel Hanford, Inc., Richland, Washington.

Kupfer, M. J., A. L. Boldt, B. A. Higley, K. M. Hodgson, L. W. Shelton, R. A. Watrous, S. L. Lambert, D. E. Place, R. M. Orme, G. L. Borsheim, N. G. Colton, M. D. LeClair, R. T. Winward, and W. W. Schulz, 1997, Standard Inventories of Chemicals and Radionuclides in Hanford Site Tank Wastes, HNF-SD-WM-TI-740, Rev. 0, Lockheed Martin Hanford Corporation for Fluor Daniel Hanford, Inc., Richland, Washington.

Shekarriz, A., J. M. Bates, R. E. Bauer, N. S. Cannon, M. A. Chieda, B. E. Hey, C. G. Linschooten, L. A. Mahoney, D. R. Rector, E. R. Siciliano, Preliminary Retained Gas Sampler Measurement Results for Hanford Waste Tanks 241-AW-101, 241-A-101, 241-AN-105, 241-AN-104, and 241-AN-103, PNNL-11450, Pacific Northwest National Laboratory, Richland, Washington.

Stauffer, L. A., 1997, Solids Level Changes for Tanks 241-AN-103, 241-AN-104, 241-AN-105, 241-AW-101, and 241-SY-103, (internal memorandum 74330-97-105), Lockheed Martin Hanford Corporation, Richland, Washington.

Watrous, R. A., and D. W. Wootan, 1997, Activity of Fuel Batches Processed Through Hanford Separations Plants, 1944 Through 1989, HNF-SD-WM-TI-794, Rev. 0, Lockheed Martin Hanford Corporation for Fluor Daniel Hanford, Inc., Richland, Washington. 
HNF-SD-WM-ER-702 Rev. 0

APPENDIX E

BIBLIOGRAPHY FOR TANK 241-AN-103

E-1 
HNF-SD-WM-ER-702 Rev. 0

This page intentionally left blank. 


\section{APPENDIX E}

\section{BIBLIOGRAPHY FOR TANK 241-AN-103}

Appendix E provides a bibliography of information that supports the characterization of tank 241-AN-103. This bibliography represents an in-depth literature search of all known information sources that provide sampling, analysis, surveillance, and modeling information, as well as processing occurrences associated with tank $241-\mathrm{AN}-103$ and its respective waste types.

The references in this bibliography are separated into three broad categories containing references broken down into subgroups. These categories and their subgroups are listed below.

\section{NON-ANALYTICAL DATA}

Ia. Models/Waste Type Inventories/Campaign Information

Ib. Fill History/Waste Transfer Records

Ic. Surveillance/Tank Configuration

Id. Sample Planning/Tank Prioritization

Ie. Data Quality Objectives/Customers of Characterization Data

\section{U. ANALYTICAL DATA - SAMPLING OF TANK WASTE AND WASTE TYPES}

IIa. Sampling of tank 241-AN-103

IIb. Sampling of 242-A Evaporator Streams

\section{COMBINED ANALYTICAL/NON-ANALYTICAL DATA}

IIIa. Inventories using both Campaign and Analytical Information (Best Estimate)

IIIb. Compendium of Existing Physical and Chemical Documented Data Sources

This bibliography is broken down into the appropriate sections of material to use, with an annotation at the end of each reference describing the information source. Where possible, a reference is provided for information sources. A majority of the information listed below may be found in the Westinghouse Hanford Company Tank Characterization and Safety Resource Center. 


\section{NON-ANALYTICAL DATA}

Ia. Models/Waste Type Inventories/Campaign Information

Agnew, S. F., J. Boyer, R. A. Corbin, T. B. Duran, J. R. Fitzpatrick, K. A. Jurgensen, T. P. Ortiz, and B. L. Young, 1997, Hanford Tank Chemical and Radionuclide Inventories: HDW Model Rev. 4, LA-UR-96-3860, Rev. 0, Los Alamos National Laboratory, Los Alamos, New Mexico.

- Document contains tank layer and supernatant models and the Historical Tank Content Estimate for Hanford Site underground waste storage tanks, as well as a list of Hanford Site waste types.

Ib. Fill History/Waste Transfer Records

Agnew, S. F., R. A. Corbin, T. B. Duran, K. A. Jurgensen, T. P. Ortiz, and B. L. Young, 1997, Waste Status and Transaction Record Summary (WSTRS) Rev. 4, LA-UR-97-311, Rev. 0, Los Alamos National Laboratory, Los Alamos, New Mexico.

- Document contains spreadsheets depicting all available data on tank additions/transfers.

Koreski, G. M., and J. N. Strode, 1996, Operational Waste Volume Projections, WHC-SD-WM-ER-029, Rev. 22, Westinghouse Hanford Company, Richland, Washington.

- Document contains account of waste transfers for double-shell tanks, including waste type and volume, source, and destination.

Ic. Surveillance/Tank Configuration

Salazar, B. E., 1994, Double-Shell Underground Waste Storage Tank Riser Survey, WHC-SD-RE-TI-093, Rev. 4, Westinghouse Hanford Company, Richland, Washington.

- Document describing double-shell underground waste tank riser survey. 
Tran, T. T., 1993, Thermocouple Status Single Shell and Double Shell Waste Tanks, WHC-SD-WM-TI-553, Rev. 0, Westinghouse Hanford Company, Richland, Washington.

- Document contains information pertaining to thermocouple trees installed in the Hanford Site underground waste tanks, such as installation date, material condition, riser number, height of individual thermocouples above the bottom of the tank, high temperature reading, frequency of surveillance, and type of thermocouple.

Id. Sample Planning/Tank Prioritization

Bell, K. E., 1993, Tank Waste Remediation System Tank Waste Characterization Plan, WHC-SD-WM-PLN-047, Rev. 1, Westinghouse Hanford Company, Richland, Washington.

- Document coordinates the activities of the tank farms and the laboratories by establishing standard sample procurement and analysis procedures, standard quality control procedures and criteria, and prioritizing tank samples.

Brown, T. M., J. W. Hunt, and L. J. Fergestrom, 1997, Tank Characterization Technical Sampling Basis, HNF-SD-WM-TA-164, Rev. 3, Lockheed Martin Hanford Company, Richland, Washington.

- Document that summarizes the technical basis for characterizing the waste in the tanks and assigns a priority number to each tank.

Grimes, G. W., 1977, Hanford Long-Term Defense High-Level Waste Management Program Waste Sampling and Characterization Plan, RHO-CD-137, Rockwell Hanford Operations, Richland, Washington.

- Early characterization planning document.

Kruger, A. A., 1996, Tank 241-AN-103 Push Mode Core Sampling and Analysis Plan, WHC-SD-WM-TSAP-105, Rev. 0, Westinghouse Hanford Company, Richland, Washington.

- Document contains detailed sampling and analysis procedure information for tank 241-AN-103 based on applicable DQOs. 
Kruger, A. A., and W. D. Winkelman, 1996, Tank 241-AN-103 Tank Characterization Plan, WHC-SD-WM-SD-TP-383, Rev. 3, Lockheed Martin Hanford Corporation, Richland, Washington.

- Document discussed any and all relevant DQOs and how they will be met for tank 241-AN-103.

Mauss, B. M., 1987, 103-AN Core Samples: Proposed Experimental Design for Dissolution Study, (internal letter 6453-87-047 to D. G. Rodenhizer on April 8), Rockwell Hanford Operations, Richland, Washington.

- Contains historical data for tank 241-AN-103.

Mauss, B. M., 1987, Revised Experimental Design for 103-Dissolution Study, (internal letter 65453-97-063 to L. C. Stegen on May 8), Rockwell Hanford Operations, Richland, Washington.

- Contains historical data for tank 241-AN-103.

Richmond, W. G., 1987, Analysis of Composice Samples from Tank 103-AN, (external letter to A. P. Toste, PNL, on May 14), Rockwell Hanford Operations, Richland, Washington.

- Contains historical data for tank 241-AN-103.

Shekarriz, A., and J. M. Bates, 1996, Sampling Plan for Tank 241-AN-103 Retained Gas Sampler Deployment, TWSMIT:080190, Pacific Northwest National Laboratory, Richland, Washington.

- Letter report providing RGS requirements input for the 241-AN-103 TSAP.

Winkelman, W. D., J. W. Hunt, and L. J. Fergestrom, 1996, FY 1997 Tank Waste Analysis Plan, WHC-SD-WM-PLN-120, Rev. 1, Lockheed Martin Hanford Corporation for Fluor Daniel Hanford, Inc., Richland, Washington.

- Document contains Tri-Party Agreement (see Ecology et al. 1994 listing in Section 5.0) requirement-driven TWRS Characterization Program information and a list of tanks addressed in fiscal year 1997. 
Ie. Data Quality Objectives (DQO) and Customers of Characterization Data

Cash, R. J., 1996a, Application of "Flammable Gas Tank Safety Program: Data Requirements for Core Sampling Analysis Developed through the Data Quality Objectives Process" Rev. 2 (internal memorandum 79300-96-028 to S. J. Eberlein, July 12), Westinghouse Hanford Company, Richland, Washington.

- Contains flammable gas retained gas sampler requirements.

Cash, R. J., 1996b, Scope Increase of "Data Quality Objective to Support Resolution of the Organic Complexant Safety Issue" Rev. 2, (internal memorandum 79300-96-029 to S. J. Eberlein, July 12), Westinghouse Hanford Company, Richland, Washington.

- Memorandum contains interim requirements for the Organic Solvents issue.

DOE-RL, 1996, Recommendation 93-5 Implementation Plan, DOE/RL-94-0001, Rev. 1, U.S. Department of Energy, Richland, Washington.

- Organic solvent description in the 93-5 implementation Plan.

Dukelow, G. T., J. W. Hunt, H. Babad, and J. E. Meacham, 1995, Tank Safety Screening Data Quality Objective, WHC-SD-WM-SP-004, Rev. 2, Westinghouse Hanford Company, Richland, Washington.

- DQO used to determine if tanks are under safe operating conditions.

Jones, T. E., and K. D. Wiemers, 1996, Data Requirements for TWRS Privatization Characterization of Potential Low Activity Waste Feed, WHC-SD-WA-SP-023, Rev. 0, Westinghouse Hanford Company, Richland, Washington.

McDuffie, N. G., and G. D. Johnson, 1995, Flammable Gas Tank Safety Program: Data Requirements for Core Sample Analysis Developed Through the Data Quality Objectives (DQO) Process, WHC-SD-WM-DQO-004, Rev. 2, Westinghouse Hanford Company, Richland Washington.

- Document provides data needs for evaluating the flammability issues in the tank. 
Mulkey, C. H., and J. M. Jones, 1995, Double-Shell Tank System Waste Analysis Plan, WHC-SD-WM-EV-053, Rev. 3, Westinghouse Hanford Company, Richland, Washington.

- Document describing requirements for double-shell tank analyses based on the compatibility and safety screening DQOs.

\section{ANALYTICAL DATA - SAMPLING OF TANK WASTE AND WASTE TYPES}

IIa. Sampling of tank 241-AN-103

Fow, C. L., 1987, Characterization of Waste from Double-Shell Tank AN-103, 7W21-87-1, Pacific Northwest Laboratory, Richland, Washington.

- Letter report containing rheological measurements from three samples from the 1986 push core sample.

Harmon, H. D., 1991, Watch List for Tanks Which May Have Hydrogen Buildup, (letter 9001478B R1 to Gerton R.E., February 8, 1991), Westinghouse Hanford Company, Richland, Washington.

- Memorandum reports the results of the slurry growth study of actual waste.

Jansky, M. T., 1984, Laboratory Support for Tank Farm Samples from Tanks 103 and $104 A N$, (internal letter 6543-84-164 to P. J. Certa on July 2), Rockwell Hanford Operations, Richland, Washington.

Mauss, B. M., 1987, Laboratory Work to be Performed on AN-103 Core Sample, (internal memorandum 65453-87-024 to L. C: Stegen, February 17), Rockwell Hanford Operation, Richland, Washington.

- Memorandum contains plans for the analysis of the 1986 push core sample.

Mudge, K. L., 1987, Core Sample Results 103-AN, (internal memorandum 65950-87-019 to K. W. Owens, January 12), Rockwell Hanford Operation, Richland, Washington.

- Memorandum reports tank conditions during the 1986 core sampling. 
Prignano, A. L., 1988, Dissolution Study of Composite from Tank AN-103, (internal memorandum 12221-PCL88-060 to Distribution, January 13), Westinghouse Hanford Company, Richland, Washington.

- Letter reports dissolution results of a waste composite from the 1986 push core sample.

Shekarriz, A., R. E. Bauer, D. R. Rector, N. S. Cannon, L. A. Mahoney, B. E. Hey, M. A. Chieda, C. G. Linshooten, J. M. Bates, F. J. Reitz, and E. R. Siciliano, 1997, Composition and Quantities of Retained Gas Measured in Hanford Waste Tanks 241-AW-101, A-101, AN-105, $A N-104$, and $A N-103$, PNNL-11450, Rev. 1, Pacific Northwest National Laboratory, Richland, Washington.

- Document contains the measurements resulting from retained gas sampling tanks.

Steen, F. H., 1997, Tank 241-AN-103, Cores 166 and 167, Analytical Results for the Final Report, HNF-SD-WM-DP-223, Rev. 0, Westinghouse Hanford Company, Richland, Washington.

- Document contains sample analyses from 1996 tank 241-AN-103 core sampling event.

Stewart, C. W., J. M. Alzheimer, M. E. Brewster, G. Chen, R. E. Mendoza, H. C. Reid, C. L. Shepard, and G. Terrones, 1996, In Situ Rheology and Gas Volume in Hanford Double-Shell Waste Tanks, PNNL-11296, Pacific Northwest National Laboratory, Richland, Washington.

- Document presents the results from operating the ball rheometer and void fraction instrument in Hanford Site waste tank 241-AN-103 and the other Flammable Gas Watch List Double-Shell Tanks.

Toste, A. P., 1987, (untitled), (external memorandum to W. G. Richmond, September 25), Pacific Northwest Laboratory, Richland, Washington.

- Memorandum contains analytical data from the 1986 push core sample.

Wilkins, N. E., 1995, Results of Vapor Space Monitoring of Flammable Gas Watch List Tanks, WHC-SD-WM-TI-682, Rev. 0A, Westinghouse Hanford Company, Richland, Washington.

- Document describing gas monitoring of double-shell tanks. 
Wilkins, N. E., R. E. Bauer, and D. M. Ogden, 1997, Results of Vapor Space Monitoring of Flammable Gas Watch List Tanks, HNF-SD-WM-TI-797, Rev. 1, Lockheed Martin Hanford Corporation for Fluor Daniel Hanford, Inc., Richland, Washington.

- Document contains results of Standard Hydrogen Monitoring Systems, vapor grab sampling, and gas characterization system monitoring of tank 241-AN-103 as well as other Flammable Gas Watch List tanks.

IIb. Sampling of 242-A Evaporator Streams

Certa, P. J., 1985, 242-A Evaporator/Crystallizer FY-84 Campaign Run 84-3 Post Run Document, RHO-SD-WM-PE-018, Rockwell Hanford Operation, Richland, Washington.

- Document describing evaporator run campaign 84-3.

Kelly, M. G., 1986, 242-A Evaporator/Crystallizer FY-1986 Campaign Run 86-2 Post-Run Document, RHO-WD-WM-PE-028, Rockwell Hanford Operation, Richland, Washington.

- Document describing evaporator run campaign 86-2.

Mauss, B. M., 1985a, The 86-2 Evaporator Campaign: Laboratory Results on the Production of Double-Shell Slurry, (internal memorandum 65453-86-038 to M. G. Kelly, March 28), Rockwell Hanford Operation, Richland, Washington.

- Memorandum containing analytical results of tank 241-103-AN sludge and evaporator process samples.

Mauss, B. M., and M. T. Jansky, 1985, 102-AW Laboratory Boildowns in Support of Evaporator Run 84-5, (internal letter 65453-85-013 to E. G. Gratney and N. L. Pontions, January 18), Rockwell Hanford Operations, Richland, Washington.

- Contains historical data for tank 241-AN-103.

Mauss, B. M., 1985b, Laboratory Support of 242-A Evaporator Run 85-2, (internal letter 65463-85-018 to N. L. Pontions, January 25), Rockwell Hanford Operations, Richland, Washington.

- Contains historical data for tank 241-AN-103. 
Pontions, N. L, 1985, 242-A Evaporator/Crystallizer FY 1985 Campaign Run 85-2 Post Run Document, RHO-SD-WM-PL-020, Rockwell Hanford Operations, Richland, Washington.

- Contains historical data for tank 241-AN-103.

\section{COMBINED ANALYTICAL/NON-ANALYTICAL DATA}

IIIa. Inventories from Campaign and Analytical Information

Agnew, S. F., J. Boyer, R. A. Corbin, T. B. Duran, J. R. Fitzpatrick, K. A. Jurgensen, T. P. Ortiz, and B. L. Young, 1997, Hanford Tank Chemical and Radionuclide Inventories: HDW Rev. 4, LA-UR-96-3860, Rev. 0, Los Alamos National Laboratory, Los Alamos, New Mexico.

- Document contains waste type summaries as well as primary chemical compound/analyte and radionuclide estimates for sludge, supernatant, and solids.

Schmittroth, F. A., Inventories for Low-Level Tank Waste, WHC-SD-WM-RPT-164, Rev. 0, Westinghouse Hanford Company, Richland, Washington.

- Document contains a global inventory based on process knowledge and radioactive decay estimations using ORIGEN2. Pu and $U$ waste contribution are taken at 1 percent of the amount used in processes. Also compares information on Tc-99 from both ORIGEN2 and analytical data.

IIIb. Compendium of data from other sources physical and chemical

Agnew, S. F. and J. G. Watkin, 1994, Estimation of Limiting Solubilities for Ionic Species in Hanford Waste Tank Supernates, LAUR-94-3590, Los Alamos National Laboratory, Los Alamos, New Mexico.

- Document gives solubility ranges used for key chemical and radionuclide components based on supernatant sample analyses. 
Brevick, C. H., L. A. Gaddis, and E. D. Johnson, 1995, Tank Waste Source Term Inventory Validation, Vol I \& II., WHC-SD-WM-ER-400, Rev. 0, Westinghouse Hanford Company, Richland, Washington.

- Document contains a quick reference to sampling information in spreadsheet or graphical form for 23 chemicals and 11 radionuclides for. all the tanks.

Brevick, C. H., J. L. Stroup, and J. W. Funk, Historical Tank Content Estimate for the Southeast Quadrant of the Hanford 200 Areas, HNF-SD-WM-ER-350, Rev. 1, Fluor Daniel Northwest, Inc. for Fluor Daniel Hanford, Inc., Richland, Washington.

- Document contain summary information from the supporting document for Tank Farms AN, AP, AW, AXY, AZ, and SY as well as in-tank photo collages and the total inventory and supernatant composite inventory estimates.

Brevick, C. H., J. L. Stroup, and J. W. Funk, 1997, Supporting Document for the Southeast Quadrant Historical Tank Farm Estimate for AN Tank Farm, HNF-SD-WM-ER-314, Rev, 1, Fluor Daniel Northwest, Inc. for Fluor Daniel Hanford, Inc., Richland, Washington.

- Document contains descriptions of riser locations and tank construction for the AN Tank Farm. It also contains descriptions of the process and temperature histories as well as the results from past sampling events.

Hall, B., 1990, Toxic Metals Concentrations of DSS and CC Containing Tanks, DSI to D. D. Stepnewski on May 16, Westinghouse Hanford Company, Richland, Washington.

- Contains toxic metal data for tank 241-AN-103.

Hanlon B. M., 1997, Waste Tank Summary Report for Month Ending March 31, 1997, HNF-EP-0182-108, Rev. 0, Lockheed Martin Hanford Corporation for Fluor Daniel Hanford, Inc., Richland, Washington.

- Document summarizing types and amounts of wastes in Hanford Site tanks. 
Husa, E. I., 1993, Hanford Site Waste Storage Tank Information Notebook, WHC-EP-0625, Westinghouse Hanford Company, Richland, Washington.

- Document contains in-tank photos as well as summaries on the tank description, leak detection system, and tank status.

Husa, E. I., 1995, Hanford Waste Tank Preliminary Dryness Evaluation, WHC-SD-WM-TI-703, Rev. 0, Westinghouse Hanford Company, Richland, Washington.

- Document gives assessment of relative dryness between tanks.

Olson, N. J., 1989, Electrochemical Testing A537 Carbon Steel Purex Scrub Solutions For Corrosion Behavior-Preliminary Report, (letter LET-011689 to D. A. Reynolds, January 16), Pacific Northwest - National Laboratory, Richland; Washington.

- Letter presents the results from corrosion testing with scrub solutions for corrosion behavior.

Prignano, A. L., 1987, Dissolution Properties of Composite From Tank 1.03-AN, RHO-SD-RE-TP-016, Rockwell Hanford Operation, Richland, Washington.

- Contains historical data for tank 241-AN-103.

Prignano, A. L., 1988, Dissolution Study of Composite for Tank 103-AN, (internal letter 12221-PCL88-060 to Distribution on January 13), Westinghouse Hanford Corporation, Richland, Washington.

- Contains historical data for tank 241-AN-103.

Shelton, L. W., 1995a, Chemical and Radionclide Inventory for Single-and Double-Shell Tanks, (internal memorandum 74A20-96-30 to D. J. Washenfelder, February 28), Westinghouse Hanford Company, Richland, Washington.

- Contains historical data for tank 241-AN-103. 
Shelton, L. W., 1995b, Chemical and Radionclide Inventory for Single- and Double-Shell Tanks, (internal memorandum 75520-95007 to R. M. Orme, August 8), Westinghouse Hanford Company, Richland, Washington.

- Memorandum contains a tank inventory estimate based on analytical information.

Shelton, L. W., 1995c, Radionuclide Inventories for Single- and Double-Shell Tanks, (internal memorandum 72320-95-002 to F. M. Cooney, February 14), Westinghouse Hanford Company, Richland, Washington.

- Memorandum contains an tank inventory estimate based on analytical information.

Van Vleet, R. J., 1993, Radionuclide and Chemical Inventories for Double-Shell Tanks, WHC-SD-WM-TI-543, Rev. 1, Westinghouse Hanford Company, Richland, Washington.

- Report containing radionuclide chemical inventories for double-shell tanks. 
DISTRIBUTION SHEET

\begin{tabular}{|c|c|c|c|c|c|}
\hline \multirow[b]{2}{*}{ Distribution } & \multirow{2}{*}{\multicolumn{3}{|c|}{ From }} & \multicolumn{2}{|l|}{ Page 1 of 2} \\
\hline & & & & \multicolumn{2}{|c|}{ Date $\quad 05 / 08 / 97$} \\
\hline \multicolumn{4}{|c|}{ Project Title/Work Order } & \multicolumn{2}{|c|}{ EDT No. EDT-617663 } \\
\hline \multicolumn{4}{|c|}{$\begin{array}{l}\text { Tank Characterization Report for Double-She11 Tank 241-AN-103, } \\
\text { HNF-SD-WM-ER-702, Rev. } 0\end{array}$} & \multicolumn{2}{|c|}{ ECN No. N/A } \\
\hline Name & MSIN & $\begin{array}{c}\text { Text } \\
\text { With All } \\
\text { Attach. }\end{array}$ & Text Only & $\begin{array}{l}\text { Attach./ } \\
\text { Appendix } \\
\text { Only }\end{array}$ & $\begin{array}{l}\text { EDT/ECN } \\
\text { Only }\end{array}$ \\
\hline
\end{tabular}

OFFSITE

Sandia Nationa 7 Laboratory

P.0. Box 5800

MS-0744, Dept. 6404

Albuquerque, NM 87815

D. Powers

Nuclear Consulting Services Inc.

P. 0. Box 29151

Columbus, OH 43229-01051

J. L. Kovach

$x$

Chemical Reaction Sub-TAP

P.0. Box 271

Lindsborg, KS 67456

B. C. Hudson

SAIC

20300 Century Boulevard. Suite 200-B

Germantown. MD 20874

H. Sutter

$x$

Los A7 amos Laboratory

CST-14 MS-J586

P. 0. BoX 1663

Los Alamos, NM 87545

S. F. Agnew

Los Alamos Technical Associates

T. T. Tran

B1-44 X

Tank Advisory Panel

102 Windham Road

Dak Ridge, TN 37830

D. 0. Campbe11

$x$ 


\section{DISTRIBUTION SHEET}

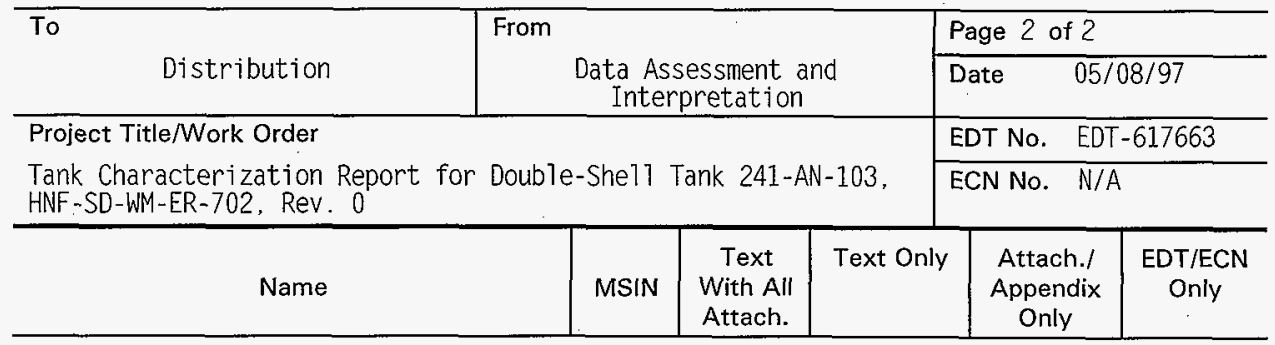

\section{ONSITE}

Department of Energy - Richland Operations
J. F. Thompson
W. S. Liou
J. A. Poppiti
N. W. Willis

K. M. Hodgson

Lockheed Martin Services, Inc.

B. G. Lauzon
Central Files
EDMC

Numatec Hanford Corporation
J. S. Garfield
J. S. Hertzel
R. A. Kirkbride
D. L. Lamberd

Pacific Northwest Nationa 1 Laboratory A. F. Noonan

K9-91 X

Rust Federal Services of Hanford. Inc. C. T. Narquis
$57-54$

$57-54$

$57-54$

S7-54

S7-14

R2-54

A2-34

S7-14

S7-14

S3-09

H0-34

S7-21

R2-12

R2-12

R2-12

R2-11

RI-5I

R1-10

R1-08

A3-88

H6-08

H5- 49

H5- 61

H5-27

H5-61 $x$
$X$
$x$
$x$

$x$
$x$
$x$
$x$
$x$
$x$
$x$
$x$
$x$

$x$
$X$
$X$
$X$
$x$

$x$

$x$
$x$
$x$
$x$
$x$
$x$
$x$
5

$x$
$X$
$X$

T6-16 $x$ 